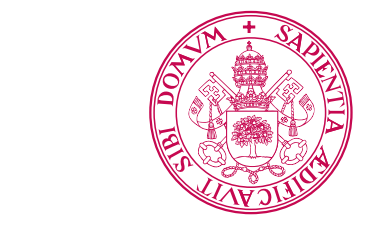

Universidad deValladolid

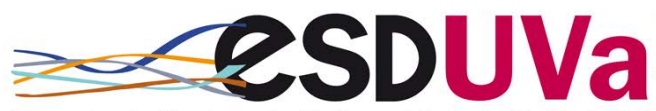

PROGRAMA DE DOCTORADO EN QUÍMICA: QUÍMICA DE SÍNTESIS, CATÁLISIS Y MATERIALES AVANZADOS

TESIS DOCTORAL:

\title{
ESTUDIO DE MÁQUINAS MOLECULARES Y DERIVADOS POLIAROMÁTICOS CON CAPACIDAD DE ESTABLECER INTERACCIONES SUPRAMOLECULARES
}

Presentada por Héctor Barbero San Juan para optar al grado de Doctor por la Universidad de Valladolid

Dirigida por:

Dr. Celedonio Manuel Álvarez González

Dr. Daniel Miguel San José 



\section{FINANCIACIÓN}
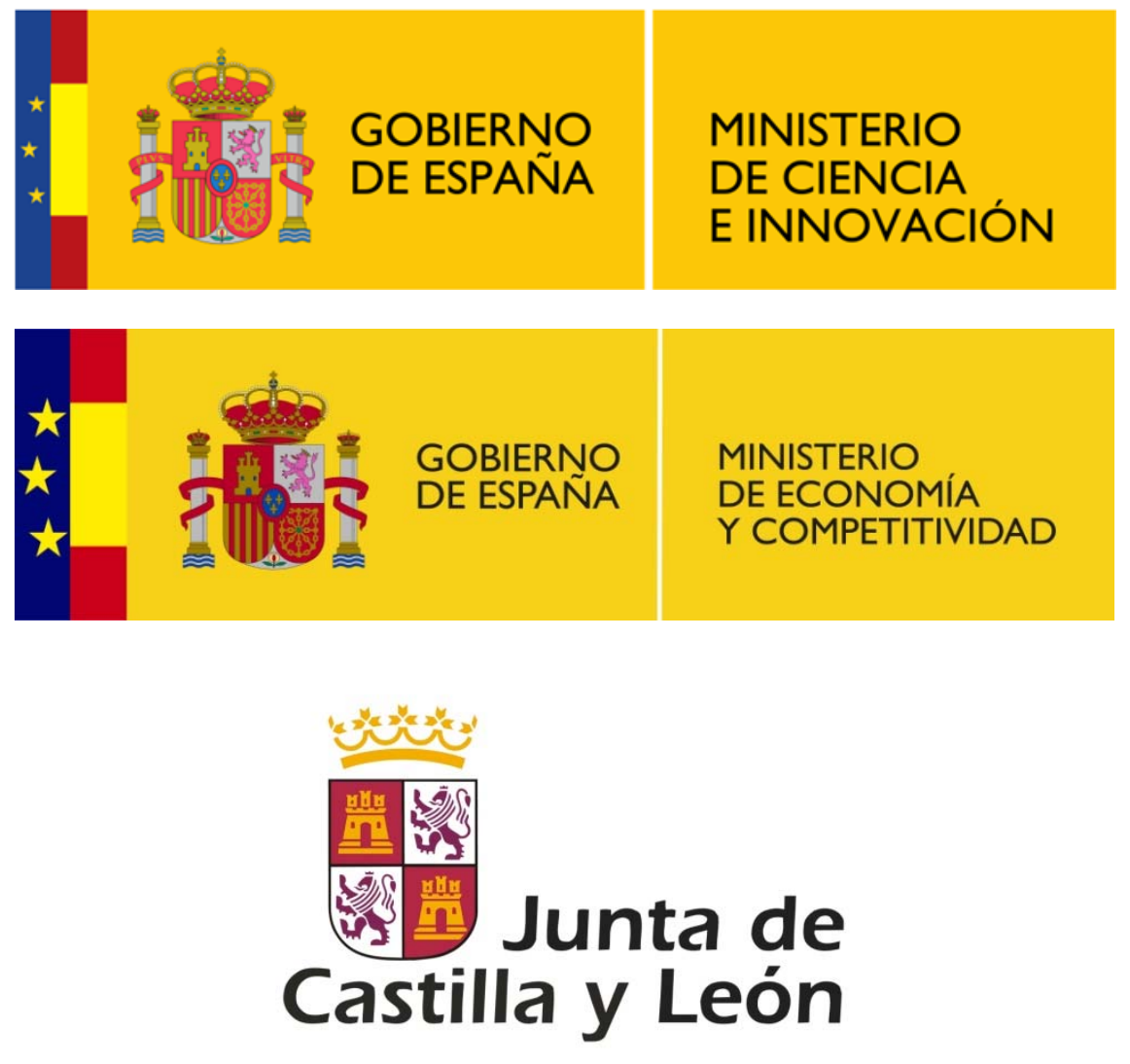

La presente Tesis Doctoral titulada "Estudio de máquinas moleculares y derivados poliaromáticos con capacidad de establecer interacciones supramoleculares" ha sido realizada gracias al apoyo económico del Ministerio de Ciencia e Innovación (proyecto CTQ2009-12111 y beca FPI durante mi formación), al Ministerio de Economía y Competitividad (proyecto CTQ2013-41067-P) y a la Junta de Castilla y León (GR Excelencia 125). 



\section{TESIS PRESENTADA COMO COMPENDIO DE PUBLICACIONES}

De acuerdo con la nueva normativa de la Universidad de Valladolid aprobada por el Consejo de Gobierno el 3 de junio de 2016 (BOCyL n 114, de 15 de junio, págs. 26299-26310), esta memoria está formada por los apartados exigidos en la misma y se presentan cuatro artículos, de los cuales tres han sido publicados, que cuentan con los requisitos necesarios y se listan a continuación (por el orden en el que posteriormente serán tratados) ${ }^{1}$ :

Artículo I: Multivalent Molecular Shuttles - Effect of Increasing the Number of Centers in Switchable Catalysts

Publicado en European Journal of Organic Chemistry: Álvarez, C. M.; Barbero, H.; Miguel, D. Eur. J. Org. Chem. 2015, 2015, 6631-6640. DOI: 10.1002/ejoc. 201500942

Artículo II: Study of $p H$-sensitive bistable molecular shuttles incorporating azobenzene as a kinetic barrier for photochemically triggered memory switching

Se enviará próximamente a Organic Chemistry Frontiers

Artículo III: Affinity modulation of photoresponsive hosts for fullerenes: light-gated corannulene tweezers

Publicado en Chemical Communications: Barbero, H.; Ferrero, S.; Álvarez-Miguel, L.; Gómez-Iglesias, P.; Miguel, D.; Álvarez, C. M.; Chem Commun. 2016, 52, 12964-12967. DOI: $10.1039 / \mathrm{c} 6 \mathrm{cc} 06445 \mathrm{k}$

Artículo IV: Synergistic Effect of Tetraaryl Porphyrins Containing Corannulene and Other Polycyclic Aromatic Fragments as Hosts for Fullerenes. Impact of $C_{60}$ in a Statistically Distributed Mixture of Atropisomers

Publicado en Journal of Organic Chemistry: Álvarez, C. M.; Barbero, H.; Ferrero, S.; Miguel, D. J. Org. Chem. 2016, 81, 6081-6086. DOI: 10.1021/acs.joc.6b00454

\section{MENCIÓN DE DOCTORADO INTERNACIONAL}

La presente memoria pretende obtener la mención de Doctorado Internacional. Para ello, esta tesis incluye toda la documentación requerida. Además, los apartados Índice, Resumen General (págs. 1-6) y Conclusiones (págs. 107-112), así como todos los Artículos (págs. 113-148) están redactados en inglés.

Debido al gran tamaño que este documento tendría si se incluyera el material suplementario de cada publicación, se ha decidido prescindir de todos ellos y reunirlos en un fichero electrónico por separado. Adicionalmente, se podrán encontrar en la página web correspondiente a la editorial de cada artículo ya publicado porque son de acceso libre. 



\section{LISTA DE ABREVIATURAS EMPLEADAS}

$-{ }^{1} \mathrm{H}$

$-{ }^{13} \mathrm{C}$

- AcOEt

- $\mathrm{AcOK}$

- $\mathrm{B}_{2} \mathrm{pin}_{2}$

- Boc

$-b r$

- BSSE

- CCD

- COSY

- CuAAC

$-d$

- DB24C8

- DCE

- DCM

- DFT

- DIAD

- dppf

- DQF COSY

- EI

- ESI

- Et

- $\mathrm{EtOH}$

- GC

- GIAO

- $\mathrm{HAcO}$

- HMBC

- HRMS

- HSQC

$-\mathrm{Hz}$

- $J$

$-K_{a}$

Protón

Carbono 13

Acetato de etilo

Acetato potásico

Diborano de bis-pinacolato

terc-Butiloxicarbonilo

Señal ancha (en RMN)

Error de Superposición de Bases (Basis Set Superposition Error)

Dispositivo de Carga Acoplada (Charge-Coupled Device)

Espectroscopía de Correlación (COrrelation SpectroscopY)

Cicloadición Azida-Alquino catalizada por Cobre(I) (Copper (I)catalyzed Azide-Alkyne Cycloaddition)

Doblete (en RMN)

Éter dibenzo[24]corona-8

Dicloroetano

Diclorometano

Teoría del Funcional de Densidad (Density Functional Theory)

Azodicarboxilato de diisopropilo

1,1'-Bis(difenilfosfino)ferroceno

COSY Filtrado a Doble Cuanto (Double Quantum Filtered COSY)

Ionización por Impacto Electrónico (Electron Impact ionization)

Ionización por Electrospray (ElectroSpray Ionization)

Etilo

Etanol

Cromatografía de Gases (Gas Chromatography)

Orbital Atómico Independiente de Medida (Gauge-Independent Atomic Orbital)

Ácido acético

Correlación Heteronuclear de Múltiple Enlace (Heteronuclear Multiple Bond Correlation)

Espectrometría de Masas de Alta Resolución (High-Resolution Mass Spectrometry)

Coherencia (o Correlación) Heteronuclear a Simple Cuanto (Heteronuclear Single Quantum Coherence)

Hercio

Constante de acoplamiento (en RMN)

Constante de asociación 


\begin{tabular}{|c|c|}
\hline - LED & Diodo Emisor de Luz (Light-Emitting Diode) \\
\hline - LRMS & $\begin{array}{l}\text { Espectrometría de Masas de Baja Resolución (Low-Resolution Mass } \\
\text { Spectrometry) }\end{array}$ \\
\hline$-\mathrm{m}$ & Multiplete (en RMN) \\
\hline - MALDI & $\begin{array}{l}\text { Desorción e Ionización por Láser Asistido por Matriz (Matrix-Assisted } \\
\text { Laser Desorption/Ionization) }\end{array}$ \\
\hline - MIM & $\begin{array}{l}\text { Molécula Entrelazada Mecánicamente (Mechanically Interlocked } \\
\text { Molecule) }\end{array}$ \\
\hline - Me & Metilo \\
\hline - $\mathrm{MeOH}$ & Metanol \\
\hline$-\mathrm{Me}_{3} \mathrm{OBF}_{4}$ & Tetrafluoroborato de trimetiloxonio \\
\hline - Ms & Mesilo (metilsulfonilo) \\
\hline$-\mathrm{MsCl}$ & Cloruro de mesilo (cloruro de metilsulfonilo) \\
\hline - MS & Espectrometría de Masas (Mass Spectrometry) \\
\hline$-\mathrm{MW}$ & Microondas (MicroWave) \\
\hline - NBS & N-bromosuccinimida \\
\hline$-\mathrm{NEt}_{3}$ & Tretilamina \\
\hline - NOESY & $\begin{array}{l}\text { Espectroscopía de Efecto Nuclear Overhauser (Nuclear Overhauser } \\
\text { Effect SpectroscopY) }\end{array}$ \\
\hline - PAH & $\begin{array}{l}\text { Hidrocarburo Policíclico } \\
\text { Hydrocarbon) }\end{array} \quad$ Aromático $\quad$ (Polycyclic $\quad$ Aromatic \\
\hline - PCM & Modelo de Continuo Polarizable (Polarizable Continuum Model) \\
\hline - ppm & Partes por millón (en RMN) \\
\hline$-\mathrm{R}$ & Resto orgánico \\
\hline - RGB & Colores primarios de la luz: rojo, verde y azul (Red Green Blue) \\
\hline - RMN & Resonancia Magnética Nuclear (en inglés, NMR) \\
\hline - ROESY & $\begin{array}{l}\text { Espectroscopía de Efecto nuclear Overhauser en Marco Rotatorio } \\
\text { (Rotating frame nuclear Overhauser Effect SpectroscopY) }\end{array}$ \\
\hline$-\mathrm{s}$ & Singlete (en RMN) \\
\hline - SCRF & Campo de Reacción Auto Consistente (Self-Consistent Reaction Field) \\
\hline$-{ }^{\mathrm{t}} \mathrm{BuONa}$ & terc-Butóxido sódico \\
\hline$-\mathrm{t}$ & Triplete (en RMN) \\
\hline - TFA & Ácido trifluoroacético \\
\hline - THF & Tetrahidrofurano \\
\hline - TLC & Cromatografía en capa fina (Thin Layer Chromatography) \\
\hline - TMS & Tetrametilsilano \\
\hline - TOCSY & Espectroscopía de Correlación Total (TOtal Correlation SpectroscopY) \\
\hline - TOF & Tiempo de Vuelo (Time Of Flight) \\
\hline - UPLC & $\begin{array}{l}\text { Cromatografía Líquida de Ultra Alto Rendimiento (Ultra-Performance } \\
\text { Liquid Chromatography) }\end{array}$ \\
\hline
\end{tabular}



- UV
Ultravioleta
- UV/Vis
Ultravioleta y Visible
- $\mathrm{Zn}(\mathrm{AcO})_{2}$
Acetato de zinc 


\section{NOTA SOBRE LA NUMERACIÓN DE LOS COMPUESTOS}

Debido al hecho de que esta memoria está construida en base a un conjunto de artículos aislados, los números de los compuestos sólo están correlacionados dentro de cada trabajo y, por tanto, sería complicado hacer referencia a ellos en esta tesis que los unifica si se usara tal numeración. Además, hay algunos compuestos comunes a dos publicaciones con números distintos, complicando aún más la identificación. Para solventar estos problemas, se ha llegado a la solución de poner una numeración doble de acuerdo con el siguiente criterio:

\section{$\mathbf{A}(\mathbf{X} / \mathbf{Y})$}

Donde:

A es el número asignado en esta tesis por orden ascendente a medida que aparecen en la parte donde se exponen los resultados obtenidos.

$\mathbf{X}$ e $\mathbf{Y}$ son los números asignados en el artículo del que proceden. Normalmente se tratará de una única cifra y, sólo en el caso en el que aparezca en dos publicaciones, tendrá dos números separados por una barra.

Además, en el bloque donde se discuten los compuestos funcionalizados con grupos poliaromáticos, la designación de los aductos supramoleculares se ha realizado de la siguiente manera:

\section{$\mathbf{A} @ \mathbf{B}$}

Donde:

$\mathrm{A}$ es $\mathrm{C}_{60}$ o $\mathrm{C}_{70}$.

B es la molécula capaz de establecer interacción con el fullereno. 


\section{ÍNDICE}

1. RESUMEN GENERAL 1

1.1. GENERAL SUMMARY

2. INTRODUCCIÓN 7

2.1. MOLÉCULAS ENTRELAZADAS MECÁNICAMENTE Y TOPOLOGÍA MOLECULAR 10

2.1.1. El enlace mecánico 11

2.1.2. Tipos de moléculas entrelazadas mecánicamente 12

2.1.2.1. Catenanos 12

2.1.2.2. Rotaxanos 12

2.1.2.3. Nudos 13

2.1.2.4.

Anillos de Salomón 13

2.1.2.5.

Anillos de Borromeo 13

2.1.2.6.

Suitanos 14

2.2. ROTAXANOS 14

2.2.1. Síntesis de rotaxanos 17

2.2.2. Interruptores moleculares basados en rotaxanos 20

2.2.2.1. Interruptores moleculares como catalizadores conmutables 22

2.3. DERIVADOS DE AZOBENCENO 24

2.3.1. Fotoisomerización reversible del azobenceno 25

2.3.2. Integración en moléculas entrelazadas mecánicamente 26

2.4. RECEPTORES DE FULLERENOS BASADOS EN MOLÉCULAS POLIAROMÁTICAS 27

2.4.1. Fullerenos y otros compuestos de carbono nanoestructurados 27

2.4.2. Coranuleno y otras moléculas poliaromáticas 29

2.4.2.1. Síntesis de coranuleno 31

2.4.2.2. Interacción de coranuleno con fullerenos __ 32

2.4.2.3. Pinzas moleculares de fullerenos que contienen unidades coranulénicas _ 32

3. OBJETIVOS 41

3.1. OBJETIVOS GENERALES 43

3.2. OBJETIVOS ESPECÍFICOS 43

4. METODOLOGÍA EMPLEADA 45

4.1. METODOLOGÍA SINTÉTICA 47

4.1.1. Métodos de purificación 47 
4.2.1. Espectroscopía de Resonancia Magnética Nuclear 48

4.2.2. Espectrometría de Masas 49

4.2.3. Espectroscopía de Absorción UV/Vis 49

4.2.4. Difracción de Rayos X de Monocristal 49

4.3. ESTUDIOS COMPUTACIONALES 50

4.4. EVALUACIÓN DE PROPIEDADES 52

4.4.1. Conmutación entre isómeros 52

4.4.1.1. Conmutación entre dos estaciones de un interruptor molecular de tipo rotaxano 52

4.4.1.2. Fotoisomerización de derivados de azobenceno 52

4.4.2. Estudio catalítico de rotaxanos 53

4.4.3. Método de variaciones continuas (Job Plots) 53

4.4.4. Estimación de constantes de asociación 53

5. RESULTADOS OBTENIDOS 55

5.1. ROTAXANOS E INTERRUPTORES MOLECULARES

5.1.1. Artículo I. Multivalent Molecular Shuttles - Effect of Increasing the Number of Centers in Switchable Catalysts

5.1.2. Artículo II. Study of $\mathrm{pH}$-sensitive bistable molecular shuttles incorporating azobenzene as a kinetic barrier for photochemically triggered memory switching 72

5.2. COMPUESTOS POLIAROMÁTICOS PLANOS Y NO PLANOS 83

5.2.1. Artículo III. Affinity modulation of photoresponsive hosts for fullerenes: light-gated corannulene tweezers

5.2.2. Artículo IV. Synergistic Effect of Tetraaryl Porphyrins Containing Corannulene and Other Polycyclic Aromatic Fragments as Host for Fullerenes. Impact of $C_{60}$ in a Statistically Distributed Mixture of Atropisomers 94

6. CONCLUSIONES 107

6.1. CONCLUSIONES GENERALES 109

6.2. CONCLUSIONES ESPECÍFICAS 109

6.3. CONCLUSIONS 111

6.3.1. GENERAL CONCLUSIONS 111

6.3.2. SPECIFIC CONCLUSIONS 111

7. ARTÍ́CULOS 113

7.1. Multivalent Molecular Shuttles - Effect of Increasing the Number of Centers in Switchable Catalysts 
7.2. Study of $\mathbf{p H}$-sensitive bistable molecular shuttles incorporating azobenzene as a kinetic barrier for photochemically triggered memory switching

7.3. Affinity modulation of photoresponsive hosts for fullerenes: light-gated corannulene tweezers

7.4. Synergistic Effect of Tetraaryl Porphyrins Containing Corannulene and Other Polycyclic Aromatic Fragments as Hosts for Fullerenes. Impact of $\mathrm{C}_{60}$ in a Statistically Distributed Mixture of Atropisomers 143

8. BIBLIOGRAFÍA 149 



\section{INDEX}

1. GENERAL SUMMARY (Spanish)___ 1

1.1. GENERAL SUMMARY (English)__ 5

2. INTRODUCTION — 7

2.1. MECHANICALLY INTERLOCKED MOLECULES AND MOLECULAR TOPOLOGY

2.1.1. The Mechanical Bond___ 11

2.1.2. Types of mechanically interlocked molecules___ 12

2.1.2.1. Catenanes__ 12

2.1.2.2. Rotaxanes___ 12

2.1.2.3. Knots__ 13

2.1.2.4. Solomon Rings ___ 13

2.1.2.5. Borromean Rings ___ 13

2.1.2.6. Suitanes

2.2. ROTAXANES

2.2.1. Rotaxanes synthesis___ 17

2.2.2. Rotaxane-based molecular shuttles__ 20

2.2.2.1. Molecular shuttles as switchable catalysts___

2.3. AZOBENZENE DERIVATIVES _ 24

2.3.1. Reversible photoisomerization of azobenzene _ 25

2.3.2. Integration in mechanically interlocked molecules___ 26

2.4. FULLERENE RECEPTORS BASED ON POLYAROMATIC MOLECULES __ 27

2.4.1. Fullerenes and other nanostructured carbon compounds__ 27

2.4.2. Corannulene and other polyaromatic molecules _ 29

2.4.2.1. Corannulene synthesis___ 31

2.4.2.2. Interaction between corannulene and fullerene _ 32

2.4.2.3. Corannulene-containing molecular tweezers as hosts for fullerenes ___ 32

3. OBJECTIVES _ 41

3.1. GENERAL OBJECTIVES___ 43

3.2. SPECIFIC OBJECTIVES___ 43

4. METHODOLOGY USED___ 45

4.1. SYNTHETICMETHODOLOGY___ 47

4.1.1. Purification methods __ 47 
4.2.1. Nuclear Magnetic Resonance Spectroscopy

4.2.2. Mass Spectrometry 49

4.2.3. UV/Vis absorption spectroscopy 49

4.2.4. Single cristal $X$ ray diffraction 49

4.3. COMPUTATIONAL STUDIES 50

4.4. PROPERTIES ASSESSMENT

4.4.1. Isomers switching 52

4.4.1.1. Switching between two stations in a rotaxane-based molecular shuttle 52

4.4.1.2. Azobenzene derivatives photoisomerization 52

4.4.2. Catalytic studies of rotaxanes 53

4.4.3. Continuous variations method (Job Plots)

4.4.4. Association constants estimation

5. OBTAINED RESULTS

\subsection{ROTAXANES AND MOLECULAR SHUTTLES}

5.1.1. Article I. Multivalent Molecular Shuttles - Effect of Increasing the Number of Centers in Switchable Catalysts

5.1.2. Article II. Study of $\mathrm{pH}$-sensitive bistable molecular shuttles incorporating azobenzene as a kinetic barrier for photochemically triggered memory switching

5.2.1. Article III. Affinity modulation of photoresponsive hosts for fullerenes: light-gated corannulene tweezers

5.2.2. Article IV. Synergistic Effect of Tetraaryl Porphyrins Containing Corannulene and Other Polycyclic Aromatic Fragments as Host for Fullerenes. Impact of $C_{60}$ in a Statistically Distributed Mixture of Atropisomers

6. CONCLUSIONS 107

6.1. GENERAL CONCLUSIONS (Spanish) 109

6.2. SPECIFIC CONCLUSIONS (Spanish) 109

6.3. CONCLUSIONS (English)

7.1. Multivalent Molecular Shuttles - Effect of Increasing the Number of Centers in Switchable Catalysts 
7.2. Study of pH-sensitive bistable molecular shuttles incorporating azobenzene as a kinetic barrier for photochemically triggered memory switching

7.3. Affinity modulation of photoresponsive hosts for fullerenes: light-gated corannulene tweezers

7.4. Synergistic Effect of Tetraaryl Porphyrins Containing Corannulene and Other Polycyclic Aromatic Fragments as Hosts for Fullerenes. Impact of $\mathrm{C}_{60}$ in a Statistically Distributed Mixture of Atropisomers 143

8. BIBLIOGRAPHY 

1. RESUMEN GENERAL 



\section{RESUMEN GENERAL}

El conjunto de trabajos presentados en esta memoria engloba la preparación de diversas familias de compuestos capaces de establecer interacciones supramoleculares, su caracterización y el estudio de sus propiedades en base a la habilidad de estas moléculas de conmutar entre isómeros, de realizar catálisis y/o de formar aductos dominados por fuerzas de dispersión.

En el primer bloque (Artículos I y II) se describirán una serie compuestos denominados rotaxanos, cuya interacción supramolecular entre los fragmentos que los constituyen se debe a enlaces de hidrógeno y fuerzas ion-dipolo y es posible conmutar entre ambas situaciones debido a la especial estructura de estas moléculas. Constituyen una vía nueva de investigación abierta en el grupo de investigación en el que me encuentro.

Así, en el Artículo I, se presentan tres rotaxanos homólogos cuya principal diferencia se debe al número de estaciones presentes y, consecuentemente, el fragmento "stopper" utilizado. Junto con ellos, se describen también otras tres moléculas que carecen del macrociclo necesario para formar una estructura entrelazada y, por tanto, la conmutación entre estaciones desaparece. Una de las partes de estas moléculas es considerada un centro con habilidades para catalizar diversas reacciones de tipo Michael vía catión iminio y se ha explotado esta capacidad, junto con la de conmutación entre estaciones.

En el Artículo II se presenta otra familia de rotaxanos, con estructuras similares a las de la publicación anterior, a la que se ha intercalado un fragmento azobenceno con una gran capacidad para fotoisomerizarse reversiblemente y se ha estudiado cómo influye este grupo en la conmutación entre estaciones.

Paralelamente, en el segundo bloque (Artículos III y IV) se amplía la familia de compuestos derivados de hidrocarburos policíclicos aromáticos de la línea de investigación iniciada con anterioridad en el grupo de investigación. La principal característica de ellos es que pueden formar aductos supramoleculares con fullerenos, principalmente las que contienen el fragmento coranuleno, cuya especial estructura tridimensional alejada de la planaridad es complementaria.

En el Artículo III se describe una familia de seis compuestos derivados de azobenceno en el que se han incorporado dos fragmentos de fenantreno, pireno o coranuleno. La extensión de su fotoisomerización, así como su reversibilidad, ha sido estudiada. Se han realizado experimentos de asociación con fullerenos, dando resultados muy positivos para aquellos derivados de coranuleno encontrando diferentes afinidades en función del isómero configuracional existente, llegando a obtenerse una pinza molecular fotosensible.

Por último, en el Artículo IV se muestra otra nueva serie de compuestos que poseen el mismo conjunto de fragmentos poliaromáticos, pero en este caso la molécula que los une es una porfirina, cuya estructura nos ha permitido integrar hasta cuatro de los mencionados fragmentos. Es conocida la capacidad de las porfirinas de establecer interacciones supramoleculares con fullerenos, pero en muy pocos casos lo hacen por sí mismas, necesitando casi siempre la existencia de una estructura tipo pinza o caja. En este caso, la unión coranuleno/porfirina ha permitido una asociación extremadamente buena en comparación con el resto de moléculas estudiadas. 


\subsection{GENERAL SUMMARY}

The main topic of this thesis, built as a set of four articles, is related to supramolecular interactions and how they can be exploited as a function of their inherent nature. Several families of compounds have been prepared and characterized. Their properties have been studied according to their ability to switch between two isomers, to catalyze and/or to establish supramolecular adducts by dispersion forces.

In the first part (Articles I and II) a set of compounds, commonly called rotaxanes, will be described. They are able to interact by means of supramolecular forces among their moieties based on hydrogen bonding and ion-dipole forces and shuttling between these situations is possible due to the special structure of these molecules. Both articles are the results of a new research line in our group which we started from the very beginning.

Thus, three homologous rotaxanes are described in Article I. Their main difference is the number of stations they possess and, consequently, the stopper fragment implied. Along with this family, a second group of compounds are presented in which the macrocycle is absent, so no interlocked structure is formed and, therefore, shuttling between stations is inexistent. Furthermore, a functional group born by these molecules is considered an organocatalytic center able to promote several Michael-type reactions through iminium cation. This capability has been exploited as a function of previously studied shuttling.

In Article II a second family of rotaxanes is described. Their structure is similar to those from the previous publication and the difference is based on the inclusion of an azobenzene fragment with the ability to be photoisomerized into a configurational isomer in a reversible process. The influence of this group has been studied in terms of shuttling between stations.

In parallel to these studies, the set of compounds bearing polycyclic aromatic hydrocarbons is extended in line with the main research line in our group. This second part comprises Articles III and IV. The main feature of these molecules is their ability to form supramolecular adducts with fullerenes, especially those containing corannulene fragment, whose special curved structure is complementary.

A family of six azobenzene-functionalized compounds attached to two polyaromatic fragments (phenanthrene, pyrene or corannulene) is described in Article III. Their photoisomerization process has been assessed as well as the reversibility of this process. Association with fullerenes have been carried out, resulting in excellent performances for corannulene derivatives and different affinities have been found as a function of the configurational isomer, finally demonstrating a photosensitized molecular tweezer.

Lastly, in Article IV, a new set of compounds is shown. They possess the same group of polyaromatic fragments, but this time the tether is a porphyrin, whose structure allowed us to integrate up to four moieties in the same molecule. It is well-known the ability of porphyrins to establish supramolecular interactions with fullerenes, but, in most cases, it is necessary to design cage-like or pincer-like structures to carry out such work. In this study, the couple corannulene/porphyrin have demonstrated to provide excellent association constants when compared to those containing other polyaromatic fragments. 
2. INTRODUCCIÓN 



\section{INTRODUCCIÓN}

La Química Supramolecular constituye actualmente un área en pleno desarrollo de la gran familia de disciplinas que componen la Química. Trata sobre la obtención de sistemas muy complejos a partir de especies relativamente sencillas que son capaces de interaccionar y auto organizarse a través de fuerzas no covalentes intermoleculares, tales como fuerzas de Van der Waals, de dispersión y electrostáticas. ${ }^{2}$

Esta filosofía tiene mucho impacto en la comunidad científica debido a que permite la fabricación de materiales nanoestructurados con alta organización que posean las propiedades deseadas. Esto se ha denominado aproximación bottom-up, en contraste con su antagonista, top down, que obtiene el material objetivo por la miniaturización progresiva de otro mayor. ${ }^{3}$

En esencia, esto es lo que ha hecho la Naturaleza desde siempre, por lo que no supone un concepto nuevo a priori. Ahora no nos parece impactante la auto organización que poseen las hebras de la doble hélice del $\mathrm{ADN},{ }^{4}$ el conocido plegamiento concreto que realiza cada proteína para desempeñar sus funciones en condiciones óptimas, ${ }^{5}$ el funcionamiento de la pareja bomba de protones / citocromo c oxidasa en nuestro organismo, ${ }^{6}$ o el funcionamiento del motor molecular kinesina, responsable del transporte celular en el citoesqueleto. ${ }^{7}$ Todos estos ejemplos suponen un fragmento muy pequeño de la diversidad de sistemas químicos existentes en los seres vivos. Son totalmente fundamentales para que la vida se desarrolle y absolutamente todos tienen un intrincado sistema de auto ensamblaje y auto organización basado en interacciones supramoleculares de distinta intensidad que conforman su estructura y funcionamiento.

${ }^{2}$ 1. (a) Balzani, V., Supramolecular Chemistry. Kluwer Academic: 1992. (b) Lehn, J.-M., Supramolecular Chemistry: Concepts and Perspectives. Wiley-VCH: Weinheim, 1995. (c) Atwood, J. L.; Davies, J. E. D.; MacNicol, D. D.; Vögtle, F.; Lehn, J.-M., Comprehensive Supramolecular Chemistry. Pergamon: Oxford, 1996. (d) Lehn, J.-M. PNAS 2002, 99, 4763-4768. (e) Atwood, J. L. S.; teed, J. W., Encyclopedia of Supramolecular Chemistry. Taylor \& Francis Group: New York, 2005. (f) Steed, J. W.; Atwood, J. L., Supramolecular Chemistry, 2nd Edition. Wiley-VCH: 2009.

${ }^{3}$ (a) Göpel, W. Adv. Mater. 1993, 5, 865-866. (b) Silva, G. A. Surg. Neurol. 2004, 61, 216-220. (c) Caruthers, S. D.; Wickline, S. A.; Lanza, G. M. Curr. Opin. Biotechnol. 2007, 18, 26-30. (d) Ozin, G. A.; Arsenault, A.; Cademartiri, L., Nanochemistry: A Chemical Approach to Nanomaterials. RSC Publishing: 2008. (e) Cademartiri, L.; Ozin, G. A., Concepts of Nanochemistry. Wiley-VCH: 2009. (f) Kerativitayanan, P.; Carrow, J. K.; Gaharwar, A. K. Adv. Healthc. Mater. 2015, 4, 1600-1627.

${ }^{4}$ (a) Franklin, R. E.; Gosling, R. G. Nature 1953, 171, 740-741. (b) Watson, J. D.; Crick, F. H. C. Nature 1953, 171, 737-738. (c) Wilkins, M. H. F.; Stokes, A. R.; Wilson, H. R. Nature 1953, 171, 738740. (d) Saenger, W., Principles of Nucleic Acid Structure. Springer: New York, 1984.

5 (a) Anfinsen, C. B. Biochem. J. 1972, 128, 737-749. (b) Alberts, B.; Johnson, A.; Lewis, J.; Raff, M.; Roberts, K.; Walter., P., Proteins. In Molecular Biology of the Cell, 4th edition, Garland Science: New York, 2002.

6 Yoshikawa, S.; Shimada, A.; Shinzawa-Itoh, K., Respiratory Conservation of Energy with Dioxygen: Cytochrome c Oxidase. In Sustaining Life on Planet Earth: Metalloenzymes Mastering Dioxygen and Other Chewy Gases, Springer International Publishing: 2015; pp 89-130.

7 (a) Vale, R. D.; Reese, T. S.; Sheetz, M. P. Cell 1985, 42, 39-50. (b) Rosenbaum, J. L.; Witman, G. B. Nat Rev Mol Cell Biol 2002, 3, 813-825. (c) Vale, R. D. Cell 2003, 112, 467-480. (d) Klumpp, S.; Lipowsky, R. PNAS 2005, 102, 17284-17289. 

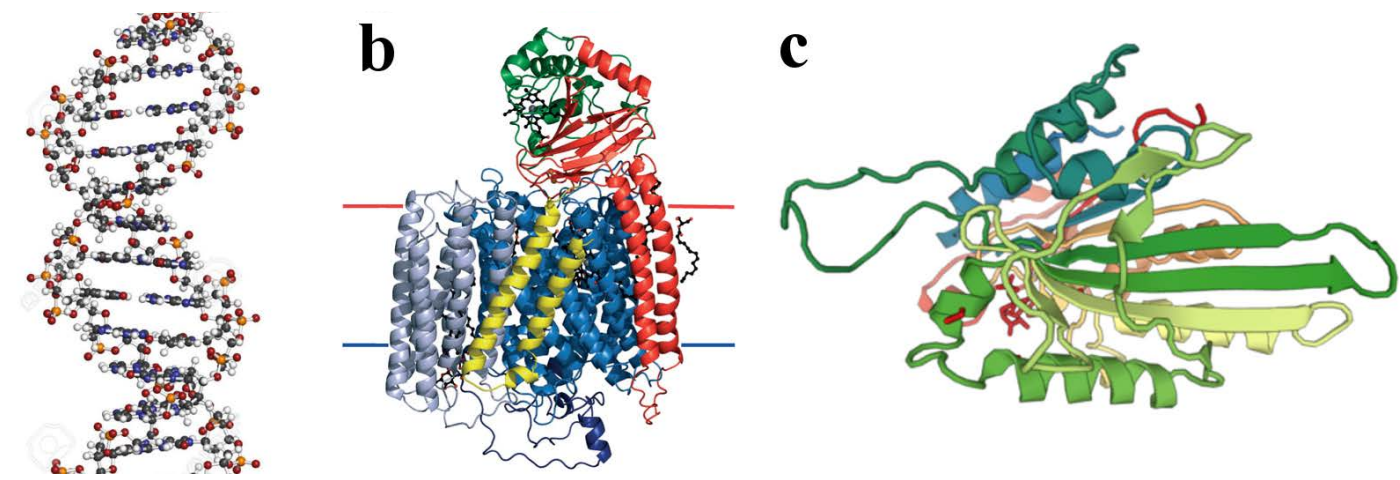

Figura 1. Algunos ejemplos de moléculas de actividad biológica con un altísimo grado de auto organización basado en interacciones supramoleculares. (a) ADN en forma B. (b) Citocromo oxidasa de tipo caa3 humana con su ubicación esquemática en una bicapa lipídica (líneas roja y azul). (c) Dominio motor de la proteína kinesina humana.

Ya desde la década de los 50 y 60, los trabajos pioneros de Cram, Lehn, y Pedersen sobre ciclofanos, criptandos, éteres corona ${ }^{8}$ y sus estudios en relación a interacciones específicas de reconocimiento molecular con la intención de comprender e imitar (o mejorar) los procesos bioquímicos naturales sentaron las bases de lo que actualmente se conoce como Química Supramolecular. Por todo ello, recibieron el Premio Nobel en Química en 1987. ${ }^{9}$

Desde entonces, se han desarrollado conceptos que actualmente son muy comunes en el lenguaje científico de un investigador químico, como son el auto ensamblaje, el efecto plantilla y la más moderna química covalente dinámica. ${ }^{10}$ Todos ellos tienen como idea subyacente la formación de asociaciones intermoleculares no covalentes previa construcción del sistema deseado y se ha constituido un área de mucho estudio en la mayoría de ramas de la ciencia.

\subsection{MOLÉCULAS ENTRELAZADAS MECÁNICAMENTE Y TOPOLOGÍA MOLECULAR}

Las Moléculas Entrelazadas Químicamente, o MIMs (Mechanically Interlocked Molecules), son especies que poseen en su estructura fragmentos que establecen interacciones supramoleculares conectados a través de uno o más enlaces mecánicos, característica especial que es consecuencia de su topología. ${ }^{11}$

${ }^{8}$ (a) Cram, D. J.; Steinberg, H. J. Am. Chem. Soc. 1951, 73, 5691-5704. (b) Pedersen, C. J. J. Am. Chem. Soc. 1967, 89, 7017-7036. (c) Dietrich, B.; Lehn, J. M.; Sauvage, J. P. Tetrahedron Lett. 1969, 10, 2885-2888. (d) Dietrich, B.; Lehn, J. M.; Sauvage, J. P. Tetrahedron Lett. 1969, 10, 2889-2892.

9 "The Nobel Prize in Chemistry 1987". Nobelprize.org. Nobel Media AB 2014. Web. 25 Aug 2016. $<$ http://www.nobelprize.org/nobel_prizes/chemistry/laureates/1987/index.html>

${ }^{10}$ (a) Rowan, S. J.; Cantrill, S. J.; Cousins, G. R. L.; Sanders, J. K. M.; Stoddart, J. F. Angew. Chem. Int. Ed. 2002, 41, 898-952. (b) Wilson, H.; Byrne, S.; Mullen, K. M. Chem. Asian J. 2015, 10, 715-721.

${ }^{11}$ (a) Amabilino, D. B.; Stoddart, J. F. Chem. Rev. 1995, 95, 2725-2828. (b) Breault, G. A.; Hunter, C. A.; Mayers, P. C. Tetrahedron 1999, 55, 5265-5293. (c) Vögtle, F.; Lukin, O.; Godt, A. Chem. Eur. J. 2004, 10, 1878-1883. (d) Badjić, J. D.; Nelson, A.; Cantrill, S. J.; Turnbull, W. B.; Stoddart, J. F. Acc. Chem. Res. 2005, 38, 723-732. (e) Amabilino, D. B.; Perez-Garcia, L. Chem. Soc. Rev. 2009, 38, 15621571 . 


\subsubsection{El enlace mecánico}

Los primeros trabajos que describían la síntesis de compuestos de este tipo proceden de los años $60,{ }^{12}$ en los que se prepararon un catenano y un rotaxano prácticamente por puro azar (Figura 2). Ya desde entonces se consideró que era necesario establecer un método para especificar la estructura de una molécula en base a su topología ${ }^{13}$ debido a que especificar la naturaleza de la conectividad de los átomos y la disposición espacial en torno a centros rígidos (átomos estereogénicos, sustitución en dobles enlaces, etc) no era suficiente.
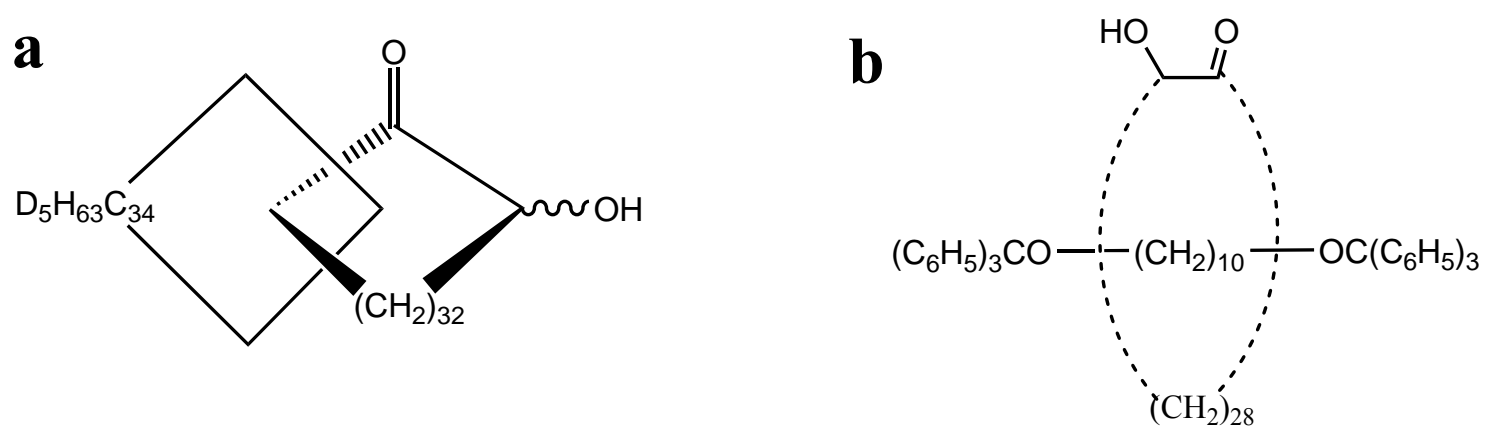

Figura 2. Primeras moléculas entrelazadas mecánicamente sintetizadas de la historia.

(a) Catenano. (b) Rotaxano.

Fruto de aquellos estudios se estableció el término del enlace mecánico. ${ }^{14}$ Consiste en la unión o uniones de dos o más entidades no conectadas a través de enlaces químicos convencionales (covalentes la mayoría de las veces) pero que, debido a la barrera estérica impuesta por la topología establecida entre ellas, no se pueden separar a menos que previamente se disocie, o al menos se distorsione significativamente, un enlace químico. Como consecuencia, esta entidad no es considerada un aducto supramolecular, sino una única molécula.

Originalmente se preparaban basándose en la pura estadística. Esto consistía en confiar que el movimiento Browniano de largas cadenas alifáticas permitiera la existencia de conformaciones accesibles energéticamente tales que, al producir la reacción final de cierre, diese como resultado la molécula entrelazada mecánicamente. Esto daba como resultado rendimientos muy bajos, en torno al $1 \%$ en el mejor de los casos.

12 (a) Wasserman, E. J. Am. Chem. Soc. 1960, 82, 4433-4434. (b) Harrison, I. T.; Harrison, S. J. Am. Chem. Soc. 1967, 89, 5723-5724. (c) Schill, G.; Zollenkopf, H. Justus Liebigs Ann. Chem. 1969, 721, 5374.

13 (a) Frisch, H. L.; Wasserman, E. J. Am. Chem. Soc. 1961, 83, 3789-3795. (b) Musso, H. Angew. Chem. 1972, 84, 270-270.

14 (a) Stoddart, J. F. Chem. Soc. Rev. 2009, 38, 1802-1820. (b) Fang, L.; Olson, M. A.; Benitez, D.; Tkatchouk, E.; Goddard Iii, W. A.; Stoddart, J. F. Chem. Soc. Rev. 2010, 39, 17-29. (c) Bruns, C. J.; Stoddart, J. F., The Mechanical Bond: A Work of Art. In Beauty in Chemistry, Fabbrizzi, L., Ed. Springer-Verlag: Heidelberg, 2011; pp 19-72. 
La estrategia actual, más óptima que un método estocástico, está basada en la síntesis dirigida. Se trata de preorganizar las entidades a través de una fuerza supramolecular débil que las una (auto ensamblaje) o de otra especie que facilite su acercamiento en la geometría adecuada (efecto plantilla) para, posteriormente, realizar una reacción final de cierre generando el enlace mecánico. ${ }^{15}$ La reacción final suele ser aquella que se produzca con el mayor rendimiento posible y en condiciones muy suaves para evitar la disrupción del sistema macromolecular previamente formado. Se dice entonces que el producto se obtiene por control cinético. Existe otra aproximación en la cual se produce una reacción de cierre reversible. De esta manera, la distribución de posibles productos va a depender de la estabilidad relativa de los mismos como consecuencia del control termodinámico establecido por los preequilibrios existentes de formación de aductos supramoleculares. Esta segunda filosofía está encuadrada en un concepto más general antes mencionado como Química Covalente Dinámica. ${ }^{10}$

Gracias a estos métodos modernos, se obtienen unos excelentes resultados llegando a obtenerse MIMs con rendimientos que superan el $90 \%$.

\subsubsection{Tipos de moléculas entrelazadas mecánicamente}

Entre todos los autores, está bastante consensuada la existencia de seis tipos principales de compuestos con la característica de poseer al menos un enlace mecánico (Figura 3):

\subsubsection{Catenanos}

Los catenanos consisten en dos o más macrociclos entrelazados mecánicamente. Su denominación deriva de la palabra latina catena en semejanza a los eslabones de una cadena macroscópica. Se suele denominar con un número delante encerrado por corchetes denotando el número de macrociclos unidos. Así, un [n]catenano consta de $n$ macrociclos. ${ }^{16}$

\subsubsection{Rotaxanos}

Los rotaxanos consisten en una o más moléculas con forma de mancuerna (esto es, alargada con extremos voluminosos) enhebrada con uno o más macrociclos. Su denominación deriva de las palabras latinas rota, o rueda, y axis, o eje, en relación a su semejanza morfológica con sus dos componentes principales. En paralelo a la denominación de los catenanos, los rotaxanos llevan el mismo índice delante denotando el número de especies entrelazadas. Así, un $[n]$ rotaxano consta de $n$ entidades en las que al menos una ha de ser el eje y otra el macrociclo. ${ }^{16}$

${ }^{15}$ (a) Aricó, F.; Badjic, J. D.; Cantrill, S. J.; Flood, A. H.; Leung, K. C.-F.; Liu, Y.; Stoddart, J. F., Templated Synthesis of Interlocked Molecules. In Templates in Chemistry II, Schalley, C. A.; Vögtle, F.; Dötz, K. H., Eds. Springer: Heidelberg, 2005; pp 203-259. (b) Stoddart, J. F.; Dichtel, W. R.; Miljanić, O. S.; Zhang, W.; Spruell, J. M.; Patel, K.; Aprahamian, I.; Heath, J. R. Acc. Chem. Res. 2008, 41, 17501761.

${ }^{16}$ (a) Vögtle, F.; Safarowsky, O.; Windisch, B.; Mohry, A. J. Prakt. Chem. 2000, 342, 437-444. (b) Yerin, A.; Wilks, E. S.; Moss, G. P.; Harada, A. Pure Appl. Chem. 2008, 80, 28. 


\subsubsection{Nudos}

A diferencia de los dos tipos anteriores, los nudos tienen la característica principal de que son moléculas no formadas por la unión de varios fragmentos, sino que son macrociclos anudados consigo mismos. ${ }^{17} \mathrm{Su}$ nombre se ha atribuido en semejanza con un nudo macroscópico. Debido a las distintas posibilidades de anudarse, estas moléculas son quirales. Su existencia experimental no es tan extendida en la literatura, por lo que no existe una manera consensuada para su notación, más allá del término "nudo de trébol" o "nudano".

\subsubsection{Anillos de Salomón}

Los anillos de Salomón son catenanos que se han entrelazado más de una vez entre ellos. Podrían considerarse un híbrido entre un catenano y un nudo. ${ }^{18} \mathrm{Su}$ nombre proviene de la semejanza con el motivo decorativo usado en muchas culturas llamado como nudo de Salomón.

\subsubsection{Anillos de Borromeo}

Los anillos de Borromeo consisten en tres anillos entrelazados de una manera tal que, si se rompe cualquiera de ellos, los otros dos se pueden separar sin disociarse ya que no estarían mecánicamente unidos. ${ }^{19}$ Denominados así en honor a una familia noble italiana que usó este motivo como su blasón. Esta disposición particular de los anillos se conoce como unión bruniana dentro de la teoría de nudos. ${ }^{20}$

17 (a) Dietrich-Buchecker, C. O.; Sauvage, J.-P. Angew. Chem. Int. Ed. 1989, 28, 189-192. (b) Dietrich-Buchecker, C. O.; Nierengarten, J. F.; Sauvage, J. P.; Armaroli, N.; Balzani, V.; De Cola, L. J. Am. Chem. Soc. 1993, 115, 11237-11244. (c) Safarowsky, O.; Nieger, M.; Fröhlich, R.; Vögtle, F. Angew. Chem. Int. Ed. 2000, 39, 1616-1618. (d) Adams, H.; Ashworth, E.; Breault, G. A.; Guo, J.; Hunter, C. A.; Mayers, P. C. Nature 2001, 411, 763-763. (e) Feigel, M.; Ladberg, R.; Engels, S.; Herbst-Irmer, R.; Fröhlich, R. Angew. Chem. Int. Ed. 2006, 45, 5698-5702. (f) Ayme, J.-F.; Beves, J. E.; Leigh, D. A.; McBurney, R. T.; Rissanen, K.; Schultz, D. Nat. Chem. 2012, 4, 15-20.

18 (a) Nierengarten, J. F.; Dietrich-Buchecker, C. O.; Sauvage, J. P. J. Am. Chem. Soc. 1994, 116, $375-$ 376. (b) Ibukuro, F.; Fujita, M.; Yamaguchi, K.; Sauvage, J.-P. J. Am. Chem. Soc. 1999, 121, 1101411015. (c) McArdle, C. P.; Vittal, J. J.; Puddephatt, R. J. Angew. Chem. 2000, 112, 3977-3980. (d) Pentecost, C. D.; Chichak, K. S.; Peters, A. J.; Cave, G. W. V.; Cantrill, S. J.; Stoddart, J. F. Angew. Chem. Int. Ed. 2007, 46, 218-222. (e) Peinador, C.; Blanco, V.; Quintela, J. M. J. Am. Chem. Soc. 2009, 131, 920-921. (f) Gil-Ramírez, G.; Leigh, D. A.; Stephens, A. J. Angew. Chem. Int. Ed. 2015, 54, 61106150.

19 (a) Cantrill, S. J.; Chichak, K. S.; Peters, A. J.; Stoddart, J. F. Acc. Chem. Res. 2004, 38, 1-9. (b) Chichak, K. S.; Cantrill, S. J.; Pease, A. R.; Chiu, S.-H.; Cave, G. W. V.; Atwood, J. L.; Stoddart, J. F. Science 2004, 304, 1308-1312. (c) Chichak, K. S.; Cantrill, S. J.; Stoddart, J. F. Chem. Commun. 2005, 3391-3393. (d) Chichak, K. S.; Peters, A. J.; Cantrill, S. J.; Stoddart, J. F. J. Org. Chem. 2005, 70, 79567962. (e) Peters, A. J.; Chichak, K. S.; Cantrill, S. J.; Stoddart, J. F. Chem. Commun. 2005, 3394-3396. (f) Thorp-Greenwood, F. L.; Kulak, A. N.; Hardie, M. J. Nat. Chem. 2015, 7, 526-531.

${ }^{20}$ Adams, C., The Knot Book American Mathematical Society: 2004. 


\subsubsection{Suitanos}

Los suitanos son la última familia en ser propuesta como tal, aunque siempre han sido considerados como rotaxanos jaula. Son rotaxanos con dos macrociclos o más, estando estos últimos todos unidos covalentemente. ${ }^{21}$ Su nombre proviene de la palabra suit, que significa traje en inglés en semejanza a la estructura y disposición de los macrociclos sobre el eje como si fuera un traje hecho a medida. Se los denomina con un número entre corchetes en mitad de la palabra denotando el número de apéndices que conforman el eje. Así, un suit $[n]$ ano tiene $n$ miembros en su eje.

$\mathbf{a}$

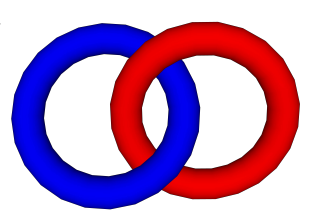

d

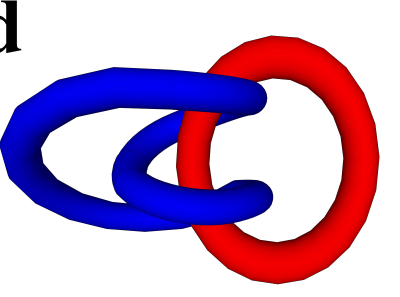

b

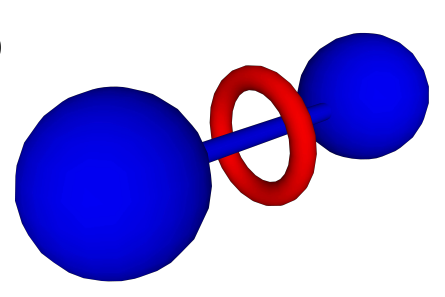

$\mathbf{e}$

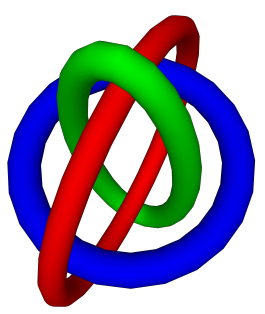

c
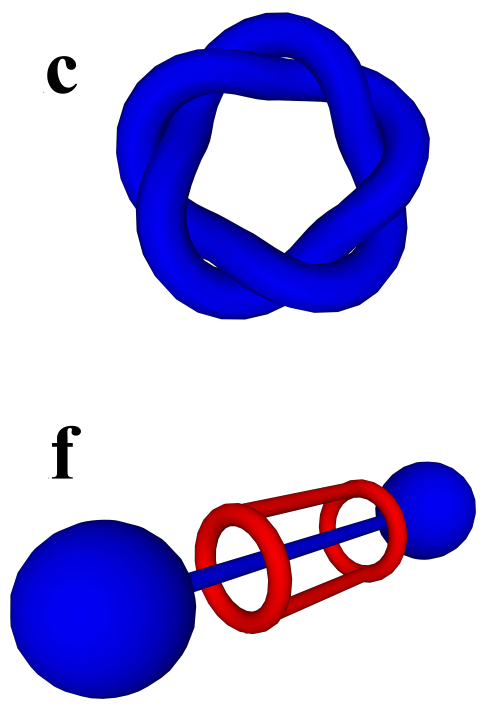

Figura 3. Diferentes tipos de MIMs. (a) [2]catenano, (b) [2] rotaxano, (c) nudo de 5 eslabones; (d) anillo de Salomón; (e) anillo de Borromeo y (f) suit[2]ano.

\subsection{ROTAXANOS}

Debido a que una parte importante del contenido de esta tesis está relacionada con el estudio de rotaxanos, a continuación se va a profundizar en la morfología y características de esta familia.

Tal y como se ha dicho anteriormente, hay dos constituyentes principales en un rotaxano. Por un lado está el anillo macrocíclico, que ha de tener un diámetro lo suficientemente grande como para ser enhebrado por el otro componente, el eje. Dicho eje posee en sus extremos unos grupos lo suficientemente voluminosos como para no permitir el desenhebrado, es decir, cumplen una función estructural y no suelen participar en la asociación supramolecular. Estos grupos son comúnmente llamados stoppers (Figura 4).

${ }^{21}$ (a) Chang, T.; Heiss, A. M.; Cantrill, S. J.; Fyfe, M. C. T.; Pease, A. R.; Rowan, S. J.; Stoddart, J. F.; Williams, D. J. Org. Lett. 2000, 2, 2943-2946. (b) Williams, A. R.; Northrop, B. H.; Chang, T.; Stoddart, J. F.; White, A. J. P.; Williams, D. J. Angew. Chem. Int. Ed. 2006, 45, 6665-6669. 


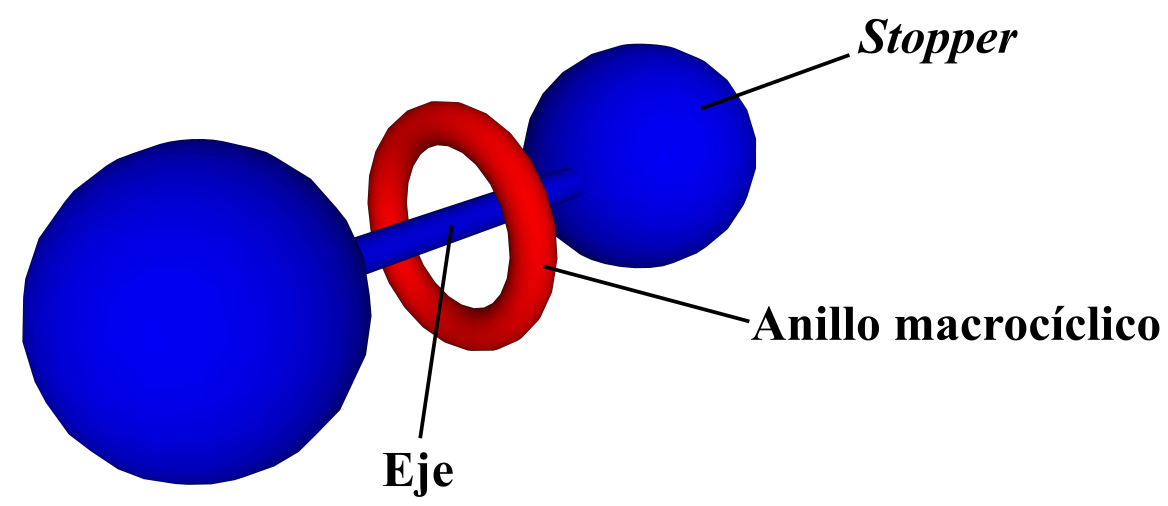

Figura 4. Elementos constituyentes de un rotaxano.

Existe un derivado análogo que en muchos casos constituye un paso previo a la síntesis de este grupo de moléculas entrelazadas mecánicamente, llamado pseudorotaxano (Figura 5). Consiste en un aducto supramolecular formado por el anillo y el eje, pero este último carece de uno o los dos fragmentos stopper, de manera que la forma enhebrada y desenhebrada están en equilibrio. Consecuentemente esta agrupación no se considera MIM, pero es importante mencionarlo en este punto debido a su gran utilidad como intermedio en el proceso sintético de rotaxanos en esta memoria.
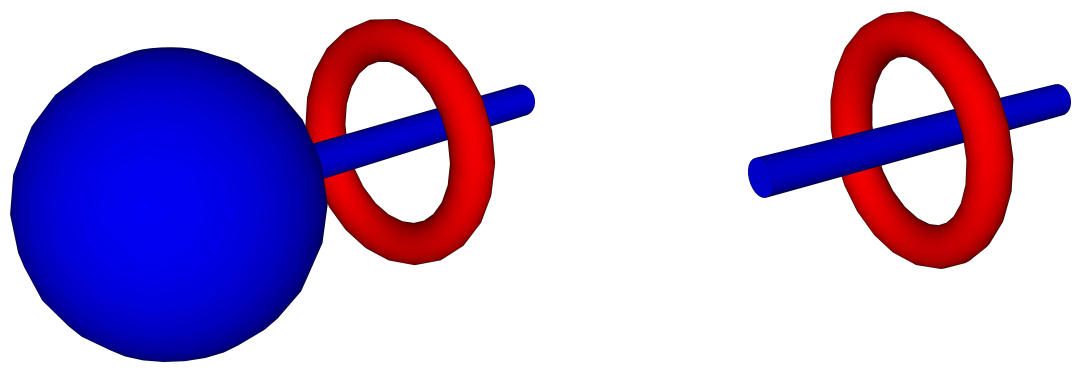

Figura 5. Pseudorotaxanos.

Hasta este punto sólo se ha hablado de la topología de estas moléculas y, de manera general, la estrategia de síntesis dirigida para prepararlos basándose en interacciones supramoleculares. Es ahora el momento de mencionar los motivos habituales encontrados en la bibliografía en la que estas fuerzas favorecen la asociación y permiten la formación de la superestructura en una situación a priori desfavorable entrópicamente. ${ }^{22}$ Todos los descritos a continuación se encuentran recogidos en la Figura 6.

${ }^{22}$ (a) Williams, D. J.; Ashton, P. R.; Ballardini, R.; Balzani, V.; Credi, A.; Gandolfi, M. T.; Menzer, S.; Perez-Garcia, L.; Prodi, L. J. Am. Chem. Soc. 1995, 117, 11171-11197. (b) Sauvage, J.-P. Acc. Chem. Res. 1998, 31, 611-619. (c) Griffiths, K. E.; Stoddart, J. F. Pure Appl. Chem. 2008, 80, 485-506. (d) Stoddart, J. F.; Colquhoun, H. M. Tetrahedron 2008, 64, 8231-8263. 
El uso de poliéteres macrocíclicos como anillos en rotaxanos es común, especialmente cuando forman complejos de inclusión con sales de amonio (a en la Figura 6) ${ }^{23}$ Los hidrógenos de dicha sal establecen puentes de hidrógeno con los oxígenos del éter corona. Otro motivo importante es el uso de metales de transición para la complejación de bipiridinas, fenantrolinas y terpiridinas (b en la Figura 6) que mantienen los ligandos cercanos en torno a un centro de manera que pueda realizarse la reacción de cierre entre ellos y generar el enlace mecánico. ${ }^{24}$

El uso de enlaces de hidrógeno o complejos con ligandos nitrogenados no se limita a lo anteriormente expuesto. Existen otras familias de compuestos capaces de establecer tales interacciones de manera eficiente, como por ejemplo isoftalimidas, capaces de asociar escuaramidas, tioureas y aniones (c en la Figura 6), o piridin-2,6-carboxamidas, que forman un ligando quelato $k-\mathrm{N} 3$ con metales de transición (d en la Figura 6) dejando al menos un hueco de coordinación para otro ligando que puede provenir de la otra especie a entrelazar. ${ }^{25}$ Estos son sólo algunos ejemplos de la amplia variedad de posibilidades que sugiere la formación de enlaces de hidrógeno o la química de la coordinación. ${ }^{26}$

Las interacciones ion-dipolo inducido también se han explotado en forma de sistemas supramoleculares $\pi$-dador/ $\pi$-aceptor, en el que normalmente la unidad $\pi$-dadora puede ir desde un simple benceno hasta un tetratiafulvaleno y la $\pi$-aceptora es una sal de piridinio, bipiridinio $o$ especies similares (e en la Figura 6). ${ }^{27}$

Por último, un motivo muy interesante usado para la síntesis de rotaxanos es el uso de ciclodextrinas o curcubiturilos como anillo macrocíclico, que poseen una cavidad hidrófoba capaz de interaccionar con muchas especies (f en la Figura 6). ${ }^{28}$

23 (a) Aston, P. R.; Glink, P. T.; Stoddart, J. F.; Tasker, P. A.; White, A. J. P.; Williams, D. J. Chem. Eur. J. 1996, 2, 729-736. (b) Stoddart, J. F.; Ashton, P. R.; Chrystal, E. J. T.; Glink, P. T.; Menzer, S.; Schiavo, C.; Spencer, N.; Tasker, P. A.; White, A. J. P.; Williams, D. J. Chem. Eur. J. 1996, 2, 709-728. (c) Ashton, P. R.; Ballardini, R.; Balzani, V.; Gómez-López, M.; Lawrence, S. E.; Martínez-Díaz, M. V.; Montalti, M.; Piersanti, A.; Prodi, L.; Stoddart, J. F.; Williams, D. J. J. Am. Chem. Soc. 1997, 119, 1064110651.

24 (a) Prikhod'ko, A. I.; Sauvage, J.-P. J. Am. Chem. Soc. 2009, 131, 6794-6807. (b) Durola, F.; Sauvage, J.-P.; Wenger, O. S. Coord. Chem. Rev. 2010, 254, 1748-1759.

25 (a) Beer, P. D.; Lankshear, M. D. Acc. Chem. Res. 2007, 40, 657-668. (b) Miyagawa, N.; Watanabe, M.; Matsuyama, T.; Koyama, Y.; Moriuchi, T.; Hirao, T.; Furusho, Y.; Takata, T. Chem. Commun. 2010, 46, 1920-1922. (c) Yang, W.; Li, Y.; Liu, H.; Chi, L.; Li, Y. Small 2012, 8, 504-516.

${ }^{26}$ Lee, C.-F.; Leigh, D. A.; Pritchard, R. G.; Schultz, D.; Teat, S. J.; Timco, G. A.; Winpenny, R. E. P. Nature 2009, 458, 314-318.

${ }^{27}$ (a) Stoddart, J. F.; Braunschweig, A. B.; Ronconi, C. M.; Han, J.-Y.; Aricó, F.; Cantrill, S. J.; Khan, S. I.; White, A. J. P.; Williams, D. J. Eur. J. Org. Chem. 2006, 2006, 1857-1866. (b) Coutrot, F.; Busseron, E. Chem. Eur. J. 2009, 15, 5186-5190. (c) Olson, M. A.; Braunschweig, A. B.; Ikeda, T.; Fang, L.; Trabolsi, A.; Slawin, A. M. Z.; Khan, S. I.; Stoddart, J. F. Org. Biomol. Chem. 2009, 7, 4391-4405. (d) Stoddart, J. F.; Zhao, Y.-L.; Liu, L.; Zhang, W.; Sue, C.-H.; Li, Q.; Miljanić, O. Š.; Yaghi, O. M. Chem. Eur. J. 2009, 15, 13356-13380.

28 (a) Sindelar, V.; Silvi, S.; Kaifer, A. E. Chem. Commun. 2006, 2185-2187. (b) Wenz, G.; Han, B.H.; Müller, A. Chem. Rev. 2006, 106, 782-817. (c) Ramalingam, V.; Urbach, A. R. Org. Lett. 2011, 13, 4898-4901. (d) Girek, T. J. Incl. Phenom. Macrocycl. Chem. 2012, 74, 1-21. 
$\mathbf{a}$<smiles>[2H][NH+]1CCCCCCCCOCCOCCOCCO1</smiles>

b

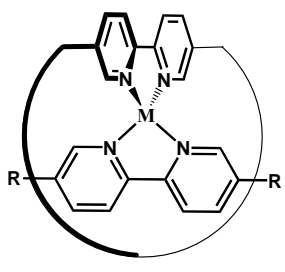

c

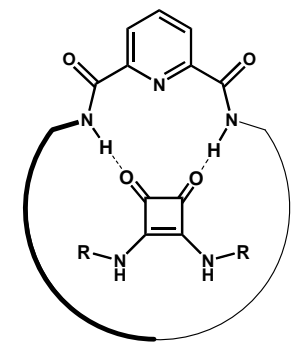

d

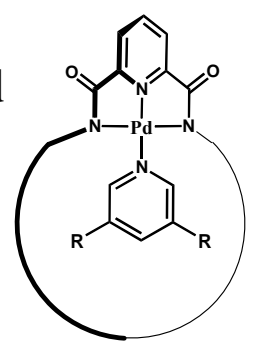

e
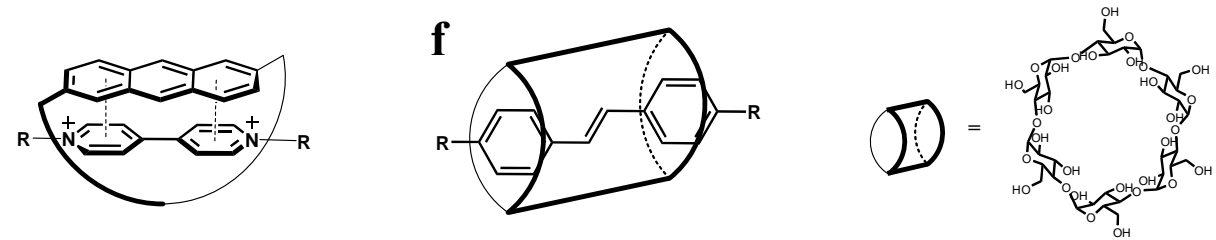

Figura 6. Motivos habituales en la preparación de rotaxanos.

\subsubsection{Síntesis de rotaxanos}

Basándonos en todo lo anteriormente descrito, a continuación se profundizará en las distintas estrategias sintéticas para la obtención de rotaxanos.

Existen al menos tres estrategias clásicas de obtención ${ }^{22 c}$ y en todas, al menos uno de los fragmentos constituyentes está completamente formado. El procedimiento "enhebrado y cierre" (threading-capping) consiste en poner en contacto el macrociclo y un eje cuyos grupos stopper están ausentes para que se produzca su auto ensamblaje. Posteriormente se produce la reacción final de cierre generando el enlace mecánico deseado (Esquema 1).
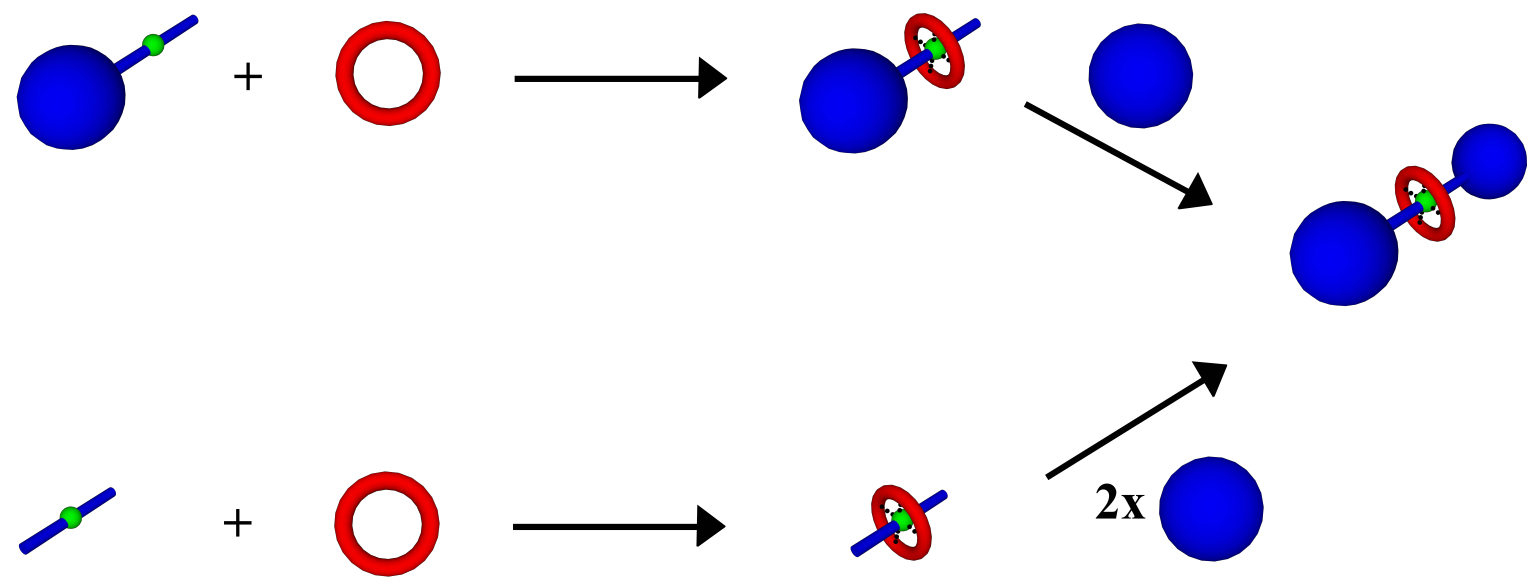

Esquema 1. Síntesis de rotaxanos por el método enhebrado y cierre. 
Esta filosofía tiene su complementaria, llamada "abrochado" (clipping). En este caso un eje ya sintetizado, es decir, que incluye los grupos stopper, se asocia por fuerzas supramoleculares a un compuesto bifuncional. Éste se termina de conectar de principio a fin usando otro compuesto bifuncional generando la molécula entrelazada mecánicamente (Esquema 2).

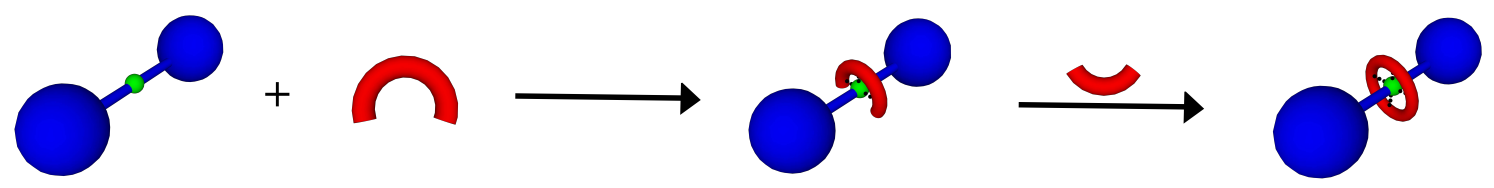

Esquema 2. Síntesis de rotaxanos por el método abrochado.

El último método, denominado "deslizamiento" (slippage), en cambio, usa tanto anillo macrocíclico como eje totalmente formados. Se ponen en contacto a elevadas temperaturas y largos tiempos con el objetivo de favorecer el movimiento Browniano en dirección a la formación del rotaxano (Esquema 3).

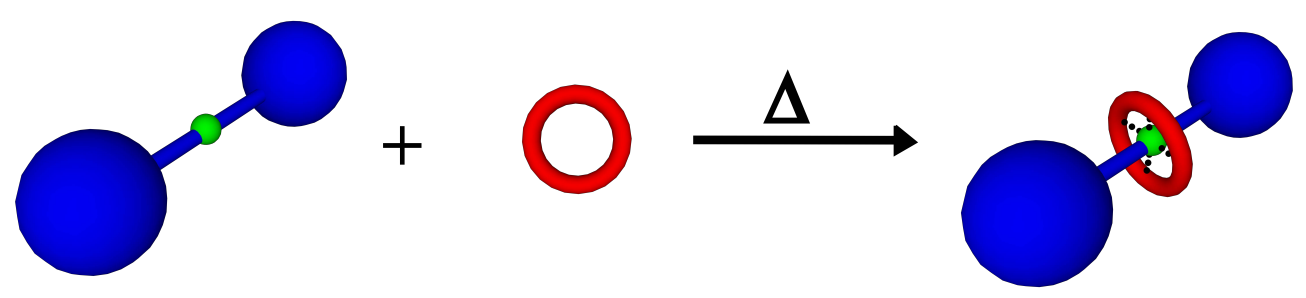

Esquema 3. Síntesis de rotaxanos por el método deslizamiento.

Además de las tres estrategias expuestas anteriormente, existen otras nuevas que han tenido menos repercusión, pero son igualmente interesantes. Es posible realizar un enhebrado con un eje y un macrociclo lo suficientemente grande como para superar la barrera estérica impuesta por el stopper sin necesidad de calentar el sistema y, posteriormente, reducir el tamaño del anillo a través de una reacción sencilla. Este método podría denominarse como "enhebrado y contracción del anillo". ${ }^{29}$ Otro análogo consiste en producir el enhebrado entre un macrociclo del tamaño adecuado y un eje totalmente formado, pero posee un grupo stopper demasiado pequeño como para impedir que el anillo sea penetrado. Una reacción posterior (por ejemplo la expansión de un anillo carbocíclico) agranda el tamaño de este grupo generándose el enlace mecánico. La diferencia con respecto al procedimiento de enhebrado y cierre consiste en que en este caso no se incorporan especies nuevas, es el propio grupo terminal que, por transposición modifica su estructura a una más voluminosa. Podría llamarse a esta estrategia como "enhebrado y agrandado del stopper". ${ }^{30}$

${ }^{29}$ Yoon, I.; Narita, M.; Shimizu, T.; Asakawa, M. J. Am. Chem. Soc. 2004, 126, 16740-16741.

${ }^{30}$ Chiu, C.-W.; Lai, C.-C.; Chiu, S.-H. J. Am. Chem. Soc. 2007, 129, 3500-3501. 
Otra estrategia desarrollada recientemente en la síntesis de rotaxanos es conocida como "plantilla con metal activo" (active metal template) en la que un metal de transición se coordina al anillo macrocíclico y cataliza la reacción de unión de dos fragmentos que conforman el eje completo. ${ }^{31}$ De esta manera, el átomo metálico participa activamente en la constitución del rotaxano y, además, permite la formación de moléculas entrelazadas cuyos fragmentos no tienen por qué tener necesariamente una fuerza supramolecular que los una o ésta es muy débil como para preparar el compuesto por los métodos tradicionales.

En este contexto, el trabajo descrito en la presente memoria hace uso de la estrategia enhebrado y cierre usando la pareja sal de amonio / éter corona cuya asociación macromolecular ha sido estudiada con profundidad desde muchas perspectivas. Se forma de manera sencilla y es muy estable, especialmente para sales de amonio secundarias bencílicas con éteres corona ${ }^{32}$

Para la reacción de cierre se decidió hacer uso de la reacción CuAAC (Copper (I)-catalyzed Azide-Alkyne Cycloaddition), la más conocida dentro de la filosofía de síntesis bautizada por Sharpless como click chemistry. ${ }^{33}$ Consiste en una cicloadición entre un grupo alquino y una azida terminales (Esquema 4). Es una reacción que se puede realizar en condiciones suaves, evitando imponer altas temperaturas favoreciendo que se mantenga el aducto supramolecular y obtener los mejores rendimientos posibles. ${ }^{34}$ Además, el triazol resultante permite posterior funcionalización a través de alquilación o coordinación de metales, por ejemplo.

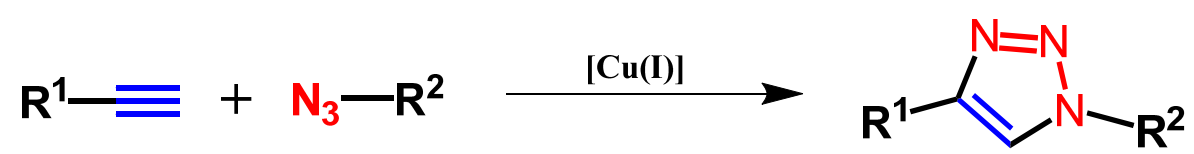

Esquema 4. Reacción CuAAC tipo click.

31 (a) Crowley, J. D.; Goldup, S. M.; Lee, A.-L.; Leigh, D. A.; McBurney, R. T. Chem. Soc. Rev. 2009, 38, 1530-1541. (b) Leigh, D. A.; Goldup, S. M.; Long, T.; McGonigal, P. R.; Symes, M. D.; Wu, J. J. Am. Chem. Soc. 2009, 131, 15924-15929. (c) Leigh, D. A.; Goldup, S. M.; McGonigal, P. R.; Ronaldson, V. E.; Slawin, A. M. Z. J. Am. Chem. Soc. 2009, 132, 315-320. (d) Crowley, J. D.; Goldup, S. M.; Gowans, N. D.; Leigh, D. A.; Ronaldson, V. E.; Slawin, A. M. Z. J. Am. Chem. Soc. 2010, 132, 62436248.

32 (a) Schneider, H.-J.; Rüdiger, V.; Solov'ev, V. P.; Kazachenko, V. P.; Raevsky, O. A. Eur. J. Org. Chem. 1999, 1999, 1847-1856. (b) Cantrill, S. J.; Fulton, D. A.; Heiss, A. M.; Pease, A. R.; Stoddart, J. F.; White, A. J. P.; Williams, D. J. Chem. Eur. J. 2000, 6, 2274-2287. (c) Clifford, T.; Abushamleh, A.; Busch, D. H. PNAS 2002, 99, 4830-4836. (d) Chiu, S.-H.; Liao, K.-S.; Su, J.-K. Tetrahedron Lett. 2004, 45, 213-216. (e) Angelo, J. P.; Sohlberg, K. J. Phys. Chem. A 2009, 113, 6724-6729. (f) Morin, J.-F.; Thibeault, D. Molecules 2010, 15, 3709-3730. (g) Schalley, C. A.; Jiang, W.; Schäfer, A.; Mohr, P. C. J. Am. Chem. Soc. 2010, 132, 2309-2320. (h) Späth, A.; König, B. Beilstein J. Org. Chem. 2010, 6, 32. (i) Payne, N. A.; Delmas, L. C.; McDowell, S. A. C.; Williams, A. R. Tetrahedron Lett. 2015, 56, 51755179.

33 (a) Kolb, H. C.; Finn, M. G.; Sharpless, K. B. Angew. Chem. Int. Ed. 2001, 40, 2004-2021. (b) Lahann, J., Click Chemistry for Biotechnology and Materials Science. John Wiley \& Sons Ltd: West Sussex, 2009; p 433.

34 (a) Stoddart, J. F.; Ivan, A.; S, M. O.; R, D. W.; Kyosuke, I.; Takuma, Y.; Takashi, K. Bull. Chem. Soc. Jpn. 2007, 80, 13. (b) Hanni, K. D.; Leigh, D. A. Chem. Soc. Rev. 2010, 39, 1240-1251. 


\subsubsection{Interruptores moleculares basados en rotaxanos}

Un interruptor molecular (traducción adaptada por nosotros del término molecular shuttle) basado en una estructura de tipo rotaxano es una especie cuyo eje posee al menos dos grupos por los que el macrociclo es capaz de establecer interacciones supramoleculares. A esos fragmentos se los denomina estaciones. Si éstas son iguales, entonces se dice que el interruptor está degenerado ya que el macrociclo estará en un equilibrio dinámico moviéndose de una a otra ininterrumpidamente, como se muestra en la Figura 7.

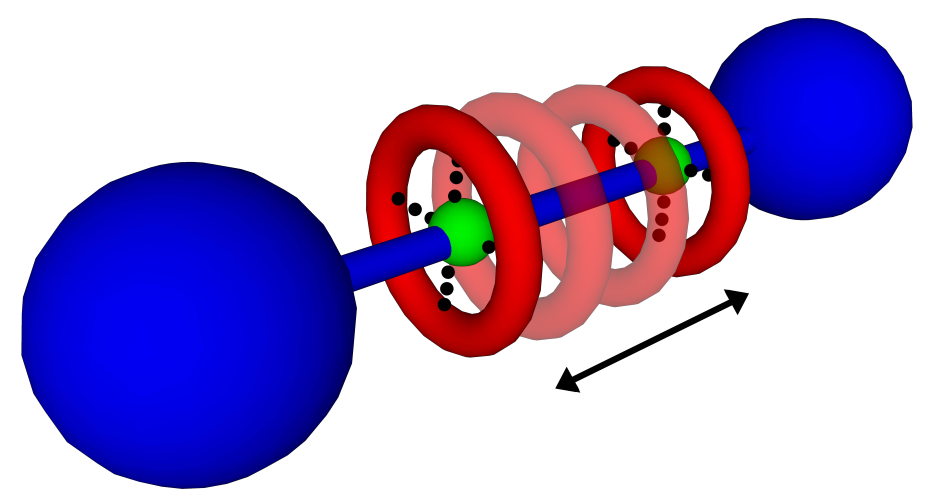

Figura 7. Modelo de un interruptor molecular basado en [2]rotaxano biestable degenerado mostrándose su equilibrio dinámico.

En cambio, si existe una diferencia de afinidad entre cada estación y el anillo, entonces éste permanecerá en una preferentemente y su movimiento dependerá de la estabilidad relativa de ambas situaciones y la energía de activación del proceso. ${ }^{35}$ En todo caso se suele aplicar a estos sistemas el término de rotaxanos multiestables debido a la posibilidad de conmutación entre sus isómeros traslacionales.

Si, además de darse la situación anteriormente expuesta, es posible variar la afinidad entre las estaciones con un estímulo externo, entonces nos encontramos con un verdadero interruptor molecular (Esquema 5).

${ }^{35}$ Fahrenbach, A. C.; Bruns, C. J.; Li, H.; Trabolsi, A.; Coskun, A.; Stoddart, J. F. Acc. Chem. Res. 2014, 47, 482-493. 

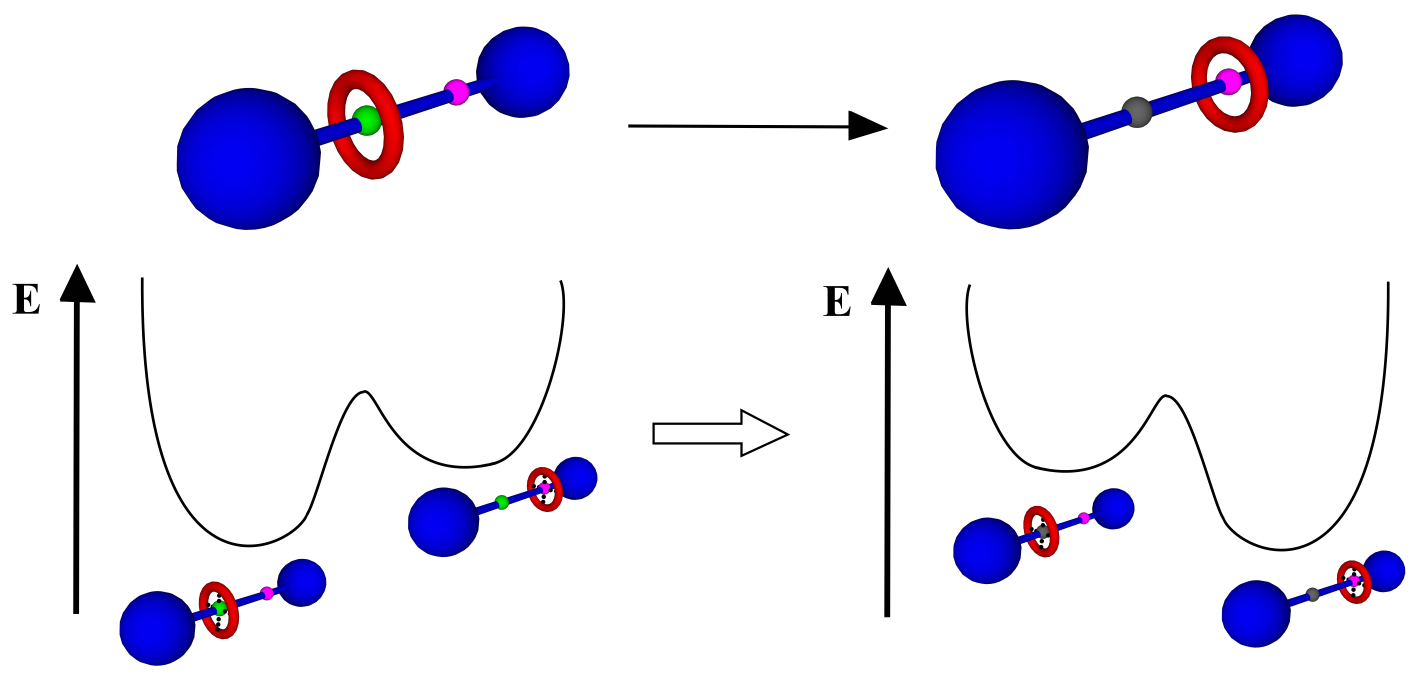

Esquema 5. Arriba: Proceso de conmutación entre los dos isómeros traslacionales de un interruptor molecular basado en un [2]rotaxano biestable. Debajo: perfil de isomerización traslacional típico de este sistema. En principio la estación coloreada de verde tiene la mayor afinidad por el macrociclo, pero cuando se aplica el estímulo externo, ésta tiene mucha menor afinidad (marcada como gris) y, por tanto, el compuesto evolucionará espontáneamente desplazando el anillo hacia la otra estación suponiendo que es posible superar la barrera energética necesaria. Dicha barrera se la suele relacionar con la naturaleza del fragmento espaciador y con el posible plegamiento del eje si éste es muy flexible.

También es posible combinar ambas características; esto es, tener un rotaxano triestable en el cual el macrociclo tiene una fuerte interacción por una estación, mientras que tiene menores afinidades por los otros dos, pero muy similares entre sí. Esto daría lugar a un interruptor propiamente dicho y a uno degenerado al mismo tiempo. ${ }^{36}$

El primer interruptor molecular fue descrito por el grupo de Stoddart ${ }^{37}$ en el que se usaba el motivo $\pi$-dador/ $\pi$-aceptor empleando un fenileno para disustituido y una sal de bipiridinio alquilada en el eje y macrociclo, respectivamente. El eje tenía como grupos espaciadores cadenas de éteres derivados del etilenglicol y, como stoppers, grupos trisisopropilsilanos (Figura 8). Debido a que el [2]rotaxano tenía dos estaciones idénticas, se trataba de un interruptor degenerado.

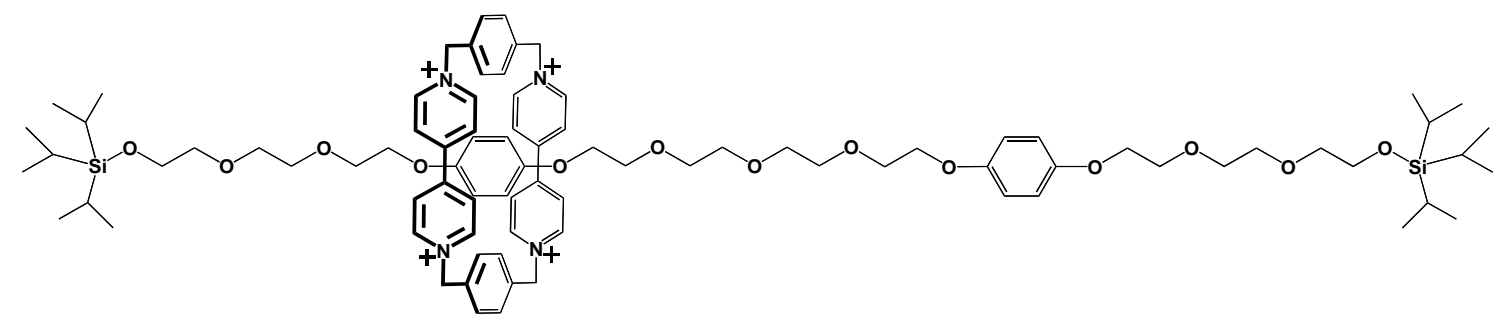

Figura 8. Primer interruptor molecular degenerado basado en un [2]rotaxano.

${ }^{36}$ Busseron, E.; Romuald, C.; Coutrot, F. Chem. Eur. J. 2010, 16, 10062-10073.

${ }^{37}$ Stoddart, J. F.; Anelli, P. L.; Spencer, N. J. Am. Chem. Soc. 1991, 113, 5131-5133. 
Desde entonces, este campo ha sido objeto de estudio intenso que llega hasta nuestros días, desarrollándose nuevos motivos de anclaje, métodos de conmutación entre estaciones, protocolos de síntesis y análisis de los mecanismos de conmutación. ${ }^{38}$ Recientemente, Sauvage, Stoddart y Feringa han sido galardonados con el Premio Nobel de Química de 2016 por su dilatada carrera científica relacionada con el diseño y síntesis de máquinas moleculares. ${ }^{39}$ Esto demuestra la importancia que tiene este campo de estudio.

\subsubsection{Interruptores moleculares como catalizadores conmutables}

Esta familia de compuestos dista mucho de ser mera curiosidad científica o juguete al servicio del investigador. El movimiento neto a nivel microscópico puede ser explotado. Así, por ejemplo, se han experimentado sus aplicaciones como transportadores de especies, ${ }^{40}$ como sensores fotoquímicos, ${ }^{41}$ elementos en dispositivos fotovoltaicos ${ }^{42}$ o puertas lógicas. ${ }^{43}$

38 Algunos ejemplos seleccionados de interruptores moleculares: (a) Balzani, V.; Stoddart, J. F.; Williams, D. J.; Ashton, P. R.; Ballardini, R.; Baxter, I.; Credi, A.; Fyfe, M. C. T.; Gandolfi, M. T.; Gómez-López, M.; Martínez-Díaz, M. V.; Piersanti, A.; Spencer, N.; Venturi, M.; White, A. J. P. J. Am. Chem. Soc. 1998, 120, 11932-11942. (b) Stoddart, J. F.; Kang, S.; Vignon, S. A.; Tseng, H.-R. Chem. Eur. J. 2004, 10, 2555-2564. (c) Garaudée, S.; Silvi, S.; Venturi, M.; Credi, A.; Flood, A. H.; Stoddart, J. F. Chem. Phys. Chem. 2005, 6, 2145-2152. (d) Loeb, S. J. Chem. Soc. Rev. 2007, 36, 226-235. (e) Coutrot, F.; Busseron, E. Chem. Eur. J. 2008, 14, 4784-4787. (f) Fioravanti, G.; Haraszkiewicz, N.; Kay, E. R.; Mendoza, S. M.; Bruno, C.; Marcaccio, M.; Wiering, P. G.; Paolucci, F.; Rudolf, P.; Brouwer, A. M.; Leigh, D. A. J. Am. Chem. Soc. 2008, 130, 2593-2601. (g) Hirose, K.; Shiba, Y.; Ishibashi, K.; Doi, Y.; Tobe, Y. Chem. Eur. J. 2008, 14, 3427-3433. (h) Bodis, P.; Panman, M. R.; Bakker, B. H.; MateoAlonso, A.; Prato, M.; Buma, W. J.; Brouwer, A. M.; Kay, E. R.; Leigh, D. A.; Woutersen, S. Acc. Chem. Res. 2009, 42, 1462-1469. (i) Durola, F.; Lux, J.; Sauvage, J.-P. Chem. Eur. J. 2009, 15, 4124-4134. (j) Lin, T.-C.; Lai, C.-C.; Chiu, S.-H. Org. Lett. 2009, 11, 613-616. (k) Phoa, K.; Neaton, J. B.; Subramanian, V. Nano Lett. 2009, 9, 3225-3229. (1) Coutrot, F.; Busseron, E. Chem. Eur. J. 2009, 15, 5186-5190. 37. (m) Chiu, S.-H.; Hsueh, S.-Y.; Lai, C.-C. Chem. Eur. J. 2010, 16, 2997-3000. (n) Sauvage, J.-P.; Collin, J.-P.; Heitz, V.; Durola, F.; Reviriego, F.; Trolez, Y. Angew. Chem. Int. Ed. 2010, 49, 10172-10175. (o) Tramontozzi, D. A.; Suhan, N. D.; Eichhorn, S. H.; Loeb, S. J. Chem. Eur. J. 2010, 16, 4466-4476. (p) Carlone, A.; Goldup, S. M.; Lebrasseur, N.; Leigh, D. A.; Wilson, A. J. Am. Chem. Soc. 2012, 134, 8321-8323. (q) Liu, L.; Liu, Y.; Liu, P.; Wu, J.; Guan, Y.; Hu, X.; Lin, C.; Yang, Y.; Sun, X.; Ma, J.; Wang, L. Chem. Sci. 2013, 4, 1701-1706. (r) Durola, F.; Heitz, V.; Reviriego, F.; Roche, C.; Sauvage, J.-P.; Sour, A.; Trolez, Y. Acc. Chem. Res. 2014, 47, 633-645. (s) Liu, P.; Li, W.; Liu, L.; Wang, L.; Ma, J. J. Phys. Chem. A 2014, 118, 9032-9044. (t) Olson, M. A. Nat. Chem. 2015, 7, 470-471.

39 "The Nobel Prize in Chemistry 2016". Nobelprize.org. Nobel Media AB 2014. Web. 9 Dec 2016. $<$ http://www.nobelprize.org/nobel_prizes/chemistry/laureates/2016/>

${ }_{40}$ (a) Hess, H.; Vogel, V. Rev. Mol. Biotechnol. 2001, 82, 67-85. (b) Makita, Y.; Kihara, N.; Takata, T. J. Org. Chem. 2008, 73, 9245-9250. (c) Lüning, U.; Hesseler, B.; Zindler, M.; Herges, R. Eur. J. Org. Chem. 2014, 2014, 3885-3901. (d) Meng, Z.; Xiang, J.-F.; Chen, C.-F. J. Am. Chem. Soc. 2016, 138, $5652-5658$.

${ }^{41}$ (a) Koyama, Y.; Matsumura, T.; Yui, T.; Ishitani, O.; Takata, T. Org. Lett. 2013, 15, 4686-4689. (b) Nitta, T.; Hess, H. Cell. Mol. Bioeng. 2013, 6, 109-115. (c) Zhang, J.-N.; Li, H.; Zhou, W.; Yu, S.-L.; Qu, D.-H.; Tian, H. Chem. Eur. J. 2013, 19, 17192-17200. (d) Sun, N.; Xiao, X.; Li, W.; Jiang, J. Adv. Sci. 2015, 2, 1500082.

42 Balzani, V. Photochem. Photobiol. Sci. 2003, 2, 459-476.

${ }^{43}$ de Silva, A. P.; McClenaghan, N. D. Chem. Eur. J. 2004, 10, 574-586. 
Conectados a nanoestructuras o sólidos es posible producir efectos a nivel macroscópico, como cambios controlados en las propiedades ópticas y mecánicas de materiales, modificaciones de la tensión superficial de líquidos y para controlar el vaciado en partículas nanoporosas que contienen compuestos de interés. ${ }^{44}$

Todos estos ejemplos aquí expuestos han llevado a llamar a este vasto grupo de moléculas entrelazadas como dispositivos, máquinas o motores moleculares por su semejanza con sus equivalentes macroscópicos, sólo que éstas operan a escala nanométrica donde las interacciones supramoleculares dominan sobre las fuerzas inerciales. ${ }^{45}$

La explotación del movimiento a través del anillo en un rotaxano con fines catalíticos también ha sido explorada desde principios de siglo. ${ }^{46}$ Pero no es hasta 2012 cuando Leigh y colaboradores estudiaron la catálisis conmutable ${ }^{47}$ posteriormente desarrollada empleando otros motivos. ${ }^{48}$ Consiste en la cobertura o exposición, por parte del anillo macrocíclico, del centro catalítico situado en el eje gracias a un estímulo externo, como se muestra en el Esquema 6. En dicho esquema, la unidad catalítica (en amarillo) está dentro de la cavidad del anillo, impidiendo que las especies a reaccionar (cubo y prisma triangular) se aproximen a dicho centro. Si se cambia la afinidad del anillo por la estación, éste se moverá a la otra, exponiendo el centro catalítico (ahora en gris) permitiendo la catálisis y generándose los productos de reacción. Si se usa exclusivamente el eje, independientemente el estado en el que esté la estación (amarillo o gris en el esquema), se producirá catálisis ya que no existe un impedimento estérico impuesto por el macrociclo.

44 (a) Schalley, C. A.; Vögtle, F.; Beizai, K. Acc. Chem. Res. 2001, 34, 465-476. (b) Hsueh, S.-Y.; Kuo, C.-T.; Lu, T.-W.; Lai, C.-C.; Liu, Y.-H.; Hsu, H.-F.; Peng, S.-M.; Chen, C.-h.; Chiu, S.-H. Angew. Chem. Int. Ed. 2010, 49, 9170-9173. (c) Fahrenbach, A. C.; Warren, S. C.; Incorvati, J. T.; Avestro, A.-J.; Barnes, J. C.; Stoddart, J. F.; Grzybowski, B. A. Adv. Mater. 2013, 25, 331-348. (d) Fu, J.; Chen, T.; Wang, M.; Yang, N.; Li, S.; Wang, Y.; Liu, X. ACS Nano 2013, 7, 11397-11408. (e) Erbas-Cakmak, S.; Leigh, D. A.; McTernan, C. T.; Nussbaumer, A. L. Chem. Rev. 2015, 115, 10081-10206. (f) Kay, E. R.; Leigh, D. A. Angew. Chem. Int. Ed. 2015, 54, 10080-10088. (g) Xue, M.; Yang, Y.; Chi, X.; Yan, X.; Huang, F. Chem. Rev. 2015, 115, 7398-7501.

${ }^{45}$ Leigh, D. A.; Zerbetto, F.; Kay, E. R. Angew. Chem. Int. Ed. 2007, 46, 72-191.

46 (a) Thordarson, P.; Bijsterveld, E. J. A.; Rowan, A. E.; Nolte, R. J. M. Nature 2003, 424, 4. (b) Takata, T.; Tachibana, Y.; Kihara, N. J. Am. Chem. Soc. 2004, 126, 3438-3439. (c) Miyagawa, N.; Watanabe, M.; Matsuyama, T.; Koyama, Y.; Moriuchi, T.; Hirao, T.; Furusho, Y.; Takata, T. Chem. Commun. 2010, 46, 1920-1922. (d) Leigh, D. A.; Lewandowski, B.; Bo, G. D.; Ward, J. W.; Papmeyer, M.; Kuschel, S.; Aldegunde, M. J.; Gramlich, P. M. E.; Heckmann, D.; Goldup, S. M.; D’Souza, D. M.; Fernandes, A. E. Science 2013, 339, 5. (e) Galli, M.; Lewis, J. E. M.; Goldup, S. M. Angew. Chem. Int. Ed. 2015, 54, 13545-13549. (f) Hoekman, S.; Kitching, M. O.; Leigh, D. A.; Papmeyer, M.; Roke, D. J. Am. Chem. Soc. 2015, 137, 7656-7659. (g) Goldup, S. M. Nat. Chem. 2016, 8, 404-406.

${ }^{47}$ Leigh, D. A.; Blanco, V.; Carlone, A.; Hänni, K. D.; Lewandowski, B. Angew. Chem. Int. Ed. 2012, 51, 5166-5169.

48 (a) Leigh, D. A.; Blanco, V.; Marcos, V.; Morales-Serna, J. A.; Nussbaumer, A. L. J. Am. Chem. Soc. 2014, 136, 4905-4908. (b) Leigh, D. A.; Marcos, V.; Wilson, M. R. ACS Catal. 2014, 4, 4490-4497. (c) Blanco, V.; Leigh, D. A.; Marcos, V. Chem. Soc. Rev. 2015, 44, 5341-5370. (d) Leigh, D. A.; Beswick, J.; Blanco, V.; De Bo, G.; Lewandowska, U.; Lewandowski, B.; Mishiro, K. Chem. Sci. 2015, 6, 140-143. (e) Kwan, C.-S.; Chan, A. S. C.; Leung, K. C.-F. Org. Lett. 2016, 18, 976-979. 

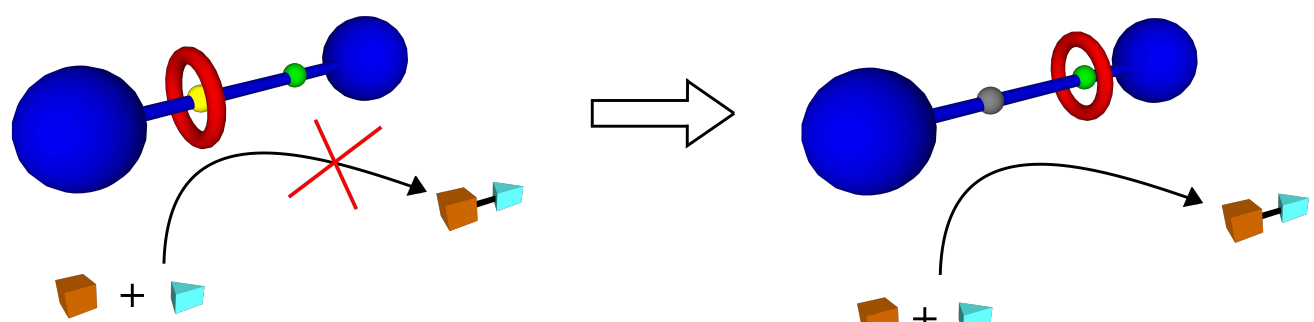

$+1$
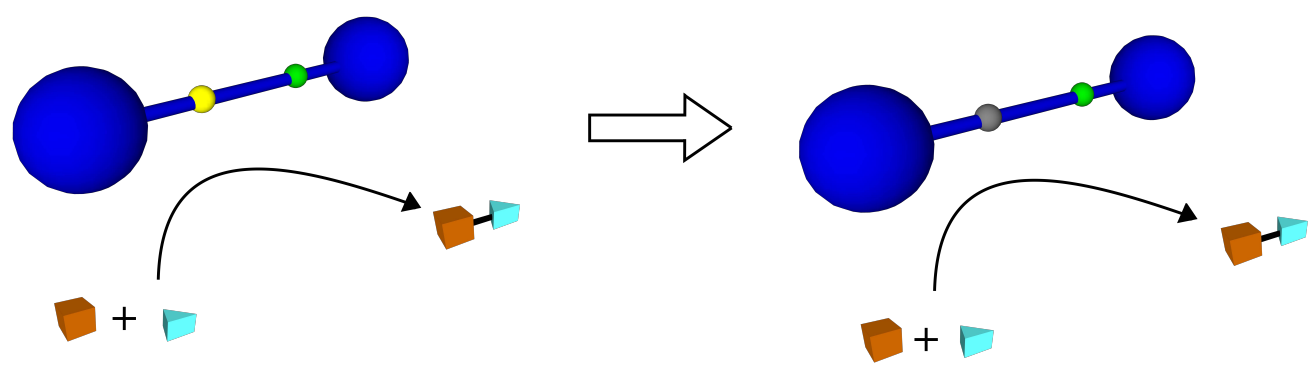

Esquema 6. Representación del funcionamiento diferencial de un organocatalizador conmutable basado en [2] rotaxano (arriba) y un organocatalizador común (abajo).

Estas máquinas moleculares permiten llegar a controlar tanto la velocidad de un proceso químico como el resultado del mismo si se usan varios centros catalíticos. El Artículo I de esta tesis tratará sobre la síntesis y estudio de rotaxanos capaces de realizar catálisis conmutable. Se estudiará el movimiento del macrociclo a lo largo del eje en interruptores moleculares con varias parejas de estaciones y se observará el impacto que produce la existencia de más centros catalíticos en su estructura.

\subsection{DERIVADOS DE AZOBENCENO}

Debido a que este especial grupo ha sido usado en dos de los trabajos descritos en esta memoria y que constituye un nexo de unión entre los dos bloques temáticos principales, se realizará aquí una breve introducción al mismo.

La molécula de azobenceno consiste en dos anillos fenílicos unidos por enlace $-\mathrm{N}=\mathrm{N}-$, como se representa en el Esquema $7 .^{49}$ Desde su descubrimiento en 1894 por Mitscherlich, ${ }^{50}$ ha suscitado el interés de muchos científicos por su capacidad de fotoisomerizarse reversiblemente y por sus interesantes aplicaciones para colorantes en la industria.

Esta molécula admite una gran variedad de tipos de funcionalizaciones ya que los grupos fenílicos se comportan de la misma manera que un grupo areno, por lo que se puede realizar todas las transformaciones que se llevan acabo en ellos. Además, los nitrógenos son capaces de formar complejos con metales de transición gracias a su capacidad dadora. ${ }^{51}$

49 McNaught, A. D.; Wilkinson, A. IUPAC. Compendium of Chemical Terminology, 2nd ed. (the "Gold Book"). Blackwell Scientific Publications: Oxford, 1997. 73.

${ }^{50}$ Mitscherlich, E. Ann. Pharma. 1834, 12, 311-314.

${ }^{51}$ Algunos ejemplos seleccionados: (a) Cope, A. C.; Siekman, R. W. J. Am. Chem. Soc. 1965, 87, 3272-3273. (b) Coe, B. J.; Blackburn, O. A. Organometallics 2011, 30, 2212-2222. (c) Coe, B. J.; Blackburn, O. A.; Helliwell, M. Organometallics 2011, 30, 4910-4923. 
La preparación de estos compuestos se puede realizar a través de muchos métodos, como por ejemplo reducción de nitroareno, condensación de nitrosoarenos y anilinas, etc. Sin embargo, uno de los más usados es el acoplamiento de sales de diazonio. ${ }^{52}$

\subsubsection{Fotoisomerización reversible del azobenceno}

La principal característica de esta especie es la isomerización fotoinducida del isómero configuracional $\mathrm{E}$ al $\mathrm{Z}$ con irradiación UV de longitudes de onda aproximadas en torno a 365 $\mathrm{nm}$ (ubicación habitual de la banda de transición $\pi-\pi^{*}$ ). El isómero $\mathrm{Z}$ es menos estable y espontáneamente evoluciona al $\mathrm{E}$ (fenómeno que se conoce como relajación térmica) a temperatura ambiente. Este proceso puede ser acelerado al aumentar la temperatura o irradiando con longitudes de onda superiores a 400nm (donde se sitúa normalmente la banda de transición $\left.n-\pi^{*}\right)$. Este proceso se encuentra reflejado en el Esquema 7. Se han estudiado los distintos mecanismo por los que este fenómeno se lleva a cabo y depende mucho de la naturaleza de sus sustituyentes. ${ }^{53}$
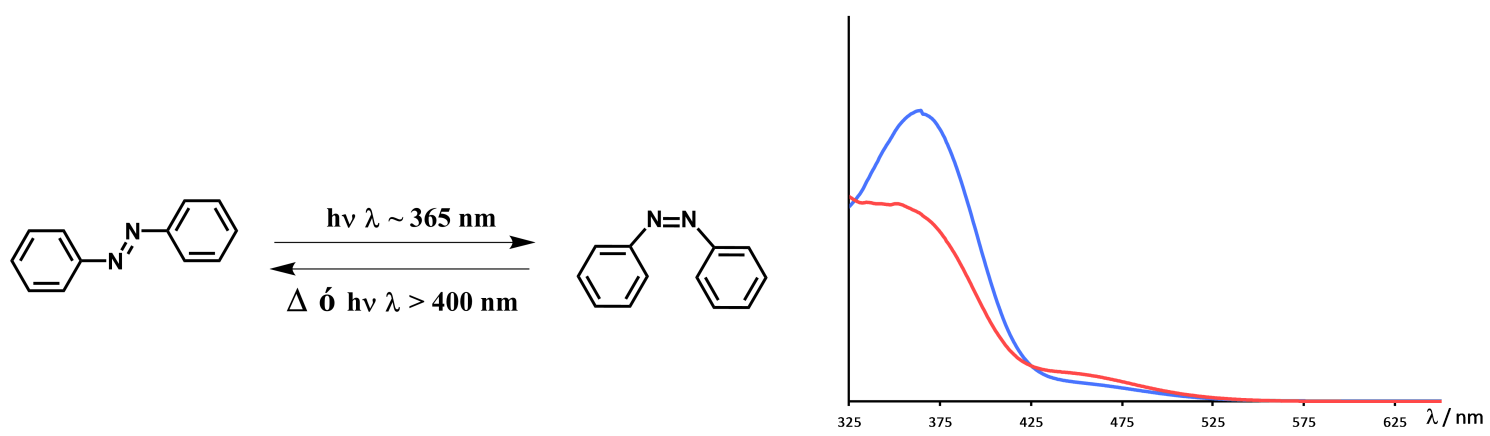

Esquema 7. Fotoisomerización reversible de la molécula de azobenceno (izquierda). Típico espectro UV/Vis de un derivado de azobenceno (derecha). En azul, isómero E; en rojo, isómero Z.

Esta capacidad ha sido usada en multitud de aplicaciones obteniéndose máquinas moleculares que responden a irradiación para modificar las características del sistema y obtener unas propiedades deseadas. ${ }^{54}$ Nosotros nos centraremos en su inclusión en moléculas entrelazadas mecánicamente y en su capacidad para formar complejos macromoleculares con fullerenos.

52 Merino, E. Chem. Soc. Rev. 2011, 40, 3835-3853.

53 (a) Cembran, A.; Bernardi, F.; Garavelli, M.; Gagliardi, L.; Orlandi, G. J. Am. Chem. Soc. 2004, 126, 3234-3243. (b) Burdette, S. C.; Bandara, H. M. D. Chem. Soc. Rev. 2012, 41, 1809-1825. (c) Schweighauser, L.; Strauss, M. A.; Bellotto, S.; Wegner, H. A. Angew. Chem. Int. Ed. 2015, 54, 1343613439.

${ }^{54}$ (a) Giordani, S.; Natali, M. Chem. Soc. Rev. 2012, 41, 4010-4029. (b) Gostl, R.; Senf, A.; Hecht, S. Chem. Soc. Rev. 2014, 43, 1982-1996. (c) Bléger, D.; Hecht, S. Angew. Chem. Int. Ed. 2015, 54, 1133811349. (d) Qu, D.-H.; Wang, Q.-C.; Zhang, Q.-W.; Ma, X.; Tian, H. Chem. Rev. 2015, 115, 7543-7588. 


\subsubsection{Integración en moléculas entrelazadas mecánicamente}

El grupo azobencénico ha sido también explotado formando parte de estructuras de catenanos y rotaxanos. ${ }^{55} \mathrm{Su}$ fotoisomerización ha servido para intentar controlar la formación de un aducto supramolecular, el movimiento del macrociclo a lo largo de un eje o incorporar un nuevo tipo de estímulo en puertas lógicas.

El ejemplo más habitual encontrado en bibliografía es la incorporación de este grupo en el eje de un rotaxano. Ya bien sea en el centro del mismo o en los extremos, puede favorecer o inhibir el movimiento del macrociclo en función de la estructura del fragmento azobencénico suponiendo un impedimento estérico o no. Otros motivos muy usados se encuentran en la formación de complejos de inclusión con ciclodextrinas o curcubiturilos, actuando el azobenceno como estación del anillo cuando se encuentra como isómero E, pero dejando de serlo una vez se fotoisomeriza al Z.
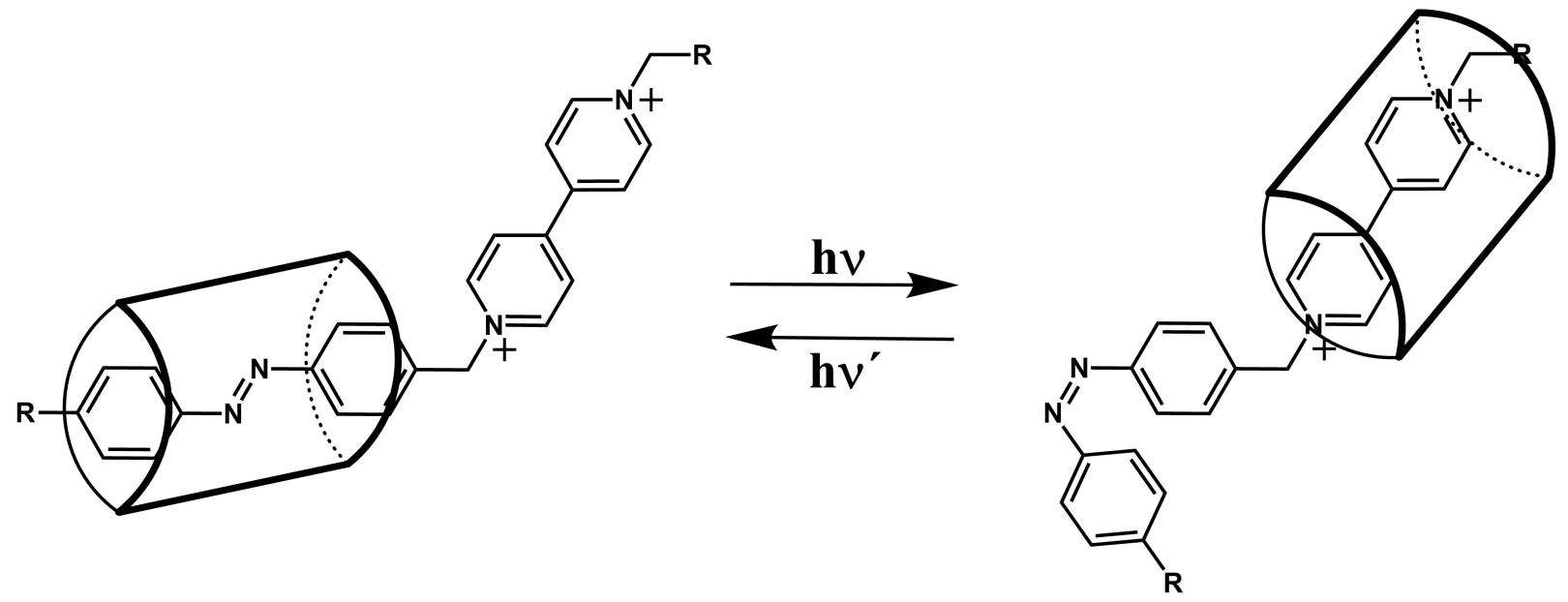

Esquema 8. Ejemplo de funcionamiento de un interruptor molecular que posee un fragmento azobencénico en el eje.

55 (a) Murakami, H.; Kawabuchi, A.; Kotoo, K.; Kunitake, M.; Nakashima, N. J. Am. Chem. Soc. 1997, 119, 7605-7606. (b) Balzani, V.; Credi, A.; Venturi, M. Chem. Soc. Rev. 2009, 38, 1542-1550. (c) Stoddart, J. F.; Coskun, A.; Friedman, D. C.; Li, H.; Patel, K.; Khatib, H. A. J. Am. Chem. Soc. 2009, 131, 2493-2495. (d) Credi, A.; Silvi, S.; Venturi, M. Chem. Commun. 2011, 47, 2483-2489. (e) Baroncini, M.; Silvi, S.; Venturi, M.; Credi, A. Angew. Chem. Int. Ed. 2012, 51, 4223-4226. (f) Stoddart, J. F.; Venturi, M.; Credi, A.; Avellini, T.; Li, H.; Coskun, A.; Barin, G.; Trabolsi, A.; Basuray, A. N.; Dey, S. K.; Silvi, S. Angew. Chem. Int. Ed. 2012, 51, 1611-1615. (g) Xue, M.; Yang, Y.; Chi, X.; Yan, X.; Huang, F. Chem. Rev. 2015, 115, 7398-7501. 
Es interesante destacar el interruptor molecular desarrollado por el grupo de Stoddart ${ }^{55 f}$ en el que el grupo de azobenceno se encuentra entre dos estaciones imponiendo un control cinético al movimiento del macrociclo cuando se encuentra como isómero Z. El fenómeno de conmutación se debe a una reacción redox y dicho control se ha hecho llamar "efecto memoria".

El Artículo II de la presente memoria tratará sobre interruptores moleculares basados en la pareja de estaciones sal de amonio/sal de triazolinio previamente estudiada en el Artículo I en los que se ha incluido un grupo azobenceno y su impacto en el movimiento del macrociclo con el objetivo de alcanzar mencionado control cinético.

\subsection{RECEPTORES DE FULLERENOS BASADOS EN MOLÉCULAS POLIAROMÁTICAS}

Dentro del concepto global de Química Supramolecular sobre el que descansa la presente tesis, hay una rama de especial estudio que trata sobre el diseño y estudio de asociaciones entre nanoestructuras de carbono y otras especies por las potenciales aplicaciones que presentan los aductos resultantes, como sistemas fotovoltaicos, sensores fotoquímicos o una mejor solubilidad en disolventes en los que dichas nanoestructuras no son solubles. ${ }^{56}$ En esta memoria se hará especial hincapié en la asociación de fullerenos con hidrocarburos policíclicos aromáticos.

\subsubsection{Fullerenos y otros compuestos de carbono nanoestructurados}

El elemento carbono posee una considerable cantidad de alótropos, siendo los más conocidos el diamante y el grafito. Este último tiene una estructura formada por capas de hexágonos fusionados. La densidad electrónica $\pi$ totalmente deslocalizada en su superficie interacciona atractivamente con la de otra apilándose de manera infinita. ${ }^{57}$ Esta interacción se conoce como apilamiento $\pi$.

Una de las familias que más está siendo estudiada por sus excelentes propiedades ${ }^{58}$ es la formada por los nanoalótropos de carbono. Se considera que están en una situación a caballo entre el concepto moléculas y material propiamente dicho, y se suelen clasificar en función de la dimensionalidad de la estructura.

${ }^{56}$ (a) Pearce, J. M. Futures 2002, 34, 663-674. (b) Luque, A.; Hegedus, S., Handbook of Photovoltaic Science and Engineering. John Wiley \& Sons: West Sussex, 2003. (c) Segura, J. L.; Martin, N.; Guldi, D. M. Chem. Soc. Rev. 2005, 34, 31-47. (d) Swanson, R. M. Science 2009, 324, 891-892. (e) Delgado, J. L.; Bouit, P.-A.; Filippone, S.; Herranz, M. A.; Martin, N. Chem. Commun. 2010, 46, 4853-4865. (f) Branker, K.; Pathak, M. J. M.; Pearce, J. M. Renew. Sust. Ener. Rev. 2011, 15, 4470-4482. (g) Pérez, E. M. Pure Appl. Chem. 2011, 83, 201-211. (h) Schneider, H.-J., Applications of Supramolecular Chemistry. CRC Press: 2012.

57 (a) Fitzer, E.; Kochling, K. H.; Boehm, H. P.; Marsh, H. Pure Appl. Chem. 1995, 67, 473. (b) Delhaes, P., Graphite and Precursors. CRC Press: 2000.

58 (a) Akasaka, T.; Wudl, F.; Nagase, S., Chemistry of Nanocarbons. John Wiley \& Sons: West Sussex, 2010. (b) Krueger, A., Carbon Materials and Nanotechnology. Wiley-VCH Verlag: Weinheim, 2010. (c) Petrukhina, M. A.; Scott, L. T., Fragments of Fullerenes and Carbon Nanotubes: Designed Synthesis, Unusual Reactions, and Coordination Chemistry. John Wiley \& Sons: Hoboken, 2011. (d) Marcaccio, M.; Paolucci, F., Making and Exploiting Fullerenes, Graphene, and Carbon Nanotubes. Springer-Verlag: Heidelberg, 2014. (e) Georgakilas, V.; Perman, J. A.; Tucek, J.; Zboril, R. Chem. Rev. 2015, 115, 4744-4822. 
Así, el grupo 0D incluye, entre otros, a los fullerenos, cuya descripción se va a profundizar a continuación. El grupo 1D continene los nanotubos de carbono ${ }^{59} \mathrm{y}$, por último, el 2D, donde está encuadrado el conocido grafeno. ${ }^{60}$

Centrando nuestra atención en el grupo 0D, los mayores representantes son los fullerenos. Conocidos también como poliarenos geodésicos ${ }^{61}$ cerrados o buckyballs, poseen estructuras en formas pseudoesféricas.

El primero en ser descubierto fue el Buckminsterfullereno, $\mathrm{C}_{60}$, nombrado así en honor al arquitecto Buckminster Fuller que popularizó el diseño de la cúpula geodésica. Sintetizado por Kroto y colaboradores en 1985 y obtenido por puro azar a partir de la irradiación láser de grafito ${ }^{62}$ ha tenido gran repercusión en las últimas décadas y ha sido objeto de estudio desde entonces. ${ }^{63}$ Por ello recibieron el premio Nobel en $1991 .{ }^{64}$

$\mathrm{Su}$ estructura posee 60 átomos de carbono distribuidos en pentágonos y hexágonos dispuestos de tal forma que cada pentágono está rodeado por cinco hexágonos (Figura 9). Fue el pionero de los poliarenos geodésicos cerrados y supuso el punto de partida para la investigación y el descubrimiento de todos sus congéneres mayores.
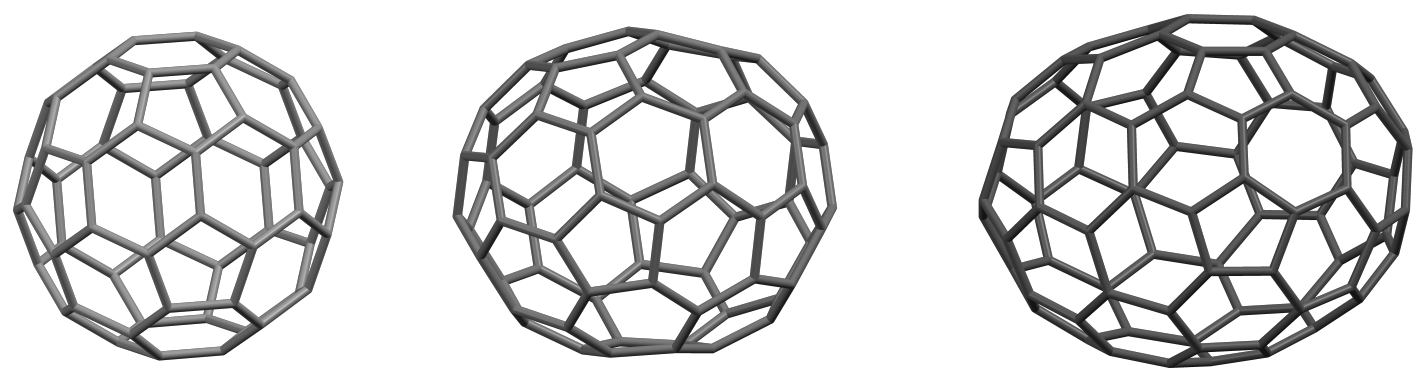

Figura 9. Estructura de las moléculas de $\mathrm{C}_{60}, \mathrm{C}_{70} \mathrm{y} \mathrm{C}_{86}$, algunos ejemplos de fullerenos.

${ }^{59}$ (a) Iijima, S. Nature 1991, 354, 56-58. (b) Bethune, D. S.; Klang, C. H.; de Vries, M. S.; Gorman, G.; Savoy, R.; Vazquez, J.; Beyers, R. Nature 1993, 363, 605-607. (c) Iijima, S.; Ichihashi, T. Nature 1993, 363, 603-605. (d) Cataldo, S.; Salice, P.; Menna, E.; Pignataro, B. Energy Environ. Sci. 2012, 5, 5919-5940. (e) De Volder, M. F. L.; Tawfick, S. H.; Baughman, R. H.; Hart, A. J. Science 2013, 339, 535-539.

60 (a) Novoselov, K. S.; Geim, A. K.; Morozov, S. V.; Jiang, D.; Zhang, Y.; Dubonos, S. V.; Grigorieva, I. V.; Firsov, A. A. Science 2004, 306, 666-669. (b) Cai, M.; Thorpe, D.; Adamson, D. H.; Schniepp, H. C. J. Mater. Chem. 2012, 22, 24992-25002. (c) Chang, D. W.; Choi, H.-J.; Filer, A.; Baek, J.-B. J. Mater. Chem. A 2014, 2, 12136-12149. (d) Fan, X.; Zhang, G.; Zhang, F. Chem. Soc. Rev. 2015, 44, 3023-3035.

${ }^{61}$ (a) Siegel, J. S.; Wu, Y.-T., Polyarenes II. Springer International Publishing: London, 2014. (b) Siegel, J. S.; Wu, Y.-T., Polyarenes I. Springer: Heidelberg, 2014.

${ }^{62}$ Kroto, H. W.; Heath, J. R.; O'Brien, S. C.; Curl, R. F.; Smalley, R. E. Nature 1985, 318, 162-163.

63 (a) Curl, R. F. Angew. Chem. Int. Ed. 1997, 36, 1566-1576. (b) Kroto, H. Angew. Chem. Int. Ed. 1997, 36, 1578-1593. (c) Smalley, R. E. Angew. Chem. Int. Ed. 1997, 36, 1594-1601.

64 "The Nobel Prize in Chemistry 1996". Nobelprize.org. Nobel Media AB 2014. Web. 12 Sep 2016. $<$ http://www.nobelprize.org/nobel_prizes/chemistry/laureates/1996/> 
Además de ser una estructura curiosa, ha demostrado tener interesantísimas aplicaciones en sistemas fotoelectrónicos ya que es un aceptor de carga excelente. Esto puede realizarse en un aducto supramolecular donde ocurre una transferencia electrónica fotoinducida desde un fragmento dador, a través de interacciones no covalentes, hacia el fullereno. ${ }^{65}$

\subsubsection{Coranuleno y otras moléculas poliaromáticas}

Las moléculas poliaromáticas planas son entidades discretas formadas por la fusión de más de un anillo bencénico y se pueden considerar como fragmentos muy pequeños de una lámina de grafito (Figura 10). ${ }^{66}$ Debido a sus propiedades ópticas y su capacidad intrínseca de establecer los apilamientos antes mencionados, al margen de su toxicidad, ${ }^{67}$ han sido muy usadas para distintas aplicaciones. $^{68}$<smiles>c1ccc2cc3ccccc3cc2c1</smiles>

b<smiles>c1ccc2c(c1)c1ccccc1c1ccccc21</smiles>

c<smiles></smiles><smiles>c1cc2ccc3cccc4ccc(c1)c2c34</smiles>

Figura 10. Algunos ejemplos de PAHs planos. (a) Antraceno. (b) Trisfenileno. (c) Perileno. (d) Pireno.

Si la fusión de anillos bencénicos incluye algún defecto como, por ejemplo, la inclusión de anillos de tamaño superior o inferior, o la exclusiva fusión en orto de los mismos, da lugar a especies poliaromáticas no planas debido a las fuerzas de repulsión generadas (Figura 11). ${ }^{69}$

65 (a) Taylor, R.; Walton, D. R. M. Nature 1993, 363, 685-693. (b) Dresselhaus, M. S.; Dresselhaus, G.; Eklund, P. C., Science of Fullerenes and Carbon Nanotubes. Academic Press: 1996. (c) Hirsch, A., Fullerenes and Related Structures. Springer: Heidelberg, 1999. (d) Guldi, D. M.; Martin, N., Fullerenes: From Synthesis to Optoelectronic Properties. Springer: Dordrecht, 2002. (e) Martin, N.; Nierengarten, J.F., Supramolecular Chemistry of Fullerenes and Carbon Nanotubes. Wiley-VCH Verlag: Weinheim, 2012.

66 (a) Moss, G. P. Pure Appl. Chem. 1998. (b) Feng, X.; Pisula, W.; Müllen, K. Pure Appl. Chem. 2009, 81, 2203.

${ }^{67}$ (a) Boström, C.-E.; Gerde, P.; Hanberg, A.; Jernström, B.; Johansson, C.; Kyrklund, T.; Rannug, A.; Törnqvist, M.; Victorin, K.; Westerholm, R. Environ. Health. Persp. 2002, 110, 451-488. (b) Baird, W. M.; Hooven, L. A.; Mahadevan, B. Environ. Mol. Mutagen. 2005, 45, 106-114.

68 Algunos ejemplos seleccionados: (a) v. Bünau, G. Ber. Bunsenges. Phys. Chem. 1970, 74, 12941295. (b) Nagao, Y. Prog. Org. Coat. 1997, 31, 43-49. (c) Manandhar, E.; Wallace, K. J. Inorg. Chim. Acta 2012, 381, 15-43. (d) Mulla, K.; Shaik, H.; Thompson, D. W.; Zhao, Y. Org. Lett. 2013, 15, 45324535. (e) Nielsen, C. B.; Holliday, S.; Chen, H.-Y.; Cryer, S. J.; McCulloch, I. Acc. Chem. Res. 2015, 48, 2803-2812. (f) Ronson, T. K.; Roberts, D. A.; Black, S. P.; Nitschke, J. R. J. Am. Chem. Soc. 2015, 137, 14502-14512. (g) Peris, E. Chem. Commun. 2016, 52, 5777-5787.

${ }^{69}$ (a) Newman, M. S.; Lednicer, D. J. Am. Chem. Soc. 1956, 78, 4765-4770. (b) Barth, W. E.; Lawton, R. G. J. Am. Chem. Soc. 1966, 88, 380-381. (b) . (c) Lawton, R. G.; Barth, W. E. J. Am. Chem. Soc. 1971, 93, 1730-1745. (d) Yamamoto, K.; Harada, T.; Nakazaki, M.; Naka, T.; Kai, Y.; Harada, S.; Kasai, N. J. Am. Chem. Soc. 1983, 105, 7171-7172. 
a

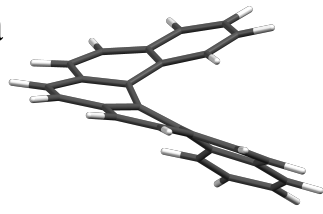

(1) b
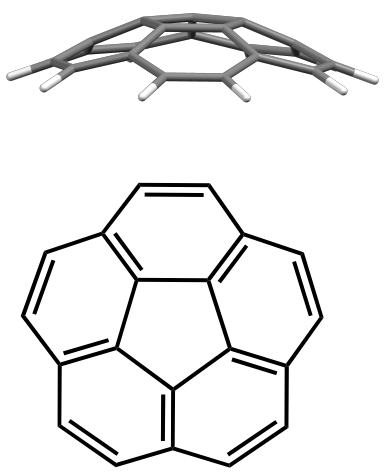

c
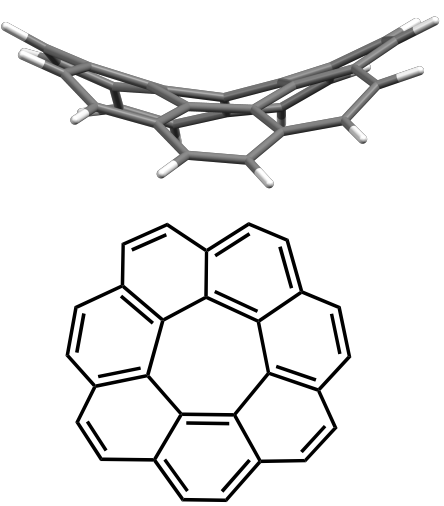

Figura 11. Algunos ejemplos de moléculas poliaromáticas no planas. (a) [6]Heliceno. (b) Coranuleno. (c) [7]Circuleno.

En concreto, el coranuleno o [5]circuleno es una molécula de fórmula $\mathrm{C}_{20} \mathrm{H}_{10}$, un poliareno geodésico abierto, o buckybowl, que consta de 5 anillos hexagonales que rodean uno pentagonal. Presenta forma tridimensional debido a la tensión producida por esta disposición particular, obligando a adaptar su estructura en forma de cuenco para aliviarla con una profundidad de $0,87 \AA$.

De esta manera, tiene dos caras diferenciadas, lo que resulta en un momento dipolar neto, pero pueden invertirse en un equilibrio fluxional que se producirá o no a temperatura ambiente dependiendo de su sustitución. ${ }^{70}$

Desde el punto de vista químico, se comporta como un areno y, por tanto, ha sido objeto de mucho estudio sintético obteniéndose derivados muy diversos. ${ }^{71}$

70 (a) Scott, L. T.; Hashemi, M. M.; Bratcher, M. S. J. Am. Chem. Soc. 1992, 114, 1920-1921. (b) Seiders, T. J.; Baldridge, K. K.; Grube, G. H.; Siegel, J. S. J. Am. Chem. Soc. 2001, 123, 517-525. (c) Nishida, S.; Morita, Y.; Ueda, A.; Kobayashi, T.; Fukui, K.; Ogasawara, K.; Sato, K.; Takui, T.; Nakasuji, K. J. Am. Chem. Soc. 2008, 130, 14954-14955. (d) Sanyal, S.; Manna, A. K.; Pati, S. K. Chem. Phys. Chem. 2014, 15, 885-893. (e) Siegel, J. S.; Wu, Y.-T., Synthesis, Structures, and Physical Properties of Aromatic Molecular-Bowl Hydrocarbons. In Polyarenes I, Springer: Heidelberg, 2014; pp 63-120.

${ }^{71}$ Algunos ejemplos seleccionados: (a) Wu, Y.-T.; Siegel, J. S. Chem. Rev. 2006, 106, 4843-4867. (b) Sygula, A. Eur. J. Org. Chem. 2011, 2011, 1611-1625. (c) Lentz, D.; Topolinski, B.; Schmidt, B. M.; Kathan, M.; Troyanov, S. I. Chem. Commun. 2012, 48, 6298-6300. (d) Stuparu, M. C. Tetrahedron 2012, 68, 3527-3531. (e) Wu, Y.-L.; Stuparu, M. C.; Boudon, C.; Gisselbrecht, J.-P.; Schweizer, W. B.; Baldridge, K. K.; Siegel, J. S.; Diederich, F. J. Org. Chem. 2012, 77, 11014-11026. (f) Furrer, F.; Linden, A.; Stuparu, M. C. Chem. Eur. J. 2013, 19, 13199-13206. (g) Lentz, D.; Topolinski, B.; Schmidt, B. M.; Schwagerus, S.; Kathan, M. Eur. J. Inorg. Chem. 2014, 2014, 5391-5405. (h) Mattarella, M.; Berstis, L.; Baldridge, K. K.; Siegel, J. S. Bioconjug. Chem. 2014, 25, 115-128. (i) Wu, D.; Shao, T.; Men, J.; Chen, X.; Gao, G. Dalton Trans. 2014, 43, 1753-1761. (j) Da Ros, S.; Linden, A.; Baldridge, K. K.; Siegel, J. S. Org. Chem. Front. 2015, 2, 626-633. (k) George, S. R. D.; Frith, T. D. H.; Thomas, D. S.; Harper, J. B. Org. Biomol. Chem. 2015, 13, 9035-9041. (1) San, L. K.; Clikeman, T. T.; Dubceac, C.; Popov, A. A.; Chen, Y.-S.; Petrukhina, M. A.; Strauss, S. H.; Boltalina, O. V. Chem. Eur. J. 2015, 21, 9488-9492. (m) Li, X.; Kang, F.; Inagaki, M. Small 2016, 12, 3206-3223. (n) Rajeshkumar, V.; Lee, Y. T.; Stuparu, M. C. Eur. J. Org. Chem. 2016, 2016, 36-40. (o) Rajeshkumar, V.; Marc, C.; Fichou, D.; Stuparu, M. C. Synlett 2016. 
También es capaz de sufrir coordinación tanto $\sigma$ como $\pi$. $^{72}$ Ésta última es particularmente interesante debido a la existencia de dos caras diferenciadas, de la posibilidad de coordinación a varios anillos y los efectos producidos sobre la curvatura de esta molécula.

\subsubsection{Síntesis de coranuleno}

Obtenido por primera vez por el grupo de Lawton en 1966 tras un proceso tedioso que conllevaba numerosas etapas sintéticas. ${ }^{73}$ Aquello motivó el desarrollo de otras metodologías para obtener esta molécula más eficazmente. Así, hubo muchos avances basados en preparación usando técnicas de pirolización a vacío, o Flash Vacuum Pyrolysis, que mejoraron notablemente el rendimiento global de esta molécula. ${ }^{74}$ Esto, además, permitió la preparación de homólogos superiores. Sin embargo, las posibles rutas sintéticas que evitasen dicha técnica continuaron en paralelo. Así, los avances producidos a finales del siglo XX, especialmente los aportados por Sygula y Rabideau, fueron los más significativos, ya que consiguieron obtener la molécula objetivo en una cantidad de pasos relativamente baja y con procedimientos asequibles para cualquier laboratorio de investigación, tal y como se muestra en el Esquema 9. ${ }^{75}$ En este protocolo se sintetiza la molécula de 2,7-dimetilnaftaleno sobre la que se va construyendo poco a poco un tercer ciclo. El éxito sobre el que esta síntesis descansa es en la doble sustitución nucleófila interna que se produce en el compuesto 1,6,7,10-tetrakis(dibromometilen)fluoranteno para dar lugar a 1,2,5,6-tetrabromocoranuleno con alto rendimiento. Se generan los dos ciclos restantes en un único paso.

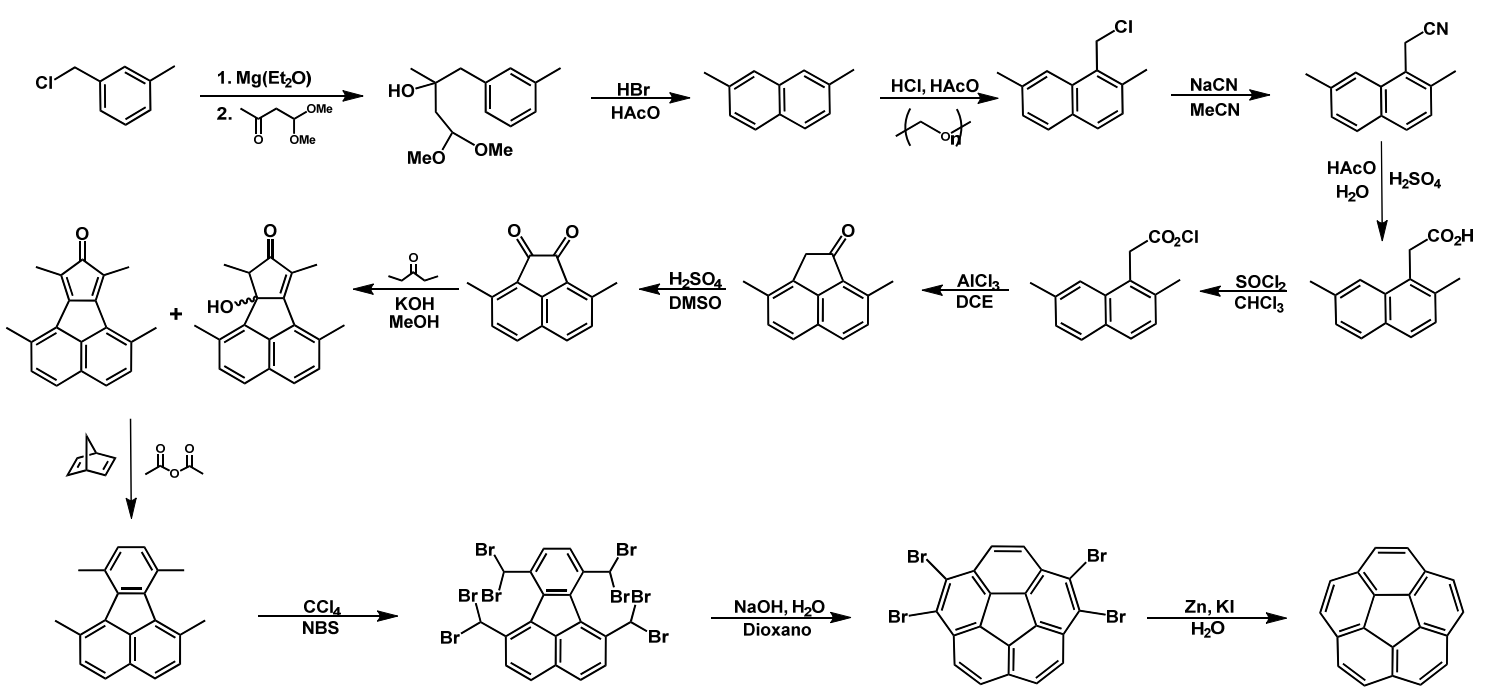

Esquema 9. Síntesis optimizada de coranuleno por el grupo de Sygula a principios del s. XXI.

${ }^{72}$ Algunos ejemplos seleccionados: (a) Seiders, T. J.; Baldridge, K. K.; O'Connor, J. M.; Siegel, J. S. J. Am. Chem. Soc. 1997, 119, 4781-4782. (b) Angelici, R. J.; Sygula, A.; Alvarez, C. M.; Sygula, R.; Rabideau, P. W. Organometallics 2003, 22, 624-626. (c) Angelici, R. J.; Sygula, A.; Vecchi, P. A.; Alvarez, C. M.; Ellern, A.; Sygula, R.; Rabideau, P. W. Organometallics 2005, 24, 4543-4552. (d) Sharp, P. R.; Lee, H. B. Organometallics 2005, 24, 4875-4877. (e) Yamada, M.; Tashiro, S.; Miyake, R.; Shionoya, M. Dalton Trans. 2013, 42, 3300-3303.

${ }^{73}$ (a) Barth, W. E.; Lawton, R. G. J. Am. Chem. Soc. 1966, 88, 380-381. (b) Lawton, R. G.; Barth, W. E. J. Am. Chem. Soc. 1971, 93, 1730-1745.

${ }^{74}$ Tsefrikas, V. M.; Scott, L. T. Chem. Rev. 2006, 106, 4868-4884.

75 (a) Siegel, J. S.; Seiders, T. J.; Elliott, E. L.; Grube, G. H. J. Am. Chem. Soc. 1999, 121, 7804-7813. (b) Sygula, A.; Rabideau, P. W. J. Am. Chem. Soc. 2000, 122, 6323-6324. (c) Xu, G.; Sygula, A.; Marcinow, Z.; Rabideau, P. W. Tetrahedron Lett. 2000, 41, 9931-9934. 
Actualmente se usa una versión modificada de este procedimiento que evita parte de las etapas iniciales produciendo una doble acilación de Friedel-Crafts en el dimetil naftaleno (Esquema 10). Esta estrategia genera dos regioisómeros, obligando a separar el no deseado derivatizándolo con el reactivo de Girard y lavando el material con agua. Se ha demostrado que este inconveniente queda totalmente superado por el ahorro en pasos sintéticos que genera. ${ }^{76}$

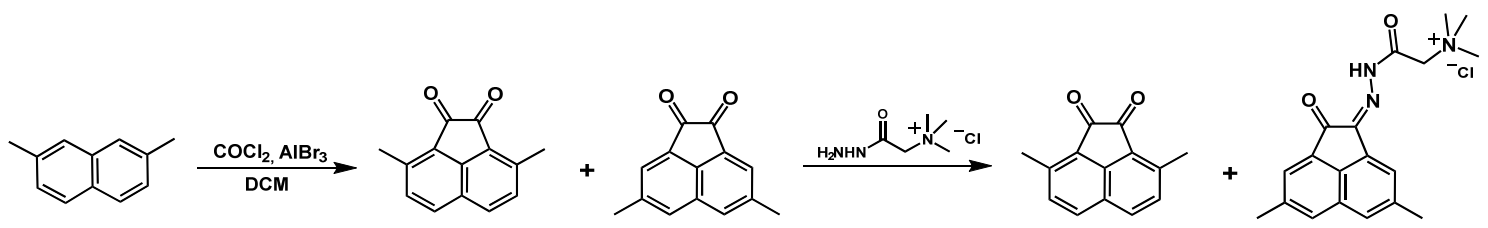

Esquema 10. Pasos clave modificados en la síntesis moderna de coranuleno. El regioisómero no deseado reacciona específicamente con el reactivo de Girard y su producto se vuelve soluble en agua, permitiendo aislar el compuesto objetivo.

\subsubsection{Interacción de coranuleno con fullerenos}

Como se ha dicho anteriormente, el coranuleno tiene momento dipolar neto. Esto, junto con su estructura curva que complementa perfectamente la superficie externa del Buckminsterfullereno, ha promovido el estudio de posibles interacciones entre este compuesto y $\mathrm{C}_{60}$ a través de fuerzas supramoleculares de apilamiento $\pi$.

En principio, la molécula en sí no es capaz de establecer el aducto en disolución o, al menos, la interacción es tan pequeña que no se puede observar. Sin embargo, sí se ha obtenido la estructura de rayos $\mathrm{X}$ de la cocristalización de una mezcla de ambos compuestos. ${ }^{77}$

Sólo un conjunto de compuestos de coranuleno sustituidos por tioéteres preparados por el grupo de Scott mostraron cambios apreciables en desplazamientos químicos durante estudios de valoración por RMN en tolueno-d8, dando constantes de asociación moderadas. ${ }^{78}$ Sin embargo, los mejores resultados se han obtenido cuando más de una unidad de coranuleno se encuentran en la misma molécula, como se describe en el siguiente apartado.

\subsubsection{Pinzas moleculares de fullerenos que contienen unidades coranulénicas}

En 2007, el grupo de Sygula introdujo un gran avance en este campo. ${ }^{79}$ Describió un compuesto llamado coloquialmente como buckycatcher que contenía dos unidades de coranuleno unidas en una disposición tal que permanecían rígidas generando una pinza molecular con una cavidad del tamaño y topología adecuados para interaccionar con fullerenos (Figura 12), dando lugar a constantes de asociación muy buenas.

${ }^{76}$ Butterfield, A. M.; Gilomen, B.; Siegel, J. S. Org. Process Res. Dev. 2012.

${ }^{77}$ Dawe, L. N.; AlHujran, T. A.; Tran, H.-A.; Mercer, J. I.; Jackson, E. A.; Scott, L. T.; Georghiou, P. E. Chem. Commun. 2012, 48, 5563-5565.

${ }^{78}$ (a) Mizyed, S.; Georghiou, P. E.; Bancu, M.; Cuadra, B.; Rai, A. K.; Cheng, P.; Scott, L. T. J. Am. Chem. Soc. 2001, 123, 12770-12774. (b) Bancu, M.; Rai, A. K.; Cheng, P.; Gilardi, R. D.; Scott, L. T. Synlett 2004, 2004, 173-176. (c) Georghiou, P. E.; Tran, A. H.; Mizyed, S.; Bancu, M.; Scott, L. T. J. Org. Chem. 2005, 70, 6158-6163.

${ }^{79}$ Sygula, A.; Fronczek, F. R.; Sygula, R.; Rabideau, P. W.; Olmstead, M. M. J. Am. Chem. Soc. 2007, 129, 3842-3843. 


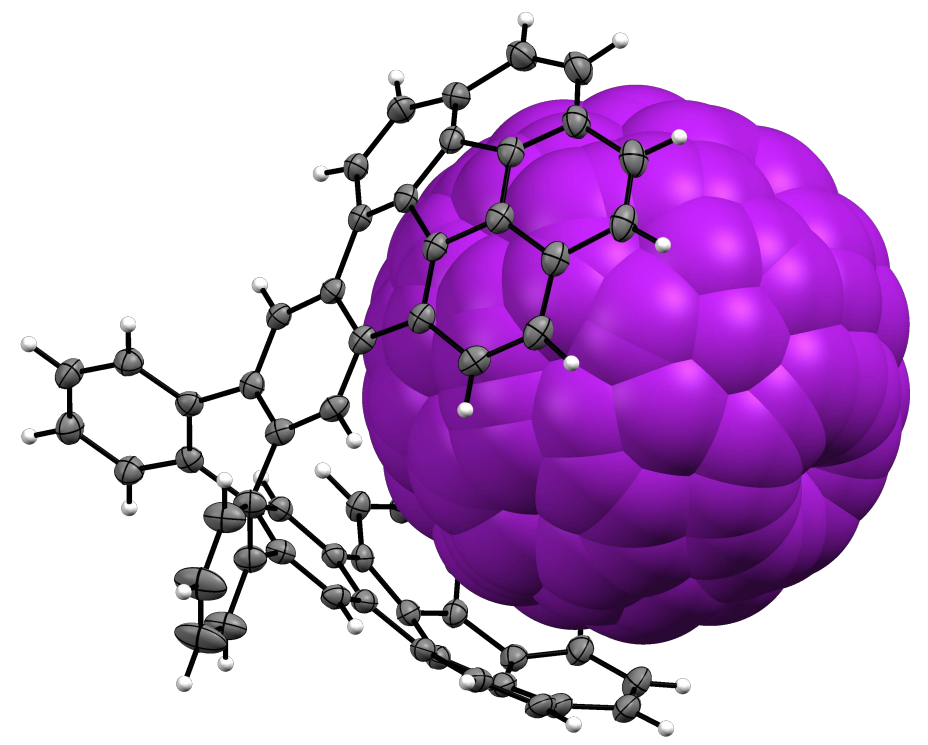

Figura 12. Estructura cristalina del aducto entre el buckcycatcher y $\mathrm{C}_{60}$.

En nuestro grupo de investigación se ha estudiado cómo aprovechar la geometría que aporta un complejo de coordinación planocuadrado para orientar dos fragmentos poliaromáticos planos y no planos a modo de pinza, ${ }^{80}$ como se muestra en la Figura 13. Así, se ha encontrado que el complejo de coordinación que posee dos grupos acetilencoranuleno, es capaz de interaccionar con fullerenos (Figura 14) con constantes de asociación considerables.

Lo más destacable de este compuesto es el hecho de que posee una diferencia de afinidad extraordinaria entre distintos fullerenos, dando lugar a una constante de asociación un orden de magnitud superior con $\mathrm{C}_{70}$ frente a $\mathrm{C}_{60}$, lo que le podría permitir discriminar entre ambos si se enfrentara a una mezcla de los mismos.
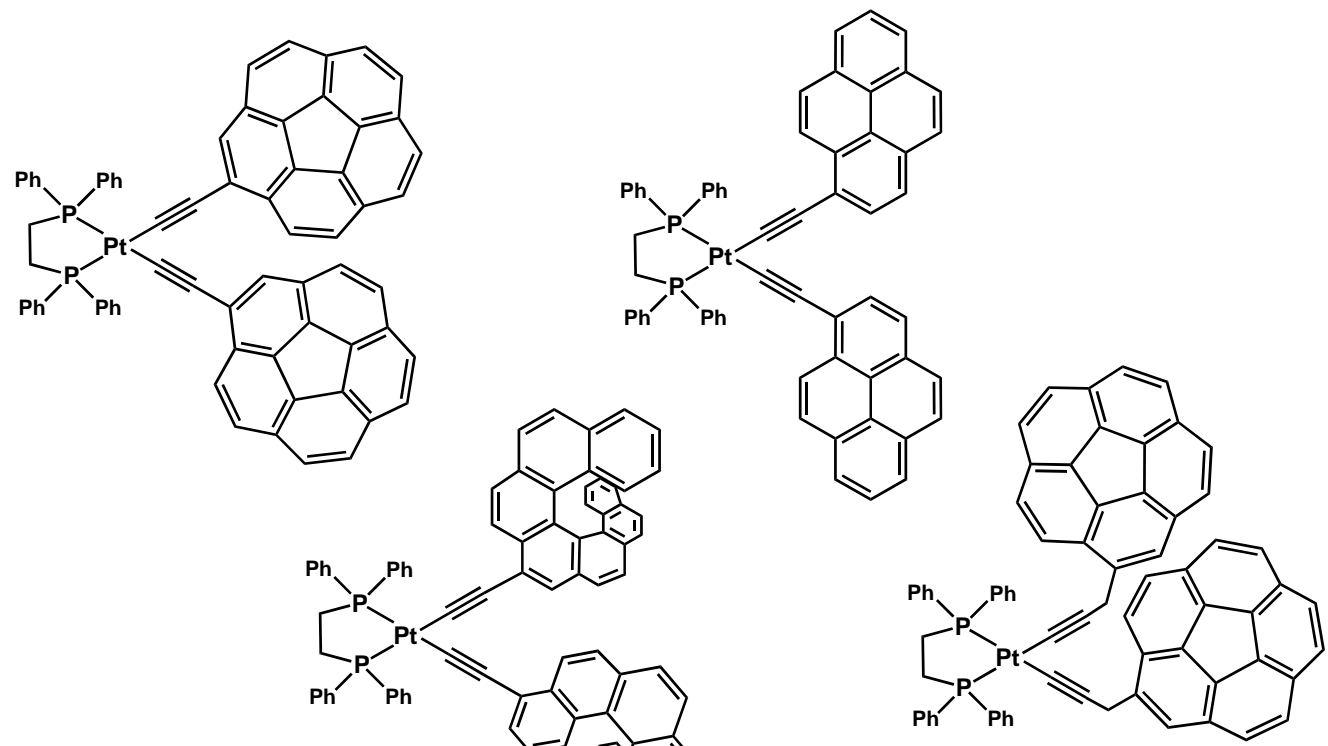

Figura 13. Complejos de platino con ligandos poliaromáticos preparados por nuestro grupo de investigación.

80 Álvarez, C. M.; García-Escudero, L. A.; García-Rodríguez, R.; Martín-Álvarez, J. M.; Miguel, D.; Rayón, V. M. Dalton Trans. 2014, 43, 15693-15696. 


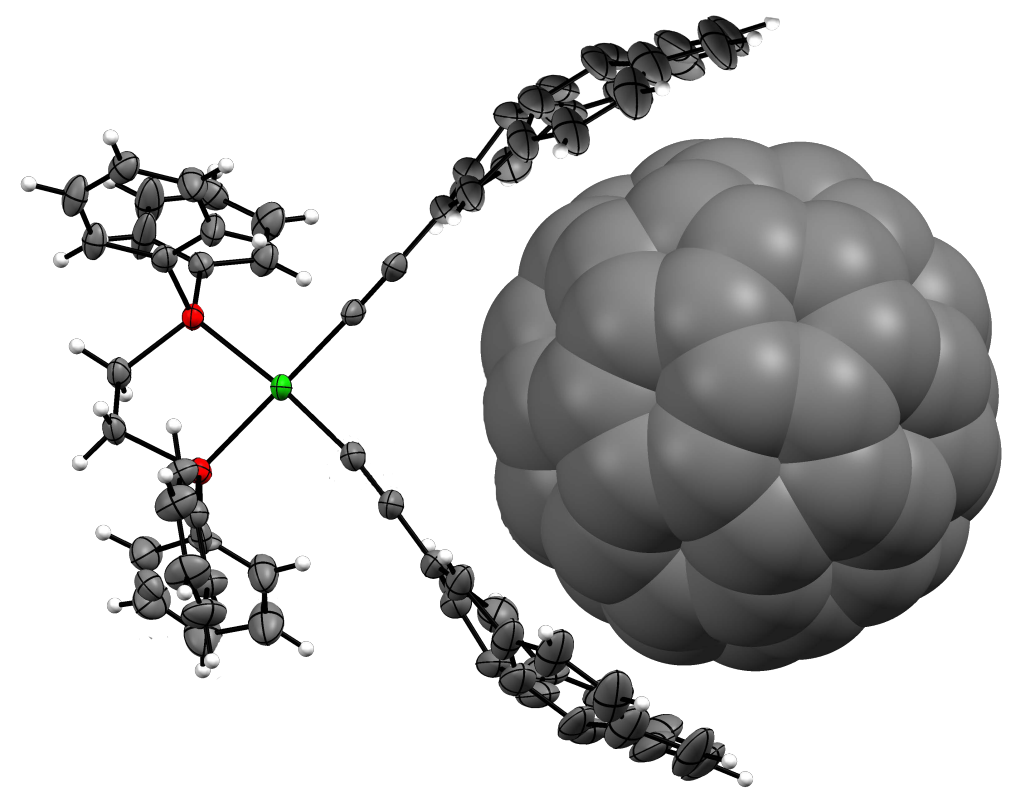

Figura 14. Estructura de rayos $\mathrm{X}$ del aducto formado por $\mathrm{C}_{60} \mathrm{y}$ el complejo de $\mathrm{Pt}$ con dos unidades acetilencoranuleno.

También se ha descrito otra familia de compuestos flexibles funcionalizados con coranuleno en los que, o bien se ha introducido una tercera unidad, o se han unido a través de otros motivos, como heliceno. De esta manera ha sido posible ensamblar unidades poliaromáticas entre sí gracias a metodologías click (Figura 15). ${ }^{81}$
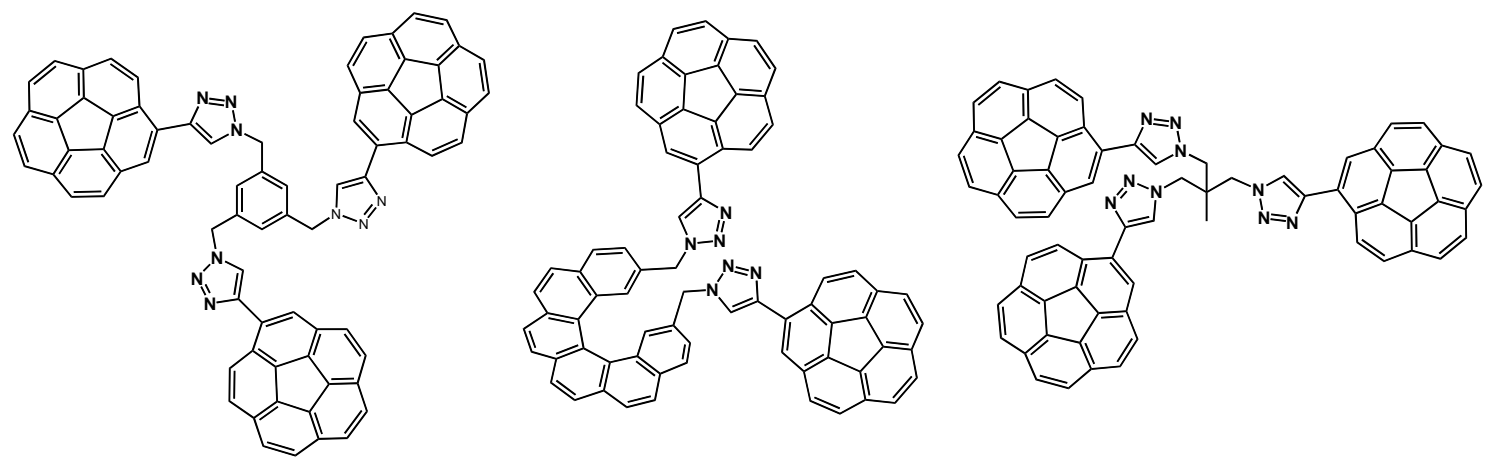

Figura 15. Compuestos flexibles derivados de coranuleno obtenidos por metodologías click en nuestro grupo de investigación.

La presencia del heliceno abre las puertas a posibles receptores quirales de fullerenos de orden superior usando enantiómeros puros del fragmento.

${ }^{81}$ (a) Álvarez, C. M.; Aullón, G.; Barbero, H.; García-Escudero, L. A.; Martínez-Pérez, C.; MartínÁlvarez, J. M.; Miguel, D. Org. Lett. 2015, 17, 2578-2581. (b) Álvarez, C. M.; Barbero, H.; Ferrero, S. J. Vis. Exp. 2016, e53954. 
Es preciso señalar otro ejemplo más en este campo, como es el trabajo de Stuparu, en el que desarrolló una familia de polímeros que contenían coranuleno capaces de establecer interacciones con $\mathrm{C}_{60}$, pudiendo dispersar muy eficientemente este nanoalótropo de carbono en agua. ${ }^{82}$ Además, el grupo de Sygula ha continuado desarrollando un conjunto de buckycatchers que comparten una estructura similar cuyas constantes de asociación han mejorado notablemente en relación al primero descrito. ${ }^{83}$ Estos ejemplos demuestran que el campo de estudio está actualmente muy vivo.

En esta memoria se pretende avanzar en el estudio de otros receptores de fullerenos que contienen unidades coranulénicas. Así, en los Artículos III y IV se describen dos conjuntos de compuestos con estas características donde dicha molécula se funcionaliza con grupos azobencénicos y con porfirinas, respectivamente.

Tal y como se describió en el apartado donde se trataron las moléculas entrelazadas mecánicamente, el fragmento azobencénico ha sido usado en los dos bloques temáticos principales y constituye un nexo de unión entre ambos, por lo que aquí se hará una pequeña reseña de su incorporación en receptores de fullerenos. Resulta interesante el hecho de que esta especie haya sido descrita en muchas aplicaciones en las que forma aductos supramoleculares, pero no existe ningún ejemplo en el que se use en sistemas para asociar fullerenos, excepto por los trabajos teóricos de Zhao y colaboradores. ${ }^{84}$ En tales estudios predicen una serie de nanoanillos basados en múltiples unidades azobencénicas capaces de asociar $\mathrm{C}_{60}{ }_{0} \mathrm{C}_{70}$ en función del estado fotoestacionario (Figura 16).
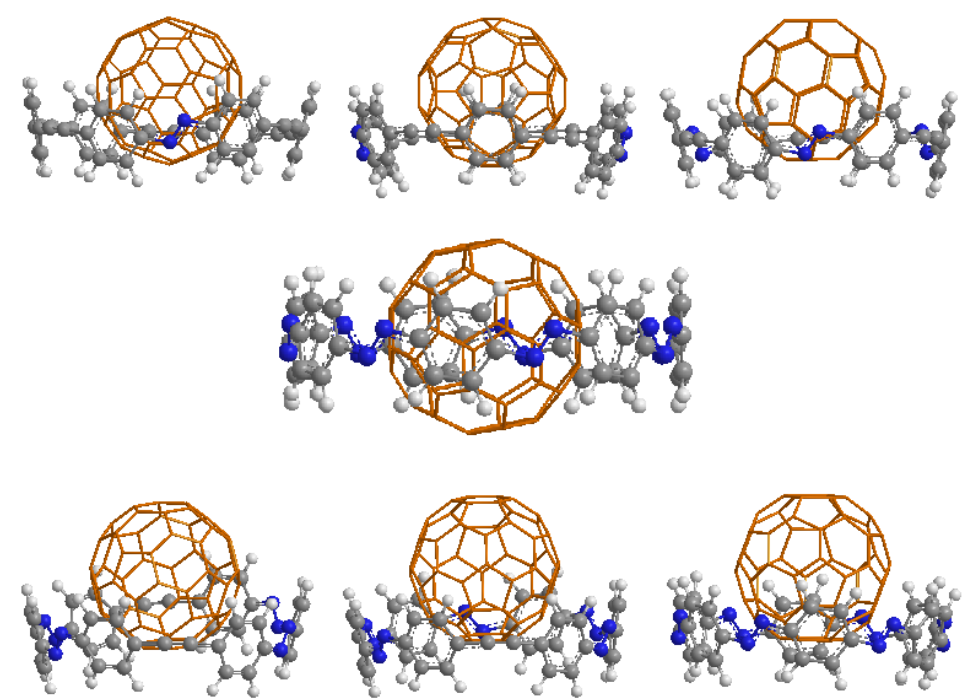

Figura 16. Predicción teórica de diversos nanoanillos basados en azobencenos capaces formar complejos de inclusión con $\mathrm{C}_{60}$.

${ }^{82}$ Stuparu, M. C. Angew. Chem. Int. Ed. 2013, 52, 7786-7790.

83 (a) Yanney, M.; Sygula, A. Tetrahedron Lett. 2013, 54, 2604-2607. (b) Abeyratne Kuragama, P. L.; Fronczek, F. R.; Sygula, A. Org. Lett. 2015, 17, 5292-5295. (c) Yanney, M.; Fronczek, F. R.; Sygula, A. Angew. Chem. Int. Ed. 2015, 54, 11153-11156. (d) Kumarasinghe, K. G. U. R.; Fronczek, F. R.; Valle, H. U.; Sygula, A. Org. Lett. 2016, 18, 3054-3057. (e) Sygula, A. Synlett 2016, 27, 2070-2080.

${ }^{84}$ (a) Yuan, K.; Guo, Y.-J.; Zhao, X. Phys. Chem. Chem. Phys. 2014, 16, 27053-27064. (b) Yuan, K.; Dang, J.-S.; Guo, Y.-J.; Zhao, X. J. Comput. Chem. 2015, 36, 518-528. 
El Artículo III de esta tesis tratará sobre la incorporación de este grupo a una molécula en forma de pinza que contiene coranulenos y el estudio de su interacción con fullerenos en función del isómero configuracional.

Por otro lado, el Artículo IV de esta tesis incluye otro gran conjunto de especies aromáticas, llamadas porfirinas, también capaces de establecer asociaciones con fullerenos y que a continuación se describirán brevemente.

Las porfirinas son una familia muy grande de compuestos heteromacrocíclicos cuya estructura básica está constituida por cuatro unidades de pirrol interconectadas entre sí por ambos carbonos $\alpha$ a través de puentes metino. Pueden estar sustituidas en los carbonos de los pirroles (posición $\beta$ ) o en los carbonos que interconectan los pirroles (posición meso, Figura 17). ${ }^{49,85}$ También es posible desprotonar los dos hidrógenos centrales, transformándose la porfirina en un ligando quelato k-N4, e introducir un metal, principalmente catiónico divalente.

La fuerte conjugación $\pi$ de sus 22 electrones proporciona una corriente de anillo tal que produce efectos anisotrópicos de apantallamiento en los protones localizados en su interior, teniendo desplazamientos químicos negativos en RMN entre -2 y -3 ppm. Por otro lado, los protones $\beta$ experimentan un gran desapantallamiento, dando lugar a desplazamientos químicos en torno a 9 ppm. ${ }^{86}$ Otro efecto de esta deslocalización es el hecho de que estos compuestos presentan bandas de absorción y emisión UV/Vis muy intensas, por lo que son muy coloreados. De hecho, su nombre proviene del griego $\pi о \rho \pi v \rho \alpha$ (porpura) cuyo significado es púrpura en castellano. El modelo de cuatro orbitales de Gouterman ${ }^{87}$ supone una teoría bastante general para explicar la aparición de las bandas de transición $\pi-\pi^{*}$ (denominadas Soret y Q) características de este tipo de compuestos (Figura 17).
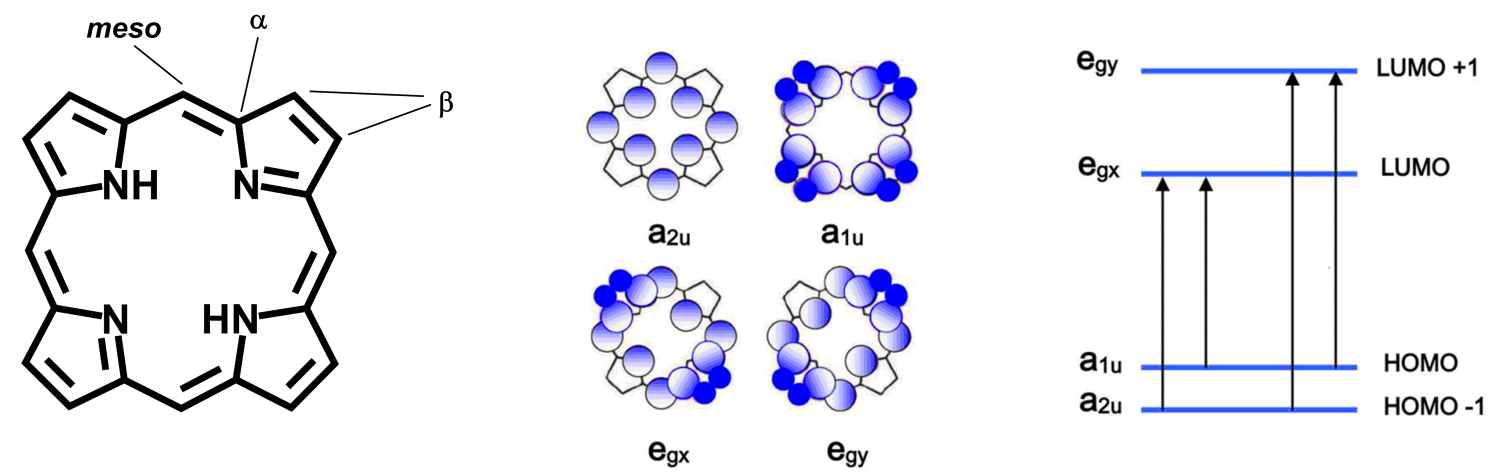

Figura 17. Estructura básica de la porfirina indicando las posiciones de interés y modelo de Gouterman de cuatro orbitales que explica las transiciones más intensas observadas en los espectros UV/Vis. Normalmente las transiciones que implican el orbital LUMO +1 son responsables de la banda de Soret y las que implican el orbital LUMO son asociadas con las bandas Q.

85 (a) Moss, G. P. Pure Appl. Chem. 1987, 59, 779-832. (b) Moss, G. P. Eur. J. Biochem. 1988, $178,277-328$

${ }^{86}$ Becker, E. D.; Bradley, R. B.; Watson, C. J. J. Am. Chem. Soc. 1961, 83, 3743-3748.

${ }^{87}$ (a) Gouterman, M. J. Chem. Phys. 1959, 30, 1139-1161. (b) Gouterman, M. J. Mol. Spectrosc. 1961, 6, 138-163. (c) Gouterman, M.; Wagnière, G. H.; Snyder, L. C. J. Mol. Spectrosc. 1963, 11, 108127. 
La estructura correcta de las porfirinas fue propuesta por primera vez por Küster en $1912,{ }^{88}$ aunque fue rechaza en un principio y no fue probada hasta 1926 por Fisher. ${ }^{89}$ Éste último ganó el premio Nobel por investigaciones relacionadas. ${ }^{90}$

La síntesis de estos compuestos depende del patrón de sustitución que presenten. De esta manera, las más sencillas son aquellas totalmente simétricas sustituidas en meso, que pueden realizarse a través de procedimientos one-pot a partir del aldehído y pirrol adecuados. Si la porfirina requiere patrones de sustitución asimétricos, el proceso ha de realizarse por pasos. En la actualidad existe una variedad notable de métodos que ha sido recopilada en distintas publicaciones. $^{91}$

El enorme interés que suscitan estas moléculas se debe a sus aplicaciones en muchos campos de la medicina, como sensores, en catálisis y como elementos en materiales fotosensibles. ${ }^{92} \mathrm{Sin}$ embargo, nuestro interés se centra en su habilidad para establecer interacciones supramoleculares con fullerenos, como se va a describir a continuación.

El estudio de posibles asociaciones supramoleculares con fullerenos ha sido motivado durante años debido a la presencia de su sistema $\pi$ deslocalizado de carácter dador, lo que permitiría usar los aductos resultantes como materiales en células fotovoltaicas. ${ }^{93}$ Una molécula de porfirina establece aductos supramoleculares con fullerenos de manera débil, muy probablemente debido a su estructura plana, que no es complementaria con la de la molécula aceptora y sólo se encuentra una prueba de su existencia en la observación de cocristalizaciones

${ }^{88}$ Kuster, W. Z. Physiol. Chem., 1912, 82, 463.

${ }^{89}$ Fischer, H.; Zeile, K. Justus Liebigs Ann. Chem. 1929, 468, 98-116.

90 "The Nobel Prize in Chemistry 1930". Nobelprize.org. Nobel Media AB 2014. Web. 14 Sep 2016. $<$ http://www.nobelprize.org/nobel_prizes/chemistry/laureates/1930/>

91 Smith, K. M., Strategies for the Synthesis of Octaalkylporphyrin Systems. In The Porphyrin Handbook, Vol. 1, Academic Press: San Diego, 2000; pp. 2-40. (b) Lindsey, J. S. Synthesis of mesoSubstituted Porphyrins, in The Porphyrin Handbook, Vol. 1, Academic Press: San Diego, 2000, pp. 4680 .

92 (a) Chou, J.-H.; Kosal, M. E.; Nalwa, H. S.; Rakow, N. A.; Suslick, K. S., Applications of Porphyrins and Metalloporphyrins to Materias Chemistry. In The Porphyrin Handbook Vol 6, Academic Press: San Diego, 2000; Vol. 6, pp 42-131. (b) Kadish, K. M.; Smith, K. M.; Guilard, R., Handbook of Porphyrin Science, Vol. 4: Phototherapy, Radioimmunotherapy and Imaging. World Scientific Publishing: Singapore, 2010. (c) Kadish, K. M.; Smith, K. M.; Guilard, R., Handbook of Porphyrin Science, Vol.10: Catalysis and Bio-Inspired Systems - Part I. World Scientific Publishing: Singapore, 2010. (d) Kadish, K. M.; Smith, K. M.; Guilard, R., Handbook of Porphyrin Science, Vol.11: Catalysis and Bio-Inspired Systems - Part 2. World Scientific Publishing: Singapore, 2010. (e) Kadish, K. M.; Smith, K. M.; Guilard, R., Handbook of Porphyrin Science, Vol.12: Applications. World Scientific Publishing: Singapore, 2010. (f) Kadish, K. M.; Smith, K. M.; Guilard, R., Handbook of Porphyrin Science, Vol.18: Applications and Materials. World Scientific Publishing: Singapore, 2010. (g) Kadish, K. M.; Smith, K. M.; Guilard, R., Handbook of Porphyrin Science, Vol.21: Catalysis. World Scientific Publishing: Singapore, 2010. (h) Burda, W. N.; Shaw, L. N.; Shepherd, M., Porphyrins: Properties and Applications. In Handbook of Porphyrins: Chemistry, Properties and Applications, Nova Science Publishers: 2012; pp 429-438.

93 (a) D'Souza, F.; Ito, O. Chem. Commun. 2009, 4913-4928. (b) de la Torre, G.; Bottari, G.; Sekita, M.; Hausmann, A.; Guldi, D. M.; Torres, T. Chem. Soc. Rev. 2013, 42, 8049-8105. 
de ambas especies. ${ }^{94}$ Quizás el único ejemplo encontrado en la literatura en el que una única porfirina es capaz de interaccionar fuertemente con un buckyball, es el descrito por Ishizuka, Kojima y colaboradores. ${ }^{95}$ El éxito de esta asociación se debe a que la porfirina se encuentra tan tensionada que adopta una geometría alejada de la planaridad, lo que le permite complementarse con la superficie externa del fullereno.

Un avance notable en esta línea de investigación apareció en la publicación de Aida, Saigo y colaboradores en 1999. ${ }^{96}$ En ese trabajo, desarrollaron dímeros de porfirinas unidos por espaciadores con el tamaño adecuado para albergar una molécula de fullereno en su cavidad.

Desde entonces, ha habido un extenso estudio en este campo encontrándose multitud de ejemplos en los que la molécula posee distintos motivos, como por ejemplo pinzas, trímeros, cajas o nanoanillos como ejemplos representativos (Figura 18) ${ }^{97}$ En todos ellos más de una porfirina está implicada y las constantes de asociación mejoran conforme más heteromacrociclos están implicados y más rígida es la estructura que genera la cavidad aceptora.

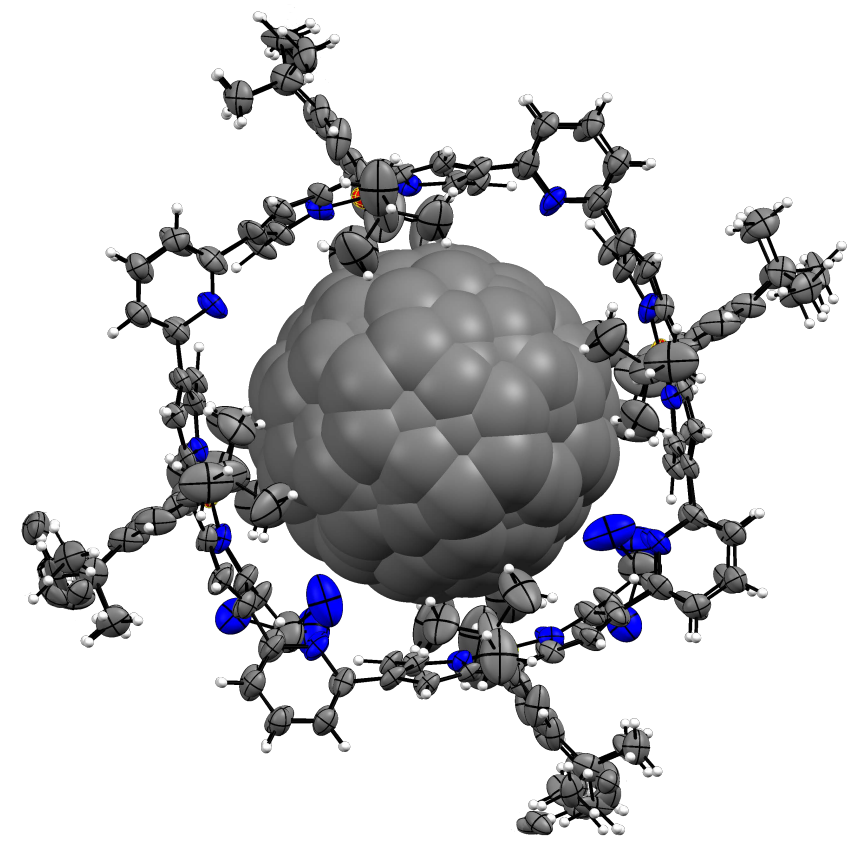

Figura 18. Ejemplo de una estructura cristalina del complejo de inclusión de un anillo formado por cuatro porfirinas y $\mathrm{C}_{60}$.

94 (a) Boyd, P. D. W.; Hodgson, M. C.; Rickard, C. E. F.; Oliver, A. G.; Chaker, L.; Brothers, P. J.; Bolskar, R. D.; Tham, F. S.; Reed, C. A. J. Am. Chem. Soc. 1999, 121, 10487-10495. (b) Evans, D. R.; Fackler, N. L. P.; Xie, Z.; Rickard, C. E. F.; Boyd, P. D. W.; Reed, C. A. J. Am. Chem. Soc. 1999, 121, 8466-8474. (c) Olmstead, M. M.; Costa, D. A.; Maitra, K.; Noll, B. C.; Phillips, S. L.; Van Calcar, P. M.; Balch, A. L. J. Am. Chem. Soc. 1999, 121, 7090-7097. (d) Ishii, T.; Aizawa, N.; Yamashita, M.; Matsuzaka, H.; Kodama, T.; Kikuchi, K.; Ikemoto, I.; Iwasa, Y. J. Chem. Soc., Dalton Trans. 2000, 44074412. (e) Song, X.; Chan, K.-S. J. Chin. Chem. Soc. 2009, 56, 667-670. (f) Obondi, C.; Rodriguez, A. A. Adv. Phys. Chem. 2010, 2010, 9.

95 Saegusa, Y.; Ishizuka, T.; Kojima, T.; Mori, S.; Kawano, M.; Kojima, T. Chem. Eur. J. 2015, 21 , 5302-5306.

96 Tashiro, K.; Aida, T.; Zheng, J.-Y.; Kinbara, K.; Saigo, K.; Sakamoto, S.; Yamaguchi, K. J. Am. Chem. Soc. 1999, 121, 9477-9478.

${ }^{97}$ (a) Boyd, P. D. W.; Reed, C. A. Acc. Chem. Res. 2005, 38, 235-242. (b) Tashiro, K.; Aida, T. Chem. Soc. Rev. 2007, 36, 189-197. (c) Li, Z.-T.; Hou, J.-L.; Li, C. Acc. Chem. Res. 2008, 41, 1343-1353. (d) Canevet, D.; Pérez, E. M.; Martín, N. Angew. Chem. Int. Ed. 2011, 50, 9248-9259. (e) Garcia-Simon, C.; Costas, M.; Ribas, X. Chem. Soc. Rev. 2016, 45, 40-62. 
No obstante, no existe ninguna publicación en la que se aproveche la capacidad aceptora del coranuleno y de porfirinas simultáneamente. Así, sólo existen unos pocos compuestos descritos en la literatura en la que ambos grupos se encuentran unidos formando enlaces covalente, pero no poseen la estructura adecuada para interaccionar con buckybowls. ${ }^{98}$ Sólo existe una predicción obtenida por cálculos computacionales realizada por el grupo de Denis que apareció casi simultáneamente con el trabajo descrito en esta memoria en el que se llega como conclusión que una porfirina simétrica con cuatro coranulenos proporcionaría unas constantes de asociación altas en base a las energías de interacción obtenidas. ${ }^{99}$

El Artículo IV describirá los resultados obtenidos en relación al uso de porfirinas funcionalizadas con coranulenos y otros PAHs con el objetivo de observar si existe cooperatividad entre ambos grupos en relación a la interacción con $\mathrm{C}_{60}$.

98 (a) Boedigheimer, H.; Ferrence, G. M.; Lash, T. D. J. Org. Chem. 2010, 75, 2518-2527. (b) Ota, K.; Tanaka, T.; Osuka, A. Org. Lett. 2014, 16, 2974-2977.

${ }^{99}$ Denis, P. A.; Yanney, M. RSC Adv. 2016, 6, 50978-50984. 
3. OBJETIVOS 



\section{OBJETIVOS}

En la presente tesis se han definido dos grandes objetivos generales que, durante el desarrollo del trabajo experimental, han permitido la concreción de diversos objetivos específicos en línea con los anteriores.

\subsection{OBJETIVOS GENERALES}

Desarrollar una metodología sintética para la obtención de interruptores moleculares basados en arquitecturas entrelazadas mecánicamente de tipo rotaxano y estudiar sus propiedades para potenciales aplicaciones.

Desarrollar una metodología sintética con el objetivo de funcionalizar grupos poliaromáticos planos y no planos, especialmente coranuleno, para así obtener moléculas con capacidad de establecer interacciones supramoleculares con fullerenos y evaluar dichas interacciones.

\subsection{OBJETIVOS ESPECÍFICOS}

Poner a punto un método de preparación de un building block a partir del cual se puedan construir distintos rotaxanos.

Preparar una familia de rotaxanos por efecto plantilla, consistente en la formación previa de un aducto supramolecular y posterior captura del macrociclo mediante el uso de metodología click chemistry.

Producir una segunda estación en el eje de los rotaxanos para obtener interruptores moleculares biestables y estudiar el proceso de conmutación.

Evaluar su uso como organocatalizadores multivalentes conmutables en reacciones de adición tipo Michael.

Incorporar un nuevo grupo funcional azobencénico a un rotaxano con el objeto de añadir un nuevo elemento de conmutación y estudiar su efecto.

Expandir el uso de derivados azobencénicos para funcionalizar compuestos poliaromáticos planos y no planos vía reacciones de acoplamiento C-C.

Estudiar la fotoisomerización de dichos derivados y evaluar su potencial uso como aceptores de fullerenos en función del isómero presente en el medio.

Poner a punto un método de preparación de porfirinas meso-tetrasutituidas y metaloporfirinas homólogas.

Desarrollar una metodología adecuada para funcionalizar dichas porfirinas con múltiples fragmentos poliaromáticos planos y no planos a través de reacciones de acoplamiento C-C.

Evaluar su capacidad para establecer interacciones supramoleculares con fullerenos. 


\section{METODOLOGÍA EMPLEADA}





\section{METODOLOGÍA EMPLEADA}

En el presente apartado se pretende abordar todo el conjunto de procedimientos realizados durante el desarrollo del trabajo experimental. Se ha dividido en cuatro apartados.

\subsection{METODOLOGÍA SINTÉTICA}

Todas las reacciones descritas en esta tesis se han realizado empleando técnicas convencionales de síntesis en un laboratorio de investigación. En los casos en los que se precisó ausencia de oxígeno o agua, se utilizaron técnicas de Schlenk usando nitrógeno como gas inerte.

Los disolventes empleados fueron adquiridos de fuentes comerciales de calidad síntesis o análisis y algunos de ellos (DCM, $\mathrm{CHCl}_{3}$, hexano y AcOEt) fueron destilados previo uso. Varios disolventes (DCM, THF, 1-4 dioxano y tolueno) fueron secados según procedimientos convencionales $^{100}$ cuando fue necesaria la ausencia de humedad en la reacción. El agua desionizada empleada fue adquirida de calidad MilliQ.

Todos los reactivos usados fueron adquiridos de fuentes comerciales y se usaron sin previa purificación, excepto para hidrocloruro de 4-(aminometil)-benzoato de metilo, que fue desprotonado previo uso.

Los catalizadores empleados, $\left[\mathrm{Cu}(\mathrm{NCMe})_{4}\right] \mathrm{PF}_{6}$ y $\left[\mathrm{PdCl}_{2}(\mathrm{dppf})\right]$ fueron preparados de acuerdo a métodos descritos en la literatura. ${ }^{101}$

El compuesto coranuleno se preparó según el método actual con ligeras modificaciones. ${ }^{76}$

Las reacciones llevadas a cabo por radiación de microondas fueron realizadas en un reactor Anton Paar Monowave 300 usando viales de $10 \mathrm{~mL}$ ó $30 \mathrm{~mL}$ a alta presión.

\subsubsection{Métodos de purificación}

La mayor parte de las separaciones se realizó por cromatografía en columna preparativa. Para ello, se empleó gel de sílice (tamaño de partícula 0,040-0,063 mm; 230-400 mesh) de Merck como fase estacionaria. Tanto las mezclas como las fracciones cromatográficas se analizaron mediante cromatografía en capa fina (TLC) con gel de sílice 60 con indicador fluorescente F254 soportado en placas de aluminio de 0,25 mm espesor de Merck. Se visualizaron bajo lámpara UV $(254 \mathrm{~nm}$ y $365 \mathrm{~nm})$ y/o se revelaron con disolución ácida de $p$ anisaldehído en EtOH seguida de breve calefacción.

100 (a) Armarego, W. L. F.; Chai, C. L. L., Purification of Laboratory Chemicals. 5th ed.; Elsevier: Bodmin, 2003. (b) Williams, D. B. G.; Lawton, M. J. Org. Chem. 2010, 75, 8351-8354.

101 (a) Anderson, G. K.; Lin, M., Bis(benzonitrile)dichloro complexes of palladium and platinum. In Reagents for Transition Metal Complex and Organometallic Syntheses, Inorganic Syntheses, Volume 28, Angelici, R. J., Ed. John Wiley \& Sons: New York, 1990; Vol. 28, pp 60-63. (b) Kubas, G. J., Tetrakis(Acetonitrile)Copper(1+) Hexafluorophosphate(1-). In Reagents for Transition Metal Complex and Organometallic Syntheses, Inorganic Syntheses, Volume 28, Angelici, R. J., Ed. John Wiley \& Sons.: New York, 1990; Vol. 28, pp 68-70. (c) Nataro, C.; Fosbenner, S. M. J. Chem. Educ. 2009, 86, 1412. 


\subsection{CARACTERIZACIÓN DE COMPUESTOS}

Tanto la identidad como la pureza de los compuestos sintetizados se analizaron gracias a las siguientes técnicas instrumentales.

\subsubsection{Espectroscopía de Resonancia Magnética Nuclear}

Los espectros de RMN fueron adquiridos en espectrómetros Agilent MR 400, DD2 500 equipado con sonda fría y VNMRS 500 ubicados en el Laboratorio de Técnicas Instrumentales de la Universidad de Valladolid. Los disolventes deuterados utilizados fueron adquiridos de fuentes comerciales y usados sin tratamiento previo. Los tubos de precisión para RMN empleados fueron de $5 \mathrm{~mm}$ de sección y de vidrio en la mayoría de los casos. Sólo en aquellos en los que se hicieron medidas de fotoisomerización, los tubos fueron de cuarzo.

Los desplazamientos químicos $(\delta)$ se dan en partes por millón $(\mathrm{ppm})$ referenciados a TMS usando la señal residual del disolvente no deuterado como referencia interna. La multiplicidad de las señales se da de acuerdo con la abreviación estándar: $\mathrm{s}=$ singlete, $\mathrm{d}=$ doblete, $\mathrm{t}=$ triplete, $\mathrm{m}=$ multiplete, $\mathrm{br}=$ señal ancha. Las constantes de acoplamiento $(J)$ en ${ }^{1} \mathrm{H}-\mathrm{RMN}$ están dadas en hercios $(\mathrm{Hz})$. Adicionalmente, se da el valor de las integrales relativas de los picos en ${ }^{1} \mathrm{H}-\mathrm{RMN}$ y la asignación completa, tanto de ${ }^{1} \mathrm{H}-\mathrm{RMN}$ como de ${ }^{13} \mathrm{C}-\mathrm{RMN}$. En los casos que involucran un derivado de azobenceno todos estos datos se dan para el isómero E.

La asignación de picos se realizó gracias a una combinación de los espectros monodimensionales $\left({ }^{1} \mathrm{H}-\mathrm{RMN}\right.$ y ${ }^{13} \mathrm{C}\left\{{ }^{1} \mathrm{H}\right\}$-RMN) con los bidimensionales convencionales: ${ }^{1} \mathrm{H}$ ${ }^{1} \mathrm{H}$ COSY, ${ }^{1} \mathrm{H}-{ }^{1} \mathrm{H}$ DQFCOSY, ${ }^{1} \mathrm{H}-{ }^{1} \mathrm{H}$ zTOCSY, ${ }^{1} \mathrm{H}-{ }^{1} \mathrm{H}$ NOESY, ${ }^{1} \mathrm{H}-{ }^{1} \mathrm{H}$ ROESY,${ }^{1} \mathrm{H}-{ }^{13} \mathrm{C}$ HSQC y ${ }^{1} \mathrm{H}-{ }^{13} \mathrm{C}$ HMBC . Excepcionalmente, en aquellos casos que, bien por su baja solubilidad, bien por el estrecho ancho espectral de las señales aromáticas, o bien por la rápida relajación de los núcleos, requirieron de condiciones especiales de adquisición y/o de secuencias de pulsos no habituales.

Las condiciones de adquisición no estándar de los espectros de ${ }^{13} \mathrm{C}\left\{{ }^{1} \mathrm{H}\right\}$-RMN fueron: pulso de $90^{\circ}$, tiempo de adquisición $=250 \mathrm{~ms}$, tiempo de relajación $=5 \mathrm{~ms}$. Incluso en estas condiciones, algunos carbonos cuaternarios no pudieron ser observados, pero sí detectados indirectamente y asignados apropiadamente gracias a espectros HMBC. Dichos carbonos se denotan con una abreviatura adicional: in.

Las secuencias de pulsos no convencionales fueron: band selective ROESY, band selective HSQC, band selective HMBC, gradient crisis HSQC y gradient crisis HMBC. ${ }^{102}$

Todos los espectros fueron analizados mediante los programas MestreNova 9.0 y VNMRj 3.2 .

102 (a) Kaerner, A.; Rabenstein, D. L. Magn. Reson. Chem. 1998, 36, 601-607. (b) Gaillet, C.; Lequart, C.; Debeire, P.; Nuzillard, J.-M. J. Magn. Reson. 1999, 139, 454-459. (c) Crouch, R.; Boyer, R. D.; Johnson, R.; Krishnamurthy, K. Magn. Reson. Chem. 2004, 42, 301-307. (d) Hu, H.; Krishnamurthy, K. Magn. Reson. Chem. 2008, 46, 683-689. 


\subsubsection{Espectrometría de Masas}

Los análisis de masas de alta y baja resolución (HRMS y LRMS, respectivamente) fueron obtenidos por el servicio de Espectrometría de Masas de la Universidad de Valladolid.

En la mayoría de casos se usó un equipo MALDI-TOF (MALDI-TOF Bruker Autoflex Speed) empleando un láser de $\mathrm{N}_{2}$ (longitud de onda de $337 \mathrm{~nm}$, con pulsos de $100 \mu \mathrm{J}$ de energía y $1 \mathrm{~ns}$ de duración), un voltaje de aceleración de $19 \mathrm{kV}$ y modo positivo lineal o reflectivo, en función de la muestra.

En otros casos se utilizó un equipo UPLC-MS (UPLC: Waters ACQUITY H-class; MS: Bruker Maxis Impact) con ionización por electrospray (ESI) en modo positivo.

En algunos casos minoritarios se empleó la técnica GC-MS (GC: 6890N Network Chromatograph con una columna HP5ms, MS: 5973 Inert spectrometer (impacto electrónico (EI) de 70 eV de energía)).

\subsubsection{Espectroscopía de Absorción UV/Vis}

Los espectros de absorción molecular UV/Vis fueron registrados en un espectrofotómetro Shimadzu UV-1603 usando como fuentes de radiación lámparas de deuterio y wolframio. Los barridos se realizaron desde $800 \mathrm{~nm}$ hasta $200 \mathrm{~nm}$ con un muestreo de $0,1 \mathrm{~nm}$ por paso. El análisis de los espectros se realizó con el software UVProbe 2.43 y el tratamiento de los datos en Microsoft Office Excel 2007. En los casos en los que se comparan espectros, están normalizados a la banda de mayor intensidad. Los coeficientes de absorción molar $(\varepsilon)^{103}$ están dados en unidades de $\mathrm{M}^{-1} \cdot \mathrm{cm}^{-1}$. Todos los espectros se realizaron en una concentración lo suficientemente baja como para asegurar que se cumple la ley de Beer-Lambert-Bouguer. Los disolventes utilizados fueron de calidad espectrofotométrica y las celdas de muestra de cuarzo.

\subsubsection{Difracción de Rayos X de Monocristal}

En los casos en los que se obtuvieron monocristales (compuestos 1(R1), 49(3a), 55(4a), $\mathbf{5 8 ( 4 b )}$ y 60(6b)) aptos para su análisis por difracción de Rayos X, fueron estudiados por Lucía Álvarez Miguel, la Dra. Patricia Gómez Iglesias y el Dr. Daniel Miguel San José.

Los datos de difracción se recogieron empleando un difractómetro Oxford Diffraction Supernova equipado con un detector de área CCD Atlas y un goniómetro kappa de cuatro círculos. Las fuentes de radiación usadas fueron fuentes microfocales de $\mathrm{Mo} \mathrm{o} \mathrm{Cu}$ con óptica multicapa.

${ }^{103}$ La IUPAC desaconseja el uso del término "extinción": Braslavsky, S. E. Pure Appl. Chem. 2007, 79, 293-465. 
La integración de datos, escalado y correcciones empíricas de absorción se llevaron a cabo con el paquete informático CrysAlisPro. ${ }^{104}$ Las estructuras se resolvieron usando métodos directos y refinadas por mínimos cuadrados contra $\mathrm{F}^{2}$ en SHELX ${ }^{105}$ operando bajo OLEX2. ${ }^{106}$ Todos los átomos diferentes de hidrógeno se refinaron anisotrópicamente mientras que los átomos de hidrógeno se colocaron en posiciones ideales y fueron refinados usando el modelo riding. En el compuesto 1(R1), el átomo de hidrógeno directamente unido al nitrógeno fue localizado, pero no se pudo refinar libremente, por lo que se fijó la distancia con AFIX 6 y se permitió rotar al enlace usando dicho nitrógeno como pivote. Los gráficos se hicieron con OLEX2 y MERCURY 3.0. ${ }^{107}$

\subsection{ESTUDIOS COMPUTACIONALES}

La mayor parte de los cálculos teóricos ha sido realizada en el clúster del Instituto de Química Teórica y Computacional (IQTC) de la Universidad de Barcelona. El resto han sido realizados en un equipo Mac Pro. El software empleado ha sido GAUSSIAN-09 ${ }^{108} \mathrm{y}$ los resultados visualizados en GAUSSVIEW 5, ${ }^{109}$ MERCURY 3.0, ${ }^{107}$ AVOGADRO 1.0.3 ${ }^{110} \mathrm{y}$ VMD 1.9.2. ${ }^{111}$ Los gráficos se renderizaron posteriormente en POV-RAY 3.7. ${ }^{112}$

En todos los estudios de los Artículos III y IV se aplicó la teoría DFT usando el funcional de Grimme B97D ${ }^{113}$ que incluye efectos de dispersión.

${ }^{104}$ CrysAlisPro-Data collection and integration software, version 1.171.37.35. Agilent Technologies UK Ltd, Oxford, UK, 2011.

${ }^{105}$ Sheldrick, G. M., Acta Cryst. 2008, A64, 112-122.

${ }^{106}$ Dolomanov, O. V.; Bourhis, L. J.; Gildea, R. J.; Howard, J. A. K.; Puschmann, H. 'OLEX2: A complete structure solution, refinement and analysis program.' J. Appl. Cryst. 2009, 42, 339-341.

${ }_{107}$ (a) Bruno, I. J.; Cole, J. C.; Edgington, P. R.; Kessler, M.; Macrae, C. F.; McCabe, P.; Pearson, J.; Taylor, R. Acta Crystallogr., Sect. B 2002, 58, 389-397. (b) Macrae, C. F.; Edgington, P. R.; McCabe, P.; Pidcock, E.; Shields, G. P.; Taylor, R.; Towler, M.; van de Streek, J. J. Appl. Crystallogr. 2006, 39, 453457. (c) Macrae, C. F.; Bruno, I. J.; Chisholm, J. A.; Edgington, P. R.; McCabe, P.; Pidcock, E.; Rodriguez-Monge, L.; Taylor, R.; van de Streek, J.; Wood, P. A. J. Appl. Crystallogr. 2008, 41, 466-470.

${ }^{108}$ Gaussian 09, Revision D.01, Frisch, M. J.; Trucks, G. W.; Schlegel, H. B.; Scuseria, G. E.; Robb, M. A.; Cheeseman, J. R.; Scalmani, G.; Barone, V.; Mennucci, B.; Petersson, G. A.; Nakatsuji, H.; Caricato, M.; Li, X.; Hratchian, H. P.; Izmaylov, A. F.; Bloino, J.; Zheng, G.; Sonnenberg, J. L.; Hada, M.; Ehara, M.; Toyota, K.; Fukuda, R.; Hasegawa, J.; Ishida, M.; Nakajima, T.; Honda, Y.; Kitao, O.; Nakai, H.; Vreven, T.; Montgomery, J. A., Jr.; Peralta, J. E.; Ogliaro, F.; Bearpark, M.; Heyd, J. J.; Brothers, E.; Kudin, K. N.; Staroverov, V. N.; Kobayashi, R.; Normand, J.; Raghavachari, K.; Rendell, A.; Burant, J. C.; Iyengar, S. S.; Tomasi, J.; Cossi, M.; Rega, N.; Millam, M. J.; Klene, M.; Knox, J. E.; Cross, J. B.; Bakken, V.; Adamo, C.; Jaramillo, J.; Gomperts, R.; Stratmann, R. E.; Yazyev, O.; Austin, A. J.; Cammi, R.; Pomelli, C.; Ochterski, J. W.; Martin, R. L.; Morokuma, K.; Zakrzewski, V. G.; Voth, G. A.; Salvador, P.; Dannenberg, J. J.; Dapprich, S.; Daniels, A. D.; Farkas, Ö.; Foresman, J. B.; Ortiz, J. V.; Cioslowski, J.; Fox, D. J. Gaussian, Inc., Wallingford CT, 2009.

${ }^{109}$ GaussView, Version 5, Dennington, R.; Keith, T.; Millam, J. Semichem Inc., Shawnee Mission, KS, 2009.

${ }^{110}$ Hanwell, M. D.; Curtis, D. E.; Lonie, D. C.; Vandermeersch, T.; Zurek, E.; Hutchison, G. R. J. Cheminform. 2012, 4, 1-17.

${ }^{111}$ Humphrey, W., Dalke, A., Schulten, K. J. Molec. Graphics, 1996, 14, 33-38.

112 Persistence of Vision Pty. Ltd. (2004). Persistence of Vision (TM) Raytracer. Persistence of Vision Pty. Ltd., Williamstown, Victoria, Australia. http://www.povray.org/

${ }^{113}$ Grimme, S. J. Comp. Chem. 2006, 27, 1787-1799. 
Los conjuntos de funciones base escogidas para todos los átomos fueron 6-311G(d,p) o 6$31 \mathrm{G}(\mathrm{d}, \mathrm{p})$ triple o doble zeta con funciones de polarización. ${ }^{114}$ Partiendo de geometrías iniciales similares a las encontradas en estructuras obtenidas por difracción de Rayos X, se optimizaron en fase gas sin ningún tipo de restricción y los mínimos encontrados fueron confirmados por su análisis vibracional. La energía libre de Gibbs se calculó a 1 atm y $298 \mathrm{~K}$. Tras eso, se recalculó la energía electrónica con conjuntos base más extendidos incluyendo funciones difusas 6$311+\mathrm{G}(\mathrm{d}, \mathrm{p})$ o $6-31+\mathrm{G}(\mathrm{d}, \mathrm{p})^{115}$ que fueron usadas para calcular la corrección counterpoise con el objetivo de evitar el error de superposición de bases (BSSE). ${ }^{116}$ La energía de interacción corregida se calculó de acuerdo a la siguiente expresión:

$$
\begin{gathered}
\Delta E_{\text {int }}^{C P}(A B)=E_{A B}^{A B}-E_{A}^{A B}-E_{B}^{A B} \\
\text { Ecuación } 1
\end{gathered}
$$

Donde $\mathrm{AB}$ es el aducto supramolecular, $\mathrm{A}$ uno de los dos miembros del aducto y $\mathrm{B}$ el otro miembro. El subíndice denota la molécula en sí y el superíndice las funciones base usadas.

Los efectos del disolvente se tuvieron en cuenta con el algoritmo SCRF usando el modelo continuo PCM con tolueno $(\varepsilon=2,37)$ como disolvente. ${ }^{117}$

Finalmente, la energía libre de Gibbs en disolución corregida se calculó con la siguiente ecuación:

$$
G_{\text {tolueno }}=G_{\text {gas }}+\left(E_{\text {tolueno }}-E_{\text {gas }}\right)
$$

\section{Ecuación 2}

Los desplazamientos químicos de protón de RMN se calcularon con el método GIAO $^{118}$ con funciones base IGLO-II ${ }^{119}$ incluidas manualmente en los ficheros de input gracias a EMSL Basis Set Exchange. ${ }^{120}$ Los desplazamientos químicos calculados se sustrajeron de los obtenidos de la molécula de TMS previamente tratada con el protocolo anteriormente descrito.

114 (a) Ditchfield, R.; Hehre, W. J.; Pople, J. A. J. Chem. Phys. 1971, 54, 724-728. (b) Hehre, W. J.; Ditchfield, R.; Pople, J. A. J. Chem. Phys. 1972, 56, 2257-2261. (b) Hariharan, P. C.; Pople, J. A. Theor. Chem. Acc. 1973, 28, 213-222. (c) Francl, M. M.; Pietro, W. J.; Hehre, W. J.; Binkley, J. S.; DeFrees, D. J.; Pople, J.A.; Gordon, M. S. J. Chem. Phys., 1982, 77, 3654-3665. (d) Binning Jr., R. C.; Curtiss, L. A. J. Comp. Chem. 1990, 11, 1206-1216. (e) Blaudeau, J.-P.; McGrath, M. P.; Curtiss, L. A.; Radom, L. J. Chem. Phys. 1997, 107, 5016-5021. (f) Rassolov, V. A.; Pople, J. A.; Ratner, M. A.; Windus, T. L. J. Chem. Phys. 1998, 109, 1223-1229. (g) Rassolov, V. A.; Ratner, M. A.; Pople, J. A.; Redfern, P. C.; Curtiss, L. A. J. Comp. Chem. 2001, 22, 976-984.

115 Clark, T.; Chandrasekhar, J.; Spitznagel, G. W.; Schleyer, P. V. R. J. Comp. Chem. 1983, 4, 294301.

116 (a) Boys, S. F.; Bernardi, F. Mol. Phys. 1970, 19, 553-566. (b) Simon S.; Duran M.; Dannenberg, J. J. J. Chem. Phys. 1996, 105, 11024-11031.

117 Tomasi, J.; Mennucci, B.; Cammi, R. Chem. Rev. 2005, 105, 2999-3093.

118 (a) London, F. J. Phys. Radium 1937, 8, 397-409. (b) McWeeny, R. Phys. Rev. 1962, 126, 1028. (c) Ditchfield, R. Mol. Phys. 1974, 27, 789-807. (d) Wolinski, K.; Hilton, J. F.; Pulay, P. J. Am. Chem. Soc. 1990, 112, 8251-60. (e) Cheeseman, J. R.; Trucks, G. W.; Keith, T. A.; Frisch, M. J. J. Chem. Phys. 1996, 104, 5497-509.

119 Kutzelnigg, W.; Fleischer, U.; Schindler, M., The IGLO-Method: Ab-initio Calculation and Interpretation of NMR Chemical Shifts and Magnetic Susceptibilities. In Deuterium and Shift Calculation, Springer Berlin Heidelberg: Berlin, Heidelberg, 1991; pp 165-262.

${ }^{120}$ (a) Feller, D. J. Comp. Chem. 1996, 17, 1571-1586. (b) Schuchardt, K.L.; Didier, B.T.; Elsethagen, T.; Sun, L.; Gurumoorthi, V.; Chase, J.; Li, J.; Windus, T.L. J. Chem. Inf. Model. 2007, 47, 1045-1052. 
El análisis de las interacciones no covalentes se llevó a cabo con el programa NCIPLOT ${ }^{121}$ en las geometrías optimizadas usando sus respectivas funciones de onda calculadas anteriormente. Los cubes generados se visualizaron en VMD 1.9.2 y el gradiente de la densidad electrónica reducido se representó frente a la densidad electrónica multiplicada por el segundo autovalor de la matriz Hessiana de dicha densidad electrónica $\left(\lambda_{2}\right)$ con GNUPLOT $5.0^{122}$ para distinguir entre las diferentes interacciones. De esta manera, todas las interacciones no covalentes se representaron de acuerdo con un esquema RGB donde el azul representa interacción atractiva fuerte, el verde interacción atractiva débil y el rojo interacción repulsiva.

Los estudios realizados en el Artículo II se llevaron a cabo mediante el método semiempírico $\mathrm{PM}^{123}$ usando los parámetros estándar integrados en el paquete informático.

\subsection{EVALUACIÓN DE PROPIEDADES}

Todos los compuestos finales obtenidos en el presente trabajo muestran un conjunto interesante de propiedades que han sido evaluadas con las siguientes metodologías.

\subsubsection{Conmutación entre isómeros}

\subsubsection{Conmutación entre dos estaciones de un interruptor molecular de tipo rotaxano}

Para mover el éter macrocíclico de la posición de la sal de amonio a la posición de la sal de triazolinio, el rotaxano se disolvió en acetona deuterada y se trató con la cantidad adecuada de una disolución $1 \mathrm{M}$ de $\mathrm{NaOH}$ en $\mathrm{D}_{2} \mathrm{O}$. Tras un período corto de agitación se registró un espectro de ${ }^{1} \mathrm{H}-\mathrm{RMN}$ para determinar si la conmutación tuvo éxito.

De manera análoga, para devolver el anillo a su posición inicial, sobre esa misma disolución se añadió la cantidad apropiada de TFA y se adquirió otro espectro de ${ }^{1} \mathrm{H}-\mathrm{RMN}$ tras breve agitación.

\subsubsection{Fotoisomerización de derivados de azobenceno}

La isomerización reversible desde la configuración $\mathrm{E}$ a la $\mathrm{Z}$ se llevó a cabo empleando una cámara PELCO UVC2 UV Cryo Chamber ( $365 \mathrm{~nm}$ ) o una linterna HQRP de 21 LEDs UV (380 $\mathrm{nm})$. Las muestras se irradiaron durante 15 minutos a temperatura ambiente y posteriormente se adquirieron espectros de ${ }^{1} \mathrm{H}-\mathrm{RMN}$ y UV/Vis para comprobar la extensión de la fotoisomerización.

Con el objetivo de volver a la configuración E inicial, las muestras se calentaron durante 30 minutos a una temperatura no superior al punto de ebullición del disolvente o se dejaron bajo luz natural visible durante mucho más tiempo (días) y se registraron espectros de ${ }^{1} \mathrm{H}-\mathrm{RMN}$ y $\mathrm{UV} / \mathrm{Vis}$.

121 (a) Yang, W.; Johnson, E. R.; Keinan, S.; Mori-Sánchez, P.; Contreras-García, J.; Cohen, A. J. J. Am. Chem. Soc. 2010, 132, 6498-6506. (b) Yang, W.; Contreras-García, J.; Johnson, E. R.; Keinan, S.; Chaudret, R.; Piquemal, J.-P.; Beratan, D. N. J. Chem. Theory Comput. 2011, 7, 625-632.

${ }_{122}$ Williams, T.; Kelley, C. and R Development Core Team (2011). Gnuplot 4.5: an interactive plotting program. URL http://gnuplot.info.

${ }^{123}$ (a) Stewart, J. J. P. J. Comput. Chem. 1989, 10, 209-220. (b) Stewart, J. J. P. J. Comput. Chem. 1989, 10, 221-264. 
En todos los casos, el material del recipiente de las muestras fue de cuarzo.

\subsubsection{Estudio catalítico de rotaxanos}

Los dos reactivos se mezclaron junto con el organocatalizador en las proporciones adecuadas y se disolvieron en el disolvente deuterado apropiado. Después se añadió una disolución $1 \mathrm{M}$ de $\mathrm{NaOH}$ en $\mathrm{D}_{2} \mathrm{O}$ en la cantidad necesaria para conmutar el interruptor molecular y se agitó vigorosamente. Se tomaron alícuotas a dos tiempos distintos y se adquirieron espectros de ${ }^{1} \mathrm{H}$ RMN para comprobar la extensión de la reacción. Con el objetivo de comprobar la identidad del producto de adición resultante, el crudo de reacción se purificó en columna cromatográfica.

\subsubsection{Método de variaciones continuas (Job Plots)}

Para obtener la estequiometría del aducto formado y así realizar un ajuste posterior a un modelo de interacción concreto se llevó a cabo el método de las variaciones continuas o Job Plots. ${ }^{124}$

Se prepararon once disoluciones manteniendo la misma concentración total de host y guest, pero variando en cada una la proporción entre ambas especies desde una fracción molar del fullereno 0 hasta 1 con un intervalo de 0,1 entre dos disoluciones consecutivas. Cada muestra fue analizada registrando espectros de ${ }^{1} \mathrm{H}-\mathrm{RMN}$ y se representó la variación de desplazamiento químico (en valor absoluto) por la fracción molar de uno de los compuestos frente a la fracción molar del mismo compuesto en Microsoft Office Excel 2007 obteniéndose una curva cuyo máximo proporciona la estequiometría del aducto. En todos los casos el máximo correspondía con un valor en el eje de abscisas de 0,5 indicando un aducto 1:1.

Se utilizó el método de Bühlmann y colaboradores ${ }^{125}$ en el que el coeficiente $\sum c / c_{\text {MAX }}$ calculado se aproxima a 6,60 asegurando la estequiometría 1:1.

\subsubsection{Estimación de constantes de asociación}

Con el objetivo de estimar las constantes de asociación supramolecular de los compuestos sintetizados con fullerenos, se aplicó el método de dilución.

Se preparó una disolución del compuesto problema de concentración $10^{-4} \mathrm{M}$ en tolueno deuterado y se transfirió un volumen conocido a un tubo de RMN. Se añadieron sucesivas alícuotas de una disolución del fullereno de concentración $10^{-3} \mathrm{M}$ y se adquirió un espectro de ${ }^{1} \mathrm{H}-\mathrm{RMN}$ a temperatura ambiente tras cada adición cubriendo un amplio intervalo de equivalentes teniendo muchos más puntos en la zona entre 0 y 2 equivalentes que en el resto.

Todos los cambios en desplazamientos químicos observados $(\Delta \delta)$ se representaron frente a la fracción molar del fullereno y la curva resultante se ajustó por un método no lineal de acuerdo con la expresión: ${ }^{126}$

$$
\Delta \delta=\Delta \delta_{\max }\left(\frac{[H G]}{\left[H_{0}\right]}\right)
$$

\section{Ecuación 3}

124 Job, P. Ann. Chim. 1928, 9, 113-203.

${ }^{125}$ Olson, E. J.; Bühlmann, P. J. Org. Chem. 2011, 76, 8406-8412.

126 (a) Fielding, L. Tetrahedron 2000, 56, 6151-6170. (b) Hirose, K. J. Incl. Phenom. Macrocycl. Chem. 2001, 39, 193-209. (c) Thordarson, P. Chem. Soc. Rev. 2011, 40, 1305-1323. 
Donde:

$$
[H G]=\frac{1}{2}\left\{\left[G_{0}\right]+\left[H_{0}\right]+\frac{1}{K_{a}}\right\}-\sqrt{\left\{\left[G_{0}\right]+\left[H_{0}\right]+\frac{1}{K_{a}}\right\}^{2}+4\left[G_{0}\right]\left[H_{0}\right]}
$$

\section{Ecuación 4}

$\left[G_{0}\right]$ es la concentración total del fullereno

$\left[H_{0}\right]$ es la concentración total de la molécula sujeta a estudio

$\Delta \delta_{\max }$ es $\Delta \delta$ en el máximo de complejación (el complejo supramolecular está formado al $100 \%)$

$\Delta \delta_{\max }$ y $K_{a}$ son parámetros optimizados durante el ajuste no lineal de la curva usando el paquete informático Statgraphics Centurion XVI 16.2.4. Todas las curvas se ajustaron considerando estequiometría 1:1. 


\section{RESULTADOS OBTENIDOS}





\section{RESULTADOS OBTENIDOS}

En esta sección se presentan los resultados divididos en dos bloques principales de acuerdo con el esquema seguido en el apartado de Introducción.

En primer lugar se aborda el estudio de diversos rotaxanos e interruptores moleculares cuya capacidad de conmutar entre isómeros variando el $\mathrm{pH}$ puede ser explotada.

En segundo lugar se abarca el conjunto de compuestos que contienen grupos poliaromáticos con capacidades para establecer asociaciones supramoleculares con fullerenos y el estudio de dichas interacciones.

\subsection{ROTAXANOS E INTERRUPTORES MOLECULARES}

\subsubsection{Artículo I. Multivalent Molecular Shuttles - Effect of Increasing the Number of Centers in Switchable Catalysts}

Tal y como se señaló en la Introducción, la formación de aductos supramoleculares a través de enlaces de hidrógeno entre sales de amonio y éteres corona es especialmente fuerte, sobre todo si se trata de sales de amonio bencílicas y DB24C8. Se decidió usar éste motivo junto con el método enhebrado y cierre para la obtención del rotaxano utilizando como reacción final, la cicloadición 1,3-dipolar entre un alquino y una azida catalizada por cobre. Esto además permitiría generar una segunda estación para el interruptor molecular con sólo metilar el triazol generado.

El procedimiento sintético para obtener los rotaxanos deseados comienza con la condensación entre 9-antracenocarboxaldehído y 4-(aminometil)benzoato de metilo cuya imina resultante se reduce inmediatamente para dar la amina secundaria 1(R1). El grupo ester se reduce al alcohol primario 2(R2) correspondiente, se protege el grupo amino obteniéndose el carbamato 3(R3/R1) para, posteriormente, formar el éter propargílico $4(\mathbf{R 4})$ como resultado del ataque nucleofílico a bromuro de propargilo. Finalmente, el grupo amino se desprotege con TFA y se intercambia el anión por hexafluorofosfato dando lugar a la sal de amonio 5(R5-H$\mathrm{PF}_{6}$ ), común en todos los rotaxanos de este trabajo (Esquema 11). El proceso consta de 6 pasos y tiene un rendimiento global del 52\%.

Los grupos stopper $8(\mathbf{S 3} / \mathbf{S 1})$, 9(S4) y 10(S5) se obtuvieron gracias a una simple reacción de sustitución nucleófila con azida sódica partiendo de los derivados bromados comerciales. En el caso del fragmento antracenilo, en cambio, se utilizó el mismo aldehído de partida del procedimiento sintético anterior, se redujo al alcohol correspondiente y se transformó en el compuesto bromado deseado (Esquema 12).

El compuesto 5( $\left(\mathbf{R 5}-\mathrm{H}_{-} \mathrm{PF}_{6}\right)$ se disolvió en $\mathrm{DCM}$ en presencia de un ligero exceso del éter macrocíclico DB24C8. Transcurrido un tiempo se tomó una alícuota de la mezcla y se analizó por ${ }^{1} \mathrm{H}-\mathrm{RMN}$ observándose que el auto ensamblaje de ambos fragmentos tenía lugar. Se añadió entonces el grupo stopper (8(S3/S1), 9(S4) ó 10(S5)), la sal de cobre $\left[\mathrm{Cu}(\mathrm{NCMe})_{4}\right] \mathrm{PF}_{6}$ y el ligando auxiliar 2,6-lutidina en las proporciones adecuadas y se agitó a temperatura ambiente el tiempo necesario. Una purificación posterior dio lugar a los compuestos 11(R6-H- $\left.\mathrm{PF}_{6}\right), \mathbf{1 2}(\mathbf{R} 7-$ $\left.\mathrm{H}-\mathrm{PF}_{6}\right)$ y 13(R8-H-PF 6$)$ con buenos rendimientos (Esquema 13). 

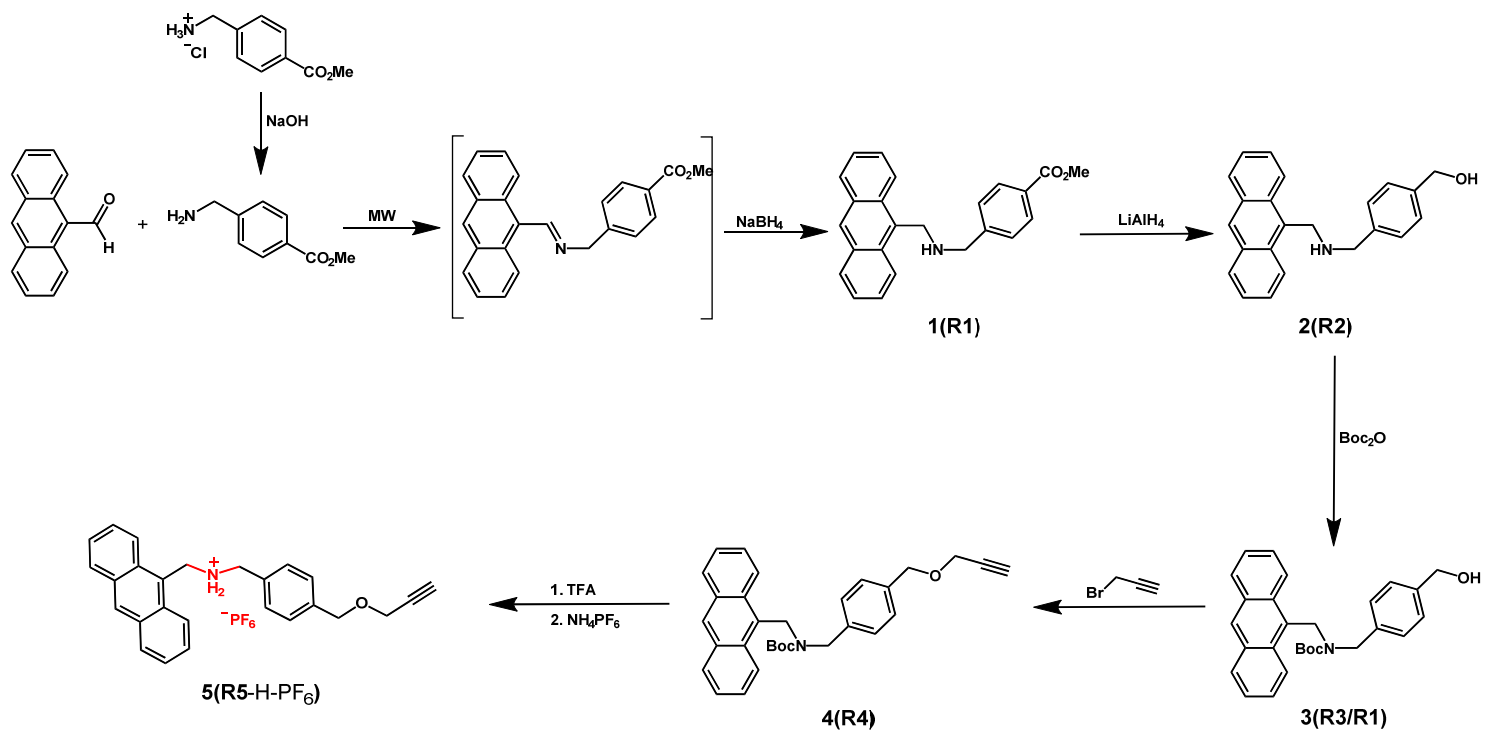

Esquema 11. Síntesis del eje común usado en todos los interruptores moleculares estudiados en el Artículo I.
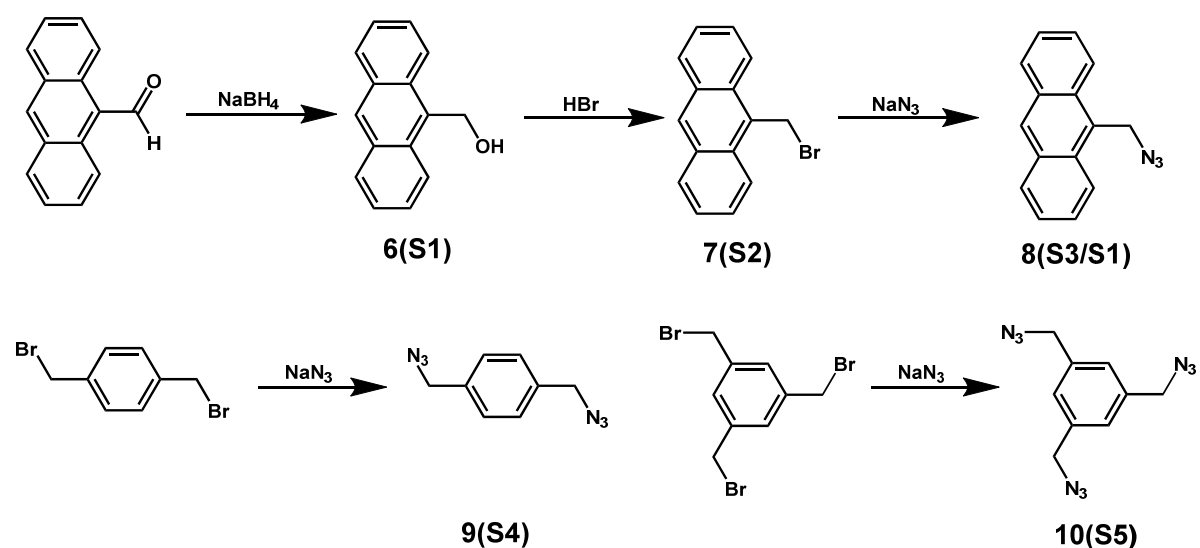

Esquema 12. Síntesis de los fragmentos stopper usados en el primer bloque.

Finalmente, se metiló el triazol resultante con $\mathrm{MeO}_{3} \mathrm{BF}_{4}$ para generar la segunda estación y convertir el rotaxano en un interruptor molecular (Figura 19). 


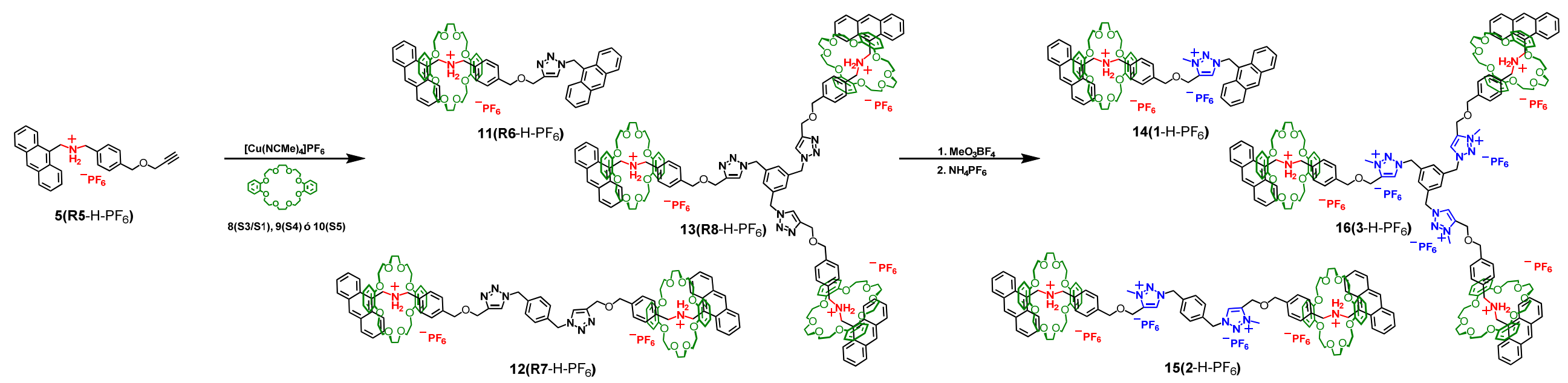

Esquema 13. Preparación de los tres rotaxanos por auto ensamblaje del eje y el anillo y posterior cierre a través de CuAAC a través de la metodología threading-capping.

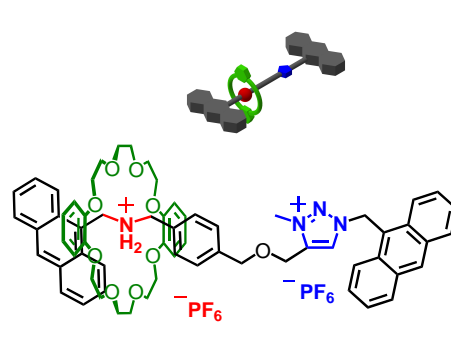

$14\left(1-H-\mathrm{PF}_{6}\right)$

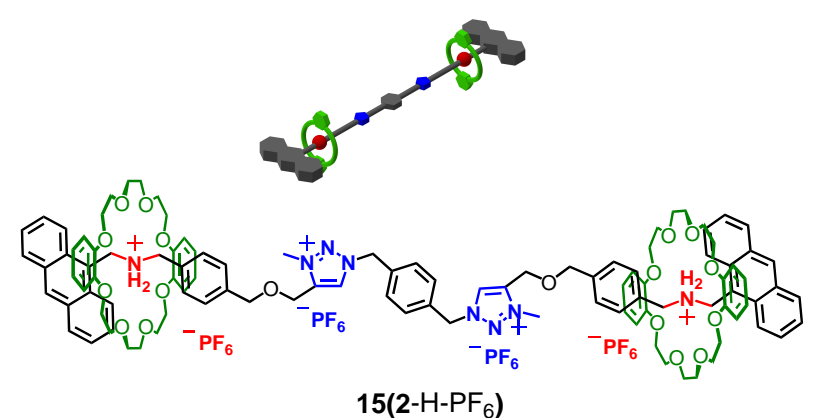

$15\left(2-H-\mathrm{PF}_{6}\right)$

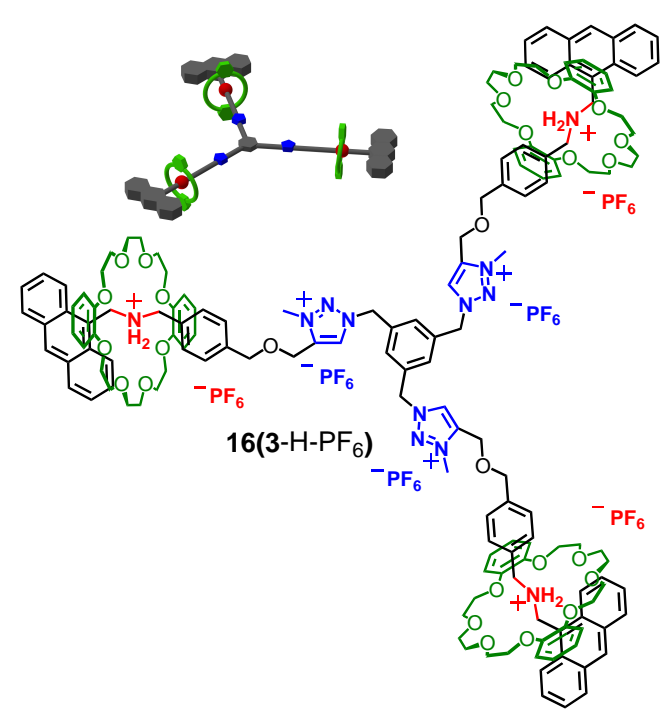

Figura 19. Familia de interruptores moleculares preparados en este trabajo. 
De manera paralela, se sintetizaron también los compuestos análogos a estos interruptores moleculares que carecen de poliéter macrocíclico. Por desgracia, la estrategia sintética empleada con anterioridad no pudo ser utilizada en este caso debido a la gran descomposición encontrada en los crudos de las reacciones de cierre y en las posteriores purificaciones, dando como resultado rendimientos muy bajos (Esquema 14). Por lo tanto, el procedimiento tuvo que ser modificado.

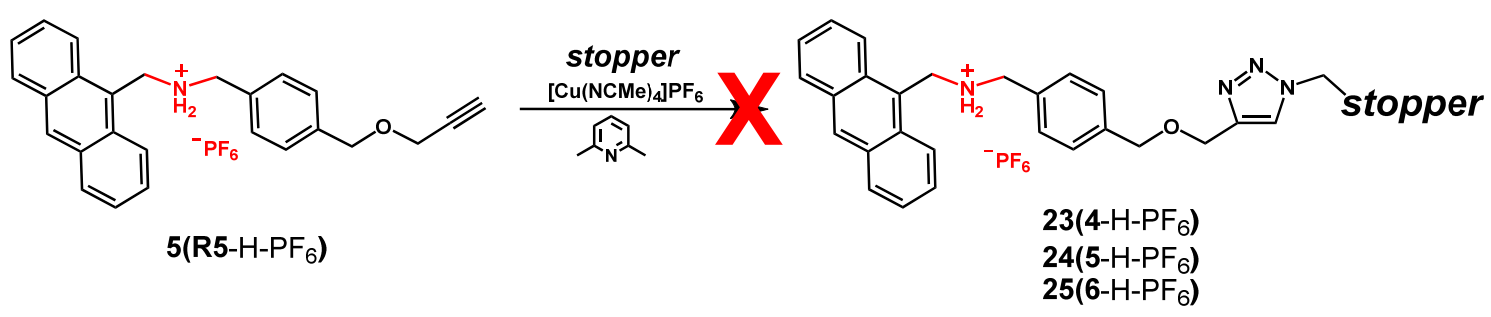

Esquema 14. Preparación sin éxito de derivados análogos a los rotaxanos no enhebrados según la metodología aplicada anteriormente.

Se partió entonces de un intermedio previo en el que el grupo amino está protegido como carbamato por el grupo Boc (4(R4)) y se realizó la reacción de cierre con el stopper adecuado. Posteriormente se metilo el triazol y se desprotegió el grupo amino dando lugar a los compuestos deseados tras un proceso de purificación con rendimientos globales moderados, aunque superiores a los obtenidos por el anterior método (Esquema 15).

Es importante destacar que estos compuestos son mucho menos estables y menos solubles en disolventes orgánicos comunes que los rotaxanos correspondientes. Este hecho demuestra la importancia del encapsulamiento de la sal de amonio por parte del anillo macrocíclico. Las estructuras de los derivados no enhebrados están esquematizadas en la Figura 20. 


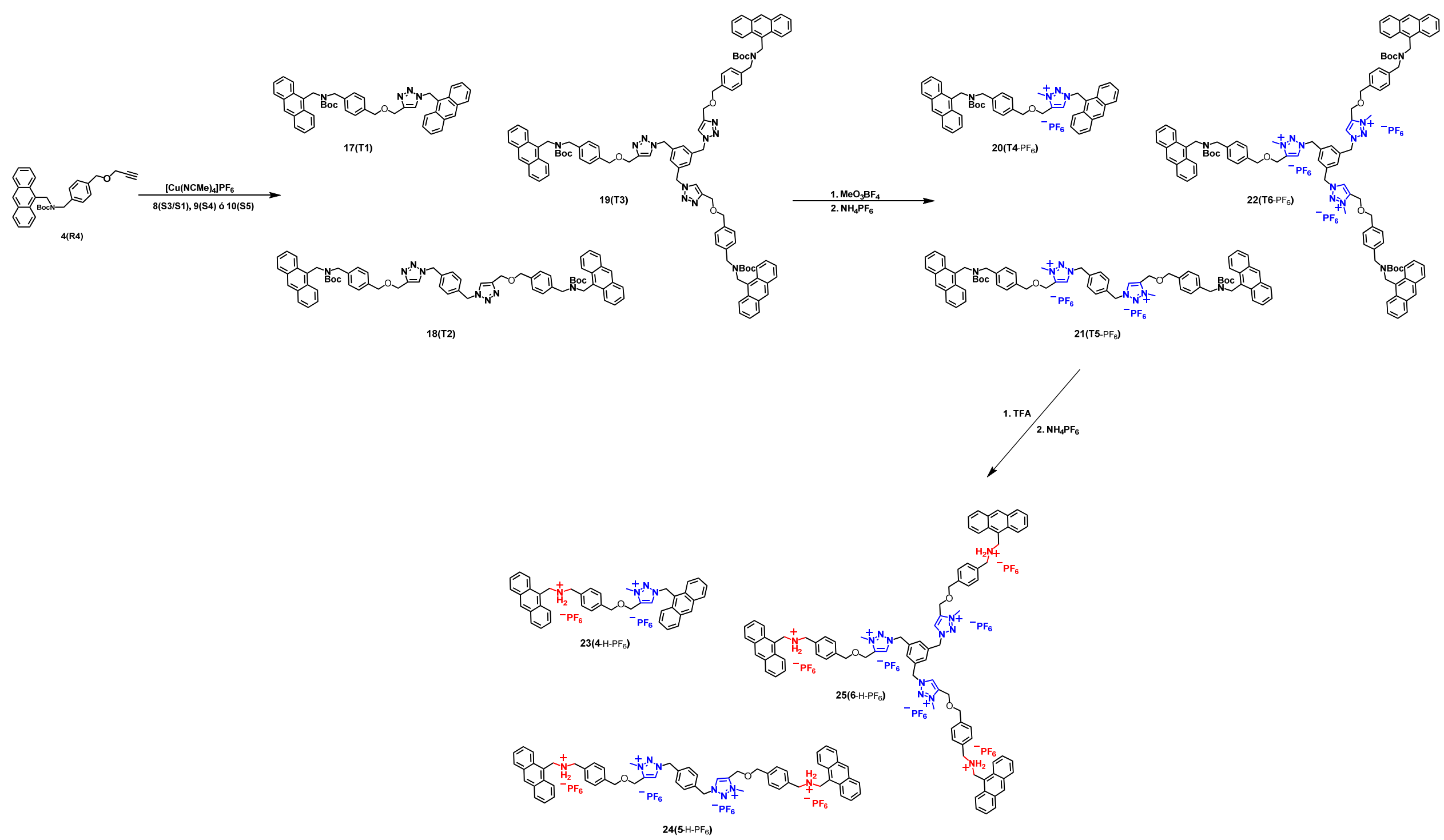

Esquema 15. Método de preparación de derivados análogos a los rotaxanos no enhebrados. 


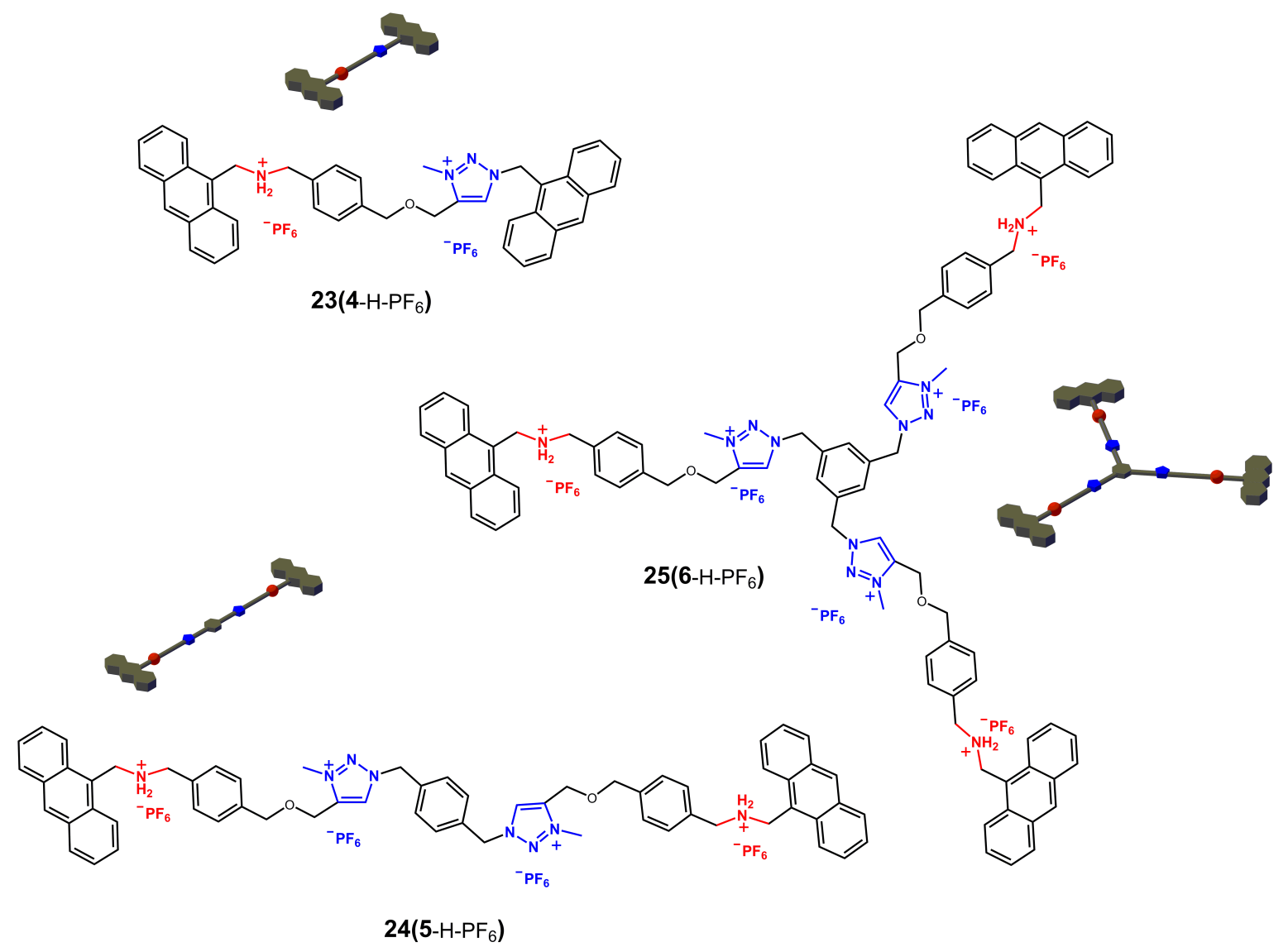

Figura 20. Familia de derivados análogos a los rotaxanos no enhebrados preparados en este trabajo.

Todos los compuestos fueron caracterizados completamente por RMN y, adicionalmente, su identidad confirmada por espectrometría de masas. El compuesto $14\left(1-\mathrm{H}-\mathrm{PF}_{6}\right)$ presenta un gran número de señales en el espectro de ${ }^{1} \mathrm{H}-\mathrm{RMN}$ debido a la baja simetría de la molécula, mientras que los espectros de $\mathbf{1 5}\left(2-\mathrm{H}_{-} \mathrm{PF}_{6}\right)$ y $\mathbf{1 6}\left(3-\mathrm{H}_{-} \mathrm{PF}_{6}\right)$ poseen un número menor de señales ya que muestran una altísima equivalencia química de los núcleos observados. De hecho, los desplazamientos químicos de estos compuestos son prácticamente equivalentes, con la salvedad de la señal aromática perteneciente al grupo fenileno que une los distintos ejes. Esta situación se repite con los derivados no enhebrados.

Las señales correspondientes a los protones bencílicos cercanos a la sal de amonio son anchas y se encuentran a campo bajo respecto de los mismos núcleos en los análogos no enhebrados. Esto normalmente indica la formación de puentes de hidrógeno entre la sal de amonio y el éter corona. ${ }^{47}$ Muy característico es el desplazamiento químico del protón de la sal de triazolinio, que se encuentra a muy bajo campo $(\sim 8 \mathrm{ppm})$ como consecuencia del gran desapantallamiento que sufre el núcleo (Figura 21). 

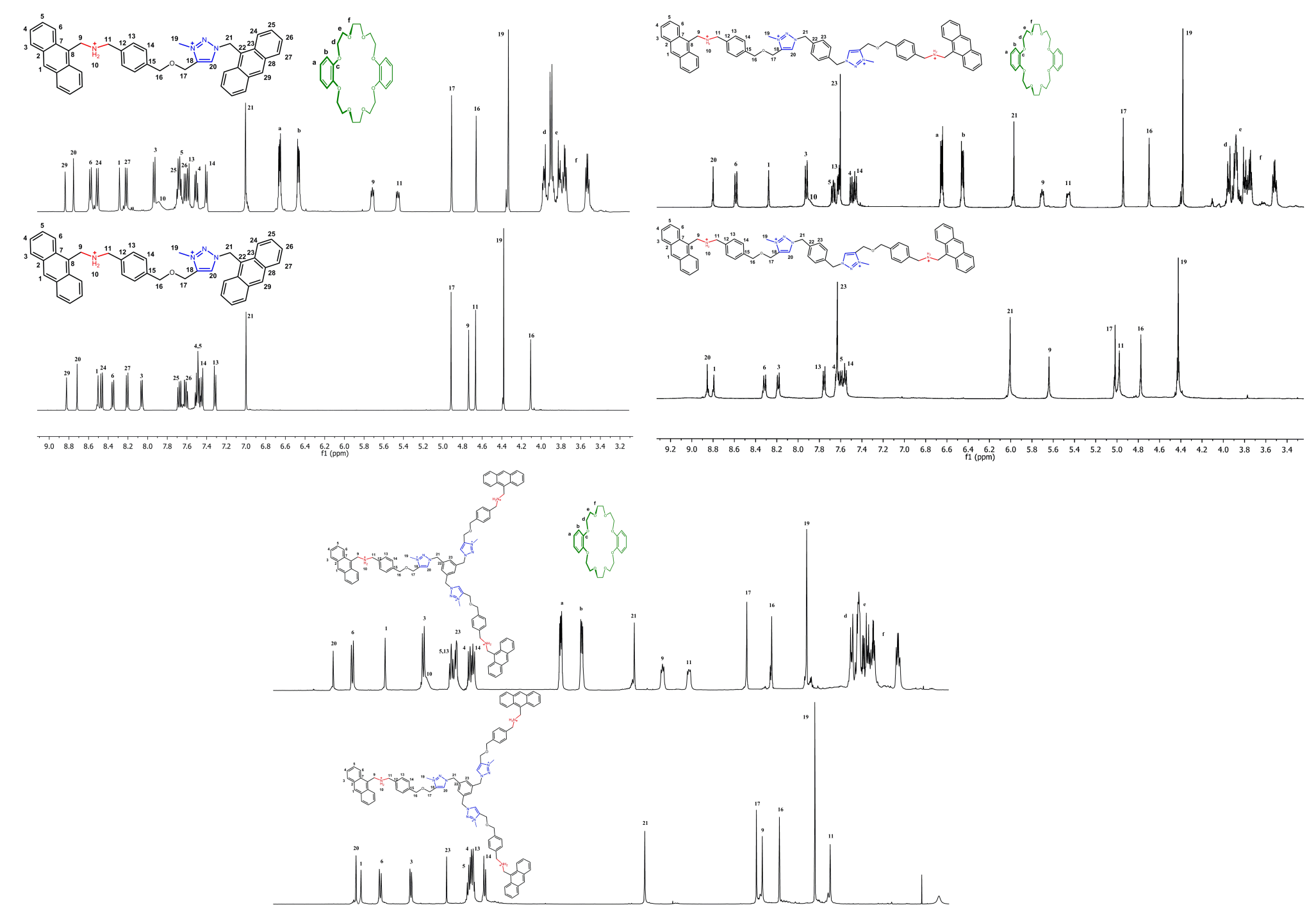

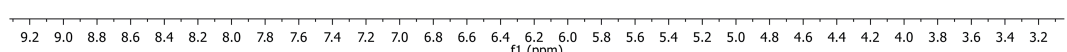

Figura 21. Comparación de espectros de ${ }^{1} \mathrm{H}-\mathrm{RMN}$ en acetona deuterada de todos los compuestos finales en este trabajo y numeración de los núcleos. Se han separado macrociclos y ejes para mayor claridad. 
La posición del anillo se pudo determinar gracias a espectros ${ }^{1} \mathrm{H}-{ }^{1} \mathrm{H}$ NOESY en los que se encontraron puntos de cruce entre protones alifáticos del éter macrocíclico y diversos protones del eje, tal y como se muestra en la Figura 22 y en la Figura 23:

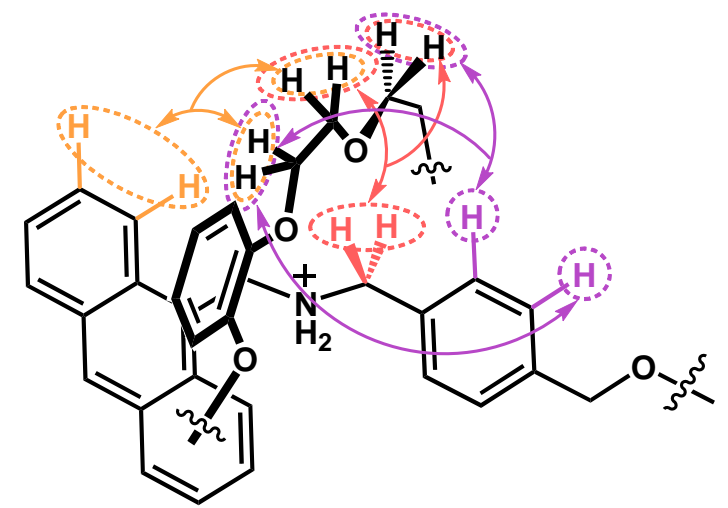

Figura 22. Principales señales NOE observadas entre protones del anillo y del eje en 14(1$\left.\mathrm{H}-\mathrm{PF}_{6}\right), \mathbf{1 5}\left(2-\mathrm{H}-\mathrm{PF}_{6}\right)$ у 16(3-H-PF 6$)$.
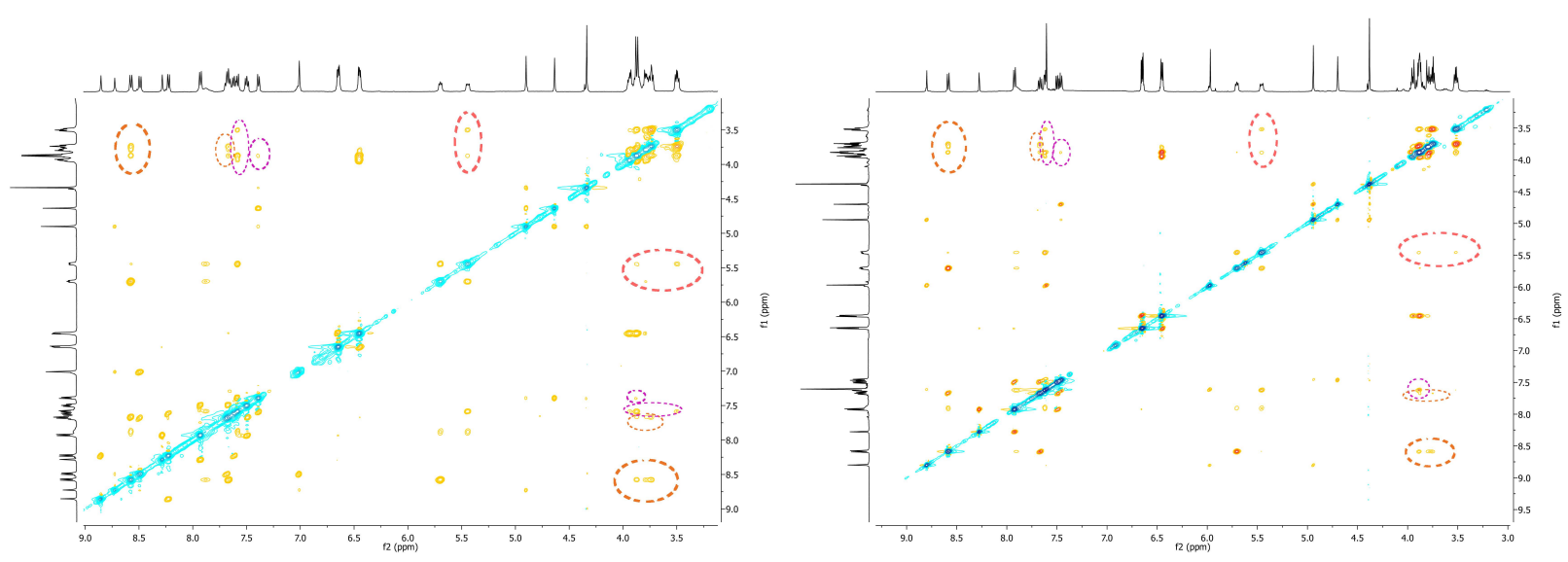

Figura 23. Espectros ${ }^{1} \mathrm{H}-{ }^{1} \mathrm{H}$ NOESY de 14(1-H-PF $)$ y 15(2-H-PF 6 ) señalando con óvalos coloreados los núcleos de eje y anillo próximos en el espacio. El espectro bidimensional de $16\left(3-\mathrm{H}_{-}-\mathrm{PF}_{6}\right)$ es análogo al de $\mathbf{1 5}\left(2-\mathrm{H}_{-} \mathrm{PF}_{6}\right)$ y se ha omitido por claridad.

Hasta la fecha de publicación de este artículo, había dos métodos principales para realizar el proceso de conmutación en este tipo de rotaxanos. ${ }^{47}$ Uno de ellos consistía en lavar una disolución orgánica del compuesto con una disolución acuosa de sosa y el otro en el uso de una resina intercambiadora de protones. Aunque el preferido por la mayoría de autores es el primero, no lo consideramos como un proceso on/off real ya que ambos requieren un procedimiento de separación posterior, decantación o filtrado, respectivamente. 
Nuestro método consiste en la adición de una cantidad adecuada de una disolución de $\mathrm{NaOH}$ $1 \mathrm{M}$ en $\mathrm{D}_{2} \mathrm{O}$ (1,1 equivalentes por grupo amonio) en una disolución del rotaxano en acetona deuterada y posterior agitación. Este medio es lo suficientemente básico como para desprotonar los grupos amonio dando lugar a aminas secundarias. Estos grupos establecen peores interacciones supramoleculares con el éter macrocíclico que la sal de triazolinio, por lo que el anillo es forzado a moverse a lo largo del eje hasta encontrar un mínimo de energía adecuado en la segunda estación. Por otro lado, la adición de un ácido fuerte no acuoso a la disolución, como TFA, reprotona la amina regenerando la sal de amonio y volviendo a forzar al macrociclo a volver a la posición inicial debido a que la primera estación vuelve a tener alta afinidad por el anillo, recuperándose la situación de partida y completando el ciclo de conmutación.

El proceso completo se ha analizado comparando los espectros monodimensionales de ${ }^{1} \mathrm{H}$ RMN y bidimensionales de ${ }^{1} \mathrm{H}-{ }^{1} \mathrm{H}$ NOESY antes y después de cada adición. De esta manera, se han podido observar cambios importantes en los desplazamientos químicos de los núcleos próximos a las estaciones así como otros cambios menores en el resto de señales.

Como era de esperar, se observan los cambios más grandes en los protones más próximos a ambas estaciones. Los protones bencílicos de la amina secundaria ( $\mathrm{H}_{9}$ y $\mathrm{H}_{11}$ en todos los casos) sufren un desplazamiento a campo alto de alrededor de $1 \mathrm{ppm}$, el protón del grupo triazolinio $\mathrm{H}_{20}$ sufre desplazamiento a campo bajo de aproximadamente $0,5 \mathrm{ppm}$ y el núcleo del grupo bencílico unido directamente al stopper $\left(\mathrm{H}_{21}\right)$, un desplazamiento a campo alto de unas $0,5 \mathrm{ppm}$. El conjunto de desplazamientos químicos pertenecientes al éter macrocíclico también varía considerablemente. En el caso de $\mathbf{1 5}\left(2-\mathrm{PF}_{6}\right)$ y $\mathbf{1 6}\left(3-\mathrm{PF}_{6}\right)$ se observa, además, un ensanchamiento de algunas señales del anillo, sugiriendo poca libertad en el movimiento Browniano de estos grupos por su mutua proximidad espacial. Adicionalmente, en $\mathbf{1 6}\left(3-\mathrm{PF}_{6}\right)$, la señal del protón del grupo triazolinio $\left(\mathrm{H}_{20}\right)$ desaparece prácticamente tras la primera adición de $\mathrm{NaOH}$ en $\mathrm{D}_{2} \mathrm{O}$, indicando intercambio por deuterio. Los otros dos rotaxanos también sufren este efecto si se dejan en disolución durante mucho tiempo. De nuevo, los espectros ${ }^{1} \mathrm{H}-{ }^{1} \mathrm{H}$ NOESY fueron clave para confirmar la nueva posición del anillo (Figura 24 y Figura 25).
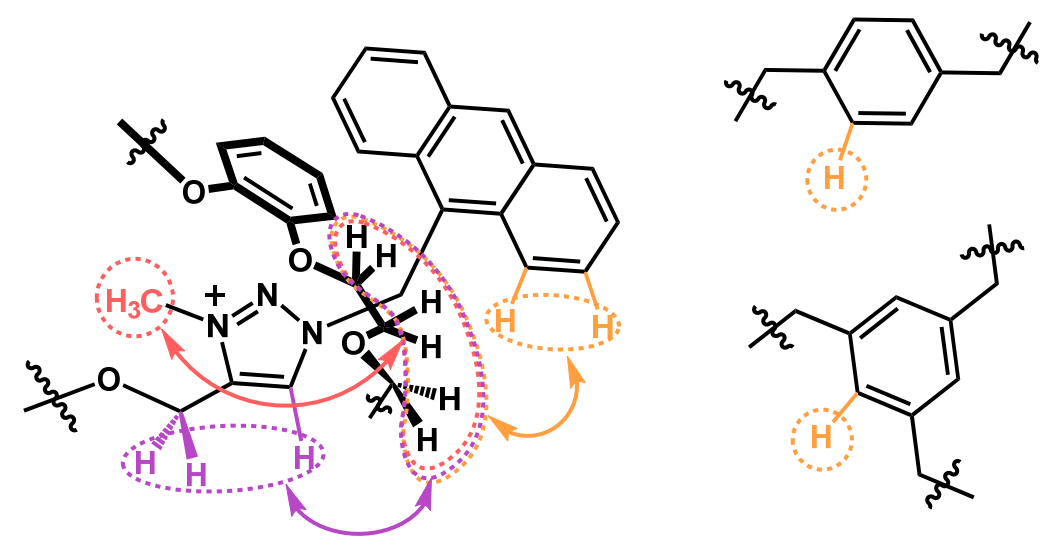

Figura 24. Principales señales NOE observadas entre protones del anillo y del eje en 14(1$\left.\mathrm{PF}_{6}\right)$, 15(2-PF $)$ y $16\left(3-\mathrm{PF}_{6}\right)$. 

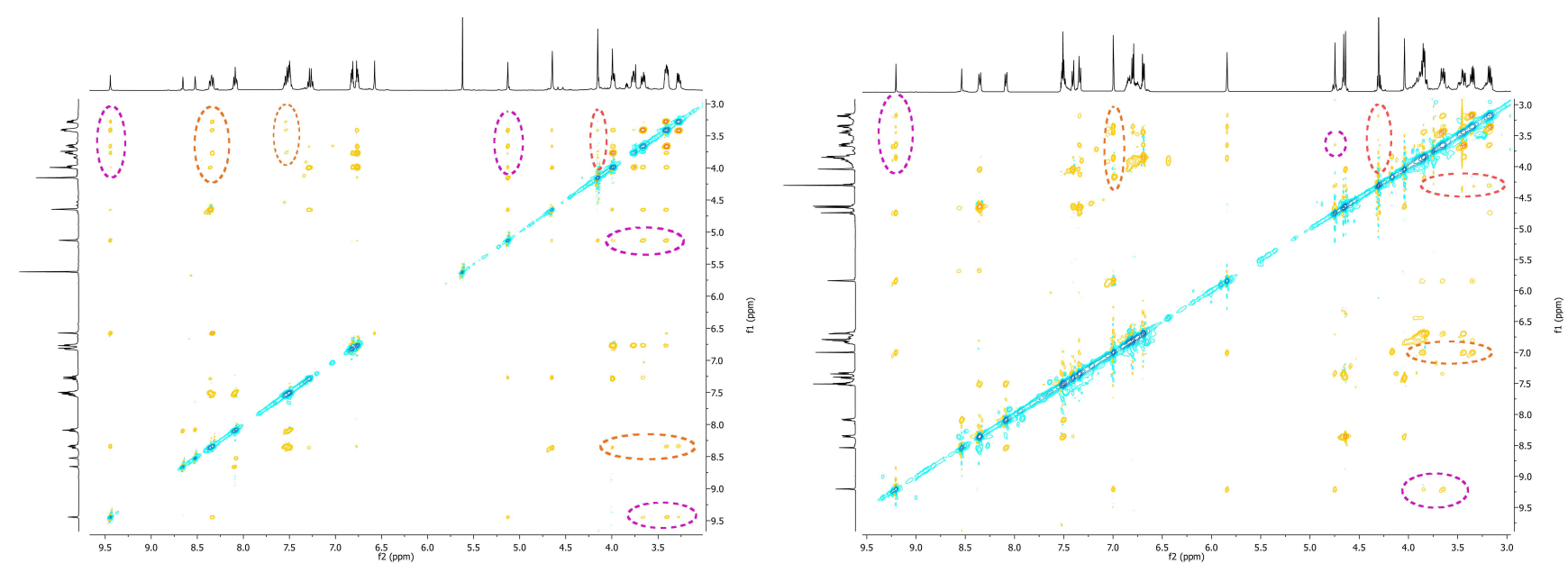

Figura 25. Espectros ${ }^{1} \mathrm{H}-{ }^{1} \mathrm{H}$ NOESY de 14(1-PF $)$ y 15(2- $\left.\mathrm{PF}_{6}\right)$ señalando con óvalos coloreados los núcleos de eje y anillo próximos en el espacio. El espectro bidimensional de $\mathbf{1 6}\left(\mathbf{3}-\mathrm{PF}_{6}\right)$ es análogo al de $\mathbf{1 5}\left(2-\mathrm{PF}_{6}\right)$ y se ha omitido por claridad.

Tras la reprotonación con TFA que recupera la posición del anillo, se obtienen unos espectros muy similares a los de partida, pero no iguales. Las diferencias en desplazamiento químico respecto del espectro original se atribuyen al efecto del contraión. ${ }^{127}$ De hecho, si una disolución del rotaxano con trifluoroacetato como anión se pone en contacto con una disolución acuosa de $\mathrm{NH}_{4} \mathrm{PF}_{6}$ y se agita vigorosamente, se obtiene el mismo espectro de partida de la fase orgánica debido a la total recuperación del anión hexafluorofosfato. Este proceso puede realizarse varias veces sin apreciarse descomposición de los rotaxanos y se encuentra resumido en la Figura 26.

${ }^{127}$ Evans, N. H.; Beer, P. D. Angew. Chem. Int. Ed. 2014, 53, 11716-11754. 


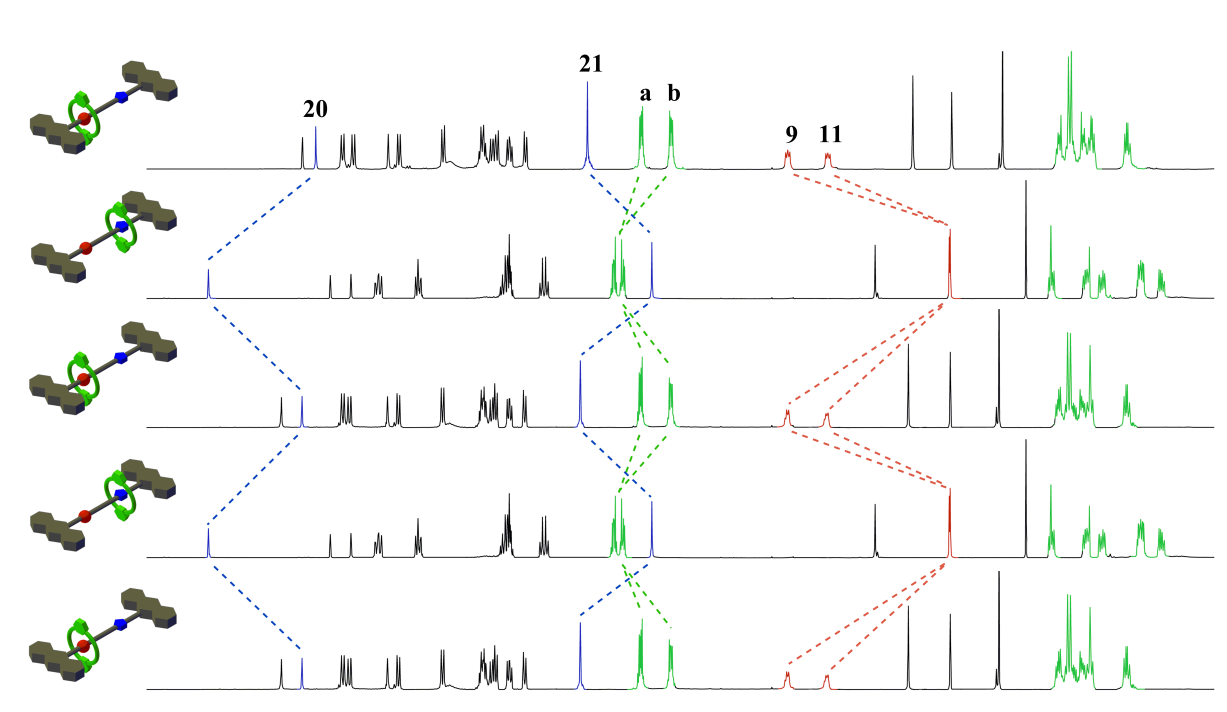

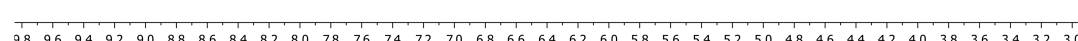

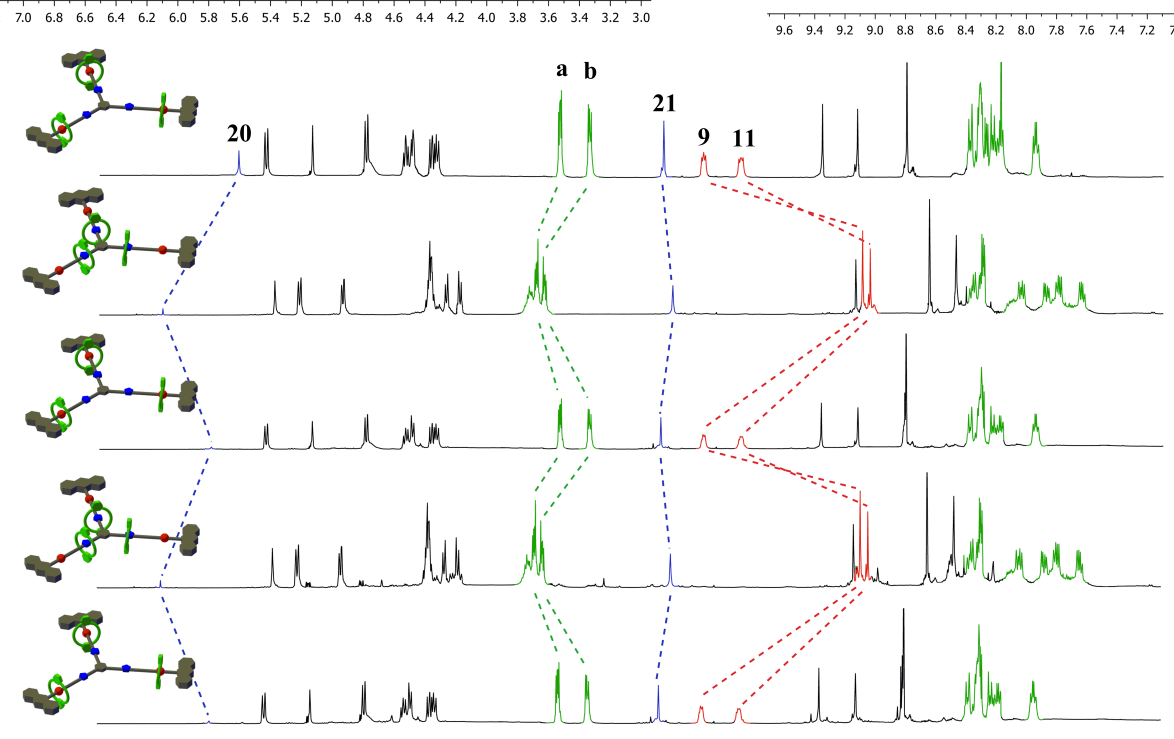

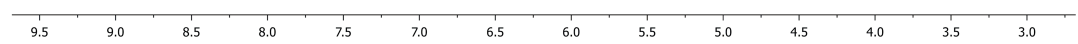

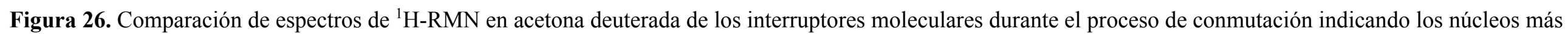
interesantes implicados en el movimiento del macrociclo. Nótese cómo nunca se recupera el espectro inicial debido a la presencia del anión trifluoroacetato. 
Una vez caracterizado todos los interruptores moleculares, junto con sus derivados análogos no enhebrados, y controlado el movimiento del éter macrocíclico a lo largo del eje, se procedió a realizar un estudio para su potencial uso como organocatalizadores. Tal y como se discutió en la parte de Introducción, es de esperar que el rotaxano no produzca catálisis cuando el anillo cubre el centro catalítico y que sí la efectúe una vez expuesto. Con el objetivo de dilucidar si la ausencia de actividad se debe al recubrimiento de la sal de amonio o al hecho de que el nitrógeno esté protonado, todos los derivados no enhebrados se sometieron al mismo estudio, así como dibencilamina a efectos de comparación. Las dos reacciones puestas a prueba se encuentran reflejadas en el Esquema 16.

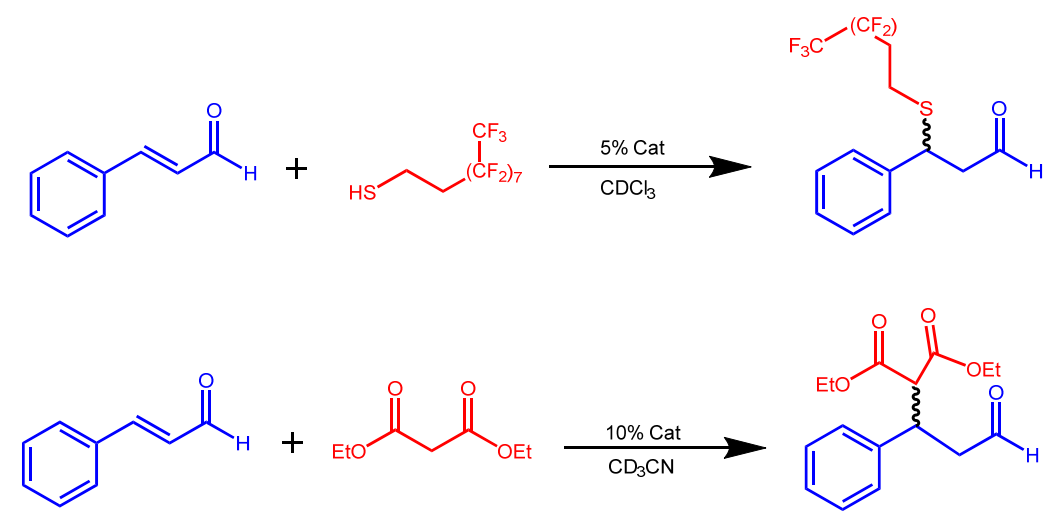

Esquema 16. Reacciones de adición tipo Michael estudiadas en el presente trabajo.

El primer caso, estudiado previamente por el grupo de $\mathrm{Leigh}^{47}$ consiste en la adición 1,4 de un tiol ${ }^{128}$ a trans-cinnamaldehído. La presencia del catalizador al $5 \%$ mol en $\mathrm{CDCl}_{3}$ y la adición de una disolución $1 \mathrm{M}$ de $\mathrm{NaOH}$ en $\mathrm{D}_{2} \mathrm{O}$ (1,2 equivalentes por centro catalítico) constituyen las condiciones óptimas para que la reacción transcurra en un tiempo razonable. Se realizaron espectros de ${ }^{1} \mathrm{H}-\mathrm{RMN}$ a dos tiempos distintos y se comparó la conversión por integración de las señales de los grupos $-\mathrm{CH}_{2}-$. Los resultados están resumidos en la Tabla 1.

Cuando los interruptores están en situación inactiva (éter macrocíclico cubriendo la sal de amonio, entradas 1-3 en la Tabla 1), no se observa reacción en absoluto. Cuando se realiza la conmutación, los interruptores moleculares tienen los centros catalíticos expuestos en forma de aminas secundarias, produciéndose catálisis (entradas 4-6 en la Tabla 1). Como se puede inferir de los datos de la tabla, el mejor organocatalizador es $16\left(3-\mathrm{PF}_{6}\right)$ debido a que posee la mejor conversión a ambos tiempos seguido de cerca por $15\left(2-\mathrm{PF}_{6}\right)$ y, por último, 14(1-PF $)_{6}$. Nótese que incluso el peor catalizador de entre todos los interruptores moleculares es mejor que la dibencilamina bajo las mismas condiciones (entrada 7 de la Tabla 1).

${ }^{128}$ El motivo por el cual se escogió el tiol fluorado representtado en el Esquema 16 se debe a que es un compuesto comercial inodoro, no volátil y tiene un espectro ${ }^{1} \mathrm{H}-\mathrm{RMN}$ muy sencillo. 
Tabla 1. Resultados observados para la adición catalizada de $1 \mathrm{H}, 1 \mathrm{H}, 2 \mathrm{H}, 2 \mathrm{H}-$ perfluorodecanotiol a trans-cinnamaldehído. ${ }^{\text {a }}$

\begin{tabular}{|c|c|c|c|}
\hline Entrada & Compuesto & $\begin{array}{c}\text { Conve rsión tras } \\
30 \text { minutos }\end{array}$ & $\begin{array}{c}\text { Conversión tras } \\
4 \text { horas }\end{array}$ \\
\hline 1 & $14\left(1-\mathrm{H}^{-} \mathrm{PF}_{6}\right)^{\mathrm{b}}$ & 0 & 0 \\
\hline 2 & $15\left(2-\mathrm{H}^{-}-\mathrm{PF}_{6}\right)^{\mathrm{b}}$ & 0 & 0 \\
\hline 3 & $16\left(3-\mathrm{H}_{-} \mathrm{PF}_{6}\right)^{\mathrm{b}}$ & 0 & 0 \\
\hline 4 & $14\left(1-\mathrm{PF}_{6}\right)$ & 50 & 72 \\
\hline 5 & $15\left(2-\mathrm{PF}_{6}\right)$ & 68 & 79 \\
\hline 6 & $16\left(3-\mathrm{PF}_{6}\right)$ & 80 & 85 \\
\hline 7 & Dibencilamina & 10 & 25 \\
\hline 8 & $23\left(4-H_{-}-F_{6}\right)^{b}$ & 8 & 21 \\
\hline 9 & $24\left(5-H_{-}-F_{6}\right)^{b}$ & 12 & 28 \\
\hline 10 & $25\left(6-\mathrm{H}^{-}-\mathrm{PF}_{6}\right)^{\mathrm{b}}$ & 20 & 35 \\
\hline 11 & $23\left(4-\mathrm{PF}_{6}\right)$ & 45 & 70 \\
\hline 12 & $24\left(5-\mathrm{PF}_{6}\right)$ & 65 & 75 \\
\hline 13 & $25\left(6-\mathrm{PF}_{6}\right)$ & 74 & 83 \\
\hline
\end{tabular}

(a) Condiciones resumidas: trans-cinnamaldehído (0,14 mmol), $1 \mathrm{H}, 1 \mathrm{H}, 2 \mathrm{H}, 2 \mathrm{H}$ perfluorodecanotiol $(0,168 \mathrm{mmol})$, catalizador $(5 \% \mathrm{~mol}), \mathrm{CDCl}_{3}(1,4 \mathrm{~mL})$ y $\mathrm{NaOH}(1,2$ equivalentes por centro catalítico). (b) Estos compuestos no fueron tratados con $\mathrm{NaOH}$.

Por otro lado, también se observa catálisis con los derivados no enhebrados, tanto en su forma inactiva como activa. Esto demuestra claramente el control del centro catalítico que ejercen los rotaxanos a través de la cobertura o exposición del mismo y no debido al mero hecho de tener la amina protonada o no. También hay que hacer notar que los derivados no enhebrados en forma de sal de amonio son mucho más lentos que sus contrapartidas desprotonadas. Esto se puede deber a que se establece un pre equilibrio de desprotonación previa activación del electrófilo que es dificultado en las condiciones dadas.

En el segundo caso se usó malonato de dietilo como nucleófilo para la síntesis de un producto de condensación mediante la formación de un enlace C-C. El único disolvente orgánico encontrado en el que la reacción transcurriera favorablemente en un tiempo razonable y, al mismo tiempo, permitiera que los rotaxanos se mantuvieran en disolución, fue acetonitrilo. Los tiempos de reacción aumentaron considerablemente, incluso doblando la cantidad de catalizador empleado. La cantidad de $\mathrm{NaOH}$ añadida para producir la conmutación de los interruptores moleculares se mantuvo. Las conversiones se determinaron por integración de las señales de aldehído en los espectros de ${ }^{1} \mathrm{H}$ RMN realizados a dos tiempos distintos. Los resultados se encuentran en la Tabla 2. 
Tabla 2. Resultados observados para la adición catalizada de malonato de dietilo a trans-cinnamaldehído. ${ }^{\mathrm{a}}$

\begin{tabular}{|c|c|c|c|}
\hline Entrada & Compuesto & $\begin{array}{c}\text { Conversión tras } \\
30 \text { minutos }\end{array}$ & $\begin{array}{c}\text { Conversión tras } \\
24 \text { horas }\end{array}$ \\
\hline 1 & $14\left(1-\mathrm{H}_{-} \mathrm{PF}_{6}\right)^{\mathrm{b}}$ & 0 & 0 \\
\hline 2 & $15\left(2-\mathrm{H}^{-} \mathrm{PF}_{6}\right)^{\mathrm{b}}$ & 0 & 0 \\
\hline 3 & $16\left(3-\mathrm{H}^{-}-\mathrm{PF}_{6}\right)^{\mathrm{b}}$ & 0 & 0 \\
\hline 4 & $14\left(1-\mathrm{PF}_{6}\right)$ & 5 & 68 \\
\hline 5 & $15\left(2-\mathrm{PF}_{6}\right)$ & 13 & 80 \\
\hline 6 & $16\left(3-P_{6}\right)$ & 20 & 100 \\
\hline 7 & Dibencilamina & 12 & 65 \\
\hline 8 & $23\left(4-\mathrm{H}_{-} \mathrm{PF}_{6}\right)^{\mathrm{b}}$ & 5 & 32 \\
\hline 9 & $24\left(5-H_{-}-\mathrm{PF}_{6}\right)^{\mathrm{b}}$ & 14 & 45 \\
\hline 10 & $25\left(6-\mathrm{H}^{-} \mathrm{PF}_{6}\right)^{\mathrm{b}}$ & 19 & 80 \\
\hline 11 & $23\left(4-\mathrm{PF}_{6}\right)$ & 6 & 65 \\
\hline 12 & $24\left(5-P_{6}\right)$ & 15 & 75 \\
\hline 13 & $25\left(6-\mathrm{PF}_{6}\right)$ & 22 & 100 \\
\hline
\end{tabular}

(a) Condiciones resumidas: trans-cinnamaldehído $(0,28 \mathrm{mmol})$, malonato de dietilo $(0,14 \mathrm{mmol})$, catalizador $(10 \% \mathrm{~mol}), \mathrm{CD}_{3} \mathrm{CN}(1,4 \mathrm{~mL})$ y $\mathrm{NaOH}(1,2$ equivalentes por centro catalítico). (b) Estos compuestos no fueron tratados con $\mathrm{NaOH}$.

Las conclusiones deducidas en este segundo experimento son análogos a los obtenidos en el primero, con la salvedad de que los derivados no enhebrados tienen el mismo comportamiento tanto si la amina se encuentra protonada como si no (entradas 8-13 en la Tabla 2). Una vez más se observó la capacidad que tienen los tres interruptores moleculares de controlar su actividad catalítica en función de la posición del anillo (entradas 1-6 en la Tabla 2), así como la mejora encontrada en rotaxanos con múltiples centros activos.

En la Figura 27 se encuentra, a modo de ilustración, espectros de ${ }^{1} \mathrm{H}-\mathrm{RMN}$ apilados donde se muestra la actividad catalítica de los interruptores moleculares preparados en este trabajo.

Este trabajo nos ha permitido inferir un conjunto de conclusiones interesante. En primer lugar, el proceso de conmutación ácido-base puede realizarse in situ evitando procedimientos de separación. En segundo lugar, la unidad fenilénica o el anillo bencénico trisustituido han demostrado ser excelentes conectores de ejes ya que los éteres macrocíclicos se pueden acomodar incluso en situaciones en las que se encuentran muy cercanos. Respecto al comportamiento catalítico, la importancia sobre la exposición o cobertura del centro activo se ha hecho evidente a lo largo de la discusión y no es debida, simplemente, a si la amina secundaria se encuentra protonada o no. Adicionalmente, se ha observado que cualquier rotaxano en este trabajo realiza su función catalítica mejor que dibencilamina en las mismas condiciones. Por último, se ha comprobado cómo el aumento del número de centros catalíticos en un interruptor molecular es un factor importante para mejorar su rendimiento; sin embargo, hay otros factores a tener en cuenta, como, por ejemplo, la flexibilidad de los rotaxanos o su solubilidad. 

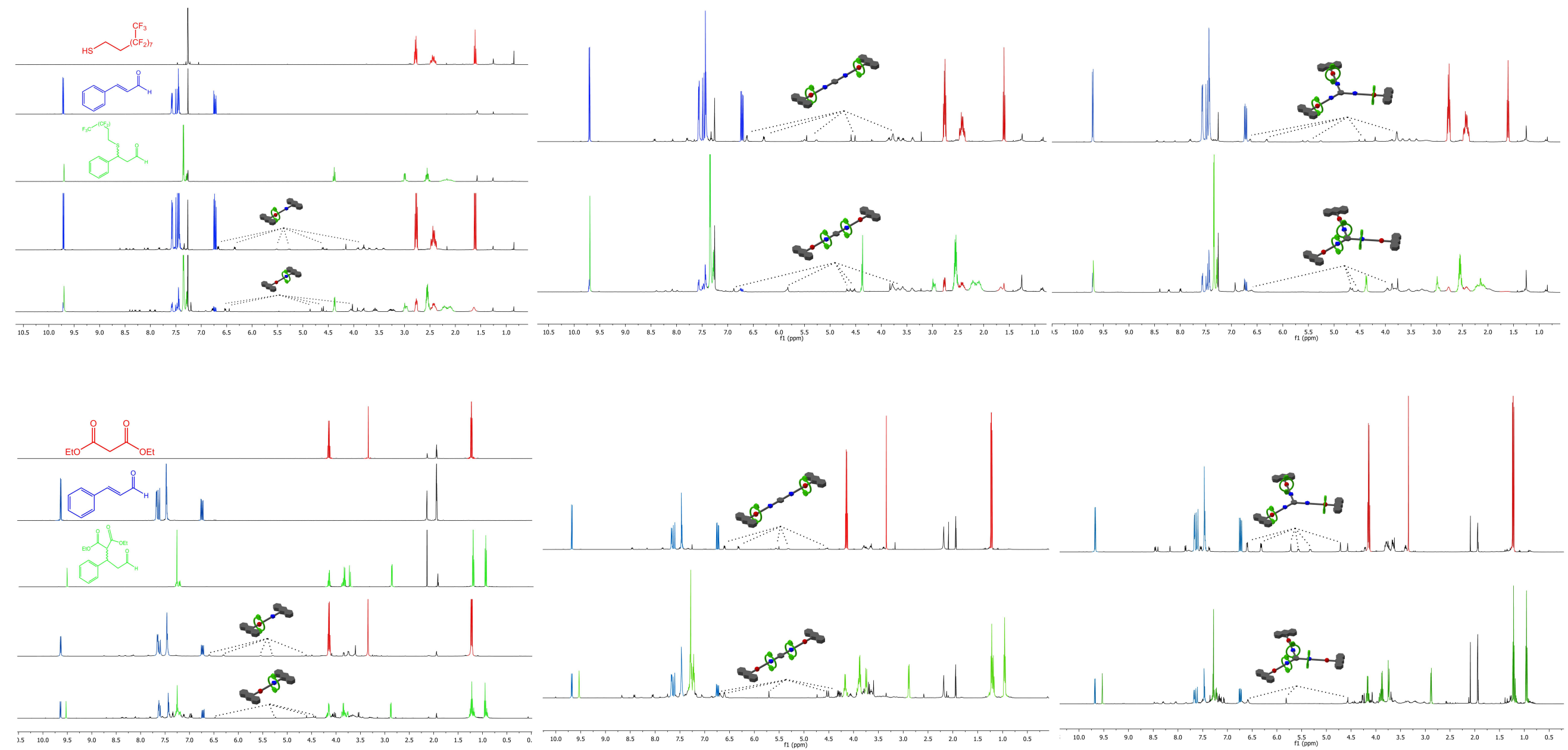

Figura 27. Comparación de espectros de ${ }^{1} \mathrm{H}-\mathrm{RMN}$ en todos los estudios catalíticos controlados por rotaxanos. En todos los casos se muestra el espectro con el catalizador inactivo primero y después con él activo tras alcanzar la conversión máxima. También se especifica, con un código de colores RGB, las señales pertenecientes a nucleófilo, electrófilo y producto de condensación en las imágenes donde está involucrado el compuesto 14(1-H-PF6). 


\subsubsection{Artículo II. Study of pH-sensitive bistable molecular shuttles incorporating azobenzene as a kinetic barrier for photochemically triggered memory switching}

En este segundo trabajo se avanza en la síntesis de nuevos rotaxanos e interruptores moleculares aprovechando las estructuras desarrolladas en el anterior e incorporando el fragmento de azobenceno entre las dos estaciones ya descritas con el objetivo de estudiar la posible barrera cinética impuesta por su isómero configuracional $Z$ en el proceso de conmutación de la posición del anillo, tal y como se describió en la parte de Introducción.

La elección de un azobenceno disustituido asimétricamente responde a las necesidades de futuras reacciones de unión de los distintos building blocks. Por un lado, el grupo fenol nos permitía acoplarlo fácilmente a $\mathbf{3}(\mathbf{R} \mathbf{3} / \mathbf{R} 1)$ y, además nos dio acceso a la formación de la molécula de azobenceno a través del método ampliamente conocido de acoplamiento de sales de diazonio. ${ }^{52}$ Por otro lado, es evidente la necesidad de ubicar un triple enlace terminal ya que la reacción de cierre iba a realizarse por metodología click aprovechando el stopper $\mathbf{8}(\mathbf{S 3} / \mathbf{S 1})$. La diferenciación en ellos se encuentra en el patrón de sustitución del grupo alquinilo (para y meta en 27(A1) y 28(A2)), a la incorporación de un pequeño espaciador para evitar conexión directa entre estaciones (grupo propargilo en 31(A5)) y a la imposición de una pequeña barrera estérica por la existencia de metilos en posiciones orto al hidroxilo (en 29(A3)). En el caso de 31(A5), se necesitó realizar una etapa adicional para formar el éter propargílico a partir del difenol 30(A4), como se muestra en el Esquema 17.
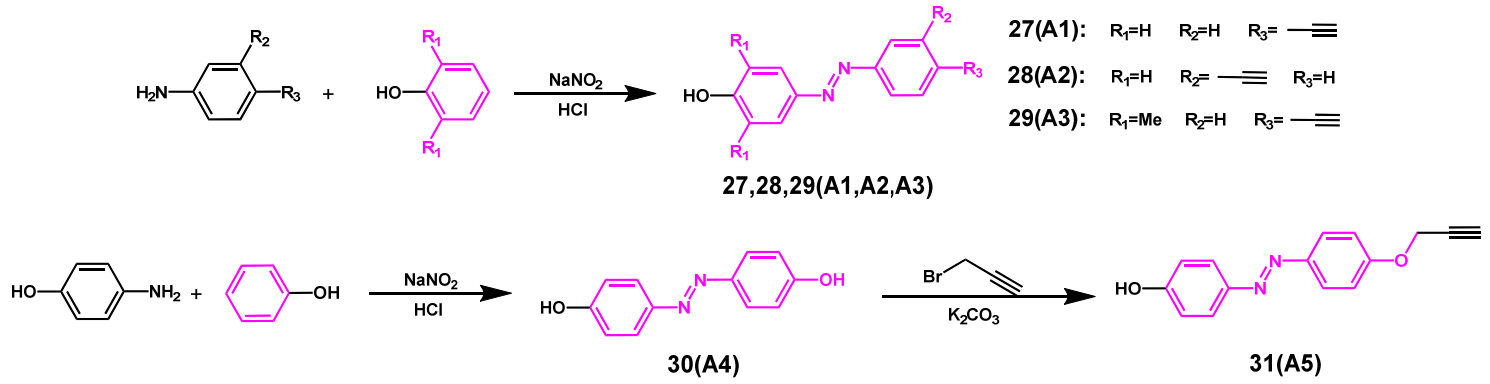

Esquema 17. Obtención de la serie de derivados de azobenceno descritos en el primer bloque.

Se llevaron a cabo dos métodos para la obtención de los éteres. En primer lugar, el procedimiento directo de acoplamiento a través de la reacción de Mitsunobu ${ }^{129}$ dio como resultado los derivados deseados, pero con rendimientos bastante bajos.

129 (a) Mitsunobu, O.; Wada, M.; Sano, T. J. Am. Chem. Soc. 1972, 94, 679-680. (b) Swamy, K. C. K.; Kumar, N. N. B.; Balaraman, E.; Kumar, K. V. P. P. Chem. Rev. 2009, 109, 2551-2651. 
El fracaso de esta reacción podría deberse a la reacción lateral producida por el ataque de la trifenilfosfina a nuestro azo derivado en vez de a DIAD, a pesar de que está mucho menos activado. En segundo lugar, se llevó a cabo la clásica transformación del grupo hidroxilo en 3(R3/R1) en un buen grupo saliente (usando un metilsulfonato en este caso) dando lugar a 26(R2) que sufrió el ataque del fenóxido correspondiente proporcionando el éter objetivo. A pesar de requerir un paso extra en el procedimiento sintético, los rendimientos obtenidos fueron razonablemente superiores recayendo gran parte del éxito en el hecho de que el mesilato 26(R2) no requiere de purificación más allá de un lavado con agua. Esta metodología está recogida en el Esquema 18.

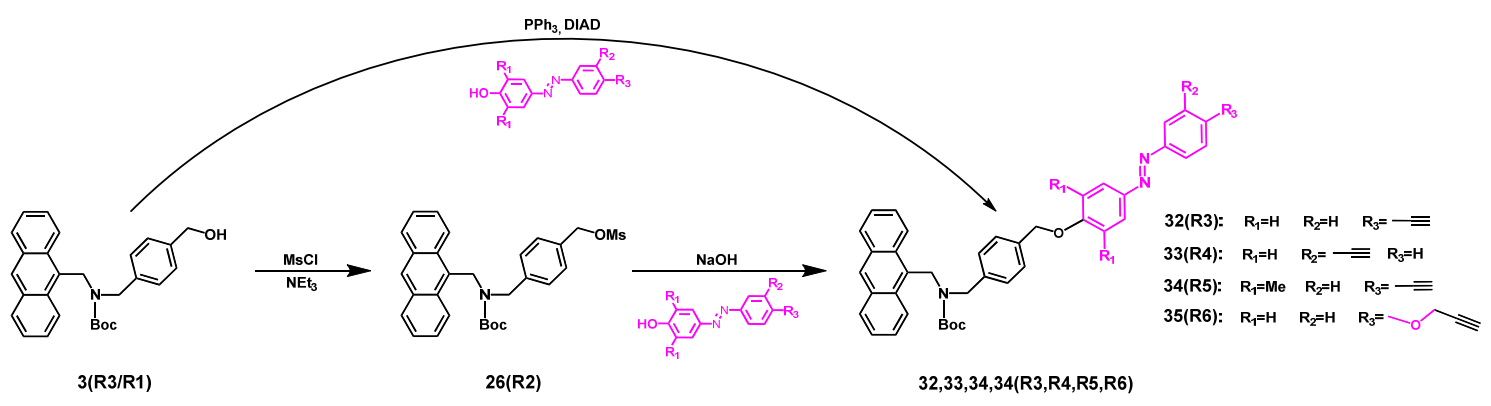

Esquema 18. Síntesis de la serie de azo éteres previos a la formación de rotaxanos.

Tal y como se realizó en el anterior trabajo de este bloque, el siguiente paso consistiría en la desprotección del grupo amino por hidrólisis del carbamato y posterior aislamiento de la sal de amonio para formar el pseudorotaxano en la siguiente transformación. Desgraciadamente, esto no pudo ser posible usando estos sistemas ya que se observó descomposición de la mayor parte del material cuando se realizó el primer ensayo con 32(R3) y los intentos por purificar el producto resultante por cromatografía en columna sólo dieron como resultado un conjunto de fracciones no caracterizadas excepto por una que, resultó ser la amina 42(7) con un rendimiento muy bajo (Esquema 19). Aunque este compuesto puede ser protonado en presencia del éter corona y así formar el pseudorotaxano objetivo, se decidió cambiar el método para intentar mejorar el procedimiento de obtención de los rotaxanos deseados.

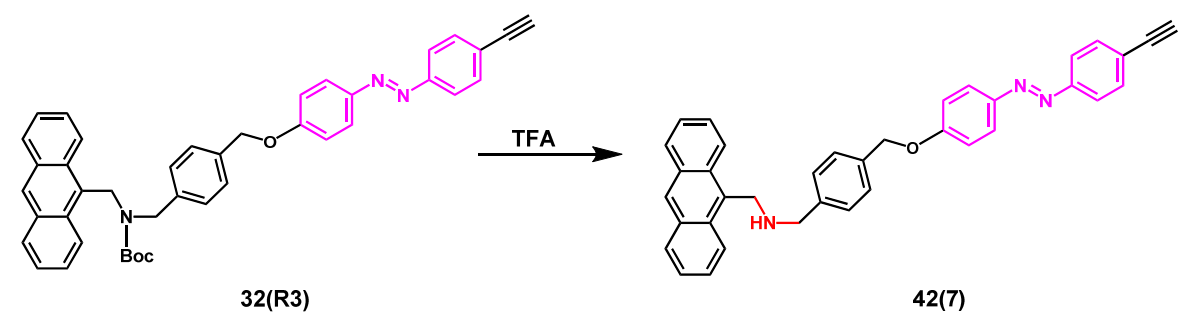

Esquema 19. Obtención de la amina secundaria 42(7) tras el intento de aislamiento de su sal de amino procedente de la desprotección del carbamato 32(R3). 
Así, se llevó a cabo la desprotección de los carbamatos en presencia del éter DB24C8 y se analizó por RMN observándose la eficiente formación del complejo de inclusión entre ambos compuestos evitando descomposición del material por la estabilización de la sal de amonio gracias al éter macrocíclico. Una vez la desprotección tuvo lugar, se eliminaron disolvente y ácido a vacío y se añadieron las especies necesarias para efectuar la reacción click de cierre final obteniendo en último término los rotaxanos deseados tras la purificación pertinente. Este procedimiento one-pot permitió saltarse un paso de aislamiento previo y los rendimientos mejoraron razonablemente (Esquema 20). La metilación posterior del triazol generó la segunda estación del interruptor molecular. Desgraciadamente, esta metodología no funcionó para 34(R5) ya que, al parecer, los grupos metilo impidieron el enhebrado a temperatura ambiente dando lugar, una vez realizada la reacción de cierre, a la amina 43(8) con bajo rendimiento junto con una descomposición masiva de la mayoría del material (Esquema 21). La calefacción del sistema con el objetivo de superar la barrera energética no resolvió el problema porque se observó la descomposición completa del compuesto.

Se realizó un estudio computacional optimizando distintos pseudorotaxanos procedentes de la desprotección de 32(R3) y 34(R5) con un método semiempírico posicionando el éter corona en distintas ubicaciones del eje. Como resultado se observó que el camino energético del proceso de enhebrado es más o menos similar hasta que se alcanza el punto crítico donde se sitúan los metilos del azobenceno. El pseudorotaxano es $10.7 \mathrm{kcal} / \mathrm{mol}$ menos estable que aquél cuyo éter corona se encuentra en la posición del doble enlace $-\mathrm{N}=\mathrm{N}-\mathrm{y}$, si no posee los metilos, sólo lo es por $4,3 \mathrm{kcal} / \mathrm{mol}$.
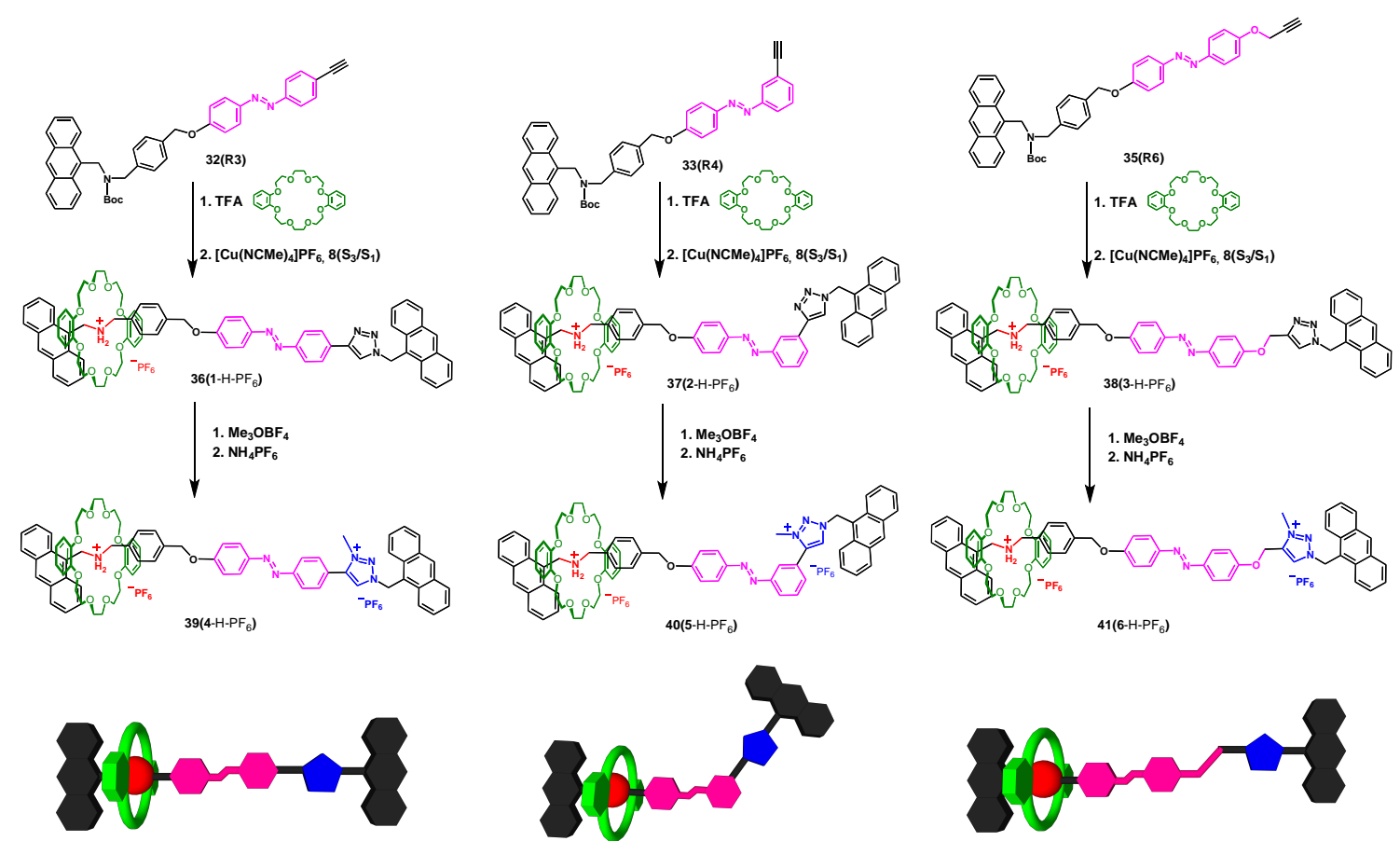

Esquema 20. Metodología one-pot para la preparación de los interruptores moleculares descritos en este trabajo. 


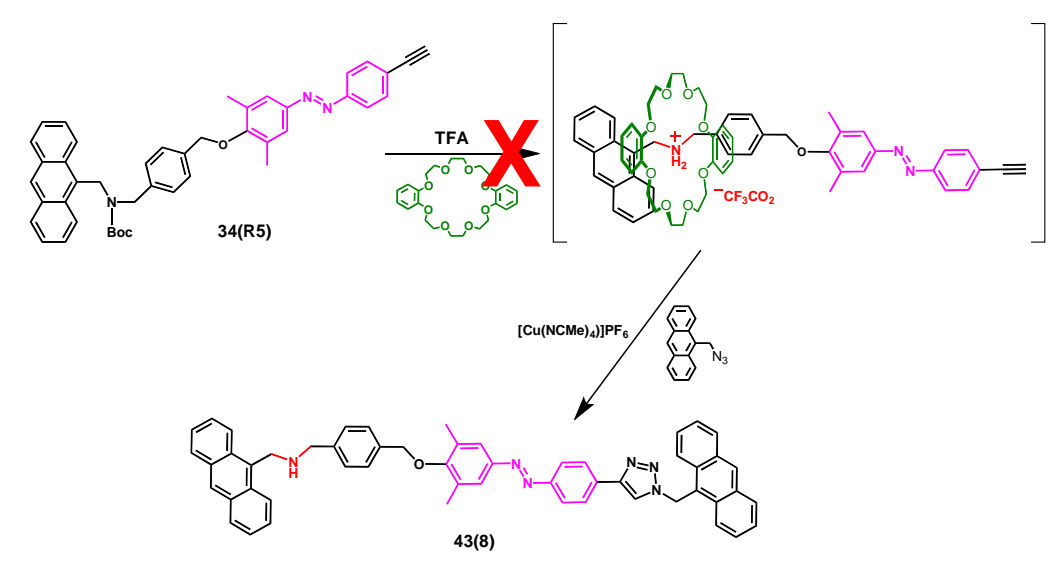

Esquema 21. Intento de formación del rotaxano proveniente de 34(R5) dando lugar al compuesto no deseado 43(8).

Aunque las barreras para ese proceso y el posterior no fueron calculadas, ${ }^{130}$ es de esperar que sean más elevadas para el sistema metilado, inhibiendo la formación del complejo de inclusión final a temperatura ambiente a través de este método de enhebrado (Figura 28).

Los tres interruptores moleculares obtenidos fueron caracterizados gracias a técnicas espectroscópicas de la misma manera que se realizó en el trabajo anterior. Los espectros ${ }^{1} \mathrm{H}$ RMN muestran un patrón muy similar al que poseen los rotaxanos catalizadores del artículo previo, destacando los protones de los grupos $-\mathrm{CH}_{2}$ - directamente conectados a la sal de amonio ( $\mathrm{H}_{9}$ y $\mathrm{H}_{11}$ en todos los casos), cuyo desplazamiento químico aparece en torno a 5,5 ppm y las señales son anchas indicando de nuevo que el rotaxano se ha formado eficientemente. También se observa el desplazamiento químico a bajo campo del protón de la sal de triazolinio (alrededor de 9,0 - 9,05 ppm) así como el gran desapantallamiento del grupo $-\mathrm{CH}_{2}$ - que conecta dicho grupo funcional y el antraceno, que aparece sobre 7,0 ppm (Figura 29).

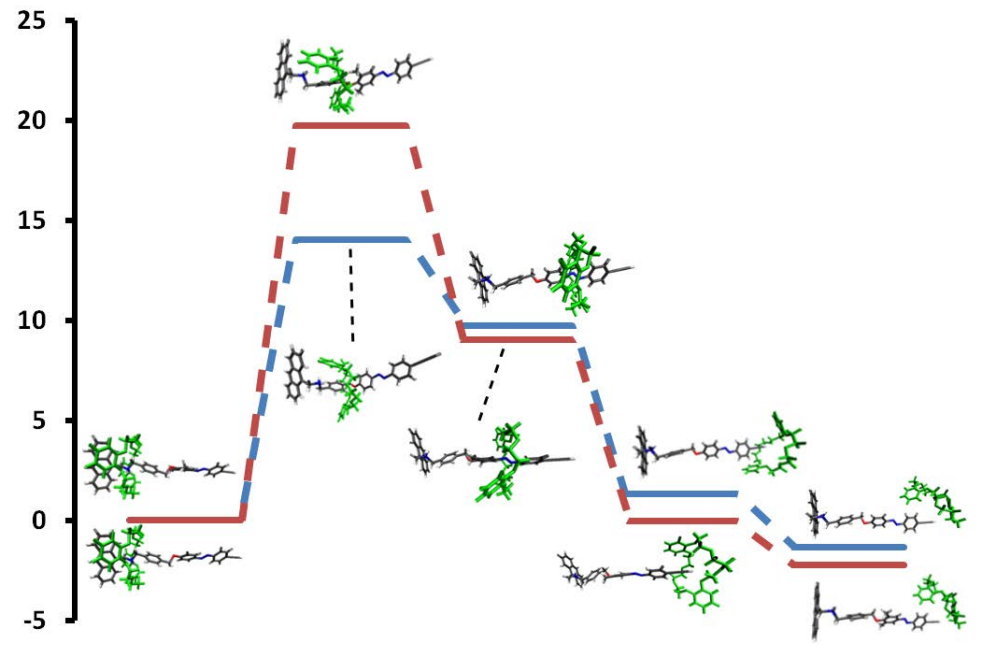

Figura 28. Perfil energético de los mínimos optimizados durante la formación del pseudorotaxano (en $\mathrm{kcal} / \mathrm{mol}$ ). Líneas azules: pseudorotaxanos tras la desprotección de 32(R3). Líneas rojas: pseudorotaxanos tras la desprotección de 34(R5).

${ }^{130}$ Es necesario hacer notar que obtener los estados de transición en este caso es una tarea muy compleja teniendo en cuenta que el proceso va a depender de múltiples coordenadas de reacción y se necesitaría mucho esfuerzo computacional (incluyendo el uso de un nivel de teoría superior y, probablemente, un método dinámico) para obtener unos resultados cuyo interés está alejado de los objetivos de este trabajo. 
Además, la posición relativa entre eje y anillo queda confirmada por los espectros bidimensionales ${ }^{1} \mathrm{H}-{ }^{1} \mathrm{H}$ NOESY y ROESY donde se encuentran señales de cruce correspondientes a los mismos núcleos cercanos en el espacio esquematizados previamente en la Figura 22.

Los espectros de absorción UV/Vis muestran el conjunto de bandas esperadas donde las transiciones $\pi-\pi^{*}$ de los grupos antraceno y azobenceno se solapan en el intervalo de 325 a 400 $\mathrm{nm}$ y la transición $\mathrm{n}-\pi^{*}$ de baja intensidad aparece en la región entre 325 y $425 \mathrm{~nm}$ (Figura 31).

Se empleó la metodología desarrollada en el anterior trabajo para producir la conmutación entre las distintas estaciones debido al éxito obtenido. Así, se añadió un volumen adecuado de una disolución de $\mathrm{NaOH} 1 \mathrm{M}$ en $\mathrm{D}_{2} \mathrm{O}$ (1,2 equivalentes) y se registró un espectro de RMN de protón apreciando cambios en todos los desplazamientos químicos, especialmente en aquellos núcleos próximos a las estaciones intervinientes en el proceso de conmutación como se puede apreciar en la Figura 30.

Todos estos cambios son esperados ya que son muy similares a los observados para la familia de interruptores moleculares estudiada en el trabajo previo. Así, la señal que corresponde al protón del triazol se desplaza a campo más bajo para 39(4-PF 6 y 41(6- $\left.\mathrm{PF}_{6}\right)$ y, curiosamente, a campo más alto para $40\left(5-\mathrm{PF}_{6}\right)$. Tal y como se preveía, este núcleo sufre intercambio con el deuterio del disolvente reduciendo dramáticamente la intensidad de la señal. El mismo efecto ocurriría si se deja el rotaxano en disolución por unas horas, independientemente de la estación en la que se encuentre el éter corona. Existen otras señales en el eje de los interruptores que sufren el mismo comportamiento, pero afortunadamente no son fundamentales para estudiar el proceso de conmutación. Otros desplazamientos químicos interesantes que sufren cambios son los procedentes de los grupos $-\mathrm{CH}_{2}-\mathrm{H}_{9}$ y $\mathrm{H}_{11}$ que se mueven hacia campo alto en torno a $1 \mathrm{ppm}$ y del metilo de la sal de triazolinio, cuyo movimiento se produce hacia campo alto alrededor de unos $0,5 \mathrm{ppm}$. La disposición relativa de las estructuras mecánicamente enlazadas también se confirmó por los espectros ${ }^{1} \mathrm{H}-{ }^{1} \mathrm{H}$ NOESY observándose las mismas señales de cruce descritas en la Figura 24 que aparecen como resultado de la cercanía en el espacio entre ambas especies.

La adición de la cantidad adecuada de TFA reprotona la amina recuperándose la sal de amonio forzando al macrociclo a moverse de nuevo a través del eje hacia esa estación. Los espectros ${ }^{1} \mathrm{H}-\mathrm{RMN}$ realizados muestran la restauración de las señales originales, pero, como era de esperar, los desplazamientos químicos no son exactamente los mismos que los registrados en la situación original debido al efecto del nuevo anión (trifluoroacetato). 


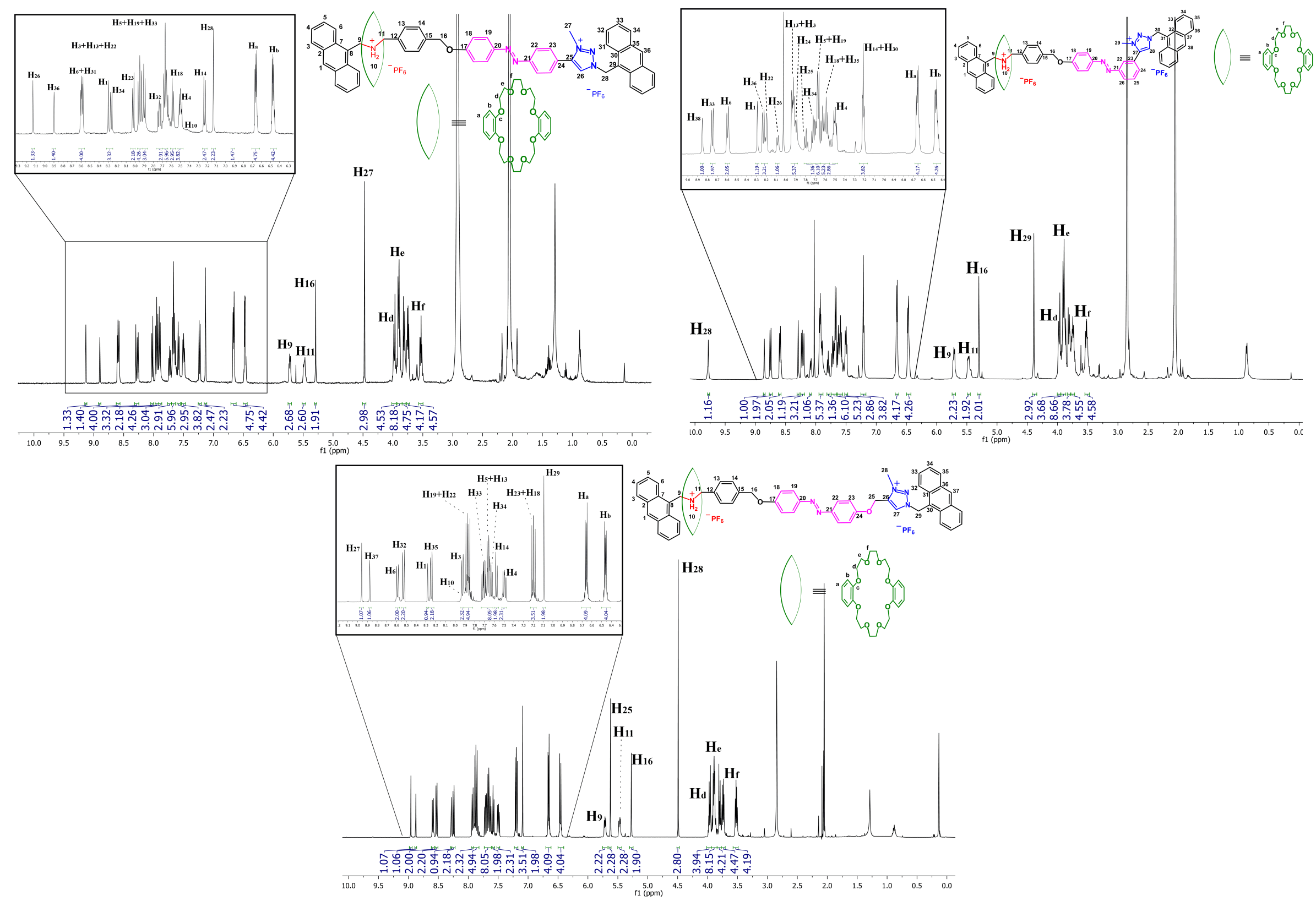

Figura 29. Espectros ${ }^{1} \mathrm{H}-\mathrm{RMN}$ en acetona deuterada de los interruptores moleculares sintetizados en este trabajo con la asignación completa. 


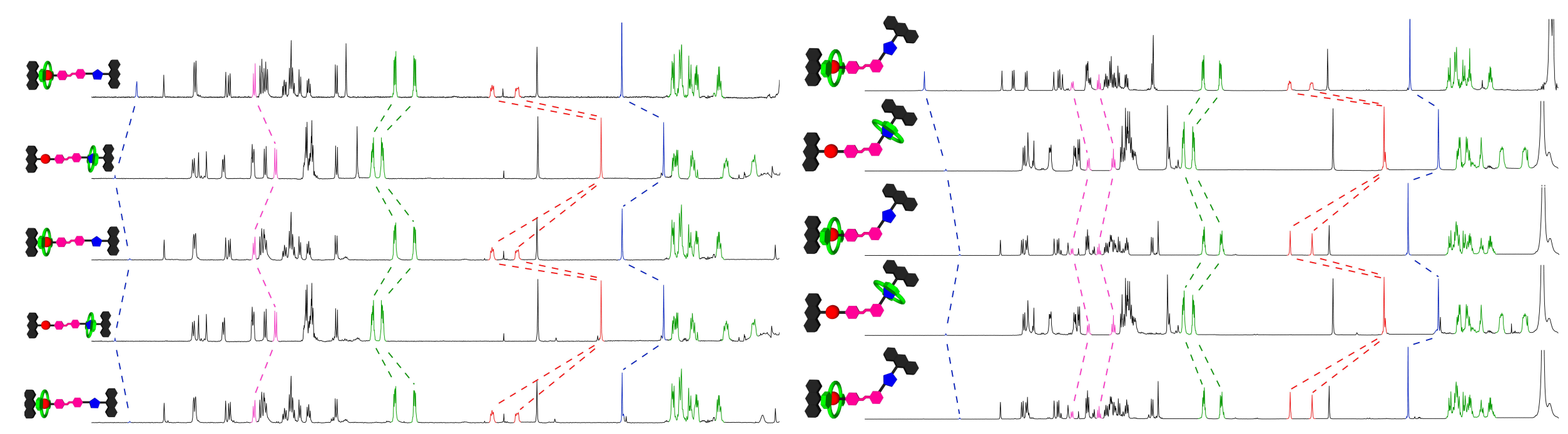

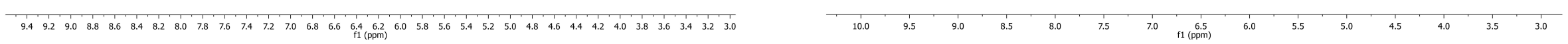

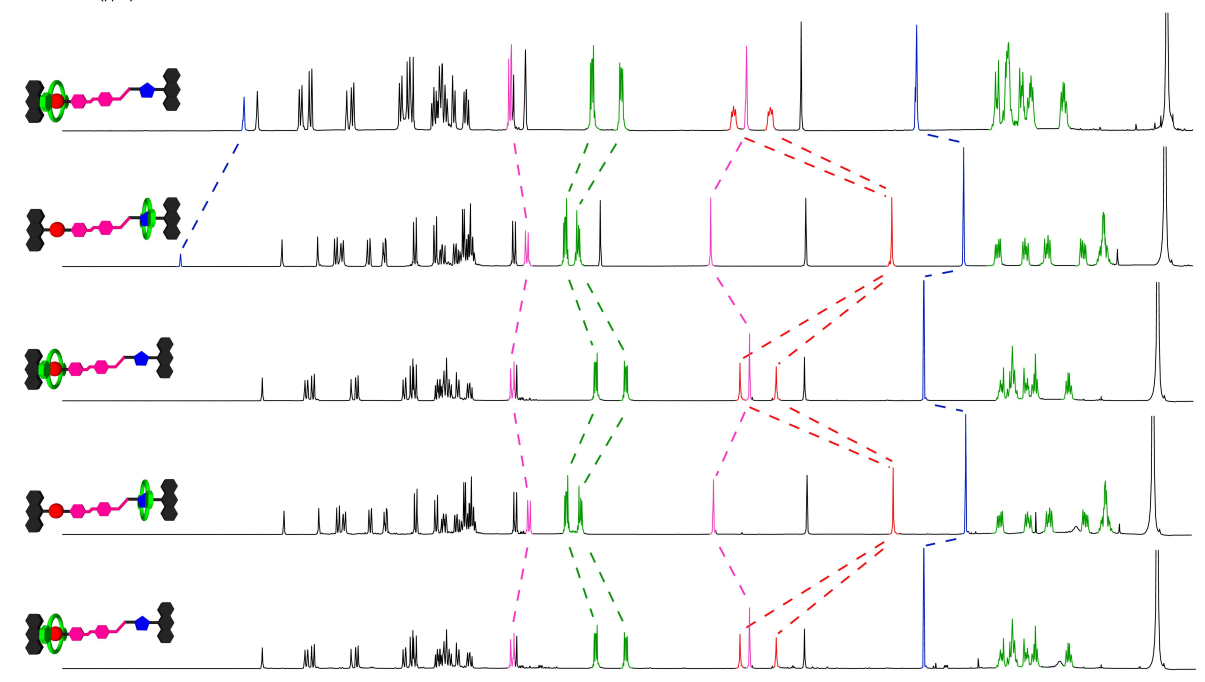

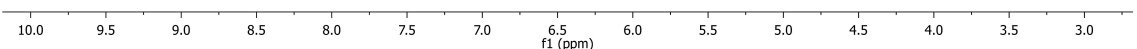

Figura 30. Comparación de espectros de ${ }^{1} \mathrm{H}-\mathrm{RMN}$ en acetona deuterada de los interruptores moleculares durante el proceso de conmutación indicando con un patrón de colores los núcleos más interesantes implicados en el movimiento del macrociclo. 


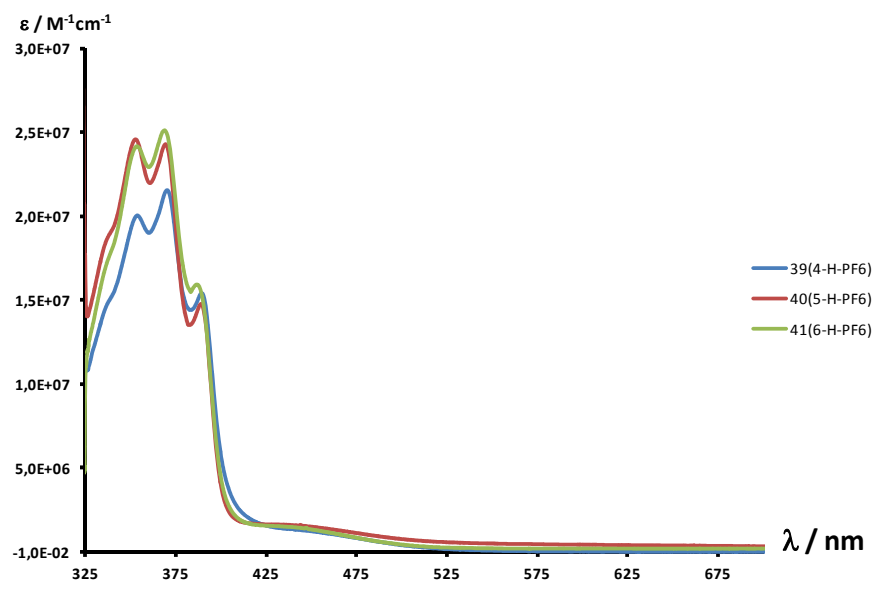

Figura 31. Espectros de absorción UV/Vis en acetona de los interruptores moleculares sintetizados en este trabajo.

No obstante, al igual que ocurría en la anterior familia de rotaxanos, si se realiza un cambio de anión por hexafluorofosfato, se obtiene exactamente el mismo espectro original. Tal y como se dijo, todos los desplazamientos químicos han cambiado con la misma magnitud con la que se modificaron en el anterior procedimiento de conmutación en el sentido contrario (por ejemplo, un cambio a campo alto pasa a campo bajo y viceversa) excepto para $\mathrm{H}_{28}$ en $\mathbf{4 0}\left(\mathbf{5}-\mathrm{H}_{-}-\mathrm{PF}_{6}\right)$ cuya señal se desplaza a campo alto en el primer proceso de conmutación y después continúa hacia campo más alto en el segundo. Sin embargo, una vez el anión trifluoroacetato está presente, los procesos subsiguientes se comportaron de la manera esperada. El procedimiento de conmutación entre estaciones se pudo realizar varias veces sin encontrar signos apreciables de descomposición.

Tras analizar el comportamiento en disolución de estos compuestos en relación al desplazamiento del anillo macrocíclico a través del eje, se procedió con el estudio del proceso de fotoisomerización, su alcance y su reversibilidad. Así, se irradiaron muestras de los tres interruptores moleculares a distintos tiempos y con diferentes longitudes de onda UV registrándose espectros de absorción UV/Vis y ${ }^{1} \mathrm{H}-\mathrm{RMN}$ (Figura 32). Los mejores resultados se obtuvieron para una longitud de onda de $365 \mathrm{~nm}$ en tiempos en torno a los 30 minutos. Longitudes de onda superiores produjeron mucha menor conversión e inferiores parecían promover la descomposición del material. La conversión aumentó con el tiempo de exposición, pero a partir de los 30 minutos se alcanzó el máximo.

En los espectros UV/Vis se observó para todos los casos la disminución de la intensidad de la señal correspondiente a las transiciones $\pi-\pi^{*}$ y el aumento de la intensidad de la banda $n-\pi^{*}$ indicando que el proceso de fotoisomerización tuvo lugar. Si la muestra se mantiene en disolución en la oscuridad, transcurrido un tiempo se recuperan las bandas originales, lo que significa que el proceso es reversible.

En relación a los espectros ${ }^{1} \mathrm{H}-\mathrm{RMN}$ se analizó la isomerización en los rotaxanos protonados y desprotonados observándose diversos cambios de mayor o menor intensidad en los desplazamientos químicos. Es interesante señalar que las señales correspondientes a los protones del éter corona no se ven prácticamente afectadas cuando éste se encuentra en la estación sal de amonio pero sí lo hacen cuando se ubica sobre la de triazolinio. Esto se debe, probablemente, a la distancia diferencial entre las estaciones y el grupo azobenceno, siendo la sal de triazolinio la más cercana. El máximo grado de conversión alcanzado fue ligeramente superior al $60 \%$ en todos los casos. 

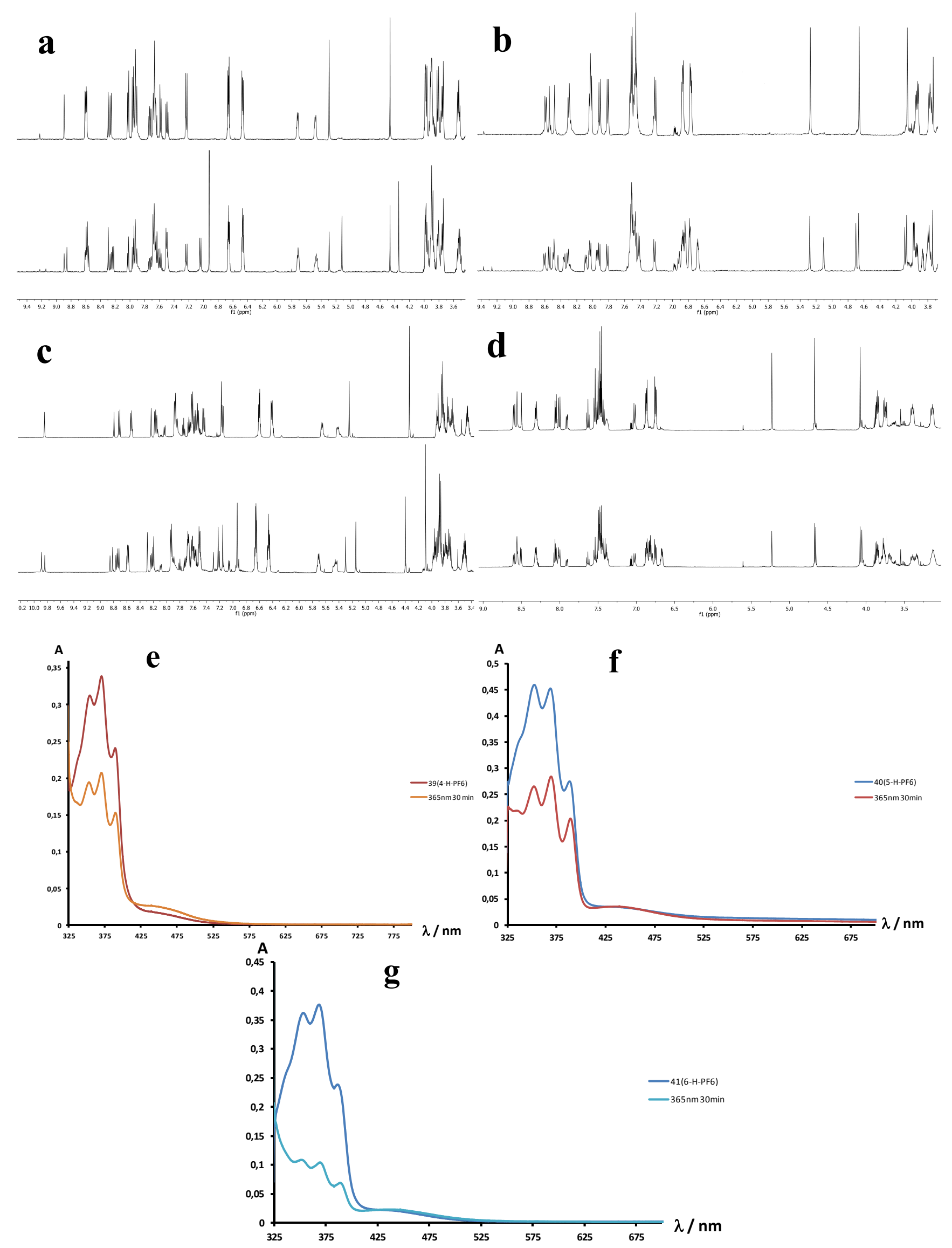

Figura 32. Conjunto de espectros ${ }^{1} \mathrm{H}-\mathrm{RMN}$ en acetona deuterada de los compuestos 39(4-H-PF $)$ (a), 39(4$\left.\mathrm{PF}_{6}\right)(\mathrm{b}), \mathbf{4 0}\left(\mathbf{5}-\mathrm{H}_{-} \mathrm{PF}_{6}\right)(\mathrm{c})$ y $\mathbf{4 0}\left(\mathbf{5}-\mathrm{PF}_{6}\right)(\mathrm{d})$ antes y después de irradiar y los espectros correspondientes UV/ Vis en acetona (e, $\mathrm{f}, \mathrm{g})$, incluyendo $\mathbf{4 1}\left(\mathbf{6}-\mathrm{H}-\mathrm{PF}_{6}\right)$. La no inclusión de los espectros de RMN de este último compuesto es deliberada (ver a continuación). 
Una vez estudiados tanto los procesos de conmutación como la fotoisomerización reversible de todos los rotaxanos, el siguiente paso consistió en el análisis de la combinación de ambos factores. Para ello, es posible realizar primero la fotoisomerización y luego producir la conmutación (en lo que se ha llamado en el artículo como forward shuttling) o invertir el orden de manera que primero se conmuta a la estación de triazolinio, luego se isomeriza y, adicionalmente, se vuelve a conmutar hacia la estación sal de amonio (llamado como backward shuttling en el artículo).

Esta metodología se realizó para todos los interruptores moleculares y, desgraciadamente, no se encontró un comportamiento positivo para $39\left(4-\mathrm{H}_{-} \mathrm{PF}_{6}\right)$ ni $\mathbf{4 0}\left(5-\mathrm{H}-\mathrm{PF}_{6}\right)$. Esto significa que el buscado "efecto memoria" no se consiguió ya que la geometría impuesta por el isómero Z, junto con la proximidad espacial de la estación de triazolinio (están directamente conectados por un enlace sencillo) no evita que el macrociclo conmute entre estaciones. El proceso completo está esquematizado en el Esquema 22.
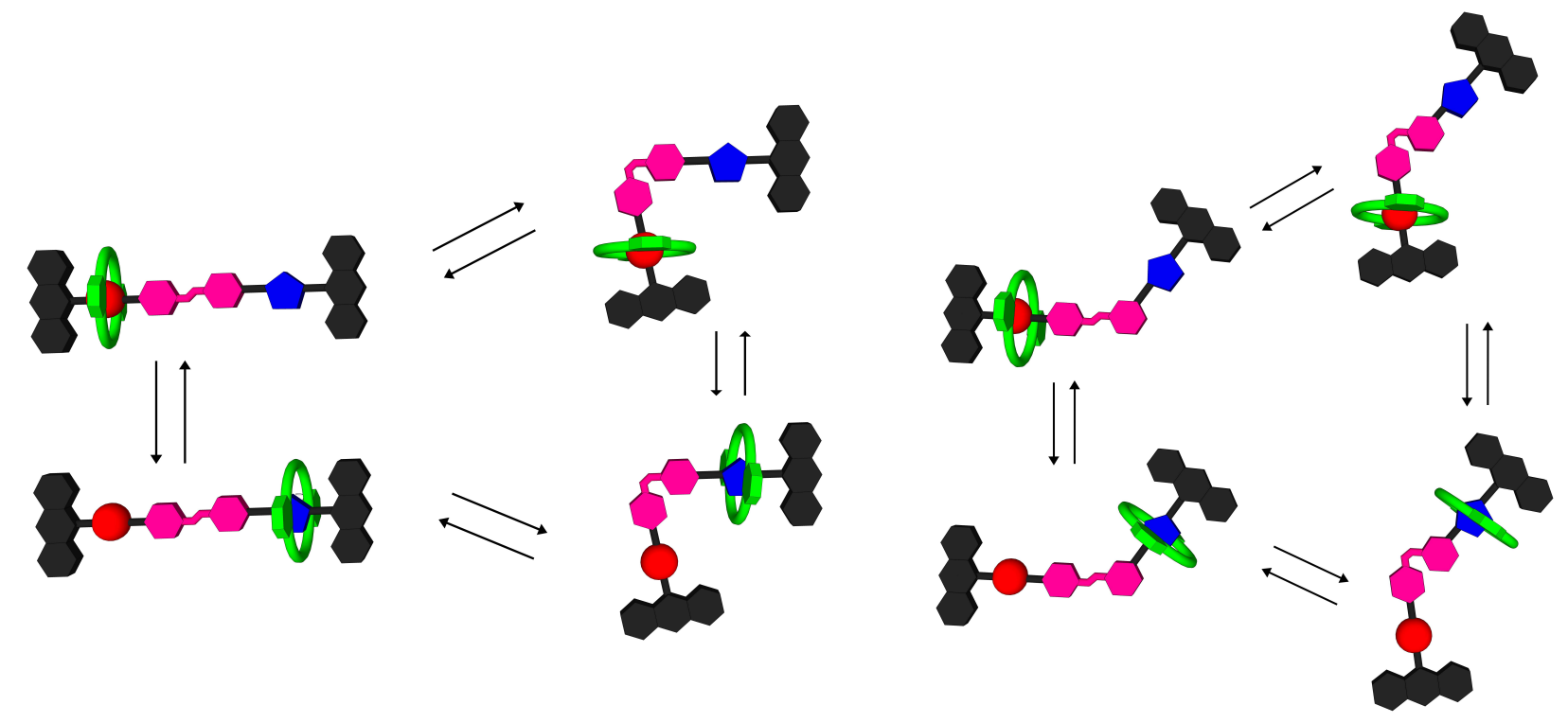

Esquema 22. Procedimiento completo de conmutación entre estaciones dependiendo del estado fotoestacionario de los compuestos $39\left(4-\mathrm{H}_{-} \mathrm{PF}_{6}\right)$ y $\mathbf{4 0}\left(5-\mathrm{H}-\mathrm{PF}_{6}\right)$ sin ningún tipo de control cinético por parte del grupo azobencénico.

El caso de 41(6-H-PF $)$ resultó ser distinto ya que, a pesar de que se encontraron señales procedentes de la conmutación de los isómeros configuracionales $\mathrm{Z}$, permanecían los desplazamientos químicos del interruptor no conmutado, mostrando un impedimento parcial por parte del grupo azobencénico, como se muestra en la Figura 33. Dado que posee la misma estructura que en los otros casos, la razón ha de deberse a la existencia del pequeño espaciador $-\mathrm{OCH}_{2}$ - incorporado entre esta unidad y la sal de triazolinio que evita la conexión directa entre ambos. 


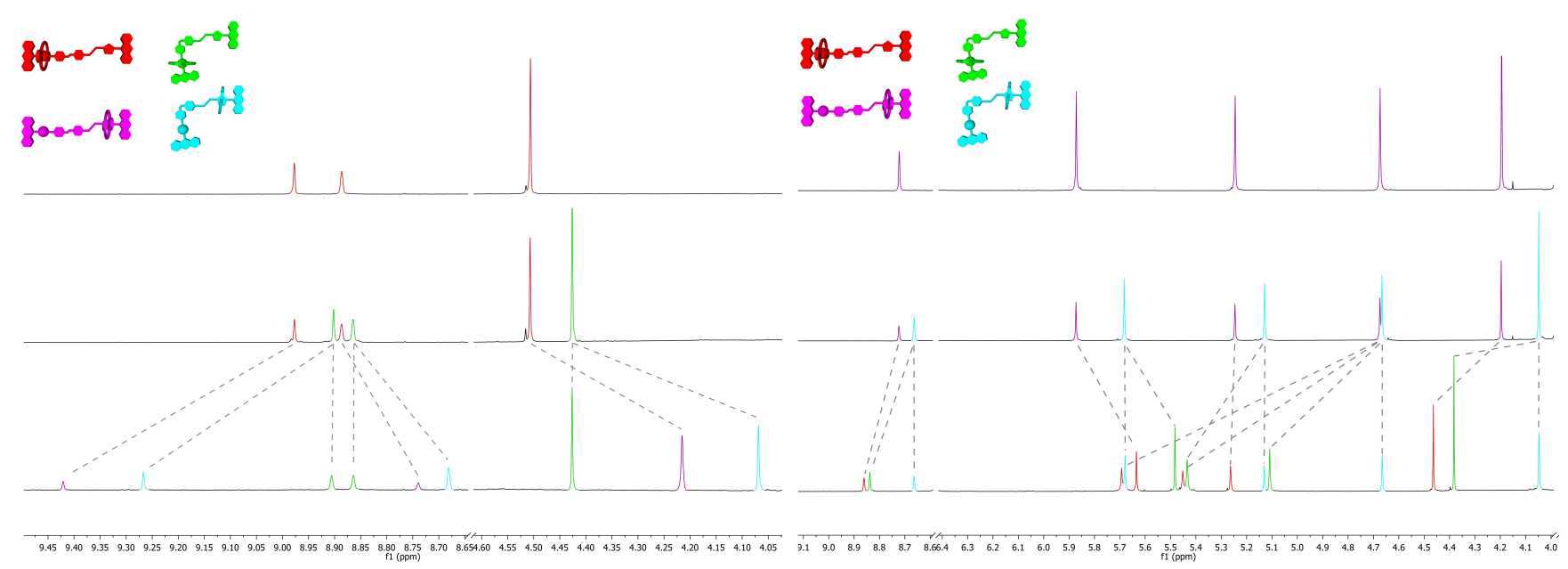

Figura 33. Diversas regiones de los espectros ${ }^{1} \mathrm{H}-\mathrm{RMN}$ en acetona deuterada de los procesos forward shuttling (izquierda) y backward shuttling (derecha) para 41(6-H-PF $)$. Arriba: espectro original; medio: tras irradiar la muestra con longitud de onda de $365 \mathrm{~nm}$ durante 30 minutos; abajo: después de conmutar a la otra estación. Se ha aplicado un código de colores especificado en la figura y se han coloreado las señales correspondientes de acuerdo con él para facilitar la identificación de los desplazamientos químicos.

Las señales remanentes evolucionaron hacia el isómero E conmutado y se estimaron las constantes cinéticas, que fueron de $7,2 \cdot 10^{-6} \mathrm{~s}^{-1}$ y $8,6 \cdot 10^{-6} \mathrm{~s}^{-1}$ para los procesos forward shuttling y backward shuttling, respectivamente, considerando una cinética de primer orden. Dado que el alrededor del $60 \%$ de las moléculas estaban isomerizadas y aproximadamente la mitad de ellas sufrieron conmutación, se estima que sólo el 30\% del material fue capaz de controlar cinéticamente tal conmutación gracias al fragmento de azobenceno. Estos resultados nos han permitido concluir que, si se desea un control cinético completo, va a ser necesario separar las estaciones del grupo azobencénico con espaciadores mayores y que habrá que incluir algún grupo voluminoso que aporte un impedimento estérico leve al movimiento del macrociclo. Nuestro diseño incluía este factor añadiendo grupos metílicos, pero no pudo prepararse el rotaxano final con la estrategia sintética diseñada. Futuros trabajos irán destinados a resolver ambos aspectos. 


\subsection{COMPUESTOS POLIAROMÁTICOS PLANOS Y NO PLANOS}

\subsubsection{Artículo III. Affinity modulation of photoresponsive hosts for fullerenes: light-gated corannulene tweezers}

En este trabajo se describe una familia de pinzas moleculares en las que dos fragmentos poliaromáticos planos y no planos están unidos por un grupo azobencénico simétrico. El método escogido ha sido el acoplamiento C-C mediante la reacción de Suzuki-Miyaura.

La preparación de los compuestos puede realizarse por dos vías en función de la situación en la que se desee ubicar el grupo boronato y el halogenuro, tal y como se muestra en el Esquema 23.

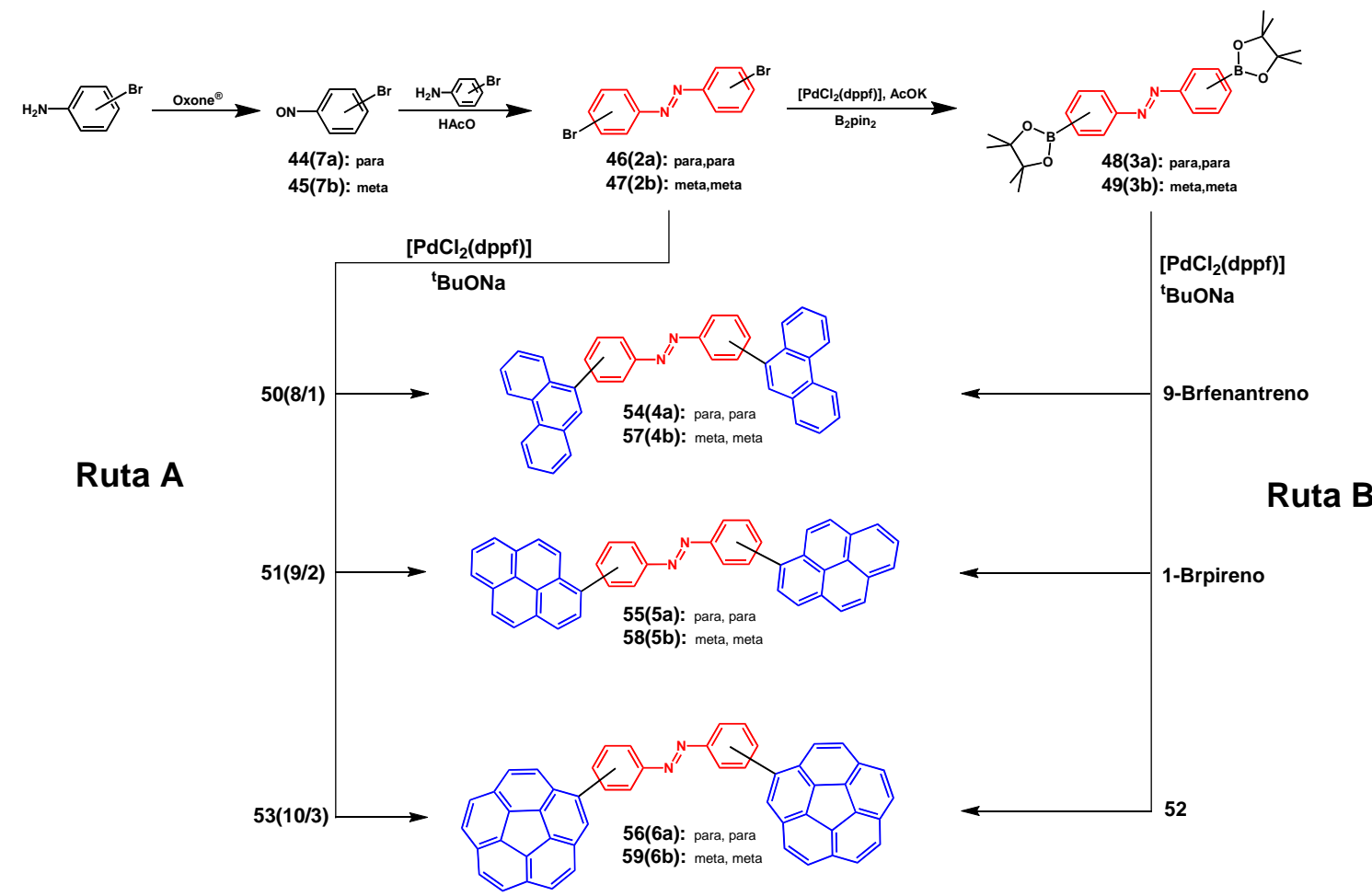

Esquema 23. Rutas sintéticas empleadas para la preparación de azobencenos funcionalizados con grupos poliaromáticos.

El proceso sintético comienza por la obtención de nitroso derivados a partir de la oxidación de $m$ - o $p$-bromo anilina y posterior reacción de Mills con la misma anilina usada en el primer paso para dar haloazobencenos simétricos. Una vez preparados esos precursores, se pueden plantean dos rutas. La Ruta A consiste en el acoplamiento C-C con los poliarenos funcionalizados como boronatos pinacolínicos, mientras que la Ruta B exige que los grupos boronato se encuentren en los derivados azobencénicos y, después, se produzca el acoplamiento C-C con los bromoarenos. 
Desde nuestra experiencia, la Ruta B se mostró como la mejor opción debido a que la purificación por cromatografía en columna en la Ruta A generaba muchas dificultades por la tendencia de los derivados halogenados de partida a eluir junto a los productos finales, disminuyendo drásticamente los rendimientos, especialmente para 56(6a). Por el contrario, la Ruta B proporcionó una mayor facilidad en la purificación de los productos. Además, la Ruta A necesitaba una etapa adicional de funcionalización en los poliarenos, mientras que la Ruta $\mathrm{B}$ sólo requería los derivados halogenados de los mismos, permitiendo evitar múltiples pasos de derivatización en los compuestos poliaromáticos. Dicha funcionalización partió de los derivados bromados comerciales en los que se sustituyó el átomo de Br por el éster borónico de pinacol. En el caso de coranuleno, éste se monobromó y se le aplicó posteriormente el mismo método que a los otros dos compuestos aromáticos, como se puede observar en el Esquema 24.

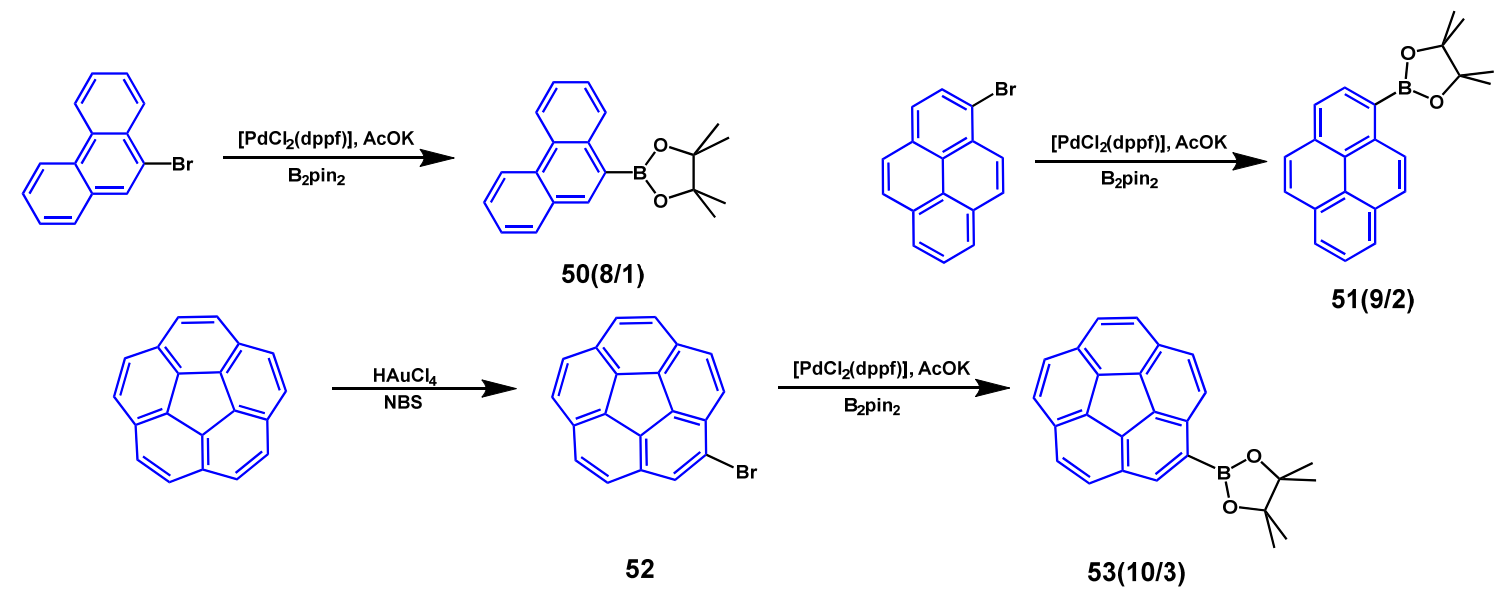

Esquema 24. Funcionalización de los compuestos policíclicos aromáticos utilizados en el Artículo III.

Todos los compuestos se caracterizaron por completo por técnicas convencionales como RMN, UV/Vis y MS (Figura 35). Los espectros de ${ }^{1} \mathrm{H}-\mathrm{RMN}$ mostraron un conjunto de señales aromáticas con un patrón complejo y con un intervalo espectral muy estrecho, lo que dificultó su asignación, que tuvo que realizarse gracias a los experimentos bidimensionales selectivos descritos con anterioridad en la sección de Metodología. 
Adicionalmente, pudieron obtenerse monocristales de los compuestos 48(3a), 54(4a), 57(4b) y 59(6b), cuya estructura se resolvió y refinó, tal y como se muestra en la Figura 34.

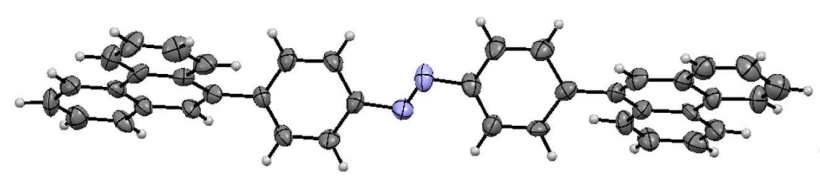

$54(4 a)$

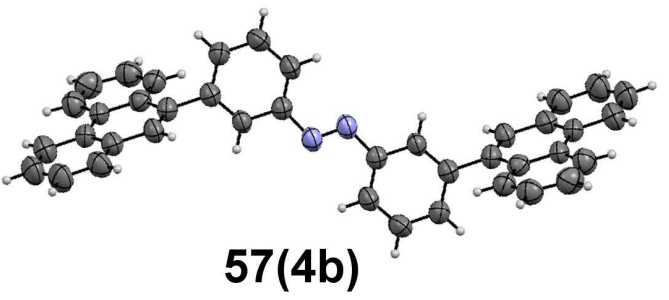

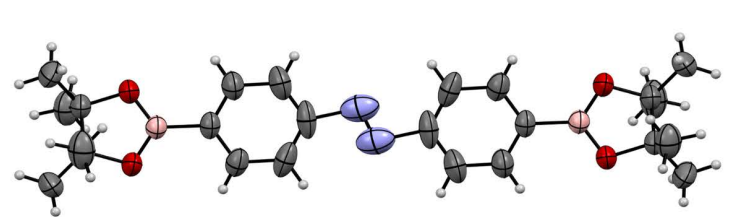

48(3a)

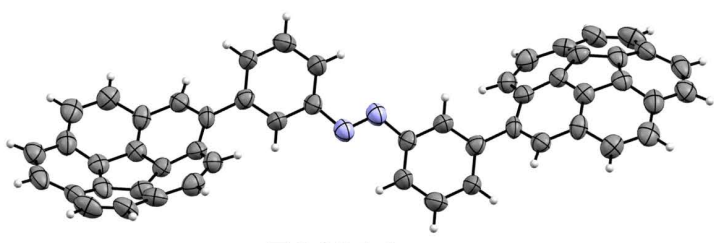

$59(6 b)$

Figura 34. Estructuras cristalinas obtenidas en este trabajo.

Como cabría esperar, los grupos fenilénicos y los átomos de nitrógeno del azobenceno están dispuestos en el mismo plano para todos los compuestos y, en los derivados con sustituyentes en meta, se encuentran en conformación relativa trans. Los ángulos dihedros $(\mathrm{CH}-\mathrm{C}-\mathrm{C}-\mathrm{CH})$ de las unidades poliaromáticas están en torno a $50^{\circ}$ respecto al azobenceno. 

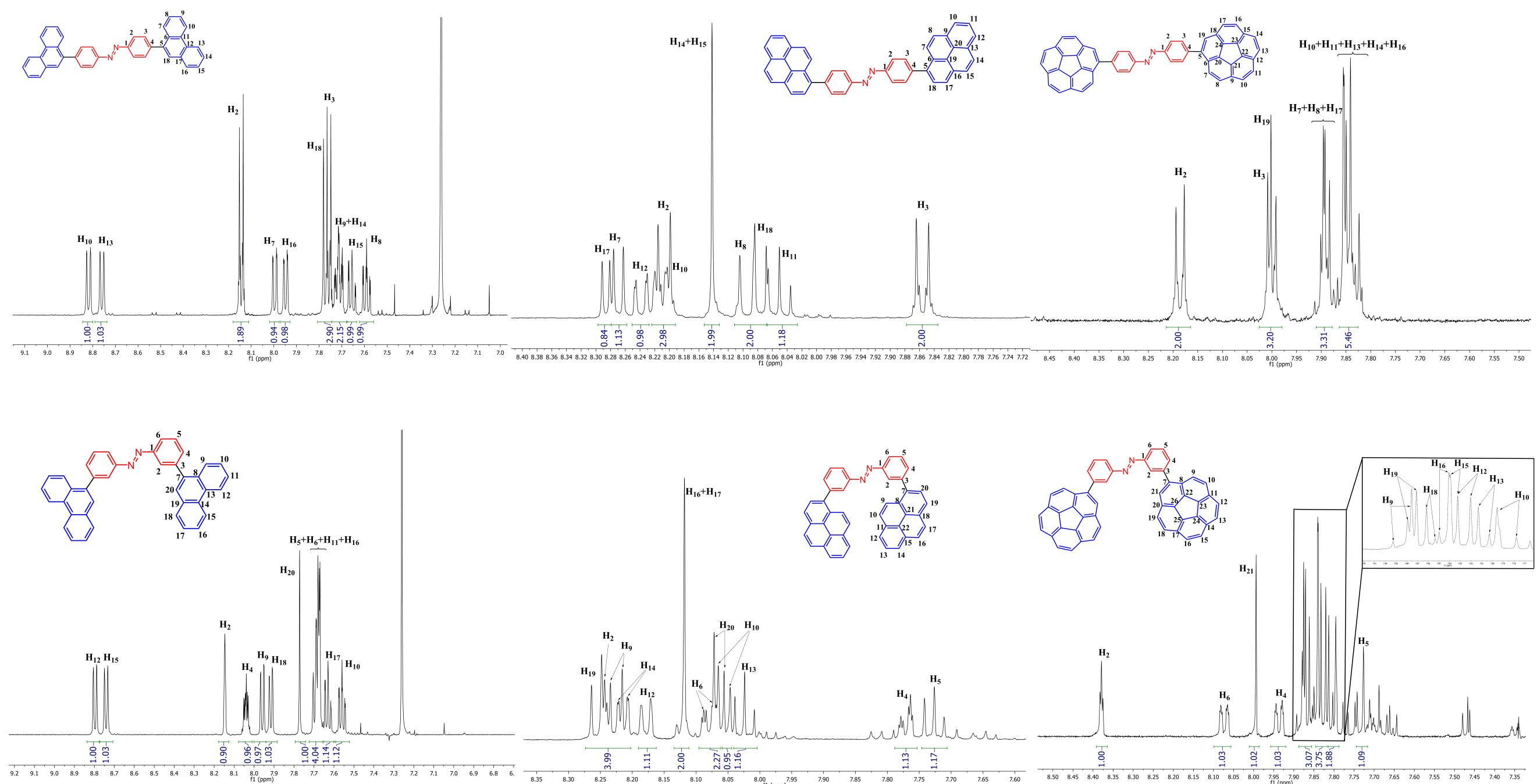

Figura 35. Comparación de espectros de ${ }^{1} \mathrm{H}-\mathrm{R} M N$ en cloroformo deuterado de todos los compuestos finales en este trabajo y numeración de los núcleos. 
Posteriormente se estudió el proceso de fotoisomerización (Esquema 25) en tolueno adquiriendo espectros UV/Vis y ${ }^{1} \mathrm{H}-\mathrm{RMN}$ tras cada etapa. Inicialmente, todos los compuestos mostraron una relación $\mathrm{E} / \mathrm{Z}$ de alrededor de $80: 20$. Si se calientan durante 30 minutos a $80^{\circ} \mathrm{C}$ es posible obtener el isómero $\mathrm{E}$ casi cuantitativamente.

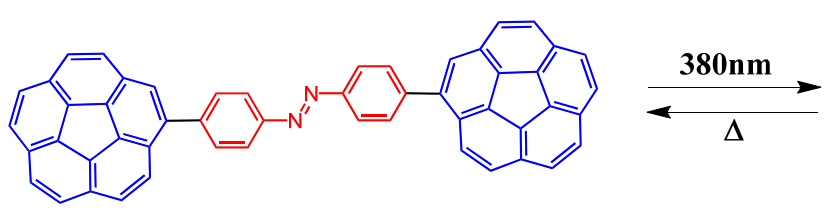

E56(E6a)

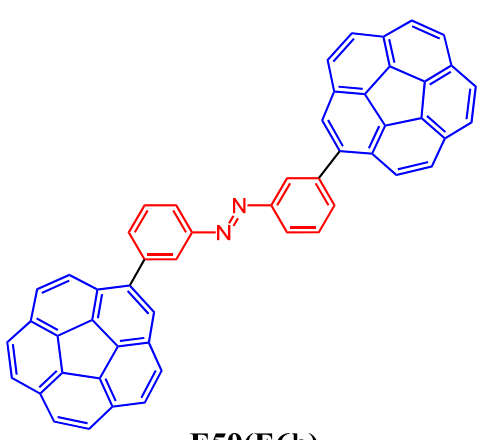

E59(E6b)

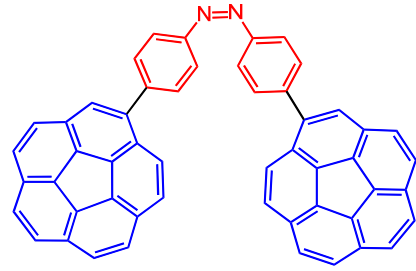

Z56(Z6a)

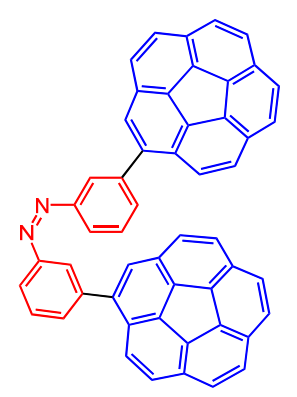

Z59(Z6b)

Esquema 25. Ejemplo de proceso de conmutación de los derivados de azobenceno funcionalizados con coranuleno y longitudes de onda de radiación UV usadas para producir una fotoisomerización eficiente. Nótese la predisposición en forma de pinza molecular para el compuesto Z56(Z6a).

La irradiación de los compuestos con luz UV a $365 \mathrm{~nm}$, longitud de onda típica para la fotoisomerización de azobencenos, ${ }^{53}$ produce mencionada isomerización, pero no es óptima para los derivados de pireno y coranuleno en para, 55(5a) y 56(6a), respectivamente. Al parecer, sus bandas de absorción $\pi \rightarrow \pi^{*}$ están desplazadas a longitudes de onda más largas. De hecho, la irradiación con luz UV a $380 \mathrm{~nm}$ produce la fotoisomerización de estos compuestos más efectivamente, tal y como se puede observar en la Figura 36 donde se muestra el ejemplo de la molécula 56(6a). 


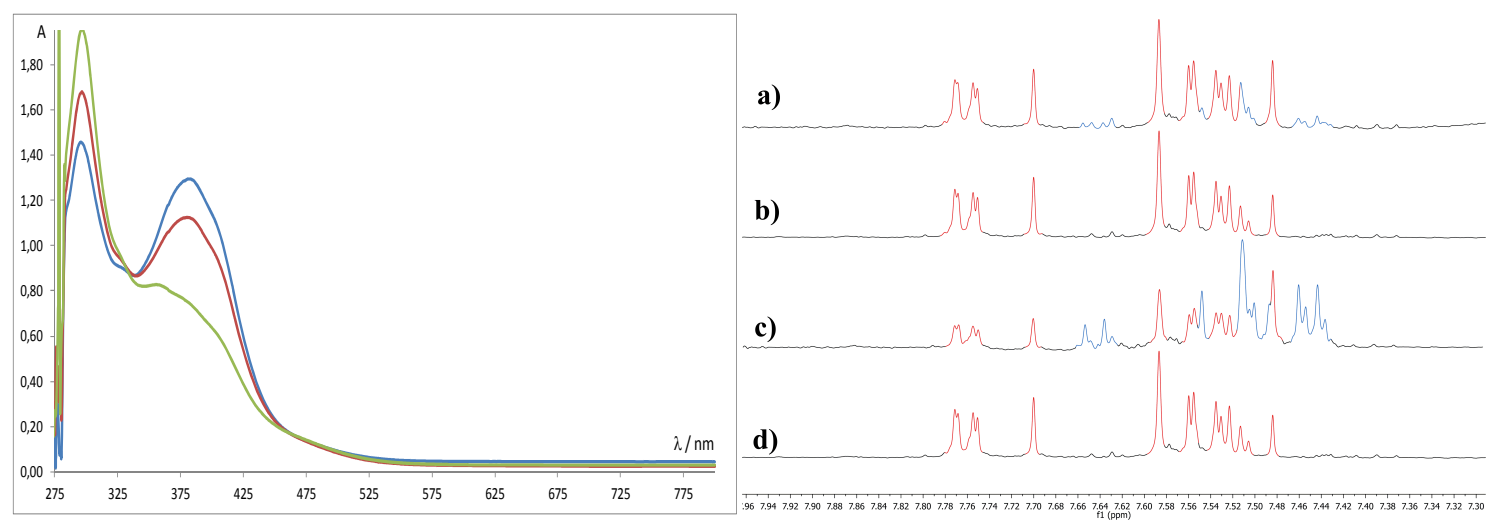

Figura 36. Izquierda: espectros $\mathrm{UV} / \mathrm{V}$ is del compuesto 56(6a) en tolueno (azul), tras la irradiación a $365 \mathrm{~nm}$ (rojo) y tras irradiar a $380 \mathrm{~nm}$ (verde). Derecha: espectros ${ }^{1} \mathrm{H}-\mathrm{RMN}$ (500 $\mathrm{MHz}$ ) de 56(6a) en tolueno-d8. a): situación inicial; b): tras calentar durante 15 minutos a $80^{\circ} \mathrm{C}$ y volver a temperatura ambiente; c): tras irradiar la muestra a $380 \mathrm{~nm}$ durante 30 minutos; d) recuperación del estado del isómero inicial después de calentar a $80^{\circ} \mathrm{C}$ durante otros 15 minutos y alcanzar temperatura ambiente de nuevo. El color rojo corresponde con el isómero E, mientras que el azul con el isómero $\mathrm{Z}$.

Por otro lado, los compuestos 58(5b) y 59(6b) poseen las bandas $\pi \rightarrow \pi^{*}$ desplazadas a longitudes de onda más cortas, en torno a $330 \mathrm{~nm}$. Sin embargo, la irradiación de los compuestos por debajo de $350 \mathrm{~nm}$ producía descomposición debido a la desaparición completa de dicha banda y la imposibilidad de recuperarla calentando la muestra o usando luz visible, indicando un proceso irreversible $\mathrm{y}$, por consiguiente, la desaparición de la unidad azobencénica. El grado de alcance de fotoisomerización de todos los compuestos se encuentra resumido en la Figura 37.
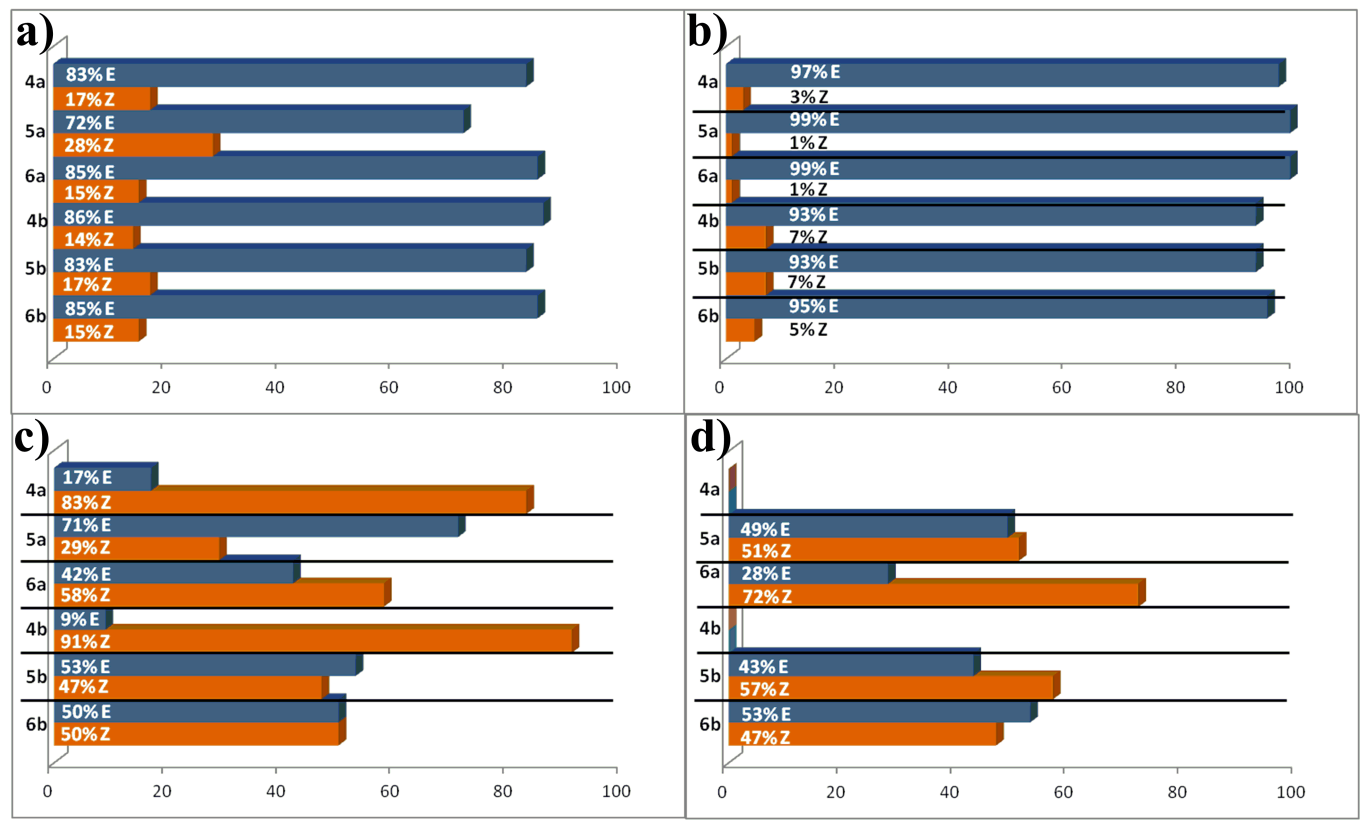

Figura 37. Estados fotoestacionarios de todos los compuestos finales descritos en este trabajo y relación $\mathrm{E} / \mathrm{Z}$ medida por integración de las señales en los espectros ${ }^{1} \mathrm{H}-\mathrm{RMN}$. a) Estado inicial; b) tras calentar a $80{ }^{\circ} \mathrm{C}$ y volver a temperatura ambiente; c) tras irradiar a $365 \mathrm{~nm}$ durante 30 minutos; d) Después de irradiar a $380 \mathrm{~nm}$ durante 30 minutos. 
Una vez caracterizados todos los compuestos y evaluado su fotoisomerización, se procedió con el análisis de su capacidad para establecer interacciones supramoleculares con fullerenos. Ninguno de los compuestos funcionalizados con fenantreno o pireno fue capaz de formar el aducto con $\mathrm{C}_{60} \mathrm{o} \mathrm{C}_{70}$ en cualquiera de los dos estados fotoestacionarios. Aunque en principio este resultado era esperado para los derivados de fenantreno (54(4a) y 57(4b)), sorprendió el hecho de que ninguno de los derivados de pireno (55(5a) y 58(5b)) diera resultados positivos, a pesar de la conocida capacidad de este fragmento poliaromático para establecer asociaciones supramoleculares con $\mathrm{C}_{70} \mathrm{y}$ nanotubos. ${ }^{131}$

Por otro lado, todos los derivados de coranuleno (56(6a) y 59(6b)) mostraron resultados satisfactorios. En primer lugar, el isómero configuracional E56(6a) (Esquema 25, arriba izquierda) es incapaz de formar aducto con ninguno de los fullerenos sometidos a estudio, debido a que su estructura no está en absoluto preorganizada como una pinza molecular porque los grupos coranuleno se encuentran muy distantes entre sí. Sin embargo, Z56(6a) (Esquema 25 , arriba derecha), cuya geometría adoptada sitúa estos fragmentos de manera que se forme una cavidad adecuada a la topología del fullereno, sí mostraron su capacidad de establecer asociación supramolecular (Figura 38).
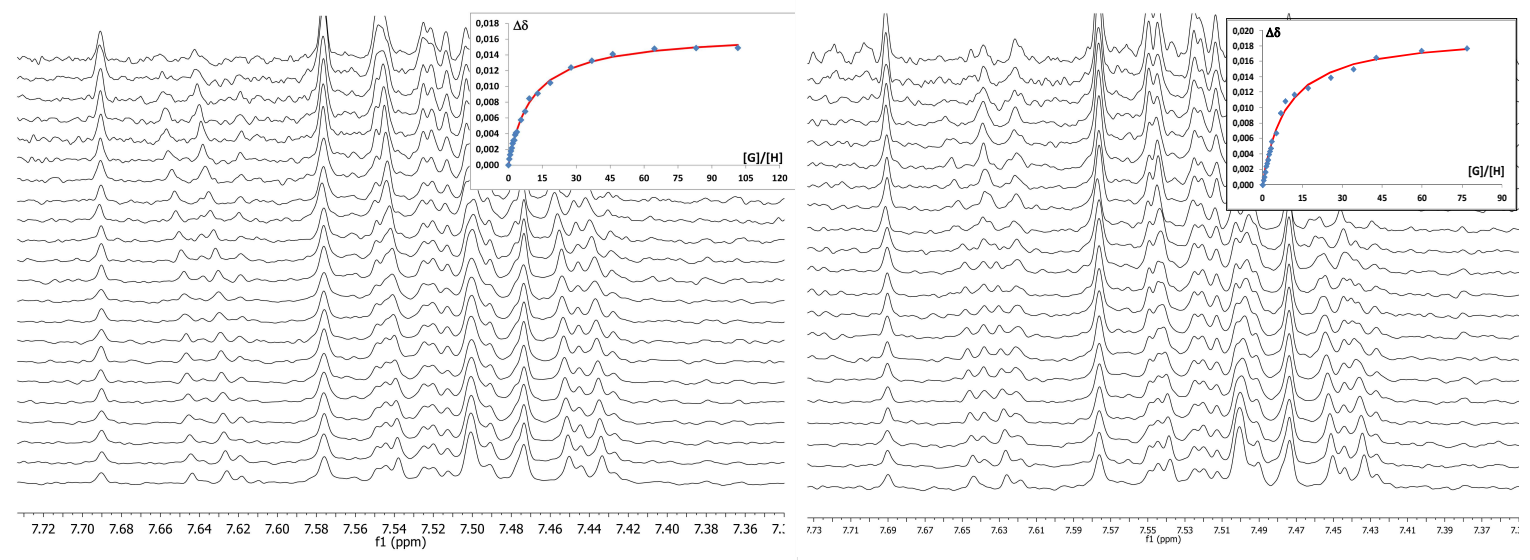

Figura 38. Espectros de ${ }^{1} \mathrm{H}-\mathrm{RMN}\left(500 \mathrm{MHz}\right.$, tolueno-d8) de la valoración de Z56(6a) con $\mathrm{C}_{60}$ (izquierda) y $\mathrm{C}_{70}$ (derecha). Los gráficos superpuestos corresponden con la representación de los cambios en desplazamiento químico observados de un núcleo frente a la variación de fracción molar del fullereno (puntos azules) y el ajuste no lineal (línea roja).

Las constantes de asociación estimadas fueron de $(2,6 \pm 0,3) \cdot 10^{3} \mathrm{M}^{-1}$ y $(2,5 \pm 0,3) \cdot 10^{3} \mathrm{M}^{-1}$ para $\mathrm{C}_{60} \mathrm{y} \mathrm{C}_{70}$ respectivamente, correspondientes con una estequiometría 1:1 de acuerdo con los Job plots elaborados. Dichas $K_{a}$ son similares a otras encontradas para compuestos biscoranulénicos ${ }^{79,83 a, 80,81}$ aunque no se observó preferencia por uno u otro fullereno debido a la similitud entre ellas. Sin embargo, este compuesto ha demostrado ser capaz de modular su afinidad por un fullereno de manera drástica debido a que en un estado fotoestacionario no puede establecer interacción, mientras que en el otro puede hacerlo con una constante razonable, convirtiéndose en una pinza molecular reversible y conmutable por luz.

131 (a) Sandanayaka, A. S. D.; Chitta, R.; Subbaiyan, N. K.; D’Souza, L.; Ito, O.; D’Souza, F. J. Phys. Chem. C 2009, 113, 13425-13432. (b) Schopf, E.; Broyer, R.; Tao, L.; Chen, Y.; Maynard, H. D. Chem. Commun. 2009, 4818-4820. (c) Bahun, G. J.; Adronov, A. J. Polym. Sci., Part A: Polym. Chem. 2010, 48, 1016-1028. (d) Komatsu, N.; Liu, G.; Wang, F.; Chaunchaiyakul, S.; Saito, Y.; Bauri, A. K.; Kimura, T.; Kuwahara, Y. J. Am. Chem. Soc. 2013, 135, 4805-4814. (e) Lopez-Moreno, A.; Perez, E. M. Chem. Commun. 2015, 51, 5421-5424. 
En el caso de 59(6b) los resultados experimentales obtenidos fueron muy diferentes. El isómero E59(6b) (Esquema 25, abajo izquierda) establece asociación supramolecular con ambos fullerenos con constantes $(5,0 \pm 0,1) \cdot 10^{2} \mathrm{M}^{-1}$ para $\mathrm{C}_{60} \mathrm{y}(8,3 \pm 0,1) \cdot 10^{2} \mathrm{M}^{-1}$ para $\mathrm{C}_{70}$ (con estequiometría 1:1, también). $\mathbf{Z 5 9}(\mathbf{6 b})$ (Esquema 25, abajo derecha) sigue poseyendo capacidades de interacción, ya que se estimaron unas $K_{a}$ de $(6,7 \pm 0,2) \cdot 10^{2} \mathrm{M}^{-1}$ y $(2,5$ $\pm 0,3) \cdot 10^{2} \mathrm{M}^{-1}$ para $\mathrm{C}_{60} \mathrm{y} \mathrm{C}_{70}$, respectivamente y, una vez más, con estequiometría 1:1 (Figura 39). Al parecer, la nueva estructura obtenida ha permitido una modificación en la afinidad de este compuesto por fullerenos. Por un lado, se ha mejorado sensiblemente la asociación con $\mathrm{C}_{60}$, aunque sigue siendo moderada $\mathrm{y}$, por otro lado, la asociación con $\mathrm{C}_{70}$ ha sido alterada negativamente, dando lugar a una disminución dramática de la constante estimada experimentalmente; sugiriendo que la nueva geometría dificulta la aproximación del fullereno.

Todos estos resultados están recogidos en la Tabla 3.
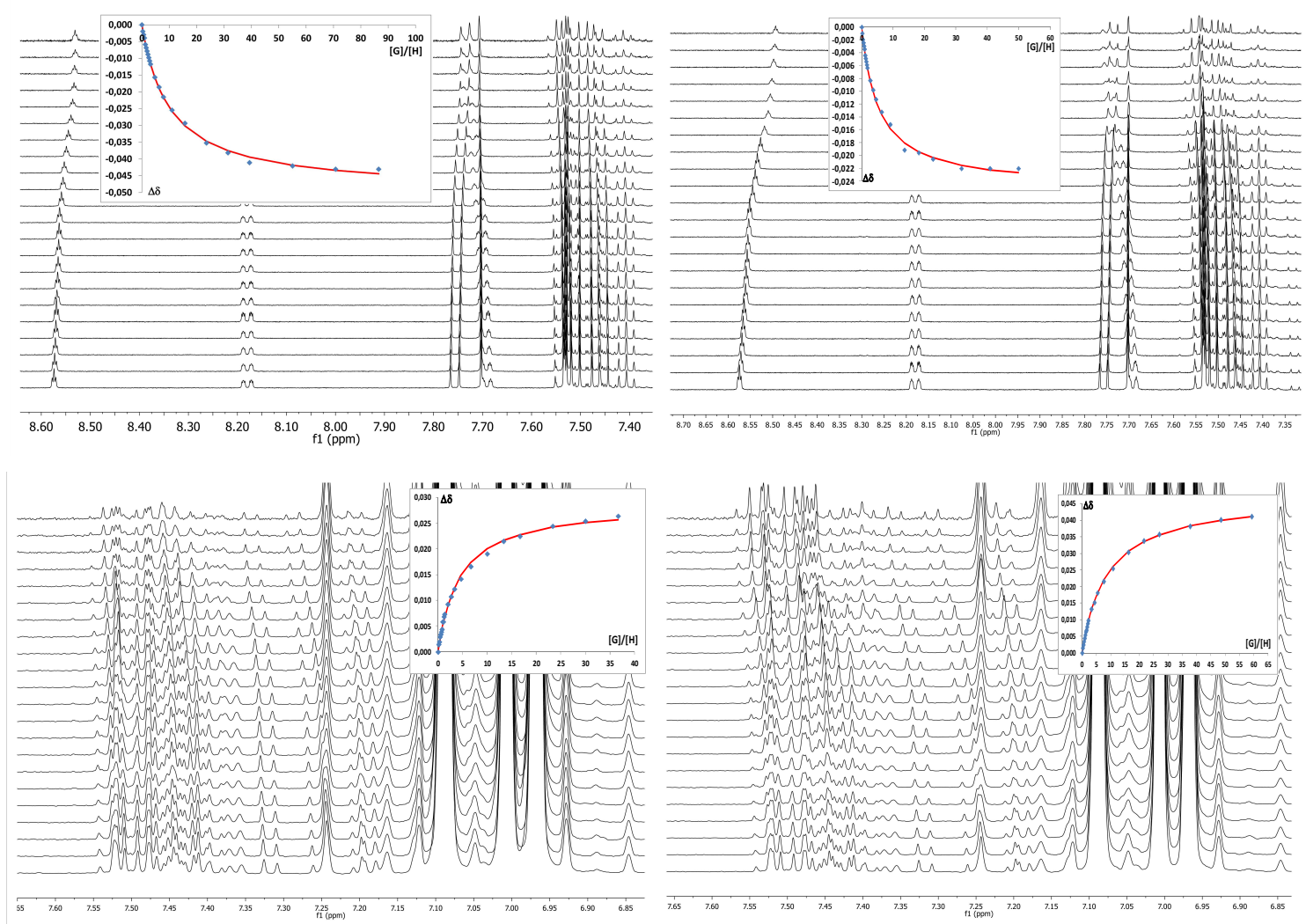

Figura 39. Espectros de ${ }^{1} \mathrm{H}-\mathrm{RMN}\left(500 \mathrm{MHz}\right.$, tolueno-d8) de la valoración de E59(6b) con $\mathrm{C}_{60}$ (arriba izquierda) y $\mathrm{C}_{70}$ (arriba derecha) y de la valoración de Z59(6b) con $\mathrm{C}_{60}$ (abajo izquierda) y $\mathrm{C}_{70}$ (abajo derecha). Los gráficos superpuestos corresponden con la representación de los cambios en desplazamiento químico observados de un núcleo frente a la variación de fracción molar del fullereno (puntos azules) y el ajuste no lineal (línea roja). 
Tabla 3. $K_{a}$ estimadas para la asociación entre los derivados azobencénicos funcionalizados con coranuleno y fullerenos, así como la modulación de la afinidad calculada entre estados fotoestacionarios.

\begin{tabular}{lccc|ccc} 
& \multicolumn{3}{c|}{$\mathrm{C}_{60}$} & \multicolumn{3}{|c}{$\mathrm{C}_{70}$} \\
& Isómero $\mathrm{E}$ & \multicolumn{2}{c|}{ Isómero Z } & Modulación & Isómero $\mathrm{E}$ & \multicolumn{2}{c}{ Isómero Z } & Modulación \\
\cline { 2 - 7 } $\mathbf{5 6 ( 6 a )}$ & 0 & $(2,6 \pm 0,3) \cdot 10^{3} \mathrm{M}^{-1}$ & $+100 \%$ & 0 & $(2,5 \pm 0,3) \cdot 10^{3} \mathrm{M}^{-1}$ & $+100 \%$ \\
$\mathbf{5 9 ( 6 b )}$ & $(5,0 \pm 0,1) \cdot 10^{2} \mathrm{M}^{-1}$ & $(8,3 \pm 0,1) \cdot 10^{2} \mathrm{M}^{-1}$ & $+66 \%$ & $(6,7 \pm 0,2) \cdot 10^{2} \mathrm{M}^{-1}$ & $(2,5 \pm 0,3) \cdot 10^{2} \mathrm{M}^{-1}$ & $-63 \%$
\end{tabular}

Aunque en la estructura cristalina del compuesto E59(6b) se observó que los fragmentos de coranuleno están dispuestos en conformación trans y, por consiguiente, el compuesto tenía una preorganización no adecuada en forma de pinza, no esperábamos una constantes un orden de magnitud más pequeñas que las encontradas para el compuesto Z56(6a) porque los grupos coranulénicos no se encuentran interaccionando entre sí y, por lo tanto, la energía de deformación a vencer no parecía un motivo decisivo para justificar el hecho.

Se optimizo la geometría de E59(6b) partiendo de su estructura cristalina y se calculó la variación de energía libre de Gibbs en disolución en función de la rotación de un enlace sencillo con el objetivo de alcanzar la organización adecuada y esperable en forma de pinza molecular. El resultado se encuentra en la Figura 40. Se obtuvo un máximo de 5,67 kcal/mol, lo suficientemente baja como para producirse a temperatura ambiente.

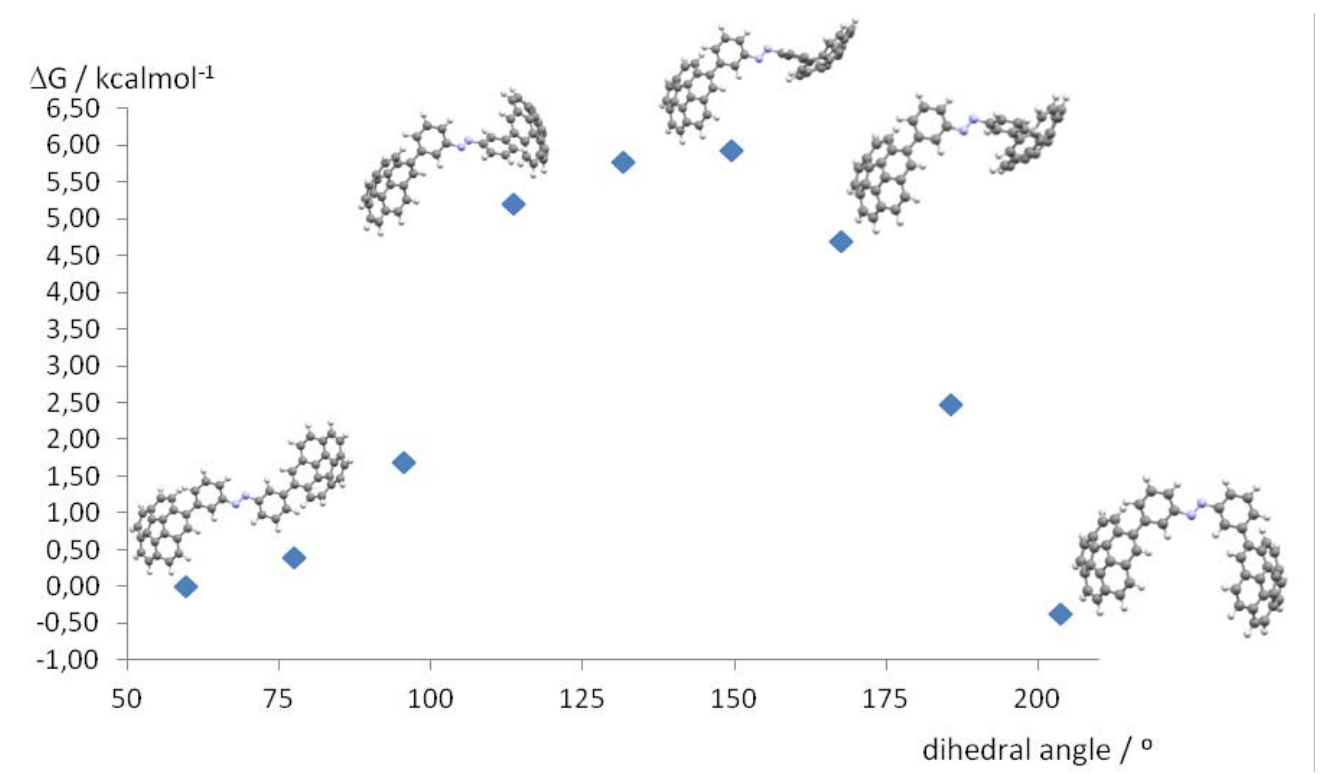

Figura 40. Perfil de energía para la rotación del ángulo diedro $\mathrm{N}=\mathrm{N}-\mathrm{C}=\mathrm{CH}$ de la molécula E59(6b) desde conformación trans a cis. Sólo se muestran cinco conformaciones por claridad.

Posteriormente se optimizó la geometría del aducto $\mathbf{C}_{60} @ \mathbf{E 5 9}(\mathbf{6 b})$ partiendo de la estructura final calculada previamente en conformación cis. La cavidad formada por los fragmentos de coranuleno es adecuada para albergar una molécula del fullereno, pero, al parecer, los hidrógenos del grupo azobencénico que apuntan a dicha cavidad parecen interferir la aproximación de $\mathrm{C}_{60}$ en vez de establecer posibles interacciones $\mathrm{CH}-\pi$. 
Esto da como resultado un aducto formado fuera de la línea ideal que establecería el fragmento de azobenceno, forzando a ambos coranulenos a adoptar una conformación inclinada tal que ambos no se encuentran en paralelo entre sí ni perpendiculares con el azobenceno, disminuyendo, así, su afinidad (Figura 41).
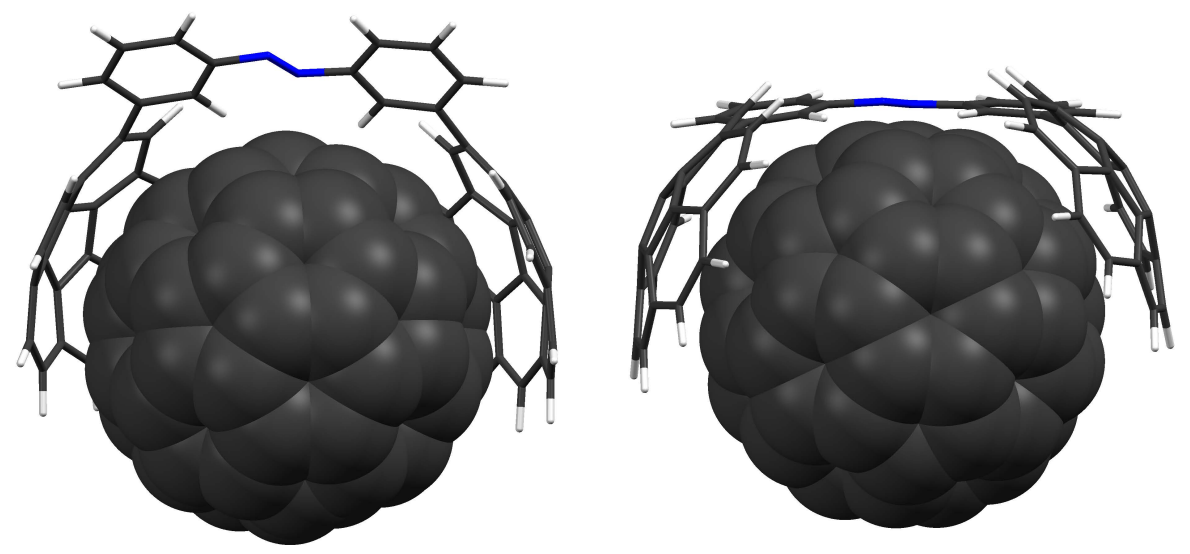

Figura 41. Geometría optimizada del aducto supramolecular $\mathbf{C}_{60} @ \mathbf{E 5 9}(6 \mathbf{b})$. Nótese que la disposición de la pinza molecular no es la adecuada para albergar la molécula del fullereno.

La energía de interacción calculada, tras corregir el error de superposición de bases, fue de $-37,1 \mathrm{kcal} / \mathrm{mol}$, muy similar a otras calculadas para otros derivados similares. ${ }^{132}$

Por otro lado, también se optimizó la geometría del aducto C60@Z56(6a) mostrando, como se esperaba, una cavidad formada por ambos extremos de la pinza, igualmente adecuada al tamaño y topología del fullereno, pero esta vez el grupo azobencénico no interfiere en absoluto en la asociación y se forma en la línea ideal del azobenceno con los coranulenos prácticamente en paralelo entre sí y en perpendicular con el fragmento que los une (Figura 42).
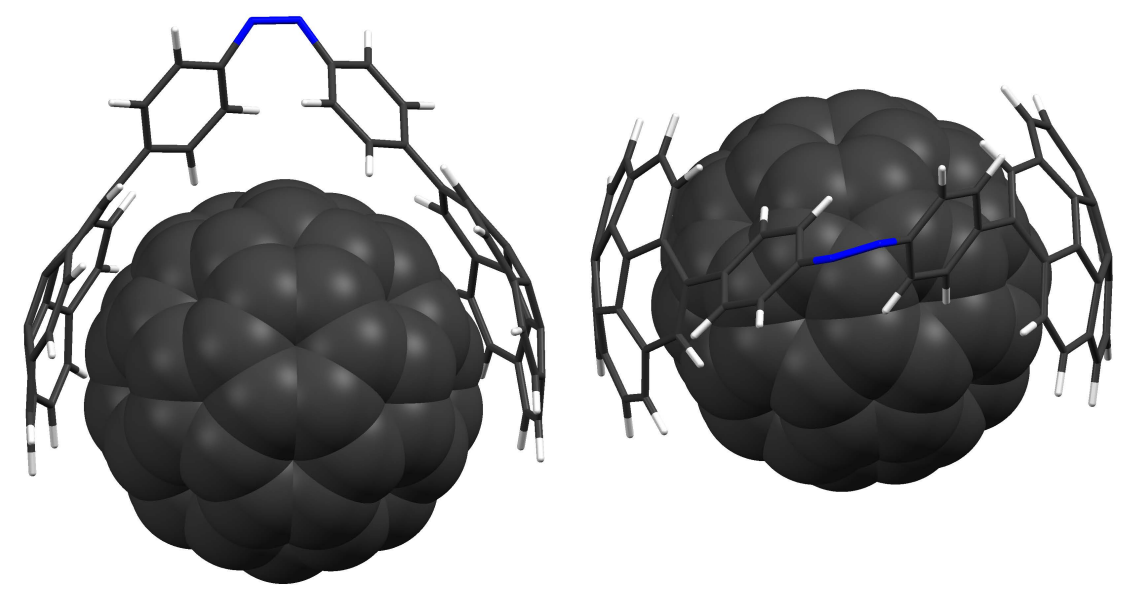

Figura 42. Geometría optimizada del aducto supramolecular $\mathbf{C}_{60} @ Z$ Z56(6a). Nótese que la disposición de la pinza molecular es prácticamente perfecta para albergar la molécula del fullereno.

132 (a) Muck-Lichtenfeld, C.; Grimme, S.; Kobryn, L.; Sygula, A. Phys. Chem. Chem. Phys. 2010, 12, 7091-7097. (b) Denis, P. A. RSC Adv. 2013, 3, 25296-25305. (c) Josa, D.; Rodríguez-Otero, J.; Cabaleiro-Lago, E. M.; Santos, L. A.; Ramalho, T. C. J. Phys. Chem. A 2014, 118, 9521-9528. 
Curiosamente, la energía de interacción obtenida fue de $-34,9 \mathrm{kcal} / \mathrm{mol}$, lo que significa 2,2 $\mathrm{kcal} / \mathrm{mol}$ más baja que la calculada para el primer aducto tratado y, sin embargo, su $K_{a}$ es muy superior. Esto sugiere que la energía de interacción no parece la principal fuerza motriz en el mecanismo de formación del aducto supramolecular. ${ }^{133}$

Estas diferencias en energía motivaron el cálculo de las interacciones no covalentes ${ }^{121}$ existentes en ambos aductos, encontrando un interesante hecho. En el aducto $\mathbf{C}_{60} @ \mathbf{E 5 9}(\mathbf{6 b})$, el fullereno se encuentra tan próximo al grupo azobencénico, que existe una interacción débil adicional por parte del mismo, mientras que en $\mathbf{C}_{60} @ \mathbf{Z 5 6}(6 \mathbf{a})$, por el contrario, se encuentra lo suficientemente alejado como para que las interacciones no covalentes se produzcan casi exclusivamente entre las dos unidades de coranuleno y la superficie de $\mathrm{C}_{60}$, tal y como se muestra en la Figura 43.
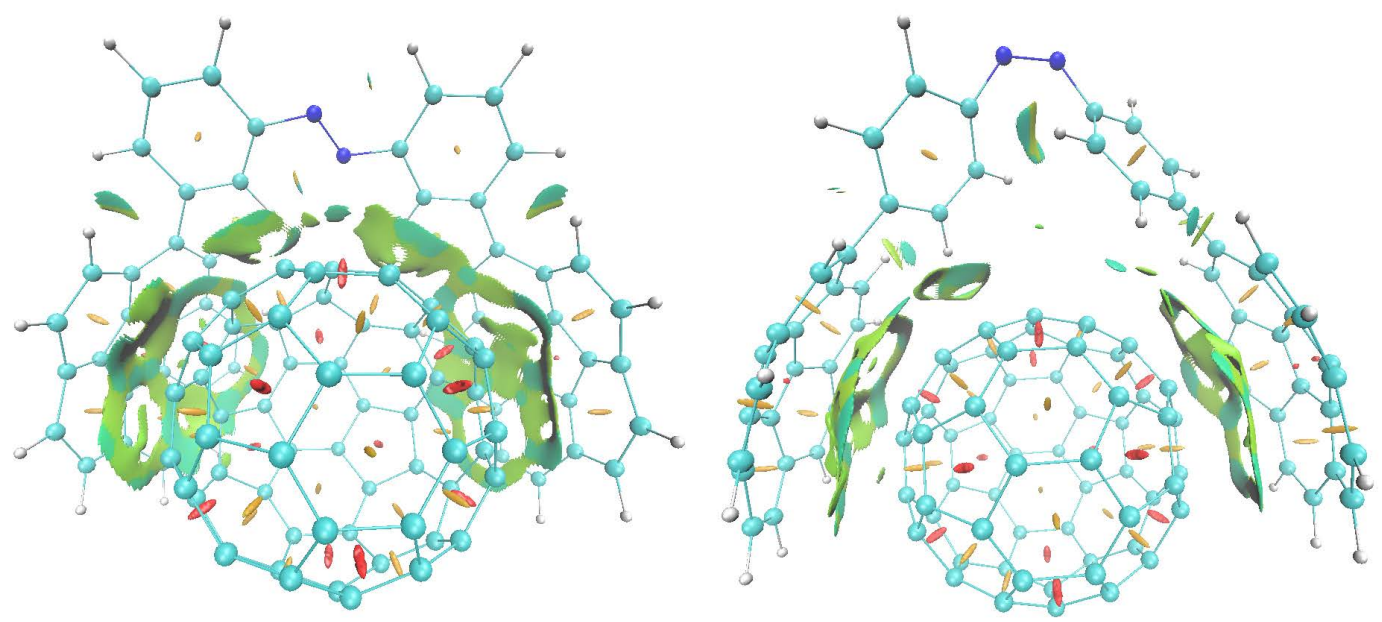

Figura 43. Isosuperficies de gradiente reducido de la densidad $(\mathrm{s}=0.3$ a.u.) de $\mathbf{C}_{60} @ \mathbf{E 5 9}(\mathbf{6 b})$ (izquierda) y de $\mathbf{C}_{60} @ Z \mathbf{Z 5 6}(6 \mathbf{6})$ (derecha). Las superficies están coloreadas de acuerdo con el sistema RGB descrito previamente en la sección de Metodología basándose en el signo del segundo autovalor de la matriz Hessiana de la densidad electrónica. De esta manera, el rojo indica repulsión, mientras que el verde significa atracción débil.

133 Grimme, S. Chem. Eur. J. 2012, 18, 9955-9964. 


\subsubsection{Artículo IV. Synergistic Effect of Tetraaryl Porphyrins Containing Corannulene and Other Polycyclic Aromatic Fragments as Host for Fullerenes. Impact of $C_{60}$ in a Statistically Distributed Mixture of Atropisomers}

En este artículo se describe una familia de porfirinas funcionalizadas en posiciones meso con grupos poliaromáticos planos y no planos tal y como se muestra en la Figura 44. Para ello, se explotó la misma reacción de acoplamiento C-C de Suzuki utilizada en el Artículo III.
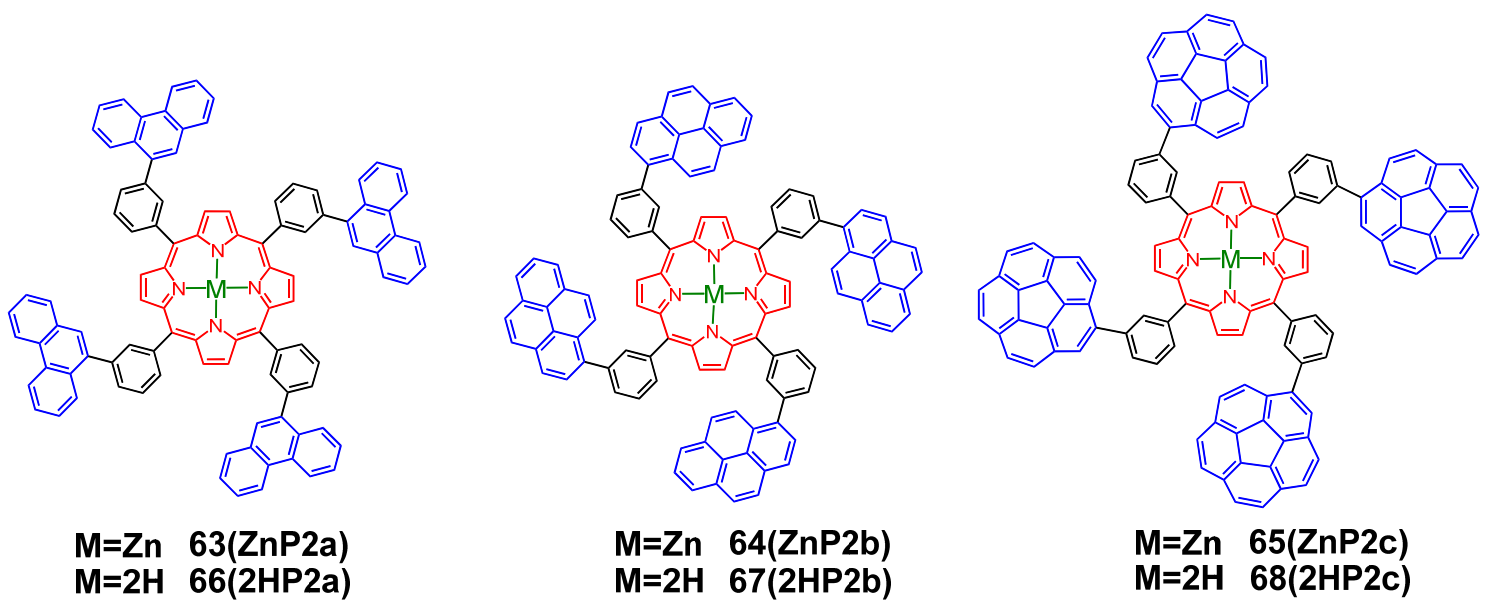

Figura 44. Moléculas descritas en este trabajo.

La síntesis de los compuestos objetivo (Esquema 26) comienza con la preparación previa del núcleo de porfirina 60(2HP1) a partir de pirrol y meta-bromobenzaldehído con la ayuda de un reactor de MW alcanzando rendimientos razonables. La reacción de Suzuki llevada a cabo con los derivados borilados poliaromáticos 50(8/1), 51(9/2) y 53(10/3) y dicha porfirina (Esquema 26, Ruta A) dio como resultado los productos esperados junto con porfirinas con el átomo de Pd coordinado (Figura 45), así como una cantidad no despreciable de subproductos de homoacoplamiento de los derivados borilados, lo que consecuentemente proporcionaba porfirinas trisustituidas, haciendo prácticamente imposible la purificación cromatográfica de los compuestos objetivo.

Para evitar la presencia de los derivados de Pd, se decidió coordinar previamente el átomo de Zn, para dar como resultado 61(ZnP1), debido a que la eliminación posterior de ese metal se puede realizar de manera sencilla (Esquema 26, Ruta B). Sin embargo, la reacción de acoplamiento $\mathrm{C}-\mathrm{C}$ cuádruple con los mismos derivados borilados poliaromáticos comentados anteriormente daba como resultado porfirinas trisustituidas junto con las tetrasutituidas cuya dificultad de purificación por cromatografía impidió la obtención de los compuestos objetivo. 


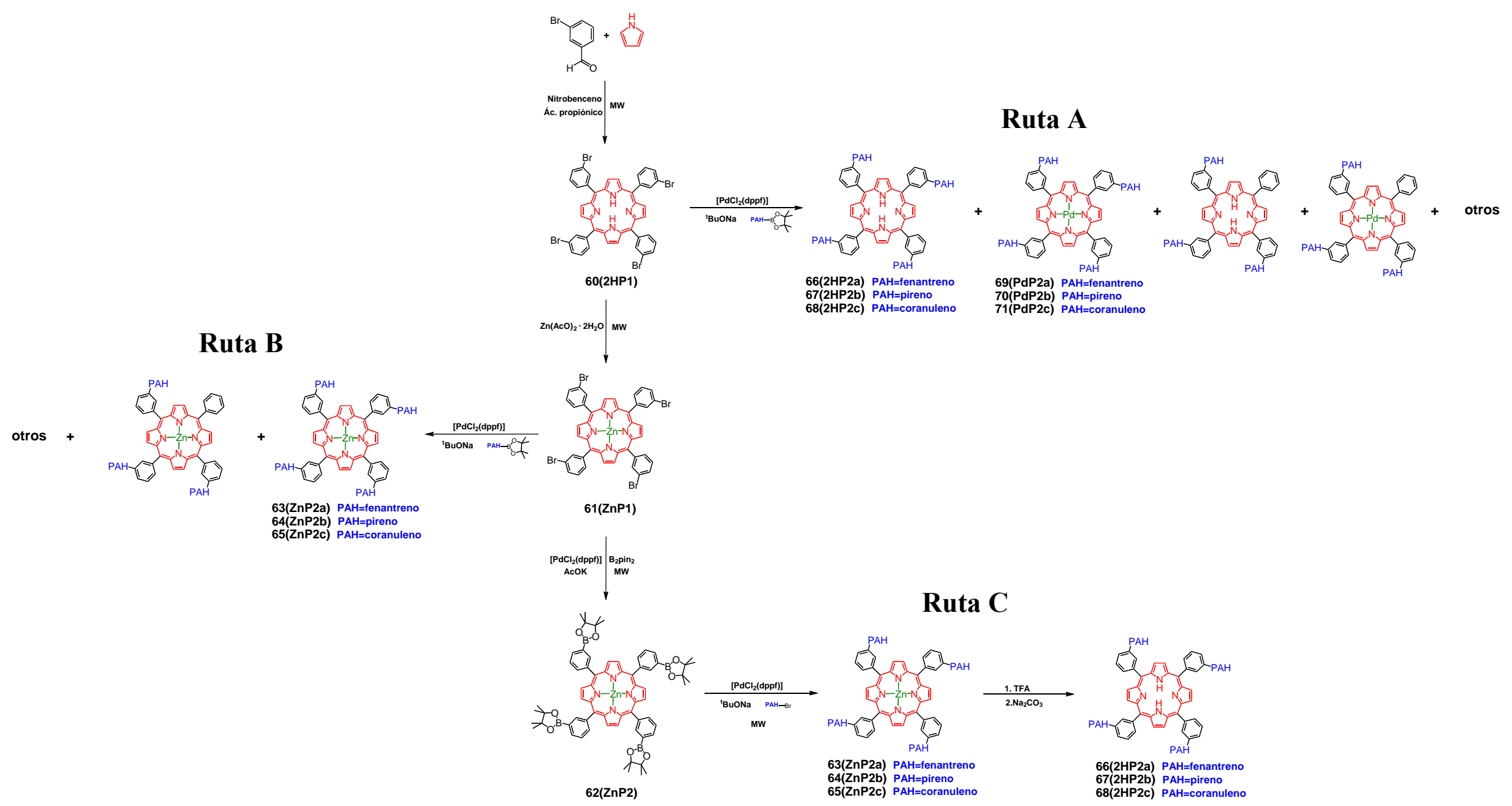

Esquema 26. Rutas sintéticas probadas para obtener las porfirinas funcionalizadas deseadas. 
Una tercera ruta de síntesis planteada (Esquema 26, Ruta C) implicó la inversión de grupos funcionales, es decir, se preparó el derivado tetraborilado 62(ZnP2) y se hizo reaccionar con los correspondientes halogenuros de arilo. Con este procedimiento se consiguieron los compuestos tetrasustituidos deseados disminuyendo notablemente la existencia de productos trisustituidos y, a su vez, evitando la incorporación del átomo de Pd en la cavidad de las porfirinas. La eliminación del átomo de $\mathrm{Zn}$ se pudo realizar de manera sencilla con TFA y posterior neutralización para dar los derivados orgánicos de manera limpia.
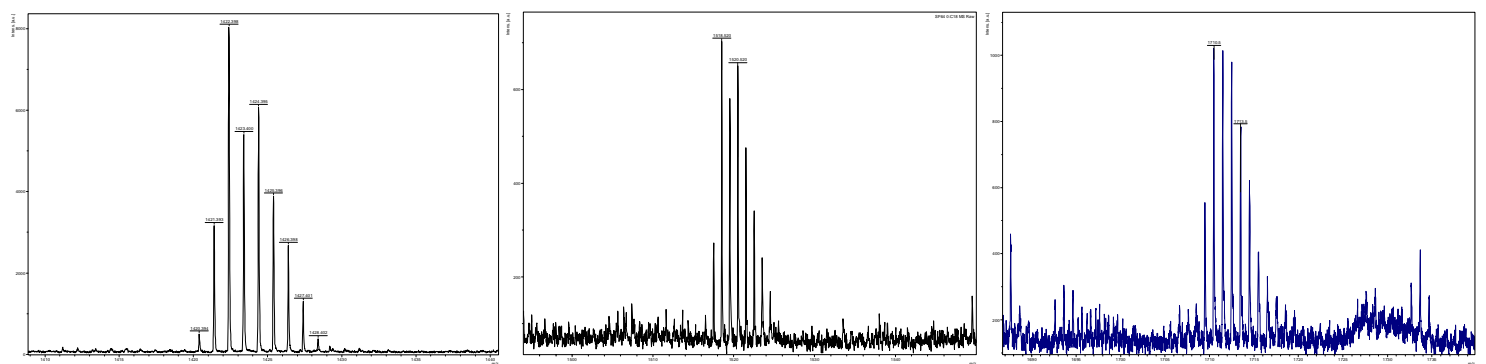

Figura 45. Espectros de masas (MALDI-TOF) de 69(PdP2a) (izquierda), 70(PdP2b) (centro) y 71(PdP2c) (derecha) indicando la existencia de estos subproductos no deseados.

Todos los compuestos nuevos se caracterizaron por completo por métodos espectroscópicos. Los espectros de UV/Vis muestran el patrón típico de este tipo de compuestos ${ }^{87}$ en los que hay una intensa banda de Soret en torno a $430 \mathrm{~nm}$ y un conjunto de bandas Q mucho menos intensas en el intervalo entre 500 y $600 \mathrm{~nm}$, tal y como se muestra en la Figura 46.

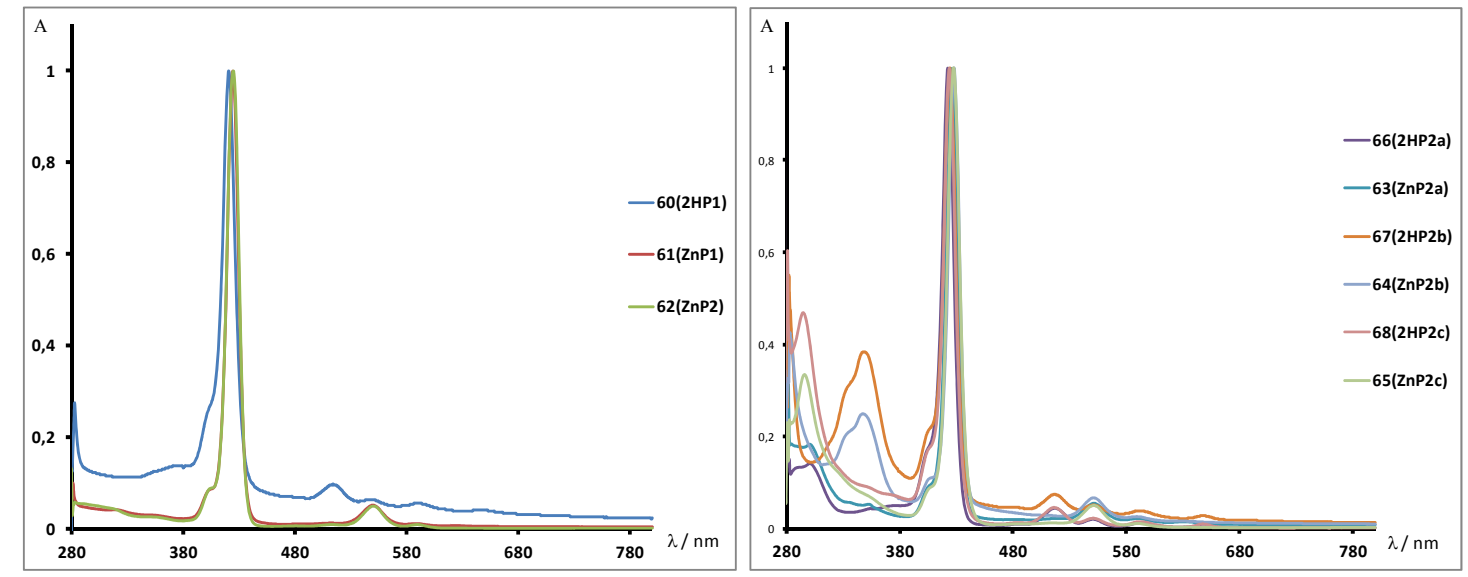

Figura 46. Espectros normalizados de absorción UV/Vis en tolueno de los compuestos preparados en este trabajo.

Los espectros ${ }^{1} \mathrm{H}-\mathrm{RMN}$ muestran la presencia de una mezcla de atropisómeros en proporción relativa a su distribución estadística, ${ }^{134}$ siendo esto especialmente evidente en los singletes correspondientes al protón $\mathrm{H}_{6}$, unido al carbono que tiene los dos carbonos adyacentes sustituidos del grupo fenilénico en la posición meso de la porfirina (Figura $47 \mathrm{y}$ Figura 48).

134 (a) Crossley, M. J.; Field, L. D.; Forster, A. J.; Harding, M. M.; Sternhell, S. J. Am. Chem. Soc. 1987, 109, 341-348. (b) Meunier, B.; R. Song; A. Robert; Bernadou, J. Analusis 1999, 27, 4. (c) Silva, A. M. S.; Tomé, A. C.; Alkorta, I.; Elguerob, J. J. Porphyrins Phthalocyanines 2011, 15, 28. 

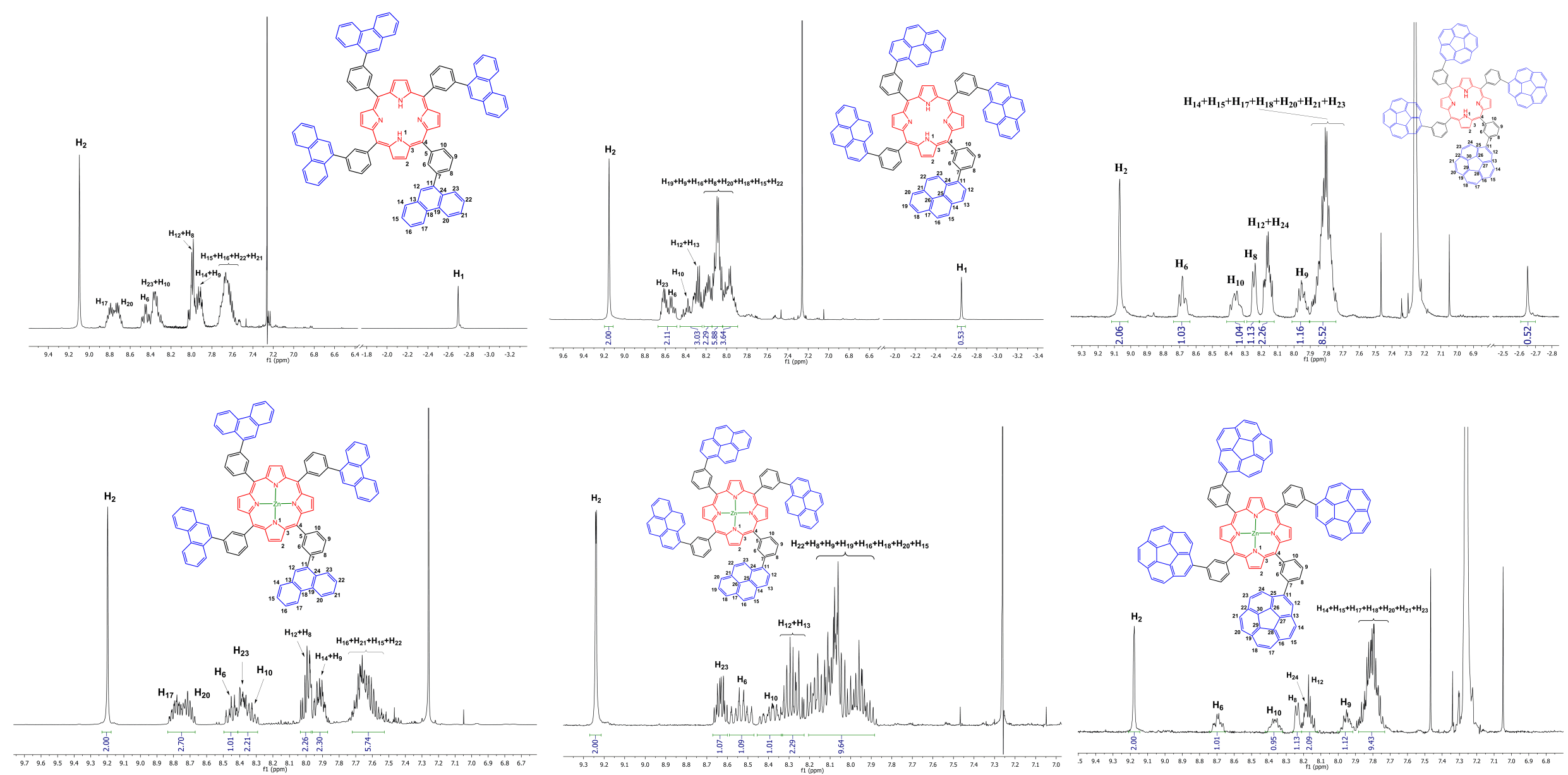

Figura 47. Espectros ${ }^{1} \mathrm{H}-\mathrm{RMN}$ en cloroformo deuterado de todos los compuestos finales obtenidos en este trabajo. 


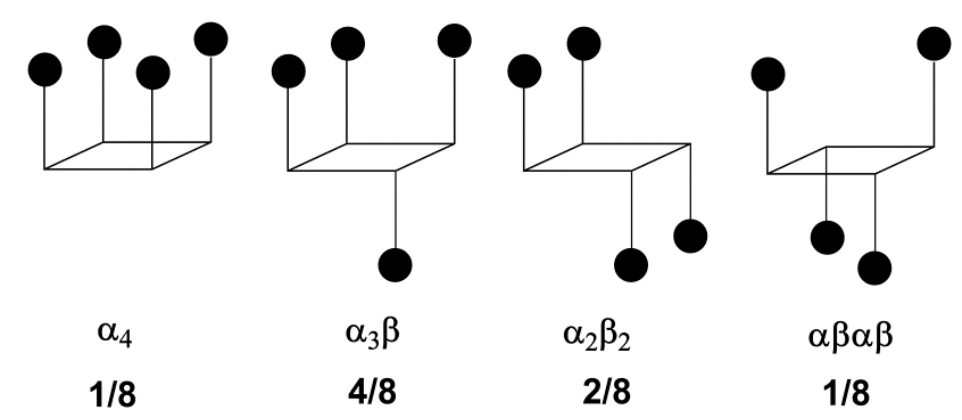

Figura 48. Representación esquemática de los distintos atropisómeros junto con su distribución estadística. El cuadrado representa el plano de la porfirina y los círculos negros representan los sustituyentes poliaromáticos.

Se realizaron diversos intentos para aislar cada atropisómero tanto por cromatografía preparativa como por HPLC, pero todos fueron infructuosos ya que en todas las fracciones se encontró la misma mezcla durante el análisis por RMN sugiriendo que la isomerización se producía durante el proceso de purificación. ${ }^{135}$

Asumiendo esta limitación, se analizó la capacidad de cada mezcla de atropisómeros de asociar $\mathrm{C}_{60}$, con el objetivo de observar el impacto en cada isómero, a través de experimentos ${ }^{1} \mathrm{H}-\mathrm{RMN}$ debido a que aquellos realizados por espectroscopía de absorción UV/Vis no permitieron distinguir los distintos isómeros rotacionales. Estos estudios se complementaron con los espectros de masas de los aductos obtenidos.

En primer lugar se realizaron los experimentos encaminados a obtener la posible estequiometría del complejo de inclusión mediante la obtención de los Job Plots correspondientes. Se usó la señal de los protones unidos directamente unidos a nitrógeno en el conjunto 66, 67, 68 (2HP2), o $\mathrm{H}_{1}$, ya que es la única que posee una anchura tal que permitió estudiar todos los isómeros en su conjunto. Todos los compuestos establecen aductos supramoleculares con $\mathrm{C}_{60}$ con estequiometría 1:1. Estos resultados fueron confirmados por los espectros de masas, ya que se observaron exclusivamente estos complejos de inclusión. Desgraciadamente, este análisis no se pudo realizar con la serie 63, 64, 65 (ZnP2) por la inexistencia de mencionados hidrógenos, sin embargo, se encontró el mismo comportamiento tras el estudio por MS, por lo que se asumió que establecen la misma estequiometría que el conjunto de porfirinas desmetaladas.

\footnotetext{
$\overline{135}$ Muchos estudios demuestran que es posible aislar cada atropisómero individualmente siempre y cuando haya un sustituyente en posición orto en el grupo fenilénico en meso de manera que la isomerización sea mucho más lenta. Para algunos ejemplos, ver la referencia 134a y: (a) Rose, E.; CardonPilotaz, A.; Quelquejeu, M.; Bernard, N.; Kossanyi, A.; Desmazieres, B. J. Org. Chem. 1995, 60, 3919-3920. (b) Spasojević, I.; Menzeleev, R.; White, P. S.; Fridovich, I. Inorg. Chem. 2002, 41, 58745881. (c) Hunter, C. A.; Misuraca, M. C.; Turega, S. M. J. Am. Chem. Soc. 2011, 133, 582-594. (d) Sun, H.; Guo, K.; Gan, H.; Li, X.; Hunter, C. A. Org. Biomol. Chem. 2015, 13, 8053-8066.
} 
En segundo lugar, se procedió con la adición de diversas alícuotas del fullereno sobre una disolución de la porfirina sujeta a estudio y adquisición posterior de un espectro de ${ }^{1} \mathrm{H}-\mathrm{RMN}$ tras cada una. Las valoraciones realizadas sobre los compuestos funcionalizados con fenantreno y pireno, 63 (ZnP2a), 64 (ZnP2b), 66 (2HP2a) y 67 (2HP2b) dieron lugar a pequeños cambios en diversos desplazamientos químicos, dando como resultado una pobre asociación. Este resultado no es sorprendente ya que este tipo de compuestos poliaromáticos planos no suelen establecer importantes interacciones supramoleculares con la molécula de Buckminsterfullereno sugiriendo que la formación del complejo de inclusión se debe fundamentalmente al núcleo de la porfirina. Por otro lado, los compuestos funcionalizados con coranuleno, 65(ZnP2c) y 68(2HP2c), mostraron grandes cambios en los desplazamientos químicos excepto para uno de los atropisómeros. Adicionalmente, se encontró el mismo patrón en mencionados cambios de $\delta$ conforme se añadían las alícuotas de la disolución de $\mathrm{C}_{60}$, especialmente para los protones $\mathrm{H}_{1}$, $\mathrm{H}_{2} \mathrm{y} \mathrm{H}_{6}$, indicando un comportamiento similar.

En tercer lugar, se realizaron estudios computacionales de todos los compuestos desmetalados, haciendo especial hincapié en 68(2HP2c), para intentar predecir los desplazamientos químicos de cada atropisómero individual y tener una hipótesis de partida con el objetivo final de distinguir los distintos isómeros rotacionales en el transcurso de la valoración. Así, la geometría de todos estos compuestos se optimizó partiendo de las estructuras idealizadas (grupos fenilénicos en meso situados perpendicularmente al plano de la porfirina) dando como resultado un conjunto de resultados interesante. Los grupos poliaromáticos del mismo lado del plano de la porfirina tienen una gran tendencia a interaccionar entre ellos deformando la estructura de la misma en el proceso de tal manera que se alcanzan conformaciones de tipo silla de montar (saddle-like), ${ }^{136}$ como se puede observar en la Figura 49.

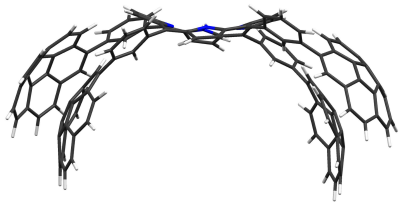

$\boldsymbol{\alpha}_{4}$

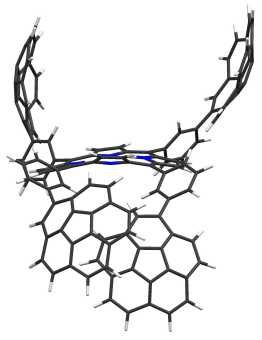

$\alpha_{2} \beta_{2}$

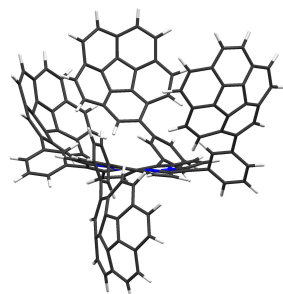

$\alpha_{3} \beta$

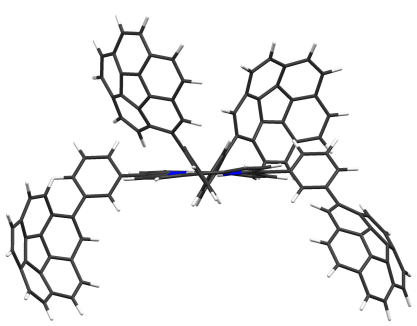

$\alpha \beta \alpha \beta$

Figura 49. Estructuras optimizadas de los distintos atropisómeros de 68(2HP2c) mostrándose la deformación del núcleo de porfirina cuanto mayor número de unidades de coranuleno se encuentra interaccionando. El resto de geometrías se encuentra en el apartado 7.4.

136 (a) Munro, O. Q.; Bradley, J. C.; Hancock, R. D.; Marques, H. M.; Marsicano, F.; Wade, P. W. J. Am. Chem. Soc. 1992, 114, 7218-7230. (b) Medforth, C. J.; Haddad, R. E.; Muzzi, C. M.; Dooley, N. R.; Jaquinod, L.; Shyr, D. C.; Nurco, D. J.; Olmstead, M. M.; Smith, K. M.; Ma, J.-G.; Shelnutt, J. A. Inorg. Chem. 2003, 42, 2227-2241. (c) Park, S. H.; Kim, S. J.; Kim, J. D.; Park, S.-B.; Huh, D. S.; Shim, Y. K.; Choe, S. J. Bull. Korean Chem. Soc. 2008, 29, 1141-1148. 
Una vez comprobados que eran mínimos por el análisis vibracional y, tras haber tenido en cuenta los efectos del disolvente, se encontró que el atropisómero más estable es $\boldsymbol{\alpha}_{4}$, mientras que le menos estable es $\boldsymbol{\alpha} \boldsymbol{\beta} \boldsymbol{\alpha} \boldsymbol{\beta}$. El resto de isómeros rotacionales tienen estabilidades intermedias, lo que sugiere que las interacciones intramoleculares entre los distintos fragmentos poliaromáticos aportan mayor estabilidad al compuesto (Tabla 4). Adicionalmente se puedo observar cómo el atropisómero $\boldsymbol{\alpha}_{4}$ parece estar mejor preorganizado (con forma de cuenco) para formar complejo de inclusión con $\mathrm{C}_{60}$ que el resto de isómeros, especialmente $\boldsymbol{\alpha} \boldsymbol{\beta} \boldsymbol{\alpha} \boldsymbol{\beta}$.

Tabla 4. Energías libres de Gibbs en disolución (en $\mathrm{kcal} / \mathrm{mol}$ ) de los 4 atropisómeros del compuesto 68(2HP2c). El resto de energías se encuentran en el apartado 7.4.

\begin{tabular}{cc}
\hline Atropisómero & $\Delta G$ \\
\hline$\alpha_{4}-68(2 H P 2 c)$ & 0,00 \\
$\alpha_{3} \beta-68(2 H P 2 c)$ & 8,38 \\
$\alpha_{2} \beta_{2}-68(2 H P 2 c)$ & 8,68 \\
$\alpha \beta \alpha \beta-68(2 H P 2 c)$ & 14,05
\end{tabular}

Las predicciones de los desplazamientos químicos del singlete $\mathrm{H}_{6}$ se promediaron excepto para $\boldsymbol{\alpha}_{3} \boldsymbol{\beta}$, cuya estructura da lugar a tres entornos químicos distintos, y se sustrajeron de los desplazamientos químicos promediados de los protones de la molécula de TMS previamente tratada computacionalmente con mismo procedimiento descrito con anterioridad para obtener el siguiente orden, de campo más bajo a campo más alto: $\alpha \beta \alpha \beta<\alpha_{3} \beta($ abajo $)<\alpha_{3} \beta($ exterior $)<\alpha_{2} \beta_{2}$ $<\alpha_{3} \beta$ (interior). Este resultado se muestra en la Figura 50.

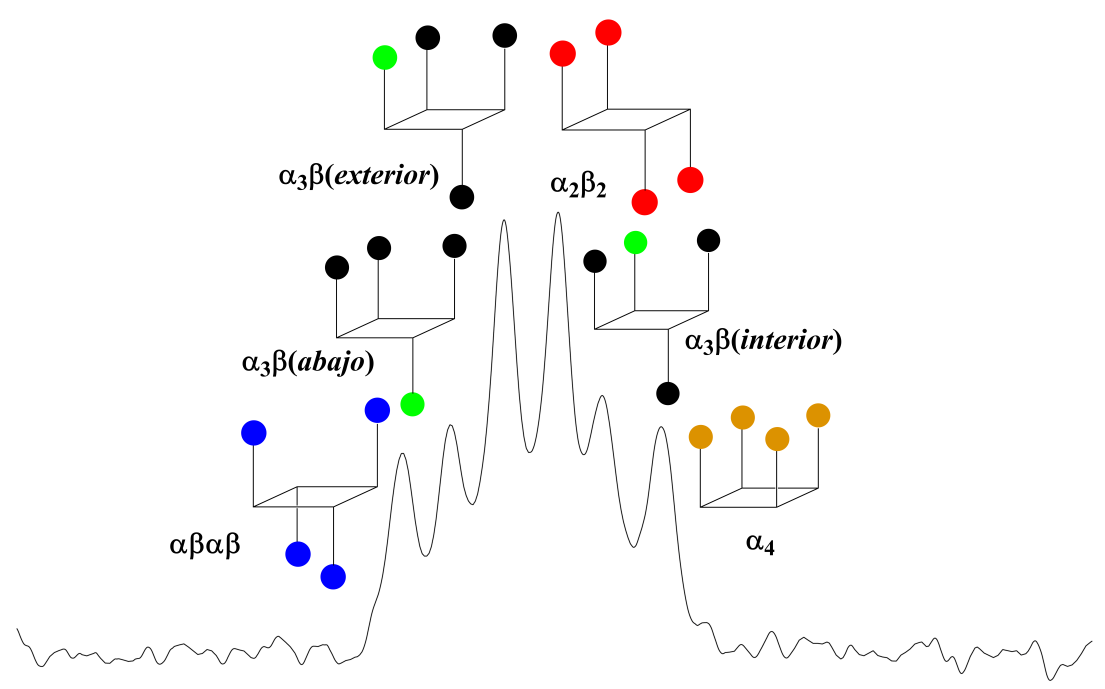

Figura 50. Espectro del singlete $\mathrm{H}_{6}$ en la molécula de 68(2HP2c) con su asignación basada en métodos computacionales. La relación de integrales es 1:1:2:2:1:1.

Partiendo de estos resultados preliminares se estudió la evolución de dicho singlete durante las sucesivas adiciones del fullereno en 65(ZnP2c) y 68(2HP2c) junto con las señales del protón $\mathrm{H}_{2}$ (conocido como $\beta$-pirrol) ya que se observó un gran desdoblamiento tras las primeras alícuotas de lo que a priori parecía un singlete. Es importante hacer notar que estos hidrógenos son equivalentes químicamente en $\boldsymbol{\alpha}_{4}$ y $\boldsymbol{\alpha} \boldsymbol{\beta} \boldsymbol{\alpha} \boldsymbol{\beta}$ pero son inequivalentes en los isómeros $\boldsymbol{\alpha}_{3} \boldsymbol{\beta}$ y $\boldsymbol{\alpha}_{2} \boldsymbol{\beta}_{2}$ dando lugar a dos desplazamientos químicos distintos. 
Primero se siguieron los cambios en los protones $\mathrm{H}_{6}$ de $\boldsymbol{\alpha}_{3} \boldsymbol{\beta}$ (abajo) y $\boldsymbol{\alpha}_{3} \boldsymbol{\beta}$ (exterior) predichos y se ajustaron las isotermas de asociación resultando unas constantes idénticas. De esta manera se asignaron definitivamente estas señales, pero no pudo confirmarse completamente porque el desplazamiento químico de $\boldsymbol{\alpha}_{3} \boldsymbol{\beta}$ (interior) se solapaba con el de $\boldsymbol{\alpha}_{2} \boldsymbol{\beta}_{2}$ durante el procedimiento de valoración (Figura 51).
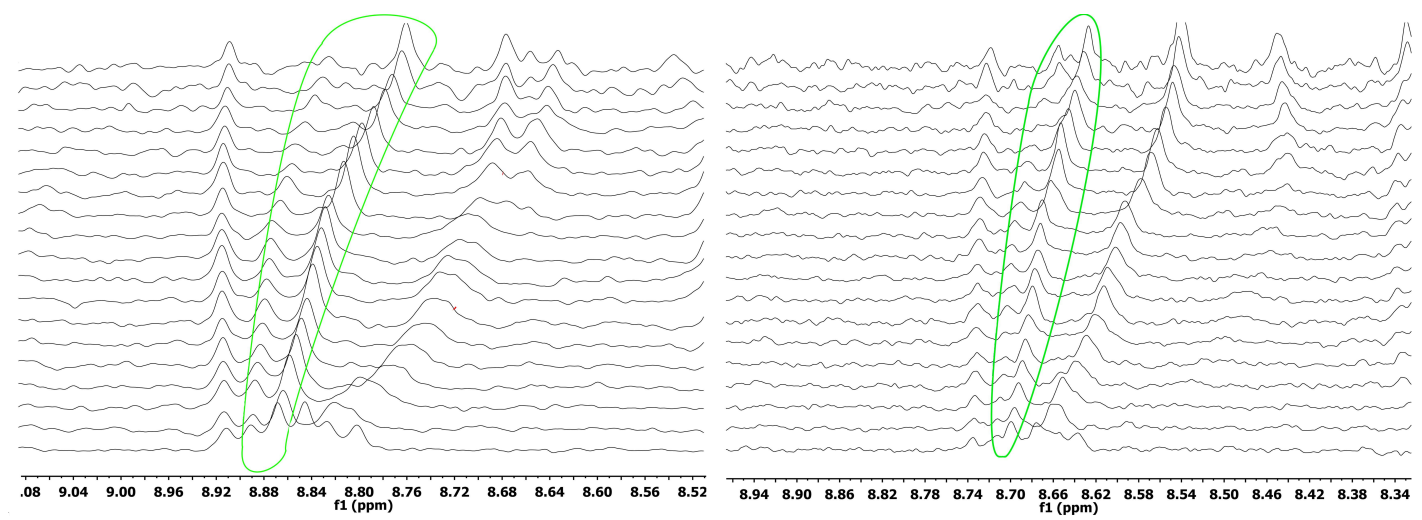

Figura 51. Espectros ${ }^{1} \mathrm{H}-\mathrm{RMN}$ en tolueno deuterado de 65(ZnP2c) (izquierda) y $68(2 \mathrm{HP2c})$ (derecha) durante la valoración con $\mathrm{C}_{60}$ mostrando la zona donde aparece $\mathrm{H}_{6}$ destacando con un óvalo verde la asignación realizada hasta ahora. El color corresponde con el patrón asignado en la Figura 50.

A continuación se analizó la evolución del singlete con integral 2 que queda por asignar definitivamente. La constante estimada es muy superior a la previamente calculada en ambos compuestos, indicando que se trata de otro atropisómero, que no puede ser otro que $\boldsymbol{\alpha}_{2} \boldsymbol{\beta}_{2}$, ya que es el único que está de acuerdo con la integral del protón $\mathrm{H}_{6}$ en relación a su distribución estadística de la mezcla (Figura 52).
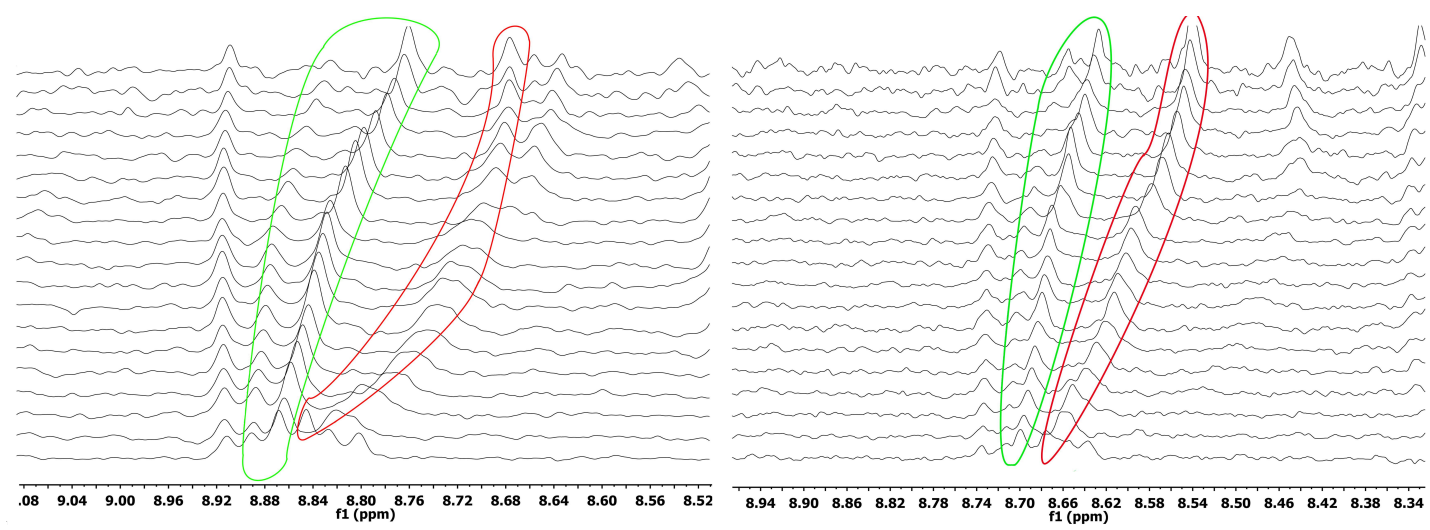

Figura 52. Espectros ${ }^{1} \mathrm{H}-\mathrm{RMN}$ en tolueno deuterado de 65(ZnP2c) (izquierda) y 68(2HP2c) (derecha) durante la valoración con $\mathrm{C}_{60}$ mostrando la zona donde aparece $\mathrm{H}_{6}$ destacando con óvalos de distinto color la asignación de dos atropisómeros realizada hasta ahora. Los colores corresponden con el patrón asignado en la Figura 50.

Llegados a este punto, quedan por asignar dos isómeros, uno cuyo singlete $\mathrm{H}_{6}$ no evoluciona prácticamente nada con las adiciones de $\mathrm{C}_{60} \mathrm{y}$ otro cuya señal se ensancha hasta tal punto de no poder observarse cambios en desplazamiento químico con claridad. Asumimos que la predicción teórica es correcta debido al alto grado de exactitud logrado hasta ahora, así como la suposición de que el atropisómero $\boldsymbol{\alpha}_{4}$ tiene una preorganización estructural tan favorable que no es posible que la señal correspondiente a su protón $\mathrm{H}_{6}$ no sufra ningún tipo de evolución (Figura $53)$. 


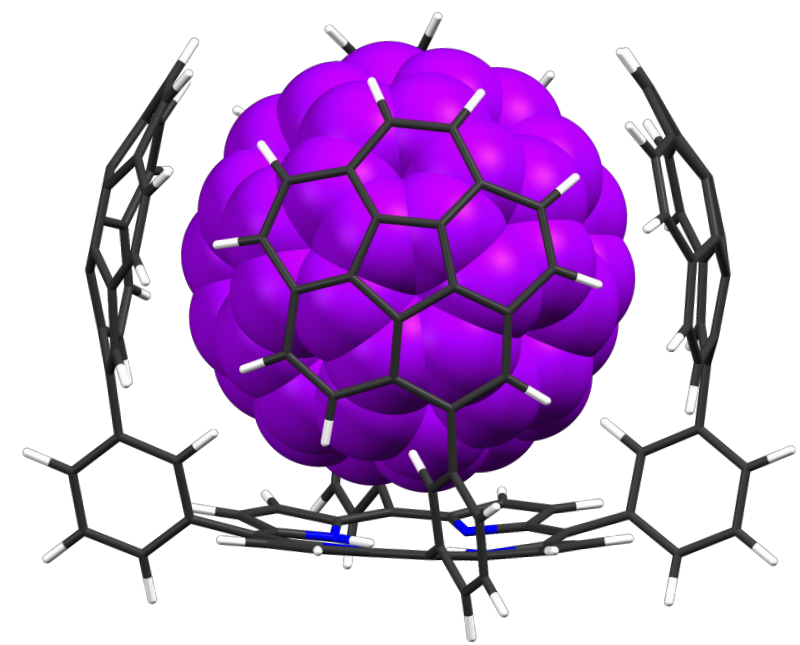

Figura 53. Estructura optimizada del aducto $\mathbf{C}_{60} @ 68(2 \mathrm{HP2c})$ mostrándose la cavidad formada por los cuatros coranulenos y el núcleo de porfirina debido a la excelente complementariedad entre los fragmentos poliaromáticos y la molécula de fullereno (de color morado). ${ }^{137}$

Además, el isómero rotacional $\boldsymbol{\alpha} \boldsymbol{\beta} \boldsymbol{\alpha} \boldsymbol{\beta}$ posee una geometría en el mínimo calculado tal que no adopta con facilidad una conformación adecuada para interaccionar con $\mathrm{C}_{60}$, luego es muy probable que no haya cambios en el desplazamiento químico de $\mathrm{H}_{6}$ durante la valoración.

En resumen, se han asignado todos los atropisómeros de 65(ZnP2c) y 68(2HP2c) en el intervalo del singlete $\mathrm{H}_{6}$, como se muestra en la Figura 54, y se han estimado las constantes de asociación de cada uno de ellos, excepto para $\boldsymbol{\alpha}_{4}$.
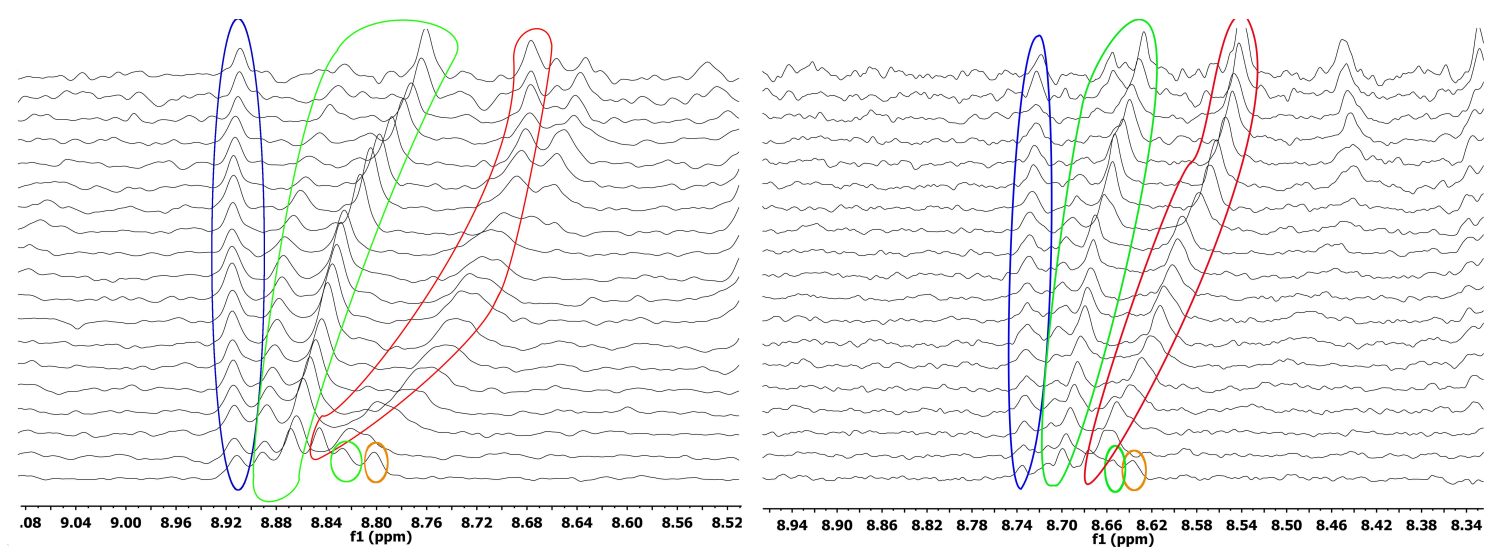

Figura 54. Asignación de atropisómeros completa de 65(ZnP2c) (izquierda) y 68(2HP2c) (derecha) durante la valoración con $\mathrm{C}_{60}$ en la zona donde aparece $\mathrm{H}_{6}$ destacando con óvalos de distinto color la asignación de cada isómero. Los colores corresponden con el patrón asignado en la Figura 50.

${ }^{137}$ Esta estructura calculada no se encuentra en el artículo publicado porque se obtuvo mucho después de su aceptación. 
Como se ha mencionado anteriormente, la evolución de los desplazamientos químicos de $\mathrm{H}_{2}$ ( $\beta$-pirrol) también fue perseguida porque se produjo un gran cambio durante la valoración y es un singlete muy alejado del resto de señales, lo que facilitó su estudio. También sirvió para evaluar la precisión de la estimación de constantes realizada en el caso de los protones $\mathrm{H}_{6}$. En este caso no se disponía de una diferenciación previa debido al solapamiento de señales de todos los atropisómeros dando lugar a un singlete o, en el mejor de los casos, dos singletes muy próximos. De acuerdo con esto, estuvimos obligados a realizar una estimación sistemática de constantes de asociación cubriendo todas las posibilidades razonables existentes.

Por suerte, resultó moderadamente sencillo encontrar los desplazamientos químicos correspondientes a los isómeros $\boldsymbol{\alpha} \boldsymbol{\beta} \boldsymbol{\alpha} \boldsymbol{\beta}$ (por existencia de un singlete sin evolución) y $\boldsymbol{\alpha}_{3} \boldsymbol{\beta}$ (por la observación de dos singletes con la misma constante que coincide con la estimada en el estudio de $\mathrm{H}_{6}$ ) debido a un menor solapamiento con el resto de señales, tal y como se muestra en la Figura 55.
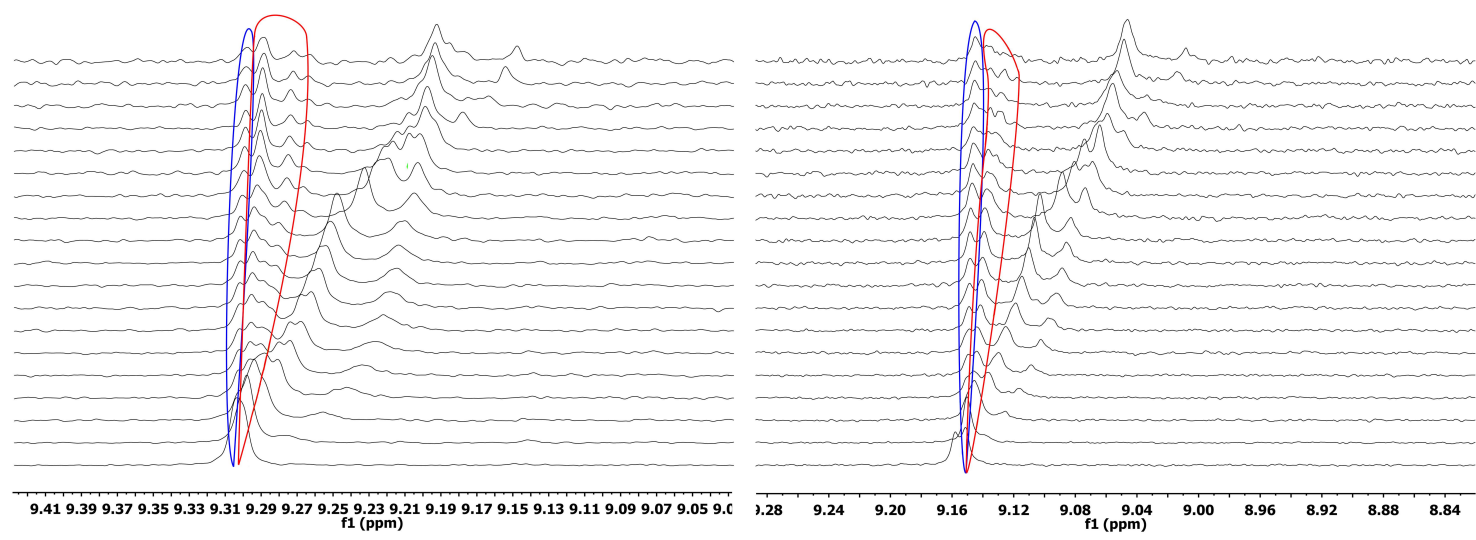

Figura 55. Espectros ${ }^{1} \mathrm{H}-\mathrm{RMN}$ en tolueno deuterado de 65(ZnP2c) (izquierda) y 68(2HP2c) (derecha) durante la valoración con $\mathrm{C}_{60}$ mostrando la zona donde aparece $\mathrm{H}_{2}$ destacando óvalos de distinto color la asignación realizada hasta ahora. El color corresponde con el patrón asignado en la Figura 50.

A continuación se estudiaron las señales que quedaban por asignar, que corresponden con los mayores cambios en los desplazamientos químicos observados. En este caso se siguió uno de los desplazamientos químicos más intensos y se encontró un cruzamiento con otra señal que inesperadamente apareció tras la primera adición, dándose por tanto dos posibilidades de asignación, como se muestra en la Figura 56. 

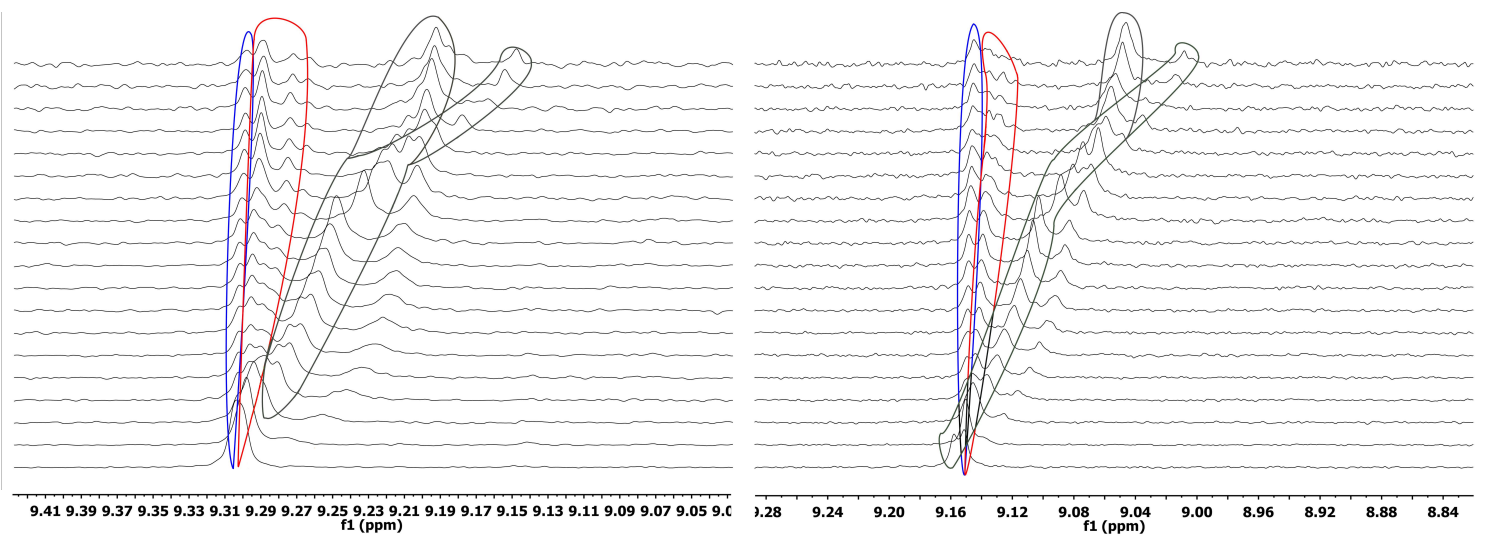

Figura 56. Espectros ${ }^{1} \mathrm{H}-\mathrm{RMN}$ en tolueno deuterado de 65(ZnP2c) (izquierda) y 68(2HP2c) (derecha) durante la valoración con $\mathrm{C}_{60}$ mostrando la zona donde aparece $\mathrm{H}_{2}$ destacando óvalos de distinto color la asignación realizada hasta ahora y las dos posibilidades de evolución de las señales más intensas que quedan por asignar. El color corresponde con el patrón asignado en la Figura 50. El gris pertenece a un atropisómero todavía no asignado.

Se ajustaron ambas posibilidades de isotermas de asociación, siendo una de ellas aquella que arrojaba una constante estimada igual a uno de los atropisómeros ya estudiados, $\boldsymbol{\alpha}_{2} \boldsymbol{\beta}_{2}$. El desplazamiento químico restante, cuya constante de asociación estimada era muy distinta a las obtenidas hasta el momento, se aceptó que procedía del isómero rotacional que quedaba por asignar, $\boldsymbol{\alpha}_{4}$. En la Figura 57 se recogen los resultados de esta discusión.
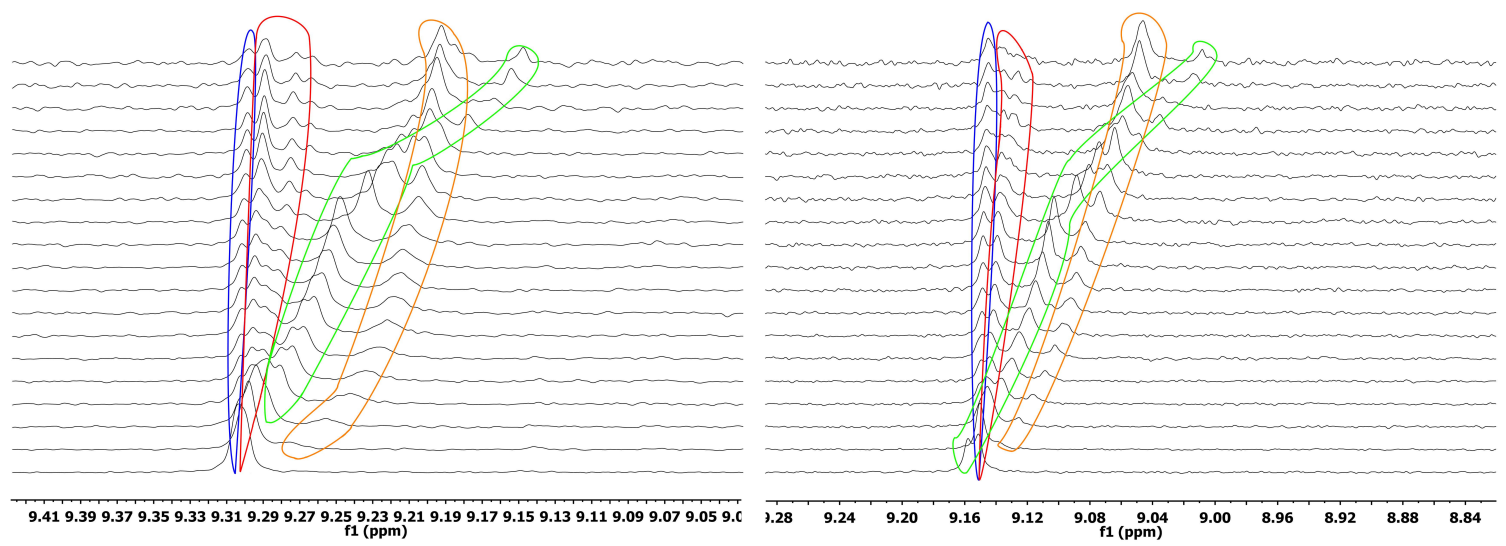

Figura 57. Asignación de atropisómeros completa de 65(ZnP2c) (izquierda) y 68(2HP2c) (derecha) durante la valoración con $\mathrm{C}_{60}$ en la zona donde aparece $\mathrm{H}_{2}$ destacando con óvalos de distinto color la asignación de cada isómero. Los colores corresponden con el patrón asignado en la Figura 50. 
Todo el protocolo previamente descrito se aplicó al resto de compuestos preparados en este trabajo cuyas unidades poliaromáticas son fenantreno y pireno obteniéndose resultados muy similares, pero con cambios en desplazamientos químicos mucho menos pronunciados. Este conjunto de estudios completo puede verse en el apartado 7.4. Todas las constantes de asociación estimadas se encuentran recogidas en la Tabla 5.

Tabla 5. Resumen de las constantes de asociación estimadas en tolueno-d8 (en $\mathrm{M}^{-1}$ ) de todas las porfirinas funcionalizadas con fragmentos poliaromáticos preparadas en este trabajo así como para cada isómero individual en la mezcla estadística de atropisómeros.

\begin{tabular}{|c|c|c|c|c|c|c|}
\hline & 63(ZnP2a) & $66(2 \mathrm{HP} 2 \mathrm{a})$ & $64(\mathrm{ZnP2b})$ & $67(2 \mathrm{HP} 2 \mathrm{~b})$ & $65(\mathrm{ZnP} 2 \mathrm{c})$ & $68(2 \mathrm{HP} 2 \mathrm{c})$ \\
\hline$\alpha_{4}$ & 180 & $\mathrm{~N} / \mathrm{A}^{a}$ & 790 & 1180 & $22330^{b}$ & $7700^{b}$ \\
\hline$\alpha_{3} \beta$ & $0^{c}$ & $0^{c}$ & $0^{c}$ & 540 & 1730 & 1820 \\
\hline$\alpha_{2} \beta_{2}$ & 240 & 130 & 690 & 1240 & 12140 & 5890 \\
\hline$\alpha \beta \alpha \beta$ & $0^{c}$ & $0^{c}$ & $0^{c}$ & $0^{c}$ & $0^{c}$ & $0^{c}$ \\
\hline Average & $\mathrm{N} / \mathrm{A}^{d}$ & $(4,5 \pm 0,05) \cdot 10^{2}$ & $\mathrm{~N} / \mathrm{A}^{d}$ & $(4,6 \pm 0,05) \cdot 10^{2}$ & $N / A^{d}$ & $(5,4 \pm 0,2) \cdot 10^{3}$ \\
\hline
\end{tabular}

Dentro de la mezcla de atropisómeros se ha observado que aquél que tiene la mejor constante de asociación es $\boldsymbol{\alpha}_{4}$, como puede esperarse por su preorganización estructural previa, seguido por $\boldsymbol{\alpha}_{2} \boldsymbol{\beta}_{2} \mathrm{y}$, por último, $\boldsymbol{\alpha}_{3} \boldsymbol{\beta}$. Puede parecer contraintuitivo el hecho de que el isómero $\boldsymbol{\alpha}_{3} \boldsymbol{\beta}$, que posee tres unidades poliaromáticas del mismo lado, sea mejor que $\boldsymbol{\alpha}_{2} \boldsymbol{\beta}_{2}$, que posee sólo dos. Es conocido que el aumento de número de grupos policíclicos aromáticos en la misma molécula no necesariamente ha de poseer una mejor asociación frente a fullerenos, sobre todo si se trata de estructuras con movilidad conformacional, ${ }^{83 a, e, 81}$ por lo que en este caso puede ocurrir lo mismo y haber otros factores mucho más decisivos, como son el factor entrópico, la solvatación del aducto o la energía de deformación. ${ }^{133}$ Por otro lado, el isómero rotacional $\boldsymbol{\alpha} \boldsymbol{\beta} \boldsymbol{\alpha} \boldsymbol{\beta}$ no parece establecer interacciones supramoleculares con la molécula de Buckminsterfullereno, como podía esperarse de su geometría optimizada computacionalmente, cuya vaga preorganización en forma de cuenco puede suponer un obstáculo importante a la hora de superar la energía de deformación necesaria para acomodar dicha molécula en su cavidad.

En el caso de las porfirinas funcionalizadas con coranuleno, se encontró que, tanto $\boldsymbol{\alpha}_{4} \mathbf{4}^{-}$ 65(ZnP2c) como $\alpha_{2} \beta_{2}-\mathbf{6 5}(\mathrm{ZnP2c})$ poseen unas constantes de asociación muy superiores a sus contrapartidas desmetaladas, $\alpha_{4}-\mathbf{6 8}(2 \mathrm{HP} 2 \mathrm{c})$ y $\boldsymbol{\alpha}_{2} \boldsymbol{\beta}_{2}-\mathbf{6 8}(2 \mathrm{HP} 2 \mathrm{c})$, respectivamente. El efecto del metal favorece la asociación supramolecular. Este hecho es sorprendente ya que es conocido que el átomo de $\mathrm{Zn}$ no suele favorecerla de tal manera ${ }^{97}$ por lo que actualmente este efecto se encuentra bajo estudio. 
Como se comentó anteriormente, la existencia de los hidrógenos $\mathrm{H}_{1}$ en la serie de compuestos desmetalados, cuyo desplazamiento químico en todos los isómeros rotacionales consiste en una señal promedio muy ancha, permitió estudiar la capacidad de la mezcla de atropisómeros de formar el complejo de inclusión con $\mathrm{C}_{60}$ en su conjunto. Así, se obtuvieron constantes del orden de $10^{2}$ para los derivados de fenantreno y pireno, mientras que fue de $10^{3}$ para el derivado de coranuleno (Tabla 5). Estos resultados apoyan la sinergia existente entre el núcleo de porfirina y las unidades del grupo poliaromático no plano a la hora de establecer aductos supramoleculares con fullerenos. Además, estos estudios han abierto la puerta al diseño de nuevas porfirinas con múltiples grupos de coranuleno y cuya estructura se asemeje a los atropisómeros $\boldsymbol{\alpha}_{4}$ (tipo picket fence) ${ }^{134 c}$ con el objetivo de mejorar esta asociación. 
6. CONCLUSIONES 



\section{CONCLUSIONES}

De la misma manera en la que se estructuró el apartado 3 de Objetivos, éste también contiene dos conclusiones principales que dan lugar a un conjunto de otras específicas.

\subsection{CONCLUSIONES GENERALES}

Se ha desarrollado una metodología de preparación de dos familias de interruptores moleculares de tipo rotaxano a través de la estrategia enhebrado y cierre con altos rendimientos y un número de etapas razonablemente corto. Se han caracterizado por completo y estudiado el movimiento del anillo macrocíclico a lo largo del eje. Adicionalmente, se han evaluado sus propiedades catalíticas y su fotoisomerización.

Se ha contribuido con el desarrollo de otra metodología sintética para funcionalizar grupos poliaromáticos planos y no planos obteniéndose compuestos particularmente complejos en muy pocos pasos y con rendimientos razonables. Todos ellos fueron caracterizados completamente y sus capacidades para establecer interacciones con fullerenos han sido evaluadas.

\subsection{CONCLUSIONES ESPECÍFICAS}

Se han puesto a punto métodos por los cuales un conjunto de building blocks de fácil acceso sintético se pueden unir para dar estructuras más complejas entrelazadas mecánicamente.

Se ha preparado una familia de rotaxanos a través de una sal de amonio común capaz de establecer un aducto supramolecular con un éter corona cuya reacción de cierre subsiguiente por metodología click dio lugar a los compuestos deseados.

Dichos rotaxanos se han transformado en interruptores moleculares por la generación de una segunda estación en el eje y se han estudiado las condiciones más sencillas por las que el movimiento del macrociclo efectivamente ocurre.

Esta familia de interruptores moleculares se ha usado para evaluar su capacidad como organocatalizores conmutables en dos reacciones tipo Michael de formación de enlaces S-C y $\mathrm{C}-\mathrm{C}$ concluyendo, que cuanto mayor número de unidades catalíticas expuestas hay en el eje, la catálisis ocurre más eficazmente.

Aprovechando la metodología sintética descrita previamente, se ha incorporado un grupo azobencénico al eje y se ha obtenido otra familia de rotaxanos que incluyen esta funcionalidad. En ese trabajo se ha prescindido del aislamiento de la sal de amonio previa a la formación del complejo de inclusión disminuyendo el número de etapas sintéticas previstas.

Todos los rotaxanos con grupo azobencénico se han transformado también en interruptores moleculares y se ha estudiado el movimiento del macrociclo en las mismas condiciones descritas en el anterior trabajo.

Se ha estudiado el efecto del grupo azobencénico con respecto al movimiento del anillo a lo largo del eje como función del isómero configuracional en el que se encuentre concluyendo que es necesaria la existencia de un espaciador más largo entre las estaciones y el fragmento de azobenceno así como grupos lo suficientemente voluminosos situados en esta unidad para obtener un control completo del funcionamiento de estas máquinas. 
Se ha expandido el uso del grupo azobenceno fucionalizándolo con moléculas poliaromáticas planas y no planas a través de reacciones de acoplamiento C-C de Suzuki-Miyaura estudiando las posibles rutas para la obtención de los compuestos deseados.

Todos los derivados azobencénicos preparados fueron estudiados en relación a su capacidad para interaccionar con fullereneos dependiendo del isómero presente encontrándose un compuesto derivado de coranuleno que es capaz de modular su afinidad por ellos de manera dramática en función del estado fotoestacionario

Se ha aprovechado la metodología desarrollada respecto al anterior trabajo para aplicarla en la funcionalización de porfirinas simétricas meso sustituidas con grupos poliaromáticos planos y no planos. Sin embargo, se ha explorado a fondo las posibilidades sintéticas que ofrecían estas estructuras para obtener los compuestos finales de manera óptima.

Todas las porfirinas descritas que poseen fragmentos poliaromáticos consisten en mezclas de atropisómeros distribuidos estadísticamente y se ha estudiado el impacto que genera la adición de $\mathrm{C}_{60}$ concluyendo que el atropisómero con los cuatro grupos apuntando hacia el mismo lado (denominada como picket fence) es el mejor anfitrión para este fullereno. También se observó que las porfirinas que poseían coranuleno en su estructura tienen mejores constantes de asociación que aquellas que no lo tienen, sugiriéndose sinergia entre ambas estructuras. 


\subsection{CONCLUSIONS}

According to the structure of part 3 (Objectives), herein two main conclusions are reported along with a set of specific conclusions.

\subsubsection{GENERAL CONCLUSIONS}

A methodology regarding the preparation of molecular shuttles have been developed. The synthetic strategy was threading-capping affording high yields with a reasonably small number of steps. All final compounds were fully characterized and the ring shuttling along the axle have been studied. Additionally, their catalytic properties and photoisomerization were assessed.

We contributed with the development of another synthetic methodology for the functionalization of planar and non-planar polyaromatic groups obtaining particularly complex compounds in very few steps. All of them were fully characterized and their capabilities to establish supramolecular interactions with fullerenes were tested.

\subsubsection{SPECIFIC CONCLUSIONS}

Several methods to easily link accessible building blocks in order to achieve more complex mechanically interlocked structures have been optimized.

A family of rotaxanes have been prepared from a common ammonium salt able to establish a supramolecular adduct with a crown ether whose subsequent final reaction by click methodology resulted in desired compounds.

Such rotaxanes where turned into molecular shuttles through the generation of a second station on the axle and the simplest conditions by which macrocycle movement effectively occurs.

This family of molecular shuttles were used to assess their capability as switchable organocatalysts in two Michael-type reactions in the formation of S-C and C-C bonds concluding that, the higher the number of exposed catalytic units on the axle, the more efficient the catalysis is.

Drawing upon the previously described synthetic methodology, an azobenzene group was incorporated in the axle and another family of rotaxanes including this function was obtained. In that work, isolation of previous ammonium salt before inclusion complex formation was avoided, diminishing the number of envisaged synthetic steps.

All azobenzene-containing rotaxanes were turned into molecular shuttles as well and macrocycle movement was studied under the same conditions that were described in the previous work.

The effect of azobenzene group was studied in terms of ring movement along the axle as a function of the configuration isomer concluding that the existence of a longer spacer between stations and azobenzene fragment as well as the presence of bulky enough substituents placed in this unit are necessary so that a total control of these machines operation. is obtained. 
The use of azobenzene group was expanded by functionalizing it with planar and non-planar polyaromatic molecules through Suzuki Miyaura C-C coupling reactions. Three possible routes for desired compounds were explored.

All of the prepared azobenzene derivatives were studied regarding their ability to interact with fullerenes as a function of the configurational isomer and we found a corannulene-derived compound able to dramatically modulate its affinity towards them depending on the photostationary state.

Previously developed synthetic methodology was applied to functionalize symmetric mesosubstituted porphyrins with planar and non-planar polyaromatic groups. Nonetheless, synthetic possibilities were thoroughly explored in order to optimally afford final compounds.

Every described porphyrin bearing polyaromatic fragments consists of statistically distributed mixture of four atropisomers and the impact of $\mathrm{C}_{60}$ additions was studied concluding that the isomer having all their substituents pointing towards the same face (known as picket fence structure) is the best host for fullerene. It was also observed that corannulene-bearing porphyrins had better association constants than those having planar groups, suggesting synergy between both structures. 
7. ARTÍCULOS 



\title{
Multivalent Molecular Shuttles - Effect of Increasing the Number of Centers in Switchable Catalysts
}

\author{
Celedonio M. Álvarez, ${ }^{*[a]}$ Héctor Barbero, ${ }^{[a]}$ and Daniel Miguel ${ }^{[a]}$
}

Keywords: Organocatalysis / Click chemistry / Rotaxanes / Molecular shuttles

A family of three molecular shuttles based upon ammonium/ triazolinium salt motifs with one, two, and three catalytic centers were prepared along with their corresponding noninterlocked threads. The switching process for all rotaxanes was tested through an in situ procedure in solution. Furthermore, their organocatalysis of two Michale-type reactions by iminium activation was tested to check whether the increase of the number of catalytic centers in the same shuttle is a decisive factor to improve their efficiencies compared with those of single-active-site organocatalytic shuttles under the same conditions.

\section{Introduction}

Rotaxanes incorporating catalytic centers in their structures have attracted the attention of many authors in this decade, ${ }^{[1]}$ and their suitability as an effective reaction platform has been demonstrated. Several switchable catalysts have been inspired ${ }^{[2]}$ by how the rates and outcomes of enzyme-catalyzed reactions can be controlled through triggerinduced events. ${ }^{[3]}$ Synthetic catalysts that could achieve similar influence in natural processes would become a scientific breakthrough.

Switchable catalysts with rotaxane-based secondary amines in their structures are of particular interest, mainly because of their potential activation modes (iminium, ${ }^{[4]}$ enamine, ${ }^{[5]}$ and trienamine $)^{[6]}$ and the ease of switching between all potential stations. They have mechanically interlocked architectures with internal motion that can be exploited to control both the rates and outcomes of several reactions by shielding and exposing a catalytic center. ${ }^{[7]}$

Moreover, multivalent catalysts based on polymers or dendrimers are generally better than their monovalent counterparts owing to their connecting frameworks. ${ }^{[8]}$

To advance the knowledge on these elegant architectures, we found it very interesting to synthesize a new family of molecular shuttles with dibenzylamine stations that can perform several kinds of reactions. The difference between them is the number of catalytic centers in their structures, and the objective was to compare their behavior in two typical Michael-type reactions, which are well-known benchmarks for comparison purposes.

[a] GIR MIOMeT, IU CINQUIMA/Química Inorgánica, Facultad de Ciencias, Universidad de Valladolid,

47005 Valladolid, Spain

E-mail: celedonio.alvarez@uva.es http://miomet.blogs.uva.es/

Supporting information for this article is available on the WWW under http://dx.doi.org/10.1002/ejoc.201500942.

\section{Results and Discussion}

\section{Synthesis and Switching Process}

All of the molecular-shuttle-based organocatalysts consist of one, two, or three axles containing dibenzylammonium and methyltriazolinium salts as binding sites along with anthracene stoppers (Figure 1). Additionally, they bear one, two, or three dibenzo-24-crown-8 (DB24C8) macrocycles, which establish very good associations with ammonium salts. ${ }^{[9]}$ Non-interlocked-thread analogues were also prepared for comparison of their spectroscopic and chemical properties (Figure 2).

The synthetic procedure for the compounds presented begins with the preparation of the ammonium salt R5-H$\mathrm{PF}_{6}$ (Scheme 1). This product was obtained in six steps from methyl 4-(aminomethyl)benzoate hydrochloride and 9anthracenecarbaldehyde with an overall yield of $52 \%$. This molecule is a common building block for all of the rotaxanes.

The strategy to obtain the threaded compounds relies on a well-known self-assembly method: the macrocyclic polyether is captured by the ammonium salt in solution followed by a copper(I)-catalyzed azide-alkyne cycloaddition (CuAAC) "click" reaction ${ }^{[10]}$ between the terminal alkyne and an azide-functionalized compound. Finally, the triazole formed was methylated to create the second binding site (Scheme 1). ${ }^{[11]}$

The preparation of the non-interlocked-thread analogues was very similar, but in these cases the amine was protected until the final step (Scheme 2). ${ }^{[12]}$ This synthesis starts with the cyclization of $\mathbf{R} \mathbf{4}$ instead of $\mathbf{R 5}-\mathrm{H}_{-}-\mathrm{PF}_{6}$ with a suitable azide and is followed by triazole methylation and a final deprotection step. It must be noted that these compounds are less soluble in common organic solvents and generally less stable than their rotaxane counterparts; therefore, the 

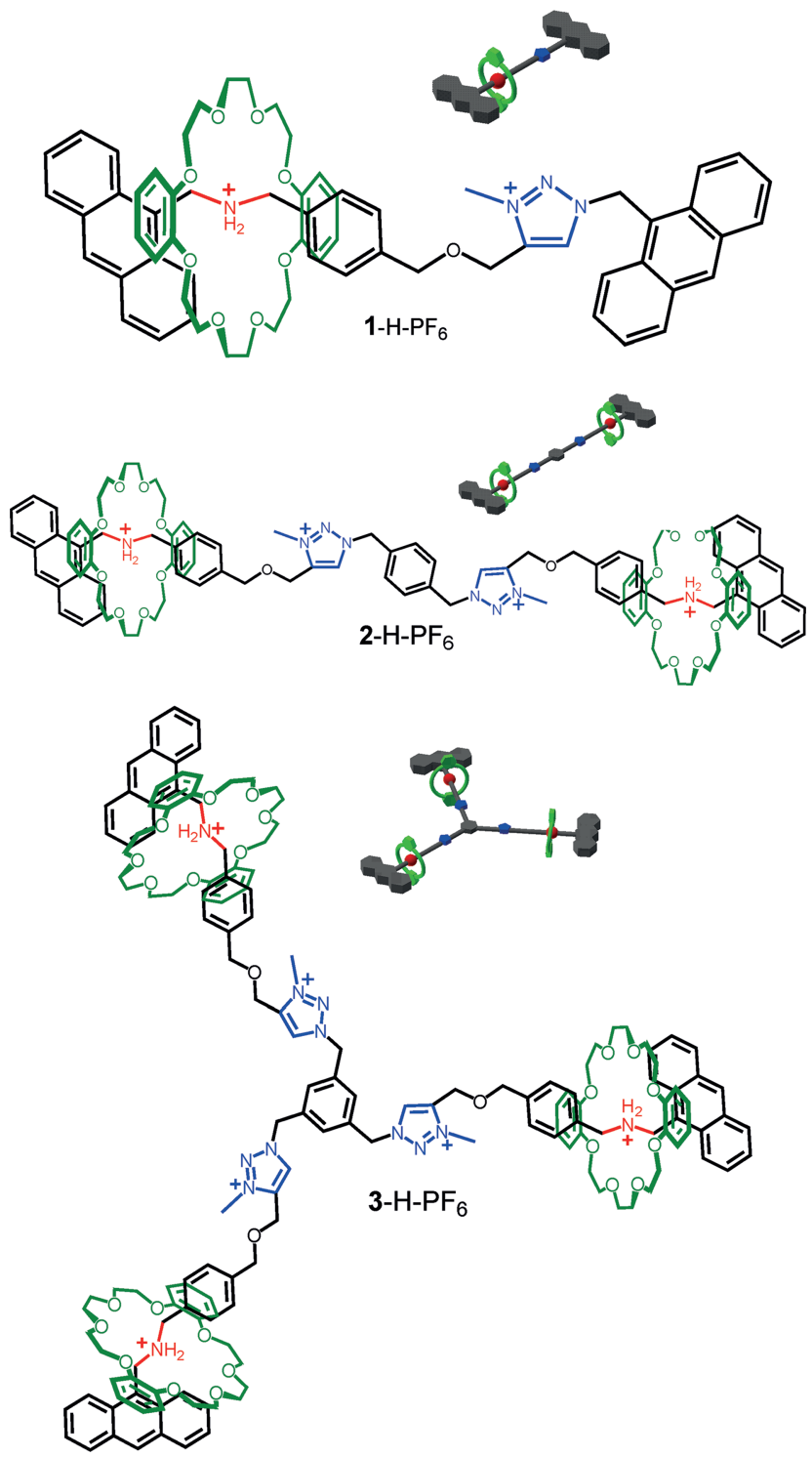

Figure 1. Molecular shuttles with one, two, and three catalytic sites reported in this study.
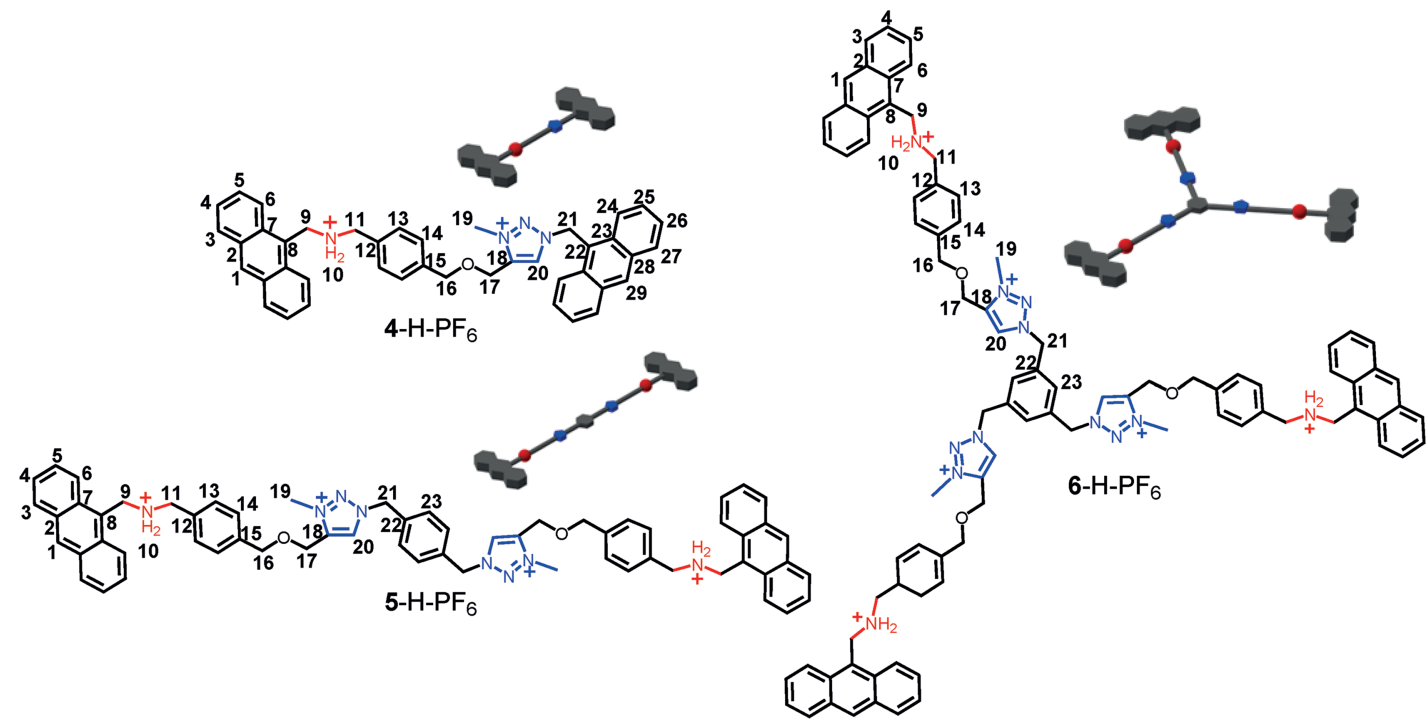
is a real on/off switching process that is performed entirely in situ without any purification step. Thus, this method was applied as follows: $\mathrm{NaOH}$ (1.1 equiv. per binding site) dissolved in $\mathrm{D}_{2} \mathrm{O}(1 \mathrm{M})$ was added to a solution of the rotaxane in $\left[\mathrm{D}_{6}\right]$ acetone under vigorous stirring, and a ${ }^{1} \mathrm{H}$ NMR spectrum was recorded after a few minutes. This medium is basic enough to deprotonate the ammonium salt to yield the amine, which is a worse binding site than the triazolinium salt; therefore, the macrocycle is forced to move along the axis to interact with the second station. The addition of $\mathrm{CF}_{3} \mathrm{CO}_{2} \mathrm{H}$ ( 1.1 equiv. per binding site) reprotonates the amine, and it is again preferable for the crown ether to recover the initial position to establish strong intermolecular interactions. The role of the anthracene moieties is to prevent undesired dethreading through their bulk. ${ }^{[13]}$

Although this method seems to be more valuable, it is not definitive, as the concentration of dissolved salts (sodium trifluoroacetate in this case) increases as the successive switching cycles are performed, and the amount of HDO severely complicates the ${ }^{1} \mathrm{H}$ NMR spectra.

For $1-\mathrm{H}-\mathrm{PF}_{6}$, the set of signals corresponding to the benzylic protons $\left(\mathrm{H}^{9}\right.$ and $\mathrm{H}^{11}$, Figure 3 ) next to the ammonium salt are broadened and shifted downfield compared with those of the non-interlocked $4-\mathrm{H}_{-} \mathrm{PF}_{6}$ (see Supporting Information). This confirms that strong hydrogen bonding occurs between the macrocycle and the ammonium salt in the axle.

Moreover, their relative position is observed in the NOESY spectrum (Figure 4, left), and the correlation

Figure 2. Non-interlocked-thread analogues. 


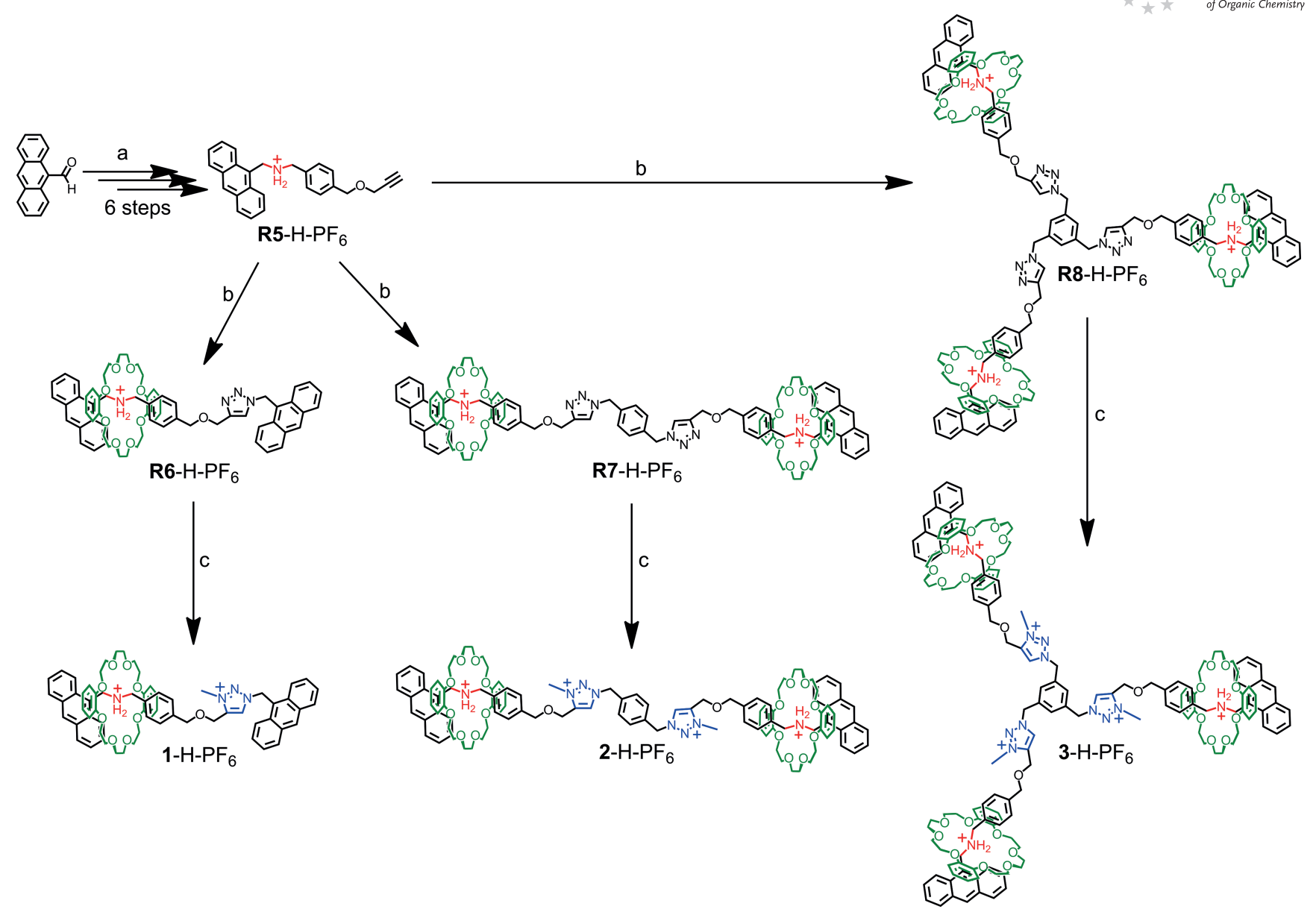

Scheme 1. Synthesis of shuttles 1-H-PF 6 , 2-H-PF , and 3-H-PF . Reagents and conditions: (a) 1. Methyl 4-(aminomethyl)benzoate hydrochloride, microwave $(\mathrm{MW}) ; 2 . \mathrm{NaBH}_{4}, \mathrm{MeOH} ; 3$. $\mathrm{LiAlH}$, THF, $-78^{\circ} \mathrm{C} ; 4 . \mathrm{Boc}_{2} \mathrm{O}, \mathrm{I}_{2}, \mathrm{MeOH} ; 5$. NaH, propargyl bromide, THF; 6 . $\mathrm{CF}_{3} \mathrm{CO}_{2} \mathrm{H}, \mathrm{NH}_{4} \mathrm{PF}_{6}, \mathrm{H}_{2} \mathrm{O} / \mathrm{DCM}$. (b) DB24C8, 9-(azidomethyl)anthracene, 1,4-bis(azidomethyl)benzene or 1,3,5-tris(azidomethyl)benzene, $\left[\mathrm{Cu}(\mathrm{NCMe})_{4}\right] \mathrm{PF}_{6}, 2$,6-lutidine, DCM. (c) $1 . \mathrm{Me}_{3} \mathrm{OBF}_{4} ; 2 . \mathrm{NH}_{4} \mathrm{PF}_{6}, \mathrm{H}_{2} \mathrm{O} / \mathrm{DCM}$.

cross-peaks between the $\mathrm{CH}_{2}$ macrocycle signals and all nuclei next to the ammonium station in the axle show the spatial proximity of both fragments.

When the rotaxane is switched (Figure 3, b), a set of signals is modified substantially along with other minor changes. As expected, the biggest changes are observed for the protons close to the shuttle stations. Thus, the most remarkable changes are for the signals of the benzylic $\mathrm{CH}_{2}$ groups next to the ammonium salt, which undergo an upfield shift of $\Delta \delta \mathrm{H}^{9}=-1.05$ and $\Delta \delta \mathrm{H}^{11}=-0.79 \mathrm{ppm}$. Furthermore, all protons near the triazolinium moiety are shifted, and the shifts for $\mathrm{H}^{20}$ and $\mathrm{H}^{21}$ are the most significant (Figure 3, $\Delta \delta \mathrm{H}_{20}=0.69 \mathrm{ppm}$ downfield and $\Delta \delta \mathrm{H}_{21}=$ $-0.41 \mathrm{ppm}$ upfield). Every signal corresponding to the macrocycle also changes (Figure 3). Additionally, the NOESY spectrum yields correlation cross-peaks between the $\mathrm{CH}_{2}$ macrocycle signals and all nuclei next to the triazolinium station, which confirms their relative situation.

After reprotonation, the ring recovers its initial position as expected, but the spectrum is not exactly like the first one recorded (Figure 3, c). Several signals have been slightly changed relative to the original ones. ${ }^{[14]}$ This effect is not surprising, as the change of counterion affects the chemical environment of nearby nuclei, ${ }^{[15]}$ and the triazolinium salt/ macrocyclic ether couple is used to bind anions in solution. ${ }^{[16]}$ The switching process can be performed several times without appreciable signs of decomposition (Figure 3, $\mathrm{d}$ and e).

This new switching procedure for $\mathbf{1}-\mathrm{H}-\mathrm{PF}_{6}$ was applied for the other shuttles. The ${ }^{1} \mathrm{H}$ NMR spectra of $2-\mathrm{H}_{-} \mathrm{PF}_{6}$ (Figure 5, left) and 3-H-PF 6 (Figure 5, right) are very similar to that of $1-\mathrm{H}-\mathrm{PF}_{6}$ but show less signals owing to the high symmetry of these shuttles. The variation in chemical shifts upon switching was analogous to that observed for the previous shuttle, but some signals corresponding to the macrocycle were broadened and, therefore, showed the expected lack of freedom in Brownian motion, possibly because of the mutual proximity of the macrocycle ethers. Additionally, for 3-H-PF 6 , the immediate exchange of the proton of the triazolinium salt $\left(\mathrm{H}^{20}\right)$ with a deuteron after the addition of the first aliquot of $\mathrm{NaOH} 1 \mathrm{M}$ in $\mathrm{D}_{2} \mathrm{O}$ showed the acidity of this proton. This effect was also observed if the other molecular shuttles were stored in solution for a long time. 


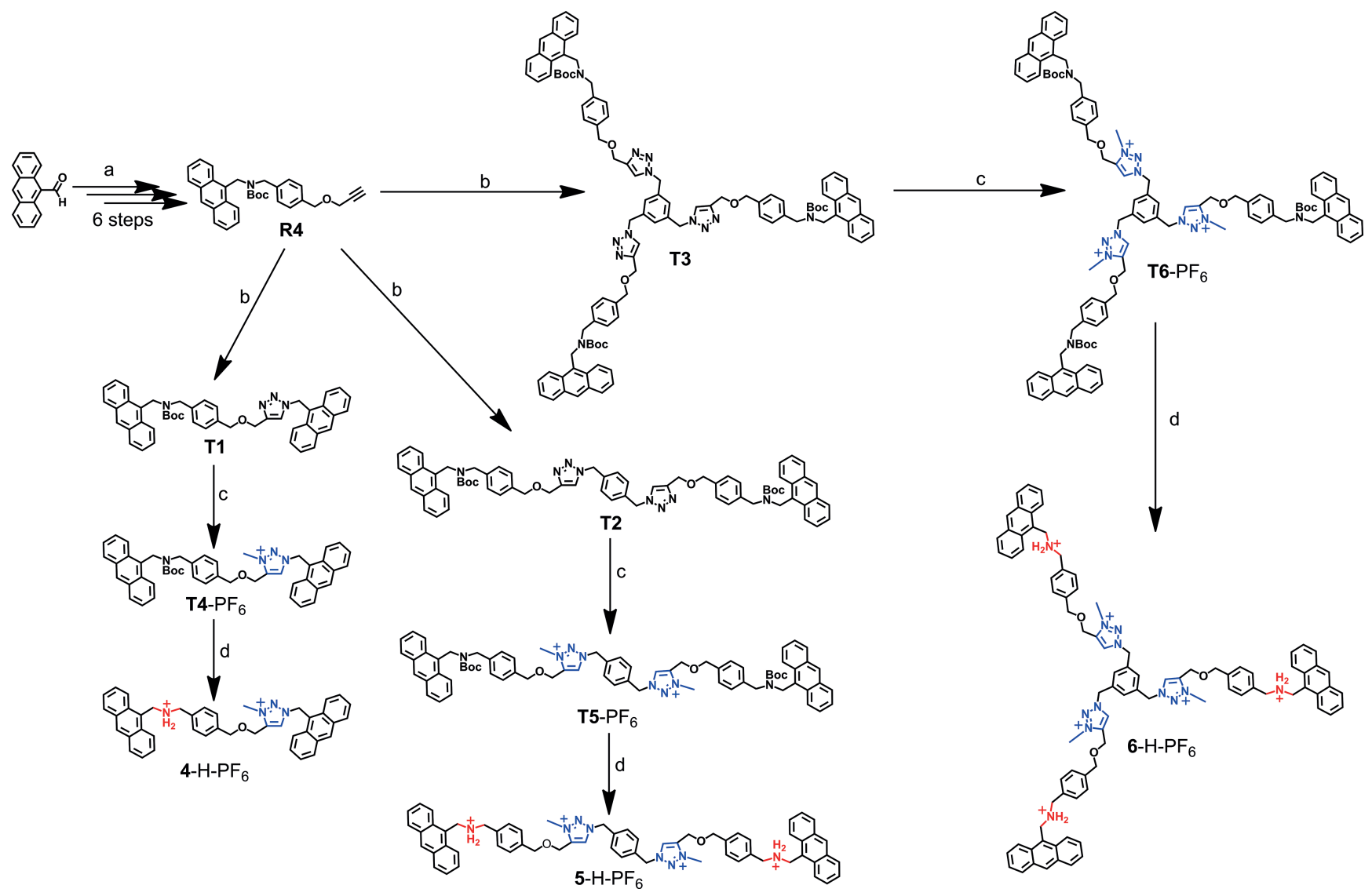

Scheme 2. Synthesis of non-interlocked threads 4-H-PF, $5-\mathrm{H}_{-} \mathrm{PF}_{6}$, and 6-H-PF 6 . Reagents and conditions: (a) 1. Methyl 4-(aminomethyl)benzoate hydrochloride, MW; 2. $\mathrm{NaBH}_{4}, \mathrm{MeOH} ; 3 . \mathrm{LiAlH}_{4}, \mathrm{THF},-78^{\circ} \mathrm{C} ; 4$. $\mathrm{Boc}_{2} \mathrm{O}, \mathrm{I}_{2}, \mathrm{MeOH} ; 5$. NaH, propargyl bromide, THF. (b) 9-(Azidomethyl)anthracene, 1,4-bis(azidomethyl)benzene or 1,3,5-tris(azidomethyl)benzene, [Cu(NCMe) 4 PF 6 , 2,6-lutidine, DCM. (c) 1. $\mathrm{Me}_{3} \mathrm{OBF}_{4} ; 2$. $\mathrm{NH}_{4} \mathrm{PF}_{6}, \mathrm{H}_{2} \mathrm{O} / \mathrm{DCM}$. (d) $\mathrm{CF}_{3} \mathrm{CO}_{2} \mathrm{H}, \mathrm{NH}_{4} \mathrm{PF}_{6}, \mathrm{H}_{2} \mathrm{O} / \mathrm{DCM}$.

(a)

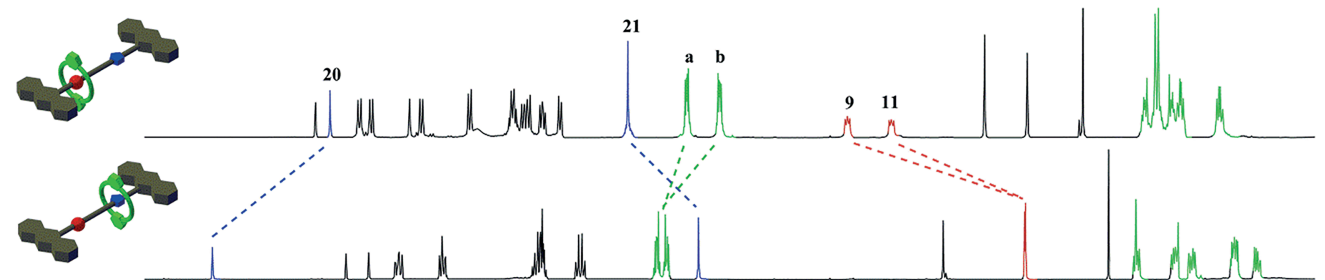

(c)

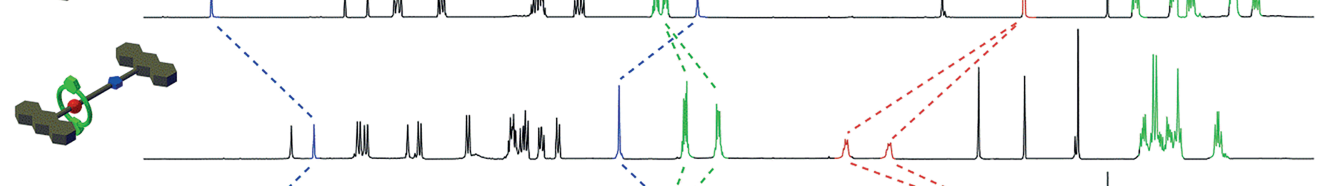

(d)

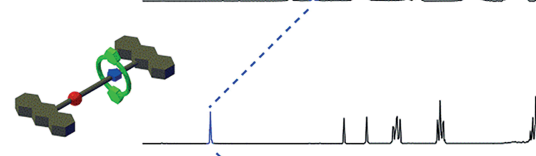

(e)

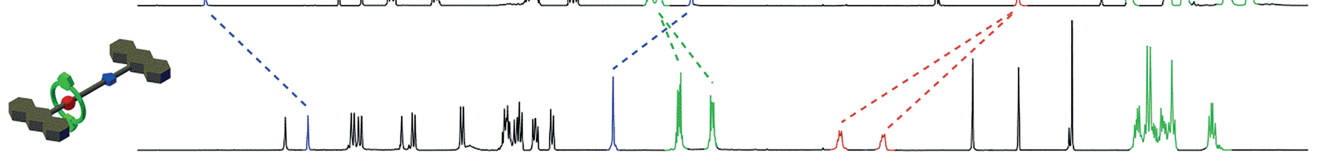

$\begin{array}{llllllllllllllllllllllllllllllllllllllllll}9.8 & 9.6 & 9.4 & 9.2 & 9.0 & 8.8 & 8.6 & 8.4 & 8.2 & 8.0 & 7.8 & 7.6 & 7.4 & 7.2 & 7.0 & 6.8 & 6.6 & 6.4 & 6.2 & 6.0 & 5.8 & 5.6 & 5.4 & 5.2 & 5.0 & 4.8 & 4.6 & 4.4 & 4.2 & 4.0 & 3.8 & 3.6 & 3.4 & 1.2 & 3.0\end{array}$

Figure 3. ${ }^{1} \mathrm{H}$ NMR spectra (500 MHz, [D $]$ acetone) of a [2]rotaxane switched twice: (a) 1- $\mathrm{H}-\mathrm{PF}_{6}$, (b) 1- $\mathrm{PF}_{6},(\mathrm{c}) \mathbf{1}-\mathrm{H}_{-} \mathrm{PF}_{6}-\mathrm{CF}_{3} \mathrm{CO}_{2},(\mathrm{~d}) \mathbf{1 -}$ $\mathrm{PF}_{6}$, (e) $1-\mathrm{H}-\mathrm{PF}_{6}-\mathrm{CF}_{3} \mathrm{CO}_{2}$. 

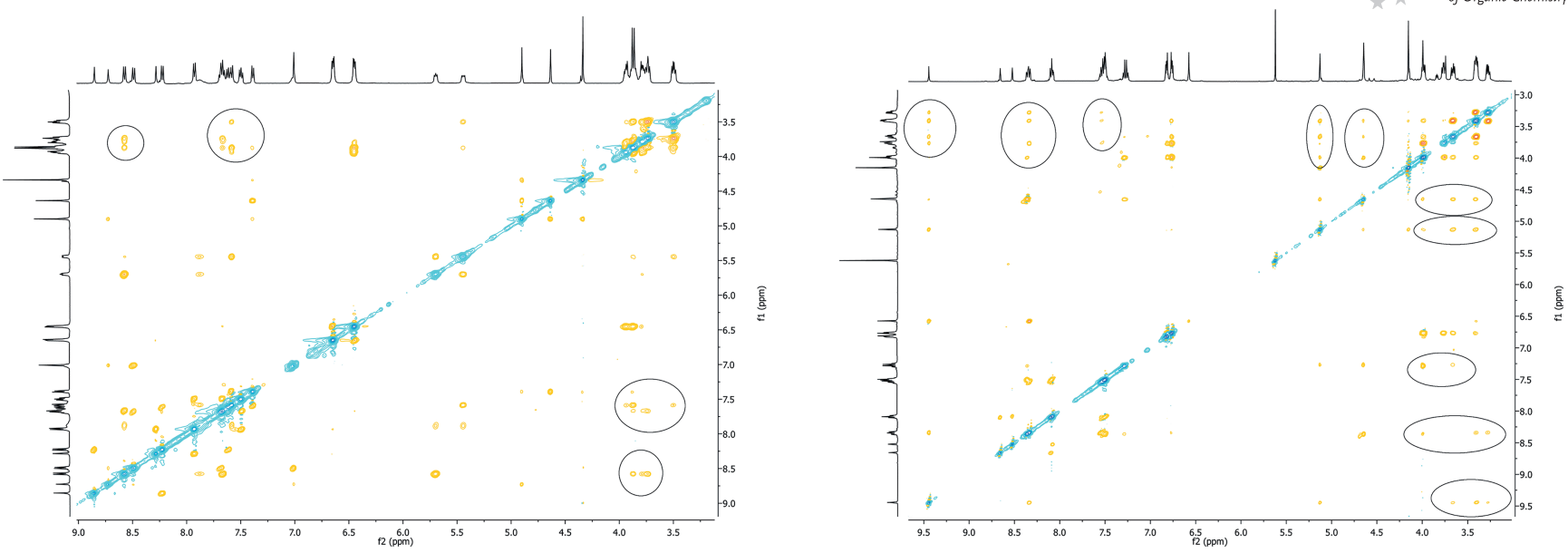

Figure 4. NOESY spectra $\left(500 \mathrm{MHz},\left[\mathrm{D}_{6}\right]\right.$ acetone $)$ of $1-\mathrm{H}_{-} \mathrm{PF}_{6}$ and $\mathbf{1}-\mathrm{PF}_{6}$. The circles depict the NOE correlations between the axle and macrocycle mechanically bonded.
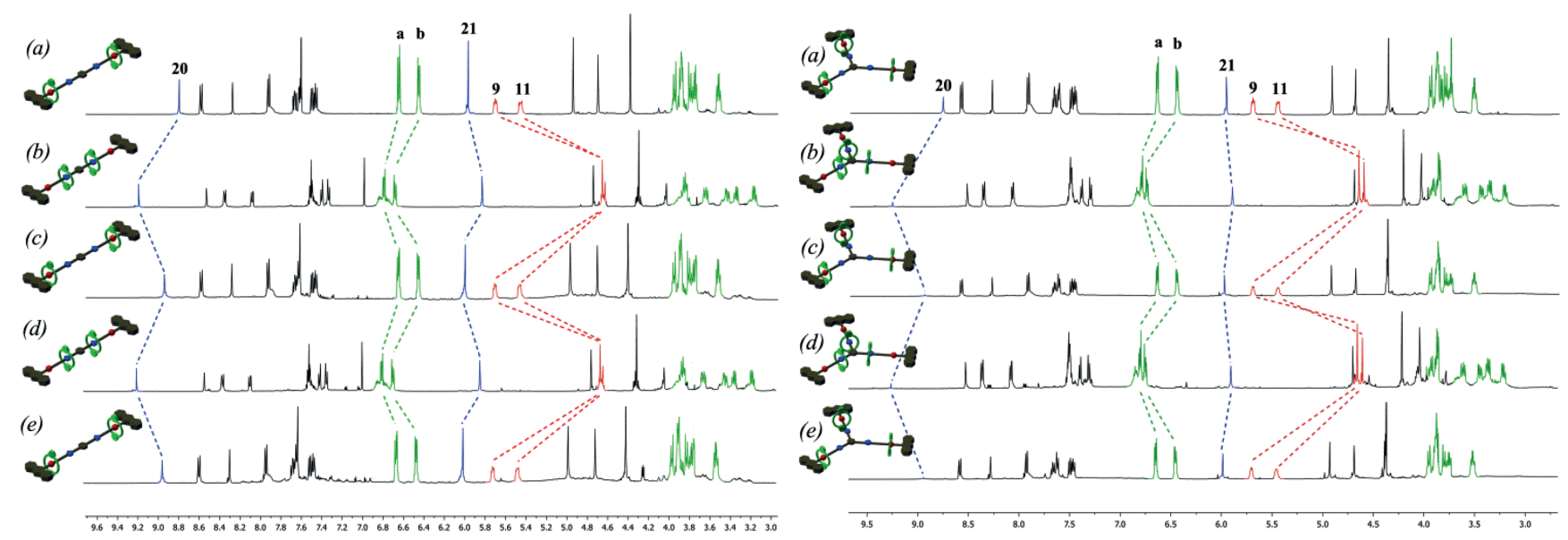

Figure 5. ${ }^{1} \mathrm{H}$ NMR spectra (500 MHz, [D $]$ ]acetone) of [3]rotaxane switched twice (left): (a) 2-H-PF, , (b) 2-PF

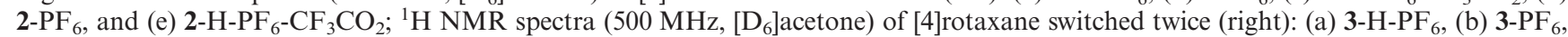
(c) $3-\mathrm{H}-\mathrm{PF}_{6}-\mathrm{CF}_{3} \mathrm{CO}_{2}$, (d) $3-\mathrm{PF}_{6}$, (e) $3 \mathrm{H}-\mathrm{PF}_{6}-\mathrm{CF}_{3} \mathrm{CO}_{2}$.

\section{Catalytic Behavior}

Once the control over the position of the macrocycles was observed in all of the molecular shuttles, two sets of catalytic tests were performed. Both were Michael-type reactions, which can be catalyzed by secondary amines through iminium activation to yield an adduct after $\mathrm{C}-\mathrm{S}$ or $\mathrm{C}-\mathrm{C}$ bond formation.

For regular ammonium-triazolinium switchable organocatalysts, the procedure works as follows: if the catalytic center is covered by the macrocycle, no reaction should be observed at all because that center is blocked, and the reactants are not allowed to access the site (Scheme 3, left), but catalysis occurs if the catalytic center is exposed (Scheme 3, right).

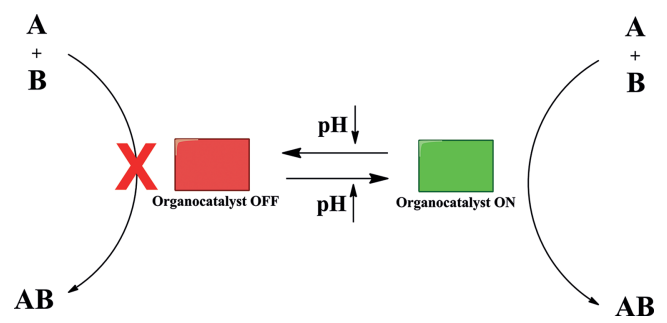

Scheme 3. Typical operation of an organocatalyst switched by $\mathrm{pH}$ changes.

The first reaction between trans-cinnamaldehyde and $1 \mathrm{H}, 1 \mathrm{H}, 2 \mathrm{H}, 2 \mathrm{H}$-perfluorodecanethiol (Table 1) has already been reported by Leigh et al. ${ }^{[7 a]}$ It was catalyzed by a molecular shuttle, and the rotaxane was switched through wash- 
ing with aqueous $\mathrm{NaOH}$. We performed the same procedure but switched the rotaxanes with the protocol described above (with a near-stoichiometric amount of $\mathrm{NaOH}$ in $\mathrm{D}_{2} \mathrm{O}$ ) to avoid possible rate enhancement owing to deprotonation of the thiol by an excess of sodium hydroxide (Table 1).

Table 1. Results observed in the first reaction. ${ }^{[a]}$

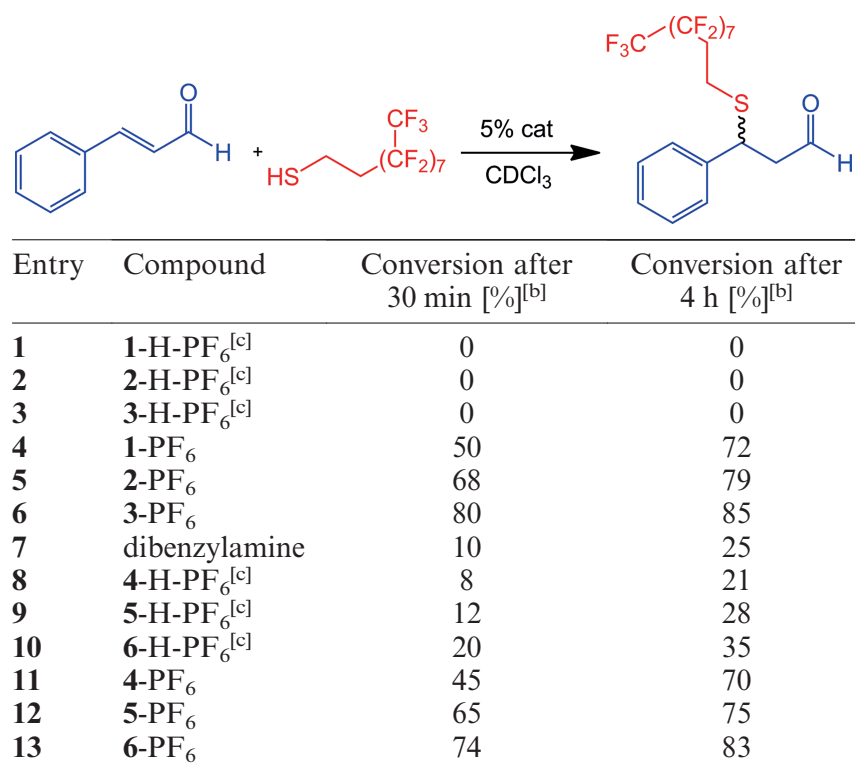

[a] Summarized conditions for all entries: trans-cinnamaldehyde (0.14 mmol), $1 H, 1 H, 2 H, 2 H$-perfluorodecanethiol $(0.168 \mathrm{mmol})$, catalyst $(5 \mathrm{~mol}-\%), \mathrm{CDCl}_{3}(1.4 \mathrm{~mL})$, and $\mathrm{NaOH}$ (1.2 equiv. for each catalytic site). [b] Determined by integration of the $\mathrm{CH}_{2}$ signals in the ${ }^{1} \mathrm{H}$ NMR spectra. [c] These compounds were not treated with $\mathrm{NaOH}$.

As expected, with the shuttles in the inactive position (Figure 6, d), that is, with the ether attached to the ammonium station, no reaction was observed. On the other hand, with the ether switched to the triazole position, the catalytic site was exposed, and the signals corresponding to the adduct appeared (Figure 6, e). All of the shuttles followed the behavior described above. This experiment was performed with non-interlocked threads in the protonated and deprotonated states for comparison.

Thus, shuttles in the inactive position cannot catalyze the reaction, as shown clearly in Table 1, Entries 1-3. However, if the shuttle is switched (Table 1, Entries 4-6), the reaction reaches a conversion of $85 \%$ for the best rotaxane, $3-\mathrm{PF}_{6}$. Interestingly, the initial rate seems to be very high and then decreases until the maximum conversion is achieved. The double rotaxane 2- $\mathrm{PF}_{6}$ (Table 1, Entry 5) is better catalyst than the single rotaxane $1-\mathrm{PF}_{6}$, and the triple rotaxane 3$\mathrm{PF}_{6}$ is the best catalyst (Table 1, Entry 6).

For the non-interlocked threads, we found the same trend as that for 4-PF $6,5-\mathrm{PF}_{6}$, and 6- $\mathrm{PF}_{6}$ (Table 1, Entries 11-13), which are the switched counterparts of the active rotaxanes with dibenzylamine catalytic sites. Each conversion is similar to the initial conversion observed for the respective rotaxane. However, the behavior of $4-\mathrm{H}_{-}-\mathrm{PF}_{6}$, 5- $-\mathrm{H}_{-} \mathrm{PF}_{6}$, and 6-H-PF ${ }_{6}$ is very different (Table 1 , Entries $8-$

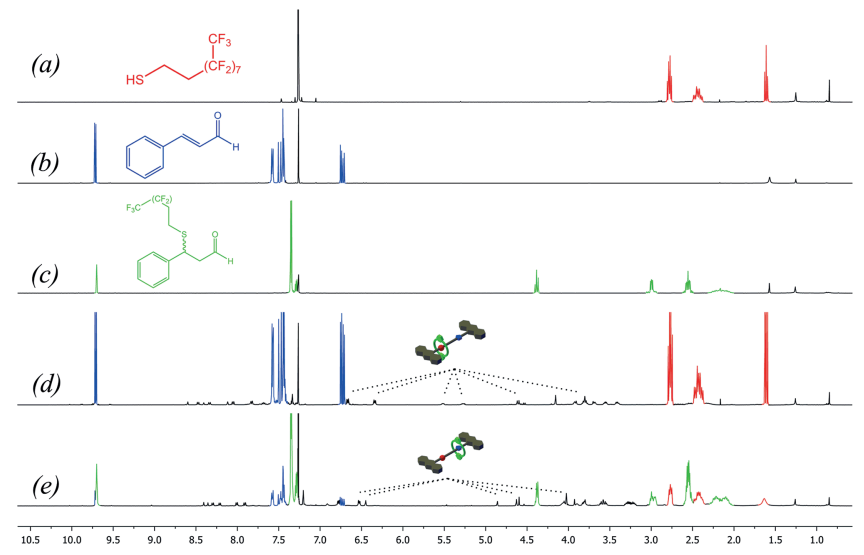

Figure 6. ${ }^{1} \mathrm{H} \mathrm{NMR}$ spectra $\left(500 \mathrm{MHz}, \mathrm{CDCl}_{3}\right)$ for the first reaction with $1-\mathrm{H}_{-} \mathrm{PF}_{6}$ as the catalyst: (a) $1 \mathrm{H}, 1 \mathrm{H}, 2 \mathrm{H}, 2 \mathrm{H}$-perfluorodecanethiol, (b) trans-cinnamaldehyde, (c) pure adduct, (d) reaction mixture with $1-\mathrm{H}_{-}-\mathrm{PF}_{6}$ inactive, and (e) reaction mixture with $1-\mathrm{PF}_{6}$ active at maximum conversion.

10). These compounds are the counterparts of inactive rotaxanes and, therefore, have ammonium salts as catalytic centers. Even with the catalysts in this state, the reaction occurred, in contrast to the behavior observed for rotaxanes in the inactive position (Table 1, Entries 1-3). This evidence shows the importance of the encapsulation of the catalytic center because non-interlocked threads can still catalyze the reaction even in the protonated state; however, if this ammonium salt is covered by the macrocycle, the shuttle cannot perform the catalysis, and the reaction is fully stopped.

Nevertheless, all of these dumbbell-shaped molecules are generally better catalysts than dibenzylamine under the same conditions (Table 1, Entry 7); therefore, their structure enhances the catalytic behavior at their active sites.

A similar protocol was applied for the second reaction, in which the nucleophile was diethyl malonate (Table 2). The reaction did not proceeded well in chlorinated solvents; therefore, we had to switch to a solvent in which the reaction occurred and the rotaxanes were soluble. The best choice was deuterated acetonitrile.

In this case, total conversion could be achieved with 3$\mathrm{PF}_{6}$ in the presence of excess aldehyde, but the reaction times are notably longer at room temperature. Again, if the shuttles are protonated, encapsulation prevents catalysis (Figure 7, d). If the shuttles are switched, catalysis occurs (Figure 7, e).

No catalysis occurred for the shuttles with the ammonium salt covered by the macrocycle (Table 2, Entries 1-3). The initial conversions for the switched shuttles (Table 2, Entries 4-6) resemble the same pattern found for the first reaction, and the best performance was observed for the triple [4]rotaxane 3-PF 6 . The corresponding non-interlocked threads (Table 2, Entries 11-13) behave in a similar way.

Once more, the protonated threads catalyzed the reaction (Table 2, Entries 8-10), and similar conversions were observed for the nonprotonated threads; therefore, these rotaxanes are very good on/off systems. 
Table 2. Results observed in the second reaction. ${ }^{[a]}$

\begin{tabular}{|c|c|c|c|}
\hline Entry & Compound & $\begin{array}{l}\text { Conversion after } \\
30 \mathrm{~min}[\%]^{[\mathrm{b}]}\end{array}$ & $\begin{array}{c}\text { Conversion after } \\
24 \mathrm{~h}^{[\mathrm{b}]}\end{array}$ \\
\hline 1 & $1-\mathrm{H}-\mathrm{PF}_{6}{ }^{[\mathrm{c}]}$ & 0 & 0 \\
\hline 2 & $2-\mathrm{H}-\mathrm{PF}_{6}{ }^{[\mathrm{c}]}$ & 0 & 0 \\
\hline 3 & $3-\mathrm{H}-\mathrm{PF}_{6}{ }^{[\mathrm{c}]}$ & 0 & 0 \\
\hline 4 & $1-\mathrm{PF}_{6}$ & 5 & 68 \\
\hline 5 & $2-\mathrm{PF}_{6}$ & 13 & 80 \\
\hline 6 & $3-\mathrm{PF}_{6}$ & 20 & 100 \\
\hline 7 & dibenzylamine & 12 & 65 \\
\hline 8 & $4-\mathrm{H}-\mathrm{PF}_{6}{ }^{[\mathrm{c}]}$ & 5 & 32 \\
\hline 9 & $5-\mathrm{H}-\mathrm{PF}_{6}{ }^{[\mathrm{c}]}$ & 14 & 45 \\
\hline 10 & 6-H-PF ${ }_{6}^{[c]}$ & 19 & 80 \\
\hline 11 & $4-\mathrm{PF}_{6}$ & 6 & 65 \\
\hline 12 & $5-\mathrm{PF}_{6}$ & 15 & 75 \\
\hline 13 & $6-\mathrm{PF}_{6}$ & 22 & 100 \\
\hline
\end{tabular}

[a] Summarized conditions for all entries: trans-cinnamaldehyde $(0.28 \mathrm{mmol})$, diethyl malonate $(0.14 \mathrm{mmol})$, catalyst $(10 \mathrm{~mol}-\%)$, $\mathrm{CD}_{3} \mathrm{CN}(1.4 \mathrm{~mL})$, and $\mathrm{NaOH}$ (1.2 equiv. for each catalytic site). [b] Determined by integration of the aldehyde signals in the ${ }^{1} \mathrm{H}$ NMR spectra. [c] These compounds were not treated with $\mathrm{NaOH}$.

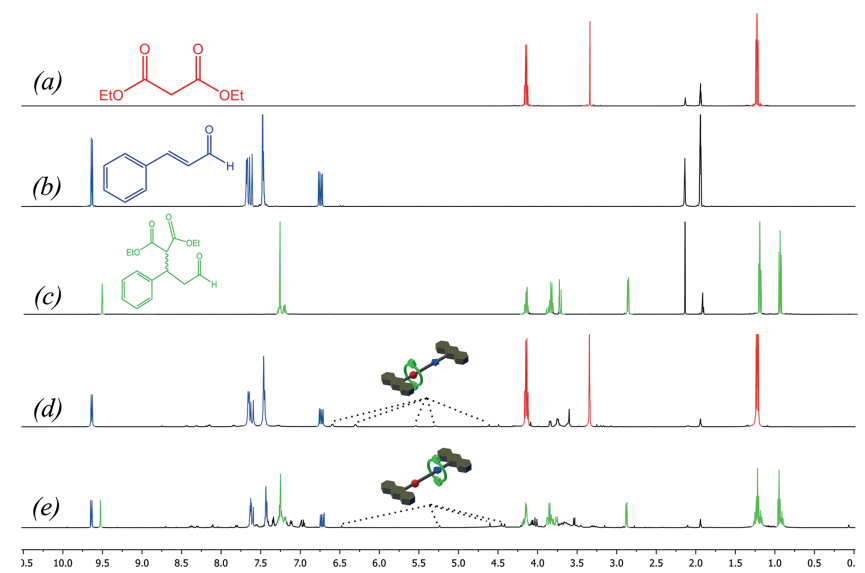

Figure 7. ${ }^{1} \mathrm{H}$ NMR spectra $\left(500 \mathrm{MHz}, \mathrm{CD}_{3} \mathrm{CN}\right)$ for the second reaction: (a) diethyl malonate, (b) trans-cinnamaldehyde, (c) pure adduct, (d) reaction mixture with $1-\mathrm{H}-\mathrm{PF}_{6}$ inactive, and (e) reaction mixture with $1-\mathrm{PF}_{6}$ active at maximum conversion.

\section{Conclusions}

A set of three pH-based molecular shuttles bearing ammonium and triazolinium groups as stations for threaded macrocycles have been prepared along with their non-interlocked analogues, and their abilities to perform switchable organocatalysis have been tested with two different Michael-type reactions.

The results provide very interesting insights: firstly, the switching process can be performed in situ to avoid separation procedures. Secondly, phenylene moieties or triply substituted benzene rings are sufficient links between axles in big shuttles, as all of the macrocyclic rings can be accom- modated in a very crowded situation. Regarding the catalytic behavior of the shuttles, the importance of covering the catalytic site is evident because catalysis is very effectively prevented if the macrocycle is situated at the ammonium station. Additionally, all of the rotaxanes perform better than dibenzylamine under the same conditions. Finally, we found that increasing the number of catalytic centers on the rotaxane is a decisive factor for the improvement of their performance.

Nevertheless, the number of sites is not the only factor to be considered, and other factors must be taken into account, for instance, the flexibility of the rotaxanes and their solubility are the most important.

These results may open a new route for the synthesis of better-designed multivalent molecular shuttles to enhance further their performance as on/off catalysts.

\section{Experimental Section}

Methyl 4-\{I(Anthracen-9-ylmethyl)amino|methyl\}benzoate (R1): Methyl-4-(aminomethyl)benzoate $(0.41 \mathrm{~g}, 2.5 \mathrm{mmol})$ was mixed with 9 -anthracenecarbaldehyde $(0.52 \mathrm{~g}, 2.5 \mathrm{mmol})$ in a $10 \mathrm{~mL}$ vial specially adapted for the microwave reactor. The mixture was heated at $140{ }^{\circ} \mathrm{C}$ for $15 \mathrm{~min}$ with vigorous stirring. After the mixture cooled to room temperature, all of the water drops that formed during the reaction were removed manually to furnish a bright red crude product at the bottom of the vial (the imine). This protocol was repeated six times, and all of the crude products were combined $(6.5 \mathrm{~g}, 18.4 \mathrm{mmol})$ and immediately dissolved in hot $\mathrm{MeOH}$ $(250 \mathrm{~mL}) . \mathrm{NaBH}_{4}(2.09 \mathrm{~g}, 55 \mathrm{mmol})$ was added carefully portionwise, and the mixture was heated under reflux for $20 \mathrm{~h}$. Then, the methanol was removed under vacuum, water $(300 \mathrm{~mL})$ was added, and the solution was extracted with dichloromethane (DCM; $4 \times 200 \mathrm{~mL}$ ). All organic phases were combined, dried with anhydrous $\mathrm{MgSO}_{4}$, filtered, and concentrated in vacuo to afford a crude product, which was purified by column chromatography $\left(\mathrm{SiO}_{2}\right.$ gel, hexane/AcOEt 3:1) to finally afford a yellow solid with spectroscopic data that agreed with those reported previously $(5.55 \mathrm{~g}, 85 \%$ yield after two steps). ${ }^{[17]}$

(4-\{[(Anthracen-9-ylmethyl)amino|methyl\}phenyl)methanol (R2): R1 $(5.33 \mathrm{~g}, 15 \mathrm{mmol})$ was dissolved in dry tetrahydrofuran (THF; $160 \mathrm{~mL}$ ) under a $\mathrm{N}_{2}$ atmosphere. The solution was degassed and cooled to $-78{ }^{\circ} \mathrm{C}$. Lithium aluminium hydride $(15 \mathrm{~mL}$ of a $1 \mathrm{M}$ solution in THF, $15 \mathrm{mmol}$ ) was added dropwise. The mixture was allowed to reach room temperature and then stirred for $3 \mathrm{~h}$. Water $(250 \mathrm{~mL})$ was added, and the solution was extracted with DCM $(3 \times 400 \mathrm{~mL})$. All of organic phases were combined, dried with anhydrous $\mathrm{MgSO}_{4}$, filtered, and concentrated under vacuum to afford an orange oil $\left(4.67 \mathrm{~g}, 95 \%\right.$ yield) that eventually solidified. ${ }^{1} \mathrm{H}$ NMR (400 MHz, $\left.\mathrm{CDCl}_{3}\right): \delta=8.40\left(\mathrm{~s}, 1 \mathrm{H}, \mathrm{H}^{1}\right), 8.22(\mathrm{~d}, J=8.6 \mathrm{~Hz}$, $\left.2 \mathrm{H}, \mathrm{H}^{6}\right), 8.00\left(\mathrm{~d}, J=7.9 \mathrm{~Hz}, 2 \mathrm{H}, \mathrm{H}^{3}\right), 7.54-7.43\left(\mathrm{~m}, 4 \mathrm{H}, \mathrm{H}^{4}\right.$, $\left.\mathrm{H}^{5}\right), 7.41$ and $7.35\left(\mathrm{AB}\right.$ system, $\left.4 \mathrm{H}, \mathrm{H}^{12}, \mathrm{H}^{13}\right), 4.69\left(\mathrm{~s}, 2 \mathrm{H}, \mathrm{H}^{15}\right)$, $4.68\left(\mathrm{~s}, 2 \mathrm{H}, \mathrm{H}^{9}\right), 4.02\left(\mathrm{~s}, 2 \mathrm{H}, \mathrm{H}^{10}\right) \mathrm{ppm} .{ }^{13} \mathrm{C} \mathrm{NMR}(100 \mathrm{MHz}$, $\left.\mathrm{CDCl}_{3}\right): \delta=139.95\left(\mathrm{C}^{14}\right), 139.81\left(\mathrm{C}^{11}\right), 131.68\left(\mathrm{C}^{2}\right), 131.59\left(\mathrm{C}^{8}\right)$, $130.46\left(\mathrm{C}^{7}\right), 129.27\left(\mathrm{C}^{3}\right), 128.70\left(\mathrm{C}^{12}\right), 127.41\left(\mathrm{C}^{1}\right), 127.30\left(\mathrm{C}^{13}\right)$, $126.21\left(\mathrm{C}^{4}\right), 125.06\left(\mathrm{C}^{5}\right), 124.26\left(\mathrm{C}^{6}\right), 65.30\left(\mathrm{C}^{15}\right), 54.08\left(\mathrm{C}^{10}\right), 45.02$ $\left(\mathrm{C}^{9}\right)$ ppm. MS (ESI+): $m / z=328[\mathrm{M}+\mathrm{H}]^{+}$(calcd. 328.17 for $\mathrm{C}_{23} \mathrm{H}_{22} \mathrm{NO}$ ).

tert-Butyl (Anthracen-9-ylmethyl)[4-(hydroxymethyl)benzyl]carbamate (R3): $\mathbf{R 2}(4.56 \mathrm{~g}, 14 \mathrm{mmol})$, di-tert-butyl dicarbonate 
( $\left.\mathrm{Boc}_{2} \mathrm{O} ; 3.04 \mathrm{~g}, 14 \mathrm{mmol}\right)$, and $\mathrm{I}_{2}(0.35 \mathrm{~g}, 1.4 \mathrm{mmol})$ were mixed under a nitrogen atmosphere and dissolved in $\mathrm{MeOH}(20 \mathrm{~mL})$. The mixture was stirred for $6 \mathrm{~h}$ at room temperature. Then, the solvent was evaporated, and the residue was redissolved in DCM $(200 \mathrm{~mL})$ and washed with an aqueous solution of $\mathrm{Na}_{2} \mathrm{~S}_{2} \mathrm{O}_{3}(5 \%, 150 \mathrm{~mL})$. The organic phase was washed again with $20 \mathrm{~mL}$ of satd. aqueous $\mathrm{NaHCO}_{3}$, dried with anhydrous $\mathrm{MgSO}_{4}$, filtered, and concentrated under reduced pressure to afford a brown oil, which was subjected to column chromatography $\left(\mathrm{SiO}_{2}\right.$ gel, hexane/AcOEt 2:1) to afford R3 as a pale yellow solid (5.39 g, 90\% yield). ${ }^{1} \mathrm{H}$ NMR (400 MHz, $\left.\mathrm{CDCl}_{3}\right): \delta=8.41\left(\mathrm{~s}, 1 \mathrm{H}, \mathrm{H}^{1}\right), 8.20\left(\mathrm{br}, 2 \mathrm{H}, \mathrm{H}^{6}\right), 7.98(\mathrm{~d}, J=$ $\left.7.8 \mathrm{~Hz}, 2 \mathrm{H}, \mathrm{H}^{3}\right), 7.52-7.35\left(\mathrm{~m}, 4 \mathrm{H}, \mathrm{H}^{4}, \mathrm{H}^{5}\right), 7.19$ (br, $\left.2 \mathrm{H}, \mathrm{H}^{16}\right)$, 6.88 (br, $\left.2 \mathrm{H}, \mathrm{H}^{15}\right), 5.56\left(\mathrm{~s}, 2 \mathrm{H}, \mathrm{H}^{9}\right), 4.65$ (s, $\left.2 \mathrm{H}, \mathrm{H}^{18}\right), 4.04$ (s, 2 $\mathrm{H}, \mathrm{H}^{13}$ ), 1.73 (br, $3 \mathrm{H}, \mathrm{H}^{12}$ ), 1.53 (br, $6 \mathrm{H}, \mathrm{H}^{12}$ ) ppm. ${ }^{13} \mathrm{C} \mathrm{NMR}$ $\left(100 \mathrm{MHz}, \mathrm{CDCl}_{3}\right): \delta=155.93\left(\mathrm{C}^{10}\right), 139.42\left(\mathrm{C}^{17}\right), 137.79\left(\mathrm{C}^{14}\right)$, $131.39\left(C^{8}\right), 131.35\left(C^{2}, C^{7}\right), 129.07\left(C^{3}\right), 128.25\left(C^{1}\right), 126.95\left(C^{15}\right)$, $126.87\left(\mathrm{C}^{16}\right), 126.12\left(\mathrm{C}^{4}\right), 124.96\left(\mathrm{C}^{5}\right), 124.43\left(\mathrm{C}^{6}\right), 80.33\left(\mathrm{C}^{11}\right)$, $65.05\left(\mathrm{C}^{18}\right), 47.55\left(\mathrm{C}^{13}\right), 40.92\left(\mathrm{C}^{9}\right), 28.48\left(\mathrm{C}^{12}\right)$ ppm. MS (ESI+): $m / z=428[\mathrm{M}+\mathrm{H}]^{+}$(calcd. 428.22 for $\mathrm{C}_{28} \mathrm{H}_{30} \mathrm{NO}_{3}$ ).

tert-Butyl (Anthracen-9-ylmethyl)\{4-I(prop-2-yn-1-yloxy)methyl]benzyl carbamate (R4): $\mathbf{R 3}(1.07 \mathrm{~g}, 2.5 \mathrm{mmol})$ and $\mathrm{NaH}(0.42 \mathrm{~g}$, $14 \mathrm{mmol})$ were placed in a Schlenk flask under a nitrogen atmosphere. Dry and degassed THF $(50 \mathrm{~mL})$ was added, and the mixture was heated under reflux for $90 \mathrm{~min}$. Then, the mixture was allowed to reach room temperature, and a solution of propargyl bromide in toluene $(80 \mathrm{wt} .-\%, 1.88 \mathrm{~mL})$ was added. This mixture was heated under reflux overnight. The reaction was then allowed to reach room temperature and concentrated under reduced pressure. DCM $(100 \mathrm{~mL})$ and water $(100 \mathrm{~mL})$ were added carefully, and the crude product was extracted with DCM $(3 \times 100 \mathrm{~mL})$. The combined organic phases were dried with anhydrous $\mathrm{MgSO}_{4}$, filtered, and concentrated to yield a brown oil, which eventually solidified and was used in the next step without further purification (1.07 g, 92\% yield). ${ }^{1} \mathrm{H}$ NMR (400 MHz, $\left.\mathrm{CDCl}_{3}\right): \delta=8.43(\mathrm{~s}, 1 \mathrm{H}$, $\left.\mathrm{H}^{1}\right), 8.20\left(\mathrm{~s}, 2 \mathrm{H}, \mathrm{H}^{6}\right), 8.00\left(\mathrm{~d}, J=7.4 \mathrm{~Hz}, 2 \mathrm{H}, \mathrm{H}^{3}\right), 7.51-7.37(\mathrm{~m}$, $\left.4 \mathrm{H}, \mathrm{H}^{4}, \mathrm{H}^{5}\right), 7.19\left(\mathrm{br}, 2 \mathrm{H}, \mathrm{H}^{16}\right), 6.89\left(\mathrm{br}, 2 \mathrm{H}, \mathrm{H}^{15}\right), 5.57$ (s, $2 \mathrm{H}$, $\left.\mathrm{H}^{9}\right), 4.58\left(\mathrm{~s}, 2 \mathrm{H}, \mathrm{H}^{18}\right), 4.17$ (s, $\left.2 \mathrm{H}, \mathrm{H}^{19}\right), 4.05$ (s, $\left.2 \mathrm{H}, \mathrm{H}^{13}\right), 2.51$ (t, $\left.J=2.3 \mathrm{~Hz}, 1 \mathrm{H}, \mathrm{H}^{21}\right), 1.73\left(\mathrm{br}, 3 \mathrm{H}, \mathrm{H}^{12^{\prime}}\right), 1.52\left(\mathrm{br}, 6 \mathrm{H}, \mathrm{H}^{12}\right.$ ) ppm. ${ }^{13} \mathrm{C}$ NMR $\left(100 \mathrm{MHz}, \mathrm{CDCl}_{3}\right): \delta=155.93\left(\mathrm{C}^{10}\right), 138.19\left(\mathrm{C}^{14}\right)$, $135.67\left(\mathrm{C}^{17}\right), 131.39\left(\mathrm{C}^{8}\right), 131.33\left(\mathrm{C}^{2}, \mathrm{C}^{7}\right), 129.07\left(\mathrm{C}^{3}\right), 128.22\left(\mathrm{C}^{1}\right)$, $128.04\left(\mathrm{C}^{16}\right), 126.85\left(\mathrm{C}^{15}\right), 126.13\left(\mathrm{C}^{4}\right), 124.96\left(\mathrm{C}^{5}\right), 124.44\left(\mathrm{C}^{6}\right)$, $79.70\left(\mathrm{C}^{20}\right), 77.24\left(\mathrm{C}^{11}\right), 74.63\left(\mathrm{C}^{21}\right), 71.29\left(\mathrm{C}^{18}\right), 56.97\left(\mathrm{C}^{19}\right), 47.54$ $\left(\mathrm{C}^{13}\right), 40.90\left(\mathrm{C}^{9}\right), 29.70\left(\mathrm{C}^{12}\right) \mathrm{ppm}$. MS (ESI+): $\mathrm{m} / \mathrm{z}=466[\mathrm{M}+$ $\mathrm{H}]^{+}$(calcd. 466.23 for $\mathrm{C}_{31} \mathrm{H}_{32} \mathrm{NO}_{3}$ ).

1-(Anthracen-9-yl)- $N$-\{4-I(prop-2-yn-1-yloxy)methyl]benzyl $\}$ methanaminium Hexafluorophosphate $\left(\mathbf{R 5}-\mathbf{H}_{-}-\mathbf{P F}_{\mathbf{6}}\right)$ : Trifluoroacetic acid $(10 \mathrm{~mL})$ was added to a solution of $\mathbf{R} 4(0.93 \mathrm{~g}, 2 \mathrm{mmol})$ in chloroform $(100 \mathrm{~mL})$, and the mixture was stirred overnight at room temperature. The solvent was then removed under vacuum, and the resulting crude product was redissolved in DCM $(20 \mathrm{~mL})$ and an aqueous solution $(10 \mathrm{~mL})$ of ammonium hexafluorophosphate $(1.63 \mathrm{~g}, 10 \mathrm{mmol})$ in MiliQ water. The biphasic mixture was stirred vigorously for $10 \mathrm{~min}$ and extracted in a separating funnel. Hexane $(20 \mathrm{~mL})$ was added to the organic phase, and the mixture was stored at $-20^{\circ} \mathrm{C}$ for $12 \mathrm{~h}$. The brown crystals were collected by filtration and dried under vacuum to afford $\mathbf{R 5}$ as a dark brownish solid $\left(0.57 \mathrm{~g}, 78 \%\right.$ yield). ${ }^{1} \mathrm{H}$ NMR (400 MHz, [D 6 ]acetone): $\delta=$ $8.79\left(\mathrm{~s}, 1 \mathrm{H}, \mathrm{H}^{1}\right), 8.31\left(\mathrm{~d}, J=8.7 \mathrm{~Hz}, 2 \mathrm{H}, \mathrm{H}^{6}\right), 8.19(\mathrm{~d}, J=8.2 \mathrm{~Hz}$, $\left.2 \mathrm{H}, \mathrm{H}^{3}\right), 7.74\left(\mathrm{~d}, J=8.1 \mathrm{~Hz}, 2 \mathrm{H}, \mathrm{H}^{12}\right), 7.68-7.56$ (AB System, 4 $\left.\mathrm{H}, \mathrm{H}^{4}, \mathrm{H}^{5}\right), 7.52\left(\mathrm{~d}, J=8.2 \mathrm{~Hz}, 2 \mathrm{H}, \mathrm{H}^{13}\right), 5.69\left(\mathrm{~s}, 2 \mathrm{H}, \mathrm{H}^{9}\right), 5.02$ (s, $\left.2 \mathrm{H}, \mathrm{H}^{10}\right), 4.66\left(\mathrm{~s}, 2 \mathrm{H}, \mathrm{H}^{15}\right), 4.25\left(\mathrm{~d}, J=2.4 \mathrm{~Hz}, 2 \mathrm{H}, \mathrm{H}^{16}\right)$, $3.02\left(\mathrm{t}, J=2.4 \mathrm{~Hz}, 1 \mathrm{H}, \mathrm{H}^{18}\right) \mathrm{ppm} .{ }^{13} \mathrm{C} \mathrm{NMR}\left(100 \mathrm{MHz},\left[\mathrm{D}_{6}\right]-\right.$ acetone): $\delta=140.94\left(\mathrm{C}^{14}\right), 132.31\left(\mathrm{C}^{2}\right), 131.90\left(\mathrm{C}^{7}\right), 131.63\left(\mathrm{C}^{1}\right)$,
$131.47\left(\mathrm{C}^{12}\right), 131.10\left(\mathrm{C}^{11}\right), 130.36\left(\mathrm{C}^{3}\right), 129.24\left(\mathrm{C}^{13}\right), 128.43\left(\mathrm{C}^{5}\right)$, $126.45\left(\mathrm{C}^{4}\right), 124.14\left(\mathrm{C}^{6}\right), 122.42\left(\mathrm{C}^{8}\right), 80.56\left(\mathrm{C}^{17}\right), 76.30\left(\mathrm{C}^{18}\right), 71.41$ $\left(\mathrm{C}^{15}\right), 58.02\left(\mathrm{C}^{16}\right), 53.06\left(\mathrm{C}^{10}\right), 44.23\left(\mathrm{C}^{9}\right) \mathrm{ppm}$. MS (ESI+): $\mathrm{m} / \mathrm{z}=$ $366\left[\mathrm{M}-\mathrm{PF}_{6}\right]^{+}$(calcd. 366.19 for $\mathrm{C}_{26} \mathrm{H}_{24} \mathrm{NO}$ ).

General Procedure for Rotaxane Synthesis (Click Reaction) to Give R6-H-PF 6 , R7-H-PF 6 , or R8-H-PF 6 : R5-H-PF 6 , S4, S5, or S6 and $\mathrm{DB} 24 \mathrm{C} 8$ were mixed in suitable proportions under an inert atmosphere and dissolved in dry and degassed DCM. The dark solution was stirred for enough time at room temperature. Then, $\left[\mathrm{Cu}(\mathrm{NCMe})_{4}\right] \mathrm{PF}_{6}$ and 2,6-lutidine were added, and the mixture was stirred at room temperature for 5 or $6 \mathrm{~d}$. After such time, the solvent was removed under vacuum, and the crude product was purified by column chromatography.

General Procedure for Rotaxane Methylation to Give Molecular Shuttles 1-H-PF 6 , 2-H-PF , or 3-H-PF $:$ R6-H-PF, R7-H-PF 6 , or R8- $-\mathrm{H}_{-} \mathrm{PF}_{6}$ and trimethyloxonium tetrafluoroborate were mixed in dry DCM under a $\mathrm{N}_{2}$ atmosphere. The mixture was degassed and stirred at room temperature until the complete dissolution of the tetrafluoroborate salt was observed. The solution was diluted with DCM, and ammonium hexafluorophosphate dissolved in MiliQ water was added. The biphasic mixture was stirred vigorously, and the organic phase was extracted, dried with anhydrous $\mathrm{MgSO}_{4}$, filtered, and concentrated to yield the expected molecular shuttle.

General Procedure for Non-Interlocked-Thread Precursors by Click Reaction to Give T1, T2 or T3: R4, S3, S4, or S5 and [Cu(NCMe) 4 $\mathrm{PF}_{6}$ were placed in a Schlenk flask under a nitrogen atmosphere. Dried and degassed DCM and 2,6-lutidine were added. The mixture was stirred at room temperature for $5 \mathrm{~d}$ and then concentrated to afford a crude product, which was purified by column chromatography.

General Procedure for the Methylation of Non-Interlocked-Thread Precursors to Give T4-PF 6 , T5-PF 6 , or T6- $\mathbf{P F}_{6}$ : Trimethyloxonium tetrafluoroborate was added to a solution of T1, T2, or T3 in dry and degassed DCM. The mixture was stirred at room temperature until the complete dissolution of the tetrafluoroborate salt was observed. The solution was then diluted with DCM, and an aqueous solution of $\mathrm{NH}_{4} \mathrm{PF}_{6}$ in MiliQ water was added. The mixture was stirred vigorously, and the organic phase was extracted, dried with anhydrous $\mathrm{MgSO}_{4}$, filtered, and concentrated under reduced pressure to afford $\mathbf{T} 4-\mathrm{PF}_{6}, \mathbf{T} 5-\mathrm{PF}_{6}$, or T6- $\mathrm{PF}_{6}$ which was used in the next step without further purification.

General Procedure for Boc Deprotection to Obtain Final Non-interlocked Threads $4-\mathrm{H}_{-} \mathrm{PF}_{6}, \mathbf{5}-\mathrm{H}-\mathrm{PF}_{6}$, and $\mathbf{6}-\mathrm{H}_{-}-\mathrm{PF}_{6}$ : Trifluoroacetic acid was added to a solution of $\mathbf{T} 4-\mathbf{P F}_{6}, \mathbf{T} 5-\mathbf{P F}_{6}$, or $\mathbf{T} 6-\mathrm{PF}_{6}$ in chloroform $(10 \mathrm{~mL})$. The dark mixture was stirred at room temperature for $30 \mathrm{~min}$. The solvents were removed, and the resulting black oil was diluted with DCM $(20 \mathrm{~mL})$. Ammonium hexafluorophosphate in MiliQ water $(15 \mathrm{~mL})$ was added, and the biphasic mixture was stirred vigorously for $30 \mathrm{~min}$. Then, both layers were separated, and the organic phase was dried with anhydrous $\mathrm{MgSO}_{4}$, filtered, and concentrated to afford a thick black oil, which was subjected to purification by column chromatography.

General Procedure for Catalytic Reaction 1: trans-Cinnamaldehyde (17.6 $\mu \mathrm{L}, \quad 0.14 \mathrm{mmol}), \quad 1 \mathrm{H}, 1 \mathrm{H}, 2 \mathrm{H}, 2 \mathrm{H}$-perfluorodecanethiol $(48.0 \mu \mathrm{L}, 0.168 \mathrm{mmol})$, and the catalyst $(5 \mathrm{~mol}-\%)$ were mixed in a round-bottom flask. $\mathrm{CDCl}_{3}(1400 \mu \mathrm{L})$ was added as well as the appropriate amount (1.2 equiv. for each catalytic site) of a $1 \mathrm{M}$ solution of $\mathrm{NaOH}$ in $\mathrm{D}_{2} \mathrm{O}$ if necessary. The mixture was stirred vigorously at room temperature. Aliquots were taken, and ${ }^{1} \mathrm{H}$ NMR spectra were recorded to check the reaction progress. Once the reaction was finished, the crude product was subjected immediately to 
purification by column chromatography $\left(\mathrm{SiO}_{2}\right.$ gel, DCM/hexane $3: 2$ ) to yield the expected addition product if the reaction was successful.

General Procedure for Catalytic Reaction 2: trans-Cinnamaldehyde $(35.2 \mu \mathrm{L}, 0.28 \mathrm{mmol})$, diethyl malonate $(21.2 \mu \mathrm{L}, 0.14 \mathrm{mmol})$, and the catalyst $(10 \mathrm{~mol}-\%)$ were mixed in a round-bottom flask. $\mathrm{CD}_{3} \mathrm{CN}(1400 \mu \mathrm{L})$ was added as well as the appropriate amount (1.2 equiv. for each catalytic site) of a $1 \mathrm{M}$ solution of $\mathrm{NaOH}$ in $\mathrm{D}_{2} \mathrm{O}$ if necessary. The mixture was stirred vigorously at room temperature. Aliquots were taken, and ${ }^{1} \mathrm{H}$ NMR spectra were recorded to check the reaction progress. Once the reaction was finished, the crude product was subjected immediately to purification by column chromatography $\left(\mathrm{SiO}_{2}\right.$ gel, hexane/AcOEt,2:1) to yield the expected addition product if the reaction was successful.

Supporting Information (see footnote on the first page of this article): A detailed overview of the complete synthesis, atom numbering, NMR spectra, and other details.

\section{Acknowledgments}

This work was funded by the Spanish Ministerio de Economía y Competitividad (MINECO) (CTQ 2013-41067-P). H. B. acknowledges with thanks the Spanish Ministerio de Educación y Ciencia (MEC) for a grant.

[1] a) P. Thordarson, E. J. Bijsterveld, A. E. Rowan, R. J. Nolte, Nature 2003, 424, 915-918; b) Y. Tachibana, N. Kihara, T. Takata, J. Am. Chem. Soc. 2004, 126, 3438-3439; c) G. Hattori, T. Hori, Y. Miyake, Y. Nishibayashi, J. Am. Chem. Soc. 2007, 129, 12930-12931; d) Y. Li, Y. Feng, Y.-M. He, F. Chen, J. Pan, Q.H. Fan, Tetrahedron Lett. 2008, 49, 2878-2881; e) N. Miyagawa, M. Watanabe, T. Matsuyama, Y. Koyama, T. Moriuchi, T. Hirao, Y. Furusho, T. Takata, Chem. Commun. 2010, 46, 1920-1922; f) Y. Suzaki, K. Shimada, E. Chihara, T. Saito, Y. Tsuchido, K. Osakada, Org. Lett. 2011, 13, 3774-3777; g) D. A. Leigh, V. Marcos, M. R. Wilson, ACS Catal. 2014, 4, 4490-4497.

[2] a) F. Würthner, J. Rebek Jr., Angew. Chem. Int. Ed. Engl. 1995, 34, 446-448; Angew. Chem. 1995, 107, 503; b) H. J. Yoon, J. Kuwabara, J.-H. Kim, C. A. Mirkin, Science 2010, 330, 6669; c) Y. Sohtome, S. Tanaka, K. Takada, T. Yamaguchi, K. Nagasawa, Angew. Chem. Int. Ed. 2010, 49, 9254-9257; d) J. Berná, M. Alajarín, R.-A. Orenes, J. Am. Chem. Soc. 2010, 132, 10741-10747; e) J. Wang, B. L. Feringa, Science 2011, 331, 1429-1432; f) M. Zirngast, E. Pump, A. Leitgeb, J. H. Albering, C. Slugove, Chem. Commun. 2011, 47, 2261-2263; g) O. B. Berryman, A. C. Sather, A. Lledó, J. Rebek Jr., Angew. Chem. Int. Ed. 2011, 50, 9400-9403; h) U. Lüning, Angew. Chem. Int. Ed. 2012, 51, 8163-8165; i) B. M. Neilson, C. W. Bielawski, J. Am. Chem. Soc. 2012, 134, 12693-12699; j) M. Schmittel, S. De, S. Pramanik, Angew. Chem. Int. Ed. 2012, 51, 3832-3836; k) M. Schmittel, S. Pramanik, S. De, Chem. Commun. 2012, 48, 11730-11732; 1) P. Viehmann, S. Hecht, Beilstein J. Org. Chem. 2012, 8, 1825-1830; m) D. Wilson, N. R. Branda, Angew. Chem. Int. Ed. 2012, 51, 5431-5434; n) B. M. Neilson, C. W. Bielawski, Chem. Commun. 2013, 49, 5453-5455; o) B. M. Neilson, C. W. Bielawski, Organometallics 2013, 32, 3121-3128; p) B. M. Neilson, C. W. Bielawski, ACS Catal. 2013, 3, 1874-1885; q) L. Osorio-Planes, C. Rodríguez-Escrich, M. A. Pericás, Org. Lett. 2014, 16, 1704-1707; r) C. M. McGuirk, C. L. Stern, C. A. Mirkin, J. Am. Chem. Soc. 2014, 136, 4689-4696.

[3] a) J. Monod, J.-P. Changeux, F. Jacob, J. Mol. Biol. 1963, 6, 306-329; b) J. A. Hardy, J. A. Wells, Curr. Opin. Struct. Biol. 2004, 14, 706-715; c) E. K. Jaffe, Trends Biochem. Sci. 2005,
30, 490-497; d) T. W. Traut, Allosteric Regulatory Enzymes, Springer, New York, 2008.

[4] A. Erkkilä, I. Majander, P. M. Pihko, Chem. Rev. 2007, 107, 5416-5470.

[5] S. Mukherjee, J. W. Yang, S. Hoffmann, B. List, Chem. Rev. 2007, 107, 5471-5569.

[6] a) I. D. Jurberg, I. Chatterjee, R. Tanert, P. Melchiorre, Chem. Commun. 2013, 49, 4869-4883; b) I. Kumar, P. Ramaraju, N. A. Mir, Org. Biomol. Chem. 2013, 11, 709-716; c) P.-Q. Chen, Y.-C. Xiao, C.-Z. Yue, Y.-C. Chen, Org. Chem. Front. 2014, 1, 490-493.

[7] a) V. Blanco, A. Carlone, K. D. Hänni, D. A. Leigh, B. Lewandowski, Angew. Chem. Int. Ed. 2012, 51, 5166-5169; b) V. Blanco, D. A. Leigh, V. Marcos, J. A. Morales-Serna, A. L. Nussbaumer, J. Am. Chem. Soc. 2014, 136, 4905-4908; c) V. Blanco, D. A. Leigh, U. Lewandowska, B. Lewandowski, V. Marcos, J. Am. Chem. Soc. 2014, 136, 15775-15780; d) J. Beswick, V. Blanco, G. De Bo, D. A. Leigh, U. Lewandowska, B. Lewandowski, K. Mishiro, Chem. Sci. 2015, 6, 140-143.

[8] a) J. W. J. Knapen, A. W. van der Made, J. C. P. de Wilde, W. N. M. van Leeuwan, P. Wijkens, D. M. Grove, G. van Koten, Nature 1994, 372, 659-663; b) R. J. Puddephatt, S. Achar, Angew. Chem. Int. Ed. Engl. 1994, 33, 847-849; Angew. Chem. 1994, 106, 895; c) H. Brunner, J. Organomet. Chem. 1995, 500, 39-46; d) R. Breinbauer, E. N. Jacobsen, Angew. Chem. Int. Ed. 2000, 39, 3604-3607; Angew. Chem. 2000, 112, 3750; e) R. Breinbauer, E. N. Jacobsen, Angew. Chem. Int. Ed. 2000, 39, 3604-3607; Angew. Chem. 2000, 112, 3750; f) A. Dahan, M. Portnoy, Org. Lett. 2003, 5, 1197-1200; g) J. Lemo, K. Heuzé, D. Astruc, Chem. Commun. 2007, 4351-4353; h) H. Hattori, K. Fujita, T. Muraki, A. Sakaba, Tetrahedron Lett. 2007, 48, 6817-6820; i) J. Yu, T. V. RajanBabu, J. R. Parquette, J. Am. Chem. Soc. 2008, 130, 7845-7847; j) J. Kassube, H. Wadepohl, L. H. Gade, Adv. Synth. Catal. 2009, 351, 607-616; k) M. Janssen, C. Müller, D. Vogt, Adv. Synth. Catal. 2009, 351, 313-318; 1) G. Jayamurugan, N. Jayaraman, Adv. Synth. Catal. 2009, 351, 2379-2390; m) D. E. Bergbreiter, J. Tian, C. Hongfa, Chem. Rev. 2009, 109, 530-582; n) J. Dennis, M. Snelders, G. van Koten, R. J. M. Klein Gebbink, J. Am. Chem. Soc. 2009, 131,11407-11416; o) T. Fujihara, S. Yoshida, J. Terao, Y. Tsuji, Org. Lett. 2009, 11, 2121-2124; p) J. Keilitz, R. Haag, Eur. J. Org. Chem. 2009, 3272-3278; q) P. Servin, R. Laurent, L. Gonsalvi, M. Tristany, M. Peruzzini, J.-P. Majoral, A.-M. Caminade, Dalton Trans. 2009, 4432-4434; r) Y. Li, Y.-M. He, Z.-W. Li, F. Zhang, Q.-H. Fan, Org. Biomol. Chem. 2009, 7, 1890$1895 ;$ s) G. Jayamurugan, C. P. Umesh, N. Jayaraman, J. Mol. Catal. A 2009, 307, 142-148; t) D. Astruc, E. Boisselier, C. Ornelas, Chem. Rev. 2010, 110, 1857-1959; u) K. Fujita, T. Ainoya, T. Tsuchimoto, H. Yasuda, Tetrahedron Lett. 2010, 51, 808-810; v) B. Natarajan, N. Jayaraman, J. Organomet. Chem. 2011, 696, 722-730; w) R. S. Bagul, N. Jayaraman, J. Organomet. Chem. 2012, 701, 27-35; x) D. Wang, D. Astruc, Coord. Chem. Rev. 2013, 257, 2317-2334; y) R. S. Bagul, N. Jayaraman, Inorg. Chim. Acta 2014, 409A, 34-52; z) L. Wang, D. J. Kiemle, C. J. Boyle, E. L. Connors, I. Gitsov, Macromolecules 2014, 47, 2199-2213; R. S. Bagul, N. Jayaraman, Polymer 2014, $55,5102-5110$.

[9] D. Thibeault, J.-F. Morin, Molecules 2010, 15, 3709-3730.

[10] a) C. W. Tornøe, C. Christensen, M. Meldal, J. Org. Chem. 2002, 67, 3057-3064; b) V. V. Rostovtsev, L. G. Green, V. V. Fokin, K. B. Sharpless, Angew. Chem. Int. Ed. 2002, 41, 25962599; Angew. Chem. 2002, 114, 2708; c) K. D. Hänni, D. A. Leigh, Chem. Soc. Rev. 2010, 39, 1240-1251.

[11] a) F. Coutrot, E. Busseron, Chem. Eur. J. 2008, 14, 4784-4787; b) F. Coutrot, C. Romuald, E. Busseron, Org. Lett. 2008, 10, 3741-3744; c) E. Busseron, C. Romuald, F. Coutrot, Chem. Eur. J. 2010, 16, 10062-10073; d) Y. Jiang, J.-B. Guo, C.-F. Chen, Org. Lett. 2010, 12, 4248-4251; e) Q. Jiang, H.-Y. Zhang, M. Han, Z.-J. Ding, Y. Liu, Org. Lett. 2010, 12, 1728-1731; f) C. Romuald, E. Busseron, F. Coutrot, J. Org. Chem. 2010, 75, 
6516-6531; g) W. Yang, Y. Li, J. Zhang, Y. Yu, T. Liu, H. Liu, Y. Li, Org. Biomol. Chem. 2011, 9, 6022-6026; h) C. Romuald, A. Ardá, C. Clavel, J. Jiménez-Barbero, F. Coutrot, Chem. Sci. 2012, 3, 1851-1857; i) E. Busseron, F. Coutrot, J. Org. Chem. 2013, 78, 4099-4106; j) C. Clavel, C. Romuald, E. Brabet, F. Coutrot, Chem. Eur. J. 2013, 19, 2982-2989; k) C. Clavel, K. Fournel-Marotte, F. Coutrot, Molecules 2013, 18, 1155311575 ; 1) S. Chao, C. Romuald, K. Fournel-Marotte, C. Clavel, F. Coutrot, Angew. Chem. Int. Ed. 2014, 53, 6914-6919.

[12] The first approach to the threads was to use the same procedure as that for the rotaxanes in the absence of DB24C8, but this led to decomposition during the purification of the crude product; therefore, the procedure was modified.

[13] a) A. C. Benniston, A. Harriman, V. M. Lynch, J. Am. Chem. Soc. 1995, 117, 5275-5291; b) P. R. Ashton, R. Ballardini, V. Balzani, I. Baxter, A. Credi, M. C. T. Fyfe, M. T. Gandolfi, M. Gómez-López, M.-V. Martínez-Díaz, A. Piersanti, N. Spencer, J. F. Stoddart, M. Venturi, A. J. P. White, D. J. Williams, J. Am. Chem. Soc. 1998, 120, 11932-11942; c) R. Ballardini, V. Balzani, W. Dehaen, A. E. Dell'Erba, F. M. Raymo, J. F. Stoddart, M. Venturi, Eur. J. Org. Chem. 2000, 65, 591-602; d) G. Giro, M. Cocchi, V. Fattori, G. Gadret, G. Ruani, M. Murgia, M. Cavallini, F. Biscarini, R. Zamboni, T. Loontjens, J. Thies, D. A. Leigh, A. F. Morales, Synth. Met. 2001, 122, 27-29; e) G. Gadret, G. Ruani, M. Cavallini, F. Biscarini, M. Murgia,
R. Zamboni, G. Giro, M. Cocchi, V. Fattori, T. Loontjens, J. Thies, D. A. Leigh, A. F. Morales, R. F. Mahrt, Appl. Surf. Sci. 2001, 175-176, 369-373.

[14] If any of the shuttles with a trifluoroacetate anion is redissolved in DCM and washed with a solution of $\mathrm{NH}_{4} \mathrm{PF}_{6}$ in MiliQ water, the original shuttle is recovered (it has the same ${ }^{1} \mathrm{H}$ NMR spectrum); therefore, the effect is produced by the anion and is reversible.

[15] a) J. L Sessler, P. Gale, W.-S. Cho, Anion Receptor Chemistry, RSC Publishing, Cambridge, UK, 2006; b) P. A. Gale, W. Dehaen (Eds.), Anion Recognition in Supramolecular Chemistry, Springer, Heidelberg, Germany, 2010; c) N. H. Evans, P. D. Beer, Angew. Chem. Int. Ed. 2014, 53, 11716-11754.

[16] a) B. R. Mullaney, A. L. Thompson, P. D. Beer, Angew. Chem. Int. Ed. 2014, 53, 11458-11462; b) B. R. Mullaney, B. E. Partridge, P. D. Beer, Chem. Eur. J. 2015, 21, 1660-1665; c) S. P. Cornes, C. H. Davies, D. Blyghton, M. R. Sambrook, P. D. Beer, Org. Biomol. Chem. 2015, 13, 2582-2587.

[17] P. R. Ashton, R. Ballardini, V. Balzani, I. Baxter, A. Credi, M. C. T. Fyfe, M. T. Gandolfi, M. Gómez-López, M. V. Martinez-Diaz, A. Piersanti, N. Spencer, J. F. Stoddart, M. Venturi, A. J. P. White, D. J. Williams, J. Am. Chem. Soc. 1998, 120, 11932-11942.

Received: July 17, 2015

Published Online: September 23, 2015 


\title{
Journal Name
}

\section{ARTICLE}

\section{Study of pH-sensitive bistable molecular shuttles incorporating azobenzene as a kinetic barrier for photochemically triggered memory switching}

Received 00th January 20xx, Accepted 00th January 20xx

DOI: $10.1039 / x 0 x \times 00000 x$

www.rsc.org/

\author{
Héctor Barbero, ${ }^{a}$ Daniel Miguel ${ }^{b}$ and Celedonio M. Álvarez*
}

\begin{abstract}
The synthesis of three bistable molecular shuttles family based on ammonium / triazolium salt motifs with DB24C8 as the macrocyclic ring incroporating an azobenzene fragment between two stations is reported. The main difference among them is the substitution pattern of azobenzene moiety. The threading followed by capping methodology was applied in a one-pot system in order to obtain final interlocked molecules and their shuttling and reversible photoisomerization was assessed in terms of spectroscopic techniques. Ultimately, the sequential combination of these two inputs were studied in order to obtain the pursued shuttling memory effect thanks to the imposition of a kinetic barrier coming from different rotaxane geometries arising from the present photostationary state. We concluded that the azobenzene unit must contain slightly bulky substituents and a little spacer connecting stations have to be used so that an effective switching gate is observed.
\end{abstract}

\section{Introduction}

The study of new synthetic molecular machines from the bottom-up approach is a hot topic in many areas of research ${ }^{1}$ due to their highly potential applications in fields such as nanotechnology or composite materials. The vast family of Mechanically Interlocked Molecules (MIMs) plays a very important role in this context because the exploitation of the interlocked species relative movement to get a result in terms of useful work. ${ }^{2}$

Azobenzene is a molecule consisting of two benzene units connected by a $-\mathrm{N}=\mathrm{N}$ - group that might undergo reversible isomerization between $\mathrm{Z}$ and $\mathrm{E}$ configurations. ${ }^{3} \mathrm{~A}$ large set of studies involving this moiety as a differential unit able to modify the whole geometry of a system has been reported in the last years. ${ }^{4}$ As a result of this structure modification, different behaviours can be observed so that a work might be extracted.

The incorporation of this interesting fragment in MIMs, especially in rotaxanes, has been tested in several systems. ${ }^{5}$ In most cases, azobenzene is located on the axle and many interesting properties have been reported. The main reason for the tethering of this molecule to a rotaxane relies on the possible kinetic control as a result of the different thermal stability of configurational isomers, adding an extra property apart from the classical shuttling thermodynamic control as a function of differential affinity among stations.

Recently, Stoddart's group described three molecular shuttles

GIR MIOMeT, IU CINQUIMA/Química Inorgánica, Facultad de Ciencias, Universidad de Valladolid, E-47011, Valladolid, Spain.

Electronic supplementary information (ESI) available. bearing an azobenzene unit (two of them degenerate) ${ }^{6}$ whose ring movement from one of them was based in a redox reaction achieving partial kinetic control by raising or lowering the energetic barrier, but this so called "memory effect" was only analyzed in terms of absorption spectra but not by NMR experiments. It is well-established that this is the most preferred technique for compounds characterization and properties studies in solution because it gives obtainable direct structure information, especially in organic chemistry. Those studies encouraged us to develop a $\mathrm{pH}$-sensitive molecular shuttle by making use of the ammonium/triazolium system (Figure 1) drawing upon the structures and methodologies peviously developed by us $^{7}$ and test the possibility of get a new family of molecular machines with shuttling kinetic control.

\section{Results and discussion}

According to model described in the previous section, we rationalized that the best candidates were azophenols so that they could be easily further bonded to an anthracene-based stopper fragment already developed ${ }^{7}$ and, at the same time, it allowed us to be prepared by well-known azo coupling tecniques. ${ }^{8}$ An alkynyl triple bond at the other end of the structure, ready for CuAAC click coupling ${ }^{9}$ with an azide, was designed.

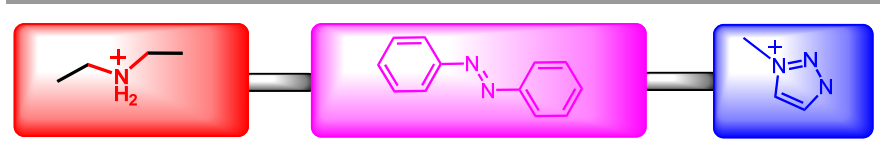

Fig. 1 Schematic model of the relative disposition of the main components in the axle for all molecular shuttles described in this work. 
<smiles>C#Cc1ccc(/N=N/c2ccc(O)cc2)cc1</smiles><smiles>C#Cc1ccc(/N=N/c2cc(C)c(O)c(C)c2)cc1</smiles>

A3<smiles>C#Cc1cccc(/N=N/c2ccc(O)cc2)c1</smiles>

A2

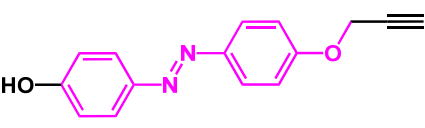

A5

Fig. 2 Set of azo groups used in this work.

Three different geometries are described (Figure 2). The most simple one is that having an alkynyl group directly connected to azo group by a single bond in para position, giving rise to a linear arrangement (A1). In addition to this, a second molecule was used having such group in meta position to test this variable in future shuttling assesment (A2). Furthermore, alkynyl and azo groups were separated by a little spacer as a $-\mathrm{OCH}_{2}$ - group so that direct connection was prevented (A3). Lastly, we made use of the first azo compound bearing two methyl positions to impose an additional steric barrier to the crown ether (A4).

\section{Synthesis strategy}

Phenol groups were subjected to two different procedures in order to obtain desired ethers (Scheme 1). On one hand, a direct Mitsunobu reaction ${ }^{10}$ with the suitable alcohol derivative was attempted. This method resulted in the expected compounds, but having very low yields (below 25\%) due, presumably, to the competitive reaction regarding the attack of $\mathrm{PPh}_{3}$ to our azo compound instead to DIAD, despite it is less activated. On the other hand a Williamson ether protocol $^{11}$ was applied. This required a previous transformation of the benzylic alcohol into a good leaving

group. This procedure added an extra step, but it was mostly preferred since the intermediate was used without purification and better yields were obtained.

Tipically, the amine group is usually isolated as an amonium salt before the inclusion complex formation with the macrocyclic ring. We carried out the same procedure for compound R3. Carbamate was hydrolized with TFA to yield the desired amonium salt, but we noticed that a large excess of acid was necessary so that deprotection occurred. Unfortunately, this gave remarkable material decomposition, so we immediately carried out column chromatography separations in order to isolate major spots observed on TLC. All fractions but one were undientified compounds, most likely fragments of the former phenolic ether. The only recognizable fraction corresponded to deprotected amine 7 in low yield (Scheme 2). This compound was protonated with the addition of TFA ( 1 eq), but we noticed increasing decomposition in solution just after a few minutes according to NMR analysis, so we immediately added DB24C8 and carried out the conditions for click chemistry. Although this methodology actually gave the expected [2] rotaxane 1-H-PF 6 , the final isolated yield was very low (below $5 \%$ ). This led us to develop an alternative onepot metholodogy consisting of carbamate deprotection in presence of DB24C8 allowing the immediately capture of the ammonium salt by the ring once formed stabilizing the compound (Scheme 3). The existence of pseudorotaxane was confirmed by ${ }^{1} \mathrm{H}-\mathrm{NMR}$ spectra of the crude. Once finished, volatiles were removed and the resulting slurry was subjected to the appropriate click chemistry conditions by using a convenient azide to finally get the desired rotaxanes in reasonable yields.

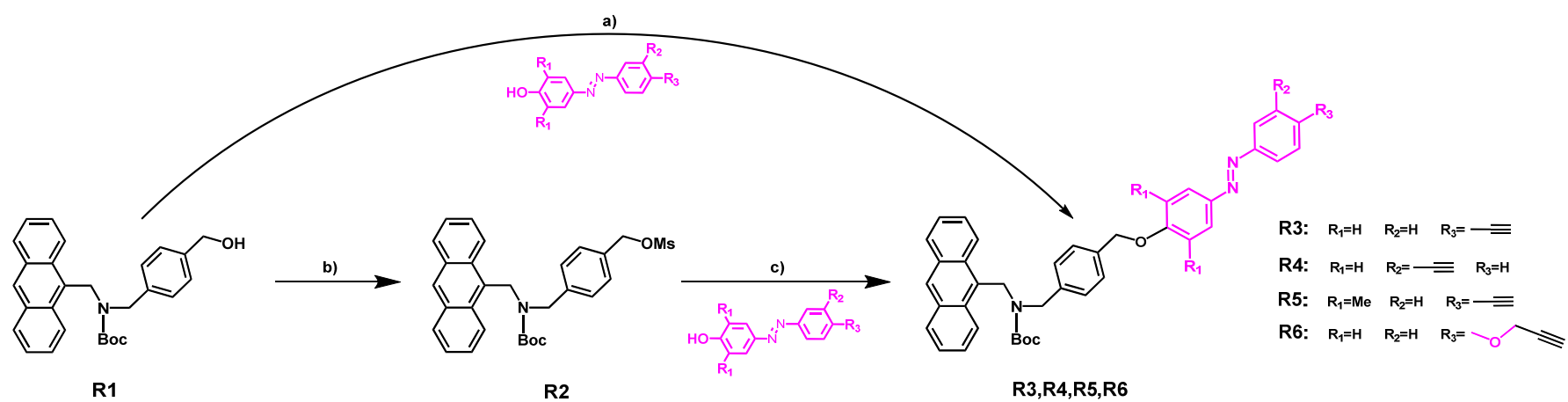

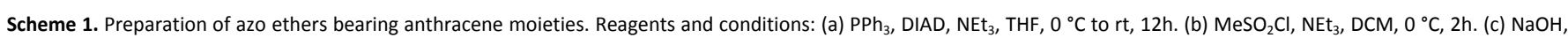
THF, reflux, $12 \mathrm{~h}$.

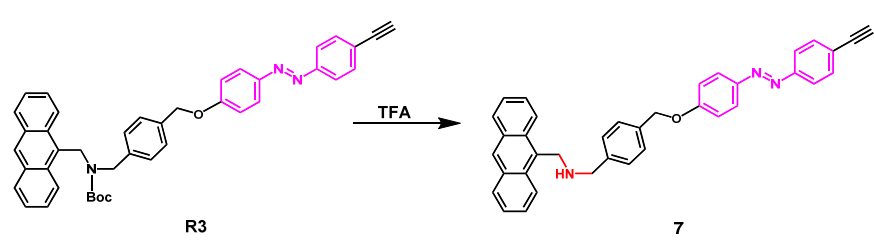

Scheme 2. Deprotection of R3 by addition of an excess of TFA yielding $\mathbf{7}$ among major material decomposition.

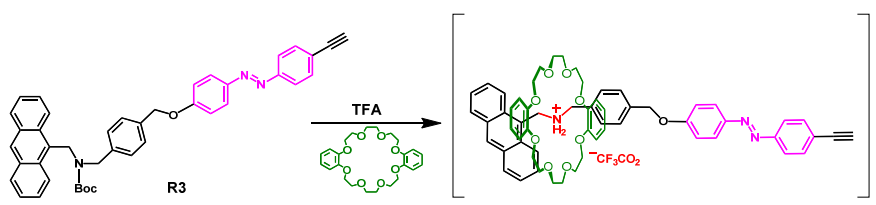

Scheme 3. Deprotection of $\mathbf{R} \mathbf{3}$ by addition of an excess of TFA in presence of DB2C8 forming a stable pseudorotaxane preventing ammonium salt decomposition and permitting the final reaction step. 
This procedure worked for R3, R4 and R6, but R5 failed to establish the adduct after deprotection, possibly due to the steric hindrance imposed by methyl substituents. Heating the mixture to overcome the energetic barrier only resulted in major decomposition. If this material is subjected to click chemistry conditions, the only recognizable isolable product was the amine 8 (Scheme 4). Semiempirical computational calculations were carried out to roughly estimate the difficulty of pseudorotaxane formations by optimizing the geometries of the inclusion complexes as a function of macrocycle podition along the axle in the ammonium salts coming from R3 and R5 whose only difference were the existence of methyl substituents (Figure 3). ${ }^{12}$ As can be seen from such scheme, the energetic profile is very similar in both cases until DB24C8 macrocycle encounters the critical point where methyl substituents are involved. In the absence of them, the minimum is only $4.3 \mathrm{kcal} / \mathrm{mol}$ less stable than the previous minimum, but it is $10.7 \mathrm{kcal} / \mathrm{mol}$ less stable in their presence. Although energetic barriers were not obtained ${ }^{13}$ they are expected to be higher for methylated product, preventing the formation of pseudorotaxane at room temperature.

Once obtained rotaxanes 1-H-PF, $2-\mathrm{H}-\mathrm{PF}_{6}$ and $3-\mathrm{H}-\mathrm{PF}_{6}$, methylation of triazole gave the second station finishing the synthetic procedure to get molecular shuttles (Scheme 5).

\section{Characterization and shuttling evaluation}

All molecular shuttles were characterized in solution by NMR experiments and UV/Vis spectra (Figures S1-S38, S54-S56 in ESI). As expected, signals around $5.5 \mathrm{ppm}$ corresponding to $\mathrm{H}_{9}$ and $\mathrm{H}_{11}$ protons, which means $-\mathrm{CH}_{2^{-}}$groups next to the ammonium salt (see ESI for atom numbering), and their pattern indicated the existence of the rotaxane, along with typical chemical shifts from DB24C8 protons.

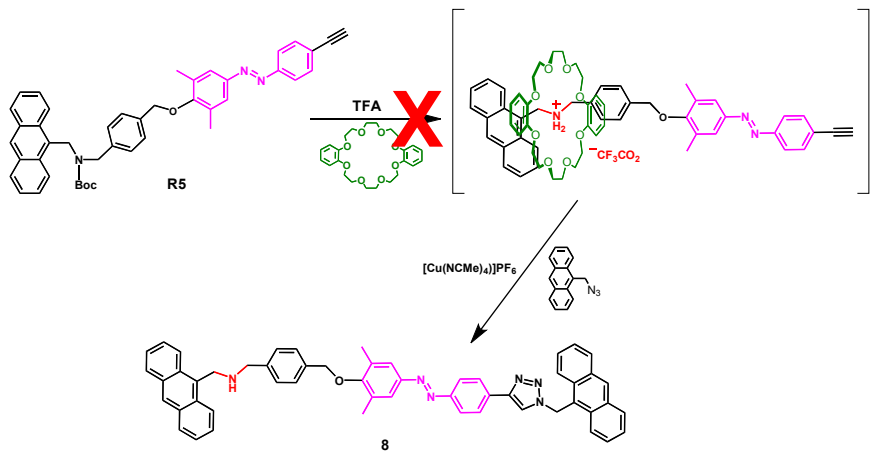

Scheme 4. Attempted synthesis of molecular shuttle from ether $\mathbf{R} 5$ resulting in $\mathbf{8}$.

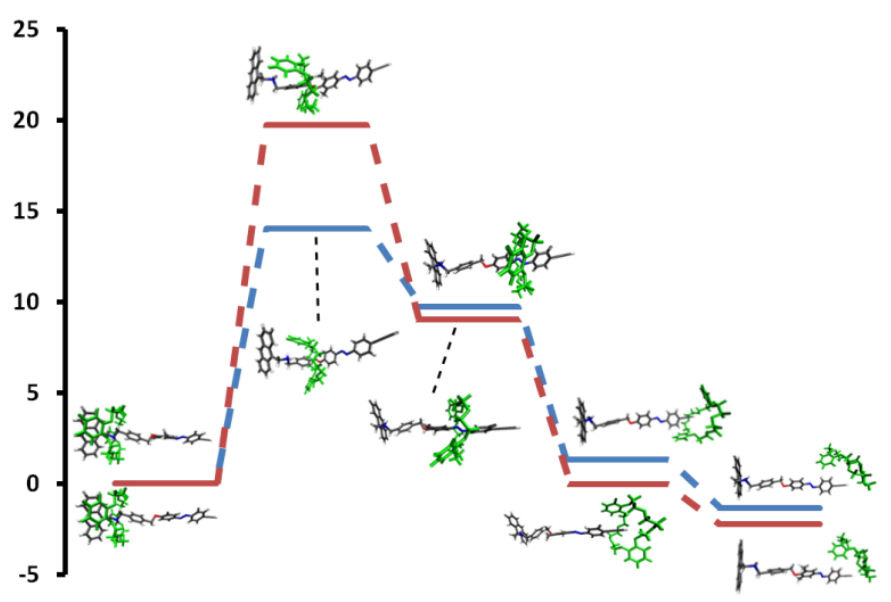

Fig.2 Energy profile of different optimized minima for the pseudorotaxane formation as a function of the macroring position (in $\mathrm{kcal} / \mathrm{mol}$ ). Blue: pseudorotaxane from R3 deprotection and DB24C8. Red: pseudorotaxane from R5 deprotection and DB24C8.

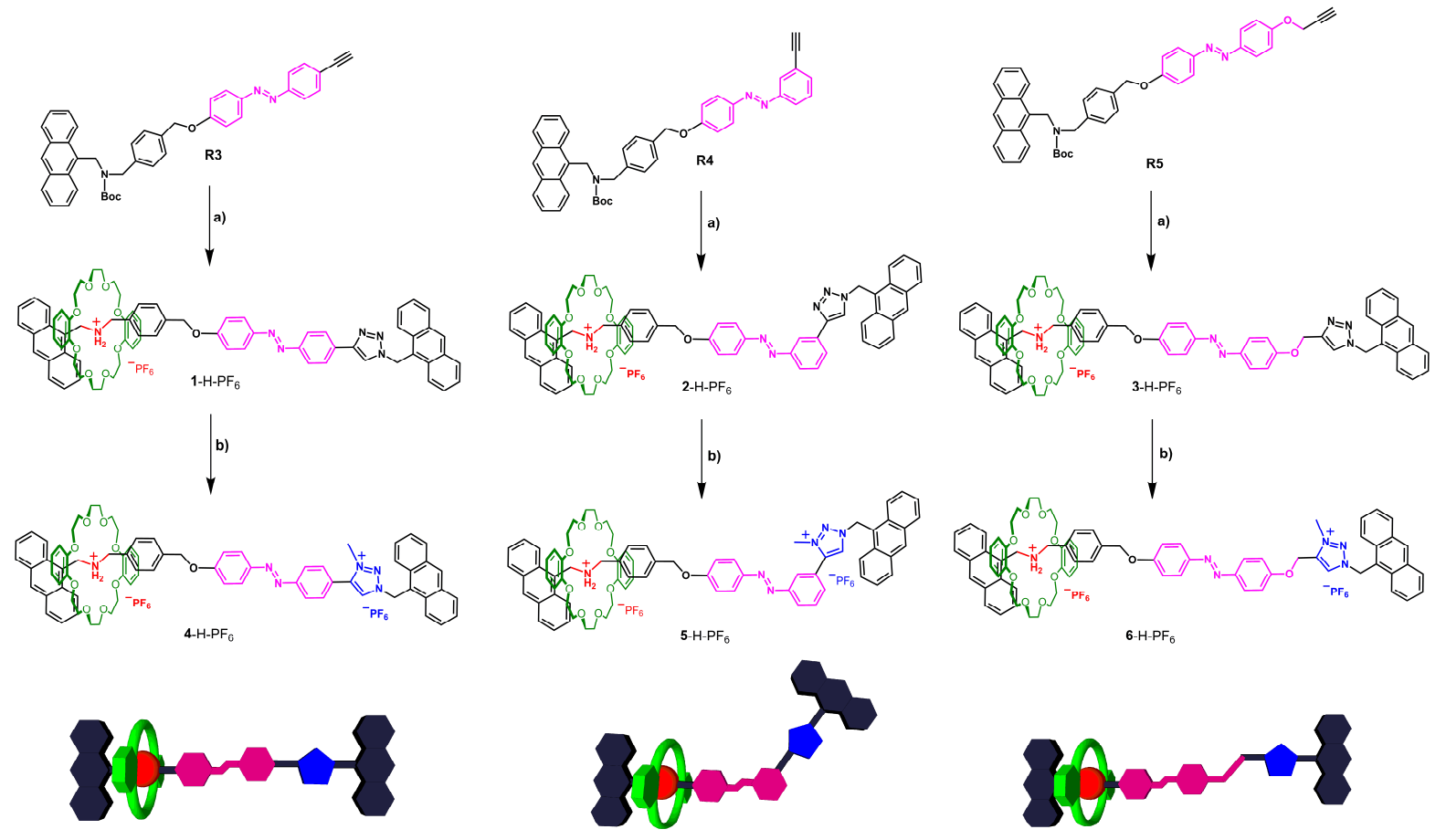

Scheme 5. Scheme 5. One-pot preparation of rotaxanes and their methylation to give the family of molecular shuttles described in this work. Reagents and conditions: (a) 1 . TFA, $\mathrm{DB} 24 \mathrm{C} 8, \mathrm{CHCl}_{3} /$ acetone, rt, 1.5h; 2. S1, $\left[\mathrm{Cu}\left(\mathrm{NCMe}_{4} \mathrm{PF}_{6}\right], 2,6\right.$-lutidine, $\mathrm{DCM}, \mathrm{rt}, 4$ days. (c) 1. $\mathrm{Me}_{3} \mathrm{OBF}_{4}, \mathrm{DCM}, \mathrm{rt}, 1.5 \mathrm{~h} ; 2 . \mathrm{NH}_{4} \mathrm{PF}$. $\mathrm{DCM} / \mathrm{H}_{2} \mathrm{O}, \mathrm{rt}, 20 \mathrm{~min}$. 
Furthermore, NOESY and ROESY spectra confirmed the relative location between axle and ring (Figures S19, S25 and S31) in ESI) UV/Vis absorption spectra were very similar among all molecular shuttles showing overlap between anthracene and azobenzene $\pi-\pi^{*}$ transitions in the region of $325 \mathrm{~nm}$ to 400 $\mathrm{nm}$. Additionaly, the band of low intensity at $450 \mathrm{~nm}$ indicated the existence of forbidden $n-\pi^{*}$ transition from azobenzene group (Figure 3 and S54-S56).

We carried out the in situ shuttling procedure previously described by $\mathrm{us}^{7}$ consisting of the addition of the suitable amount of a $\mathrm{NaOH}$ solution in $\mathrm{D}_{2} \mathrm{O}$ in order to neutralize the ammonium salt and force the crown ether to move so that it establish a better interaction with triazolium salt. All chemical shifts in their ${ }^{1} \mathrm{H}-\mathrm{NMR}$ spectra changed, especially those next to both stations. Signals from $\mathrm{H}_{9}$ and $\mathrm{H}_{11}$ changed around 1 ppm towards high field and their pattern turned from multiplet to singlet. On the other hand, $\mathrm{H}_{27}, \mathrm{H}_{29}$ and $\mathrm{H}_{28}$ signals from 4- $-\mathrm{PF}_{6}, 5-\mathrm{H}-\mathrm{PF}_{6}$ and $\mathbf{6}-\mathrm{H}-\mathrm{PF}_{6}$, respectively, (methyl group in triazolium station) moved almost $0.5 \mathrm{ppm}$ towards high field as well. The most remarkably change in chemical shift was observed for the other proton of triazolium salt station, $\mathrm{H}_{26}$, $\mathrm{H}_{28}$ and $\mathrm{H}_{27}$ in $4-\mathrm{H}-\mathrm{PF}_{6}, 5-\mathrm{H}-\mathrm{PF}_{6}$ and $6-\mathrm{H}-\mathrm{PF}_{6}$, respectively. This signal is the outermost peak at low field appearing in a chemical shift over $9 \mathrm{ppm}$. Once the macroring is shuttled, that signal moved to low field in $4-\mathrm{H}-\mathrm{PF}_{6}$ and $6-\mathrm{H}-\mathrm{PF}_{6}$ and, interestingly, to high field in the case of $\mathbf{5}-\mathrm{H}_{-} \mathrm{PF}_{6}$. All changes occurred in the range of $0.2-0.5 \mathrm{ppm}$. Additionally, this signal suffered an exchange with deuteron dramatically lowering its intensity in all cases, especially in $6-\mathrm{PF}_{6}$, whose peak could not be observed anymore beyond that shuttling step. This effect was also detected if any protonated molecular shuttle is kept in deuterated solution for long times. Some other proton signals suffered the same effect too. Apart from chemical shift changes, the location of DB24C8 was confirmed thanks to NOESY experiments (Figures S22, S28, and S34).

If TFA is added to switched molecular shuttles, the secondary amine is reprotonated so that the ammonium salt is recovered, making the macrocycle to move again to the initial position and giving the initial ${ }^{1} \mathrm{H}-\mathrm{NMR}$ spectra, completing the cycle. Nonetheless, a close inspection of those spectra demonstrates that all signals slightly differs from the initial situations. This was according to our initial assumption due to the fact that the anion is different and this effect was previously observed. ${ }^{7,14,15}$ If an anion exchange to ${ }^{-} \mathrm{PF}_{6}$ is performed, the exact initial spectra are recovered. Interestingly, proton $\mathrm{H}_{28}$ in $\mathbf{5}-\mathrm{H}-\mathrm{PF}_{6}$ underwent evolution to high field instead of the expected low-field shift giving the most difference in chemical shift if compared to the initial molecular shuttle $(|\Delta \delta|=0.38 \mathrm{ppm}$ while for other signals $|\Delta \delta|<0.1 \mathrm{ppm})$. Then, the evolution of this chemical shift resembled that observed for $4-\mathrm{H}-\mathrm{PF}_{6}$ in further shuttling cycles.

The procedure described above could be carried out for several times without appreciable signs of decomposition. Two of these cycles are depicted in Figure 4.

\section{Photoisomerization}

After the assessment of the shuttling process in terms of $\mathrm{pH}$ changes, we carried out several experiments in order to observe reversible photoisomerization of azobenzenes units. Firstly, UV/Vis absorption spectra were recorded during the process. Irradiation with a UV lamp at $365 \mathrm{~nm}$ gave remarkable changes in all bands. The bands in the range of $325 \mathrm{~nm}$ to 400 $\mathrm{nm}\left(\pi-\pi^{*}\right.$ transitions) decreased in intensity while the band at $450 \mathrm{~nm}$ increased, as expected, according the typical behaviour of azobenzene derivatives ${ }^{3}$ (see Figure 5 as an example; all the other spectra are gathered in ESI, Figures S57S59). Irradiation was performed at different times concluding that the longer the time, the better the conversion. However, once reached a time around 30 minutes, no longer evolution was observed. Samples were irradiatied at longer wavelengths (380 $\mathrm{nm})$, but worse performance was obtained. Irradiation at shorter wavelengths $(330 \mathrm{~nm}$ ) only led to decompositions due to the disappearance of $n-\pi^{*}$ transition bands that could not be recovered indicating azobenzene decomposition. The reversibility of this process was checked as well by leaving the sample in the dark for some time and recording a spectrum afterwards, yielding the same initial bands.

Photoisomerization was tested by NMR techniques as well. A ${ }^{1} \mathrm{H}-\mathrm{NMR}$ spectrum was recorded before and after irradiation giving rise to chemical shift changes in many protons, especially those next to the azobenzene unit. In protonated molecular shuttles all signals from DB24C8 moiety were not substantially modified. This is reasonable due to the distance between the ammonium station and the azobenzene spacer. In fact, azobenzene signals along with directly connected triazolium salt group were the most affected (see Figure 6 as an example; all the other spectra are gathered in ESI, Figures S42, S44 and S46).

In the case of deprotonated shuttles (macrocycle on triazolium station) many signals have suffered changes upon irradiation. This time, those chemical shifts corresponding to crown ether protons have been changed as well. According to the previous findings, the position of this unit is on triazolium station, which is much closer to azobenzene spacer than ammonium station,

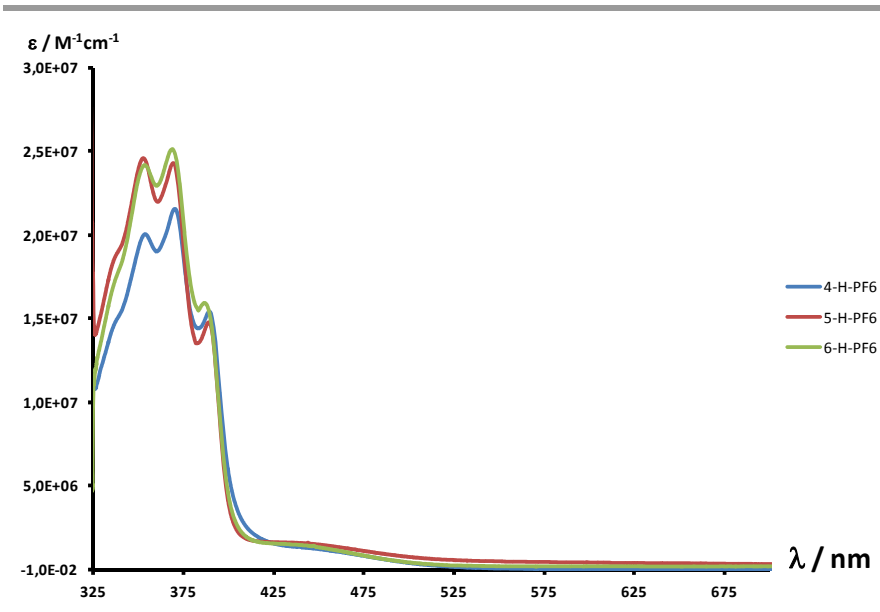

Fig. 3 UV/Vis absorption spectra of molecular shuttles in acetone. 


\section{Journal Name}

\section{ARTICLE}
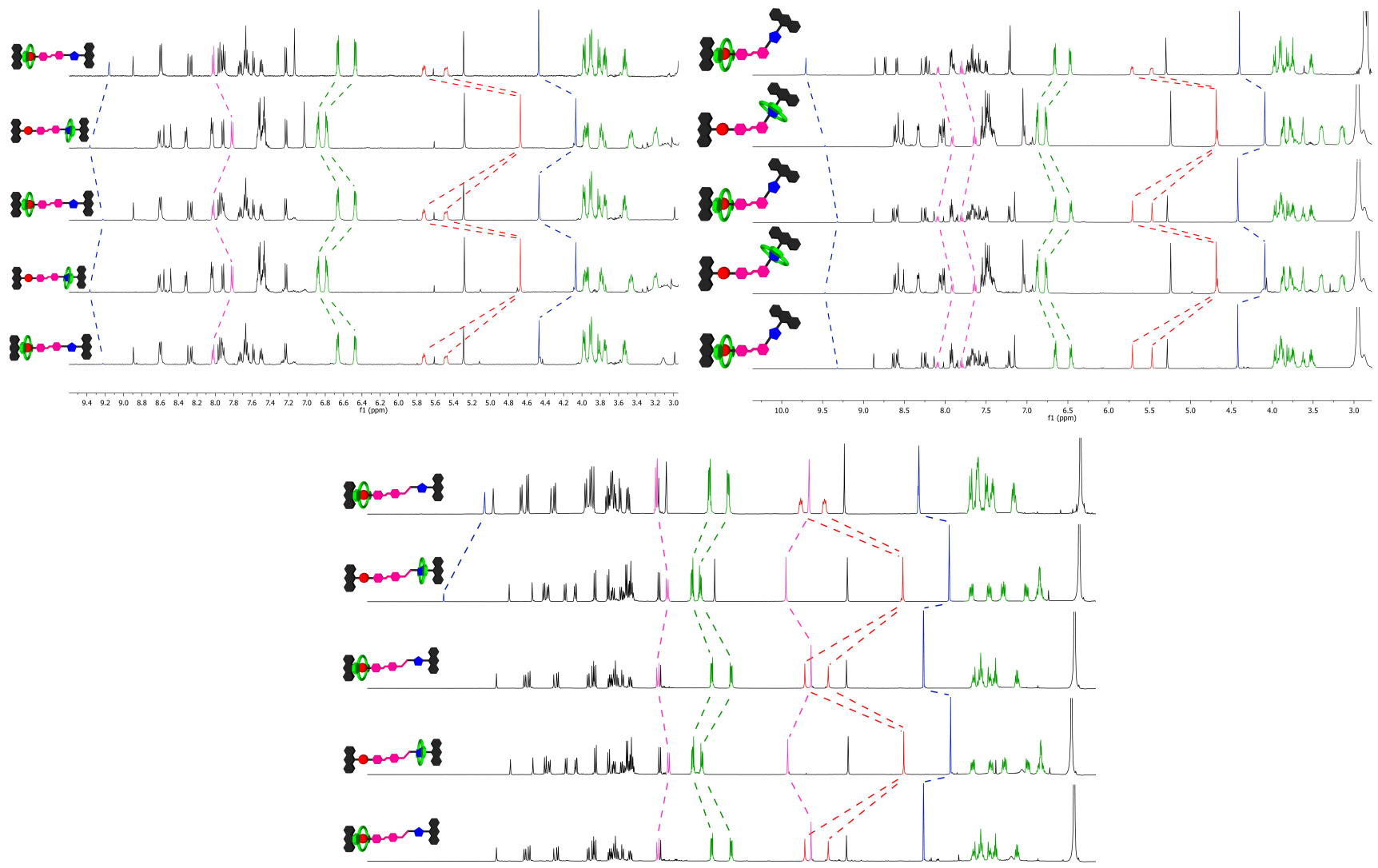

Fig. $4{ }^{1} \mathrm{H}$-NMR spectra $\left(500 \mathrm{MHz}\right.$, acetone- $\mathrm{d}_{6}$ ) of all three molecular shuttles prepared in this work and the two-step sequence of macrocyclic ring shuttling along the axis. Some signals were coloured according to the chromatic definition of each group at the beginning of the text in order to show significant chemical shift changes.

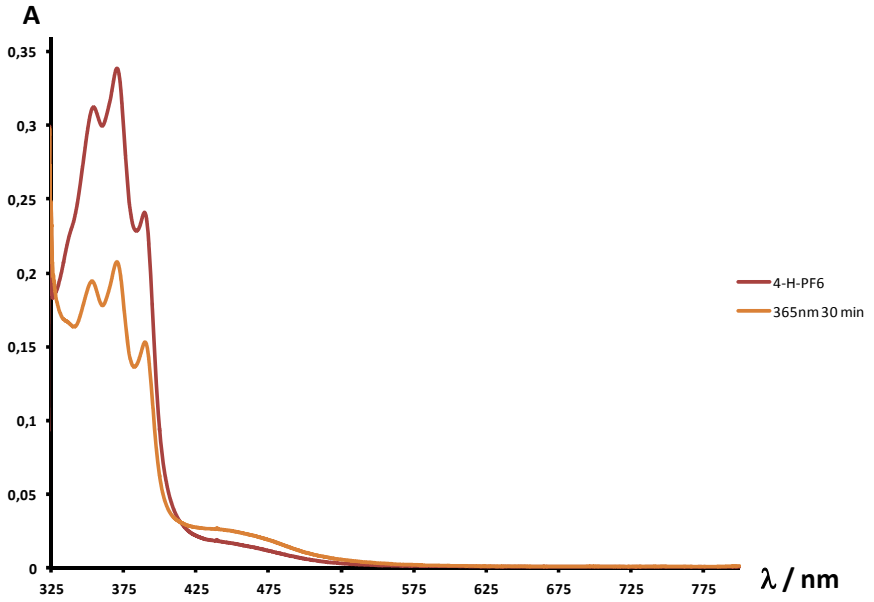

Fig. 5 UV/Vis absorption spectra in acetone of 4-H-PF6 (brown) and after irradiation at $365 \mathrm{~nm}$ for $30 \mathrm{~min}$.

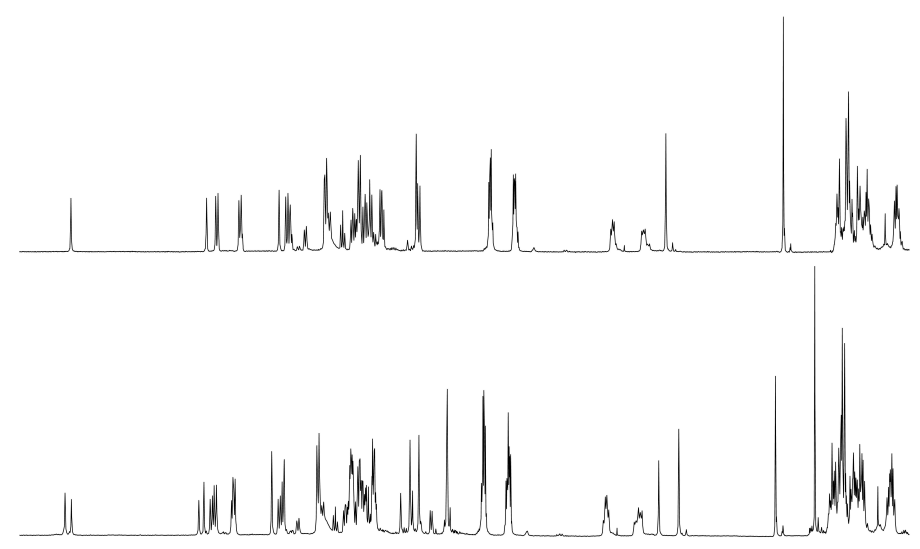

Fig. $6{ }^{1} \mathrm{H}-\mathrm{NMR}$ spectra $\left(500 \mathrm{MHz}\right.$, acetone- $\left.\mathrm{d}_{6}\right)$ of $\mathbf{5}-\mathrm{H}-\mathrm{PF}_{6}$ before (up) and after irradiation with UV at $365 \mathrm{~nm}$ (down). 

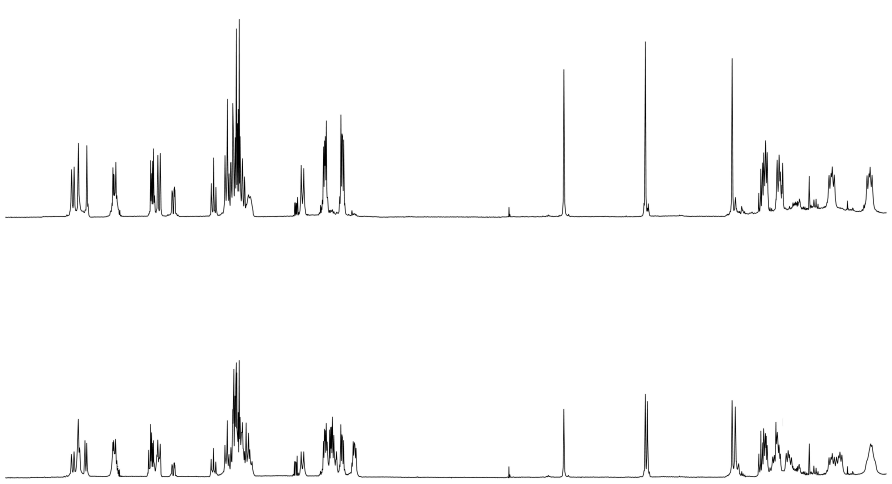

Fig. $7{ }^{1} \mathrm{H}-\mathrm{NMR}$ spectra (500 MHz, acetone- $\mathrm{d}_{6}$ ) of $5-\mathrm{PF}_{6}$ before (up) and after Fig. $7{ }^{1} \mathrm{H}-\mathrm{NMR}$ spectra $(500 \mathrm{MHz}$, acet
irradiation with UV at $365 \mathrm{~nm}$ (down).

inducing such changes (see Figure 7 as an example; all the other spectra are gathered in ESI, Figures S43, S45 and $\mathrm{S} 47)$. The maximum conversion from $\mathrm{E}$ to $\mathrm{Z}$ isomer was $62 \%$, $65 \%$ and $63 \%$ for $4-\mathrm{H}_{-}-\mathrm{PF}_{6}, 5-\mathrm{H}-\mathrm{PF}_{6}$ and $6-\mathrm{H}-\mathrm{PF}_{6}$, respectively. Longer irradiation time beyond $\mathbf{3 0}$ minutes were tested, but no further conversion was achieved. If irradiated samples were kept in the dark at room temperature for a long time their original ${ }^{1} \mathrm{H}-\mathrm{NMR}$ spectra were recovered demonstrating the reversibility of the isomerization.

Once evaluated photoisomerization processes by two techniques we carried out macrocycle shuttling experiments in $Z$ isomers starting from both stations. We called 'forward shuttling' as the movement of the macrocycle through the axle from the ammonium to the triazolium station and 'backward shuttling' that one beginning on triazolium group towards ammonium station.

Unfortunately, both forward and backward shuttling on $\mathrm{Z}$ isomers of $4-\mathrm{H}-\mathrm{PF}_{6}$ and $\mathbf{5}-\mathrm{H}-\mathrm{PF}_{6}$ were allowed as in previous experiments with $\mathrm{E}$ isomers. The desired memory effect was not observed, possibly due to the connection between azobenzene and triazolium units which is just a single bond. This, along with the poor efficiency of $Z$ azobenzene isomer as a steric barrier for the macrocycle could be the reasons why the memory effect is prevented. The whole shuttling and photoisomerization procedures are depicted in Scheme 6.

The case of 6-H-PF6 was slightly different. Upon photoisomerization and addition of $\mathrm{NaOH}$ (forward shuttling), we observed chemical shifts coming from $\mathbf{Z 6}-\mathrm{H}-\mathrm{PF}_{6}$ and $\mathbf{Z 6}-\mathrm{PF}_{6}$, along with the expected $\mathrm{EG}_{-} \mathrm{PF}_{6}$ from the remaining isomer (Figure 8). Azobenzene in Z configuration partially prevented the ring shuttling. Thermal relaxation was measured so that a slow movement of DB24C8 from amine station to triazolium station occurred. We are not able to establish whether the memory effect comes from the slow shuttling rate imposed by the rotaxane geometry in $\mathrm{Z}$ isomer or from relaxation to $\mathrm{E}$ isomer in which it is known that shuttling occurs, nevertheless, we could follow the intensity enhancement of final E6-PF 6 isomer and obtain a rate constant of 7.2.10-6 $\mathrm{s}^{-1}$. The same procedure was applied for backward shuttling by inverting the steps (first switching to triazolium station in basic media, then
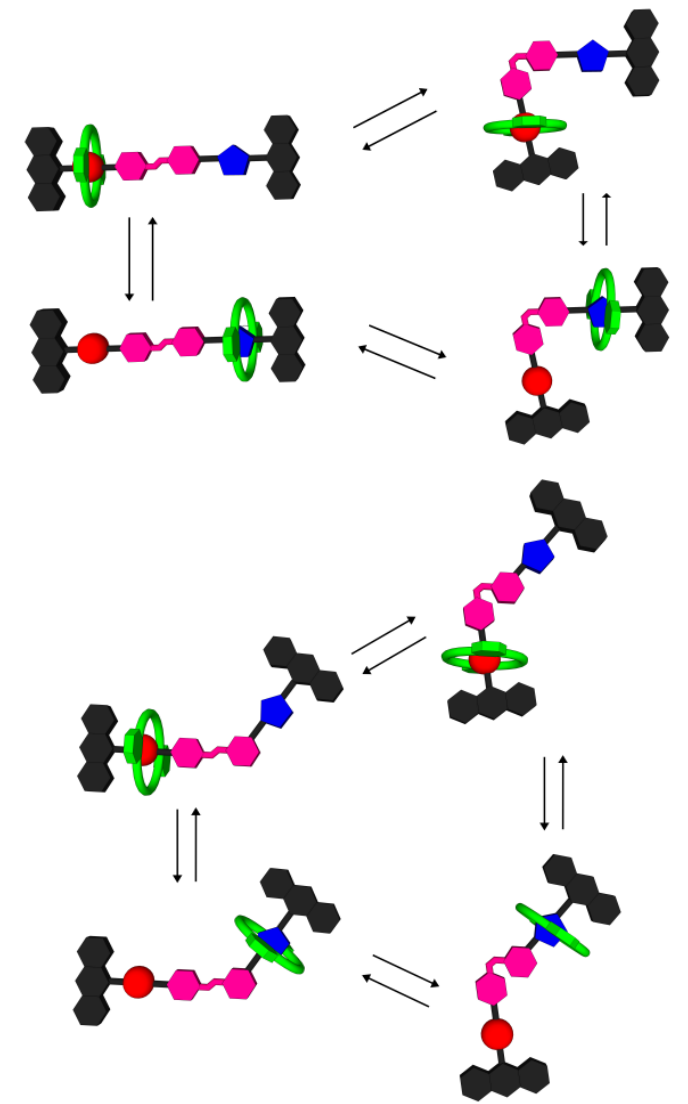

Scheme 6. Complete shuttling process in 4-H-PF6 and 5-H-PF6 whose Z isomers could not prevent macrocycle movement along the axle.

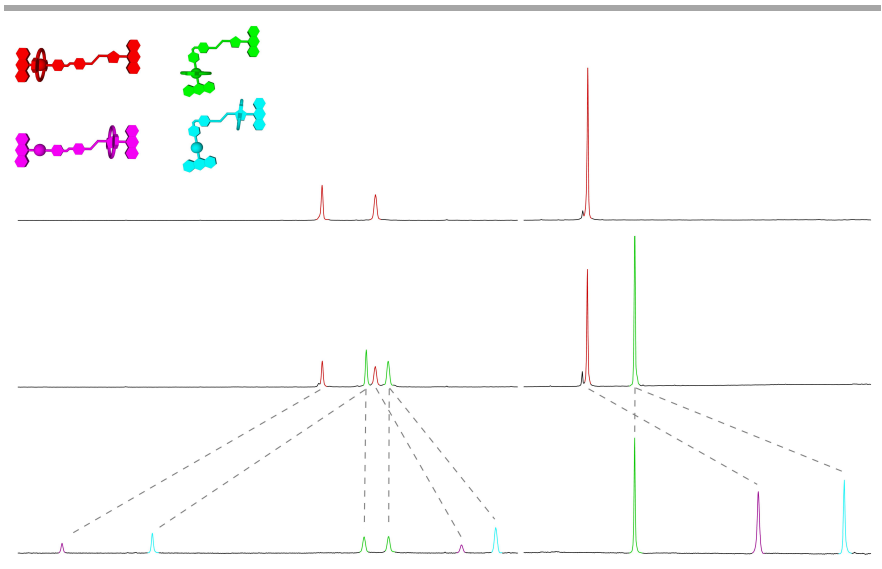

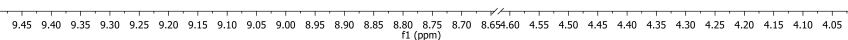

Fig. 8 Partial ${ }^{1} \mathrm{H}-\mathrm{NMR}$ spectra $\left(500 \mathrm{MHz}\right.$, acetone- $\mathrm{d}_{6}$ ) of $6-\mathrm{H}-\mathrm{PF}_{6}$ forward shuttling showing selected probe protons $\mathrm{H}_{27}, \mathrm{H}_{28}$ and $\mathrm{H}_{37}$ due to their simplicity. All four potential isomers were coloured to facilitate identification. Top: E6-H-PF 6 . Middle: after irradiation at $365 \mathrm{~nm}$ for $30 \mathrm{~min}$ showing the presence of $\mathbf{Z 6}-\mathrm{H}-\mathrm{PF}_{6}$. Bottom: after addition of $\mathrm{NaOH}$, observing the existence of $\mathrm{E6}_{-}-\mathrm{PF}_{6}, \mathbf{Z 6}-\mathrm{H}-\mathrm{PF}_{6}$, and $\mathbf{Z 6}-\mathrm{PF}_{6}$.

irradiation to get $\mathbf{Z}$ isomer and, finally, switching again with acidic media). However, this effect occurred partially, because Z6- $-\mathrm{PF}_{6}$ signals appeared, along with E6-H-PF 6 peaks coming from remaining isomer. Thermal relaxations was also measured yielding a constant of $8.6 \cdot 10^{-6} \mathrm{~s}^{-1}$. 

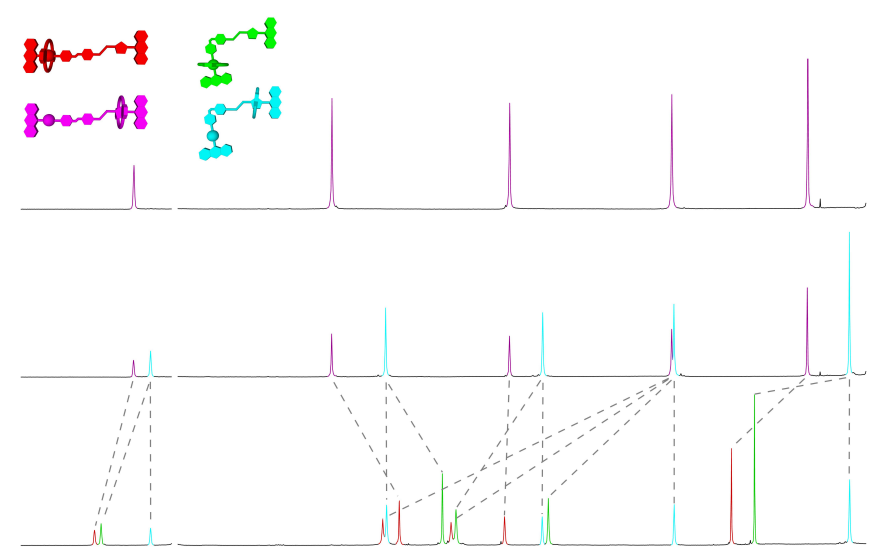

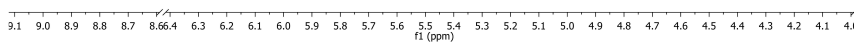

Fig. 9 Partial ${ }^{1} \mathrm{H}-\mathrm{NMR}$ spectra $\left(500 \mathrm{MHz}\right.$, acetone- $\mathrm{d}_{6}$ ) of $6-\mathrm{PF}_{6}$ backward shuttling showing selected probe protons $\mathrm{H}_{9}, \mathrm{H}_{11}, \mathrm{H}_{16}, \mathrm{H}_{25}, \mathrm{H}_{28}$ and $\mathrm{H}_{37}$ due to their simplicity. All four potential isomers were coloured to facilitate identification. Top: E6- $\mathrm{PF}_{6}$. Middle: after irradiation at $365 \mathrm{~nm}$ for 30 min showing the presence of Z6- $\mathrm{PF}_{6}$. Bottom: after addition of TFA, observing the existence of $\mathbf{E 6}-\mathrm{H}-\mathrm{PF}_{6}, \mathbf{Z 6}-\mathrm{H}-\mathrm{PF}_{6}$, and $\mathbf{Z 6}-\mathrm{PF}_{6}$.

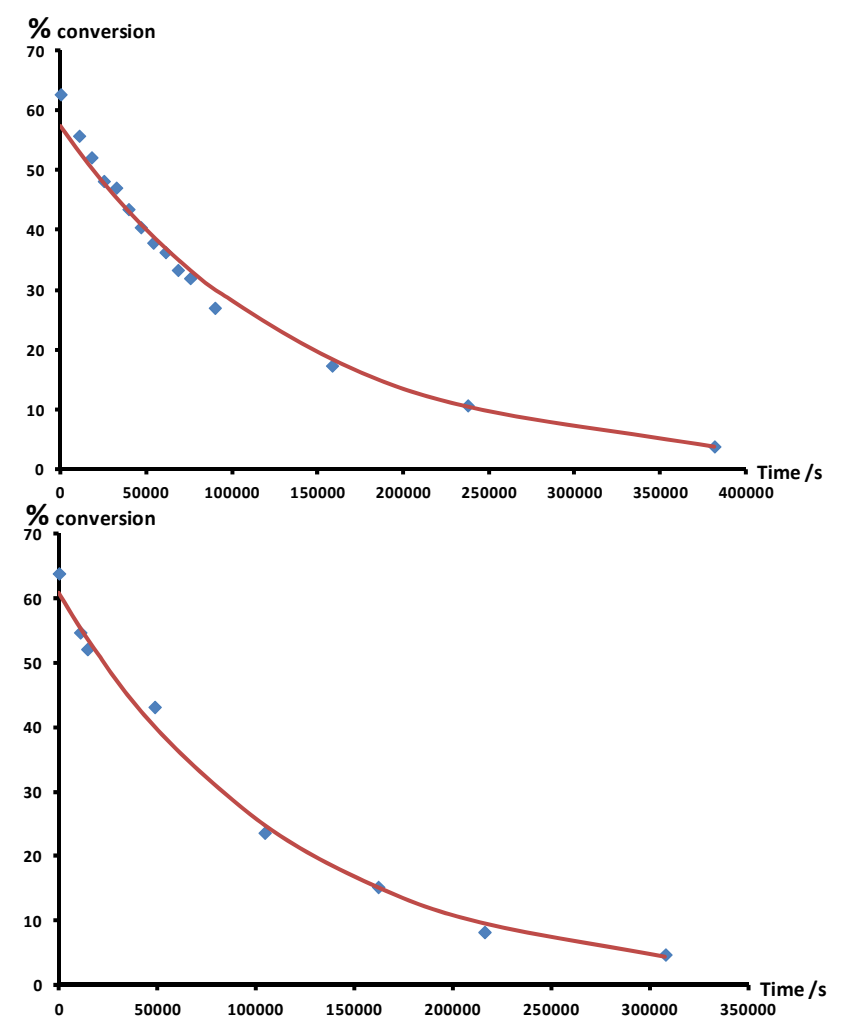

Fig. 10 Time-dependent memory effect of $6-\mathrm{H}_{-} \mathrm{PF}_{6}$ forward shuttling (up) and 6-PF backward shuttling (down). Blue squares: experimental data arising from integration in ${ }^{1} \mathrm{H}-\mathrm{NMR}$ spectra; red line: exponential fitting.

In light of these findings it is clear that kinetic control of shuttling procedure is only achieved provided that stations are not directly connected, but we can conclude that this arrangement is not totally optimum since a bulky substituent must be attached to azobenzene moiety in order to control the process more efficiently.

\section{Conclusions}

Herein we report a family of three molecular shuttles based upon ammonium salt / triazolium salt stations by using DB24C8 as a ring incorporating an azobenzene group between both units. The preparation of rotaxanes could be achieved in a one-pot method from a partially built axle through amine deprotection in presence of crown ether and a final click reaction with the suitable stopper. Characterization, shuttling procedure and reversible photoisomerization were studied. Finally, we combined the Z/E switch with $\mathrm{pH}$ changes in order to assess the performance of all rotaxanes. Those compounds having the triazolium group directly connected to azobenzene (4-H-PF 6 and $\left.5-\mathrm{H}-\mathrm{PF}_{6}\right)$ did not control kinetically the shuttling process, but, if a little spacer is used $\left(5-\mathrm{H}_{-}-\mathrm{PF}_{6}\right)$, such control takes place, but not totally, concluding that a bulky substituent in azobenzene group must be attached in order to get optimum memory effect. We tried it by using an ortho dimethylazobenzene unit, but the methodology designed for the synthesis of rotaxanes did not work in this case due to the fact that pseudorotaxane was never formed, possibly because of the barrier imposed by methyl substituents so that DB24C8 could never be threaded.

\section{Experimental}

\section{General methods}

All reagents were purchased from regular suppliers and used without further purification. Solvents were either used as purchased or dried according to procedures described elsewhere. ${ }^{16}$ All reactions were carried out under inert atmosphere with standard Schlenk techniques. Column chromatography separations were carried out by using Silica gel 60 (particle size 0.040-0.063 mm; 230-400 mesh; Merck, Germany) as the stationary phase and TLCs were performed on precoated silica gel plates $\left(0.25 \mathrm{~mm}\right.$ thick, $60 \mathrm{~F}_{254}$, Merck, Germany) and observed under UV light or by dipping in $\mathrm{p}$ anisaldehyde. NMR spectra were recorded on an Agilent DD2 $500 \mathrm{MHz}$ equipped with a cryoprobe instrument or on an Agilent MR $400 \mathrm{MHz} .{ }^{1} \mathrm{H}$ and ${ }^{13} \mathrm{C}$ NMR chemical shifts are reported in parts per million (ppm) and are referenced to TMS, using solvents as an internal reference. Coupling constants (J) are reported in hertz $(\mathrm{Hz})$. Standard abbreviations used to indicate multiplicity: $\mathrm{s}=$ singlet, $\mathrm{d}=$ doublet, $\mathrm{t}=$ triplet, $\mathrm{m}=$ multiplet, br $=$ broad. ${ }^{1} \mathrm{H}$ and ${ }^{13} \mathrm{C}$ assignments were performed by utilizing 2D NMR methods (DQFCOSY, NOESY, ROESY, HSQC and $\mathrm{HMBC}$ ). High resolution mass spectra were recorded at mass spectrometry service of the Laboratory of Instrumental Techniques of the University of Valladolid. An UPLC-MS system (UPLC: Waters ACQUITY H-class UPLC; MS: Bruker Maxis Impact) by electrospray ionization (ESI positive and negative) was used. UV/vis spectra were recorded with a Shimadzu UV1603 using spectrophotometric-grade solvents. Absorption spectral wavelengths $(\lambda)$ are reported in nanometers $(\mathrm{nm})$, and molar absorption coefficient $(\varepsilon)$ are reported in $\mathrm{M}-1 \mathrm{~cm}-1$. Photoisomerization experiments were carried out at room 
temperature with a PELCO UVC2 UV Cryo Chamber at room temperature using quartz NMR tubes for all samples.

\section{Synthetic procedures and characterization}

\section{Preparation of initial building blocks based on azo compounds}

Compounds A1, A2, A3, A4 and $\mathbf{A} 5$ were prepared according to methods published elsewhere ${ }^{17}$ and their spectral data are in agreement to those previously described.

Preparation of other initial building blocks

Compounds R1 and $\mathbf{S 1}$ were prepared according to procedures developed by us. ${ }^{7}$

4-(((anthracen-9-ylmethyl)(tert-butoxycarbonyl) amino)methyl) benzyl methanesulfonate (R2)

Alcohol R1 (128 mg, $0.3 \mathrm{mmol}$ ) was placed in a Schlenk flask under inert atmosphere. Then, it was dissolved in dry DCM (9 $\mathrm{mL}$ ) and the solution was degassed. The flask was put in an ice bath. $\mathrm{NEt}_{3}(35 \mu \mathrm{L}, 0.45 \mathrm{mmol})$ was added and, subsequently, $\mathrm{MeSOCl}_{2}(63 \mu \mathrm{L}, 0.45 \mathrm{mmol})$. The mixture was stirred at low temperature for 2 hours before allowing it to reach room temperature. Water $(10 \mathrm{~mL})$ was added and extracted with more DCM $(3 \times 10 \mathrm{~mL})$. All organic fractions were combined and concentrated under vacuum to finally give a yellow oil which was used in the next step without further purification. (144 mg, 95\% yield). ${ }^{1} \mathrm{H}$ NMR (500 MHz, CDCl3) $\delta 8.42$ (s, $1 \mathrm{H}$, $\left.\mathrm{H}_{1}\right), 8.18\left(\mathrm{br}, 2 \mathrm{H}, \mathrm{H}_{6}\right), 7.99\left(\mathrm{~d}, J=7.8 \mathrm{~Hz}, 2 \mathrm{H}, \mathrm{H}_{3}\right), 7.56-7.36$ $\left(\mathrm{m}, 4 \mathrm{H}, \mathrm{H}_{4}+\mathrm{H}_{5}\right), 7.24\left(\mathrm{br}, 2 \mathrm{H}, \mathrm{H}_{16}\right), 6.88\left(\mathrm{br}, 2 \mathrm{H}, \mathrm{H}_{15}\right), 5.57(\mathrm{br}$ $\left.2 \mathrm{H}, \mathrm{H}_{9}\right), 5.18\left(\mathrm{~s}, 2 \mathrm{H}, \mathrm{H}_{18}\right), 4.06\left(\mathrm{br}, 2 \mathrm{H}, \mathrm{H}_{13}\right), 2.92\left(\mathrm{~s}, 3 \mathrm{H}, \mathrm{H}_{19}\right)$, $1.73\left(\mathrm{br}, 3 \mathrm{H}, \mathrm{H}_{12^{\prime}}\right), 1.50\left(\mathrm{br}, 6 \mathrm{H}, \mathrm{H}_{12}\right) .{ }^{13} \mathrm{C}\left\{{ }^{1} \mathrm{H}\right\} \mathrm{NMR}(126 \mathrm{MHz}$, $\left.\mathrm{CDCl}_{3}\right) \delta 155.95\left(\mathrm{C}_{10}\right), 140.18\left(\mathrm{C}_{14}\right), 131.83\left(\mathrm{C}_{17}\right), 131.43 \mathrm{C}_{2}+\mathrm{C}_{7}$ $\left.+C_{8}\right), 129.27\left(C_{3}\right), 128.84\left(C_{16}\right), 128.47\left(C_{1}\right), 127.28\left(C_{15}\right)$, $126.34\left(C_{5}\right), 125.11\left(C_{4}\right), 124.39\left(C_{6}\right), 80.55\left(C_{11}\right), 71.49\left(C_{18}\right)$, $47.80\left(C_{13}\right), 41.19\left(C_{9}\right), 38.48\left(C_{19}\right), 28.55\left(C_{12}\right)$. HRMS (ESI-TOF): $\mathrm{m} / \mathrm{z} 528.1821[\mathrm{M}+\mathrm{Na}]^{+}$(calcd 528.1821 for $\mathrm{C}_{29} \mathrm{H}_{31} \mathrm{NNaO}_{5} \mathrm{~S}$ ).

\section{General procedure for the synthesis of phenolic ethers R3-R6}

Appropriate phenol (A1, A2, $\mathbf{A} 3$ or $\mathbf{A} 5,0.3 \mathrm{mmol}$ ) and wellcrushed sodium hydroxide $(500 \mathrm{mg}, 12.5 \mathrm{mmol}$ ) were mixed in a Schlenk flask under inert atmosphere. THF was added $(6 \mathrm{~mL})$ and the mixtures was degassed, sonicated and refluxed for 30 minutes. After such time, the deep red mixture was allowed to reach room temperature. On a separate Schlenk, a solution of mesylate $\mathbf{R} \mathbf{2}(0.25 \mathrm{mmol})$ in THF $(6 \mathrm{~mL})$ is prepared under inert atmosphere and added to the first mixture through a cannula. The resulting final mixture was refluxed overnight under nitrogen atmosphere. Once finished, it was allowed to reach room temperature and solvent was removed in vacuo. The crude was then partitioned in water and DCM (30 mL, each). Extracted with more DCM $(3 \times 40 \mathrm{~mL})$, all organic fractions were combined and subjected to a column chromatography to obtain the desired compound.

tert-butyl-(anthracen-9-ylmethyl) (4-((4-((4-ethynylphenyl) diazenyl)phenoxy)methyl)benzyl)carbamate (R3)

Column chromatography conditions $\left(\mathrm{SiO}_{2}\right.$ gel, hexane/AcOEt 3:1). (123mg, 65\% yield), red solid. ${ }^{1} \mathrm{H}$ NMR $\left(400 \mathrm{MHz}, \mathrm{CDCl}_{3}\right) \delta$ $8.43\left(\mathrm{~s}, 1 \mathrm{H}, \mathrm{H}_{1}\right), 8.20\left(\mathrm{br}, 2 \mathrm{H}, \mathrm{H}_{6}\right), 8.00\left(\mathrm{~d}, J=7.5 \mathrm{~Hz}, 2 \mathrm{H}, \mathrm{H}_{3}\right)$, $7.95\left(\mathrm{~d}, J=8.9 \mathrm{~Hz}, 2 \mathrm{H}, \mathrm{H}_{21}\right), 7.85\left(\mathrm{~d}, J=8.2 \mathrm{~Hz}, 2 \mathrm{H}, \mathrm{H}_{24}\right), 7.63(\mathrm{~d}$, $\left.J=8.2 \mathrm{~Hz}, 2 \mathrm{H}, \mathrm{H}_{25}\right), 7.49-7.38\left(\mathrm{~m}, 4 \mathrm{H}, \mathrm{H}_{4}+\mathrm{H}_{5}\right), 7.26(\mathrm{br}, 2 \mathrm{H}$ $\left.\mathrm{H}_{16}\right), 7.10\left(\mathrm{~d}, J=8.9 \mathrm{~Hz}, 2 \mathrm{H}, \mathrm{H}_{20}\right), 6.93\left(\mathrm{br}, 2 \mathrm{H}, \mathrm{H}_{15}\right), 5.58(\mathrm{br}$, $\left.2 \mathrm{H}, \mathrm{H}_{9}\right), 5.12\left(\mathrm{~s}, 2 \mathrm{H}, \mathrm{H}_{8}\right), 4.07\left(\mathrm{br}, 2 \mathrm{H}, \mathrm{H}_{13}\right), 3.22\left(\mathrm{~s}, 1 \mathrm{H}, \mathrm{H}_{28}\right)$, $1.72\left(\mathrm{br}, 3 \mathrm{H}, \mathrm{H}_{12}\right), 1.52\left(\mathrm{br}, 6 \mathrm{H}, \mathrm{H}_{12}\right) .{ }^{13} \mathrm{C}\left\{{ }^{1} \mathrm{H}\right\}$ NMR $(101 \mathrm{MHz}$, $\left.\mathrm{CDCl}_{3}\right) \delta 161.66\left(\mathrm{C}_{19}\right), 156.02\left(\mathrm{C}_{10}\right), 152.49\left(\mathrm{C}_{23}\right), 147.26\left(\mathrm{C}_{22}\right)$, $138.70\left(C_{14}\right), 134.90\left(C_{17}\right), 133.08\left(C_{25}\right), 131.47\left(C_{2}+C_{7}+C_{8}\right)$, $129.23\left(C_{3}\right), 128.41\left(C_{1}\right), 127.55\left(C_{16}\right), 127.22\left(C_{15}\right), 126.28\left(C_{5}\right)$, $125.10\left(C_{4}+C_{21}\right), 124.54\left(C_{6}\right), 124.12\left(C_{26}\right), 122.69\left(C_{24}\right), 115.33$ $\left(C_{20}\right), 83.56\left(C_{27}\right), 80.50\left(C_{11}\right), 79.28\left(C_{28}\right), 70.23\left(C_{18}\right), 47.71$ $\left(\mathrm{C}_{13}\right), 41.09\left(\mathrm{C}_{9}\right), 28.61\left(\mathrm{C}_{12}\right)$. HRMS (ESI-TOF): m/z 654.2732 $[\mathrm{M}+\mathrm{Na}]^{+}$(calcd 654.2727 for $\mathrm{C}_{42} \mathrm{H}_{37} \mathrm{~N}_{3} \mathrm{NaO}_{3}$ ). tert-butyl-(anthracen-9-ylmethyl)(4-((4-((3-ethynylphenyl) diazenyl)phenoxy)methyl)benzyl)carbamate (R4)

Column chromatography conditions $\left(\mathrm{SiO}_{2}\right.$ gel, hexane/AcOEt 3:1). (123mg, 65\% yield), red solid. ${ }^{1} \mathrm{H}$ NMR $\left(500 \mathrm{MHz}, \mathrm{CDCl}_{3}\right) \delta$ $8.43\left(\mathrm{~s}, 1 \mathrm{H}, \mathrm{H}_{1}\right), 8.20\left(\mathrm{br}, 1 \mathrm{H}, \mathrm{H}_{6}\right), 8.04-7.97\left(\mathrm{~m}, 3 \mathrm{H}, \mathrm{H}_{24}+\mathrm{H}_{3}\right)$, $7.95\left(\mathrm{~d}, J=9.1 \mathrm{~Hz}, 2 \mathrm{H}, \mathrm{H}_{21}\right), 7.88\left(\mathrm{dt}, J=8.0,1.3 \mathrm{~Hz}, 1 \mathrm{H}, \mathrm{H}_{28}\right)$, $7.56\left(\mathrm{dt}, J=7.6,1.3 \mathrm{~Hz}, 1 \mathrm{H}, \mathrm{H}_{26}\right), 7.49-7.36\left(\mathrm{~m}, 5 \mathrm{H}, \mathrm{H}_{27}+\mathrm{H}_{4}+\right.$ $\left.\mathrm{H}_{5}\right), 7.29\left(\mathrm{br}, 2 \mathrm{H}, \mathrm{H}_{16}\right), 7.10\left(\mathrm{~d}, J=9.1 \mathrm{~Hz}, 2 \mathrm{H}, \mathrm{H}_{20}\right), 6.94(\mathrm{br}, 2 \mathrm{H}$, $\left.\mathrm{H}_{15}\right), 5.58\left(\mathrm{br}, 2 \mathrm{H}, \mathrm{H}_{9}\right), 5.12\left(\mathrm{~s}, 2 \mathrm{H}, \mathrm{H}_{18}\right), 4.06\left(\mathrm{br}, 2 \mathrm{H}, \mathrm{H}_{13}\right), 3.14$ $\left(\mathrm{s}, 1 \mathrm{H}, \mathrm{H}_{30}\right), 1.73\left(\mathrm{br}, 3 \mathrm{H}, \mathrm{H}_{12^{\prime}}\right), 1.52\left(\mathrm{br}, 6 \mathrm{H}, \mathrm{H}_{12}\right) .{ }^{13} \mathrm{C}$ NMR $(126$ $\left.\mathrm{MHz}, \mathrm{CDCl}_{3}\right) \delta 161.63\left(\mathrm{C}_{19}\right), 156.03\left(\mathrm{C}_{10}\right), 152.63\left(\mathrm{C}_{23}\right), 147.11$ $\left(C_{22}\right), 138.72\left(C_{14}\right), 134.90\left(C_{17}\right), 133.82\left(C_{26}\right), 131.52\left(C_{2}+C_{8}\right)$, $131.45\left(C_{23}\right), 129.19\left(C_{3}+C_{27}+C_{7}\right), 128.41\left(C_{1}\right), 127.56\left(C_{16}\right)$, $127.19\left(C_{15}\right), 126.29\left(C_{5}\right), 126.14\left(C_{24}\right), 125.08\left(C_{4}+C_{21}\right), 124.56$ $\left(C_{6}\right), 123.44\left(C_{28}\right), 123.13\left(C_{25}\right), 115.31\left(C_{20}\right), 83.19\left(C_{29}\right), 80.44$ $\left(C_{11}\right), 77.91\left(C_{30}\right), 70.13\left(C_{18}\right), 47.73\left(C_{13}\right), 41.03\left(C_{9}\right), 28.59$ $\left(\mathrm{C}_{12}\right)$. HRMS (ESI-TOF): $\mathrm{m} / \mathrm{z} 654.2721[\mathrm{M}+\mathrm{Na}]^{+}$(calcd 654.2727 for $\mathrm{C}_{42} \mathrm{H}_{37} \mathrm{~N}_{3} \mathrm{NaO}_{3}$ ).

tert-butyl-(anthracen-9-ylmethyl)(4-((4-((4-ethynylphenyl) diazenyl)-2,6-dimethylphenoxy)methyl)benzyl)carbamate (R5)

Column chromatography conditions $\left(\mathrm{SiO}_{2}\right.$ gel, hexane/AcOEt $4: 1)$. (66mg, 40\% yield), deep red solid. ${ }^{1} \mathrm{H}$ NMR $(500 \mathrm{MHz}$, $\left.\mathrm{CDCl}_{3}\right) \delta 8.45\left(\mathrm{~s}, 1 \mathrm{H}, \mathrm{H}_{1}\right), 8.22\left(\mathrm{br}, 2 \mathrm{H}, \mathrm{H}_{6}\right), 8.00(\mathrm{~d}, J=7.8 \mathrm{~Hz}$, $\left.2 \mathrm{H}, \mathrm{H}_{3}\right), 7.86\left(\mathrm{~d}, J=8.7 \mathrm{~Hz}, 2 \mathrm{H}, \mathrm{H}_{25}\right), 7.66\left(\mathrm{~s}, 2 \mathrm{H}, \mathrm{H}_{22}\right), 7.63(\mathrm{~d}, J$ $\left.=8.7 \mathrm{~Hz}, 2 \mathrm{H}, \mathrm{H}_{26}\right), 7.50-7.40\left(\mathrm{~m}, 4 \mathrm{H}, \mathrm{H}_{4}+\mathrm{H}_{5}\right), 7.32(\mathrm{br}, 2 \mathrm{H}$, $\left.\mathrm{H}_{16}\right), 6.96\left(\mathrm{br}, 2 \mathrm{H}, \mathrm{H}_{15}\right), 5.58\left(\mathrm{br}, 2 \mathrm{H}, \mathrm{H}_{9}\right), 4.85\left(\mathrm{~s}, 2 \mathrm{H}, \mathrm{H}_{18}\right), 4.07$ (br, $\left.2 \mathrm{H}, \mathrm{H}_{13}\right), 3.22\left(\mathrm{~s}, 1 \mathrm{H}, \mathrm{H}_{29}\right), 2.40\left(\mathrm{~s}, 6 \mathrm{H}, \mathrm{H}_{21}\right), 1.73(\mathrm{br}, 3 \mathrm{H}$, $\left.\mathrm{H}_{12^{\prime}}\right), 1.52\left(\mathrm{br}, 6 \mathrm{H}, \mathrm{H}_{12}\right) .{ }^{13} \mathrm{C}\left\{{ }^{1} \mathrm{H}\right\} \mathrm{NMR}\left(126 \mathrm{MHz}, \mathrm{CDCl}_{3}\right) \delta 158.67$ $\left(C_{19}\right), 152.30\left(C_{24}\right), 148.75\left(C_{23}\right), 135.64\left(C_{17}\right), 132.96\left(C_{26}\right)$, $132.10\left(C_{20}\right), 131.33\left(C_{7}+C_{2}\right), 129.09\left(C_{3}\right), 128.27\left(C_{1}\right), 127.93$ $\left(C_{16}\right), 126.95\left(C_{15}\right), 126.16\left(C_{4}\right.$ or $\left.C_{5}\right), 124.97\left(C_{4}\right.$ or $\left.C_{5}\right), 124.45$ $\left(C_{6}\right), 124.24\left(C_{27}\right), 123.69\left(C_{22}\right), 122.61\left(C_{25}\right), 83.34\left(C_{28}\right), 80.28$ $\left(C_{11}\right), 79.25\left(C_{29}\right), 74.08\left(C_{18}\right), 47.57\left(C_{13}\right), 40.85\left(C_{9}\right), 28.44$ $\left(\mathrm{C}_{12}\right), 16.73\left(\mathrm{C}_{21}\right)$. HRMS (ESI-TOF): $\mathrm{m} / \mathrm{z} 682.3022[\mathrm{M}+\mathrm{Na}]^{+}$ (calcd 682.3040 for $\mathrm{C}_{44} \mathrm{H}_{41} \mathrm{~N}_{3} \mathrm{NaO}_{3}$ ).

tert-butyl (E)-(anthracen-9-ylmethyl)(4-((4-((4-(prop-2-yn-1-yloxy) phenyl)diazenyl)phenoxy)methyl)benzyl)carbamate (R6)

Column chromatography conditions $\left(\mathrm{SiO}_{2}\right.$ gel, hexane/AcOEt 5:1). (99mg, 60\% yield), deep red solid. ${ }^{1} \mathrm{H}$ NMR $(500 \mathrm{MHz}$, $\left.\mathrm{CDCl}_{3}\right) \delta 8.42\left(\mathrm{~s}, 1 \mathrm{H}, \mathrm{H}_{1}\right), 8.20\left(\mathrm{br}, 2 \mathrm{H}, \mathrm{H}_{6}\right), 8.00(\mathrm{~d}, J=7.7 \mathrm{~Hz}$ $\left.2 \mathrm{H}, \mathrm{H}_{3}\right), 7.91\left(\mathrm{~d}, J=7.5 \mathrm{~Hz}, 2 \mathrm{H}, \mathrm{H}_{21}\right.$ or $\left.\mathrm{H}_{24}\right), 7.90(\mathrm{~d}, J=7.5 \mathrm{~Hz}$, $2 \mathrm{H}, \mathrm{H}_{21}$ or $\left.\mathrm{H}_{24}\right), 7.50-7.38\left(\mathrm{~m}, 4 \mathrm{H}, \mathrm{H}_{4}+\mathrm{H}_{5}\right), 7.28\left(\mathrm{br}, 2 \mathrm{H}, \mathrm{H}_{16}\right)$, $7.09\left(\mathrm{~d}, J=7.5 \mathrm{~Hz}, 4 \mathrm{H}, \mathrm{H}_{20}+\mathrm{H}_{25}\right), 6.92\left(\mathrm{br}, 2 \mathrm{H}, \mathrm{H}_{15}\right), 5.59(\mathrm{br}$, $\left.2 \mathrm{H}, \mathrm{H}_{9}\right), 5.11\left(\mathrm{~s}, 2 \mathrm{H}, \mathrm{H}_{18}\right), 4.77\left(\mathrm{~d}, J=1.8 \mathrm{~Hz}, 2 \mathrm{H}, \mathrm{H}_{27}\right), 4.06(\mathrm{br}$, $\left.2 \mathrm{H}, \mathrm{H}_{13}\right), 2.57\left(\mathrm{t}, J=1.8 \mathrm{~Hz}, 1 \mathrm{H}, \mathrm{H}_{29}\right), 1.74\left(\mathrm{br}, 2 \mathrm{H}, \mathrm{H}_{12^{\prime}}\right), 1.53(\mathrm{br}$, $\left.6 \mathrm{H}, \mathrm{H}_{12}\right) .{ }^{13} \mathrm{C}\left\{{ }^{1} \mathrm{H}\right\}$ NMR $\left(126 \mathrm{MHz}, \mathrm{CDCl}_{3}\right) \delta 160.84\left(\mathrm{C}_{19}\right), 159.38$ $\left(C_{26}\right), 155.91\left(C_{8}\right.$ or $\left.C_{14}\right), 147.60\left(C_{22}\right.$ or $\left.C_{23}\right), 147.17\left(C_{22}\right.$ or $\left.C_{23}\right)$, 
$138.51\left(C_{8}\right.$ or $\left.C_{14}\right), 134.93\left(C_{17}\right), 131.32\left(C_{2}+C_{7}\right), 129.10\left(C_{3}\right)$, $128.27\left(C_{1}\right), 127.43\left(C_{16}\right), 127.04\left(C_{15}\right), 126.16\left(C_{5}\right), 124.97\left(C_{4}\right)$ $124.50-124.40\left(C_{6}+C_{21}+C_{23}+C_{24}\right), 115.12\left(C_{20}+C_{25}\right), 80.32$ $\left(C_{11}\right), 78.13\left(C_{28}\right), 75.94\left(C_{29}\right), 70.04\left(C_{18}\right), 56.00\left(C_{27}\right), 47.62$ $\left(C_{13}\right), 40.91\left(C_{9}\right), 28.46\left(C_{12}\right)$. HRMS (ESI-TOF): m/z 684.2830 $[\mathrm{M}+\mathrm{Na}]^{+}$(calcd 684.2833 for $\mathrm{C}_{43} \mathrm{H}_{39} \mathrm{~N}_{3} \mathrm{NaO}_{4}$ ).

Alternate procedure for the synthesis of phenolic ethers R3-R6 by Mitsunobu reaction

Benzyl alcohol R1 (107 mg, $0.25 \mathrm{mmol}$ ) and $\mathrm{PPh}_{3}(79 \mathrm{mg}, 0.3$ $\mathrm{mmol}$ ) were placed together in a Schlenk flask and dissolved in $5 \mathrm{~mL}$ of dry THF. The solution was degassed and cooled down to $0{ }^{\circ} \mathrm{C}$. Then, DIAD (59 $\left.\mu \mathrm{L}, 0.3 \mathrm{mmol}\right)$ was added. In a separate Schlenk, the appropriate phenol (A1, A2, A3 or A5, $0.3 \mathrm{mmol}$ ) dissolved in dry THF (4 mL) was added dropwise through a cannula to the previous mixture. After the addition, the resulting mixture was allowed to reach room temperature and stirred overnight. Once finished, all volatiles were removed in vacuo and the crude was purified by column chromatography ( $\mathrm{SiO}_{2}$ gel, hexane/AcOEt 2:1 in all cases) to give the expected product with yields below $25 \%$.

General procedure for the preparation of rotaxanes $1-\mathrm{H}-\mathrm{PF}_{6}, 2-\mathrm{H}-$ $\mathrm{PF}_{6}$ and 3-H-PF

Appropriate phenol ether ( $\mathbf{R} \mathbf{3}, \mathbf{R} \mathbf{4}$ or $\mathbf{R 6}, 0.15 \mathrm{mmol}$ ) was dissolved in a mixture of $\mathrm{CHCl}_{3}(13 \mathrm{~mL})$ and acetone $(4 \mathrm{~mL})$ in the presence of $\mathrm{DB} 24 \mathrm{C} 8(101 \mathrm{mg}, 0.23 \mathrm{mmol})$ under inert atmosphere. Then, TFA was added $(9 \mathrm{~mL})$ all at once. The colour turned from yellow/orange to deep red immediately. The mixture was stirred at room temperature for 1.5 hours. After such time, it was thoroughly concentrated in vacuo to give a red slurry which was redissolved in dried and degassed DCM (15 mL). [Cu(NCMe) $)_{4}$ PF 6 (56 mg, $\left.0.15 \mathrm{mmol}\right), \mathbf{S 1}(35 \mathrm{mg}$, $0.15 \mathrm{mmol})$, and 2,6-lutidine $(1.7 \mu \mathrm{L}, 0.015 \mathrm{mmol})$ were added under $\mathrm{N}_{2}$ atmosphere and the mixture was vigorously stirred for 4 days. Then, volatiles were removed and the resulting crude was purified by column chromatography $\left(\mathrm{SiO}_{2}\right.$ gel, $\mathrm{DCM} / \mathrm{MeOH}$ 95:5).

1-H-PF 6

(102mg, 50\% yield), orange solid. ${ }^{1} \mathrm{H}$ NMR $(500 \mathrm{MHz}$, acetone$\left.d_{6}\right) \delta 8.75\left(\mathrm{~s}, 1 \mathrm{H}, \mathrm{H}_{35}\right), 8.65\left(\mathrm{~d}, J=9.1 \mathrm{~Hz}, 2 \mathrm{H}, \mathrm{H}_{30}\right), 8.58(\mathrm{~d}, J=$ $\left.8.9 \mathrm{~Hz}, 2 \mathrm{H}, \mathrm{H}_{6}\right), 8.27\left(\mathrm{~s}, 1 \mathrm{H}, \mathrm{H}_{1}\right), 8.22\left(\mathrm{~s}, 1 \mathrm{H}, \mathrm{H}_{26}\right), 8.19(\mathrm{~d}, J=7.9$ $\left.\mathrm{Hz}, 1 \mathrm{H}, \mathrm{H}_{33}\right), 7.96\left(\mathrm{~d}, J=8.7 \mathrm{~Hz}, 2 \mathrm{H}, \mathrm{H}_{23}\right), 7.92(\mathrm{~d}, J=8.4 \mathrm{~Hz}, 2 \mathrm{H}$, $\left.\mathrm{H}_{3}\right), 7.87\left(\mathrm{~d}, J=9.1 \mathrm{~Hz}, 2 \mathrm{H}, \mathrm{H}_{13}\right), 7.82\left(\mathrm{~d}, J=8.7 \mathrm{~Hz}, 2 \mathrm{H}, \mathrm{H}_{22}\right)$, $7.71-7.63\left(\mathrm{~m}, 6 \mathrm{H}, \mathrm{H}_{31}+\mathrm{H}_{5}+\mathrm{H}_{19}\right), 7.61-7.54\left(\mathrm{~m}, 4 \mathrm{H}, \mathrm{H}_{32}+\right.$ $\left.\mathrm{H}_{18}\right), 7.48\left(\mathrm{dd}, J=8.4,6.5 \mathrm{~Hz}, 2 \mathrm{H}, \mathrm{H}_{4}\right), 7.16(\mathrm{~d}, J=9.1 \mathrm{~Hz}, 2 \mathrm{H}$, $\left.\mathrm{H}_{14}\right), 6.77\left(\mathrm{~s}, 2 \mathrm{H}, \mathrm{H}_{27}\right), 6.67-6.61\left(\mathrm{~m}, 4 \mathrm{H}, \mathrm{H}_{\mathrm{a}}\right), 6.48-6.42(\mathrm{~m}$, $\left.4 \mathrm{H}, \mathrm{H}_{\mathrm{b}}\right), 5.72-5.68\left(\mathrm{~m}, 2 \mathrm{H}, \mathrm{H}_{9}\right), 5.48-5.43\left(\mathrm{~m}, 2 \mathrm{H}, \mathrm{H}_{11}\right), 5.27$ $\left(\mathrm{s}, 2 \mathrm{H}, \mathrm{H}_{16}\right), 4.00-3.93\left(\mathrm{~m}, 4 \mathrm{H}, \mathrm{H}_{\mathrm{d}}\right), 3.91-3.83\left(\mathrm{~m}, 8 \mathrm{H}, \mathrm{H}_{\mathrm{d}^{\prime}}+\right.$ $\left.\mathrm{H}_{\mathrm{e}}\right), 3.80-3.76\left(\mathrm{~m}, 4 \mathrm{H}, \mathrm{H}_{\mathrm{f}}\right), 3.75-3.70\left(\mathrm{~m}, 4 \mathrm{H}, \mathrm{H}_{\mathrm{f}}\right) .{ }^{13} \mathrm{C}\left\{{ }^{1} \mathrm{H}\right\}$ NMR $\left(126 \mathrm{MHz}\right.$, acetone- $\left.\mathrm{d}_{6}\right) \delta 162.08\left(\mathrm{C}_{15}\right), 152.69\left(\mathrm{C}_{21}\right)$, $147.92\left(C_{12}\right), 147.73\left(C_{c}\right), 147.17\left(C_{25}\right), 138.09\left(C_{17}\right), 134.31$ $\left(C_{24}\right), 134.06\left(C_{20}\right), 132.56\left(C_{28}+C_{34}\right), 131.78\left(C_{7}+C_{29}\right), 131.64$ $\left(C_{1}+C_{2}\right), 130.44\left(C_{35}\right), 130.29\left(C_{3}\right), 130.22\left(C_{33}\right), 128.59\left(C_{18}\right)$, $128.34\left(C_{19}\right), 128.19\left(C_{31}\right), 128.03\left(C_{5}\right), 126.86\left(C_{23}\right), 126.28$ $\left(C_{32}\right), 125.87\left(C_{4}\right), 125.38\left(C_{13}\right), 124.86\left(C_{6}\right), 124.58\left(C_{30}\right), 123.78$ $\left(C_{22}\right), 122.32\left(C_{8}\right), 121.93\left(C_{a}\right), 121.72\left(C_{26}\right), 116.38\left(C_{14}\right), 112.80$ $\left(C_{b}\right), 71.84\left(C_{f}\right), 71.31\left(C_{e}\right), 70.27\left(C_{16}\right), 68.77\left(C_{d}\right), 53.42\left(C_{11}\right)$,
$47.03\left(C_{27}\right), 46.30\left(C_{9}\right)$. HRMS (ESI-TOF): $\mathrm{m} / \mathrm{z} 1213.5439$ [M$\left.\mathrm{PF}_{6}\right]^{+}$(calcd 1213,5434 for $\mathrm{C}_{76} \mathrm{H}_{73} \mathrm{~N}_{6} \mathrm{O}_{9}$ ).

\section{2-H-PF}

(102mg, 60\% yield), orange solid. ${ }^{1} \mathrm{H}$ NMR $(500 \mathrm{MHz}$, acetone$\left.d_{6}\right) \delta 8.75\left(\mathrm{~s}, 1 \mathrm{H}, \mathrm{H}_{37}\right), 8.66\left(\mathrm{~d}, J=8.9 \mathrm{~Hz}, 2 \mathrm{H}, \mathrm{H}_{32}\right), 8.58(\mathrm{~d}, J=$ $\left.9.0 \mathrm{~Hz}, 2 \mathrm{H}, \mathrm{H}_{6}\right), 8.30\left(\mathrm{t}, J=1.7 \mathrm{~Hz}, 1 \mathrm{H}, \mathrm{H}_{22}\right), 8.28\left(\mathrm{~s}, 1 \mathrm{H}, \mathrm{H}_{1}\right)$, $8.25\left(\mathrm{~s}, 1 \mathrm{H}, \mathrm{H}_{28}\right), 8.19\left(\mathrm{~d}, J=8.5 \mathrm{~Hz}, 1 \mathrm{H}, \mathrm{H}_{35}\right), 7.93(\mathrm{~d}, J=8.2 \mathrm{~Hz}$, $\left.1 \mathrm{H}, \mathrm{H}_{3}\right), 7.89-7.85\left(\mathrm{~m}, 4 \mathrm{H}, \mathrm{H}_{24}\right.$ or $\left.\mathrm{H}_{25}+\mathrm{H}_{10}+\mathrm{H}_{13}\right), 7.74(\mathrm{dt}, J=$ 7.9, $\left.1.4 \mathrm{~Hz}, 1 \mathrm{H}, \mathrm{H}_{26}\right), 7.70-7.64\left(\mathrm{~m}, 5 \mathrm{H}, \mathrm{H}_{5}+\mathrm{H}_{33}+\mathrm{H}_{24}\right.$ or $\left.\mathrm{H}_{25}\right)$, $7.61-7.55\left(\mathrm{~m}, 4 \mathrm{H}, \mathrm{H}_{34}+\mathrm{H}_{18}\right), 7.52-7.47\left(\mathrm{~m}, 4 \mathrm{H}, \mathrm{H}_{4}+\mathrm{H}_{19}\right)$, $7.17\left(\mathrm{~d}, J=9.0 \mathrm{~Hz}, 2 \mathrm{H}, \mathrm{H}_{14}\right), 6.77\left(\mathrm{~s}, 2 \mathrm{H}, \mathrm{H}_{29}\right), 6.68-6.63(\mathrm{~m}$, $\left.4 \mathrm{H}, \mathrm{H}_{\mathrm{a}}\right), 6.49-6.43\left(\mathrm{~m}, 4 \mathrm{H}, \mathrm{H}_{\mathrm{b}}\right), 5.74-5.68\left(\mathrm{~m}, 2 \mathrm{H}, \mathrm{H}_{9}\right), 5.51-$ $5.43\left(\mathrm{~m}, 2 \mathrm{H}, \mathrm{H}_{11}\right), 5.29\left(\mathrm{~s}, 2 \mathrm{H}, \mathrm{H}_{16}\right), 3.99-3.93\left(\mathrm{~m}, 4 \mathrm{H}, \mathrm{H}_{\mathrm{d}}\right), 3.92$ - $3.84\left(\mathrm{~m}, 8 \mathrm{H}, \mathrm{H}_{\mathrm{d}^{\prime}}+\mathrm{H}_{\mathrm{e}}\right), 3.82-3.76\left(\mathrm{~m}, 4 \mathrm{H}, \mathrm{H}_{\mathrm{e}^{\prime}}\right), 3.76-3.69(\mathrm{~m}$, $\left.4 \mathrm{H}, \mathrm{H}_{\mathrm{f}}\right), 3.55-3.48\left(\mathrm{~m}, 4 \mathrm{H}, \mathrm{H}_{\mathrm{f}^{\prime}}\right) .{ }^{13} \mathrm{C}\left\{{ }^{1} \mathrm{H}\right\} \mathrm{NMR}(126 \mathrm{MHz}$, acetone- $\left.d_{6}\right) \delta 161.27\left(\mathrm{C}_{15}\right), 152.94\left(\mathrm{C}_{17}\right), 146.93\left(\mathrm{C}_{12}\right), 146.85$ $\left(C_{c}\right), 146.37\left(C_{27}\right), 137.20\left(C_{7}\right), 133.17\left(C_{20}\right), 132.35\left(C_{2}\right), 131.66$ $\left(C_{36}\right), 130.90\left(C_{31}\right), 130.75\left(C_{1}\right), 129.54\left(C_{37}\right), 129.52\left(C_{4}\right.$ or $\left.C_{9}\right)$, $129.40\left(C_{3}\right), 129.31\left(C_{35}\right), 127.70\left(C_{18}\right.$ or $\left.C_{34}\right), 127.48\left(C_{13}, C_{24}\right.$ or $\left.C_{25}\right), 127.41\left(C_{21}\right), 127.26\left(C_{5}\right.$ or $\left.C_{33}\right), 127.14\left(C_{5}\right.$ or $\left.C_{33}\right), 125.36$ $\left(C_{18}\right.$ or $\left.C_{34}\right), 125.22\left(C_{30}\right), 124.97\left(C_{4}\right.$ or $\left.C_{19}\right), 124.56\left(C_{13}, C_{24}\right.$ or $\left.\mathrm{C}_{25}\right), 123.96\left(\mathrm{C}_{6}\right), 123.71\left(\mathrm{C}_{32}\right), 121.56\left(\mathrm{C}_{26}\right), 121.44\left(\mathrm{C}_{8}\right), 121.04$ $\left(C_{a}\right), 120.60\left(C_{28}\right), 119.09\left(C_{22}\right), 115.52\left(C_{14}\right), 111.92\left(C_{b}\right), 70.95$ $\left(C_{f}\right), 70.42\left(C_{e}\right), 69.38\left(C_{16}\right), 67.89\left(C_{d}\right), 52.54\left(C_{11}\right), 46.12\left(C_{29}\right)$, $45.42\left(\mathrm{C}_{9}\right)$. HRMS (ESI-TOF): $\mathrm{m} / \mathrm{z} 1213.5450$ [M-PF $\left._{6}\right]^{+}$(calcd 1213,5434 for $\mathrm{C}_{76} \mathrm{H}_{73} \mathrm{~N}_{6} \mathrm{O}_{9}$ ).

\section{3-H-PF 6}

(83mg, $40 \%$ yield), orange solid. ${ }^{1} \mathrm{H}$ NMR $(500 \mathrm{MHz}$, acetone$\left.d_{6}\right) \delta 8.72\left(\mathrm{~s}, 1 \mathrm{H}, \mathrm{H}_{36}\right), 8.62\left(\mathrm{~d}, J=8.9 \mathrm{~Hz}, 2 \mathrm{H}, \mathrm{H}_{31}\right), 8.58(\mathrm{~d}, J=$ $\left.9.0 \mathrm{~Hz}, 2 \mathrm{H}, \mathrm{H}_{6}\right), 8.27\left(\mathrm{~s}, 1 \mathrm{H}, \mathrm{H}_{1}\right), 8.17(\mathrm{dd}, J=8.5,0.7 \mathrm{~Hz}, 2 \mathrm{H}$, $\left.\mathrm{H}_{34}\right), 7.92\left(\mathrm{~d}, J=8.5 \mathrm{~Hz}, 2 \mathrm{H}, \mathrm{H}_{3}\right), 7.88\left(\mathrm{br}, 2 \mathrm{H}, \mathrm{H}_{10}\right), 7.84(\mathrm{~d}, J=$ $\left.9.0 \mathrm{~Hz}, 2 \mathrm{H}, \mathrm{H}_{19}\right), 7.82\left(\mathrm{~s}, 1 \mathrm{H}, \mathrm{H}_{27}\right), 7.79\left(\mathrm{~d}, J=9.0 \mathrm{~Hz}, 2 \mathrm{H}, \mathrm{H}_{22}\right)$, $7.69-7.63\left(\mathrm{~m}, 6 \mathrm{H}, \mathrm{H}_{5}+\mathrm{H}_{13}+\mathrm{H}_{32}\right), 7.59-7.55\left(\mathrm{~m}, 4 \mathrm{H}, \mathrm{H}_{14}+\right.$ $\left.\mathrm{H}_{33}\right), 7.51-7.46\left(\mathrm{~m}, 2 \mathrm{H}, \mathrm{H}_{4}\right), 7.15\left(\mathrm{~d}, J=9.0 \mathrm{~Hz}, 2 \mathrm{H}, \mathrm{H}_{18}\right), 7.09$ $\left(\mathrm{d}, J=9.0 \mathrm{~Hz}, 2 \mathrm{H}, \mathrm{H}_{23}\right), 6.71\left(\mathrm{~s}, 2 \mathrm{H}, \mathrm{H}_{28}\right), 6.68-6.62\left(\mathrm{~m}, 4 \mathrm{H}, \mathrm{H}_{\mathrm{a}}\right)$, $6.47-6.42\left(\mathrm{~m}, 4 \mathrm{H}, \mathrm{H}_{\mathrm{b}}\right), 5.74-5.67\left(\mathrm{~m}, 2 \mathrm{H}, \mathrm{H}_{9}\right), 5.50-5.42(\mathrm{~m}$, $\left.2 \mathrm{H}, \mathrm{H}_{11}\right), 5.26\left(\mathrm{~s}, 2 \mathrm{H}, \mathrm{H}_{16}\right), 5.14\left(\mathrm{~s}, 2 \mathrm{H}, \mathrm{H}_{25}\right), 3.99-3.92(\mathrm{~m}, 4 \mathrm{H}$, $\left.\mathrm{H}_{\mathrm{d}}\right), 3.92-3.84\left(\mathrm{~m}, 8 \mathrm{H}, \mathrm{H}_{\mathrm{d}^{\prime}}+\mathrm{H}_{\mathrm{e}}\right), 3.80-3.76\left(\mathrm{~m}, 4 \mathrm{H}, \mathrm{H}_{\mathrm{e}^{\prime}}\right), 3.75$ - $3.69\left(\mathrm{~m}, 4 \mathrm{H}, \mathrm{H}_{\mathrm{f}}\right), 3.54-3.48\left(\mathrm{~m}, 4 \mathrm{H}, \mathrm{H}_{\mathrm{f}^{\prime}}\right) .{ }^{13} \mathrm{C}\left\{{ }^{1} \mathrm{H}\right\}$ NMR $(126$ $\mathrm{MHz}$, acetone- $\left.d_{6}\right) \delta 160.68\left(\mathrm{C}_{17}+\mathrm{C}_{24}\right), 147.01\left(\mathrm{C}_{20}\right.$ or $\left.\mathrm{C}_{21}\right)$, $146.89\left(C_{20}\right.$ or $\left.C_{21}\right), 146.84\left(C_{c}\right), 142.99\left(C_{26}\right), 137.27\left(C_{15}\right)$, $133.13\left(C_{12}\right), 131.62\left(C_{2}\right.$ or $C_{7}$ or $C_{29}$ or $C_{30}$ or $\left.C_{35}\right), 130.88\left(C_{2}\right.$ or $C_{7}$ or $C_{29}$ or $C_{30}$ or $\left.C_{35}\right), 130.78\left(C_{1}\right), 130.74\left(C_{2}\right.$ or $C_{7}$ or $C_{29}$ or $C_{30}$ or $\left.C_{35}\right), 129.49\left(C_{36}\right), 129.40\left(C_{3}\right), 129.31\left(C_{34}\right), 127.69\left(C_{14}\right.$ or $\left.C_{33}\right), 127.44\left(C_{5}\right.$ or $C_{13}$ or $\left.C_{32}\right), 127.28\left(C_{5}\right.$ or $C_{13}$ or $\left.C_{32}\right)$, $127.14\left(C_{5}\right.$ or $C_{13}$ or $\left.C_{32}\right), 125.36\left(C_{14}\right.$ or $\left.C_{33}\right), 124.98\left(C_{4}\right), 124.11$ $\left(C_{19}\right.$ or $\left.C_{22}\right), 124.09\left(C_{19}\right.$ or $\left.C_{22}\right), 123.96\left(C_{6}\right), 123.66\left(C_{31}\right)$, $123.49\left(C_{27}\right), 121.43\left(C_{8}\right), 121.04\left(C_{a}\right), 115.42\left(C_{18}\right), 115.05\left(C_{23}\right)$, $111.90\left(C_{b}\right), 70.94\left(C_{f}\right), 70.41\left(C_{e}\right), 69.34\left(C_{16}\right), 67.87\left(C_{d}\right), 61.65$ $\left(\mathrm{C}_{25}\right), 52.53\left(\mathrm{C}_{11}\right), 45.84\left(\mathrm{C}_{28}\right), 45.41\left(\mathrm{C}_{9}\right)$. HRMS (ESI-TOF): $\mathrm{m} / \mathrm{z}$ $1243.5523\left[\mathrm{M}-\mathrm{PF}_{6}\right]^{+}$(calcd 1243,5539 for $\mathrm{C}_{77} \mathrm{H}_{75} \mathrm{~N}_{6} \mathrm{O}_{10}$ ). General procedure for the preparation of molecular shuttles 4-H$\mathrm{PF}_{6}, 5-\mathrm{H}-\mathrm{PF}_{6}$ and 6-H-PF

Rotaxane (1- $-\mathrm{PF}_{6}, \mathbf{2}-\mathrm{H}-\mathrm{PF}_{6}$ or $\left.\mathbf{3}-\mathrm{H}-\mathrm{PF}_{6} .0 .01 \mathrm{mmol}\right)$ was placed in a Schlenk flask under inert atmosphere and dissolved in 5 $\mathrm{mL}$ of dry DCM. To this solution, $\mathrm{Me}_{3} \mathrm{OBF}_{4}(2.4 \mathrm{mg}, 0.017$ $\mathrm{mmol}$ ) was added and the mixture was stirred four 1.5 hours. 
Then, it was diluted with DCM $(7 \mathrm{~mL})$ and a solution of $\mathrm{NH}_{4} \mathrm{PF}_{6}$ $(16.3 \mathrm{mg}, 0.1 \mathrm{mmol})$ in deionized water $(7 \mathrm{~mL})$ was poured. The biphasic mixture was vigorously stirred for 20 minutes and extracted with more DCM $(3 \times 7 \mathrm{~mL})$. All combined organic phases were concentrated and quickly purified by column chromatography $\left(\mathrm{SiO}_{2}\right.$ gel, $\mathrm{DCM} / \mathrm{MeOH}$ gradient elution from 95:5 to 75:25). Yellowish orange solid obtained in all cases.

\section{4-H-PF 6}

(13 mg, 85\% yield). ${ }^{1} \mathrm{H}$ NMR (500 MHz, acetone- $\left.d_{6}\right) \delta 9.13(\mathrm{~s}$, $\left.1 \mathrm{H}, \mathrm{H}_{26}\right), 8.90\left(\mathrm{~s}, 1 \mathrm{H}, \mathrm{H}_{36}\right), 8.67-8.57\left(\mathrm{~m}, 4 \mathrm{H}, \mathrm{H}_{6}+\mathrm{H}_{31}\right), 8.30(\mathrm{~s}$, $\left.1 \mathrm{H}, \mathrm{H}_{1}\right), 8.27\left(\mathrm{~d}, J=8.5 \mathrm{~Hz}, 2 \mathrm{H}, \mathrm{H}_{34}\right), 8.02\left(\mathrm{~d}, J=8.6 \mathrm{~Hz}, 2 \mathrm{H}, \mathrm{H}_{23}\right)$, $7.98-7.87\left(\mathrm{~m}, 6 \mathrm{H}, \mathrm{H}_{13}+\mathrm{H}_{3}+\mathrm{H}_{22}\right), 7.78-7.70\left(\mathrm{~m}, 2 \mathrm{H}, \mathrm{H}_{32}\right)$, $7.70-7.62\left(\mathrm{~m}, 6 \mathrm{H}, \mathrm{H}_{5}+\mathrm{H}_{19}+\mathrm{H}_{33}\right), 7.58\left(\mathrm{~d}, J=8.1 \mathrm{~Hz}, 2 \mathrm{H}, \mathrm{H}_{18}\right)$, $7.52-7.46\left(\mathrm{~m}, 2 \mathrm{H}, \mathrm{H}_{4}\right), 7.43\left(\mathrm{br}, 2 \mathrm{H}, \mathrm{H}_{10}\right), 7.23(\mathrm{~d}, J=9.1 \mathrm{~Hz}$, $\left.2 \mathrm{H}, \mathrm{H}_{14}\right), 7.13\left(\mathrm{~s}, 2 \mathrm{H}, \mathrm{H}_{28}\right), 6.71-6.60\left(\mathrm{~m}, 4 \mathrm{H}, \mathrm{H}_{\mathrm{a}}\right), 6.52-6.41$ $\left(\mathrm{m}, 4 \mathrm{H}, \mathrm{H}_{\mathrm{b}}\right), 5.78-5.66\left(\mathrm{~m}, 2 \mathrm{H}, \mathrm{H}_{9}\right), 5.51-5.44\left(\mathrm{~m}, 2 \mathrm{H}, \mathrm{H}_{11}\right)$, $5.29\left(\mathrm{~s}, 2 \mathrm{H}, \mathrm{H}_{16}\right), 4.47\left(\mathrm{~s}, 3 \mathrm{H}, \mathrm{H}_{27}\right), 4.01-3.96\left(\mathrm{~m}, 4 \mathrm{H}, \mathrm{H}_{\mathrm{d}}\right), 3.93$ $-3.86\left(\mathrm{~m}, 8 \mathrm{H}, \mathrm{H}_{\mathrm{d}^{\prime}}+\mathrm{H}_{\mathrm{e}}\right), 3.84-3.78\left(\mathrm{~m}, 4 \mathrm{H}, \mathrm{H}_{\mathrm{e}^{\prime}}\right), 3.78-3.72(\mathrm{~m}$, $\left.4 \mathrm{H}, \mathrm{H}_{\mathrm{f}}\right), 3.57-3.51\left(\mathrm{~m}, 4 \mathrm{H}, \mathrm{H}_{\mathrm{f}^{\prime}}\right) .{ }^{13} \mathrm{C}\left\{{ }^{1} \mathrm{H}\right\}$ NMR $(126 \mathrm{MHz}$, acetone- $\left.d_{6}\right) \delta 161.96\left(C_{15}\right), 153.88\left(C_{21}\right), 146.95\left(C_{12}\right), 146.86$ $\left(C_{26}+C_{c}\right), 142.19\left(C_{24}\right), 137.06\left(C_{17}\right), 133.24\left(C_{20}\right), 131.60\left(C_{35}\right)$, $131.43\left(C_{30}\right), 130.91\left(C_{2}\right), 130.74\left(C_{1}+C_{22}+C_{36}\right), 130.24\left(C_{25}\right)$, $129.41\left(C_{34}\right), 129.32\left(C_{3}\right), 127.79\left(C_{32}\right), 127.73\left(C_{18}\right), 127.49\left(C_{5}\right.$ or $C_{19}$ or $\left.C_{33}\right), 127.14\left(C_{5}\right.$ or $C_{19}$ or $\left.C_{33}\right), 125.55\left(C_{5}\right.$ or $C_{19}$ or $\left.C_{33}\right)$, $125.04\left(C_{13}\right), 124.98\left(C_{4}\right), 124.64\left(C_{29}\right), 124.01\left(C_{6}\right.$ or $\left.C_{31}\right)$, $123.97\left(C_{6}\right.$ or $\left.C_{31}\right), 123.03\left(C_{23}\right), 122.44\left(C_{8}\right), 121.45\left(C_{30}\right)$, $121.05\left(C_{a}\right), 115.61\left(C_{14}\right), 111.93\left(C_{b}\right), 70.96\left(C_{f}\right), 70.43\left(C_{e}\right)$, $69.50\left(C_{16}\right), 67.90\left(C_{d}\right), 52.54\left(C_{11}\right), 49.73\left(C_{28}\right), 45.43\left(C_{9}\right), 38.79$ $\left(\mathrm{C}_{27}\right)$. HRMS (ESI-TOF): $\mathrm{m} / \mathrm{z}$ 1373,5329 $\quad\left[\mathrm{M}-\mathrm{PF}_{6}\right]^{+} \quad$ (calcd 1373,5310 for $\left.\mathrm{C}_{77} \mathrm{H}_{76} \mathrm{~F}_{6} \mathrm{~N}_{6} \mathrm{O}_{9} \mathrm{P}\right)$.

\section{5-H-PF}

(12 mg, 80\% yield). ${ }^{1} \mathrm{H}$ NMR (500 MHz, acetone- $\left.d_{6}\right) \delta 9.78$ $\left(\mathrm{s}, 1 \mathrm{H}, \mathrm{H}_{28}\right), 8.85\left(\mathrm{~s}, 1 \mathrm{H}, \mathrm{H}_{38}\right), 8.75\left(\mathrm{~d}, J=8.9 \mathrm{~Hz}, 2 \mathrm{H}, \mathrm{H}_{33}\right)$, $8.59\left(\mathrm{~d}, J=8.7 \mathrm{~Hz}, 2 \mathrm{H}, \mathrm{H}_{6}\right), 8.29\left(\mathrm{~s}, 1 \mathrm{H}, \mathrm{H}_{1}\right), 8.23(\mathrm{~d}, J=$ $\left.8.5 \mathrm{~Hz}, 2 \mathrm{H}, \mathrm{H}_{36}\right), 8.20\left(\mathrm{~s}, 1 \mathrm{H}, \mathrm{H}_{22}\right), 8.08(\mathrm{~d}, J=7.9 \mathrm{~Hz}, 1 \mathrm{H}$, $\left.\mathrm{H}_{26}\right), 7.95-7.91\left(\mathrm{~m}, 4 \mathrm{H}, \mathrm{H}_{13}+\mathrm{H}_{3}\right), 7.90(\mathrm{~d}, J=7.9 \mathrm{~Hz}, 1 \mathrm{H}$, $\left.\mathrm{H}_{24}\right), 7.79\left(\mathrm{t}, J=7.9 \mathrm{~Hz}, 1 \mathrm{H}, \mathrm{H}_{25}\right), 7.75-7.66\left(\mathrm{~m}, 6 \mathrm{H}, \mathrm{H}_{34}+\right.$ $\left.\mathrm{H}_{5}+\mathrm{H}_{19}\right), 7.65-7.55\left(\mathrm{~m}, 4 \mathrm{H}, \mathrm{H}_{18}+\mathrm{H}_{35}\right), 7.53-7.47(\mathrm{~m}$, $\left.2 \mathrm{H}, \mathrm{H}_{4}\right), 7.23-7.19\left(\mathrm{~m}, 4 \mathrm{H}, \mathrm{H}_{14}+\mathrm{H}_{30}\right), 6.68-6.63(\mathrm{~m}$, $\left.4 \mathrm{H}, \mathrm{H}_{\mathrm{a}}\right), 6.50-6.44\left(\mathrm{~m}, 4 \mathrm{H}, \mathrm{H}_{\mathrm{b}}\right), 5.76-5.67\left(\mathrm{~m}, 2 \mathrm{H}, \mathrm{H}_{9}\right)$, $5.50-5.41\left(\mathrm{~m}, 2 \mathrm{H}, \mathrm{H}_{11}\right), 5.30\left(\mathrm{~s}, 2 \mathrm{H}, \mathrm{H}_{16}\right), 4.39(\mathrm{~s}, 3 \mathrm{H}$, $\left.\mathrm{H}_{29}\right), 4.01-3.94\left(\mathrm{~m}, 4 \mathrm{H}, \mathrm{H}_{\mathrm{d}}\right), 3.93-3.85\left(\mathrm{~m}, 8 \mathrm{H}, \mathrm{H}_{\mathrm{d}^{\prime}}+\right.$ $\left.\mathrm{H}_{\mathrm{e}}\right), 3.83-3.79\left(\mathrm{~m}, 4 \mathrm{H}, \mathrm{H}_{\mathrm{e}^{\prime}}\right), 3.78-3.71\left(\mathrm{~m}, 4 \mathrm{H}, \mathrm{H}_{\mathrm{f}}\right), 3.55$ - $3.49\left(\mathrm{~m}, 4 \mathrm{H}, \mathrm{H}_{\mathrm{f}^{\prime}}\right) .{ }^{13} \mathrm{C}\left\{{ }^{1} \mathrm{H}\right\} \mathrm{NMR}\left(126 \mathrm{MHz}\right.$, acetone- $\left.d_{6}\right) \delta$ $161.84\left(C_{15}\right), 152.84\left(C_{21}\right), 146.85\left(C_{c}+C_{12}\right), 142.73\left(C_{27}\right)$, $137.06\left(C_{17}\right), 133.25\left(C_{20}\right), 131.60\left(C_{37}\right), 131.44\left(C_{32}\right)$, $131.25\left(C_{24}\right), 130.90\left(C_{2}\right), 130.76\left(C_{1}+C_{38}\right), 130.46\left(C_{25}\right)$, $129.87\left(C_{28}\right), 129.40\left(C_{36}+C_{3}\right.$ or $\left.C_{13}\right), 127.86\left(C_{5}\right.$ or $C_{18}$ or $\mathrm{C}_{19}$ or $\mathrm{C}_{34}$ or $\left.\mathrm{C}_{35}\right), 127.73\left(\mathrm{C}_{5}\right.$ or $\mathrm{C}_{18}$ or $\mathrm{C}_{19}$ or $\mathrm{C}_{34}$ or $\left.\mathrm{C}_{35}\right)$, $127.48\left(C_{5}\right.$ or $C_{18}$ or $C_{19}$ or $C_{34}$ or $\left.C_{35}\right), 127.17\left(C_{5}\right.$ or $C_{18}$ or $\mathrm{C}_{19}$ or $\mathrm{C}_{34}$ or $\left.\mathrm{C}_{35}\right), 125.57\left(\mathrm{C}_{34}\right.$ or $\left.\mathrm{C}_{35}\right), 124.99\left(\mathrm{C}_{3}\right.$ or $\mathrm{C}_{13}+$ $\left.\mathrm{C}_{4}+\mathrm{C}_{26}\right), 124.12\left(\mathrm{C}_{23}\right), 123.98\left(\mathrm{C}_{6}\right), 123.81\left(\mathrm{C}_{33}\right), 123.61$ $\left(C_{22}\right), 122.15\left(C_{31}\right), 121.45\left(C_{8}\right), 121.04\left(C_{a}\right), 115.61\left(C_{14}\right)$, $111.92\left(C_{b}\right), 78.32\left(C_{16}\right), 70.97\left(C_{f}\right), 70.43\left(C_{e}\right), 67.90\left(C_{d}\right)$, $52.54\left(C_{11}\right), 49.85\left(C_{30}\right), 45.42\left(C_{9}\right), 38.64\left(C_{29}\right)$. HRMS (ESITOF): $\mathrm{m} / \mathrm{z} \quad 1373,5412 \quad\left[\mathrm{M}-\mathrm{PF}_{6}\right]^{+} \quad$ (calcd 1373,5310 for $\left.\mathrm{C}_{77} \mathrm{H}_{76} \mathrm{~F}_{6} \mathrm{~N}_{6} \mathrm{O}_{9} \mathrm{P}\right)$.

\section{6-H-PF 6}

(15 mg, 95\% yield). ${ }^{1} \mathrm{H}$ NMR (500 MHz, acetone- $d_{6}$ ) $\delta 8.96(\mathrm{~s}$, $\left.1 \mathrm{H}, \mathrm{H}_{27}\right), 8.88\left(\mathrm{~s}, 1 \mathrm{H}, \mathrm{H}_{37}\right), 8.59\left(\mathrm{~d}, J=9.0 \mathrm{~Hz}, 2 \mathrm{H}, \mathrm{H}_{6}\right), 8.53(\mathrm{~d}, J=$ $\left.8.9 \mathrm{~Hz}, 2 \mathrm{H}, \mathrm{H}_{32}\right), 8.28\left(\mathrm{~s}, 1 \mathrm{H}, \mathrm{H}_{1}\right), 8.25\left(\mathrm{~d}, J=8.4 \mathrm{~Hz}, 2 \mathrm{H}, \mathrm{H}_{35}\right)$, $7.93\left(\mathrm{~d}, J=8.5 \mathrm{~Hz}, 2 \mathrm{H}, \mathrm{H}_{3}\right), 7.90\left(\mathrm{br}, 2 \mathrm{H}, \mathrm{H}_{10}\right), 7.89-7.83(\mathrm{~m}$, $\left.4 \mathrm{H}, \mathrm{H}_{19}+\mathrm{H}_{22}\right), 7.73-7.69\left(\mathrm{~m}, 2 \mathrm{H}, \mathrm{H}_{33}\right), 7.69-7.65\left(\mathrm{~m}, 4 \mathrm{H}, \mathrm{H}_{5}+\right.$ $\left.\mathrm{H}_{13}\right), 7.64-7.62\left(\mathrm{~m}, 2 \mathrm{H}, \mathrm{H}_{34}\right), 7.58\left(\mathrm{~d}, J=8.3 \mathrm{~Hz}, 2 \mathrm{H}, \mathrm{H}_{14}\right), 7.52-$ $7.47\left(\mathrm{~m}, 2 \mathrm{H}, \mathrm{H}_{4}\right), 7.22-7.17\left(\mathrm{~m}, 4 \mathrm{H}, \mathrm{H}_{23}+\mathrm{H}_{18}\right), 7.09\left(\mathrm{~s}, 2 \mathrm{H}, \mathrm{H}_{29}\right)$, $6.68-6.63\left(\mathrm{~m}, 4 \mathrm{H}, \mathrm{H}_{\mathrm{a}}\right), 6.48-6.44\left(\mathrm{~m}, 4 \mathrm{H}, \mathrm{H}_{\mathrm{b}}\right), 5.73-5.70(\mathrm{~m}$, $\left.2 \mathrm{H}, \mathrm{H}_{9}\right), 5.62\left(\mathrm{~s}, 2 \mathrm{H}, \mathrm{H}_{25}\right), 5.49-5.45\left(\mathrm{~m}, 2 \mathrm{H}, \mathrm{H}_{11}\right), 5.27(\mathrm{~s}, 2 \mathrm{H}$, $\left.\mathrm{H}_{16}\right), 4.49\left(\mathrm{~s}, 3 \mathrm{H}, \mathrm{H}_{28}\right), 4.00-3.94\left(\mathrm{~m}, 4 \mathrm{H}, \mathrm{H}_{\mathrm{d}}\right), 3.93-3.86(\mathrm{~m}$, $\left.8 \mathrm{H}, \mathrm{H}_{\mathrm{d}^{\prime}}+\mathrm{H}_{\mathrm{e}}\right), 3.82-3.77\left(\mathrm{~m}, 4 \mathrm{H}, \mathrm{H}_{\mathrm{e}^{\prime}}\right), 3.77-3.72\left(\mathrm{~m}, 4 \mathrm{H}, \mathrm{H}_{\mathrm{f}}\right)$, $3.55-3.49\left(\mathrm{~m}, 4 \mathrm{H}, \mathrm{H}_{\mathrm{f}}\right) .{ }^{13} \mathrm{C}\left\{{ }^{1} \mathrm{H}\right\}$ NMR $\left(126 \mathrm{MHz}\right.$, acetone- $\left.d_{6}\right) \delta$ $161.03\left(C_{17}\right), 159.19\left(C_{24}\right), 147.78\left(C_{21}\right), 146.93\left(C_{20}\right), 146.84$ $\left(C_{c}\right), 139.86\left(C_{26}\right), 137.20\left(C_{15}\right), 133.17\left(C_{12}\right), 131.57\left(C_{36}\right)$, $131.29\left(C_{2}, C_{7}, C_{8}, C_{30}\right.$ or $\left.C_{31}\right), 131.11\left(C_{37}\right), 130.89\left(C_{2}, C_{7}, C_{8}\right.$, $C_{30}$ or $\left.C_{31}\right), 130.75\left(C_{1}\right), 129.92\left(C_{27}\right), 129.57\left(C_{35}\right), 129.40\left(C_{3}\right)$, $128.06\left(C_{33}\right), 127.69\left(C_{14}\right), 127.44\left(C_{5}\right.$ or $\left.C_{13}\right), 127.15\left(C_{5}\right.$ or $\left.C_{13}\right)$, $125.64\left(C_{34}\right), 124.99\left(C_{4}\right), 124.31\left(C_{19}\right.$ or $\left.C_{22}\right), 124.21\left(C_{19}\right.$ or $\left.C_{22}\right), 123.97\left(C_{6}\right), 123.09\left(C_{32}\right), 121.44\left(C_{2}, C_{7}, C_{8}, C_{30}\right.$ or $\left.C_{31}\right)$, $121.36\left(C_{2}, C_{7}, C_{8}, C_{30}\right.$ or $\left.C_{31}\right), 121.04\left(C_{a}\right), 115.42\left(C_{18}\right), 115.26$ $\left(C_{23}\right), 111.91\left(C_{b}\right), 70.96\left(C_{f}\right), 70.42\left(C_{e}\right), 69.41\left(C_{16}\right), 67.88\left(C_{d}\right)$, $58.31\left(C_{25}\right), 52.54\left(C_{11}\right), 49.97\left(C_{29}\right), 45.42\left(C_{9}\right), 38.50\left(C_{28}\right)$. HRMS (ESI-TOF): $\mathrm{m} / \mathrm{z} 629.2893\left[\mathrm{M}-2 \mathrm{PF}_{6}\right]^{2+}$ (calcd 629.2884 for $\mathrm{C}_{78} \mathrm{H}_{78} \mathrm{~N}_{6} \mathrm{O}_{10}$ ).

Synthesis of 1-(anthracen-9-yl)-N-(4-((4-((4-ethynylphenyl) diazenyl)phenoxy)methyl)benzyl)methanamine (7) in an attempt to prepare the corresponding ammonium salt

Phenol ether $\mathbf{R 3}$ (95 mg, $0.15 \mathrm{mmol}$ ) was dissolved in $\mathrm{CHCl}_{3}(10$ $\mathrm{mL}$ ) follwed by the addition of TFA $(5 \mathrm{~mL})$. The colour turned from orange to deep red. Stirred for $30 \mathrm{~min}$ and checked by ${ }^{1} \mathrm{H}-\mathrm{NMR}$ to show major decomposition. All volatiles were removed and the resulting slurry was immediately subjected to a separation by column chromatography $\left(\mathrm{SiO}_{2}\right.$ gel, Hex/AcOEt gradient elution from 2:1 to 1:2) to finally recover a yellow solid corresponding to deprotected amine $(24 \mathrm{mg}, 30 \%$ yield). ${ }^{1} \mathrm{H}$ NMR $\left(400 \mathrm{MHz} \mathrm{CDCl}_{3}\right) \delta 8.41\left(\mathrm{~s}, 1 \mathrm{H}, \mathrm{H}_{1}\right), 8.22(\mathrm{~d}, \mathrm{~J}=$ $\left.8.6 \mathrm{~Hz}, 2 \mathrm{H}, \mathrm{H}_{6}\right), 8.01\left(\mathrm{~d}, \mathrm{~J}=7.9 \mathrm{~Hz}, 2 \mathrm{H}, \mathrm{H}_{3}\right), 7.93(\mathrm{~d}, \mathrm{~J}=9.0 \mathrm{~Hz}$, $\left.2 \mathrm{H}, \mathrm{H}_{19}\right), 7.84\left(\mathrm{~d}, \mathrm{~J}=8.4 \mathrm{~Hz}, 2 \mathrm{H}, \mathrm{H}_{22}\right), 7.62\left(\mathrm{~d}, \mathrm{~J}=8.4 \mathrm{~Hz}, 2 \mathrm{H}, \mathrm{H}_{23}\right)$, $7.53-7.38\left(\mathrm{~m}, 8 \mathrm{H}, \mathrm{H}_{4}+\mathrm{H}_{5}+\mathrm{H}_{13}+\mathrm{H}_{14}\right), 7.10(\mathrm{~d}, \mathrm{~J}=9.0 \mathrm{~Hz}, 2 \mathrm{H}$, $\left.\mathrm{H}_{18}\right), 5.18\left(\mathrm{~s}, 2 \mathrm{H}, \mathrm{H}_{16}\right), 4.73\left(\mathrm{~s}, 2 \mathrm{H}, \mathrm{H}_{9}\right), 4.06\left(\mathrm{~s}, 2 \mathrm{H}, \mathrm{H}_{11}\right), 3.21$ (s, $\left.1 \mathrm{H}, \mathrm{H}_{26}\right) .{ }^{13} \mathrm{C}\left\{{ }^{1} \mathrm{H}\right\}$ NMR (101 MHz, $\left.\mathrm{cdcl}_{3}\right) \delta 161.67\left(\mathrm{C}_{17}\right), 152.50$ $\left(C_{21}\right), 147.26\left(C_{20}\right), 140.34\left(C_{12}\right), 135.42\left(C_{15}\right), 133.08\left(C_{23}\right)$, $131.69\left(C_{2}, C_{7}\right.$ or $\left.C_{8}\right), 131.36\left(C_{2}, C_{7}\right.$ or $\left.C_{8}\right), 130.49\left(C_{2}, C_{7}\right.$ or $\left.C_{8}\right)$, $129.30\left(C_{3}\right), 128.87\left(C_{4}, C_{5}, C_{13}\right.$ or $\left.C_{14}\right), 127.87\left(C_{4}, C_{5}, C_{13}\right.$ or $\left.C_{14}\right), 127.51\left(C_{1}\right), 126.25\left(C_{4}, C_{5}, C_{13}\right.$ or $\left.C_{14}\right), 125.09\left(C_{4}, C_{5}, C_{13}\right.$ or $\left.C_{14}+C_{19}\right), 124.23\left(C_{6}\right), 124.11\left(C_{24}\right), 122.69\left(C_{22}\right), 115.34$ $\left(C_{18}\right), 83.57\left(C_{25}\right), 79.25\left(C_{26}\right), 70.31\left(C_{16}\right), 53.93\left(C_{11}\right), 44.93$ $\left(\mathrm{C}_{9}\right)$. HRMS (ESI-TOF): $\mathrm{m} / \mathrm{z} 532.2384[\mathrm{M}+\mathrm{H}]^{+}$(calcd 532.2383 for $\mathrm{C}_{37} \mathrm{H}_{30} \mathrm{~N}_{3} \mathrm{O}$ ).

Synthesis of 1-(anthracen-9-y))-N-(4-((4-((4-(1-(anthracen-9-

ylmethyl)-1H-1,2,3-triazol-4-yl)phenyl)diazenyl)-2,6-

dimethylphenoxy)methyl) benzyl)methanamine (8) in an attempt to prepare the threaded compound with DB2C8

Compound $\mathbf{R 5}$ ( $99 \mathrm{mg}, 0.15 \mathrm{mmol}$ ) was dissolved in a mixture of $\mathrm{CHCl}_{3}(13 \mathrm{~mL})$ and acetone $(4 \mathrm{~mL})$ in the presence of DB24C8 
(101mg, $0.23 \mathrm{mmol}$ ) under inert atmosphere. Then, TFA was added $(9 \mathrm{~mL})$ all at once. The colour turned from yellow/orange to deep red immediately. The mixture was stirred at room temperature for 1.5 hours. If checked by ${ }^{1} \mathrm{H}$ NMR major decomposition is observed and the presence of pseudorotaxane is inexistent. After such time, it was thoroughly concentrated in vacuo to give a red slurry which was redissolved in dried and degassed DCM (15 mL). [Cu(NCMe) $\left.{ }_{4}\right]_{P F}$ (56 mg, $0.15 \mathrm{mmol}$ ), S1 (35 mg, $0.15 \mathrm{mmol}$ ), and 2,6-lutidine $(1.7 \mu \mathrm{L}, 0.015 \mathrm{mmol})$ were added under $\mathrm{N}_{2}$ atmosphere and the mixture was vigorously stirred for 4 days. Then, volatiles were removed and the resulting crude was purified by column chromatography $\left(\mathrm{SiO}_{2}\right.$ gel, $\mathrm{CHCl}_{3} / \mathrm{MeOH}$ gradient elution from 95:5 to 80:20) to give a red solid (35 mg, $30 \%$ yield). ${ }^{1} \mathrm{H}$ NMR (500 MHz, acetone- $\left.d_{6}\right) \delta 8.76\left(\mathrm{~s}, 1 \mathrm{H}, \mathrm{H}_{36}\right)$, $8.66\left(\mathrm{~d}, J=9.0 \mathrm{~Hz}, 2 \mathrm{H}, \mathrm{H}_{31}\right), 8.51\left(\mathrm{~s}, 1 \mathrm{H}, \mathrm{H}_{1}\right), 8.37(\mathrm{~d}, J=9.1 \mathrm{~Hz}$, $\left.2 \mathrm{H}, \mathrm{H}_{3}\right), 8.23\left(\mathrm{~s}, 1 \mathrm{H}, \mathrm{H}_{27}\right), 8.19\left(\mathrm{~d}, J=8.7 \mathrm{~Hz}, 2 \mathrm{H}, \mathrm{H}_{34}\right), 8.06(\mathrm{~d}, J$ $\left.=7.4 \mathrm{~Hz}, 2 \mathrm{H}, \mathrm{H}_{6}\right), 7.98\left(\mathrm{~d}, J=8.7 \mathrm{~Hz}, 2 \mathrm{H}, \mathrm{H}_{23}\right), 7.86(\mathrm{~d}, J=8.7 \mathrm{~Hz}$, $\left.2 \mathrm{H}, \mathrm{H}_{24}\right), 7.74-7.66\left(\mathrm{~m}, 2 \mathrm{H}, \mathrm{H}_{32}\right), 7.63\left(\mathrm{~s}, 2 \mathrm{H}, \mathrm{H}_{20}\right), 7.62-7.57$ $\left(\mathrm{m}, 2 \mathrm{H}, \mathrm{H}_{33}\right), 7.57-7.44\left(\mathrm{~m}, 8 \mathrm{H}, \mathrm{H}_{4}+\mathrm{H}_{5}+\mathrm{H}_{13}+\mathrm{H}_{14}\right), 6.77(\mathrm{~s}$, $\left.2 \mathrm{H}, \mathrm{H}_{28}\right), 4.97\left(\mathrm{~s}, 2 \mathrm{H}, \mathrm{H}_{16}\right), 4.72\left(\mathrm{~s}, 2 \mathrm{H}, \mathrm{H}_{11}\right), 4.12\left(\mathrm{~s}, 2 \mathrm{H}, \mathrm{H}_{9}\right)$, $2.38\left(\mathrm{~s}, 6 \mathrm{H}, \mathrm{H}_{19}\right) .{ }^{13} \mathrm{C}\left\{{ }^{1} \mathrm{H}\right\}$ NMR $(126 \mathrm{MHz}$, acetone) $\delta 158.59$ $\left(C_{17}\right), 151.87\left(C_{25}\right), 148.65\left(C_{21}\right), 146.27\left(C_{26}\right), 142.28\left(C_{7}\right)$, $136.17\left(C_{15}\right), 133.57\left(C_{22}\right), 132.13\left(C_{12}\right), 131.66\left(C_{2}+C_{35}\right)$, $130.91\left(C_{30}\right), 130.47\left(C_{8}\right), 129.53\left(C_{36}\right), 129.31\left(C_{34}\right), 128.83\left(C_{6}\right)$, $128.38\left(\mathrm{C}_{4}, \mathrm{C}_{5}, \mathrm{C}_{13}\right.$ or $\left.\mathrm{C}_{14}\right), 128.21\left(\mathrm{C}_{4}, \mathrm{C}_{5}, \mathrm{C}_{13}\right.$ or $\left.\mathrm{C}_{14}\right), 127.29$ $\left(C_{32}\right), 126.87\left(C_{1}\right), 125.97\left(C_{23}\right), 125.71\left(C_{4}, C_{5}, C_{13}\right.$ or $\left.C_{14}\right)$, $125.37\left(C_{33}\right), 124.92\left(C_{4}, C_{5}, C_{13}\right.$ or $\left.C_{14}\right), 124.70\left(C_{3}\right), 123.68$ $\left(C_{31}\right), 123.31\left(C_{20}\right), 122.96\left(C_{24}\right), 120.85\left(C_{27}\right), 73.92\left(C_{16}\right), 53.62$ $\left(C_{9}\right), 46.13\left(C_{28}\right), 44.76\left(C_{11}\right), 15.92\left(C_{19}\right)$. HRMS (ESI-TOF): $\mathrm{m} / \mathrm{z}$ $792.3581[\mathrm{M}+\mathrm{H}]^{+}$(calcd 792.3577 for $\mathrm{C}_{54} \mathrm{H}_{45} \mathrm{~N}_{6} \mathrm{O}$ ).

\section{Acknowledgements}

This work was funded by the Spanish Ministerio de Economía y Competitividad (CTQ 2013-41067-P). H. B. acknowledges with thanks a MEC-FPI grant.

\section{Notes and references}

${ }^{1}$ (a) V. Balzani, A. Credi and M. Venturi, Molecular Devices and Machines - A Journey into the Nano World., Wiley-VCH, Weinheim, 2003. (b) M. Schliwa, Molecular Motors WileyVCH, 2006. (c) V. Balzani, A. Credi and M. Venturi, Molecular Devices and Machines: Concepts and Perspectives for the Nanoworld, Second Edition, Wiley-VCH Verlag, Weinheim, 2008. (d) B. L. Feringa and W. R. Browne, Molecular Switches, Revised and Enlarged Edition, Wiley, 2011. (e) Many, From Non-Covalent Assemblies to Molecular Machines, Wiley-VCH, Weinheim, 2011. (f) J. Wang, Nanomachines: Fundamentals and Applications, Wiley-VCh Verlag, Weinheim, 2013. (g) A. Credi, S. Silvi and M. Venturi, Molecular Machines and Motors, Springer International Publishing, London, 2014.

2 (a) H. Hess and V. Vogel, Rev. Mol. Biotechnol., 2001, 82, 67-85.(b) C. A. Schalley, F. Vögtle and K. Beizai, Acc. Chem. Res., 2001, 34, 465-476. (c) V. Balzani, Photochem. Photobiol.
Sci., 2003, 2, 459-476. (d) A. P. de Silva and N. D. McClenaghan, Chem. Eur. J., 2004, 10, 574-586. (e) D. A. Leigh, F. Zerbetto and E. R. Kay, Angew. Chem. Int. Ed., 2007, 46, 72-191. (f) Y. Makita, N. Kihara and T. Takata, J. Org. Chem., 2008, 73, 9245-9250. (g) A. C. Fahrenbach, S. C. Warren, J. T. Incorvati, A.-J. Avestro, J. C. Barnes, J. F. Stoddart and B. A. Grzybowski, Adv. Mater., 2013, 25, 331348. (h) V. Blanco, D. A. Leigh and V. Marcos, Chem. Soc. Rev., 2015, 44, 5341-5370. (i) T. Nitta and H. Hess, Cell. Mol. Bioeng., 2013, 6, 109-115. (j) U. Lüning, B. Hesseler, M. Zindler and R. Herges, Eur. J. Org. Chem., 2014, 2014, 38853901. (k) S. Erbas-Cakmak, D. A. Leigh, C. T. McTernan and A. L. Nussbaumer, Chem. Rev., 2015, 115, 10081-10206. (I) E. R. Kay and D. A. Leigh, Angew. Chem. Int. Ed., 2015, 54, 1008010088. (m) Z. Meng, J.-F. Xiang and C.-F. Chen, J. Am. Chem. Soc., 2016, 138, 5652-5658.

${ }^{3}$ (a) A. Cembran, F. Bernardi, M. Garavelli, L. Gagliardi and G. Orlandi, J. Am. Chem. Soc., 2004, 126, 3234-3243. (b) S. C. Burdette and H. M. D. Bandara, Chem. Soc. Rev., 2012, 41, 1809-1825. (c) L. Schweighauser, M. A. Strauss, S. Bellotto and H. A. Wegner, Angew. Chem. Int. Ed., 2015, 54, 1343613439.

${ }^{4}$ (a) S. Giordani and M. Natali, Chem. Soc. Rev., 2012, 41, 4010-4029. (b) E. Merino and M. Ribagorda, Beilstein J. Org. Chem., 2012, 8, 20. (c) R. Gostl, A. Senf and S. Hecht, Chem. Soc. Rev., 2014, 43, 1982-1996. (d) D. Bléger and S. Hecht, Angew. Chem. Int. Ed., 2015, 54, 11338-11349. (e) D.-H. Qu, Q.-C. Wang, Q.-W. Zhang, X. Ma and H. Tian, Chem. Rev., 2015, 115, 7543-7588.

${ }^{5}$ (a) H. Murakami, A. Kawabuchi, K. Kotoo, M. Kunitake and N. Nakashima, J. Am. Chem. Soc., 1997, 119, 7605-7606. (b) V. Balzani, A. Credi and M. Venturi, Chem. Soc. Rev., 2009, 38, 1542-1550. (c) A. Credi, S. Silvi and M. Venturi, Chem. Commun., 2011, 47, 2483-2489. (d) M. Baroncini, S. Silvi, M. Venturi and A. Credi, Angew. Chem. Int. Ed., 2012, 51, 42234226. (e) M. Xue, Y. Yang, X. Chi, X. Yan and F. Huang, Chem. Rev., 2015, 115, 7398-7501.

${ }^{6}$ (a) J. F. Stoddart, A. Coskun, D. C. Friedman, H. Li, K. Patel and H. A. Khatib, J. Am. Chem. Soc., 2009, 131, 2493-2495. (b) J. F. Stoddart, M. Venturi, A. Credi, T. Avellini, H. Li, A. Coskun, G. Barin, A. Trabolsi, A. N. Basuray, S. K. Dey and S. Silvi, Angew. Chem. Int. Ed., 2012, 51, 1611-1615.

${ }^{7}$ C. M. Álvarez, H. Barbero and D. Miguel, Eur. J. Org. Chem., 2015, 2015, 6631-6640.

${ }^{8}$ E. Merino, Chem. Soc. Rev., 2011, 40, 3835-3853.

${ }^{9}$ (a) H. C. Kolb, M. G. Finn and K. B. Sharpless, Angew. Chem. Int. Ed., 2001, 40, 2004-2021. (b) J. F. Stoddart, A. Ivan, M. O. S, D. W. R, I. Kyosuke, Y. Takuma and K. Takashi, Bull. Chem. Soc. Jpn., 2007, 80, 13. (c) J. Lahann, Click Chemistry for Biotechnology and Materials Science, John Wiley \& Sons Ltd, West Sussex, 2009. (d) K. D. Hanni and D. A. Leigh, Chem. Soc. Rev., 2010, 39, 1240-1251.

${ }^{10}$ (a) O. Mitsunobu, M. Wada and T. Sano, J. Am. Chem. Soc., 1972, 94, 679-680. (b) K. C. K. Swamy, N. N. B. Kumar, E. Balaraman and K. V. P. P. Kumar, Chem. Rev., 2009, 109, 2551-2651. 
${ }^{11}$ (a) A. Williamson, Philos. Mag., 1850, 37, 350-356. (b) S. Mandal, S. Mandal, S. K. Ghosh, P. Sar, A. Ghosh, R. Saha and B. Saha, RSC Adv., 2016, 6, 69605-69614.

12 See ESI for more details about the theoretical model followed.

${ }^{13}$ Obtention of transition states is a very difficult issue, especillay in cases similar to this one where there are many degrees of freedom and potential energy surface is a function of multiple reaction coordinates. According to that, excessive efforts should be applied to compute data that are far from the main topic of this work-

14 a) J. L Sessler, P. Gale and W.-S. Cho, Anion Receptor Chemistry, RSC Publishing, Cambridge, UK, 2006. b) P. A. Gale, W. Dehaen, Anion Recognition in Supramolecular Chemistry, Springer, Heidelberg, Germany, 2010. c) N.H. Evans, P.D Beer, Angew. Chem. Int. Ed. 2014, 53, 1171611754.

15 a) B. R. Mullaney, A. L. Thompson, P. D. Beer, Angew. Chem. Int. Ed. 2014, 53, 11458-11462- b) B. R. Mullaney, B. E. Partridge, P. D. Beer, Chem. Eur. J. 2015, 21, 1660-1665. c) S. P. Cornes, C. H. Davies, D. Blyghton, M. R. Sambrook, P. D. Beer, Org. Biomol. Chem. 2015, 13, 2582-2587.

${ }^{16}$ (a) W. L. F. Armarego and C. L. L. Chai, Purification of Laboratory Chemicals, Elsevier, Bodmin, 5th edn., 2003. (b) D. B. G. Williams and M. Lawton, J. Org. Chem., 2010, 75, 8351-8354.

17 (a) S. Nishihara, K. Watari and H. Kouzai, J. Jap. Soc. Col. Mater., 2004, 77, 355-358. (b) Z. Jiang, X. Chen, J. Zhang, H. Zhang, G. Shi, Y. Li and Y. Song, Dyes and Pigments, 2008, 77, 223-228. (c) X. Xue, J. Zhu, Z. Zhang, Z. Cheng, Y. Tu and X. Zhu, Polymer, 2010, 51, 3083-3090. (d) V. Chandrasekaran and T. K. Lindhorst, Chem. Commun., 2012, 48, 7519-7521. (e) Z. Xie, H. He, Y. Deng, X. Wang and C. Liu, J. Mater. Chem. C, 2013, 1, 1791-1797. (f) A. Bergen, S. Rudiuk, M. Morel, T. Le Saux, H. Ihmels and D. Baigl, Nano Lett., 2016, 16, 773780 . 


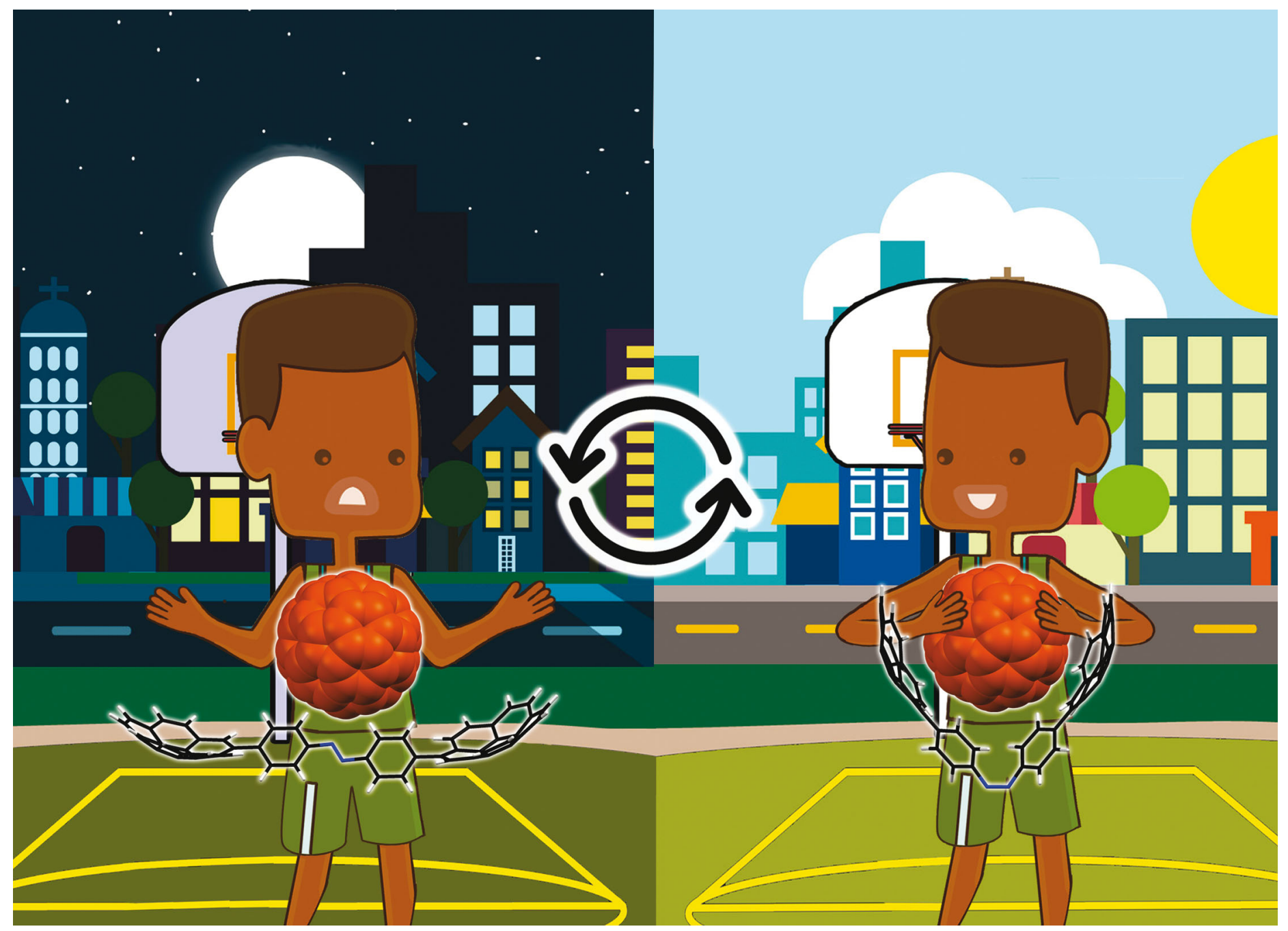

Showcasing research from Dr Alvarez and co-workers, MIOMeT Lab, IU CINQUIMA/Química Inorgánica, Facultad de Ciencias, Universidad de Valladolid, Valladolid, Spain.

Affinity modulation of photoresponsive hosts for fullerenes: light-gated corannulene tweezers

A new azobenzene-based molecular tweezer bearing corannulene subunits has been prepared. Its ability to modulate the interaction with fullerenes has been assessed demonstrating an on/off behaviour. The work is completed with a set of polyaromatic units tethered by azobenzene group. 


\section{COMMUNICATION}

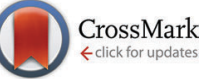

Cite this: Chem. Commun., 2016, 52, 12964

Received 4th August 2016,

Accepted 6th September 2016

DOI: $10.1039 / \mathrm{c} 6 \mathrm{cc} 06445 \mathrm{k}$

www.rsc.org/chemcomm

\section{Affinity modulation of photoresponsive hosts for fullerenes: light-gated corannulene tweezers $\dagger$}

\author{
Héctor Barbero, Sergio Ferrero, Lucía Álvarez-Miguel, Patricia Gómez-Iglesias, \\ Daniel Miguel and Celedonio M. Álvarez*
}

Six azobenzene derivatives bearing polyaromatic fragments have been prepared and their reversible photoisomerization has been assessed. Corannulene-functionalized molecules have demonstrated excellent switchable hosting abilities towards fullerenes in which an interesting range of affinities has been found. The success of this design relies upon the reversible formation and destruction of tweezer-like structures.

The study of supramolecular chemistry has been a hot topic in science since the end of last century, ${ }^{1}$ and a great development of a wide variety of concepts has been made in order to get a deep understanding of the assembly among molecular entities through weak and reversible interactions. One of such concepts consists of using molecular tweezers as hosts for recognizing a variety of molecules, in which fullerenes occupy an important position. ${ }^{2}$ The main reason to achieve such an interaction relies upon a complementarity between a curved fullerene and the receptor molecule, whose structure matches the surface of the guest. Several approaches have been carried out, one of them being the use of corannulene $e^{3}$ due to its nonplanar structure, commonly known as buckybowl. Convex and concave faces have different electron densities, allowing dipolar interactions and giving rise to interesting properties. ${ }^{4}$

Of particular interest are receptors that can modulate or even change totally their affinity by an external stimulus, such as light. ${ }^{5}$ One of the most used photoresponsive moieties is azobenzene, which can be switched between both configurational isomers with a simple input of light. ${ }^{6}$ Thus, several studies have been performed using this molecule in order to carry out considerably different works, ${ }^{7}$ but none of them dealt with corannulene-fullerene interactions. ${ }^{8}$ Herein, we present the first corannulene derivatives that contain azobenzene as a tether resulting in photoresponsive tweezers. Along with these

GIR MIOMeT, IU CINQUIMA/Química Inorgánica, Facultad de Ciencias,

Universidad de Valladolid, E-47011, Valladolid, Spain.

E-mail: celedonio.alvarez@uva.es

$\dagger$ Electronic supplementary information (ESI) available. CCDC 1494861-1494864. For ESI and crystallographic data in CIF or other electronic format see DOI: 10.1039/c6cc06445k

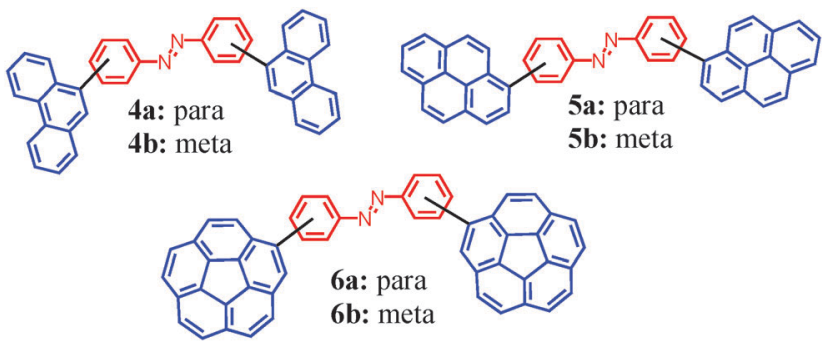

Fig. 1 Six azobenzene derivatives prepared in this work.

compounds, other planar polycyclic aromatic hydrocarbon derivatives, such as phenanthrene and pyrene, have been prepared in the same way in order to complete the whole family and for comparison purposes (Fig. 1).

The preparation of all azobenzene-containing PAHs followed two different routes depending on the location of pinacol boronate groups prior to Suzuki-Miyaura C-C coupling (see the $\mathrm{ESI} \dagger$ for a more detailed discussion about the most preferable chosen route).

Compounds 4, 5 and 6 were fully characterized using NMR, UV-Vis absorption spectra and mass spectra (see the ESI $\dagger$ ). Single crystals suitable for X-ray diffraction of phenanthrene compounds $4 \mathbf{a}$ and $\mathbf{4 b}$ and corannulene derivative $\mathbf{6 b}$ were obtained, showing their structures in the solid state, as depicted in Fig. 2. Phenylene moieties and nitrogen atoms of the azobenzene are placed in the same plane in all compounds, whereas phenanthrene and corannulene substituents have a $\mathrm{CH}-\mathrm{C}-\mathrm{C}-\mathrm{CH}$

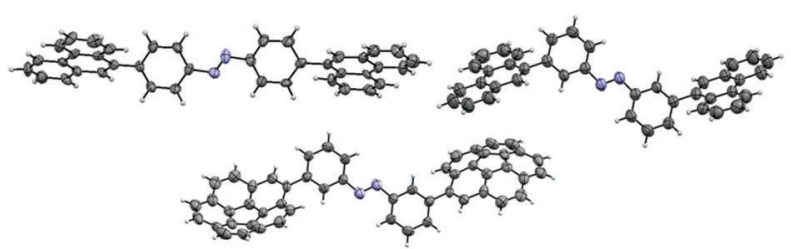

Fig. 2 Crystal structures of $\mathbf{4 a}$ and $\mathbf{4 b}$ (top), and $\mathbf{6 b}$ (bottom). Ellipsoids at $50 \%$ probability. 

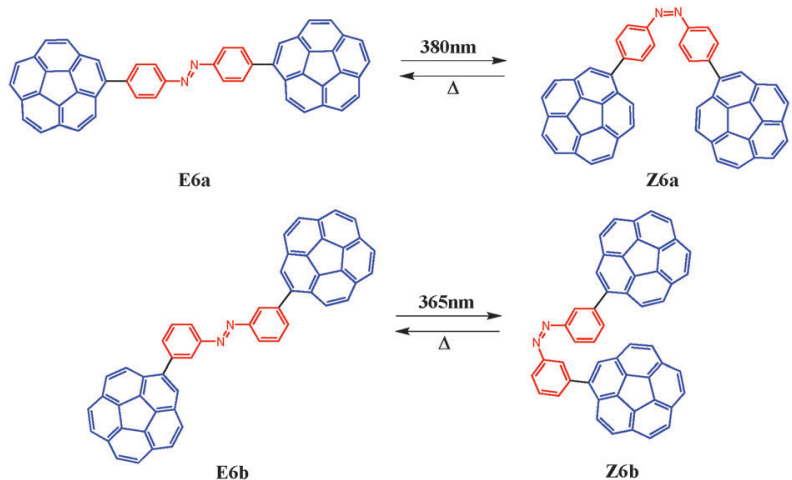

Scheme 1 Example of the switching process between corannulenefunctionalized azobenzenes, depicting tweezer-like preorganization for $6 \mathbf{a}$ upon conversion to the $Z$ isomer.

dihedral angle of around 50 degrees with respect to azobenzene; $50.4^{\circ}$ (in 4a), $59.5^{\circ}$ (in $4 \mathbf{b}$ ) and $47.21^{\circ}$ (in 6b). In meta-substituted molecules, both substituents are in an expected trans configuration to avoid steric constraints of the cis configuration.

Photoisomerization (Scheme 1) was studied in toluene. UV-Vis absorption and ${ }^{1} \mathrm{H}-\mathrm{NMR}$ spectra were recorded to check the process. Initially, all compounds show an $E / Z$ ratio of around $80: 20$. After heating for 30 minutes at $80{ }^{\circ} \mathrm{C}$, this ratio is shifted to $95: 5$. Irradiation with $365 \mathrm{~nm}$ at room temperature gives rise to signals at higher fields, indicating the presence of $Z$ isomers with different $E / Z$ ratios (Fig. S75, ESI $\dagger$ ). It's known that this wavelength is commonly used for switching azobenzene derivatives, but is not optimum for our corannulene and pyrene-substituted azobenzenes (5 and 6) whose $\pi \rightarrow \pi^{*}$ band appears at longer wavelengths for para substituted and shorter for meta substituted. Indeed, if irradiated at $380 \mathrm{~nm}$, compounds $\mathbf{5 a}$ and $\mathbf{6 a}$ are more efficiently switched as it can be seen in the UV-Vis spectrum (Fig. 3 and Fig. S71, S73, ESI $\dagger$ ) and, more explicitly, in their ${ }^{1} \mathrm{H}-\mathrm{NMR}$ spectra (Fig. 4 and Fig. S55-S58, ESI $\dagger$ ).

After determining the structures of all compounds and the extent of photoisomerization, all compounds were subjected to preliminary supramolecular association tests in both photostationary states by recording ${ }^{1} \mathrm{H}-\mathrm{NMR}$ spectra in toluene- $\mathrm{d}_{8}$. The addition of an excess of $\mathrm{C}_{60}$ or $\mathrm{C}_{70}$ can produce chemical shift changes indicating supramolecular association events.

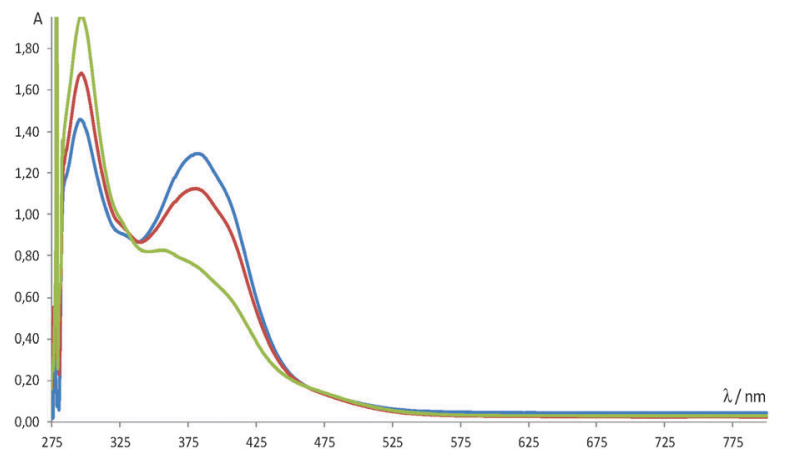

Fig. 3 UV-Vis spectra of $\mathbf{6 a}$ in toluene (blue), after irradiation for 15 minutes at $365 \mathrm{~nm}$ (red) and after irradiation for 15 minutes at $380 \mathrm{~nm}$ (green).

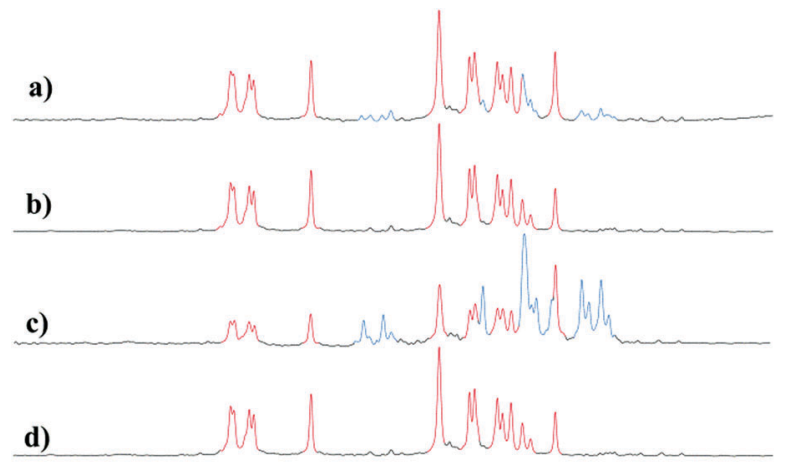

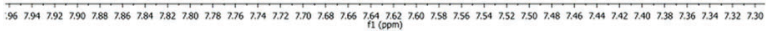

Fig. $4{ }^{1} \mathrm{H}-\mathrm{NMR}$ spectra $(500 \mathrm{MHz})$ of $\mathbf{6 a}$ in toluene-d 8 . (a) Initial spectrum; (b) after being heated at $80^{\circ} \mathrm{C}$ for 15 minutes and cooled down to rt; (c) after irradiation at $380 \mathrm{~nm}$ for 30 minutes; (d) thermal conversion to the initial state by heating the sample at $80{ }^{\circ} \mathrm{C}$ for other 15 minutes and cooling down to rt. The red color corresponds to the $E$ isomer and the blue color to the $Z$ isomer.

As expected, compounds 4 showed no appreciable changes in chemical shift. Moreover, compounds $\mathbf{5}$ followed the same trend despite the ability of pyrene to establish strong association with polyaromatic architectures. ${ }^{9}$ We expected, at least, a subtle interaction with the central part of $\mathrm{C}_{70}$ but that was not observed. On the other hand, corannulene-functionalized azobenzenes $\mathbf{6}$ gave positive results. In the case of $\mathbf{6 a}$ we found a great explicit affinity modulation towards fullerenes.

Compound E6a does not establish supramolecular association with $\mathrm{C}_{60}$ or $\mathrm{C}_{70}$ at all. ${ }^{1} \mathrm{H}-\mathrm{NMR}$ spectra recorded after additions of fullerenes remained unchanged (Fig. S88 and S89, ESI $\dagger)$. As expected, its structure is not suitable to host the guest in a tweezer-like manner (Scheme 1, top left). However, Z6a (Scheme 1, top right) is capable of hosting fullerenes with estimated association constants $\left(K_{\mathrm{a}}\right)$ of $(2.6 \pm 0.3) \times 10^{3} \mathrm{M}^{-1}$ and $(2.5 \pm 0.3) \times 10^{3} \mathrm{M}^{-1}$ for $\mathrm{C}_{60}$ and $\mathrm{C}_{70}$, respectively (Fig. 5 and Fig. S90-S95, ESI $\dagger$ ), which are similar to those obtained for other bis-corannulene derivatives. ${ }^{3 b, d_{2} f, h}$ This molecule forms $1: 1$ adducts with both fullerenes and no discrimination is observed since both constants are almost identical. In light of these results, an on-off behavior is suggested that can be repeated for several cycles, making this compound a light-gated supramolecular tweezer.

Compound E6b, in contrast, shows a very different behavior (Scheme 1, bottom left). Supramolecular association is established with $\mathrm{C}_{60}$ and $\mathrm{C}_{70}$ having constants of $(5.0 \pm 0.1) \times 10^{2} \mathrm{M}^{-1}$ and $(8.3 \pm 0.1) \times 10^{2} \mathrm{M}^{-1}$ in toluene- $\mathrm{d}_{8}$, respectively (Fig. 6 and Fig. S76-S81, ESI $\dagger$ ), corresponding to a $1: 1$ stoichiometry according to Job plots. The switched form of that compound, Z6b (Scheme 1, bottom right), shows abilities to host fullerenes too. The association constants of $\mathbf{C}_{60} @ \mathbf{Z 6 b}$ and $\mathbf{C}_{\mathbf{7 0}} @ \mathbf{Z 6 b}$ were estimated to be $(6.7 \pm 0.2) \times 10^{2} \mathrm{M}^{-1}$ and $(2.5 \pm 0.3) \times 10^{2} \mathrm{M}^{-1}$ (1:1 stoichiometry). The new structure has affected its ability to host fullerenes. The affinity for $\mathrm{C}_{60}$ is still moderate, although it is slightly better than before. In the case of $\mathrm{C}_{70}$ we found a constant notably worse than that estimated for E6b. Its affinity 


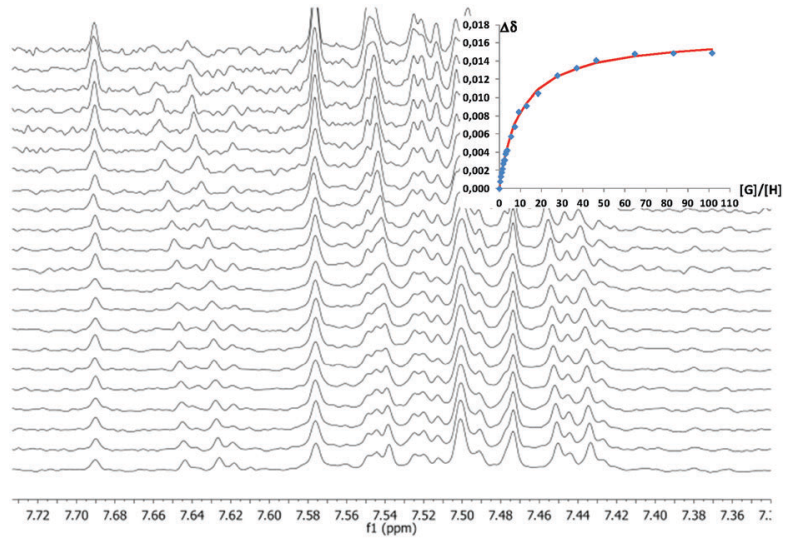

Fig. $5{ }^{1} \mathrm{H}$-NMR spectra $\left(500 \mathrm{MHz}\right.$, toluene- $\left.d_{8}\right)$ in the supramolecular titration of Z6a with $\mathrm{C}_{60}$. Inset: Plot of changes in chemical shift against $[G] /[H]$, where $G$ is $C_{60}$ and $H$ is $\mathbf{Z 6 a}$. The red line corresponds to the nonlinear fitting of $\Delta \delta$ for one proton to a $1: 1$ binding isotherm.

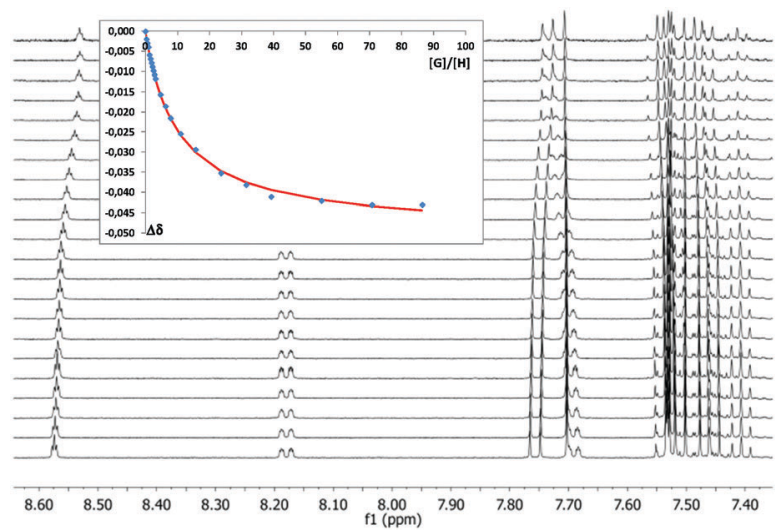

Fig. $6{ }^{1} \mathrm{H}-\mathrm{NMR}$ spectra $\left(500 \mathrm{MHz}\right.$, toluene- $\left.\mathrm{d}_{8}\right)$ in the supramolecular titration of E6b with $\mathrm{C}_{60}$. Inset: Plot of changes in chemical shift against $[G] /[H]$, where $G$ is $C_{60}$ and $H$ is $\mathbf{E} 6 \mathbf{b}$. The red line corresponds to the nonlinear fitting of $\Delta \delta$ for one proton to a $1: 1$ binding isotherm.

has been decreased by around $70 \%$. We reasoned that the new structure of switched $\mathbf{6 b}$ makes harder the approximation of $\mathrm{C}_{70}$ due to the lack of space and poor host preorganization (Fig. S97, $\mathbf{E S I} \dagger$ ). Due to the fact that E6b is not initially preorganized as a host, this compound must undergo a conformational twist from trans to cis, implying an additional deformation energy to be overcome before establishing the supramolecular association, eventually giving smaller $K_{\mathrm{a}}$ when compared to Z6a, but we did not expect a difference of one order of magnitude.

Therefore, we conducted computational calculations and optimized its geometry in the trans conformation from the structure observed in single crystals (see the ESI $\dagger$ for the theoretical model followed). Thus, the rotation of the $\mathrm{N}=\mathrm{N}-\mathrm{C}=\mathrm{CH}$ dihedral angle was calculated in toluene and a maximum at $5.67 \mathrm{kcal} \mathrm{mol}^{-1}$ was achieved, low enough to be easily reachable at room temperature (Fig. S98, ESI $\dagger$ ), because it is not hampered by other intramolecular associations (corannulene-corannulene intramolecular $\pi$ stacking, for instance). Then, we minimized the structure of the adduct $\mathbf{C}_{60} @ \mathbf{E} 6 \mathbf{b}$ at the same level of theory (Fig. 7).
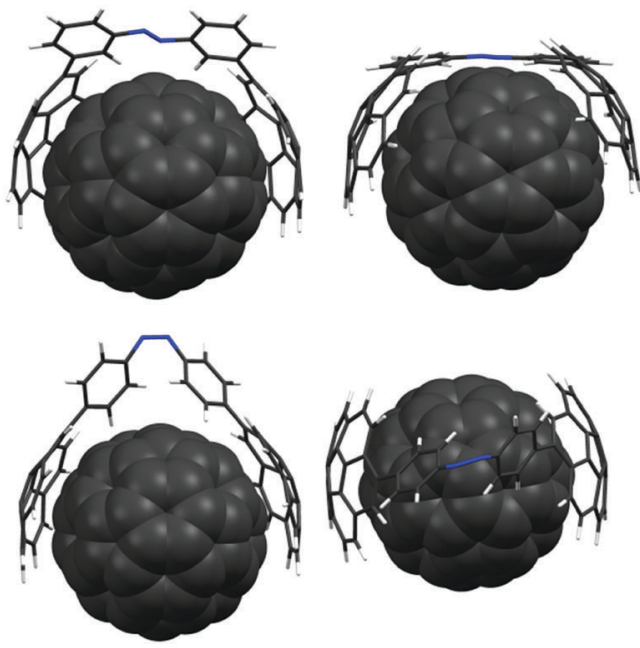

Fig. 7 Optimized geometry of $\mathbf{C}_{60} @ E 6 b$ (top) and $\mathbf{C}_{60} @$ @Z6a (bottom).

The cavity formed by both corannulene subunits is suitable to host a fullerene, but $\mathrm{CH}$ hydrogens of azobenzenes pointing towards substituents seem to interfere with guest approximation and the adduct is formed out of the ideal azobenzene line, forcing both corannulenes to adopt a bent conformation, and, consequently, diminishing the affinity.

An interaction energy between $\mathrm{C}_{60}$ and the host molecule of $-37.1 \mathrm{kcal} \mathrm{mol}^{-1}$ was achieved after taking into account basis set superposition error (see the ESI $\dagger$ regarding how this calculation is done). This energy is very similar to those calculated for other corannulene derivatives. ${ }^{10}$

The geometry of the $\mathbf{C}_{\mathbf{6 0}} @ \mathbf{Z 6 a}$ adduct was also optimized (Fig. 6) at the same level of theory giving rise to an expected geometry showing a very good complementarity between the fullerene and the host molecule. In this case, the adduct is formed in the ideal azobenzene line having both corannulene subunits almost perpendicular to it and parallel between them. This fact along with the better preorganized structure could be the reasons for its better affinity. Interestingly, the calculated interaction energy is $-34.9 \mathrm{kcal} \mathrm{mol}^{-1}$; this means $2.2 \mathrm{kcal} \mathrm{mol}^{-1}$ less than that calculated for $\mathbf{C}_{\mathbf{6 0}} @ \mathbf{E} \mathbf{6 b}$. This unexpected slight decrease encouraged us to map the interactions between the host and the guest. Non-covalent interactions ${ }^{11}$ were analyzed in both
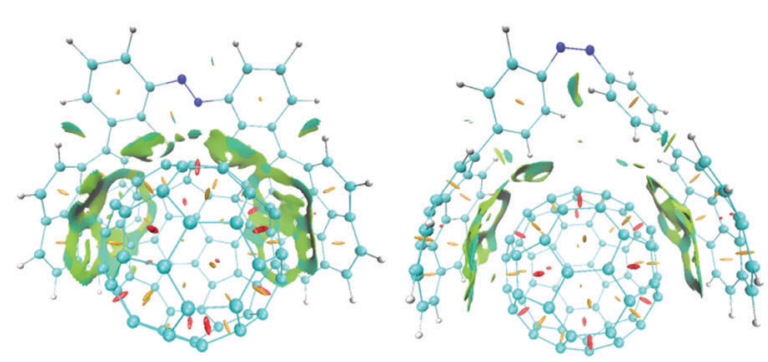

Fig. 8 Gradient isosurfaces ( $s=0.3$ a.u.) of $\mathbf{C}_{60}$ @E6b (left) and $\mathbf{C}_{60} @ \mathbf{a Z 6 a}$ (right). The surfaces are colored on a blue-green-red scale according to values of $\operatorname{sign}\left(\lambda_{2}\right) \rho$. Red indicates repulsion, while green means weak attraction. 
optimized models, revealing a more attractive interaction in $\mathbf{C}_{60} @ \mathbf{E 6 b}$ in which the isosurface almost covers half of the fullerene surface, whereas in $\mathbf{C}_{60} @ \mathbf{Z 6 a}$, it substantially lies at corannulene fragments (Fig. 8). It seems that the azobenzene tether contributes to interaction energy while $\mathrm{C}_{60}$ is close to it, but this contribution disappears when $\mathrm{C}_{60}$ moves away.

The effect of additional stabilization by the fragment connecting corannulene subunits has been pointed out by Sygula and coworkers. ${ }^{3 i, j}$

In summary, we have described six new azobenzene derivatives bearing polycyclic aromatic fragments and assessed their ability to modulate their affinities towards fullerenes. One of these compounds, $\mathbf{6 a}$, has demonstrated to be an effective on/off light-gated molecular tweezer.

This work was funded by the Spanish Ministerio de Economía y Competitividad (CTQ 2013-41067-P). H. B. acknowledges with thanks a MEC-FPI grant.

\section{Notes and references}

1 (a) J. L. Atwood and J. W. Steed, Encyclopedia of Supramolecular Chemistry, Taylor \& Francis Group, New York, 2005; (b) J. W. Steed, D. R. Turner and K. J. Wallace, Core Concepts in Supramolecular Chemistry and Nanochemistry, John Wiley and Sons, West Sussex, UK, 2007.

2 (a) T. Kawase and H. Kurata, Chem. Rev., 2006, 106, 5250-5273; (b) K. Tashiro and T. Aida, Chem. Soc. Rev., 2007, 36, 189-197; (c) E. M. Pérez and N. Martín, Chem. Soc. Rev., 2008, 37, 1512-1519; (d) M. Hardouin-Lerouge, P. Hudhomme and M. Sallé, Chem. Soc. Rev., 2011, 40, 30-43; (e) N. Martín and E. M. Pérez, Pure Appl. Chem., 2010, 82, 523-533; $(f)$ N. Martín and J.-F. Nierengarten, Supramolecular Chemistry of Fullerenes and Carbon Nanotubes, Wiley-VCH Verlag GmbH, Weinheim, Germany, 2012.

3 (a) S. Mizyed, P. E. Geourghiou, M. Bancu, B. Cuadra, A. K. Rai, P. Cheng and L. T. Scott, J. Am. Chem. Soc., 2001, 123, 12770-12774; (b) A. Sygula, F. R. Fronczek, R. Sygula, P. W. Rabideau and M. M. Olmstead, J. Am. Chem. Soc., 2007, 129, 3842-3843; (c) M. C. Stuparu, J. Polym. Sci., Part A: Polym. Chem., 2012, 50, 2641-2649; (d) M. Yanney and A. Sygula, Tetrahedron Lett., 2013, 54, 2604-2607; (e) M. C. Stuparu, Angew. Chem., Int. Ed., 2013, 52, 7786-7790; $(f)$ V. H. Le, M. Yanney, M. McGuire, A. Sygula and E. A. Lewis, J. Phys. Chem. B, 2014, 118, 11956-11964; ( $g$ ) C. M. Álvarez, L. A. GarcíaEscudero, R. García-Rodriguez, J. M. Martin-Álvarez, D. Miguel and V. M. Rayón, Dalton Trans., 2014, 43, 15693-15696; (h) C. M. Álvarez, G. Aullón, H. Barbero, L. A. García-Escudero, C. Martínez-Pérez, J. M. Martín-Álvarez and D. Miguel, Org. Lett., 2015, 17, 2578-2581; (i) M. Yanney, F. R. Fronczek and A. Sygula, Angew. Chem., Int. Ed., 2015, 54, 11153-11156; $(j)$ P. L. Abeyratne Kuragama, F. R. Fronczek and A. Sygula, Org. Lett., 2015, 17, 5292-5295; $(k)$ K. G. U. R. Kumarasinghe, F. R. Fronczek, H. U. Valle and A. Sygula, Org. Lett., 2016, 18, 3054-3057; (l) C. M. Álvarez, H. Barbero, S. Ferrero and D. Miguel, J. Org. Chem., 2016, 81, 6081-6086.

4 (a) L. T. Scott, M. M. Hashemi and M. S. Bratcher, J. Am. Chem. Soc., 1992, 114, 1920-1921; (b) A. Sygula, H. E. Folsom, R. Sygula, A. H. Abdourazak, Z. Marcinow, F. R. Fronczek and P. W. Rabideau, J. Chem. Soc., Chem. Commun., 1994, 2571-2572; (c) F. J. Lovas, R. J. McMahon, J.-U. Grabow, M. Schnell, J. Mack, L. T. Scott and R. L. Kuczkowski, J. Am. Chem. Soc., 2005, 127, 4345-4349; (d) Y.-T. Wu, T. Hayama, K. K. Baldrige, A. Linden and J. S. Siegel,
J. Am. Chem. Soc., 2006, 128, 6870-6884; (e) Y.-T. Wu and J. S. Siegel, Chem. Rev., 2006, 106, 4843-4867; $(f)$ V. M. Tsefrikas and L. T. Scott, Chem. Rev., 2006, 106, 4868-4884; ( $g$ ) Y.-T. Wu, D. Bandera, R. Maag, A. Linden, K. K. Baldridge and J. S. Siegel, J. Am. Chem. Soc., 2008, 130, 10729-10739; (h) Y.-T. Wu and J. S. Siegel, Top. Curr. Chem., 2014, 349, 63-120.

5 (a) V. Balzani, A. Credi and M. Venturi, Molecular Devices and Machines: Concepts and Perspectives for the Nanoworld, Wiley-VCH Verlag GmbH, Weinheim, Germany, 2nd edn, 2008; (b) B. L. Feringa and W. R. Browne, Molecular Switches, Wiley-VCH Verlag GmbH, Weinheim, Germany, 2nd edn, 2011; (c) J. Wang, Nanomachines: Fundamentals and Applications, Wiley-VCH Verlag $\mathrm{GmbH}$, Weinheim, Germany, 2013; (d) D.-H. Qu, Q.-C. Wang, Q.-W. Zhang, X. Ma and H. Tian, Chem. Rev., 2015, 115, 7543-7588; (e) D. Bléger and S. Hecht, Angew. Chem., Int. Ed., 2015, 54, 11338-11349.

6 (a) H. M. D. Bandara and S. C. Burdette, Chem. Soc. Rev., 2012, 41, 1809-1825; (b) E. Merino and M. Ribagorda, Beilstein J. Org. Chem., 2012, 8, 1071-1090.

7 For some recent examples of architectures containing azobenzenes that do useful work see: (a) T. Ogoshi, K. Kida and T. Yamagishi, J. Am. Chem. Soc., 2012, 134, 20146-20150; (b) G. Yu, C. Han, Z. Zhang, J. Chen, X. Yan, B. Zheng, S. Liu and F. Huang, J. Am. Chem. Soc., 2012, 134, 8711-8717; (c) L. Osorio-Planes, M. Espelt, M. A. Pericàs and P. Ballester, Chem. Sci., 2014, 5, 4260-4264; (d) J. Park, L.-B. Sun, Y.-P. Chen, Z. Perry and H.-C. Zhou, Angew. Chem., Int. Ed., 2014, 53, 5842-5846; (e) J. P. Van der Berg, W. A. Velema, W. Szymanski, A. J. M. Driessen and B. L. Feringa, Chem. Sci., 2015, 6, 3593-3598; $(f)$ M. Baroncini, S. d'Agostino, G. Bergamini, P. Ceroni, A. Comotti, P. Sozzani, I. Bassanetti, F. Grepioni, T. M. Hernandez, S. Silvi, M. Venturi and A. Credi, Nat. Chem., 2015, 7, 634-640; $(g)$ M. Lohse, K. Nowosinski, N. L. Traulsen, A. J. Achazi, L. K. S. von Krbek, B. Paulus, C. A. Schalley and S. Hecht, Chem. Commun., 2015, 51, 9777-9780; (h) E. Anger and S. P. Fletcher, Eur. J. Org. Chem., 2015, 3651-3655; (i) M. Dommaschk, M. Peters, F. Gutzeit, C. Schütt, C. Näther, F. D. Sönnichsen, S. Tiwari, C. Riedel, S. Boretius and R. Herges, J. Am. Chem. Soc., 2015, 137, 7552-7555; $(j)$ J. P. Van der Berg, W. A. Velema, W. Szymanski, A. J. M. Driessen and B. L. Feringa, Chem. Sci., 2015, 6, 3593-3598; (k) M. Xue, Y. Yang, X. Chi, X. Yan and F. Huang, Chem. Rev., 2015, 115, 7398-7501; (l) S. Erbas-Cakmak, D. A. Leigh, C. T. McTernan and A. L. Nussbaumer, Chem. Rev., 2015, 115, 10081-10206.

8 K. Yuan, Y.-J. Guo and X. Zhao, Phys. Chem. Chem. Phys., 2014, 16, 27053-27064.

9 (a) Q. Yang, L. Shuai, J. Zhou, F. Lu and X. Pan, J. Phys. Chem. B, 2008, 112, 12934-12939; (b) C. Ehli, G. M. A. Rahman, N. Jux, D. Balbinot, D. M. Guldi, F. Paolucci, M. Marcaccio, D. Paolucci, M. Melle-Franco, F. Zerbetto, S. Campidelli and M. Prato, J. Am. Chem. Soc., 2006, 128, 11222-11231; (c) Y.-L. Zhao, L. Hu, G. Grüner and J. F. Stoddart, J. Am. Chem. Soc., 2008, 130, 16996-17003; (d) A. S. D. Sandanayaka, R. Chitta, N. K. Subbaiyan, L. D'Souza, O. Ito and F. D'Souza, J. Phys. Chem. C, 2009, 113, 13425-13432; (e) C. B. Kc, G. N. Lim and F. D'Souza, Angew. Chem., Int. Ed., 2015, 54, 5088-5092; $(f)$ A. Lopez-Moreno and E. M. Perez, Chem. Commun., 2015, 51, 5421-5424.

10 (a) C. Mück-Lichtenfeld, S. Grimme, L. Kobryn and A. Sygula, Phys. Chem. Chem. Phys., 2010, 12, 7091-7097; (b) P. A. Denis, RSC Adv., 2013, 3, 25296-25305; (c) D. Josa, J. Rodríguez-Otero, E. M. Cabaleiro-Lago, L. A. Santos and T. C. Ramalho, J. Phys. Chem. A, 2014, 118, 9521-9528.

11 (a) W. Yang, E. R. Johnson, S. Keinan, P. Mori-Sánchez, J. ContrerasGarcía and A. J. Cohen, J. Am. Chem. Soc., 2010, 132, 6498-6506; (b) W. Yang, J. Contreras-García, E. R. Johnson, S. Keinan, R. Chaudret, J.-P. Piquemal and D. N Beratan, J. Chem. Theory Comput., 2011, 7, 625-632. 
142 


\title{
Synergistic Effect of Tetraaryl Porphyrins Containing Corannulene and Other Polycyclic Aromatic Fragments as Hosts for Fullerenes. Impact of $\mathrm{C}_{60}$ in a Statistically Distributed Mixture of Atropisomers
}

\author{
Celedonio M. Álvarez,* Héctor Barbero, Sergio Ferrero, and Daniel Miguel \\ GIR MIOMeT, IU CINQUIMA/Química Inorgánica, Facultad de Ciencias, Universidad de Valladolid, E-47011 Valladolid, Spain
}

Supporting Information

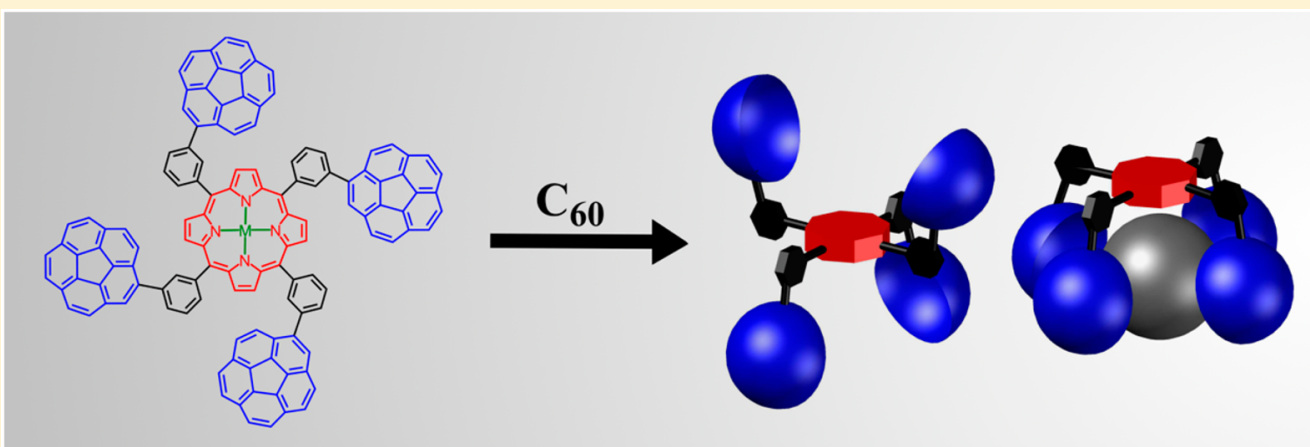

ABSTRACT: Symmetric meso-tetraarylporphyrins bearing phenanthrene, pyrene, and corannulene moieties in meta positions have been synthesized in a straightforward procedure under microwave irradiation by quadruple Suzuki-Miyaura reactions. Their ${ }^{1} \mathrm{H}$ NMR spectra showed the typical pattern of four atropisomers distributed according to their statistical ratio not properly separable due to their fast isomerization. Their ability to bind buckminsterfullerene has been tested with the whole mixture, and different behaviors have been found, $\alpha_{4}$ isomer corannulene-substituted porphyrins being the best hosts in the family.

$\mathrm{P}$ orphyrins are a vast family of naturally occurring macrocycles having multiple functions in living beings. Many applications have been developed ${ }^{1}$ by using these astounding architectures, one of them being their use as hosts for fullerenes, especially for $\mathrm{C}_{60}$, which is considered a very promising and potentially useful building block for new electronic devices. ${ }^{2}$ The main approach in order to bind fullerenes by means of supramolecular forces has consisted of building structures with more than one porphyrin, yielding molecular tweezers, trimers, or strapped porphyrins leading to very high affinities. ${ }^{3}$ However, there are not examples in which a single porphyrin shows binding abilities to $\mathrm{C}_{60}$ beyond cocrystallization, ${ }^{4}$ except in the work of Ishizuka and Kojima et al., ${ }^{5}$ whose curved porphyrins were able to carry out such a task.

In our research regarding corannulene-functionalized molecules to act as fullerene hosts, ${ }^{6}$ we decided to link this molecule to a porphyrin scaffold in order to add the association abilities of corannulene ${ }^{7}$ to the porphyrin core. Additionally, other planar aromatic fragments were linked to the porphyrin core as well and tested for comparison purposes.

Compounds 2HP1 and ZnP1 could be synthesized with the help of a microwave reactor with a modified method reported elsewhere. ${ }^{8}$ From that point we reasoned that the Suzuki reaction from borylated polyaromatic fragments with known Schlenk techniques would lead to $\mathbf{2 H P 2 a - c}$ and ZnP2a-c families (see Schemes S1-S3 in the Supporting Information for a description of all routes attempted); however, a great amount of homocoupling byproducts were obtained as well as nonnegligible amounts of porphyrins with $\mathrm{Pd}$ in their cavity (Figures S57-S59 in the Supporting Information). This mixture leads to a very cumbersome chromatographic separations. Therefore, we turned to a synthetic route in which the pinacol boronate fragment was located in the porphyrin core and reacted with brominated polyaromatic fragments as depicted in Scheme 1. In order to obtain free base porphyrins, removal of the $\mathrm{Zn}$ atom was preferred. Additionally, all coupling reactions were carried out with microwave irradiation because the reaction times were significantly improved. ${ }^{9}$

New porphyrins were fully characterized by spectroscopic methods. All of them have the expected Soret bands around $430 \mathrm{~nm}$ and Q bands between 500 and $600 \mathrm{~nm}$ (Figures S67S70 in the Supporting Information) in their absorption spectra. ${ }^{1} \mathrm{H}$ NMR spectra show clearly the presence of statistically distributed atropisomers, ${ }^{10}$ especially for $\mathrm{H}_{6}$ singlets (see the Supporting Information for the numbering of the system), as depicted in Figure 1. Attempts to separate each atropisomer individually, by both preparative methods and HPLC, were unfruitful, even though the HPLC chromatogram clearly

Received: March 1, 2016

Published: June 16, 2016 
Scheme 1. Best Synthetic Route toward Porphyrin Targets ${ }^{a}$
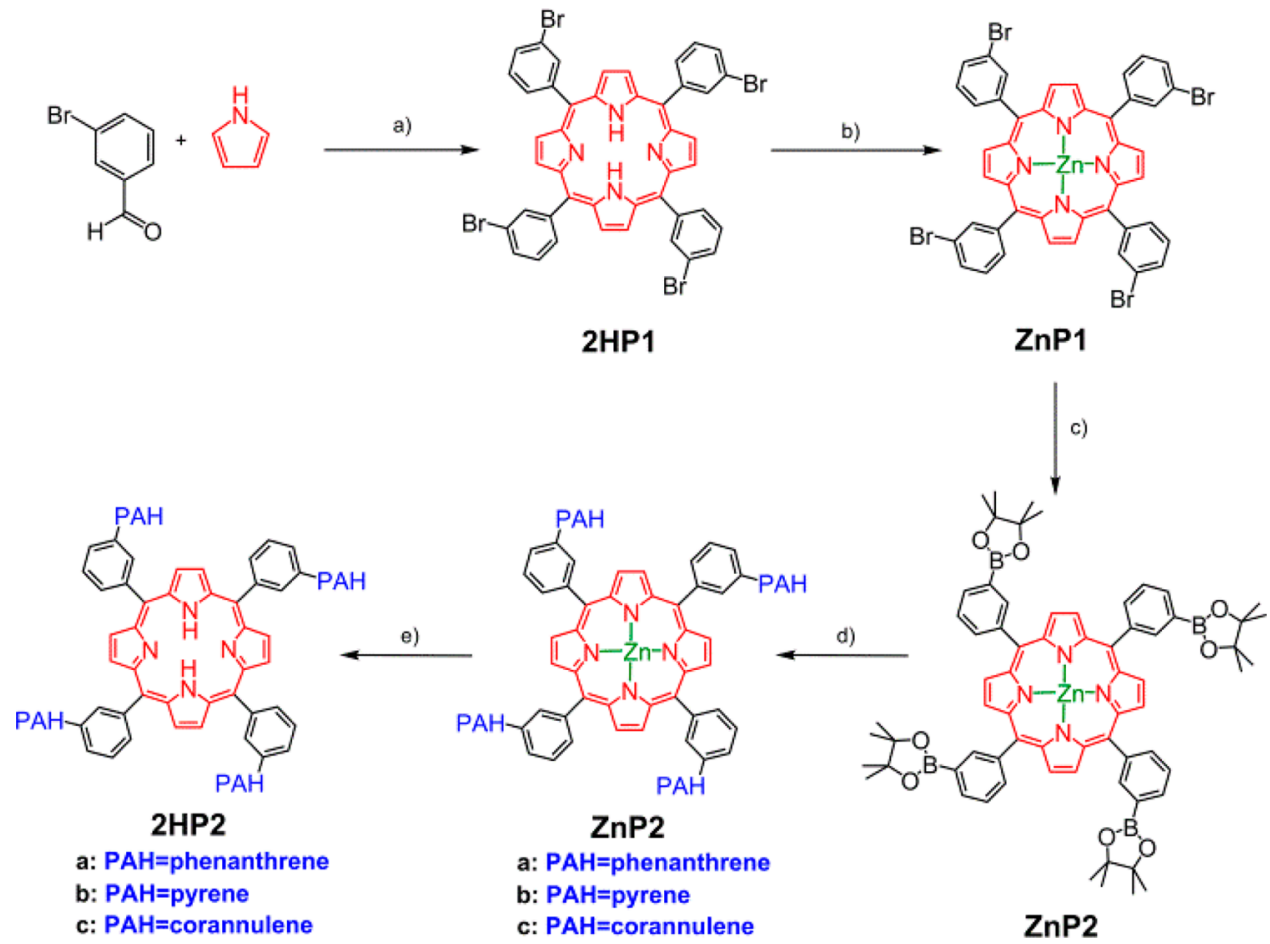

${ }^{a}$ Reagents and conditions: (a) propionic acid, nitrobenzene, $\mathrm{MW}, 200{ }^{\circ} \mathrm{C}$; (b) $\mathrm{Zn}(\mathrm{OAc}){ }_{2} \cdot 2 \mathrm{H}_{2} \mathrm{O}, \mathrm{CHCl}_{3}, \mathrm{MW}, 120{ }^{\circ} \mathrm{C} ;(\mathrm{c}) \mathrm{B}_{2} \mathrm{pin}_{2},\left[\mathrm{PdCl}_{2}(\mathrm{dppf})\right]$, AcOK, dioxane, MW; (d) $\left[\mathrm{PdCl}_{2}(\mathrm{dppf})\right]$, ${ }^{\mathrm{B}} \mathrm{BuONa}$, toluene, $\mathrm{MW}$; (e) $\mathrm{CF}_{3} \mathrm{CO}_{2} \mathrm{H}$.

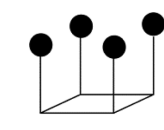

$\alpha_{4}$

$1 / 8$

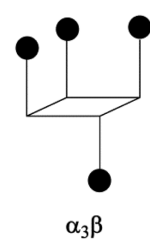

$4 / 8$

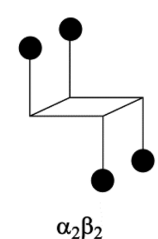

$2 / 8$

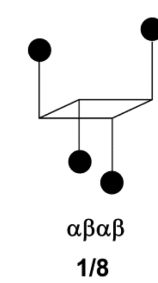

Figure 1. Schematic representation of the atropisomers and their statistical distribution.

showed four separate peaks. NMR tests of each fraction indicated the presence of the mixture, showing that isomerization occurred even at low temperature. ${ }^{11}$

Notwithstanding such limitations, we decided to analyze the ability of the whole mixture to bind $\mathrm{C}_{60}$ and, at the same time, to observe potential differences among all individual atropisomers. ${ }^{1} \mathrm{H}$ NMR was chosen to be the technique because, unlike UV/vis experiments, it is able to distinguish all isomers. This was complemented by mass spectrometry experiments. Before performing any titration, we first attempted to determine the stoichiometry of the adduct. Job plots were carried out for all compounds; however, only $\mathrm{H}_{1}$ was observed as an average signal due to its inherent broadness, so that only the 2 HP2 set could be properly analyzed (Figures S77-S79 in the Supporting Information). Surprisingly, all porphyrins showed 1:1 adduct formation and this was confirmed by MS (Figures S60-S66 in the Supporting Information). We assumed that this stoichiometry was maintained in $\mathbf{Z n P 2}$ derivatives because only 1:1 adducts were located in all mass spectra. Titrations of phenanthrene and pyrene-substituted porphyrins ZnP2a,b and 2HP2a,b provided several small chemical shift changes, indicating supramolecular association for some atropisomers. This was not expected, because it is known that compounds bearing these types of PAHs do not establish adducts with $\mathrm{C}_{60}$ due to their low shape complementarity; $^{12}$ this suggests that the host-guest formation is taking place due mainly to the porphyrin core. ${ }^{13}$ On the other hand, corannulene derivatives, 2HP2c and Zn2P2c, showed great chemical shift changes, except for one atropisomer. Interestingly, we observed the same pattern in all chemical shift changes, and especially for $\mathrm{H}_{1}, \mathrm{H}_{2}$, and $\mathrm{H}_{6}$ hydrogens in all compounds. This suggests a similar behavior.

In order to assign signals for each atropisomer, we carried out computational studies ${ }^{14}$ for the $2 \mathrm{HP2}$ set of isomers and the optimized structures provided interesting features. Intramolecular association of PAH units at the same side is so preferred that it leads to deformation of the porphyrin, reaching a saddlelike conformation for the most stable isomers, $\boldsymbol{\alpha}_{4}$. On the other hand, $\boldsymbol{\alpha} \boldsymbol{\beta} \boldsymbol{\alpha} \boldsymbol{\beta}$ isomers are the least stable and PAH moieties are not interacting among them. This lack of interaction could be the reason for the low stability. Thus, a quasi-planar porphyrin core as well as vague preorganization as host are observed, as seen in Figure 2 for 2HP2c. The other atropisomers present intermediate situations and stabilities (Tables S1-S3 and Figures S80-S91 in the Supporting Information).

Theoretical NMR predictions for the $\mathrm{H}_{6}$ proton signal in 2HP2c gave, from lowest to highest field, the following order: $\alpha \beta \alpha \beta<\alpha_{3} \beta$ (bottom) $<\alpha_{3} \beta$ (outer) $<\alpha_{2} \beta_{2}<\alpha_{3} \beta$ (inner) $<\alpha_{4}$, as depicted in Figure 3.

From that starting point, we followed the evolution of singlet $\mathrm{H}_{6}$ during titration with $\mathrm{C}_{60}$. Simultaneously, the evolution of singlet $\mathrm{H}_{2}$ proton ( $\beta$-pyrrole) could be traced due to a great disruption observed upon addition of initial aliquots. It must be noted that these $\mathrm{H}_{2}$ protons are all equivalent for $\boldsymbol{\alpha} \boldsymbol{\beta} \boldsymbol{\alpha} \boldsymbol{\beta}$ and $\boldsymbol{\alpha}_{4}$ isomers, but there are two nonequivalent protons for $\boldsymbol{\alpha}_{3} \boldsymbol{\beta}$ and 


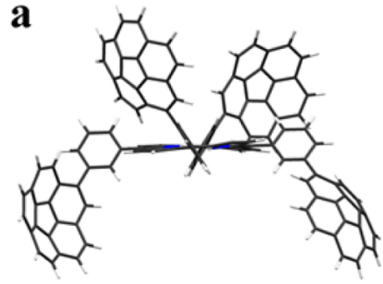

b

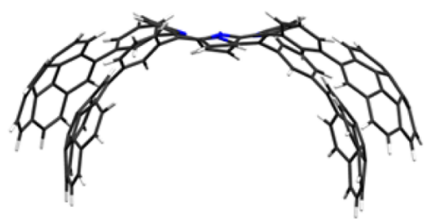

Figure 2. Optimized structures of (a) $\alpha \beta \alpha \beta 2 \mathrm{HP} 2 \mathrm{c}$ and (b) $\boldsymbol{\alpha}_{4} 2 \mathrm{HP} 2 \mathrm{c}$.

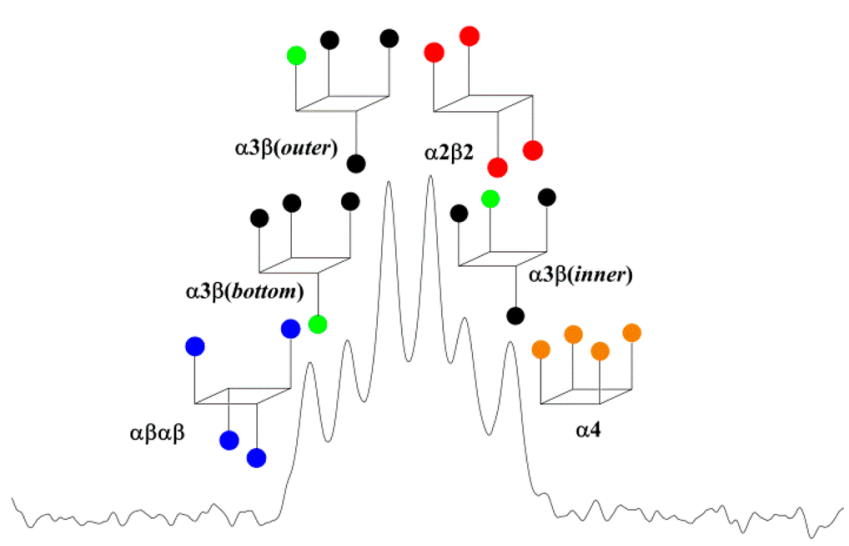

Figure 3. Simulated $\mathrm{H}_{6}$ resonance in toluene of the 2HP2c pattern and theoretical assignments with an integral ratio of $1: 1: 2: 2: 1: 1$.

$\boldsymbol{\alpha}_{2} \boldsymbol{\beta}_{2}$ isomers. Additionally, this procedure was applied to ZnP2c to check similar behavior and reproducibility.

First of all, predicted $\boldsymbol{\alpha}_{3} \boldsymbol{\beta}$ (bottom) and $\boldsymbol{\alpha}_{3} \boldsymbol{\beta}$ (outer) $\mathrm{H}_{6}$ chemical shift changes were followed and their curves fitted, yielding equal constants. However, $\boldsymbol{\alpha}_{3} \boldsymbol{\beta}$ (inner) $\mathrm{H}_{6}$ could not be followed due to spectral overlap with $\boldsymbol{\alpha}_{2} \boldsymbol{\beta}_{2} \mathrm{H}_{6}$ during titration. Once those proton signals were located and determined, another atropisomer could be directly assigned, $\boldsymbol{\alpha}_{2} \boldsymbol{\beta}_{2}$. Its $\mathrm{H}_{6}$ signal is that left with a ratio of 2 (Figure 3 ). The titration for this atropisomer gave a very different constant (3-fold increase for 2HP2c and 1 order of magnitude in $\mathrm{ZnP2c}$ ). Thus, at this point, just two minor signals corresponding to $\boldsymbol{\alpha} \boldsymbol{\beta} \boldsymbol{\alpha} \boldsymbol{\beta}$ and $\boldsymbol{\alpha}_{4}$ isomers were left to be matched with computational results. It was assumed that the theoretical assignment was correct due to the accuracy observed for the other predictions. Unfortunately, the $\mathrm{H}_{6}$ proton for the $\boldsymbol{\alpha}_{4}$ atropisomer could not be followed because it became broadened after the first addition. These findings are all gathered in Figure 4.

On the other hand, $\mathrm{H}_{2}$ proton ( $\beta$-pyrrole) evolution was followed as well in order to identify each signal in the course of titration experiments. Each chemical shift change observed was systematically subjected to a fitting process covering all possibilities until the association constants matched those previously estimated for $\mathrm{H}_{6}$. Fortunately, signals corresponding to $\boldsymbol{\alpha} \boldsymbol{\beta} \boldsymbol{\alpha} \boldsymbol{\beta}$ and $\boldsymbol{\alpha}_{3} \boldsymbol{\beta}$ isomers, especially for $\mathrm{ZnP2c}$, were located easily due to the lack of spectral overlap during titration. Spectral changes of the $\boldsymbol{\alpha}_{2} \boldsymbol{\beta}_{2}$ atropisomer (which, depending on FID processing, can be seen as one large broad signal or two resolved shifts) were also easily followed. A crossover with a broad chemical shift change that unexpectedly increased at the beginning of the titration was observed in the last additions. This new signal was accepted to come from the remaining $\boldsymbol{\alpha}_{4}$ atropisomer; therefore, we carried out the same fitting procedure, resulting in a new association constant. This procedure is shown in Figure 5. ${ }^{15}$
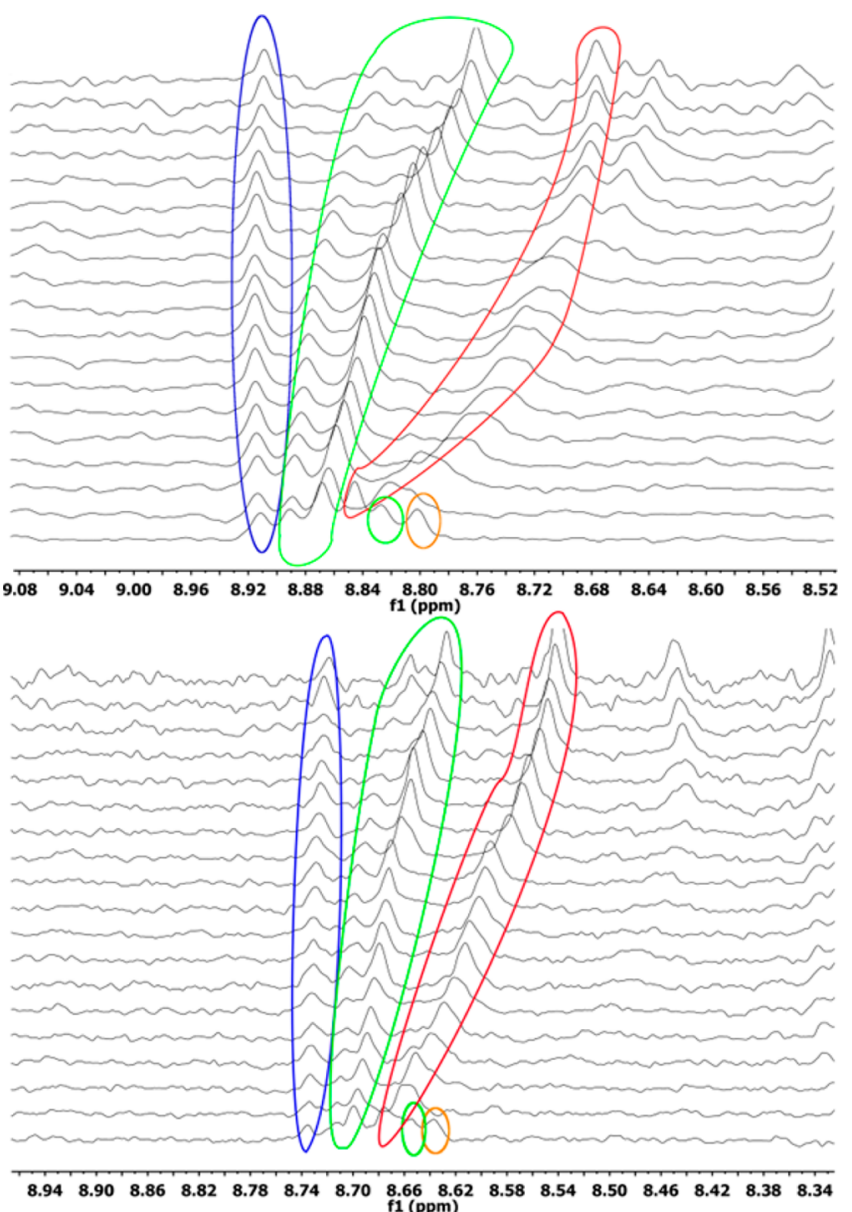

Figure 4. ${ }^{1} \mathrm{H}$ NMR showing $\mathrm{H}_{6}$ chemical shifts in deuterated toluene of $\mathrm{ZnP2c}$ (up) and 2HP2c (bottom) upon addition of aliquots of $\mathrm{C}_{60}$. Colored domains enclose the assignments according to the pattern established in Figure 3. Note the overlap of the $\mathrm{H}_{6}$ (inner) proton in the $\boldsymbol{\alpha}_{3} \boldsymbol{\beta}$ isomer with the $\mathrm{H}_{6}$ proton in the $\boldsymbol{\alpha}_{2} \boldsymbol{\beta}_{2}$ isomer and the "disappearance" of the $\mathrm{H}_{6}$ proton in the $\boldsymbol{\alpha}_{4}$ isomer after the first addition.

This protocol was applied for phenanthrene- and pyrenesubstituted porphyrins (ZnP2a, ZnP2b, 2HP2a, and 2HP2b), resulting in very similar findings, but less pronounced changes of chemical shifts were observed.

These results were not consistent with our initial assumptions, because we thought that $\boldsymbol{\alpha} \boldsymbol{\beta} \boldsymbol{\alpha} \boldsymbol{\beta} 2 \mathrm{HP} 2 \mathrm{c}$ ideally had a good preorganization in a double-tweezer mode and could lead to moderate association. However, we did not find chemical shift changes great enough to be measured in any of all $\boldsymbol{\alpha} \boldsymbol{\beta} \boldsymbol{\alpha} \boldsymbol{\beta}$ atropisomers. Furthermore, as stated previously, structures of optimized minima clearly showed that preorganization of this atropisomer to adapt a fullerene shape is not efficient, while other atropisomer preorganizations are much more efficient.

The best results were obtained for corannulene-functionalized porphyrins; especially ZnP2c. $\alpha_{4} \mathrm{ZnP2c}$, and $\boldsymbol{\alpha}_{2} \boldsymbol{\beta}_{2} \mathrm{ZnP2c}$ showed the best association constants in the mixture of atropisomers, which are 22330 and $12140 \mathrm{M}^{-1}$, respectively. The presence of the metal favors supramolecular adduct formation.

As commented above, 2HP2 (free base) porphyrins possess averaged signals coming from internal hydrogens $\left(\mathrm{H}_{1}\right)$ and could be followed during titration experiments; therefore, an 

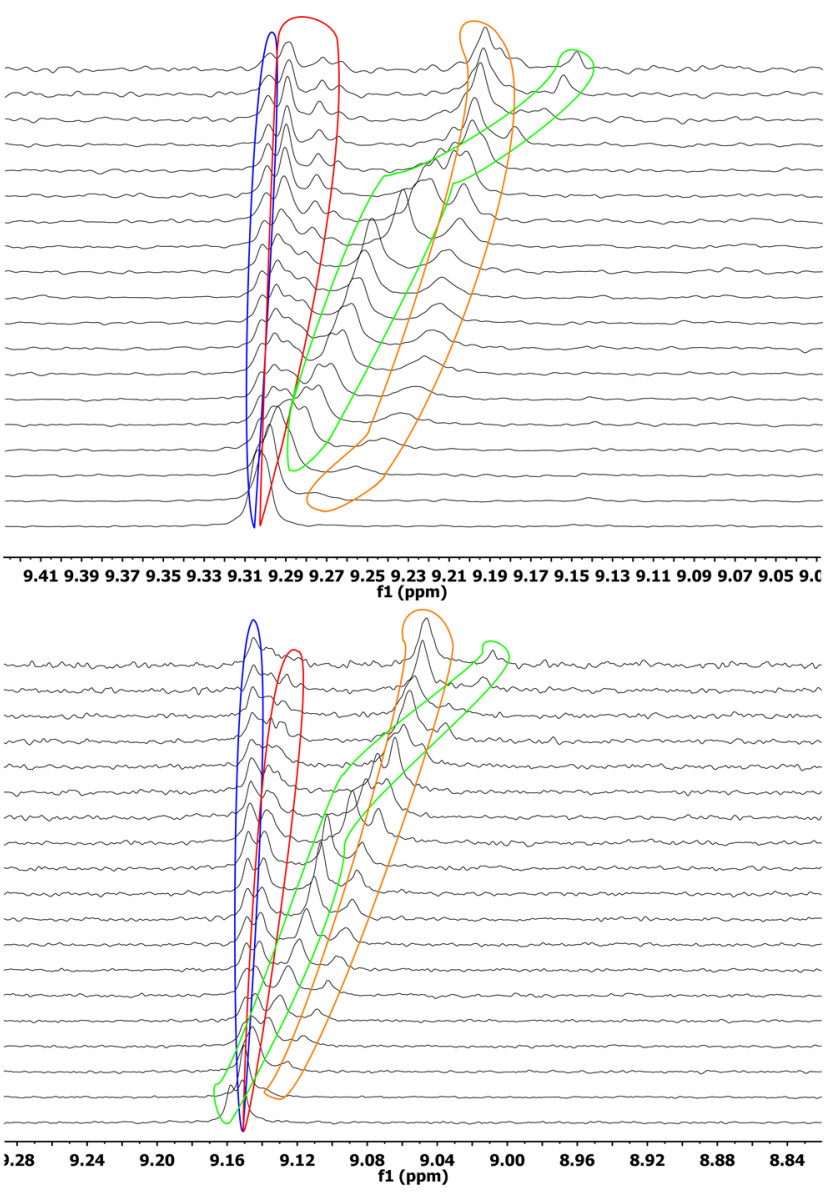

Figure 5. ${ }^{1} \mathrm{H}$ NMR showing $\mathrm{H}_{2}$ chemical shifts in deuterated toluene of $\mathrm{ZnP2c}$ (top) and 2HP2c (bottom) upon addition of aliquots of $\mathrm{C}_{60}$. Colored domains enclose the assignments according to the pattern established in Figure 3. Note the crossover between the green and yellow domains.

average association constant could be given, leading to the conclusion that our corannulene-based porphyrins are very good hosts for $\mathrm{C}_{60}$ because their association constants are 1 order of magnitude greater than those bearing planar polycyclic hydrocarbons. In light of these findings, synergy between a porphyrin core and well-adapted corannulene groups to bind buckminsterfullerene is suggested. All of the estimated constants are gathered in Table 1.

In summary, a set of six tetrasubstituted porphyrins in meso positions with polycyclic aromatic hydrocarbons have been prepared very easily by using microwave irradiation. The existence of a statistically distributed mixture of atropisomers was identified by NMR and assigned with the help of a combination of titration experiments of the whole mixture with
$\mathrm{C}_{60}$ and computational studies. Complexation studies were carried out, and a great difference was observed for each individual atropisomer, the best hosts being $\boldsymbol{\alpha}_{4}$ and $\boldsymbol{\alpha}_{2} \boldsymbol{\beta}_{2}$ porphyrins bearing corannulene as a substituent, whose crude estimated association constants were among the highest of those reported for single-porphyrin hosts. Theoretical calculations have suggested that preorganization of corannulene moieties in a tweezerlike fashion are crucial. This initial research has opened new possibilities to design more complex "picket fence" ( $\boldsymbol{\alpha}_{4}$ atropisomer) porphyrins having multiple corannulene fragments with the objective to increase their affinity toward fullerenes thanks to a synergy between the porphyrin and nonplanar polycyclic hydrocarbon.

\section{EXPERIMENTAL SECTION}

All reagents were purchased from the usual vendors and used without further purification. Solvents were either used as purchased or dried according to procedures described elsewhere. ${ }^{16}$ Microwave reactions were carried out with an Anton Paar Monowave 300 Reactor. Column chromatography was carried out using silica gel 60 (particle size $0.040-0.063 \mathrm{~mm} ; 230-400$ mesh) as the stationary phase, and TLC was performed on precoated silica gel plates $(0.25 \mathrm{~mm}$ thick, $60 \mathrm{~F} 254)$ and observed under UV light and/or dipping in anisaldehyde. NMR spectra were recorded on 400 and $500 \mathrm{MHz}$ instruments. ${ }^{1} \mathrm{H}$ and ${ }^{13} \mathrm{C}$ NMR chemical shifts $(\delta)$ are reported in parts per million (ppm) and are referenced to TMS, using the solvent as an internal reference. Coupling constants $(J)$ are reported in hertz $(\mathrm{Hz})$. Standard abbreviations used to indicate multiplicity: $\mathrm{s}=$ singlet, $\mathrm{d}=$ doublet, $\mathrm{t}$ $=$ triplet, $\mathrm{m}=$ multiplet, $\mathrm{br}=$ broad. ${ }^{13} \mathrm{C}\left\{{ }^{1} \mathrm{H}\right\}$ spectra for all porphyrin derivatives had to be recorded with the following acquisition parameters due to their fast relaxation: a $90^{\circ}$ pulse, a relaxation delay of $5 \mathrm{~ms}$, and an acquisition time of $250 \mathrm{~ms} .{ }^{1} \mathrm{H}$ and ${ }^{13} \mathrm{C}$ assignments were performed by utilizing $2 \mathrm{D}$ NMR methods: bandselective HSQC and band-selective HMBC. High-resolution mass spectra were recorded with a MALDI-TOF system with an $\mathrm{N}_{2}$ laser (337 nm, pulse energy $100 \mu \mathrm{J}, 1 \mathrm{~ns}$ ). An acceleration voltage of $19 \mathrm{kV}$ and a reflector positive mode were used. UV/vis absorption spectral wavelengths $(\lambda)$ are reported in nanometers $(\mathrm{nm})$, and molar absorption coefficient $(\varepsilon)$ are reported in $\mathrm{M}^{-1} \mathrm{~cm}^{-1}$. Corannulene and bromocorannulene were prepared according to literature procedures. $^{17}$

General Method for Polyaromatic Boronate Ester Preparation. Bromoarene $(0.4 \mathrm{mmol})$, bis(pinacolato)diboron $(152 \mathrm{mg}, 0.6$ $\mathrm{mmol}),\left[\mathrm{PdCl}_{2}(\mathrm{dppf})\right](15 \mathrm{mg}, 0.02 \mathrm{mmol})$, and AcOK (118 mg, 1.2 $\mathrm{mmol}$ ) were mixed in a Schlenk flask under an inert atmosphere. A 2 $\mathrm{mL}$ portion of dry dioxane was added, and the mixture was degassed. Then, the mixture was refluxed overnight. Solvent was removed under vacuum before purification by column chromatography with $\mathrm{SiO}_{2}$ gel and hexane/AcOEt as eluent (5/1 for 9-(pinacolatoboron)phenanthrene (1), 20/1 for 1-(pinacolatoboron)pyrene (2) and 1(pinacolatoboron) corannulene (3)). Yields were over $85 \%$. Spectral data are in agreement with those published. ${ }^{18}$

2HP1. Pyrrole (277 $\mu \mathrm{L}, 4 \mathrm{mmol})$, 3-bromobenzaldehyde ( $468.0 \mu \mathrm{L}$, $4 \mathrm{mmol})$, propionic acid $(12 \mathrm{~mL}, 160 \mathrm{mmol})$, and nitrobenzene $(7$ $\mathrm{mL}, 68 \mathrm{mmol}$ ) were mixed in a sealed reactor specifically designed for

Table 1. Summary of $K_{\mathrm{a}}$ of New Porphyrins vs $\mathrm{C}_{60}$ Estimated in Toluene- $d_{8}$

\begin{tabular}{lllllll} 
& ZnP2a & \multicolumn{1}{c}{ 2HP2a } & ZnP2b & 2HP2b & ZnP2c & 2HP2c \\
$\boldsymbol{\alpha}_{4}$ & 180 & $\mathrm{~N} / \mathrm{A}^{a}$ & 790 & 1180 & $22330^{b}$ & $7700^{b}$ \\
$\boldsymbol{\alpha}_{3} \boldsymbol{\beta}$ & $0^{d}$ & $0^{d}$ & $0^{d}$ & 540 & 1730 & 1820 \\
$\boldsymbol{\alpha}_{2} \boldsymbol{\beta}_{2}$ & 240 & 130 & 690 & 1240 & 12140 & 5890 \\
$\boldsymbol{\alpha} \boldsymbol{\beta} \boldsymbol{\alpha} \boldsymbol{\beta}$ & $0^{d}$ & $0^{d}$ & $0^{d}$ & $0^{d}$ & $0^{d}$ & $0^{d}$ \\
average & $\mathrm{N} / \mathrm{A}^{c}$ & $(4.5 \pm 0.05) \times 10^{2}$ & $\mathrm{~N} / \mathrm{A}^{c}$ & $(4.6 \pm 0.05) \times 10^{2}$ & $\mathrm{~N}^{c}$ & $(5.4 \pm 0.2) \times 10^{3}$
\end{tabular}

${ }^{a}$ Could not be estimated due to spectral overlap. ${ }^{b}$ Determined from the $\Delta \delta$ change of $\mathrm{H}_{2} \beta$-pyrrole protons only. ${ }^{c}$ Not measured (no average signal). ${ }^{d} \Delta \delta$ too low to allow a reasonable estimate of a constant. 
microwave irradiation. The mixture was stirred inside a microwave reactor at $200{ }^{\circ} \mathrm{C}$ for $15 \mathrm{~min}$ (a pressure of 5 bar was achieved). The resulting black crude product obtained after cooling to room temperature was taken up in $\mathrm{MeOH}(50 \mathrm{~mL})$, precipitating a purple solid. The product was filtered in a Büchner funnel and washed thoroughly with more $\mathrm{MeOH}$. The solid was collected, placed in an oven, and kept under reduced pressure $\left(200{ }^{\circ} \mathrm{C}, 24 \mathrm{~h}, 50 \mathrm{mbar}\right)$ to finally give pure $2 \mathrm{HP1}$, whose spectroscopic data agree with those reported $^{19}$ (0.295 g, 32\% yield).

$\mathrm{ZnP1}$. 2HP1 $(0.250 \mathrm{~g}, 0.268 \mathrm{mmol})$ and $\mathrm{Zn}(\mathrm{AcO})_{2} \cdot 2 \mathrm{H}_{2} \mathrm{O}(0.294 \mathrm{~g}$, $1.34 \mathrm{mmol}$ ) were mixed in a sealed reactor specifically designed for microwave irradiation and dissolved in $12 \mathrm{~mL}$ of $\mathrm{CHCl}_{3}$. The solution was stirred at $130{ }^{\circ} \mathrm{C}$ for $2.5 \mathrm{~h}$ (a pressure of 6 bar was achieved). Once the reaction was complete, solvent was removed in vacuo. The resulting solid was washed several times with water and dried to give a purple solid corresponding to $\mathrm{ZnP1}$ (0.203 g, $76 \%$ yield). ${ }^{1} \mathrm{H}$ NMR $\left(500 \mathrm{MHz}, \mathrm{CDCl}_{3}\right): \delta 8.96\left(\mathrm{~s}, 2 \mathrm{H}, \mathrm{H}_{2}\right), 8.38\left(\mathrm{~s}, 1 \mathrm{H}, \mathrm{H}_{6}\right), 8.16(\mathrm{br}, 1 \mathrm{H}$, $\left.\mathrm{H}_{10}\right), 7.95\left(\mathrm{~d}, J=8.2 \mathrm{~Hz}, 1 \mathrm{H}, \mathrm{H}_{8}\right), 7.64\left(\mathrm{~d}, J=8.2 \mathrm{~Hz}, 1 \mathrm{H}, \mathrm{H}_{9}\right)$. ${ }^{13} \mathrm{C}\left\{{ }^{1} \mathrm{H}\right\}$ NMR $\left(101 \mathrm{MHz}, \mathrm{CDCl}_{3}\right): \delta 150.0\left(\mathrm{C}_{3}\right), 144.5\left(\mathrm{C}_{5}\right), 137.1$ $\left(\mathrm{C}_{6}\right), 132.9\left(\mathrm{C}_{10}\right), 132.1\left(\mathrm{C}_{2}\right), 130.9\left(\mathrm{C}_{8}\right), 128.0\left(\mathrm{C}_{9}\right), 110.0\left(\mathrm{C}_{4}\right)$. HRMS (MALDI-TOF): $\mathrm{m} / z$ 987.8010 $[\mathrm{M}]^{+}$(calcd 987.8026 for $\left.\mathrm{C}_{44} \mathrm{H}_{24} \mathrm{Br}_{4} \mathrm{~N}_{4} \mathrm{Zn}\right)$. UV/vis (toluene): $\lambda 404$ ( $\left.\varepsilon=17033\right), 424(\varepsilon=$ 195165), 550 ( $\varepsilon=10220), 588(\varepsilon=2308)$.

ZnP2. ZnP1 (80 mg, $0.08 \mathrm{mmol})$, bis(pinacolato)diboron $(122 \mathrm{mg}$, $0.48 \mathrm{mmol}),\left[\mathrm{PdCl}_{2}(\mathrm{dppf})\right](12 \mathrm{mg}, 0.016 \mathrm{mmol})$, and $\mathrm{AcOK}(94 \mathrm{mg}$, $0.96 \mathrm{mmol}$ ) were mixed in a reactor specifically designed for microwave irradiation inside a two-necked round-bottom flask in order to place the mixture under an inert atmosphere. A $1.8 \mathrm{~mL}$ portion of dry dioxane and $116 \mu \mathrm{L}$ of dry pyridine were added, and the mixture was degassed before sealing the vial. Then, the mixture was stirred inside the microwave reactor at $150{ }^{\circ} \mathrm{C}$ for $2 \mathrm{~h}$ (a pressure of 3 bar was achieved). Solvent was removed under vacuum, and the resulting dark residue was redissolved in a $\mathrm{CHCl}_{3} / \mathrm{MeOH} 95 / 5$ mixture before the solution was quickly passed through a plug of $\mathrm{SiO}_{2}$ gel. The deep red filtrate was concentrated to afford a purple solid (85 mg, 90\% yield). ${ }^{1} \mathrm{H}$ NMR ( $\left.500 \mathrm{MHz}, \mathrm{CDCl}_{3}\right): \delta 8.91\left(\mathrm{~s}, 2 \mathrm{H}, \mathrm{H}_{2}\right), 8.66$ $\left(\right.$ br, $\left.1 \mathrm{H}, \mathrm{H}_{6}\right), 8.31\left(\mathrm{~d}, J=7.5 \mathrm{~Hz}, 1 \mathrm{H}, \mathrm{H}_{10}\right), 8.21(\mathrm{~d}, J=7.5 \mathrm{~Hz}, 1 \mathrm{H}$, $\left.\mathrm{H}_{8}\right), 7.76\left(\mathrm{t}, J=7.5 \mathrm{~Hz}, 1 \mathrm{H}, \mathrm{H}_{9}\right), 1.38\left(\mathrm{~s}, 12 \mathrm{H}, \mathrm{H}_{12}\right) .{ }^{13} \mathrm{C}\left\{{ }^{1} \mathrm{H}\right\} \mathrm{NMR}$ $\left(126 \mathrm{MHz}, \mathrm{CDCl}_{3}\right): \delta 150.3\left(\mathrm{C}_{3}\right), 142.5\left(\mathrm{C}_{5}\right), 140.5\left(\mathrm{C}_{6}\right), 137.1$ $\left(\mathrm{C}_{10}\right), 133.9\left(\mathrm{C}_{8}\right), 132.1\left(\mathrm{C}_{2}\right), 127.8\left(\mathrm{C}_{7}\right), 126.1\left(\mathrm{C}_{9}\right), 121.1\left(\mathrm{C}_{4}\right)$, 84.1 $\left(\mathrm{C}_{11}\right), 25.1\left(\mathrm{C}_{12}\right)$. HRMS (MALDI-TOF): $m / z 1180.5043[\mathrm{M}]^{+}$ (calcd 1180.5047 for $\left.\mathrm{C}_{68} \mathrm{H}_{72} \mathrm{~B}_{4} \mathrm{~N}_{4} \mathrm{O}_{8} \mathrm{Zn}\right)$. UV/vis (toluene) $\lambda 402(\varepsilon=$ 17927), 425 ( $\varepsilon=324268), 550(\varepsilon=16098), 590(\varepsilon=3049)$.

General Procedure for Quadruple C-C Cross Coupling. The porphyrin derivative (2HP1, ZnP1, or ZnP2; $0.02 \mathrm{mmol}$ ), PAH derivative (9-bromophenanthrene, 1-bromopyrene, bromocorannulene, 1-3; $0.08 \mathrm{mmol}),\left[\mathrm{PdCl}_{2}(\mathrm{dppf})\right](11.7 \mathrm{mg}, 0.016 \mathrm{mmol})$, and ${ }^{t} \mathrm{BuONa}(23.1 \mathrm{mg}, 0.24 \mathrm{mmol})$ were mixed in a reactor specifically designed for microwave irradiation inside a two-necked round-bottom flask in order to place the mixture under an inert atmosphere. A 2.2 $\mathrm{mL}$ portion of dry and degassed toluene and $30 \mu \mathrm{L}$ of dry pyridine were added before sealing the vial. The mixture was then sonicated thoroughly and stirred vigorously inside the microwave reactor at 130 ${ }^{\circ} \mathrm{C}$ for $1 \mathrm{~h}$ (a pressure of 3 bar was achieved). After this time, solvent was removed under vacuum and the black residue was subjected to purification by column chromatography, yielding a pure purple solid corresponding with the expected product.

ZnP2a. Chromatography conditions: $\mathrm{SiO}_{2}$ gel, hexane/AcOEt gradient elution (10/1-5/1-1/1 AcOEt). 98\% yield. ${ }^{1} \mathrm{H}$ NMR (500 $\left.\mathrm{MHz}, \mathrm{CDCl}_{3}\right): \delta 9.20\left(\mathrm{~s}, 2 \mathrm{H}, \mathrm{H}_{2}\right), 8.85-8.75\left(\mathrm{~m}, 1 \mathrm{H}, \mathrm{H}_{17}\right), 8.75-8.65$ $\left(\mathrm{m}, 1 \mathrm{H}, \mathrm{H}_{20}\right), 8.50-8.41\left(\mathrm{~m}, 1 \mathrm{H}, \mathrm{H}_{6}\right), 8.41-8.35\left(\mathrm{~m}, 1 \mathrm{H}, \mathrm{H}_{23}\right), 8.35-$ $8.27\left(\mathrm{~m}, 1 \mathrm{H}, \mathrm{H}_{10}\right), 8.05-7.96\left(\mathrm{~m}, 2 \mathrm{H}, \mathrm{H}_{12}+\mathrm{H}_{8}\right), 7.96-7.86(\mathrm{~m}, 2 \mathrm{H}$, $\left.\mathrm{H}_{14}+\mathrm{H}_{9}\right)$, 7.73-7.51 (m, 4H, $\left.\mathrm{H}_{16}+\mathrm{H}_{21}+\mathrm{H}_{15}+\mathrm{H}_{22}\right) .{ }^{13} \mathrm{C}\left\{{ }^{1} \mathrm{H}\right\} \mathrm{NMR}$ $\left(126 \mathrm{MHz}, \mathrm{CDCl}_{3}\right): \delta 150.4\left(\mathrm{C}_{3}\right), 143.1\left(\mathrm{C}_{5}\right), 139.2\left(\mathrm{C}_{7}\right), 138.7$ $\left(\mathrm{C}_{11}\right), 136.4\left(\mathrm{C}_{6}\right), 133.7\left(\mathrm{C}_{10}\right), 132.3\left(\mathrm{C}_{2}\right), 131.7\left(\mathrm{C}_{24}\right), 131.4\left(\mathrm{C}_{19}\right)$, $130.9\left(\mathrm{C}_{13}\right), 130.2\left(\mathrm{C}_{18}\right), 129.4\left(\mathrm{C}_{8}\right), 128.8\left(\mathrm{C}_{14}\right), 128.2\left(\mathrm{C}_{12}\right), 127.1-$ $126.7\left(\mathrm{C}_{9}+\mathrm{C}_{15}+\mathrm{C}_{16}+\mathrm{C}_{21}+\mathrm{C}_{22}+\mathrm{C}_{23}\right), 123.1\left(\mathrm{C}_{17}\right), 122.6\left(\mathrm{C}_{20}\right)$, $121.1\left(\mathrm{C}_{4}\right)$. HRMS (MALDI): $m / z 1380.4075[\mathrm{M}]^{+}$(calcd 1380.4104 for $\left.\mathrm{C}_{100} \mathrm{H}_{60} \mathrm{~N}_{4} \mathrm{Zn}\right)$. UV/vis (toluene): $\lambda 300(\varepsilon=38806), 400(\varepsilon=$ 14179), 426 ( $\varepsilon=211940), 552(\varepsilon=11642), 590(\varepsilon=4627)$.
ZnP2b. Chromatography conditions: $\mathrm{SiO}_{2}$ gel, hexane/AcOEt gradient elution (5/1-1/1 AcOEt). 92\% yield. ${ }^{1} \mathrm{H}$ NMR (500 MHz, $\left.\mathrm{CDCl}_{3}\right): \delta 9.26-9.21\left(\mathrm{~m}, 2 \mathrm{H}, \mathrm{H}_{2}\right), 8.68-8.59\left(\mathrm{~m}, 1 \mathrm{H}, \mathrm{H}_{23}\right), 8.59-$ $8.47\left(\mathrm{~m}, 1 \mathrm{H}, \mathrm{H}_{6}\right), 8.45-8.35\left(\mathrm{~m}, 1 \mathrm{H}, \mathrm{H}_{10}\right), 8.35-8.22\left(\mathrm{~m}, 2 \mathrm{H}, \mathrm{H}_{12}+\right.$ $\left.\mathrm{H}_{13}\right), 8.22-7.86\left(\mathrm{~m}, 8 \mathrm{H}, \mathrm{H}_{22}+\mathrm{H}_{8}+\mathrm{H}_{9}+\mathrm{H}_{19}+\mathrm{H}_{16}+\mathrm{H}_{18}+\mathrm{H}_{20}+\right.$ $\left.\mathrm{H}_{15}\right) .{ }^{13} \mathrm{C}\left\{{ }^{1} \mathrm{H}\right\}$ NMR $\left(126 \mathrm{MHz}, \mathrm{CDCl}_{3}\right): \delta 150.4\left(\mathrm{C}_{3}\right), 143.3\left(\mathrm{C}_{5}\right)$, $139.5\left(\mathrm{C}_{7}\right), 137.6\left(\mathrm{C}_{11}\right), 137.0\left(\mathrm{C}_{6}\right), 133.6\left(\mathrm{C}_{10}\right), 132.2\left(\mathrm{C}_{2}\right), 131.6-$ $130.7\left(\mathrm{C}_{26}+\mathrm{C}_{17}+\mathrm{C}_{21}+\mathrm{C}_{14}\right), 129.8\left(\mathrm{C}_{8}\right), 128.9\left(\mathrm{C}_{24}\right), 128.1\left(\mathrm{C}_{12}\right)$, $127.8\left(\mathrm{C}_{22}\right.$ or $\left.\mathrm{C}_{16}\right), 127.5\left(\mathrm{C}_{22}\right.$ or $\left.\mathrm{C}_{16}\right), 126.7\left(\mathrm{C}_{9}\right), 126.0\left(\mathrm{C}_{19}\right), 125.4$ $\left(\mathrm{C}_{23}\right), 125.2-124.8\left(\mathrm{C}_{13}+\mathrm{C}_{15}+\mathrm{C}_{18}+\mathrm{C}_{20}+\mathrm{C}_{25}\right), 121.0\left(\mathrm{C}_{4}\right)$. HRMS (MALDI): $m / z$ 1476.4104 [M] $]^{+}$(calcd 1476.4104 for $\left.\mathrm{C}_{108} \mathrm{H}_{60} \mathrm{~N}_{4} \mathrm{Zn}\right)$. $\mathrm{UV} / \mathrm{vis}$ (toluene): $\lambda 329(\varepsilon=36774), 347(\varepsilon=48548), 405(\varepsilon=$ 20806), 428 ( $\varepsilon=195161), 551(\varepsilon=12903), 591(\varepsilon=4839)$.

$\mathrm{ZnP2c}$. Chromatography conditions: $\mathrm{SiO}_{2}$ gel, hexane/AcOEt gradient elution (5:1-1:1 AcOEt $\left.\mathrm{CHCl}_{3}\right) .85 \%$ yield. ${ }^{1} \mathrm{H}$ NMR (500 $\left.\mathrm{MHz}, \mathrm{CDCl}_{3}\right): \delta 9.18\left(\mathrm{~s}, 2 \mathrm{H}, \mathrm{H}_{2}\right), 8.74-8.65\left(\mathrm{~m}, 1 \mathrm{H}, \mathrm{H}_{6}\right), 8.42-8.31$ $\left(\mathrm{m}, 1 \mathrm{H}, \mathrm{H}_{10}\right), 8.28-8.21\left(\mathrm{~m}, 1 \mathrm{H}, \mathrm{H}_{8}\right), 8.21-8.11\left(\mathrm{~m}, 2 \mathrm{H}, \mathrm{H}_{24}+\mathrm{H}_{12}\right)$, 8.00-7.90 (m, $\left.1 \mathrm{H}, \mathrm{H}_{9}\right), 7.90-7.72\left(\mathrm{~m}, 7 \mathrm{H}, \mathrm{H}_{14}+\mathrm{H}_{15}+\mathrm{H}_{17}+\mathrm{H}_{18}+\right.$ $\left.\mathrm{H}_{20}+\mathrm{H}_{21}+\mathrm{H}_{23}\right){ }^{13} \mathrm{C}\left\{{ }^{1} \mathrm{H}\right\}$ NMR $\left(126 \mathrm{MHz}, \mathrm{CDCl}_{3}\right): \delta 150.3\left(\mathrm{C}_{3}\right)$, $145.2\left(\mathrm{C}_{25}\right.$ or $\left.\mathrm{C}_{27}\right), 143.5\left(\mathrm{C}_{5}\right), 137.7\left(\mathrm{C}_{7}\right), 136.2\left(\mathrm{C}_{6}\right), 133.9\left(\mathrm{C}_{10}\right)$, $132.1\left(\mathrm{C}_{2}\right), 123.0\left(\mathrm{C}_{25}\right.$ or $\left.\mathrm{C}_{27}\right), 129.0\left(\mathrm{C}_{8}\right), 127.5\left(\mathrm{C}_{24}\right), 127.2-126.7$ $\left(\mathrm{C}_{9}+\mathrm{C}_{14}+\mathrm{C}_{15}+\mathrm{C}_{17}+\mathrm{C}_{18}+\mathrm{C}_{20}+\mathrm{C}_{21}+\mathrm{C}_{23}\right), 126.2\left(\mathrm{C}_{12}\right), 120.9$ $\left(\mathrm{C}_{4}\right)$. HRMS (MALDI): $\mathrm{m} / z$ 1668,4133 [M] ${ }^{+}$(calcd 1668,4104 for $\left.\mathrm{C}_{124} \mathrm{H}_{60} \mathrm{~N}_{4} \mathrm{Zn}\right)$. UV/vis (toluene): $\lambda 296(\varepsilon=146667), 400(\varepsilon=$ 27407), 427 ( $\varepsilon=438889), 551(\varepsilon=22037), 590(\varepsilon=4630)$.

General Procedure for Zn Removal. Zinc porphyrin derivatives (ZnP2a, ZnP2b, ZnP2c; $0.02 \mathrm{mmol}$ ) were dissolved in $2 \mathrm{~mL}$ of DCM. To the deep red solution was added $2 \mathrm{~mL}$ of $\mathrm{CF}_{3} \mathrm{CO}_{2} \mathrm{H}$ at once, and the mixture immediately turned green. The mixture was stirred at room temperature overnight. Then, it was diluted with $6 \mathrm{~mL}$ of DCM and a saturated solution of $\mathrm{Na}_{2} \mathrm{CO}_{3}$ in water was added portionwise with vigorous stirring until the evolution of gas ceased and the organic layer became deep red again. This layer was separated from the aqueous phase and washed three times with water. The solution was finally dried with anhydrous $\mathrm{MgSO}_{4}$, filtered, and concentrated to afford the expected demetalated product as a purple solid.

2HP2a. From quadruple cross coupling of 1 and 2HP1: chromatography conditions $\mathrm{SiO}_{2}$ gel, hexane/AcOEt gradient elution (10:1-5:1-1:1 AcOEt); 73\% yield. From demetalation of ZnP2a: quantitative yield. ${ }^{1} \mathrm{H}$ NMR $\left(500 \mathrm{MHz}, \mathrm{CDCl}_{3}\right): \delta 9.10\left(\mathrm{~s}, 2 \mathrm{H}, \mathrm{H}_{2}\right)$, 8.85-8.76 (m, $\left.1 \mathrm{H}, \mathrm{H}_{17}\right), 8.76-8.66\left(\mathrm{~m}, 1 \mathrm{H}, \mathrm{H}_{20}\right), 8.50-8.40(\mathrm{~m}, 1 \mathrm{H}$, $\left.\mathrm{H}_{6}\right), 8.40-8.26\left(\mathrm{~m}, 2 \mathrm{H}, \mathrm{H}_{23}+\mathrm{H}_{10}\right), 8.06-7.95\left(\mathrm{~m}, 2 \mathrm{H}, \mathrm{H}_{12}+\mathrm{H}_{8}\right)$, 7.95-7.85 (m, 2H, $\left.\mathrm{H}_{23}+\mathrm{H}_{8}\right), 7.75-7.56\left(\mathrm{~m}, 4 \mathrm{H}, \mathrm{H}_{22}+\mathrm{H}_{21}+\mathrm{H}_{15}+\right.$ $\left.\mathrm{H}_{16}\right),-2.70$ (br, $\left.0.5 \mathrm{H}, \mathrm{H}_{1}\right) \cdot{ }^{13} \mathrm{C}\left\{{ }^{1} \mathrm{H}\right\}$ NMR $\left(126 \mathrm{MHz}, \mathrm{CDCl}_{3}\right): \delta$ $142.4\left(\mathrm{C}_{5}\right), 139.4\left(\mathrm{C}_{7}\right), 138.6\left(\mathrm{C}_{11}\right), 136.4\left(\mathrm{C}_{6}\right), 133.9\left(\mathrm{C}_{10}\right), 131.7$ $\left(\mathrm{C}_{24}\right), 131.4\left(\mathrm{C}_{13}\right), 130.9\left(\mathrm{C}_{19}\right), 130.2\left(\mathrm{C}_{18}\right), 129.6\left(\mathrm{C}_{8}\right), 128.9\left(\mathrm{C}_{14}\right)$, $128.2\left(\mathrm{C}_{12}\right), 127.0\left(\mathrm{C}_{23}\right), 126.8-126.7\left(\mathrm{C}_{9}+\mathrm{C}_{22}+\mathrm{C}_{16}+\mathrm{C}_{21}+\mathrm{C}_{15}\right)$, $123.2\left(\mathrm{C}_{17}\right), 122.7\left(\mathrm{C}_{20}\right), 120.2\left(\mathrm{C}_{4}\right)$. HRMS (MALDI): $\mathrm{m} / \mathrm{z}$ $1318.4946[\mathrm{M}]^{+}$(calcd 1318.4969 for $\left.\mathrm{C}_{100} \mathrm{H}_{62} \mathrm{~N}_{4}\right)$. UV/vis (toluene): $\lambda 300$ ( $\varepsilon=35455), 400(\varepsilon=34364), 422(\varepsilon=250727), 517(\varepsilon=$ $11091), 551(\varepsilon=5091)$.

2HP2b. From quadruple cross coupling of 2 and 2HP1: chromatography conditions $\mathrm{SiO}_{2}$ gel, hexane/AcOEt gradient elution (5:1-1:1 - AcOEt); 57\% yield. From demetalation of $\mathbf{Z n P 2 b}$ : quantitative yield. ${ }^{1} \mathrm{H}$ NMR $\left(500 \mathrm{MHz}, \mathrm{CDCl}_{3}\right): \delta 9.15\left(\mathrm{~s}, 2 \mathrm{H}, \mathrm{H}_{2}\right)$, 8.68-8.56 (m, $\left.1 \mathrm{H}, \mathrm{H}_{23}\right), 8.56-8.48\left(\mathrm{~m}, 1 \mathrm{H}, \mathrm{H}_{6}\right), 8.45-8.34(\mathrm{~m}, 1 \mathrm{H}$, $\left.\mathrm{H}_{10}\right), 8.34-8.23\left(\mathrm{~m}, 2 \mathrm{H}, \mathrm{H}_{12}+\mathrm{H}_{13}\right), 8.23-7.88\left(\mathrm{~m}, 8 \mathrm{H}, \mathrm{H}_{19}+\mathrm{H}_{9}+\right.$ $\left.\mathrm{H}_{16}+\mathrm{H}_{8}+\mathrm{H}_{20}+\mathrm{H}_{18}+\mathrm{H}_{15}+\mathrm{H}_{22}\right),-2.65\left(\mathrm{br}, 0.5 \mathrm{H}, \mathrm{H}_{1}\right) .{ }^{13} \mathrm{C}\left\{{ }^{1} \mathrm{H}\right\}$ NMR $\left(126 \mathrm{MHz}, \mathrm{CDCl}_{3}\right): \delta 142.5\left(\mathrm{C}_{5}\right), 139.8\left(\mathrm{C}_{7}\right), 137.4\left(\mathrm{C}_{11}\right)$, $137.0\left(\mathrm{C}_{6}\right), 133.7\left(\mathrm{C}_{10}\right), 131.6-130.7\left(\mathrm{C}_{26}+\mathrm{C}_{17}+\mathrm{C}_{21}+\mathrm{C}_{14}\right), 130.2$ $\left(\mathrm{C}_{8}\right), 128.9\left(\mathrm{C}_{24}\right), 128.1\left(\mathrm{C}_{12}\right), 127.9\left(\mathrm{C}_{22}\right), 127.6\left(\mathrm{C}_{16}\right), 126.9\left(\mathrm{C}_{9}\right)$, $126.1\left(\mathrm{C}_{19}\right), 125.3-124.9\left(\mathrm{C}_{13}+\mathrm{C}_{15}+\mathrm{C}_{18}+\mathrm{C}_{20}+\mathrm{C}_{23}+\mathrm{C}_{25}\right), 120.2$ $\left(\mathrm{C}_{4}\right)$. HRMS (MALDI): $\mathrm{m} / z$ 1414.4981 [M] ${ }^{+}$(calcd 1414.4969 for $\left.\mathrm{C}_{108} \mathrm{H}_{62} \mathrm{~N}_{4}\right) . \mathrm{UV} /$ vis (toluene): $\lambda 333(\varepsilon=28462), 348(\varepsilon=35692)$, 405 ( $\varepsilon=19846), 424$ ( $\varepsilon=93077), 517$ ( $\varepsilon=6923)$, $551(\varepsilon=4769)$.

2HP2c. From quadruple cross coupling of 3 and 2HP1: chromatography conditions $\mathrm{SiO}_{2}$ gel, hexane/AcOEt gradient elution (5:1-1:1 AcOEt $\left.\mathrm{CHCl}_{3}\right)$; 47\% yield. From demetalation of $\mathbf{Z n P 2 c}$ : quantitative yield. ${ }^{1} \mathrm{H}$ NMR $\left(500 \mathrm{MHz}, \mathrm{CDCl}_{3}\right): \delta 9.07\left(\mathrm{~s}, 2 \mathrm{H}, \mathrm{H}_{2}\right)$, $8.72-8.63\left(\mathrm{~m}, 1 \mathrm{H}, \mathrm{H}_{6}\right), 8.41-8.31\left(\mathrm{~m}, 1 \mathrm{H}, \mathrm{H}_{10}\right), 8.27-8.21(\mathrm{~m}, 1 \mathrm{H}$, 
$\mathrm{H}_{8}$ ), 8.20-8.11 (m, $\left.2 \mathrm{H}, \mathrm{H}_{12}+\mathrm{H}_{24}\right), 8.00-7.90\left(\mathrm{~m}, 1 \mathrm{H}, \mathrm{H}_{9}\right), 7.90-$ $7.70\left(\mathrm{~m}, 7 \mathrm{H}, \mathrm{H}_{14}+\mathrm{H}_{15}+\mathrm{H}_{17}+\mathrm{H}_{18}+\mathrm{H}_{20}+\mathrm{H}_{21}+\mathrm{H}_{23}\right),-2.65(\mathrm{br}$, $\left.0.5 \mathrm{H}, \mathrm{H}_{1}\right) .{ }^{13} \mathrm{C}\left\{{ }^{1} \mathrm{H}\right\}$ NMR $\left(126 \mathrm{MHz}, \mathrm{CDCl}_{3}\right): \delta 142.8\left(\mathrm{C}_{5}\right), 141.5$ $\left(\mathrm{C}_{25}\right.$ or $\left.\mathrm{C}_{27}\right), 138.2\left(\mathrm{C}_{7}\right), 136.3\left(\mathrm{C}_{6}\right), 135.9\left(\mathrm{C}_{11}\right), 135.5\left(\mathrm{C}_{10}\right), 134.1$ $\left(\mathrm{C}_{2}\right), 131.1\left(\mathrm{C}_{25}\right.$ or $\left.\mathrm{C}_{27}\right), 129.5\left(\mathrm{C}_{8}\right), 127.7\left(\mathrm{C}_{24}\right), 127.6-126.8\left(\mathrm{C}_{9}+\right.$ $\left.\mathrm{C}_{14}+\mathrm{C}_{15}+\mathrm{C}_{17}+\mathrm{C}_{18}+\mathrm{C}_{20}+\mathrm{C}_{21}+\mathrm{C}_{23}\right), 126.6\left(\mathrm{C}_{12}\right), 120.1\left(\mathrm{C}_{4}\right)$. HRMS (MALDI): $m / z$ 1606,4985 [M] ${ }^{+}$(calcd 1606,4969 for $\left.\mathrm{C}_{124} \mathrm{H}_{62} \mathrm{~N}_{4}\right) . \mathrm{UV} /$ vis (toluene): $\lambda 294(\varepsilon=131250), 404(\varepsilon=$ 47000), 424 ( $\varepsilon=279750), 516(\varepsilon=13000), 551(\varepsilon=6500)$.

General Procedure for Complexation Measurements. A $10^{-4}$ $M$ solution of each compound in deuterated toluene was prepared, and a known volume was transferred to a NMR tube $(500 \mu \mathrm{L})$. It was titrated by adding known portions of a stock solution of $\mathrm{C}_{60}\left(10^{-3} \mathrm{M}\right)$ in deuterated toluene covering a wide range of amounts. A ${ }^{1} \mathrm{H}$ NMR spectrum was recorded at room temperature after each addition. Once all data were obtained, changes in chemical shifts $(\Delta \delta)$ for selected protons $\left(\mathrm{H}_{1}, \mathrm{H}_{2}\right.$. and/or $\left.\mathrm{H}_{6}\right)$ were plotted as a function of guest molar fraction and the resulting curve was fitted by a nonlinear method.

\section{ASSOCIATED CONTENT}

\section{S Supporting Information}

The Supporting Information is available free of charge on the ACS Publications website at DOI: 10.1021/acs.joc.6b00454.

Overview of both synthetic methods, atom numbering, one- and two-dimensional NMR spectra of all new compounds, MS spectra, UV-vis spectra, details of association constant estimation, and computational calculations (PDF)

\section{AUTHOR INFORMATION}

\section{Corresponding Author}

*E-mail: celedonio.alvarez@uva.es.

\section{Notes}

The authors declare no competing financial interest.

\section{ACKNOWLEDGMENTS}

This work was funded by the Spanish Ministerio de Economía y Competitividad (CTQ 2013-41067-P). H.B. acknowledges with thanks an MEC-FPI grant. Allocation of computer facilities at IQTCUB is also acknowledged.

\section{REFERENCES}

(1) (a) The Porphyrin Handbook; Kadish, K. M., Smith, K. M., Guilard, R, Eds.; Elsevier: Amsterdam, 2000; 20 volumes (particularly interesting Vol. 6: Applications: Past, Present and Future). (b) Handbook of Porphyrin Science; Kadish, K. M., Smith, K. M., Guilard, R., Eds.; World Scientific: Singapore, 2010; 35 volumes (particularly interesting Vols. 1, 4, 10, 11, 12, 18, 21, 27, 28, 33, 34).

(2) Bonifazi, D.; Enger, O.; Diederich, F. Chem. Soc. Rev. 2007, 36, 390-414 and references therein.

(3) (a) Fang, X.; Zhu, Y.-Z.; Zheng, J.-Y. J. Org. Chem. 2014, 79, 1184-1191. (b) Zhu, B.; Chen, H.; Lin, W.; Ye, Y.; Wu, J.; Li, S. J. Am. Chem. Soc. 2014, 136, 15126-15129. (c) García-Simón, C.; GarciaBorràs, M.; Gómez, L.; Parella, T.; Osuna, S.; Juanhuix, J.; Imaz, I.; Maspoch, D.; Costas, M.; Ribas, X. Nat. Commun. 2014, 5, 5557.

(4) (a) Olmstead, M. M.; Costa, D. A.; Maitra, K.; Noll, B. C.; Phillips, S. L.; Van Calcar, P. M.; Balch, A. L. J. Am. Chem. Soc. 1999, 121, 7090-7097. (b) Evans, D. R.; Fackler, N. L. P.; Xie, Z.; Rickard, C. E. F.; Boyd, P. D. W.; Reed, C. A. J. Am. Chem. Soc. 1999, 121, 8466-8474. (c) Boyd, P. D. W.; Hodgson, M. C.; Rickard, C. E. F.; Oliver, A. G.; Chaker, L.; Brothers, P. J.; Bolskar, R. D.; Tham, F. S.; Reed, C. A. J. Am. Chem. Soc. 1999, 121, 10487-10495.

(5) Saegusa, Y.; Ishizuka, T.; Kojima, T.; Mori, S.; Kawano, M.; Kojima, T. Chem. - Eur. J. 2015, 21, 5302-5306.

(6) (a) Álvarez, C. M.; García-Escudero, L. A.; García-Rodríguez, R.; Martín-Álvarez, J. M.; Miguel, D.; Rayón, V. M. Dalton Trans. 2014,
43, 15693-15696. (b) Álvarez, C. M.; Aullón, G.; Barbero, H.; GarcíaEscudero, L. A.; Martínez-Pérez, C.; Martín-Álvarez, J. M.; Miguel, D. Org. Lett. 2015, 17, 2578-2581.

(7) (a) Stuparu, M. C. Angew. Chem., Int. Ed. 2013, 52, 7786-7790. (b) Yanney, M.; Fronczek, F. R.; Sygula, A. Angew. Chem., Int. Ed. 2015, 54, 11153-11156. (c) Abeyratne Kuragama, P. L.; Fronczek, F. R.; Sygula, A. Org. Lett. 2015, 17, 5292-5295.

(8) (a) Chauhan, S. M. S.; Sahoo, B. B.; Srinivas, K. A. Synth. Commun. 2001, 31, 33-37. (b) Nascimento, B. F. O.; Pineiro, M.; Gonsalves, A. M. R.; Silva, M. R.; Beja, A. M.; Paixão, J. A. J. Porphyrins Phthalocyanines 2007, 11, 77-84. (c) De Paula, R.; Faustino, M. A. F.; Pinto, D. C. G. A.; Neves, M. G. P. M. S.; Cavaleiro, J. A. S. J. Heterocycl. Chem. 2008, 45, 453-459.

(9) For instance, the Miyaura reaction was complete after $2 \mathrm{~h}$, while $72 \mathrm{~h}$ was needed to reach the same conversion with reflux by conventional methods.

(10) (a) Crossley, M. J.; Field, L. D.; Forster, A. J.; Harding, M. M.; Sternhell, S. J. Am. Chem. Soc. 1987, 109, 341-348. (b) Song, R.; Robert, A.; Bernadou, J.; Meunier, B. Analusis 1999, 27, 464-467. (c) Tomé, A. C.; Silva, A. M. S.; Alkorta, I.; Elguero, J. J. Porphyrins Phthalocyanines 2011, 15, 1-28.

(11) There are many works in which all atropisomers could be isolated, but all authors stated that only ortho-substituted porphyrins gave satisfactory results. For a few examples, see ref 10a and: (a) Rose, E.; Cardon-Pilotaz, A.; Quelquejeu, M.; Bernard, N.; Kossanyi, A. J. Org. Chem. 1995, 60, 3919-3920. (b) Spasojević, I.; Menzeleev, R.; White, P. S.; Fridovich, I. Inorg. Chem. 2002, 41, 5874-5881. (c) Hunter, C. A.; Misuraca, M. C.; Turega, S. M. J. Am. Chem. Soc. 2011, 133, 582-594. (d) Sun, H.; Guo, K.; Gan, H.; Li, X.; Hunter, C. A. Org. Biomol. Chem. 2015, 13, 8053-8066.

(12) (a) Zhao, Y.-L.; Stoddart, J. F. Acc. Chem. Res. 2009, 42, 11611171. (b) Gavrel, G.; Jousselme, B.; Filoramo, A.; Campidelli, S. Top. Curr. Chem. 2013, 348, 95-126.

(13) Porphyrins 2HP1, ZnP1, and $\mathbf{Z n P 2}$ were also tested to investigate their properties to bind buckminsterfullerene, but both MS and ${ }^{1} \mathrm{H}$ NMR spectra gave negative results, indicating that $\mathrm{PAH}$ subunits slightly contribute to form the adduct.

(14) See the Supporting Information for details about the computational model followed.

(15) Additional possibilities were also tested, implying other initial assignments for $\mathrm{H}_{6}$ as reported by Sternhell et al. or Bernardou and Meunier et al., ${ }^{10 a, b}$ but none of them were reasonable in our case, due mainly to inconsistencies in fitting of the curves and no reproducibility in comparison to $\mathrm{H}_{2}$ chemical shift changes.

(16) (a) Purification of Laboratory Chemicals, 5th ed.; Armarego, W. L. E., Chai, C. L. L., Eds.; Butterworth-Heinemann: London, 2003. (b) Williams, D. B. G.; Lawton, M. J. Org. Chem. 2010, 75, 83518354.

(17) (a) Topolinski, B.; Schmidt, B. M.; Kathan, M.; Troyanov, S. I.; Lentz, D. Chem. Commun. 2012, 48, 6298-6300. (b) Butterfield, A. M.; Gilomen, B.; Siegel, J. S. Org. Process Res. Dev. 2012, 16, 664-676.

(18) (a) Boller, T. M.; Murphy, J. M.; Hapke, M.; Ishiyama, T.; Miyaura, N.; Hartwig, J. F. J. Am. Chem. Soc. 2005, 127, 14263-14278. (b) Beinhoff, M.; Weigel, W.; Jurczok, M.; Rettig, W.; Modrakowski, C.; Brüdgam, I.; Hartl, H.; Schlüter, A. D. Eur. J. Org. Chem. 2001, 2001, 3819-3829. (c) Wegner, H. A.; Scott, L. T.; de Meijere, A. J. Org. Chem. 2003, 68, 883-887.

(19) Hunter, C. A.; Misuraca, M. C.; Turega, S. M. J. Am. Chem. Soc. 2011, 133, 582-594. 


\section{BIBLIOGRAFÍA}





\title{
8. BIBLIOGRAFÍA
}

\begin{abstract}
${ }^{1}$ Los requisitos singulares de la revista del Artículo IV exigen que se incluyan explícitamente las geometrías de todos los compuestos calculados en la Supporting Information. En nuestro caso esto alarga dicho documento alrededor de 38 páginas de manera artificial. Con el objetivo de no agrandar el tamaño de esta tesis en su formato impreso de manera innecesaria, se ha prescindido de todo ese material, mientras que la versión electrónica de este documento contendrá toda la extensión.
\end{abstract}

${ }^{2}$ 1. (a) Balzani, V., Supramolecular Chemistry. Kluwer Academic: 1992. (b) Lehn, J.-M., Supramolecular Chemistry: Concepts and Perspectives. Wiley-VCH: Weinheim, 1995. (c) Atwood, J. L.; Davies, J. E. D.; MacNicol, D. D.; Vögtle, F.; Lehn, J.-M., Comprehensive Supramolecular Chemistry. Pergamon: Oxford, 1996. (d) Lehn, J.-M. PNAS 2002, 99, 4763-4768. (e) Atwood, J. L. S.; teed, J. W., Encyclopedia of Supramolecular Chemistry. Taylor \& Francis Group: New York, 2005. (f) Steed, J. W.; Atwood, J. L., Supramolecular Chemistry, 2nd Edition. Wiley-VCH: 2009.

3 (a) Göpel, W. Adv. Mater. 1993, 5, 865-866. (b) Silva, G. A. Surg. Neurol. 2004, 61, 216-220. (c) Caruthers, S. D.; Wickline, S. A.; Lanza, G. M. Curr. Opin. Biotechnol. 2007, 18, 26-30. (d) Ozin, G. A.; Arsenault, A.; Cademartiri, L., Nanochemistry: A Chemical Approach to Nanomaterials. RSC Publishing: 2008. (e) Cademartiri, L.; Ozin, G. A., Concepts of Nanochemistry. Wiley-VCH: 2009. (f) Kerativitayanan, P.; Carrow, J. K.; Gaharwar, A. K. Adv. Healthc. Mater. 2015, 4, 1600-1627.

${ }^{4}$ (a) Franklin, R. E.; Gosling, R. G. Nature 1953, 171, 740-741. (b) Watson, J. D.; Crick, F. H. C. Nature 1953, 171, 737-738. (c) Wilkins, M. H. F.; Stokes, A. R.; Wilson, H. R. Nature 1953, 171, 738740. (d) Saenger, W., Principles of Nucleic Acid Structure. Springer: New York, 1984.

5 (a) Anfinsen, C. B. Biochem. J. 1972, 128, 737-749. (b) Alberts, B.; Johnson, A.; Lewis, J.; Raff, M.; Roberts, K.; Walter., P., Proteins. In Molecular Biology of the Cell, 4th edition, Garland Science: New York, 2002.

6 Yoshikawa, S.; Shimada, A.; Shinzawa-Itoh, K., Respiratory Conservation of Energy with Dioxygen: Cytochrome c Oxidase. In Sustaining Life on Planet Earth: Metalloenzymes Mastering Dioxygen and Other Chewy Gases, Springer International Publishing: 2015; pp 89-130.

${ }^{7}$ (a) Vale, R. D.; Reese, T. S.; Sheetz, M. P. Cell 1985, 42, 39-50. (b) Rosenbaum, J. L.; Witman, G. B. Nat Rev Mol Cell Biol 2002, 3, 813-825. (c) Vale, R. D. Cell 2003, 112, 467-480. (d) Klumpp, S.; Lipowsky, R. PNAS 2005, 102, 17284-17289.

8 (a) Cram, D. J.; Steinberg, H. J. Am. Chem. Soc. 1951, 73, 5691-5704. (b) Pedersen, C. J. J. Am. Chem. Soc. 1967, 89, 7017-7036. (c) Dietrich, B.; Lehn, J. M.; Sauvage, J. P. Tetrahedron Lett. 1969, 10, 2885-2888. (d) Dietrich, B.; Lehn, J. M.; Sauvage, J. P. Tetrahedron Lett. 1969, 10, 2889-2892.

9 "The Nobel Prize in Chemistry 1987". Nobelprize.org. Nobel Media AB 2014. Web. 25 Aug 2016. $<$ http://www.nobelprize.org/nobel_prizes/chemistry/laureates/1987/index.html>

${ }^{10}$ (a) Rowan, S. J.; Cantrill, S. J.; Cousins, G. R. L.; Sanders, J. K. M.; Stoddart, J. F. Angew. Chem. Int. Ed. 2002, 41, 898-952. (b) Wilson, H.; Byrne, S.; Mullen, K. M. Chem. Asian J. 2015, 10, 715-721.

${ }^{11}$ (a) Amabilino, D. B.; Stoddart, J. F. Chem. Rev. 1995, 95, 2725-2828. (b) Breault, G. A.; Hunter, C. A.; Mayers, P. C. Tetrahedron 1999, 55, 5265-5293. (c) Vögtle, F.; Lukin, O.; Godt, A. Chem. Eur. J. 2004, 10, 1878-1883. (d) Badjić, J. D.; Nelson, A.; Cantrill, S. J.; Turnbull, W. B.; Stoddart, J. F. Acc. Chem. Res. 2005, 38, 723-732. (e) Amabilino, D. B.; Perez-Garcia, L. Chem. Soc. Rev. 2009, 38, $1562-$ 1571.

12 (a) Wasserman, E. J. Am. Chem. Soc. 1960, 82, 4433-4434. (b) Harrison, I. T.; Harrison, S. J. Am. Chem. Soc. 1967, 89, 5723-5724. (c) Schill, G.; Zollenkopf, H. Justus Liebigs Ann. Chem. 1969, 721, 5374.

${ }^{13}$ (a) Frisch, H. L.; Wasserman, E. J. Am. Chem. Soc. 1961, 83, 3789-3795. (b) Musso, H. Angew. Chem. 1972, 84, 270-270.

14 (a) Stoddart, J. F. Chem. Soc. Rev. 2009, 38, 1802-1820. (b) Fang, L.; Olson, M. A.; Benitez, D.; Tkatchouk, E.; Goddard Iii, W. A.; Stoddart, J. F. Chem. Soc. Rev. 2010, 39, 17-29. (c) Bruns, C. J.; Stoddart, J. F., The Mechanical Bond: A Work of Art. In Beauty in Chemistry, Fabbrizzi, L., Ed. Springer-Verlag: Heidelberg, 2011; pp 19-72.

15 (a) Aricó, F.; Badjic, J. D.; Cantrill, S. J.; Flood, A. H.; Leung, K. C.-F.; Liu, Y.; Stoddart, J. F., Templated Synthesis of Interlocked Molecules. In Templates in Chemistry II, Schalley, C. A.; Vögtle, F.; Dötz, K. H., Eds. Springer: Heidelberg, 2005; pp 203-259. (b) Stoddart, J. F.; Dichtel, W. R.; Miljanić, O. S.; Zhang, W.; Spruell, J. M.; Patel, K.; Aprahamian, I.; Heath, J. R. Acc. Chem. Res. 2008, 41, 17501761 . 
${ }^{16}$ (a) Vögtle, F.; Safarowsky, O.; Windisch, B.; Mohry, A. J. Prakt. Chem. 2000, 342, 437-444. (b) Yerin, A.; Wilks, E. S.; Moss, G. P.; Harada, A. Pure Appl. Chem. 2008, 80, 28.

17 (a) Dietrich-Buchecker, C. O.; Sauvage, J.-P. Angew. Chem. Int. Ed. 1989, 28, 189-192. (b) Dietrich-Buchecker, C. O.; Nierengarten, J. F.; Sauvage, J. P.; Armaroli, N.; Balzani, V.; De Cola, L. J. Am. Chem. Soc. 1993, 115, 11237-11244. (c) Safarowsky, O.; Nieger, M.; Fröhlich, R.; Vögtle, F. Angew. Chem. Int. Ed. 2000, 39, 1616-1618. (d) Adams, H.; Ashworth, E.; Breault, G. A.; Guo, J.; Hunter, C. A.; Mayers, P. C. Nature 2001, 411, 763-763. (e) Feigel, M.; Ladberg, R.; Engels, S.; Herbst-Irmer, R.; Fröhlich, R. Angew. Chem. Int. Ed. 2006, 45, 5698-5702. (f) Ayme, J.-F.; Beves, J. E.; Leigh, D. A.; McBurney, R. T.; Rissanen, K.; Schultz, D. Nat. Chem. 2012, 4, 15-20.

${ }^{18}$ (a) Nierengarten, J. F.; Dietrich-Buchecker, C. O.; Sauvage, J. P. J. Am. Chem. Soc. 1994, 116, $375-$ 376. (b) Ibukuro, F.; Fujita, M.; Yamaguchi, K.; Sauvage, J.-P. J. Am. Chem. Soc. 1999, 121, 1101411015. (c) McArdle, C. P.; Vittal, J. J.; Puddephatt, R. J. Angew. Chem. 2000, 112, 3977-3980. (d) Pentecost, C. D.; Chichak, K. S.; Peters, A. J.; Cave, G. W. V.; Cantrill, S. J.; Stoddart, J. F. Angew. Chem. Int. Ed. 2007, 46, 218-222. (e) Peinador, C.; Blanco, V.; Quintela, J. M. J. Am. Chem. Soc. 2009, 131, 920-921. (f) Gil-Ramírez, G.; Leigh, D. A.; Stephens, A. J. Angew. Chem. Int. Ed. 2015, 54, 61106150 .

${ }^{19}$ (a) Cantrill, S. J.; Chichak, K. S.; Peters, A. J.; Stoddart, J. F. Acc. Chem. Res. 2004, 38, 1-9. (b) Chichak, K. S.; Cantrill, S. J.; Pease, A. R.; Chiu, S.-H.; Cave, G. W. V.; Atwood, J. L.; Stoddart, J. F. Science 2004, 304, 1308-1312. (c) Chichak, K. S.; Cantrill, S. J.; Stoddart, J. F. Chem. Commun. 2005, 3391-3393. (d) Chichak, K. S.; Peters, A. J.; Cantrill, S. J.; Stoddart, J. F. J. Org. Chem. 2005, 70, 79567962. (e) Peters, A. J.; Chichak, K. S.; Cantrill, S. J.; Stoddart, J. F. Chem. Commun. 2005, 3394-3396. (f) Thorp-Greenwood, F. L.; Kulak, A. N.; Hardie, M. J. Nat. Chem. 2015, 7, 526-531.

${ }^{20}$ Adams, C., The Knot Book American Mathematical Society: 2004.

${ }^{21}$ (a) Chang, T.; Heiss, A. M.; Cantrill, S. J.; Fyfe, M. C. T.; Pease, A. R.; Rowan, S. J.; Stoddart, J. F.; Williams, D. J. Org. Lett. 2000, 2, 2943-2946. (b) Williams, A. R.; Northrop, B. H.; Chang, T.; Stoddart, J. F.; White, A. J. P.; Williams, D. J. Angew. Chem. Int. Ed. 2006, 45, 6665-6669.

22 (a) Williams, D. J.; Ashton, P. R.; Ballardini, R.; Balzani, V.; Credi, A.; Gandolfi, M. T.; Menzer, S.; Perez-Garcia, L.; Prodi, L. J. Am. Chem. Soc. 1995, 117, 11171-11197. (b) Sauvage, J.-P. Acc. Chem. Res. 1998, 31, 611-619. (c) Griffiths, K. E.; Stoddart, J. F. Pure Appl. Chem. 2008, 80, 485-506. (d) Stoddart, J. F.; Colquhoun, H. M. Tetrahedron 2008, 64, 8231-8263.

${ }^{23}$ (a) Aston, P. R.; Glink, P. T.; Stoddart, J. F.; Tasker, P. A.; White, A. J. P.; Williams, D. J. Chem. Eur. J. 1996, 2, 729-736. (b) Stoddart, J. F.; Ashton, P. R.; Chrystal, E. J. T.; Glink, P. T.; Menzer, S.; Schiavo, C.; Spencer, N.; Tasker, P. A.; White, A. J. P.; Williams, D. J. Chem. Eur. J. 1996, 2, 709-728. (c) Ashton, P. R.; Ballardini, R.; Balzani, V.; Gómez-López, M.; Lawrence, S. E.; Martínez-Díaz, M. V.; Montalti, M.; Piersanti, A.; Prodi, L.; Stoddart, J. F.; Williams, D. J. J. Am. Chem. Soc. 1997, 119, 1064110651.

24 (a) Prikhod'ko, A. I.; Sauvage, J.-P. J. Am. Chem. Soc. 2009, 131, 6794-6807. (b) Durola, F.; Sauvage, J.-P.; Wenger, O. S. Coord. Chem. Rev. 2010, 254, 1748-1759.

25 (a) Beer, P. D.; Lankshear, M. D. Acc. Chem. Res. 2007, 40, 657-668. (b) Miyagawa, N.; Watanabe, M.; Matsuyama, T.; Koyama, Y.; Moriuchi, T.; Hirao, T.; Furusho, Y.; Takata, T. Chem. Commun. 2010, 46, 1920-1922. (c) Yang, W.; Li, Y.; Liu, H.; Chi, L.; Li, Y. Small 2012, 8, 504-516.

${ }^{26}$ Lee, C.-F.; Leigh, D. A.; Pritchard, R. G.; Schultz, D.; Teat, S. J.; Timco, G. A.; Winpenny, R. E. P. Nature 2009, 458, 314-318.

${ }^{27}$ (a) Stoddart, J. F.; Braunschweig, A. B.; Ronconi, C. M.; Han, J.-Y.; Aricó, F.; Cantrill, S. J.; Khan, S. I.; White, A. J. P.; Williams, D. J. Eur. J. Org. Chem. 2006, 2006, 1857-1866. (b) Coutrot, F.; Busseron, E. Chem. Eur. J. 2009, 15, 5186-5190. (c) Olson, M. A.; Braunschweig, A. B.; Ikeda, T.; Fang, L.; Trabolsi, A.; Slawin, A. M. Z.; Khan, S. I.; Stoddart, J. F. Org. Biomol. Chem. 2009, 7, 4391-4405. (d) Stoddart, J. F.; Zhao, Y.-L.; Liu, L.; Zhang, W.; Sue, C.-H.; Li, Q.; Miljanić, O. Š.; Yaghi, O. M. Chem. Eur. J. 2009, 15, 13356-13380.

${ }^{28}$ (a) Sindelar, V.; Silvi, S.; Kaifer, A. E. Chem. Commun. 2006, 2185-2187. (b) Wenz, G.; Han, B.H.; Müller, A. Chem. Rev. 2006, 106, 782-817. (c) Ramalingam, V.; Urbach, A. R. Org. Lett. 2011, 13, 4898-4901. (d) Girek, T. J. Incl. Phenom. Macrocycl. Chem. 2012, 74, 1-21.

${ }^{29}$ Yoon, I.; Narita, M.; Shimizu, T.; Asakawa, M. J. Am. Chem. Soc. 2004, 126, 16740-16741.

${ }^{30}$ Chiu, C.-W.; Lai, C.-C.; Chiu, S.-H. J. Am. Chem. Soc. 2007, 129, 3500-3501. 
31 (a) Crowley, J. D.; Goldup, S. M.; Lee, A.-L.; Leigh, D. A.; McBurney, R. T. Chem. Soc. Rev. 2009, 38, 1530-1541. (b) Leigh, D. A.; Goldup, S. M.; Long, T.; McGonigal, P. R.; Symes, M. D.; Wu, J. J. Am. Chem. Soc. 2009, 131, 15924-15929. (c) Leigh, D. A.; Goldup, S. M.; McGonigal, P. R.; Ronaldson, V. E.; Slawin, A. M. Z. J. Am. Chem. Soc. 2009, 132, 315-320. (d) Crowley, J. D.; Goldup, S. M.; Gowans, N. D.; Leigh, D. A.; Ronaldson, V. E.; Slawin, A. M. Z. J. Am. Chem. Soc. 2010, 132, 62436248.

32 (a) Schneider, H.-J.; Rüdiger, V.; Solov'ev, V. P.; Kazachenko, V. P.; Raevsky, O. A. Eur. J. Org. Chem. 1999, 1999, 1847-1856. (b) Cantrill, S. J.; Fulton, D. A.; Heiss, A. M.; Pease, A. R.; Stoddart, J. F.; White, A. J. P.; Williams, D. J. Chem. Eur. J. 2000, 6, 2274-2287. (c) Clifford, T.; Abushamleh, A.; Busch, D. H. PNAS 2002, 99, 4830-4836. (d) Chiu, S.-H.; Liao, K.-S.; Su, J.-K. Tetrahedron Lett. 2004, 45, 213-216. (e) Angelo, J. P.; Sohlberg, K. J. Phys. Chem. A 2009, 113, 6724-6729. (f) Morin, J.-F.; Thibeault, D. Molecules 2010, 15, 3709-3730. (g) Schalley, C. A.; Jiang, W.; Schäfer, A.; Mohr, P. C. J. Am. Chem. Soc. 2010, 132, 2309-2320. (h) Späth, A.; König, B. Beilstein J. Org. Chem. 2010, 6, 32. (i) Payne, N. A.; Delmas, L. C.; McDowell, S. A. C.; Williams, A. R. Tetrahedron Lett. 2015, 56, 51755179.

33 (a) Kolb, H. C.; Finn, M. G.; Sharpless, K. B. Angew. Chem. Int. Ed. 2001, 40, 2004-2021. (b) Lahann, J., Click Chemistry for Biotechnology and Materials Science. John Wiley \& Sons Ltd: West Sussex, 2009; p 433.

${ }^{34}$ (a) Stoddart, J. F.; Ivan, A.; S, M. O.; R, D. W.; Kyosuke, I.; Takuma, Y.; Takashi, K. Bull. Chem. Soc. Jpn. 2007, 80, 13. (b) Hanni, K. D.; Leigh, D. A. Chem. Soc. Rev. 2010, 39, 1240-1251.

${ }^{35}$ Fahrenbach, A. C.; Bruns, C. J.; Li, H.; Trabolsi, A.; Coskun, A.; Stoddart, J. F. Acc. Chem. Res. 2014, 47, 482-493.

${ }^{36}$ Busseron, E.; Romuald, C.; Coutrot, F. Chem. Eur. J. 2010, 16, 10062-10073.

${ }^{37}$ Stoddart, J. F.; Anelli, P. L.; Spencer, N. J. Am. Chem. Soc. 1991, 113, 5131-5133.

38 Algunos ejemplos seleccionados de interruptores moleculares: (a) Balzani, V.; Stoddart, J. F.; Williams, D. J.; Ashton, P. R.; Ballardini, R.; Baxter, I.; Credi, A.; Fyfe, M. C. T.; Gandolfi, M. T.; Gómez-López, M.; Martínez-Díaz, M. V.; Piersanti, A.; Spencer, N.; Venturi, M.; White, A. J. P. J. Am. Chem. Soc. 1998, 120, 11932-11942. (b) Stoddart, J. F.; Kang, S.; Vignon, S. A.; Tseng, H.-R. Chem. Eur. J. 2004, 10, 2555-2564. (c) Garaudée, S.; Silvi, S.; Venturi, M.; Credi, A.; Flood, A. H.; Stoddart, J. F. Chem. Phys. Chem. 2005, 6, 2145-2152. (d) Loeb, S. J. Chem. Soc. Rev. 2007, 36, 226-235. (e) Coutrot, F.; Busseron, E. Chem. Eur. J. 2008, 14, 4784-4787. (f) Fioravanti, G.; Haraszkiewicz, N.; Kay, E. R.; Mendoza, S. M.; Bruno, C.; Marcaccio, M.; Wiering, P. G.; Paolucci, F.; Rudolf, P.; Brouwer, A. M.; Leigh, D. A. J. Am. Chem. Soc. 2008, 130, 2593-2601. (g) Hirose, K.; Shiba, Y.; Ishibashi, K.; Doi, Y.; Tobe, Y. Chem. Eur. J. 2008, 14, 3427-3433. (h) Bodis, P.; Panman, M. R.; Bakker, B. H.; MateoAlonso, A.; Prato, M.; Buma, W. J.; Brouwer, A. M.; Kay, E. R.; Leigh, D. A.; Woutersen, S. Acc. Chem. Res. 2009, 42, 1462-1469. (i) Durola, F.; Lux, J.; Sauvage, J.-P. Chem. Eur. J. 2009, 15, 4124-4134. (j) Lin, T.-C.; Lai, C.-C.; Chiu, S.-H. Org. Lett. 2009, 11, 613-616. (k) Phoa, K.; Neaton, J. B.; Subramanian, V. Nano Lett. 2009, 9, 3225-3229. (1) Coutrot, F.; Busseron, E. Chem. Eur. J. 2009, 15, 5186-5190. 37. (m) Chiu, S.-H.; Hsueh, S.-Y.; Lai, C.-C. Chem. Eur. J. 2010, 16, 2997-3000. (n) Sauvage, J.-P.; Collin, J.-P.; Heitz, V.; Durola, F.; Reviriego, F.; Trolez, Y. Angew. Chem. Int. Ed. 2010, 49, 10172-10175. (o) Tramontozzi, D. A.; Suhan, N. D.; Eichhorn, S. H.; Loeb, S. J. Chem. Eur. J. 2010, 16, 4466-4476. (p) Carlone, A.; Goldup, S. M.; Lebrasseur, N.; Leigh, D. A.; Wilson, A. J. Am. Chem. Soc. 2012, 134, 8321-8323. (q) Liu, L.; Liu, Y.; Liu, P.; Wu, J.; Guan, Y.; Hu, X.; Lin, C.; Yang, Y.; Sun, X.; Ma, J.; Wang, L. Chem. Sci. 2013, 4, 1701-1706. (r) Durola, F.; Heitz, V.; Reviriego, F.; Roche, C.; Sauvage, J.-P.; Sour, A.; Trolez, Y. Acc. Chem. Res. 2014, 47, 633-645. (s) Liu, P.; Li, W.; Liu, L.; Wang, L.; Ma, J. J. Phys. Chem. A 2014, 118, 9032-9044. (t) Olson, M. A. Nat. Chem. 2015, 7, 470-471.

39 "The Nobel Prize in Chemistry 2016". Nobelprize.org. Nobel Media AB 2014. Web. 9 Dec 2016. $<$ http://www.nobelprize.org/nobel_prizes/chemistry/laureates/2016/>

40 (a) Hess, H.; Vogel, V. Rev. Mol. Biotechnol. 2001, 82, 67-85. (b) Makita, Y.; Kihara, N.; Takata, T. J. Org. Chem. 2008, 73, 9245-9250. (c) Lüning, U.; Hesseler, B.; Zindler, M.; Herges, R. Eur. J. Org. Chem. 2014, 2014, 3885-3901. (d) Meng, Z.; Xiang, J.-F.; Chen, C.-F. J. Am. Chem. Soc. 2016, 138, $5652-5658$.

${ }^{41}$ (a) Koyama, Y.; Matsumura, T.; Yui, T.; Ishitani, O.; Takata, T. Org. Lett. 2013, 15, 4686-4689. (b) Nitta, T.; Hess, H. Cell. Mol. Bioeng. 2013, 6, 109-115. (c) Zhang, J.-N.; Li, H.; Zhou, W.; Yu, S.-L.; Qu, D.-H.; Tian, H. Chem. Eur. J. 2013, 19, 17192-17200. (d) Sun, N.; Xiao, X.; Li, W.; Jiang, J. Adv. Sci. 2015, 2, 1500082.

42 Balzani, V. Photochem. Photobiol. Sci. 2003, 2, 459-476.

${ }^{43}$ de Silva, A. P.; McClenaghan, N. D. Chem. Eur. J. 2004, 10, 574-586. 
${ }^{44}$ (a) Schalley, C. A.; Vögtle, F.; Beizai, K. Acc. Chem. Res. 2001, 34, 465-476. (b) Hsueh, S.-Y.; Kuo, C.-T.; Lu, T.-W.; Lai, C.-C.; Liu, Y.-H.; Hsu, H.-F.; Peng, S.-M.; Chen, C.-h.; Chiu, S.-H. Angew. Chem. Int. Ed. 2010, 49, 9170-9173. (c) Fahrenbach, A. C.; Warren, S. C.; Incorvati, J. T.; Avestro, A.-J.; Barnes, J. C.; Stoddart, J. F.; Grzybowski, B. A. Adv. Mater. 2013, 25, 331-348. (d) Fu, J.; Chen, T.; Wang, M.; Yang, N.; Li, S.; Wang, Y.; Liu, X. ACS Nano 2013, 7, 11397-11408. (e) Erbas-Cakmak, S.; Leigh, D. A.; McTernan, C. T.; Nussbaumer, A. L. Chem. Rev. 2015, 115, 10081-10206. (f) Kay, E. R.; Leigh, D. A. Angew. Chem. Int. Ed. 2015, 54, 10080-10088. (g) Xue, M.; Yang, Y.; Chi, X.; Yan, X.; Huang, F. Chem. Rev. 2015, 115, 7398-7501.

${ }^{45}$ Leigh, D. A.; Zerbetto, F.; Kay, E. R. Angew. Chem. Int. Ed. 2007, 46, 72-191.

${ }^{46}$ (a) Thordarson, P.; Bijsterveld, E. J. A.; Rowan, A. E.; Nolte, R. J. M. Nature 2003, 424, 4. (b) Takata, T.; Tachibana, Y.; Kihara, N. J. Am. Chem. Soc. 2004, 126, 3438-3439. (c) Miyagawa, N.; Watanabe, M.; Matsuyama, T.; Koyama, Y.; Moriuchi, T.; Hirao, T.; Furusho, Y.; Takata, T. Chem. Commun. 2010, 46, 1920-1922. (d) Leigh, D. A.; Lewandowski, B.; Bo, G. D.; Ward, J. W.; Papmeyer, M.; Kuschel, S.; Aldegunde, M. J.; Gramlich, P. M. E.; Heckmann, D.; Goldup, S. M.; D’Souza, D. M.; Fernandes, A. E. Science 2013, 339, 5. (e) Galli, M.; Lewis, J. E. M.; Goldup, S. M. Angew. Chem. Int. Ed. 2015, 54, 13545-13549. (f) Hoekman, S.; Kitching, M. O.; Leigh, D. A.; Papmeyer, M.; Roke, D. J. Am. Chem. Soc. 2015, 137, 7656-7659. (g) Goldup, S. M. Nat. Chem. 2016, 8, 404-406.

${ }^{47}$ Leigh, D. A.; Blanco, V.; Carlone, A.; Hänni, K. D.; Lewandowski, B. Angew. Chem. Int. Ed. 2012, 51, 5166-5169.

${ }^{48}$ (a) Leigh, D. A.; Blanco, V.; Marcos, V.; Morales-Serna, J. A.; Nussbaumer, A. L. J. Am. Chem. Soc. 2014, 136, 4905-4908. (b) Leigh, D. A.; Marcos, V.; Wilson, M. R. ACS Catal. 2014, 4, 4490-4497. (c) Blanco, V.; Leigh, D. A.; Marcos, V. Chem. Soc. Rev. 2015, 44, 5341-5370. (d) Leigh, D. A.; Beswick, J.; Blanco, V.; De Bo, G.; Lewandowska, U.; Lewandowski, B.; Mishiro, K. Chem. Sci. 2015, 6, 140-143. (e) Kwan, C.-S.; Chan, A. S. C.; Leung, K. C.-F. Org. Lett. 2016, 18, 976-979.

${ }^{49}$ McNaught, A. D.; Wilkinson, A. IUPAC. Compendium of Chemical Terminology, 2nd ed. (the "Gold Book"). Blackwell Scientific Publications: Oxford, 1997. 73.

${ }^{50}$ Mitscherlich, E. Ann. Pharma. 1834, 12, 311-314.

${ }^{51}$ Algunos ejemplos seleccionados: (a) Cope, A. C.; Siekman, R. W. J. Am. Chem. Soc. 1965, 87, 3272-3273. (b) Coe, B. J.; Blackburn, O. A. Organometallics 2011, 30, 2212-2222. (c) Coe, B. J.; Blackburn, O. A.; Helliwell, M. Organometallics 2011, 30, 4910-4923.

${ }_{52}^{52}$ Merino, E. Chem. Soc. Rev. 2011, 40, 3835-3853.

53 (a) Cembran, A.; Bernardi, F.; Garavelli, M.; Gagliardi, L.; Orlandi, G. J. Am. Chem. Soc. 2004, 126, 3234-3243. (b) Burdette, S. C.; Bandara, H. M. D. Chem. Soc. Rev. 2012, 41, 1809-1825. (c) Schweighauser, L.; Strauss, M. A.; Bellotto, S.; Wegner, H. A. Angew. Chem. Int. Ed. 2015, 54, 1343613439.

${ }^{54}$ (a) Giordani, S.; Natali, M. Chem. Soc. Rev. 2012, 41, 4010-4029. (b) Gostl, R.; Senf, A.; Hecht, S. Chem. Soc. Rev. 2014, 43, 1982-1996. (c) Bléger, D.; Hecht, S. Angew. Chem. Int. Ed. 2015, 54, 1133811349. (d) Qu, D.-H.; Wang, Q.-C.; Zhang, Q.-W.; Ma, X.; Tian, H. Chem. Rev. 2015, 115, 7543-7588.

${ }^{55}$ (a) Murakami, H.; Kawabuchi, A.; Kotoo, K.; Kunitake, M.; Nakashima, N. J. Am. Chem. Soc. 1997, 119, 7605-7606. (b) Balzani, V.; Credi, A.; Venturi, M. Chem. Soc. Rev. 2009, 38, 1542-1550. (c) Stoddart, J. F.; Coskun, A.; Friedman, D. C.; Li, H.; Patel, K.; Khatib, H. A. J. Am. Chem. Soc. 2009, 131, 2493-2495. (d) Credi, A.; Silvi, S.; Venturi, M. Chem. Commun. 2011, 47, 2483-2489. (e) Baroncini, M.; Silvi, S.; Venturi, M.; Credi, A. Angew. Chem. Int. Ed. 2012, 51, 4223-4226. (f) Stoddart, J. F.; Venturi, M.; Credi, A.; Avellini, T.; Li, H.; Coskun, A.; Barin, G.; Trabolsi, A.; Basuray, A. N.; Dey, S. K.; Silvi, S. Angew. Chem. Int. Ed. 2012, 51, 1611-1615. (g) Xue, M.; Yang, Y.; Chi, X.; Yan, X.; Huang, F. Chem. Rev. 2015, 115, 7398-7501.

${ }^{56}$ (a) Pearce, J. M. Futures 2002, 34, 663-674. (b) Luque, A.; Hegedus, S., Handbook of Photovoltaic Science and Engineering. John Wiley \& Sons: West Sussex, 2003. (c) Segura, J. L.; Martin, N.; Guldi, D. M. Chem. Soc. Rev. 2005, 34, 31-47. (d) Swanson, R. M. Science 2009, 324, 891-892. (e) Delgado, J. L.; Bouit, P.-A.; Filippone, S.; Herranz, M. A.; Martin, N. Chem. Commun. 2010, 46, 4853-4865. (f) Branker, K.; Pathak, M. J. M.; Pearce, J. M. Renew. Sust. Ener. Rev. 2011, 15, 4470-4482. (g) Pérez, E. M. Pure Appl. Chem. 2011, 83, 201-211. (h) Schneider, H.-J., Applications of Supramolecular Chemistry. CRC Press: 2012.

57 (a) Fitzer, E.; Kochling, K. H.; Boehm, H. P.; Marsh, H. Pure Appl. Chem. 1995, 67, 473. (b) Delhaes, P., Graphite and Precursors. CRC Press: 2000. 
58 (a) Akasaka, T.; Wudl, F.; Nagase, S., Chemistry of Nanocarbons. John Wiley \& Sons: West Sussex, 2010. (b) Krueger, A., Carbon Materials and Nanotechnology. Wiley-VCH Verlag: Weinheim, 2010. (c) Petrukhina, M. A.; Scott, L. T., Fragments of Fullerenes and Carbon Nanotubes: Designed Synthesis, Unusual Reactions, and Coordination Chemistry. John Wiley \& Sons: Hoboken, 2011. (d) Marcaccio, M.; Paolucci, F., Making and Exploiting Fullerenes, Graphene, and Carbon Nanotubes. Springer-Verlag: Heidelberg, 2014. (e) Georgakilas, V.; Perman, J. A.; Tucek, J.; Zboril, R. Chem. Rev. 2015, 115, 4744-4822.

59 (a) Iijima, S. Nature 1991, 354, 56-58. (b) Bethune, D. S.; Klang, C. H.; de Vries, M. S.; Gorman, G.; Savoy, R.; Vazquez, J.; Beyers, R. Nature 1993, 363, 605-607. (c) Iijima, S.; Ichihashi, T. Nature 1993, 363, 603-605. (d) Cataldo, S.; Salice, P.; Menna, E.; Pignataro, B. Energy Environ. Sci. 2012, 5, 5919-5940. (e) De Volder, M. F. L.; Tawfick, S. H.; Baughman, R. H.; Hart, A. J. Science 2013, 339, 535-539.

${ }^{60}$ (a) Novoselov, K. S.; Geim, A. K.; Morozov, S. V.; Jiang, D.; Zhang, Y.; Dubonos, S. V.; Grigorieva, I. V.; Firsov, A. A. Science 2004, 306, 666-669. (b) Cai, M.; Thorpe, D.; Adamson, D. H.; Schniepp, H. C. J. Mater. Chem. 2012, 22, 24992-25002. (c) Chang, D. W.; Choi, H.-J.; Filer, A.; Baek, J.-B. J. Mater. Chem. A 2014, 2, 12136-12149. (d) Fan, X.; Zhang, G.; Zhang, F. Chem. Soc. Rev. 2015, 44, 3023-3035.

61 (a) Siegel, J. S.; Wu, Y.-T., Polyarenes II. Springer International Publishing: London, 2014. (b) Siegel, J. S.; Wu, Y.-T., Polyarenes I. Springer: Heidelberg, 2014.

${ }^{62}$ Kroto, H. W.; Heath, J. R.; O'Brien, S. C.; Curl, R. F.; Smalley, R. E. Nature 1985, 318, 162-163.

63 (a) Curl, R. F. Angew. Chem. Int. Ed. 1997, 36, 1566-1576. (b) Kroto, H. Angew. Chem. Int. Ed. 1997, 36, 1578-1593. (c) Smalley, R. E. Angew. Chem. Int. Ed. 1997, 36, 1594-1601.

64 "The Nobel Prize in Chemistry 1996". Nobelprize.org. Nobel Media AB 2014. Web. 12 Sep 2016. $<$ http://www.nobelprize.org/nobel_prizes/chemistry/laureates/1996/>

65 (a) Taylor, R.; Walton, D. R. M. Nature 1993, 363, 685-693. (b) Dresselhaus, M. S.; Dresselhaus, G.; Eklund, P. C., Science of Fullerenes and Carbon Nanotubes. Academic Press: 1996. (c) Hirsch, A., Fullerenes and Related Structures. Springer: Heidelberg, 1999. (d) Guldi, D. M.; Martin, N., Fullerenes: From Synthesis to Optoelectronic Properties. Springer: Dordrecht, 2002. (e) Martin, N.; Nierengarten, J.F., Supramolecular Chemistry of Fullerenes and Carbon Nanotubes. Wiley-VCH Verlag: Weinheim, 2012.

${ }^{66}$ (a) Moss, G. P. Pure Appl. Chem. 1998. (b) Feng, X.; Pisula, W.; Müllen, K. Pure Appl. Chem. 2009, 81, 2203.

${ }^{67}$ (a) Boström, C.-E.; Gerde, P.; Hanberg, A.; Jernström, B.; Johansson, C.; Kyrklund, T.; Rannug, A.; Törnqvist, M.; Victorin, K.; Westerholm, R. Environ. Health. Persp. 2002, 110, 451-488. (b) Baird, W. M.; Hooven, L. A.; Mahadevan, B. Environ. Mol. Mutagen. 2005, 45, 106-114.

68 Algunos ejemplos seleccionados: (a) v. Bünau, G. Ber. Bunsenges. Phys. Chem. 1970, 74, 12941295. (b) Nagao, Y. Prog. Org. Coat. 1997, 31, 43-49. (c) Manandhar, E.; Wallace, K. J. Inorg. Chim. Acta 2012, 381, 15-43. (d) Mulla, K.; Shaik, H.; Thompson, D. W.; Zhao, Y. Org. Lett. 2013, 15, 45324535. (e) Nielsen, C. B.; Holliday, S.; Chen, H.-Y.; Cryer, S. J.; McCulloch, I. Acc. Chem. Res. 2015, 48, 2803-2812. (f) Ronson, T. K.; Roberts, D. A.; Black, S. P.; Nitschke, J. R. J. Am. Chem. Soc. 2015, 137, 14502-14512. (g) Peris, E. Chem. Commun. 2016, 52, 5777-5787.

${ }^{69}$ (a) Newman, M. S.; Lednicer, D. J. Am. Chem. Soc. 1956, 78, 4765-4770. (b) Barth, W. E.; Lawton, R. G. J. Am. Chem. Soc. 1966, 88, 380-381. (b) . (c) Lawton, R. G.; Barth, W. E. J. Am. Chem. Soc. 1971, 93, 1730-1745. (d) Yamamoto, K.; Harada, T.; Nakazaki, M.; Naka, T.; Kai, Y.; Harada, S.; Kasai, N. J. Am. Chem. Soc. 1983, 105, 7171-7172.

${ }^{70}$ (a) Scott, L. T.; Hashemi, M. M.; Bratcher, M. S. J. Am. Chem. Soc. 1992, 114, 1920-1921. (b) Seiders, T. J.; Baldridge, K. K.; Grube, G. H.; Siegel, J. S. J. Am. Chem. Soc. 2001, 123, 517-525. (c) Nishida, S.; Morita, Y.; Ueda, A.; Kobayashi, T.; Fukui, K.; Ogasawara, K.; Sato, K.; Takui, T.; Nakasuji, K. J. Am. Chem. Soc. 2008, 130, 14954-14955. (d) Sanyal, S.; Manna, A. K.; Pati, S. K. Chem. Phys. Chem. 2014, 15, 885-893. (e) Siegel, J. S.; Wu, Y.-T., Synthesis, Structures, and Physical Properties of Aromatic Molecular-Bowl Hydrocarbons. In Polyarenes I, Springer: Heidelberg, 2014; pp 63-120. 
${ }^{71}$ Algunos ejemplos seleccionados: (a) Wu, Y.-T.; Siegel, J. S. Chem. Rev. 2006, 106, 4843-4867. (b) Sygula, A. Eur. J. Org. Chem. 2011, 2011, 1611-1625. (c) Lentz, D.; Topolinski, B.; Schmidt, B. M.; Kathan, M.; Troyanov, S. I. Chem. Commun. 2012, 48, 6298-6300. (d) Stuparu, M. C. Tetrahedron 2012, 68, 3527-3531. (e) Wu, Y.-L.; Stuparu, M. C.; Boudon, C.; Gisselbrecht, J.-P.; Schweizer, W. B.; Baldridge, K. K.; Siegel, J. S.; Diederich, F. J. Org. Chem. 2012, 77, 11014-11026. (f) Furrer, F.; Linden, A.; Stuparu, M. C. Chem. Eur. J. 2013, 19, 13199-13206. (g) Lentz, D.; Topolinski, B.; Schmidt, B. M.; Schwagerus, S.; Kathan, M. Eur. J. Inorg. Chem. 2014, 2014, 5391-5405. (h) Mattarella, M.; Berstis, L.; Baldridge, K. K.; Siegel, J. S. Bioconjug. Chem. 2014, 25, 115-128. (i) Wu, D.; Shao, T.; Men, J.; Chen, X.; Gao, G. Dalton Trans. 2014, 43, 1753-1761. (j) Da Ros, S.; Linden, A.; Baldridge, K. K.; Siegel, J. S. Org. Chem. Front. 2015, 2, 626-633. (k) George, S. R. D.; Frith, T. D. H.; Thomas, D. S.; Harper, J. B. Org. Biomol. Chem. 2015, 13, 9035-9041. (1) San, L. K.; Clikeman, T. T.; Dubceac, C.; Popov, A. A.; Chen, Y.-S.; Petrukhina, M. A.; Strauss, S. H.; Boltalina, O. V. Chem. Eur. J. 2015, 21, 9488-9492. (m) Li, X.; Kang, F.; Inagaki, M. Small 2016, 12, 3206-3223. (n) Rajeshkumar, V.; Lee, Y. T.; Stuparu, M. C. Eur. J. Org. Chem. 2016, 2016, 36-40. (o) Rajeshkumar, V.; Marc, C.; Fichou, D.; Stuparu, M. C. Synlett 2016.

${ }^{72}$ Algunos ejemplos seleccionados: (a) Seiders, T. J.; Baldridge, K. K.; O'Connor, J. M.; Siegel, J. S. J. Am. Chem. Soc. 1997, 119, 4781-4782. (b) Angelici, R. J.; Sygula, A.; Alvarez, C. M.; Sygula, R.; Rabideau, P. W. Organometallics 2003, 22, 624-626. (c) Angelici, R. J.; Sygula, A.; Vecchi, P. A.; Alvarez, C. M.; Ellern, A.; Sygula, R.; Rabideau, P. W. Organometallics 2005, 24, 4543-4552. (d) Sharp, P. R.; Lee, H. B. Organometallics 2005, 24, 4875-4877. (e) Yamada, M.; Tashiro, S.; Miyake, R.; Shionoya, M. Dalton Trans. 2013, 42, 3300-3303.

${ }^{73}$ (a) Barth, W. E.; Lawton, R. G. J. Am. Chem. Soc. 1966, 88, 380-381. (b) Lawton, R. G.; Barth, W. E. J. Am. Chem. Soc. 1971, 93, 1730-1745.

${ }^{74}$ Tsefrikas, V. M.; Scott, L. T. Chem. Rev. 2006, 106, 4868-4884.

${ }^{75}$ (a) Siegel, J. S.; Seiders, T. J.; Elliott, E. L.; Grube, G. H. J. Am. Chem. Soc. 1999, 121, 7804-7813.

(b) Sygula, A.; Rabideau, P. W. J. Am. Chem. Soc. 2000, 122, 6323-6324. (c) Xu, G.; Sygula, A.; Marcinow, Z.; Rabideau, P. W. Tetrahedron Lett. 2000, 41, 9931-9934.

${ }^{76}$ Butterfield, A. M.; Gilomen, B.; Siegel, J. S. Org. Process Res. Dev. 2012.

${ }^{77}$ Dawe, L. N.; AlHujran, T. A.; Tran, H.-A.; Mercer, J. I.; Jackson, E. A.; Scott, L. T.; Georghiou, P. E. Chem. Commun. 2012, 48, 5563-5565.

${ }^{78}$ (a) Mizyed, S.; Georghiou, P. E.; Bancu, M.; Cuadra, B.; Rai, A. K.; Cheng, P.; Scott, L. T. J. Am. Chem. Soc. 2001, 123, 12770-12774. (b) Bancu, M.; Rai, A. K.; Cheng, P.; Gilardi, R. D.; Scott, L. T. Synlett 2004, 2004, 173-176. (c) Georghiou, P. E.; Tran, A. H.; Mizyed, S.; Bancu, M.; Scott, L. T. J. Org. Chem. 2005, 70, 6158-6163.

${ }^{79}$ Sygula, A.; Fronczek, F. R.; Sygula, R.; Rabideau, P. W.; Olmstead, M. M. J. Am. Chem. Soc. 2007, 129, 3842-3843.

80 Álvarez, C. M.; García-Escudero, L. A.; García-Rodríguez, R.; Martín-Álvarez, J. M.; Miguel, D.; Rayón, V. M. Dalton Trans. 2014, 43, 15693-15696.

${ }^{81}$ (a) Álvarez, C. M.; Aullón, G.; Barbero, H.; García-Escudero, L. A.; Martínez-Pérez, C.; MartínÁlvarez, J. M.; Miguel, D. Org. Lett. 2015, 17, 2578-2581. (b) Álvarez, C. M.; Barbero, H.; Ferrero, S. J. Vis. Exp. 2016, e53954.

${ }^{82}$ Stuparu, M. C. Angew. Chem. Int. Ed. 2013, 52, 7786-7790.

83 (a) Yanney, M.; Sygula, A. Tetrahedron Lett. 2013, 54, 2604-2607. (b) Abeyratne Kuragama, P. L.; Fronczek, F. R.; Sygula, A. Org. Lett. 2015, 17, 5292-5295. (c) Yanney, M.; Fronczek, F. R.; Sygula, A. Angew. Chem. Int. Ed. 2015, 54, 11153-11156. (d) Kumarasinghe, K. G. U. R.; Fronczek, F. R.; Valle, H. U.; Sygula, A. Org. Lett. 2016, 18, 3054-3057. (e) Sygula, A. Synlett 2016, 27, 2070-2080.

${ }^{84}$ (a) Yuan, K.; Guo, Y.-J.; Zhao, X. Phys. Chem. Chem. Phys. 2014, 16, 27053-27064. (b) Yuan, K.; Dang, J.-S.; Guo, Y.-J.; Zhao, X. J. Comput. Chem. 2015, 36, 518-528.

85 (a) Moss, G. P. Pure Appl. Chem. 1987, 59, 779-832. (b) Moss, G. P. Eur. J. Biochem. 1988, 178, 277-328.

${ }^{86}$ Becker, E. D.; Bradley, R. B.; Watson, C. J. J. Am. Chem. Soc. 1961, 83, 3743-3748.

${ }^{87}$ (a) Gouterman, M. J. Chem. Phys. 1959, 30, 1139-1161. (b) Gouterman, M. J. Mol. Spectrosc. 1961, 6, 138-163. (c) Gouterman, M.; Wagnière, G. H.; Snyder, L. C. J. Mol. Spectrosc. 1963, 11, 108127.

${ }^{88}$ Kuster, W. Z. Physiol. Chem., 1912, 82, 463.

${ }^{89}$ Fischer, H.; Zeile, K. Justus Liebigs Ann. Chem. 1929, 468, 98-116.

90 "The Nobel Prize in Chemistry 1930". Nobelprize.org. Nobel Media AB 2014. Web. 14 Sep 2016. $<$ http://www.nobelprize.org/nobel_prizes/chemistry/laureates/1930/> 
91 Smith, K. M., Strategies for the Synthesis of Octaalkylporphyrin Systems. In The Porphyrin Handbook, Vol. 1, Academic Press: San Diego, 2000; pp. 2-40. (b) Lindsey, J. S. Synthesis of mesoSubstituted Porphyrins, in The Porphyrin Handbook, Vol. 1, Academic Press: San Diego, 2000, pp. 4680 .

92 (a) Chou, J.-H.; Kosal, M. E.; Nalwa, H. S.; Rakow, N. A.; Suslick, K. S., Applications of Porphyrins and Metalloporphyrins to Materias Chemistry. In The Porphyrin Handbook Vol 6, Academic Press: San Diego, 2000; Vol. 6, pp 42-131. (b) Kadish, K. M.; Smith, K. M.; Guilard, R., Handbook of Porphyrin Science, Vol. 4: Phototherapy, Radioimmunotherapy and Imaging. World Scientific Publishing: Singapore, 2010. (c) Kadish, K. M.; Smith, K. M.; Guilard, R., Handbook of Porphyrin Science, Vol.10: Catalysis and Bio-Inspired Systems - Part I. World Scientific Publishing: Singapore, 2010. (d) Kadish, K. M.; Smith, K. M.; Guilard, R., Handbook of Porphyrin Science, Vol.11: Catalysis and Bio-Inspired Systems - Part 2. World Scientific Publishing: Singapore, 2010. (e) Kadish, K. M.; Smith, K. M.; Guilard, R., Handbook of Porphyrin Science, Vol.12: Applications. World Scientific Publishing: Singapore, 2010. (f) Kadish, K. M.; Smith, K. M.; Guilard, R., Handbook of Porphyrin Science, Vol.18: Applications and Materials. World Scientific Publishing: Singapore, 2010. (g) Kadish, K. M.; Smith, K. M.; Guilard, R., Handbook of Porphyrin Science, Vol.21: Catalysis. World Scientific Publishing: Singapore, 2010. (h) Burda, W. N.; Shaw, L. N.; Shepherd, M., Porphyrins: Properties and Applications. In Handbook of Porphyrins: Chemistry, Properties and Applications, Nova Science Publishers: 2012; pp 429-438.

93 (a) D'Souza, F.; Ito, O. Chem. Commun. 2009, 4913-4928. (b) de la Torre, G.; Bottari, G.; Sekita, M.; Hausmann, A.; Guldi, D. M.; Torres, T. Chem. Soc. Rev. 2013, 42, 8049-8105.

94 (a) Boyd, P. D. W.; Hodgson, M. C.; Rickard, C. E. F.; Oliver, A. G.; Chaker, L.; Brothers, P. J.; Bolskar, R. D.; Tham, F. S.; Reed, C. A. J. Am. Chem. Soc. 1999, 121, 10487-10495. (b) Evans, D. R.; Fackler, N. L. P.; Xie, Z.; Rickard, C. E. F.; Boyd, P. D. W.; Reed, C. A. J. Am. Chem. Soc. 1999, 121, 8466-8474. (c) Olmstead, M. M.; Costa, D. A.; Maitra, K.; Noll, B. C.; Phillips, S. L.; Van Calcar, P. M.; Balch, A. L. J. Am. Chem. Soc. 1999, 121, 7090-7097. (d) Ishii, T.; Aizawa, N.; Yamashita, M.; Matsuzaka, H.; Kodama, T.; Kikuchi, K.; Ikemoto, I.; Iwasa, Y. J. Chem. Soc., Dalton Trans. 2000, 44074412. (e) Song, X.; Chan, K.-S. J. Chin. Chem. Soc. 2009, 56, 667-670. (f) Obondi, C.; Rodriguez, A. A. Adv. Phys. Chem. 2010, 2010, 9.

95 Saegusa, Y.; Ishizuka, T.; Kojima, T.; Mori, S.; Kawano, M.; Kojima, T. Chem. Eur. J. 2015, 21, $5302-5306$

96 Tashiro, K.; Aida, T.; Zheng, J.-Y.; Kinbara, K.; Saigo, K.; Sakamoto, S.; Yamaguchi, K. J. Am. Chem. Soc. 1999, 121, 9477-9478.

${ }^{97}$ (a) Boyd, P. D. W.; Reed, C. A. Acc. Chem. Res. 2005, 38, 235-242. (b) Tashiro, K.; Aida, T. Chem. Soc. Rev. 2007, 36, 189-197. (c) Li, Z.-T.; Hou, J.-L.; Li, C. Acc. Chem. Res. 2008, 41, 1343-1353. (d) Canevet, D.; Pérez, E. M.; Martín, N. Angew. Chem. Int. Ed. 2011, 50, 9248-9259. (e) Garcia-Simon, C.; Costas, M.; Ribas, X. Chem. Soc. Rev. 2016, 45, 40-62.

98 (a) Boedigheimer, H.; Ferrence, G. M.; Lash, T. D. J. Org. Chem. 2010, 75, 2518-2527. (b) Ota, K.; Tanaka, T.; Osuka, A. Org. Lett. 2014, 16, $2974-2977$.

${ }^{99}$ Denis, P. A.; Yanney, M. RSC Adv. 2016, 6, 50978-50984.

100 (a) Armarego, W. L. F.; Chai, C. L. L., Purification of Laboratory Chemicals. 5th ed.; Elsevier: Bodmin, 2003. (b) Williams, D. B. G.; Lawton, M. J. Org. Chem. 2010, 75, 8351-8354.

101 (a) Anderson, G. K.; Lin, M., Bis(benzonitrile)dichloro complexes of palladium and platinum. In Reagents for Transition Metal Complex and Organometallic Syntheses, Inorganic Syntheses, Volume 28, Angelici, R. J., Ed. John Wiley \& Sons: New York, 1990; Vol. 28, pp 60-63. (b) Kubas, G. J., Tetrakis(Acetonitrile)Copper(1+) Hexafluorophosphate(1-). In Reagents for Transition Metal Complex and Organometallic Syntheses, Inorganic Syntheses, Volume 28, Angelici, R. J., Ed. John Wiley \& Sons.: New York, 1990; Vol. 28, pp 68-70. (c) Nataro, C.; Fosbenner, S. M. J. Chem. Educ. 2009, 86, 1412.

102 (a) Kaerner, A.; Rabenstein, D. L. Magn. Reson. Chem. 1998, 36, 601-607. (b) Gaillet, C.; Lequart, C.; Debeire, P.; Nuzillard, J.-M. J. Magn. Reson. 1999, 139, 454-459. (c) Crouch, R.; Boyer, R. D.; Johnson, R.; Krishnamurthy, K. Magn. Reson. Chem. 2004, 42, 301-307. (d) Hu, H.; Krishnamurthy, K. Magn. Reson. Chem. 2008, 46, 683-689.

${ }^{103}$ La IUPAC desaconseja el uso del término "extinción": Braslavsky, S. E. Pure Appl. Chem. 2007, 79, 293-465.

${ }^{104}$ CrysAlisPro-Data collection and integration software, version 1.171.37.35. Agilent Technologies UK Ltd, Oxford, UK, 2011.

105 Sheldrick, G. M., Acta Cryst. 2008, A64, 112-122.

106 Dolomanov, O. V.; Bourhis, L. J.; Gildea, R. J.; Howard, J. A. K.; Puschmann, H. 'OLEX2: A complete structure solution, refinement and analysis program.' J. Appl. Cryst. 2009, 42, 339-341. 
107 (a) Bruno, I. J.; Cole, J. C.; Edgington, P. R.; Kessler, M.; Macrae, C. F.; McCabe, P.; Pearson, J.; Taylor, R. Acta Crystallogr., Sect. B 2002, 58, 389-397. (b) Macrae, C. F.; Edgington, P. R.; McCabe, P.; Pidcock, E.; Shields, G. P.; Taylor, R.; Towler, M.; van de Streek, J. J. Appl. Crystallogr. 2006, 39, 453457. (c) Macrae, C. F.; Bruno, I. J.; Chisholm, J. A.; Edgington, P. R.; McCabe, P.; Pidcock, E.; Rodriguez-Monge, L.; Taylor, R.; van de Streek, J.; Wood, P. A. J. Appl. Crystallogr. 2008, 41, 466-470.

${ }^{108}$ Gaussian 09, Revision D.01, Frisch, M. J.; Trucks, G. W.; Schlegel, H. B.; Scuseria, G. E.; Robb, M. A.; Cheeseman, J. R.; Scalmani, G.; Barone, V.; Mennucci, B.; Petersson, G. A.; Nakatsuji, H.; Caricato, M.; Li, X.; Hratchian, H. P.; Izmaylov, A. F.; Bloino, J.; Zheng, G.; Sonnenberg, J. L.; Hada, M.; Ehara, M.; Toyota, K.; Fukuda, R.; Hasegawa, J.; Ishida, M.; Nakajima, T.; Honda, Y.; Kitao, O.; Nakai, H.; Vreven, T.; Montgomery, J. A., Jr.; Peralta, J. E.; Ogliaro, F.; Bearpark, M.; Heyd, J. J.; Brothers, E.; Kudin, K. N.; Staroverov, V. N.; Kobayashi, R.; Normand, J.; Raghavachari, K.; Rendell, A.; Burant, J. C.; Iyengar, S. S.; Tomasi, J.; Cossi, M.; Rega, N.; Millam, M. J.; Klene, M.; Knox, J. E.; Cross, J. B.; Bakken, V.; Adamo, C.; Jaramillo, J.; Gomperts, R.; Stratmann, R. E.; Yazyev, O.; Austin, A. J.; Cammi, R.; Pomelli, C.; Ochterski, J. W.; Martin, R. L.; Morokuma, K.; Zakrzewski, V. G.; Voth, G. A.; Salvador, P.; Dannenberg, J. J.; Dapprich, S.; Daniels, A. D.; Farkas, Ö.; Foresman, J. B.; Ortiz, J. V.; Cioslowski, J.; Fox, D. J. Gaussian, Inc., Wallingford CT, 2009.

109 Gauss View, Version 5, Dennington, R.; Keith, T.; Millam, J. Semichem Inc., Shawnee Mission, KS, 2009.

${ }^{110}$ Hanwell, M. D.; Curtis, D. E.; Lonie, D. C.; Vandermeersch, T.; Zurek, E.; Hutchison, G. R. J. Cheminform. 2012, 4, 1-17.

${ }^{111}$ Humphrey, W., Dalke, A., Schulten, K. J. Molec. Graphics, 1996, 14, 33-38.

112 Persistence of Vision Pty. Ltd. (2004). Persistence of Vision (TM) Raytracer. Persistence of Vision Pty. Ltd., Williamstown, Victoria, Australia. http://www.povray.org/

113 Grimme, S. J. Comp. Chem. 2006, 27, 1787-1799.

114 (a) Ditchfield, R.; Hehre, W. J.; Pople, J. A. J. Chem. Phys. 1971, 54, 724-728. (b) Hehre, W. J.; Ditchfield, R.; Pople, J. A. J. Chem. Phys. 1972, 56, 2257-2261. (b) Hariharan, P. C.; Pople, J. A. Theor. Chem. Acc. 1973, 28, 213-222. (c) Francl, M. M.; Pietro, W. J.; Hehre, W. J.; Binkley, J. S.; DeFrees, D. J.; Pople, J.A.; Gordon, M. S. J. Chem. Phys., 1982, 77, 3654-3665. (d) Binning Jr., R. C.; Curtiss, L. A. J. Comp. Chem. 1990, 11, 1206-1216. (e) Blaudeau, J.-P.; McGrath, M. P.; Curtiss, L. A.; Radom, L. J. Chem. Phys. 1997, 107, 5016-5021. (f) Rassolov, V. A.; Pople, J. A.; Ratner, M. A.; Windus, T. L. J. Chem. Phys. 1998, 109, 1223-1229. (g) Rassolov, V. A.; Ratner, M. A.; Pople, J. A.; Redfern, P. C.; Curtiss, L. A. J. Comp. Chem. 2001, 22, 976-984. 301 .

${ }^{115}$ Clark, T.; Chandrasekhar, J.; Spitznagel, G. W.; Schleyer, P. V. R. J. Comp. Chem. 1983, 4, 294-

116 (a) Boys, S. F.; Bernardi, F. Mol. Phys. 1970, 19, 553-566. (b) Simon S.; Duran M.; Dannenberg, J. J. J. Chem. Phys. 1996, 105, 11024-11031.

117 Tomasi, J.; Mennucci, B.; Cammi, R. Chem. Rev. 2005, 105, 2999-3093.

118 (a) London, F. J. Phys. Radium 1937, 8, 397-409. (b) McWeeny, R. Phys. Rev. 1962, 126, 1028. (c) Ditchfield, R. Mol. Phys. 1974, 27, 789-807. (d) Wolinski, K.; Hilton, J. F.; Pulay, P. J. Am. Chem. Soc. 1990, 112, 8251-60. (e) Cheeseman, J. R.; Trucks, G. W.; Keith, T. A.; Frisch, M. J. J. Chem. Phys. 1996, 104, 5497-509.

119 Kutzelnigg, W.; Fleischer, U.; Schindler, M., The IGLO-Method: Ab-initio Calculation and Interpretation of NMR Chemical Shifts and Magnetic Susceptibilities. In Deuterium and Shift Calculation, Springer Berlin Heidelberg: Berlin, Heidelberg, 1991; pp 165-262.

${ }^{120}$ (a) Feller, D. J. Comp. Chem. 1996, 17, 1571-1586. (b) Schuchardt, K.L.; Didier, B.T.; Elsethagen, T.; Sun, L.; Gurumoorthi, V.; Chase, J.; Li, J.; Windus, T.L. J. Chem. Inf. Model. 2007, 47, 1045-1052.

121 (a) Yang, W.; Johnson, E. R.; Keinan, S.; Mori-Sánchez, P.; Contreras-García, J.; Cohen, A. J. J. Am. Chem. Soc. 2010, 132, 6498-6506. (b) Yang, W.; Contreras-García, J.; Johnson, E. R.; Keinan, S.; Chaudret, R.; Piquemal, J.-P.; Beratan, D. N. J. Chem. Theory Comput. 2011, 7, 625-632.

${ }_{122}$ Williams, T.; Kelley, C. and R Development Core Team (2011). Gnuplot 4.5: an interactive plotting program. URL http://gnuplot.info.

123 (a) Stewart, J. J. P. J. Comput. Chem. 1989, 10, 209-220. (b) Stewart, J. J. P. J. Comput. Chem. 1989, 10, 221-264.

124 Job, P. Ann. Chim. 1928, 9, 113-203.

${ }^{125}$ Olson, E. J.; Bühlmann, P. J. Org. Chem. 2011, 76, 8406-8412.

126 (a) Fielding, L. Tetrahedron 2000, 56, 6151-6170. (b) Hirose, K. J. Incl. Phenom. Macrocycl. Chem. 2001, 39, 193-209. (c) Thordarson, P. Chem. Soc. Rev. 2011, 40, 1305-1323.

${ }^{127}$ Evans, N. H.; Beer, P. D. Angew. Chem. Int. Ed. 2014, 53, 11716-11754. 
${ }^{128}$ El motivo por el cual se escogió el tiol fluorado representtado en el Esquema 16 se debe a que es un compuesto comercial inodoro, no volátil y tiene un espectro ${ }^{1} \mathrm{H}-\mathrm{RMN}$ muy sencillo.

${ }^{129}$ (a) Mitsunobu, O.; Wada, M.; Sano, T. J. Am. Chem. Soc. 1972, 94, 679-680. (b) Swamy, K. C. K.; Kumar, N. N. B.; Balaraman, E.; Kumar, K. V. P. P. Chem. Rev. 2009, 109, 2551-2651.

${ }^{130}$ Es necesario hacer notar que obtener los estados de transición en este caso es una tarea muy compleja teniendo en cuenta que el proceso va a depender de múltiples coordenadas de reacción y se necesitaría mucho esfuerzo computacional (incluyendo el uso de un nivel de teoría superior y, probablemente, un método dinámico) para obtener unos resultados cuyo interés está alejado de los objetivos de este trabajo.

131 (a) Sandanayaka, A. S. D.; Chitta, R.; Subbaiyan, N. K.; D’Souza, L.; Ito, O.; D’Souza, F. J. Phys. Chem. C 2009, 113, 13425-13432. (b) Schopf, E.; Broyer, R.; Tao, L.; Chen, Y.; Maynard, H. D. Chem. Commun. 2009, 4818-4820. (c) Bahun, G. J.; Adronov, A. J. Polym. Sci., Part A: Polym. Chem. 2010, 48, 1016-1028. (d) Komatsu, N.; Liu, G.; Wang, F.; Chaunchaiyakul, S.; Saito, Y.; Bauri, A. K.; Kimura, T.; Kuwahara, Y. J. Am. Chem. Soc. 2013, 135, 4805-4814. (e) Lopez-Moreno, A.; Perez, E. M. Chem. Commun. 2015, 51, 5421-5424.

132 (a) Muck-Lichtenfeld, C.; Grimme, S.; Kobryn, L.; Sygula, A. Phys. Chem. Chem. Phys. 2010, 12, 7091-7097. (b) Denis, P. A. RSC Adv. 2013, 3, 25296-25305. (c) Josa, D.; Rodríguez-Otero, J.; Cabaleiro-Lago, E. M.; Santos, L. A.; Ramalho, T. C. J. Phys. Chem. A 2014, 118, 9521-9528.

${ }^{133}$ Grimme, S. Chem. Eur. J. 2012, 18, 9955-9964.

134 (a) Crossley, M. J.; Field, L. D.; Forster, A. J.; Harding, M. M.; Sternhell, S. J. Am. Chem. Soc. 1987, 109, 341-348. (b) Meunier, B.; R. Song; A. Robert; Bernadou, J. Analusis 1999, 27, 4. (c) Silva, A. M. S.; Tomé, A. C.; Alkorta, I.; Elguerob, J. J. Porphyrins Phthalocyanines 2011, 15, 28.

${ }^{135}$ Muchos estudios demuestran que es posible aislar cada atropisómero individualmente siempre y cuando haya un sustituyente en posición orto en el grupo fenilénico en meso de manera que la isomerización sea mucho más lenta. Para algunos ejemplos, ver la referencia 134a y: (a) Rose, E.; Cardon-Pilotaz, A.; Quelquejeu, M.; Bernard, N.; Kossanyi, A.; Desmazieres, B. J. Org. Chem. 1995, 60, 3919-3920. (b) Spasojević, I.; Menzeleev, R.; White, P. S.; Fridovich, I. Inorg. Chem. 2002, 41, 58745881. (c) Hunter, C. A.; Misuraca, M. C.; Turega, S. M. J. Am. Chem. Soc. 2011, 133, 582-594. (d) Sun, H.; Guo, K.; Gan, H.; Li, X.; Hunter, C. A. Org. Biomol. Chem. 2015, 13, 8053-8066.

136 (a) Munro, O. Q.; Bradley, J. C.; Hancock, R. D.; Marques, H. M.; Marsicano, F.; Wade, P. W. J. Am. Chem. Soc. 1992, 114, 7218-7230. (b) Medforth, C. J.; Haddad, R. E.; Muzzi, C. M.; Dooley, N. R.; Jaquinod, L.; Shyr, D. C.; Nurco, D. J.; Olmstead, M. M.; Smith, K. M.; Ma, J.-G.; Shelnutt, J. A. Inorg. Chem. 2003, 42, 2227-2241. (c) Park, S. H.; Kim, S. J.; Kim, J. D.; Park, S.-B.; Huh, D. S.; Shim, Y. K.; Choe, S. J. Bull. Korean Chem. Soc. 2008, 29, 1141-1148.

${ }^{137}$ Esta estructura calculada no se encuentra en el artículo publicado porque se obtuvo mucho después de su aceptación. 
